\title{
Les amphores grecques dans le nord de l'Italie
}

Échanges commerciaux entre les Apennins et les Alpes aux époques archaïque et classique

\section{Federica Sacchetti}

DOI : 10.4000/books.pccj. 1244

Éditeur : Publications du Centre Camille Jullian, Éditions Errance

Lieu d'édition : Aix-en-Provence

Année d'édition : 2012

Date de mise en ligne : 13 février 2020

Collection : Bibliothèque d'archéologie méditerranéenne et africaine

ISBN électronique : 9782957155798

\section{Sbooks}

http://books.openedition.org

\section{Édition imprimée}

Date de publication : 15 mars 2013

ISBN : 9782877724869

Nombre de pages : 287

\section{Référence électronique}

SACCHETTI, Federica. Les amphores grecques dans le nord de l'Italie : Échanges commerciaux entre les Apennins et les Alpes aux époques archaïque et classique. Nouvelle édition [en ligne]. Aix-en-Provence : Publications du Centre Camille Jullian, 2012 (généré le 21 décembre 2020). Disponible sur Internet <http://books.openedition.org/pccj/1244>. ISBN : 9782957155798. DOl : https://doi.org/10.4000/ books.pccj.1244.

(C) Publications du Centre Camille Jullian, 2012

Conditions d'utilisation

http://www.openedition.org/6540 


\section{Bibliothèque d'Archéologie Méditerranéenne et Africaine 10}


Dans la lignée des anciens Travaux du Centre Camille Jullian, la Bibliothèque d'Archéologie Méditerranéenne et Africaine (BiAMA) regroupe des travaux (monographies, actes de colloques, ouvrages collectifs) en relation avec les programmes scientifiques du Centre Camille Jullian, sur l'histoire et l'archéologie de la Gaule méridionale, de l'Afrique du Nord et du bassin méditerranéen. La BiAMA peut comprendre des sous-séries, comme la collection Études massaliètes (EtMassa).

Responsable légal :

Marie-Brigitte Carre, Directrice du CCJ

Directeur de la publication :

Henri Tréziny

Comité de pilotage :

Marie-Brigitte Carre, Xavier Delestre, Henri Tréziny

Conception graphique :

Véronique Gémonet

Mise en page de ce volume :

V. Gémonet, Lucien Rivet, Sylvie Saulnier

\section{Comité de lecture :}

Ph.Borgard(CCJ,CNRS),S.Bouffier(CCJ,Aix-MarseilleUniversité), J.-P. Brun (Collège de France), M-Br. Carre (CCJ, CNRS), X. Delestre (CCJ, DRAC PACA), D. Garcia (CCJ, AMU, Inst. Univ. de France), M. Griesheimer (CCJ, AMU), A. Hermary (CCJ, AMU), Ph. Jockey (CCJ, AMU), M. Lombardo (Professeur à l'Université de Lecce), T. S. Loseby (Professeur à l'Université de Sheffield), J.-M. Mignon (Service archéologique départemental du Vaucluse), P. Pomey (CCJ, CNRS), L. Rivet (CCJ, CNRS), J. Sanmarti (Professeur à l'Université de Barcelone), J.-Chr. Sourisseau (CCJ, AMU), H. Tréziny (CCJ, CNRS), C. Virlouvet (École française de Rome), E. Voutiras (Professeur à l'Université de Thessalonique).

Conseiller scientifique pour ce volume : Jean-Christophe Sourisseau.

(C) 2012 pour tous pays,

Édition Errance, éditeur du groupe Actes Sud,

BP 9003813633 Arles Cédex

Tel. 04-88-65-92-05 / 06-99-30-70-87

Fax : 0143293488

Courriel : contact@editions-errance.fr

http://www.libairie-epona.fr

Centre Camille Jullian

Maison Méditerranéenne des Sciences de l'Homme

5 rue du Château de l'Horloge. BP 647, 13094 Aix-en-Provence Cedex 2

ISBN : 978-2-87772-486-9

ISSN : 2101-2849

Illustrations de couverture: dessin d'amphore grecque occidentale de forme « $1 \alpha$ » et photo d'un fond d'amphore d'Adria du même type avec graffito (ㅇ F. Sacchetti) ; conception graphique St. Renaut (CNRS/LAMPEA) Illustration 4ème de couverture : Adria, nécropole de Cà Cima, Tombe 11 (ㅇ Soprintendenza per i Beni Archeologici del Veneto).

\section{Publications du Centre Camille Jullian}

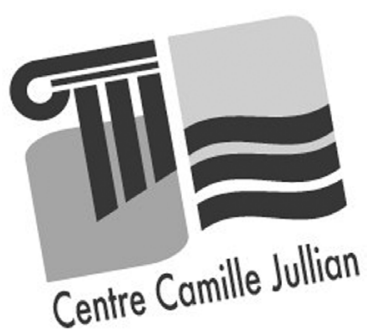

Ouvrage financé par

le programme européen Marie Curie

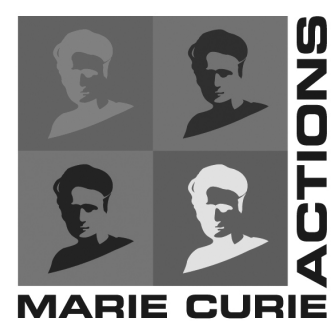

AGAME - Archaic and classical Greek Amphoras in north-western Mediterranean area and central Europe : diffusion, origin and contents (2010-2012)

Intra-European Fellowships (IEF) Call : FP7-PEOPLE-2009-IEF, Marie Curie «Promoting Science» - reference number : 252132

Envoyer les manuscrits à :

Henri Tréziny

Bibliothèque d'Archéologie Méditerranéenne et Africaine

Centre Camille Jullian

Maison Méditerranéenne des Sciences de l'Homme

5 rue du Château de l'Horloge. BP 647,

13094 Aix-en-Provence Cedex 2 


\section{LES AMPHORES GRECQUES DANS LE NORD DE L'ITALIE}

ÉCHANGES COMMERCIAUX ENTRE LES APENNINS

ET LES ALPES AUX ÉPOQUES ARCHAÏQUE ET CLASSIQUE

Federica SACCHETTI 


\section{Sommaire}

\section{PREMIÈRE PARTIE :}

\section{Bilan des études sur les amphores grecques des époques archaïque et classique}

CHAPITRE 1 :

Les amphores corinthiennes et de type corinthien

1. Les amphores de type corinthien $A$ et $A^{\prime}$

2. Les amphores de type corinthien $B$ ou corcyréen

\section{CHAPITRE 2 :}

Les productions grecques occidentales archaïques

1. Les amphores de « forme $1 \alpha$ »

2. Les amphores de «forme $2 »$

\section{CHAPITRE 3 :}

Les amphores attiques et de type attique « à la brosse »

\section{CHAPITRE 4 :}

Les productions de l'Égée septentrionale

1. La production attribuée à Mendé

2. La production attribuée à Thasos

3. Les productions des centres nord-égéens non identifiés

4. Contenu

5. Diffusion en Méditerranée occidentale

\section{CHAPITRE 5 :}

Les productions de l'Égée orientale

2. La production attribuée à Clazomènes

3. Les amphores de type samien, milésien, et du groupe « Samos-Milet » 


\section{DEUXIÈME PARTIE :}

\section{Les amphores grecques de l'Italie du nord}

\section{CHAPITRE 1 :}

Le littoral et le territoire d'Adria ......

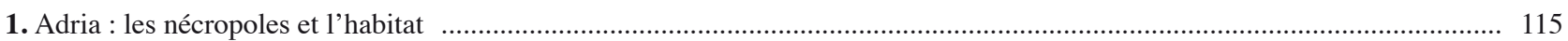

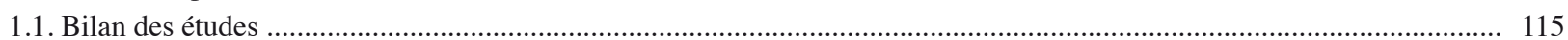

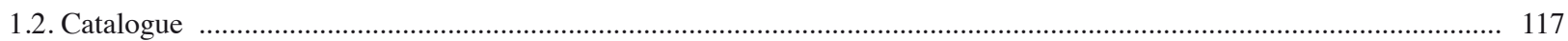

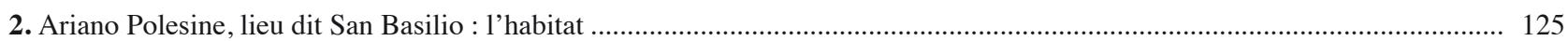

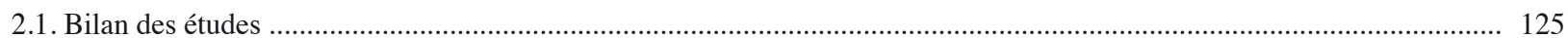

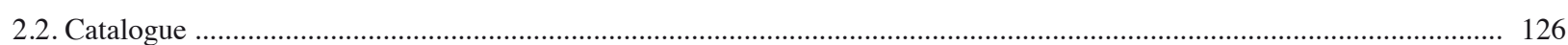

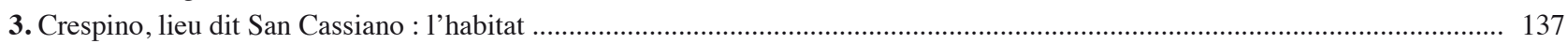

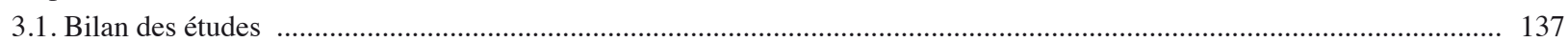

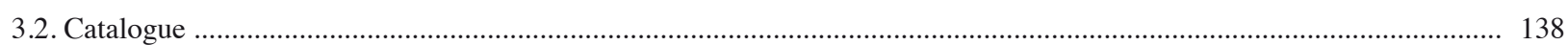

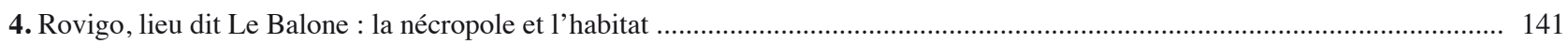

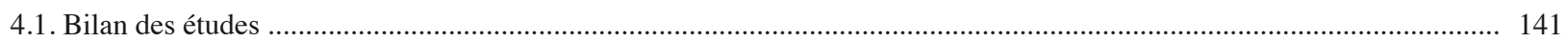

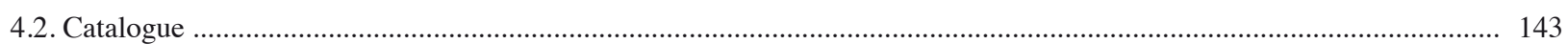

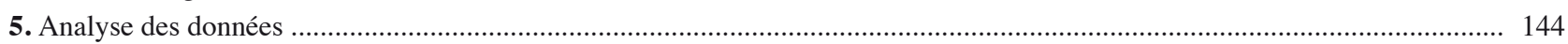

\section{CHAPITRE 2 :}

Spina : les nécropoles de Valle Trebba et de Valle Pega et l'habitat de Valle Lepri de Comacchio

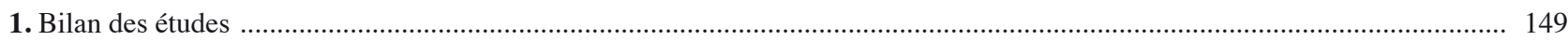

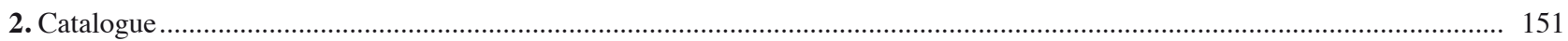

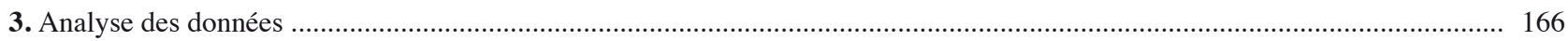

\section{CHAPITRE 3 :}

Bagnolo San Vito, lieu dit Forcello : l'habitat

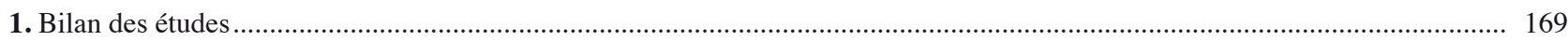

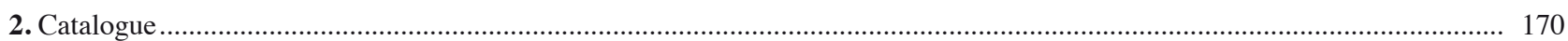

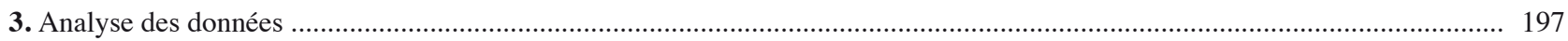

\section{CHAPITRE 4 :}

Bologne/Felsina : l'acropole et l'habitat

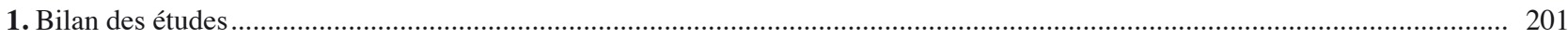

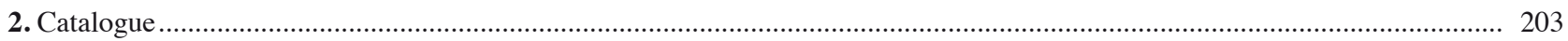

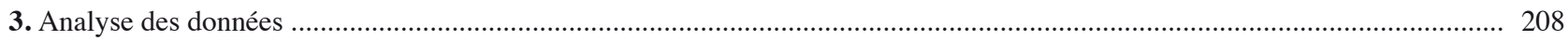

\section{CHAPITRE 5 :}

Marzabotto : l'habitat

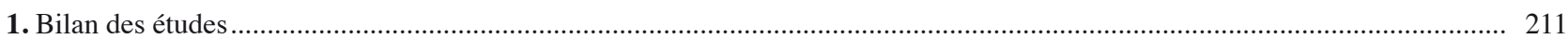

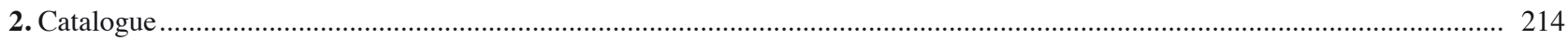

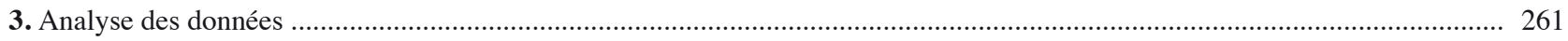

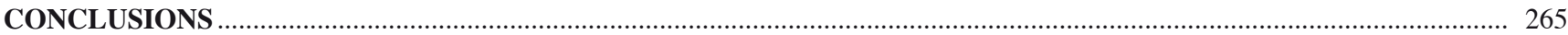

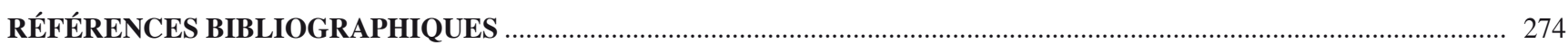


Alla memoria di mio padre, Franco, e a mia madre, Nerina Gobbi 


\section{Remerciements}

Aix-en-Provence 21.04.2012

Cet ouvrage sur les amphores grecques dans le Nord de l'Italie est issu d'une Thèse de Doctorat en « Scienze Archeologiche » soutenue le 23 mars 2006 à l'Université de Padoue (Italie) devant un jury composé de Giovanni Uggeri (Professeur, Université de Rome, La Sapienza - Président du jury), Emanuele Papi (Professeur, Université de Sienne), Marisa Bonamici (Professeur, Université de Pise), Emanuela Montanari (Professeur, Université de Trieste). Pour cette thèse j'ai bénéficié pendant trois ans d'une bourse de l'Université de Padoue.

Cette publication en est une version remaniée suite à l'expérience acquise au cours de ces dernières années, après mon parcours de doctorat. Elle est également complétée par l'intégration de matériels découverts, ou rendus accessibles à l'étude, postérieurement à la conclusion de ma thèse. J'ai pu y parvenir en bénéficiant d'un post-doc de l’Université de Provence au Centre Camille Jullian d'Aix-en-Provence.

Je tiens à témoigner ma reconnaissance à tous ceux qui ont contribué, de diverses manières, à l'aboutissement de cette étude et à sa publication.

Mes remerciements vont aux membres du jury de Thèse pour les remarques et les conseils qu'ils ont formulés lors de la soutenance. À Giuseppe Sassatelli (Professeur, Université de Bologne) pour m'avoir confié ce sujet de recherche novateur dans le cadre des études sur le Nord de l'Italie. À Raffaele C. de Marinis (Professeur, Université de Milan), qui déjà au moment de mon cursus à la Scuola di Specializzazione in Archeologia de l'Université de Bologne m'a permis de faire mes premiers pas dans l'étude d'un sujet dont il a été pionnier pour ce secteur géographique, en me montrant sans aucune réserve les matériels de ses fouilles du Forcello, tant publiés qu'inédits. À Bruno d'Agostino (Professeur, Université l'Orientale de Naples) pour m'avoir donné accès au matériel alors inédit de ses fouilles des remparts d'époque archaïque de Cumes. À Emanuele Greco, directeur de la Scuola Archeologica Italiana di Atene, pour m'avoir accueilli à l'occasion de mes séjours d'étude en Grèce et notamment à la SAIA, et à Jack L. Davis, directeur de l'American School of Classical Studies at Athens, pour m'avoir ouvert les portes de la Stoa of Attalos conservant les matériels des fouilles de l'Agora d'Athènes. Mes remerciements vont également à Alessandro Naso (Professeur, Université d'Innsbruck) pour m'avoir invitée sur la fouille de Milet, où il a en charge l'étude des amphores de Kalabaktepe. De cette première invitation, depuis 2006, est née ma collaboration avec l'équipe internationale des fouilles allemandes de la cité, collaboration pour laquelle je sais gré à Volkmar von Graeve (Professeur, Université de Bochum).

Je n'aurais pas pu acquérir une spécialisation dans le domaine des amphores grecques sans l'aide précieuse de Mark Lawall (Professeur, University of Manitoba - Canada), spécialiste reconnu dans ce champ de recherche. C'est grâce à sa disponibilité, à sa forte compétence dans ce domaine et à sa profonde connaissance des amphores et des contextes des fouilles de l'Agora d'Athènes, sujet de son Ph.D., que j'ai pu me former à l'étude de productions amphoriques qui posent encore beaucoup de problèmes, notamment celles du $\mathrm{V}^{\mathrm{e}} \mathrm{s}$. av. J.-C. Ces problèmes sont davantage contraignants lorsqu'il s'agit d'étudier des collections en provenance de contextes d'habitat, comme c'est le cas dans le Nord de l'Italie, présentant un degré de fragmentation important. Sans son accompagnement, notamment dans l'identification visuelle des pâtes, ce travail n'aurait pas pu aboutir.

Je suis également reconnaissante envers Jean-Christophe Sourisseau (Maître de Conférence à l'Université d'AixMarseille), avec qui j'ai l'opportunité de travailler depuis mon arrivée à Aix en profitant de sa compétence sur les productions grecques occidentales d'époque archaïque. Je le remercie pour m'avoir éclairée sur l'identification visuelle des pâtes qui caractérisent les différents ateliers de la Méditerranée occidentale, surtout à travers les matériels des fouilles de Marseille. Grâce à sa disponibilité, ces matériels, qui avaient fait l'objet de sa thèse, ont pu être 
intégrés dans un programme d'analyse sur les résidus organiques actuellement en cours dans le cadre du projet européen dont je suis responsable, supporté par une Marie Curie Intra European fellowship (IEF - 7th European Community Framework Programme).

Je remercie Sandro De Maria (Professeur, Université de Bologne), Erika Vecchietti et Elisa Esquilini pour m'avoir montré les échantillons des amphores en provenance des fouilles de Phoiniké soumis à analyse pétrographique, pour en avoir partagé avec moi les résultats encore inédits et pour la discussion que nous avons pu avoir sur des échantillons de comparaison que j'ai pu leur apporter.

Je voudrais témoigner toute ma reconnaissance à Simonetta Bonomi (actuellement Soprintendente Archeologo pour la Calabre), avec qui j'ai eu la possibilité de travailler lorsqu'elle était directrice du Museo Archeologico Nazionale d'Adria, pour la grande disponibilité dont elle a fait preuve et pour la rapidité avec laquelle elle a bien voulu me confier l'étude des amphores en provenance de fouilles très récentes et encore inédites qu'elle a dirigées. Je lui suis également reconnaissante pour le soutien qu'elle m'a témoigné dans certains moments difficiles pendant le travail qui a abouti à la publication de cet ouvrage.

Mes remerciements vont aux différentes institutions et chercheurs qui m'ont accordé l'autorisation d'étudier les matériels inédits d'Adria, San Basilio d'Ariano Polesine, San Cassiano de Crespino (prov. de Rovigo), Padoue :

La Soprintendenza per i Beni Archeologici del Veneto, particulièrement Maurizia De Min (ancienne Soprintendente Archeologo), directrice des premières fouilles de San Basilio, Luciano Salzani (Ispettore Archeologo) et Daniele Vitali (Professeur, Université de Dijon), directeurs des plus récentes fouilles de San Basilio, Maurizio Harari (Professeur, Université de Pavie), directeur de la fouille de San Cassiano, et Raffaele Peretto, directeur du Museo dei Grandi Fiumi de Rovigo, où les matériels du site sont conservés, et enfin Vincenzo Tinè et Giovanna Gambacurta, respectivement actuels Soprintendente Archeologo del Veneto et directrice du Museo Archeologico Nazonale de Adria, pour avoir donné leur autorisation à la publications des images.

Je remercie la Soprintendenza per $i$ Beni Archeologici dell'Emilia-Romagna, pour l'autorisation d'étudier les matériels de Bologne et de Marzabotto (prov. de Bologne), particulièrement Marina Calvani, Luigi Malnati et Filippo Maria Gambari, qui pendant ces années se sont succédé à la direction de cette Surintendance, Enzo Lippolis (actuellement Professeur, Université La Sapienza de Rome) et Paola Desantis, directeurs successifs du Museo Etrusco P. Aria de Marzabotto. Mes remerciements et mon amitié vont aussi à tous les fonctionnaires, collègues et amis du Museo Civico Archeologico de Bologne, où est conservé une partie des matériels des fouilles de Bologne, et notamment à l'ancienne directrice du Musée, Cristiana Morigi Govi.

Je souhaite également remercier tous ceux qui par leurs conseil, aide, soutien et collaboration ont contribué à la réussite et à l'aboutissement de ce travail :

Sergio Sani, Ispettore Onorario de la Soprintendenza per i Beni Archeologici dell'Emilia-Romagna, pour ces renseignements précieux, dont il n'y a pas de traces écrites, que seule l'expérience professionnelle qui a été la sienne pouvaient m'apporter. Vincenzo Baldoni, pour le partage que nous avons eu pendant nos années de doctorat, un partage fait tant de soutien amical que de confrontation scientifique. Tout le personnel du Museo Archeologico Nazionale d'Adria, le personnel du Museo Etrusco P. Aria de Marzabotto et celui du Museo Civico Archeologico de Bologne que j'ai eu la chance de rencontrer pendant les nombreuses années de travail, sur le terrain et dans les musées. Vincent Dumas (CNRS - CCJ), Lucile Delavault, Aura Fossati, qui ont collaboré au traitement informatique des images. Véronique Gémonet (CNRS - CCJ), Lucien Rivet (CNRS - CCJ) et Sylvie Saulnier (chercheur associé CCJ), qui se sont occupés de l'édition de cet ouvrage et Stéphane Renault (CNRS - LAMPEA), qui en a projeté la couverture.

Je suis particulièrement reconnaissante envers Dominique Garcia et Marie-Brigitte Carre, directeurs successifs du Centre Camille Jullian, Henri Tréziny, directeur de la collection BiAMA, et tous les membres du laboratoire qui depuis mon arrivée ont facilité mon intégration au sein de l'équipe et la réalisation de ce travail.

Cet ouvrage est dédié à ma famille, et notamment à ma mère et à mon père, précocement disparu pendant que je préparais cette publication. À eux, qui m'ont permis de faire mes études, laissée libre dans mes choix et qui m'ont depuis toujours soutenue et encouragée sans réserves, vont toutes mes pensées et mes remerciements les plus sincères et inconditionnels. 


\section{Crédits}

\section{ILLUSTRATIONS}

L'échelle des dessins des illustrations de la première partie est au dixième pour les amphores complètes et au quart pour les fragments.

Dans la deuxième partie, l'échelle des dessins des planches, sauf mention contraire, est au quart. La numérotation des dessins des planches correspond à la numérotation des fiches du catalogue.

\section{CRÉDITS GRAPHIQUES POUR LES DESSINS DES MATÉRIELS INÉDITS}

Adria, San Basilio : dessins des matériels (C) Leonardo Di Simone (Museo Archeologico Nazionale di Adria) ; graffiti (C) Federica Sacchetti. Padoue : (C) Leonardo Di Simone.

San Cassiano, Bologne, Marzabotto : (C) Federica Sacchetti.

\section{RÉFÉRENCES POUR LES DESSINS DES MATÉRIELS PRÉCÉDEMMENT PUBLIÉS}

\section{DAO CCJ}

Adria : AD3, d'après Toniolo 2000.

San Basilio : SB2, SB31, SB32, d'après Salzani, Vitali 2003 ; SB4, SB6, SB29, SB34 d'après Cattaneo Cassano 1995.

Spina : d'après De Luca De Marco 1979 ; Baldoni 1984 ; Desantis 1989 ; Desantis 1993.

Forcello : d'après de Marinis 1988 ; Id. 2007.

Bologna/Felsina : FE3, d'après Baldoni, Morico, Pini 2007.

Marzabotto : MA1, MA90, MA112, MA113, d'après Iscrizioni e graffiti ; MA76, MA83-MA88, d'après F. H. Massa-Pairault, in MassaPairault 1997.

Poviglio, Case Carpi : CC1, d'après Macellari 1990. 


\section{Introduction}

\section{État général des études sur les amphores commerciales grecques des époques archaïque et classique}

Les amphores commerciales grecques des époques archaïque et classique constituent une catégorie d'importations restée à l'écart des études traditionnelles, qui ont longtemps privilégié la céramique décorée par rapport à d'autres productions d'une moindre valeur artistique. Néanmoins, à partir des années 1980, de nombreux chercheurs ont souligné le caractère partiel et insuffisant des reconstructions historiques à caractère économique et commercial construites sur cette base, en montrant que ces objets ne devaient représenter qu'une « cargaison d'accompagnement » par rapport aux marchandises qui constituaient, elles, l'objet primaire des échanges, c'est-à-dire toutes les denrées alimentaires et les produits de première nécessité qui entrent dans la sphère du biotos ${ }^{1}$.

Lors de son intervention à l'occasion du Colloque Flotte e commercio greco, cartaginese ed etrusco nel Mar Tirreno, M. Gras (1988) exprimait une perplexité explicite face à l'amphorologie en tant que science en soi. On était alors à un moment charnière où le problème de l'étude des amphores commerciales d'époque archaïque en particulier était soumis à l'attention du monde scientifique au travers d'une série d'importantes initiatives, comme le colloque Il commercio etrusco arcaico et l'exposition qui l'accompagnait en $1983^{2}$, le colloque international Recherches sur les amphores grecques organisé l'année suivante par l'école française d'Athènes, ou encore en 1989 la table ronde de Lattes Les amphores de Marseille grecque spécialement consacrée à la production massaliète. C'est à ce moment particulier, caractérisé par la «redécouverte » d'une catégorie d'objets longtemps abandonnée à une place marginale, que M. Gras a voulu mettre en garde la communauté scientifique sur le risque d'une spécialisation technique excessive du problème qui aurait pu détourner les archéologues de la vision historique globale et de la véritable finalité de l'étude des conteneurs de transport. À la même occasion, M. Gras a également souligné le risque d'une schématisation excessive des données disponibles qu'il jugeait particulièrement insuffisantes pour établir des classifications typologiques crédibles, du moins au moment où il rédige son article, c'est-à-dire vers le milieu des années 1980.

La perplexité exprimée par M. Gras n'était évidemment pas sans fondements, mais le fait que les amphores de transport grecques archaïques et classiques constituaient un domaine presque complètement inexploré et dont l'étude se révélait plus que jamais urgente, était ressenti au même moment comme une exigence scientifique désormais incontournable de la part de plusieurs institutions scientifiques. Ce fut le cas du Centro di Studio per l'Archeologia Etrusco-Italica (CNR), promoteur sous la direction de M. Cristofani, du Colloque et de l'Exposition associée de 1983 (cf. supra), du Centre Jean-Bérard de Naples, éditeur de la collection Cahiers des Amphores Archaïques et Classiques $^{3}$ puis promoteur d'un programme d'analyses pétrographiques sur certaines productions occidentales d'époques archaïque et classique, jusqu'aux travaux des institutions scientifiques du Midi de la Gaule sur le problème des amphores de Marseille et celles de la Mer Noire ${ }^{4}$. Toutes ces initiatives commencèrent alors à donner de l'épaisseur à l'analyse de cette catégorie d'objets particuliers sans valeur artistique et dont l'importance ne résidait pas tellement dans l'objet en soi et dans sa valeur intrinsèque, mais plutôt dans le contenu qu'il transportait, en

1. Sur cet argument, développé en premier lieu par A. Mele (1979, notamment p. 102), cf. également Martelli 1985 ; Ead. 1989 ; Sassatelli 1993 ; Gras 1996.

2. Pour le colloque cf. Commercio etrusco arcaico ; pour l'exposition, cf. Rizzo 1990. Plus récemment le thème du commerce étrusque a été repris à l'occasion d'un colloque qui n'a toutefois pas réservé une attention particulière aux amphores de transport (Etruschi e il Mediterraneo). 3. Dans la collection ont été publiées les deux monographies de M. Cavalier (1985) et de N. Di Sandro (1986) dédiées respectivement aux amphores de Lipari et à celles du Scarico Gosetti de Pithécusses.

4. Cf. Amphores de Marseille grecque et Production et commerce. 
répondant aux principes posés dès les années 1960 par G. Vallet et Fr. Villard sur la distinction bien connue entre 《vases marchandise » et «vases conteneurs ${ }^{5}$. Les groupes de travail qui se constituèrent à ces occasions durent effectuer une véritable recherche de « première main » consistant dans la révision des données d'archives et surtout dans des vérifications dans les dépôts archéologiques, car même dans le cas d'exemplaires complets qui faisaient partie d'ensembles funéraires des nécropoles d'Étrurie méridionale, on avait souvent perdu au cours des années toute référence à leur contexte ${ }^{6}$.

C'est en Grèce que les premières études spécialement consacrées au matériel amphorique grec du $\mathrm{V}^{\mathrm{e}}$ siècle ont été initiées par l'American School of Classical Studies d'Athènes dans le cadre des fouilles de l'Agora. Grâce aux contextes exceptionnels des puits de l'agora d'Athènes, V. Grace se fit promotrice et initiatrice des premières recherches systématiques sur les amphores appartenant à un cadre culturel et chronologique presque complètement inexploré du fait de la rareté des exemplaires publiés. En effet, si les amphores grecques archaïques plus anciennes étaient assez bien attestées grâce aux découvertes des nécropoles, comme celles de contextes occidentaux mentionnés plus haut, il n'existait pas la même base documentaire pour les amphores de la seconde moitié du VI et du V ${ }^{\mathrm{e}} \mathrm{s}$. av. J.-C. Les cultures méditerranéennes abandonnant progressivement l'habitude de déposer des offrandes alimentaires dans les tombes ${ }^{7}$, et donc parfois des amphores, la connaissance des productions amphoriques grecques devait alors nécessairement se baser uniquement sur les découvertes des fouilles urbaines dont les objets, récupérés souvent dans un état très fragmentaire, compromettaient l'élaboration d'une classification correcte et exhaustive.

\section{État des études sur les amphores commerciales grecques dans l’Italie septentrionale}

Dans un contexte marqué par l'absence presque complète de découvertes amphoriques en milieu funéraire, la fouille et la publication rapide du matériel de l'oppidum préromain de Gênes et surtout les découvertes tout à fait inattendues de l'habitat étrusque du Forcello à Bagnolo San Vito, où dans une aire fouillée relativement réduite on avait trouvé de nombreux fragments d'amphores, attirèrent l'attention des spécialistes sur le problème de l'identification et de l'étude du matériel amphorique grec des époques archaïque et classique dans ces sites du secteur étrusco-padan, où les importations de céramique fines et figurée du monde grec avaient jusqu'alors monopolisé l'intérêt des chercheurs. C'est dans ce contexte que furent signalées et recensées les découvertes de l'habitat de Spina, site privilégié pour les importations méditerranéennes ${ }^{8}$.

Le cadre de notre recherche cumulait donc les handicaps : le matériel de l'Italie septentrionale se présentait dans un état de conservation évidemment fragmentaire parce qu'issu de contextes d'habitats, mais aussi s'attachait à une phase chronologique, la fin du $\mathrm{VI}^{\mathrm{e}}$ et le $\mathrm{V}^{\mathrm{e}} \mathrm{s}$. av. J.-C., particulièrement problématique au regard des connaissances acquises limitées dans l'étude des conteneurs de transport de production grecque.

Compte tenu de la situation particulière des études du secteur, ce travail est donc articulé en deux parties distinctes.

La première partie est une révision des études spécialisées sur les amphores grecques d'époque tardo-archaïque et classique.

La deuxième partie est consacrée à la présentation et à l'étude du matériel amphorique des $\mathrm{VI}^{\mathrm{e}}$ et $\mathrm{V}^{\mathrm{e}} \mathrm{s}$. av. J.-C. de l'Étrurie padane et de l'Italie septentrionale et a consisté dans le recensement et l'analyse du matériel publié, ainsi que dans l'étude de corpus inédits.

5. Vallet, Villard 1961.

6. Cf. la Premessa de P. Pelagatti in Rizzo 1990.

7. Pour ce problème voir Colonna 1985. Les seuls contextes funéraires du V V s. av. J.-C. où fut conservé l'usage de la déposition d'amphores sont constitués par les kourganes Scythes (cf. en dernier lieu Monachov 2003a, avec la bibliographie antérieure). Assez récemment, les nécropoles de la chora de Métaponte ont aussi restitué des amphores, utilisées dans les tombes, soit pour des sépultures à enchytrismos, soit comme éléments du mobilier funéraire (Morter, Leonard 1998).

8. Pour Gênes, Milanese 1987 ; pour le Forcello, De Marinis 1988 et 2007 (avec bibliographie antérieure) ; pour l'habitat de Spina, Desantis 1989, précédé et suivi par l'édition partielle des amphores de la nécropole : De Luca De Marco 1979 ; Desantis 1993 ; Ead. 1996. 

Première Partie

BILAN DES ÉTUDES

SUR LES AMPHORES GRECQUES

DES ÉPOQUES ARCHAÏQUE

ET CLASSIQUE

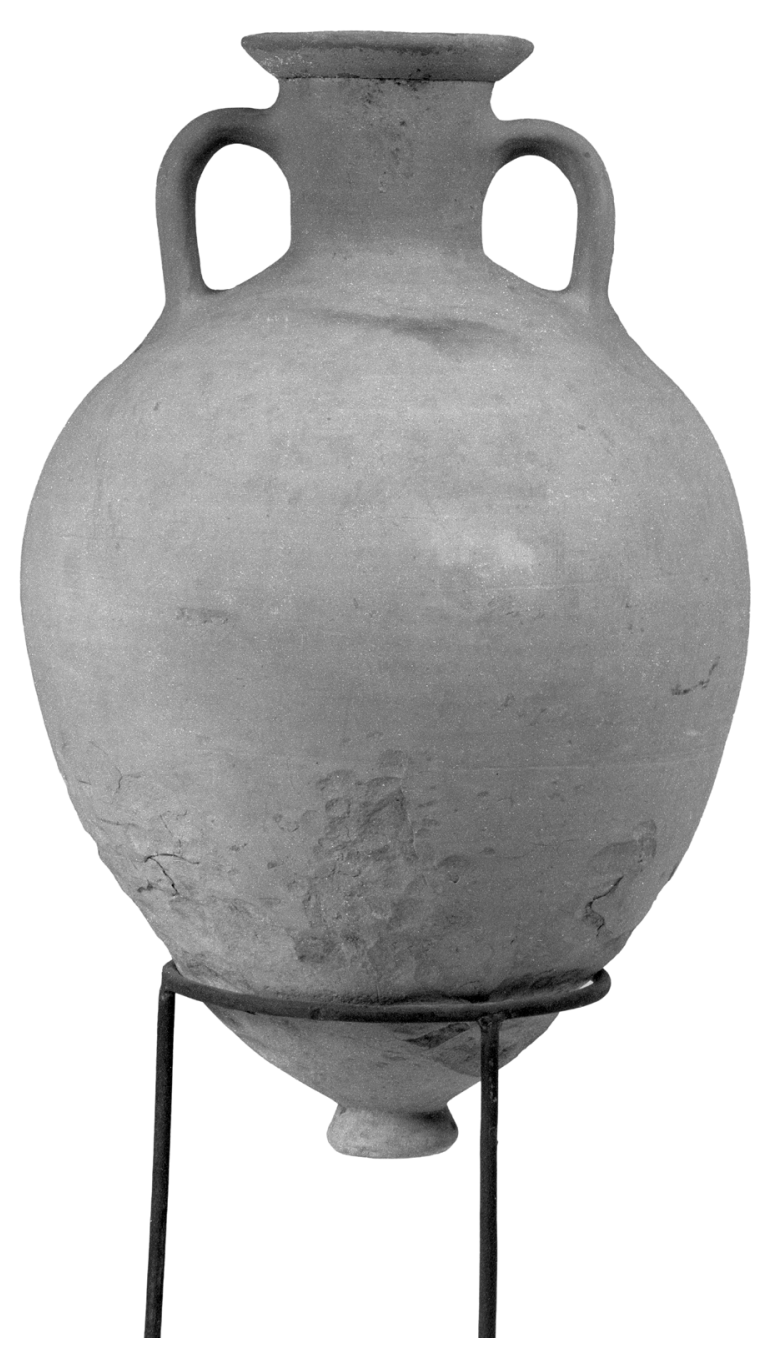





\section{Chapitre 1}

\section{Les amphores corinthiennes et de type corinthien}

$\mathrm{L}$ a cité de Corinthe a vu naître et se développer une importante production d'amphores au sein de laquelle, dès les années 1930, on a cru pouvoir distinguer deux formes distinctes, distinction reprise et formalisée par C. G. Koehler, à la fin des années 1970 et au début des années 1980. Ces deux formes sont connues sous l'appellation Corinthian A et Corinthian $B^{9}$.

C'est donc essentiellement à l'école Américaine que nous devons les travaux typo-chronologiques pionniers sur ces productions et leur attribution à Corinthe ; à leurs résultats se sont ajoutées, plus récemment, les contributions de l'école Britannique d'Athènes dans le domaine des analyses physico-chimiques ${ }^{10}$. L'impulsion donnée par V. Grace a eu une suite remarquable à travers les études de C. G. Koehler, qui ont progressé grâce aux fouilles menées à Corinthe par l'école Américaine à la fin des années 1970 et notamment à la découverte du Punic Amphora Building, où on a découvert non seulement de nombreuses amphores importées du $\mathrm{V}^{\mathrm{e}} \mathrm{s}$. av. J.-C., mais aussi plusieurs amphores qui furent considérées de production locale. Sur la base de l'étude morphologique et de l'examen visuel de la pâte des amphores appartenant à cette production locale, C. G. Koehler a

9. Bien que quelques contributions des années 1930 distinguent déjà les deux formes principales supposées être produites à Corinthe (Pease 1937, n 200-201 ; Campbell 1938, p. 604-605), la définition des deux groupes - Corinthian A et Corinthian $B$ - a été introduite pour la première fois par C. G. Koehler (1978, 1979 et 1981).

10. Pour les études publiées par l'école Américaine d'Athènes, à part les ouvrages déjà cités (cf. n.9), on consultera les travaux de V. Grace, notamment: Grace 1953, p. 108-109, n $164-166$ et Ead. 1961. Dans le sillage de ces premières études, voir les travaux encore fondamentaux de C. G. Koehler, notamment: Koehler 1978a ; Ead.1978b ; Ead. 1979 ; Ead. 1981 ; Ead. 1982 ; Ead. 1986 ; Ead. 1992. Les études récentes menées par l'école Britannique d'Athènes dans le cadre du Fitch Laboratory sont dus essentiellement à R. E. Jones $(1986$, p. 712) et à I. K. Whitbread (1986, p. 95-101 ; Id. 1988, p. 221-222 ; Id. 1995, surtout p. 255293) qui reposent en particulier sur l'étude pétrographique des argiles et des dégraissants. proposé l'existence de trois séries qu'elle a appelées Type A, Type A' et Type B. Les trois séries peuvent être réduites à deux familles de formes principales, le groupe dit des Corinthian A et celui dit des Corinthian B, bien distinctes par leur morphologie, mais dont la production serait en partie contemporaine. Alors que la production locale de la forme A n'a jamais été discutée, puisqu'elle s'intègre parfaitement dans la tradition céramique de Corinthe, tant d'un point de vue morphologique que pour les caractéristiques de la pâte, l'attribution à la même cité de la production de la forme B, objet de nombreuses études et de débats scientifiques complexes, a été mise en doute par V. Grace la première, qui proposait déjà, plusieurs années avant les études approfondies de C. G. Koehler, de la rattacher à la colonie corinthienne de Corcyre ${ }^{11}$. Cette forme constituerait donc un type pour partie «extra-régional » car, tout en reconnaissant à Corinthe une production d'amphores de forme $\mathrm{B}$, on sait aujourd'hui qu'il existe des éléments nombreux et de natures différentes qui montrent que ces amphores ont été produites par plusieurs centres de fabrication connus (probablement Corcyre) ou encore non identifiés. Corinthe serait donc la seule cité grecque de l'aire égéenne qui ait été productrice en même temps d'une forme amphorique «locale» et d'une forme de nature « extra-régionale ».

Dans l'arc chronologique retenu par notre étude, les deux formes et les trois séries d'amphores attribuées par C. G. Koehler à la ville de Corinthe - c'est-à-dire la forme «locale », dans les deux séries A et A', et la forme « extra-régionale » $\mathrm{B}$ - étaient toutes en phase de production.

Dans les paragraphes qui suivent nous présenterons une synthèse des études typologiques et chronologiques concernant chacune des trois séries d'amphores actuellement attribuées à la cité de Corinthe, suivie par la description de la pâte et de la discussion relative aux lieux de production.

11. Grace 1953, p. 108-109, n 164-166. 


\section{Les amphores de type corinthien $\mathrm{A}$ et $\mathrm{A}^{\prime}$}

\subsection{Le type A}

Les amphores corinthiennes de type A constituent la série la plus ancienne des productions corinthiennes car leur fabrication commence dès le milieu du $\mathrm{VIII}^{\mathrm{e}} \mathrm{s}$. av. J.-C. et se poursuit sans interruption jusqu'à la fin du IV ${ }^{\mathrm{e}}$ ou le début du $\mathrm{III}^{\mathrm{e}} \mathrm{s}$. av. J.-C. Les exemplaires les plus archaïques d'amphores corinthiennes A proviennent de Corinthe et des nécropoles anciennes de Sicile et de Grande Grèce, notamment Métaponte, Géla ou Camarine. Elles étaient encore très largement exportées entre la fin du $\mathrm{VI}^{\mathrm{e}}$ et la première moitié du $\mathrm{V}^{\mathrm{e}} \mathrm{s}$. av. J.-C., comme le montrent les nombreux exemplaires répertoriés notamment en Méditerranée occidentale. à partir du milieu du V ${ }^{\mathrm{e}}$ s. av. J.-C., en revanche, C. G. Koehler suppose une sensible diminution des exportations et donc probablement de la production du type A, car la plupart des exemplaires connus des $\mathrm{V}^{\mathrm{e}}$ et $\mathrm{IV}^{\mathrm{e}} \mathrm{s}$. av. J.-C. proviennent de contextes corinthiens ${ }^{12}$. Les découvertes récentes des nécropoles des $\mathrm{V}^{\mathrm{e}}$ et $\mathrm{IV}^{\mathrm{e}} \mathrm{s}$. dans la chora de Métaponte confirment cette hypothèse, puisque la plupart des amphores recueillies dans cette nécropole sont de type $\mathrm{A}^{\prime 13}$.

L'étude de la morphologie des amphores du type A, qui s'inscrit parfaitement dans la tradition morphologique des storage jars géométriques locales ${ }^{14}$, a permis de définir une évolution typologique générale sur la base de laquelle il est possible de déterminer la datation des différentes variantes. Si nous ne disposons pas encore d'une véritable séquence typo-chronologique précise pour les périodes les plus anciennes de la production (VIII ${ }^{\mathrm{e}}-\mathrm{VII}{ }^{\mathrm{e}} \mathrm{s}$. av. J.-C.), l'évolution de la forme A, à partir du début du $\mathrm{VI}^{\mathrm{e}} \mathrm{s}$. av. J.-C. et jusqu'à la disparition des exemplaires de ce type, est désormais beaucoup plus articulée et ancrée à une chronologie plus resserrée. Les données archéologiques à notre disposition montrent que le développement de la forme A ne se présente pas de manière tout à fait linéaire. Ce développement a dû consister en une évolution lente et continue, caractérisée

12. Koehler 1981, p. 454, 457 ; Ead. 1992, p. 269-270 et n. 13 ; Lawall 1995, p. 61.

13. Morter, Leonard 1998. Sur plus d'une trentaine d'amphores corinthiennes signalées dans les nécropoles de Pantanello et Saldone, seules deux sont de forme A.

14. Sur la genèse et le développement de ces amphores durant la période géométrique, voir: Pfaff 1988, p.29-31. Sur la relation étroite et la continuité à l'intérieur d'une même tradition entre les storage jars géométriques et les amphores corinthiennes $\mathrm{A}$, voir : Koehler 1981, p.451, pl.98. a. aussi par la coexistence de plusieurs types et variantes, à l'intérieur de laquelle il est parfois difficile de distinguer un schéma évolutif progressif et une articulation chronologique précise. L'ancrage à des chronologies mieux définies des types et des variantes actuellement identifiés devrait nous permettre à l'avenir, soit de reconnaître une plus grande continuité de développement de la série ou bien, au contraire, de confirmer l'existence de formes différentes mais contemporaines, peut-être attribuables à la production de différents ateliers locaux.

\subsubsection{Bilan des études}

Les conteneurs les plus anciens, datables du milieu du VIII ${ }^{e}$ et du courant du VII ${ }^{\mathrm{e}}$ s. av. J.-C., sont assez facilement reconnaissables par leur forme trapue et massive. Ils présentent un profil de forme globulaire se rétrécissant assez graduellement vers le fond. Dans les exemplaires datables avant le milieu du $\mathrm{VI}^{\mathrm{e}} \mathrm{s}$. av. J.-C., le pied ne se distingue pas nettement de la panse et présente une forme arrondie d'un diamètre considérable, il est creux à l'intérieur et aplati au-dessous. Les diamètres de l'embouchure et du col de ces amphores sont également remarquables. Le col, de forme cylindrique, est encore court, ainsi que les anses verticales qui présentent une courbure plus ou moins accentuée. Elles présentent une section ronde et sont implantées, en bas, sur l'épaule fuyante et, en haut, légèrement au-dessous d'une lèvre lourde. La lèvre est caractérisée par une forme à marli saillant, horizontale, au sommet plat, au bord extérieur mouluré ou émoussé et à section carrée ou, plus rarement, trapézoïdale ${ }^{15}$.

La chronologie du début de série des amphores corinthiennes $\mathrm{A}$, tout comme la séquence chronologique au cours de l'époque archaïque, est illustrée par les exemplaires trouvés dans les principaux contextes d'utilisation de ces produits dans la période de l'expansion du commerce corinthien vers l'Occident. Les plus anciens produits manufacturés (fig. 1) sont en effet attestés à l'intérieur des sépultures des côtes sud-orientale et centrale de Sicile et de Grande Grèce, notamment à Métaponte et à Géla, où entre la fin du VIII ${ }^{\mathrm{e}}$ et le milieu du VII ${ }^{\mathrm{e}} \mathrm{s}$. av. J.-C. ils étaient remployés comme urnes cinéraires ou pour la pratique funéraire de l'enchytrismos ${ }^{\mathbf{1 6}}$. Des exemplaires du troisième et du dernier quart du

15. Pour quelques exemplaires d'amphores corinthiennes de type A du VII ${ }^{\mathrm{e}}$ s. av. J.-C., voir: Koehler 1978a fig. 1.a, 3.a ; Ead. 1981, pl. 98. b-c. Pour des exemplaires datables du VI s. av. J.-C., cf. : Ead. 1978a, fig. 1.b ; Di Sandro 1981b, fig. II.1 ; Koehler 1981, pl. 98. d-e.

16. Pour Métaponte et Géla et les attestations de la fin VIII ${ }^{\mathrm{e}}$-milieu VII ${ }^{\mathrm{e}}$ s. av. J.-C., voir : Koehler 1981, p. 451, pl. 98.b et Ead. 1978a, fig. 3.a. 


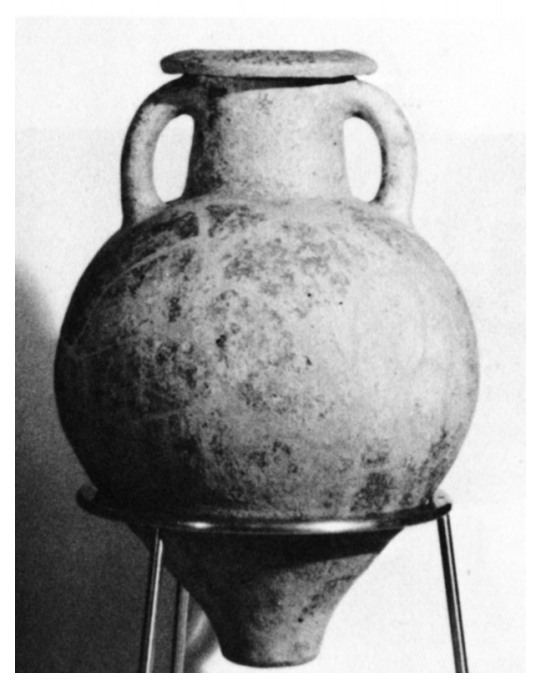

Fig. 1. Amphore de type corinthien A. Metaponto (?) (D'après Koehler 1981, pl. 98.b).

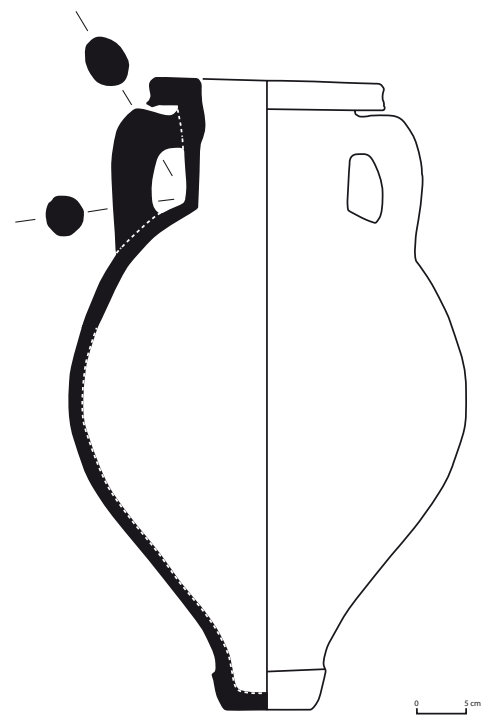

Fig. 2. Amphore de type corinthien A. Corinthe, inv. C-62-644 (d'après Koehler 1978a, fig. 1.a).

$\mathrm{VII}^{\mathrm{e}}$ s., qui témoignent d'après C. G. Koehler d'un premier stade évolutif de la forme (fig. 2), ont été trouvés dans un contexte de Corinthe, l'Anaploga Well ${ }^{17}$, et pour le $\mathrm{VI}^{\mathrm{e}} \mathrm{s}$. av. J.-C. ${ }^{18}$, les nombreux exemplaires de la nécropole de Rifriscolaro permettent aujourd'hui

17. Pour la seconde moitié du VII' s. av. J.-C.: Koehler 1978a fig. 1.a; Ead.1981, pl. 98.c; Ead. 1992, n. 9, pl. 1.a,

18. Pour la fin du VII et pour le VI ${ }^{\mathrm{e}}$ s. av. J.-C. : Koehler 1981, p. 451-452, pl. $98 . c-$ e. d'appréhender l'évolution typo-chronologique de la série jusqu'au début du $\mathrm{V}^{\mathrm{e}} \mathrm{s}$. av. J.-C. ${ }^{19}$.

Le fort potentiel du matériel de la nécropole de Rifriscolaro à Camarine pour la définition de la séquence typo-chronologique des amphores corinthiennes A du $\mathrm{VI}^{\mathrm{e}} \mathrm{s}$. av. J.-C., déjà souligné en son temps par C. G. Koehler ${ }^{20}$, à permis à J.-Chr. Sourisseau de fournir une importante contribution à la sériation typologique de la classe au cours de l'époque archaïque, surtout pour le $\mathrm{VI}^{\mathrm{e}}$ s. av. J.-C. : six types, dont un est subdivisé en trois variantes, situés entre le début du $\mathrm{VI}^{\mathrm{e}}$ et le début du $\mathrm{V}^{\mathrm{e}} \mathrm{s}$. av. J.-C. ont été caractérisés ${ }^{21}$.

Le type 1 de Camarine-Rifriscolaro (fig. 3, type 1) est défini comme un conteneur au profil général sphérique mais légèrement allongé dans sa partie inférieure. Il présente une lèvre du type à marli, dont le sommet est légèrement arrondi et convexe, la face inférieure rectiligne et à peu près horizontale, le profil extérieur vertical ou légèrement concave et l'épaisseur plus ou moins accentuée à la jonction avec le col. Le col présente une hauteur inférieure par rapport à sa largeur et se rétrécit vers le haut en prenant une forme tronconique. Les anses, massives et de section ronde, sont implantées immédiatement au-dessous du bord et retombent sur l'épaule près de la jonction avec le col, ce qui peut leur donner par conséquent un profil légèrement rentrant. La panse, sphérique, se rétrécit vers le fond et se termine en un large pied de forme cylindrique, creux à l'intérieur, aplati au-dessous et avec un profil extérieur marqué par une légère inflexion carénée ${ }^{22}$.

Le type, correspondant aux plus anciens exemplaires décrits par C. G. Koehler et qu'elle date du $\mathrm{VII}^{\mathrm{e}} \mathrm{s}$. av. J.-C., pourrait avoir connu, d'après J.-Chr. Sourisseau, une utilisation jusqu'aux premières années du VI ${ }^{\text {e }}$ s. av. J.-C. Ainsi, en plus des exemplaires de la nécropole de Camarine où, conformément à la date de fondation traditionnelle (598 av. J.-C.), on ne connait pas

19. Sourisseau 2006.

20. Koehler 1981, p. 451, n. 10, et Ead. 1992, n. 9.

21. La classification typo-chronologique proposée par J.-Chr. Sourisseau, comme il l'a lui-même indiqué, est encore susceptible de modifications, car les contextes de la nécropole de Rifriscolaro à Camarine sont encore en cours d'étude. Par conséquent, la présentation de l'ensemble des typologies amphoriques ainsi que leur séquence chronologique dans le cadre d'une typologie du site attendent encore les confrontations nécessaires qui viendront de l'étude des associations de matériels susceptibles de fournir une datation. Malgré cela, la contribution préliminaire de J.-Chr. Sourisseau, en l'état actuel des recherches, s'avère fondamentale pour l'étude des amphores corinthiennes $\mathrm{A}$ du $\mathrm{VI}^{\mathrm{e}} \mathrm{s}$. av. J.-C. et j'estime qu'il faut lui réserver un éclairage particulier à l'intérieur de ce travail.

22. Sourisseau 2006, p. 135, fig. 2, 8. Le type correspond à Koehler 1979, pl. 1, n 1-4, et Ead. 1981, p. 451, pl. 98. b. 


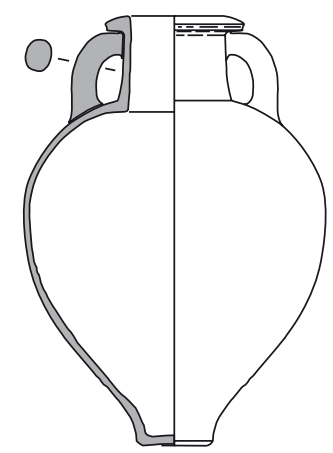

Type 1

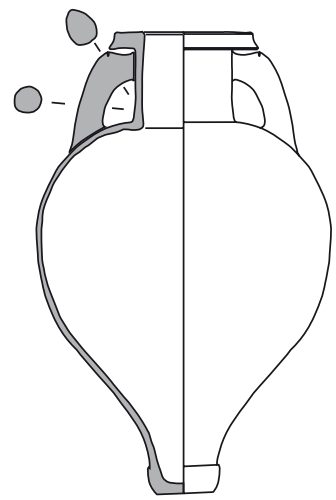

Type 2

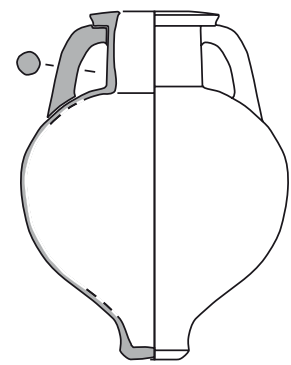

Type 3

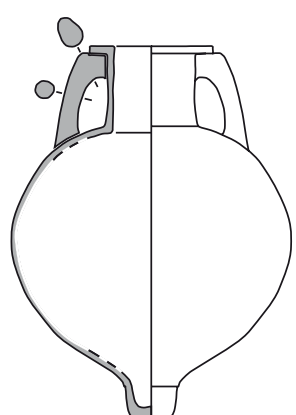

Type 4A

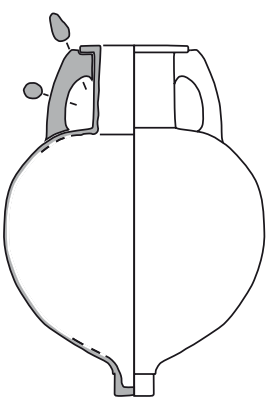

Type 4B

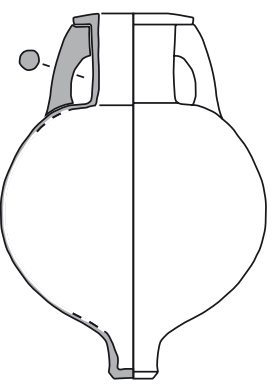

Type 4C

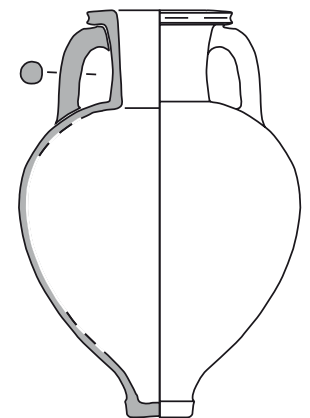

Type 5

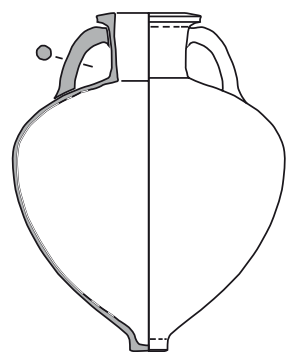

Type 6

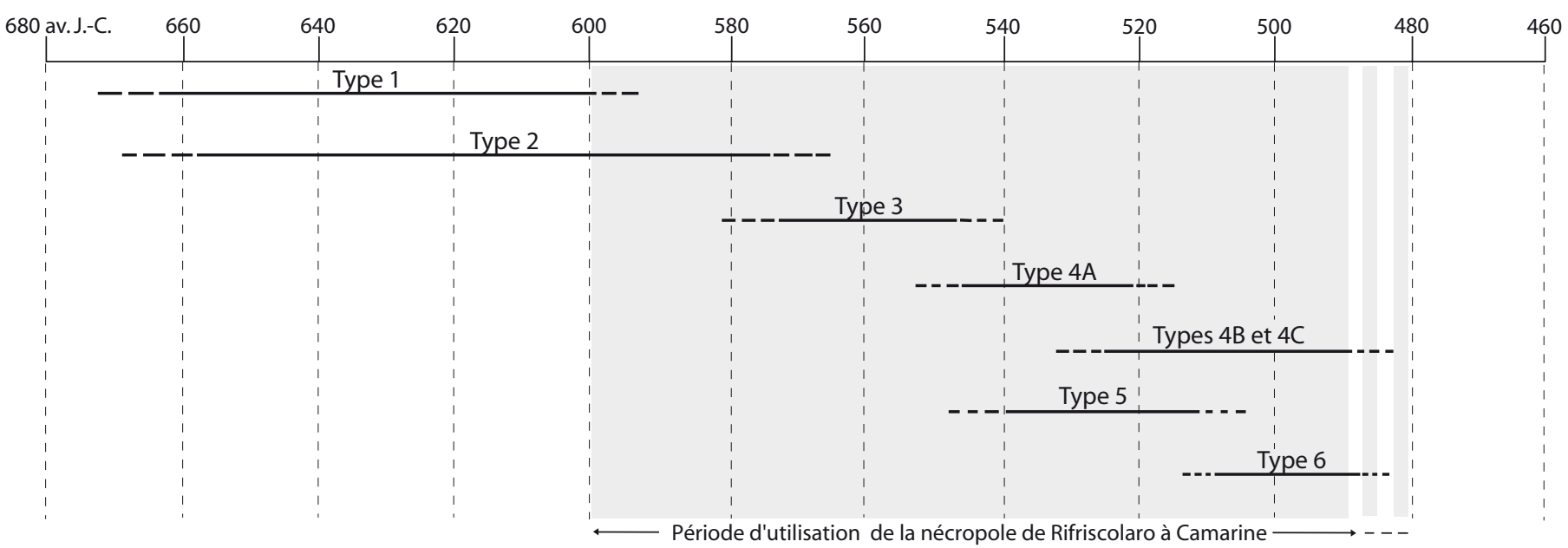

Fig. 3. Tableau typo-chronologique des amphores corinthiennes A de la nécropole de Camarina-Rifriscolaro (d'après Sourisseau 2006, fig. 8). 
de matériel antérieur au début du $\mathrm{VI}^{\mathrm{e}} \mathrm{s}$. av. J.-C. ${ }^{23}$, une lèvre qu'on peut assimiler à cette forme a été trouvée dans les fouilles de Marseille, également fondée vers 600 av. J.-C. ${ }^{24}$. Une séquence chronologique plus articulée des amphores qu'on peut rattacher à ce type dans le courant du VII ${ }^{\mathrm{e}}$ s. av. J.-C., ainsi qu'une éventuelle définition morphologique d'un ou de plusieurs sous-types du début $\mathrm{du} \mathrm{VI}^{\mathrm{e}} \mathrm{s}$. sont cependant impossibles à déterminer en l'état actuel ${ }^{25}$.

Le type 2 de Camarine-Rifriscolaro (fig. 3, type 2) est un conteneur de forme encore sphérique mais qui se rétrécit davantage vers le fond. La lèvre à marli présente une section quadrangulaire avec un profil extérieur concave ; le sommet de la lèvre horizontal ou légèrement rentrant est caractérisé par un chanfrein aussi bien à l'extrémité interne que, plus souvent, à l'extrémité externe, et la surface inférieure légèrement concave présente un angle extérieur souvent saillant. Le col, large, est massif et de forme cylindrique. Les anses présentent, selon l'endroit, un épaississement ou un aplatissement, ce qui leur donne une double section : circulaire dans la partie inférieure et elliptique dans la partie supérieure. Elles se rattachent au col juste au-dessous de la lèvre, avec un resserrement évident de leur section. Le pied, toujours de forme cylindrique, creux à l'intérieur et aplati au-dessous, est moins large que dans le type précédent et présente un profil arrondi en bas et caréné en haut ${ }^{26}$.

Le début de la production de ce type, déjà défini par C. G. Koehler comme une première évolution morphologique de la forme $\mathrm{A}$, est attesté à Corinthe peu avant le milieu du VII ${ }^{\mathrm{e}} \mathrm{s}$. av. J.-C. et, dans les contextes de référence les mieux documentés en Occident, quelques amphores de ce type sont datées de la deuxième moitié du siècle ${ }^{27}$. Le matériel de Camarine permet de fixer la limite chronologique la plus basse de la production de ce type au premier quart du VI ${ }^{\mathrm{e}} \mathrm{s}$. av. J.-C., en accord avec la chronologie proposée par C. G. Koehler et compte

23. Sur cet aspect, voir la mise au point récent de P. Pelagatti (2006) et de C. W. Neeft (2006) sur la céramique corinthienne.

24. Pour une liste d'amphores attribuables à ce type et datées entre le second et le dernier quart du VII e s. av. J.-C., voir Sourisseau 2006, n. 33. À ce répertoire, on peut aussi ajouter une amphore corinthienne A du «Tumulo della Speranza » de Caére, datée entre la fin du premier et le deuxième quart du VII ${ }^{\mathrm{e}} \mathrm{s}$. av. J.-C. sur la base du mobilier (Rizzo 1990, p. 55, III.1, fig. 345). Pour le fragment de Marseille daté du début du VIe s. av. J.-C., Sourisseau 1997, II, p. 130 , fig. $45, \mathrm{n}^{\circ} 1$.

25. Sourisseau 2006, p. 142.

26. Sourisseau 2006, p. 135-137, fig. 3, 8. Le type correspond à Koehler 1979 pl. 1, n 6, pl. 2, n 9-12, et Ead.1981, p. 452, pl. 98, c. 27. Pour une liste de ces attestations les plus archaïques, Sourisseau 2006, p. $136-137$, n $^{\circ} 37-39$. tenu des associations observées avec la céramique corinthienne ${ }^{28}$.

Le type 3 de Camarine-Rifriscolaro (fig. 3, type 3) correspond à un conteneur de forme sphérique, massive et assez trapue, dont la largeur maximale est souvent situé au niveau de l'épaule. La lèvre, du type à marli, présente une section quadrangulaire plutôt allongée et est caractérisée par une légère inclinaison vers l'extérieur. Le sommet de la lèvre peut donc présenter un profil légèrement convexe avec, à ses extrémités de petits chanfrein ou, au contraire, un léger épaississement au niveau du col. Le bord extérieur de la lèvre est convexe de manière moins accentuée et son épaisseur est sensiblement inférieure par rapport au type précédent. Le col, de forme cylindrique, est large et sa hauteur souvent inférieure à sa largeur. Pour les anses, de possibles variations ont été observées : dans certains cas, elles présentent, dans la partie supérieure, un épaississement lié à un écrasement latéral, ce qui leur confère, comme dans le cas du type précédent, une double section ronde et elliptique. Elles ont cependant une courbure moins accentuée, et donc un profil plus rectiligne. Elles sont généralement implantées, dans la partie haute, juste contre la surface inférieure de la lèvre. Dans d'autres cas, les anses conservent leur section ronde ainsi qu'un profil plus courbe et sont implantées, en haut, sous la lèvre. Le pied cylindrique, creux à l'intérieur et plat au-dessous, présente un profil caréné relativement bas ${ }^{29}$.

La chronologie de ce type, généralement daté entre la fin du premier et le deuxième quart du $\mathrm{VI}^{\mathrm{e}} \mathrm{s}$. av. J.-C. d'après le travail de C. G. Koehler, a été récemment confirmée et précisée sur la base des contextes de Camarine. Dans la nécropole de Rifriscolaro, en l'état actuel, cette forme n'est jamais attestée en association avec du matériel datable du premier ou du dernier quart $\mathrm{du} \mathrm{VI}{ }^{\mathrm{e}} \mathrm{s}$. av. J.-C., ce qui laisse supposer une diffusion entre 580/570 et 550/540 av. J.-C. ${ }^{30}$.

Le type 4 de Camarine-Rifriscolaro (fig. 3, type 4A-C) présente une réduction des dimensions et une finesse des parois ainsi que des détails morphologiques dénotant une évidente rupture au niveau technique de cette forme par rapport aux types précédents ${ }^{31}$. La lèvre est du type à marli à section quadrangulaire et, bien qu'elle soit parfois légèrement pendante vers l'extérieur, elle est généralement horizontale. Le col, cylindrique et

28. Sourisseau 2006, p. 136-137, n. 40 ; Koehler 1981,p. 451, n. 11, pl. 98, c ; Ead. 1992, n. 9, pl. 1.a ; Neeft 2006.

29. Sourisseau 2006, p. 137-138, fig. 4, 8. Le type correspond à Koehler 1979 pl. 3, n 20, et Ead. 1981, p. 452, pl. 98.d.

30. Koehler 1981, p. 452, pl. 98.d ; Sourisseau 2006, p. 138.

31. La plus grande finesse des amphores du type 4 a été observée notamment pour les variantes $4 \mathrm{~B}$ et $4 \mathrm{C}$. La variante $4 \mathrm{~A}$ peut, en revanche, encore présenter un aspect plus massif. 
élancé, présente une hauteur normalement supérieure à sa largeur. Les anses, minces, montrent une nette courbure supérieure caractérisée par un épaississement lié à un écrasement latéral déjà observé pour les deux types précédents. Elle est cependant aplatie, dans sa partie supérieure, au niveau de la lèvre à laquelle elle est en général directement attachée, et présente un rétrécissement évident de la section dans la zone centrale et non dans la zone inférieure. Le panse, nettement sphérique, se rétrécit sensiblement vers le pied qui, en fonction de certains détails morphologiques, a permis de distinguer trois variantes du type.

La variante $4 \mathrm{~A}$ est caractérisée par un pied de forme cylindrique à tronconique étroite, creux à l'intérieur, aplati au-dessous, au profil arrondi et se distinguant de la panse par un ressaut placé assez haut.

La variante 4B présente un pied creux de forme cylindrique étroite, aplati au-dessous, au profil rectiligne et se distinguant de la panse par un ressaut généralement bien marqué.

La variante $4 \mathrm{C}$ présente un pied cylindrique, creux à l'intérieur, aplati au-dessous, et le bord inférieur au profil légèrement caréné ${ }^{32}$.

La chronologie de ces trois formes a été située entre le milieu du $\mathrm{VI}^{\mathrm{e}}$ et le début $\mathrm{du} \mathrm{V}^{\mathrm{e}} \mathrm{s}$. av. J.-C. par C. G. Koehler qui, bien que manquant d'éléments de comparaison chronologique précis, a proposé de les considérer, sur la base du style, comme des productions se succédant l'une après l'autre suivant une évolution cohérente et linéaire ${ }^{33}$. Des découvertes récentes dans des contextes datés permettent maintenant, d'une part d'en établir un encadrement chronologique plus sûr confirmant pour partie la proposition de C. G. Koehler, et de l'autre, de supposer la coexistence partielle des trois formes, en écartant l'idée de leur succession dans le temps.

Le type 4 semble en effet avoir fait son apparition vers le milieu ou, au plus bas, dans le troisième quart du $\mathrm{VI}^{\mathrm{e}}$ s. av. J.-C., du moins dans sa variante $4 \mathrm{~A}$, comme le montrent certains éléments contextuels de Camarine, et avoir eu une continuité d'utilisation au moins jusqu'à 490 av. J.-C., date proposée pour l'épave de Gela qui transportait des amphores de ce type, notamment la variante $4 \mathrm{~B}^{34}$. Il serait toutefois hasardeux pour l'instant de déterminer une succession éventuelle des variantes

32. Sourisseau 2006, p. 138-139, fig. 5, 8. La variante A du type 4 de Camarine-Rifriscolaro trouve un parallèle dans Koehler 1981, pl. 98.e ; la variante 4B correspond à Ibid., pl. 98.f ; la variante 4C à Ibid., pl. 98.g.

33. Koehler 1979 p. 14-15.

34. Pour la nécropole de Camarina (T. 449 encore inédite), où une amphore de ce type se trouvait en association avec de la céramique corinthienne du milieu ou du troisième quart du $\mathrm{VI}^{\mathrm{e}} \mathrm{s}$. av. J.-C., voir du type 4 et de leur chronologie relative, compte tenu du fait que les trois variantes sont présentes sans distinction dans des contextes datables entre le milieu du $\mathrm{VI}^{\mathrm{e}}$ et le premier quart du $\mathrm{V}^{\mathrm{e}} \mathrm{s}$. av. J.-C. ${ }^{35}$.

Le type 5 de Camarine-Rifriscolaro (fig. 3, type 5) plutôt rare, est décrit comme un conteneur dont la morphologie générale est proche de celle des types 2 et 3 . Il a cependant été distingué du fait de l'absence des caractéristiques morphologiques de détail propres à chacun des deux types. La lèvre, du type à marli horizontal, présente une section quadrangulaire généralement sans chanfrein. Le col, de forme cylindrique, a une hauteur plus ou moins égale à la largeur. Les anses, à section ronde, sont implantées sur le col immédiatement au-dessous de la lèvre et descendent plus ou moins obliquement sur l'épaule. La partie inférieure de la panse se rétrécit progressivement en descendant vers le pied de forme cylindrique. Celui-ci est creux à l'intérieur et présente un profil caréné. Le fond peut être complètement plat ou présenter un profil caractérisé par une légère convexité centrale ${ }^{36}$.

La rareté de ce type d'amphore rend son encadrement chronologique particulièrement difficile. Les données dont nous disposons actuellement, provenant en particulier de contextes du sud de la France, suggèrent un arc chronologique de diffusion compris entre le milieu du $\mathrm{VI}^{\mathrm{e}}$ et le premier quart du $\mathrm{V}^{\mathrm{e}}$ S. av. J.-C. ${ }^{37}$.

Le type 6 de Camarine-Rifriscolaro (fig. 3, type 6) correspond à un conteneur encore sphérique mais à profil plus rétréci vers le bas par rapport aux types précédents du fait des dimensions plus réduites du pied. Celui-ci est toujours de forme cylindrique et creux à l'intérieur et se distingue maintenant nettement de la partie inférieure de la panse. Il est moins profond et est privé du profil externe caréné traditionnel. La lèvre est du type à marli, horizontal, ou plus souvent légèrement pendante, et présente une section trapézoïdale avec une légère concavité de son extrémité. Le col, de hauteur et largeur réduites, peut avoir une forme cylindrique, tronconique ou un profil concave. Les anses, plutôt fines, sont du type à section circulaire. Elles sont implantées, en haut, immédiatement au-dessous de la lèvre, tandis qu'en bas

Sourisseau 2006, p. 139, n. 47 ; pour l'épave de Gela, Panvini 2001, p. 131-132, pl. XXII, n 128-129, 132, et XXIII, n 135.

35. Pour un répertoire de ces contextes voir Sourisseau 2006, n. 48. J.-Chr. Sourisseau avance cependant l'hypothèse que la variante 4A ait fait son apparition de manière précoce, vers le milieu du $\mathrm{VI}^{\mathrm{e}} \mathrm{s}$. av. J.-C., pour disparaître au début, ou au plus tard, dans le courant du dernier quart du siècle, tandis que les variantes $4 \mathrm{~B}$ et $4 \mathrm{C}$, à son avis tout à fait contemporaines, auraient été fabriquées entre le dernier quart du VI' s. av. J.-C. et 490 av. J.-C.. (Ibid., p. 139).

36. Sourisseau 2006, p. 140, fig. 6, 8. Ce type pourrait correspondre à Koehler 1979 pl. 6, n 34.

37. Pour ces limites chronologiques et les contextes relatifs, voir Sourisseau 2006, p. 150, n. 50-51. 
la jonction sur l'épaule est très éloignée de la base du col, ce qui entraîne une allure oblique assez prononcée du profil des anses. La minceur des parois de ce type les rapproche des conteneurs du type $4^{38}$. D'après J.-Chr. Sourisseau, le type 6 illustre une forme autonome par rapport à la ligne évolutive des amphores corinthiennes A et, plus exactement, constituerait la première étape de la série A', qui, comme nous le verrons plus avant, se développe dans le courant du $\mathrm{V}^{\mathrm{e}} \mathrm{s}$. av. J.-C. ${ }^{39}$.

Pour la chronologie du type 6, C. G. Koehler a proposé, sur une base stylistique, les années 540-520 av. J.-C. Des découvertes récentes suggèrent de faire descendre au moins la limite chronologique plus basse de cette forme aux années 490-480 av. J.-C. Une amphore du type 6 faisait en effet partie de la cargaison de l'épave de Géla, datée précisément autour de $490 \mathrm{av.} \mathrm{J.-C.}{ }^{40}$. C'est aussi vers la fin du $\mathrm{VI}^{\mathrm{e}}$ et le début du $\mathrm{V}^{\mathrm{e}} \mathrm{s}$. av. J.-C. que renvoient les exemplaires de la nécropole de Camarine ainsi que ceux d'un contexte bien daté de Zancle. Bien qu'on ne manque pas d'attestations d'amphores du type 6 dans des contextes compris dans une fourchette chronologique plus ample, d'après J.-Chr. Sourisseau, la limite chronologique la plus haute doit aussi être abaissée autour de 510 av. J.-C. ${ }^{41}$.

Comme l'avait déjà pensé et proposé C. G. Koehler, sur une base stylistique, l'évolution morphologique des amphores corinthiennes $\mathrm{A}$ du $\mathrm{VI}^{\mathrm{e}} \mathrm{s}$. av. J.-C., illustrée par les découvertes de Camarine, confirme sur une base archéologique, l'existence d'un développement progressif de la forme, des types les plus anciens jusqu'aux types datés de la période de transition entre la fin du $\mathrm{VI}^{\mathrm{e}}$ et le début du $\mathrm{V}^{\mathrm{e}} \mathrm{s}$. av. J.-C. Les contextes de Camarine permettent également de mieux distinguer la morphologie de chacun des types et d'en préciser la chronologie, ainsi que d'observer la coexistence de variantes contemporaines du même type qui se situent à la transition entre le $\mathrm{VI}^{\mathrm{e}}$ et le $\mathrm{V}^{\mathrm{e}} \mathrm{s}$. av. J.-C.

Il est intéressant de noter que pour ce moment problématique du passage au $\mathrm{V}^{\mathrm{e}} \mathrm{s}$. av. J.-C., M. Lawall ${ }^{42}$, sur la base de l'étude des amphores corinthiennes A de l'Agora d'Athènes et notamment sur l'observation de l'évolution des lèvres, aboutit à des conclusions analogues.

38. Sourisseau 2006, p. 140-141, fig. 7-8. Ce type correspond à Koehler 1979 p. 16, pl. 6, n 35-36, 38.

39. Sourisseau 2006, n. 57.

40. Panvini 2001, p. 131, pl. XXII, $\mathrm{n}^{\circ} 130$

41. Pour Camarine : T. 611A de la nécropole de Rifriscolaro, encore inédite (Sourisseau 2006, n. 54, avec un répertoire d'autres contextes de la seconde moitié du VI ${ }^{\mathrm{e}}$ ou la première moitié du V $\mathrm{V}^{\mathrm{e}} \mathrm{s}$. av. J.-C.) ; pour Zancle, Tigano 1999, p. 146, fig. 9, n 127.

42. Lawall 1995, p. 58-61, fig. 9-10.
En suivant le schéma évolutif proposé par C. G. Koehler, il observe que le phénomène d'inclinaison de la lèvre vers l'extérieur semble commencer dans le premier quart $\mathrm{du} \mathrm{V}^{\mathrm{e}} \mathrm{s}$. av. J.-C. Toutefois, les exemplaires provenant des contextes clos de l'Agora suggèrent une période, si brève soit-elle, de coexistence des lèvres à marli horizontal et à marli oblique, qu'il distingue respectivement sous l'appellation des variantes $\mathrm{A} / 1$ et $\mathrm{A} / 2$.

La variante $\mathrm{A} / 1$ (fig. 4) serait caractérisée par des détails morphologiques encore archaïsants, tels la lèvre à marli horizontal ou légèrement oblique, un profil fin, ni moulurée ni chanfreiné comme pour les types plus anciens, et un pied cylindrique à profil externe rectiligne. La variante A/1 de l'Agora pourrait correspondre aux types $4 \mathrm{~A}$ et $4 \mathrm{~B}$ de Camarine-Rifriscolaro à lèvre à marli horizontal.

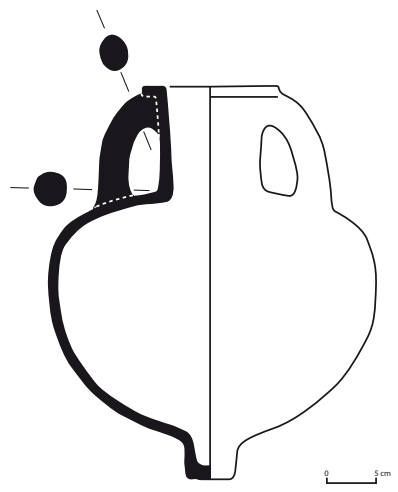

Fig. 4. Amphore de type corinthien A. Athènes, Agora, inv. P12795 (d'après Koehler 1978a, fig. 1.b).

La variante A/2 (fig. 5-6) présente des éléments plus innovants par rapport aux types plus archaïques et même à la variante $\mathrm{A} / 1$, tels que la lèvre à marli oblique vers l'extérieur et la base cylindrique étroite à profil externe concave et pied chanfreiné. Ces éléments spécifiques seraient à la base de l'évolution morphologique de la production corinthienne du $\mathrm{V}^{\mathrm{e}} \mathrm{s}$. av. J.-C., dont l'évolution dans le courant du siècle serait caractérisée par une évolution régulière et continue, sans ruptures morphologiques nettes. L'évolution typo-chronologique ne serait perceptible que sur la base de la variation des proportions entre les différentes parties du conteneur ${ }^{43}$.

43. Le conservatisme morphologique de la variante $\mathrm{A} / 2$ dans le courant du $\mathrm{V}^{\mathrm{e}} \mathrm{s}$. av. J.-C. est documenté notamment par deux exemplaires complets de Corinthe : C-37-2037 (Koehler 1978a fig. 1.c ; Ead. 1981, pl.98.g) et C-34-933 (Koehler 1981, fig. 1.d, pl.98.h), datés respectivement du début et du troisième quart du siècle. Pour ces deux amphores, voir en dernier lieu Lawall 1995, p. 61, respectivement fig. 9 et 10 . 


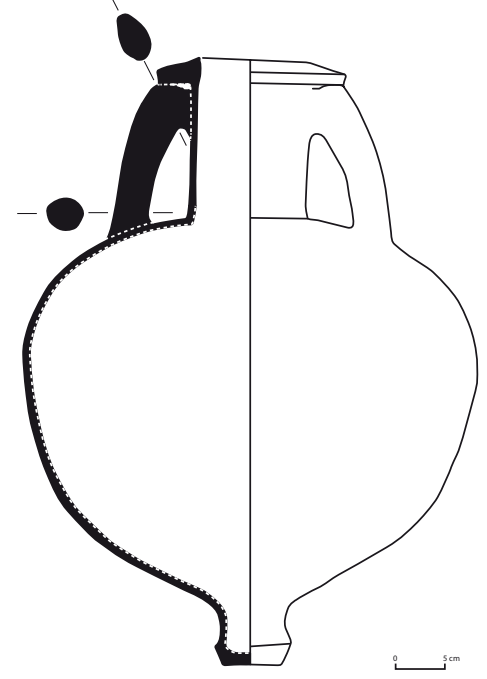

Fig. 5. Amphore de type corinthien A. Corinthe, inv. C-37-2037 (d'après Koehler 1978a, fig. 1.c).

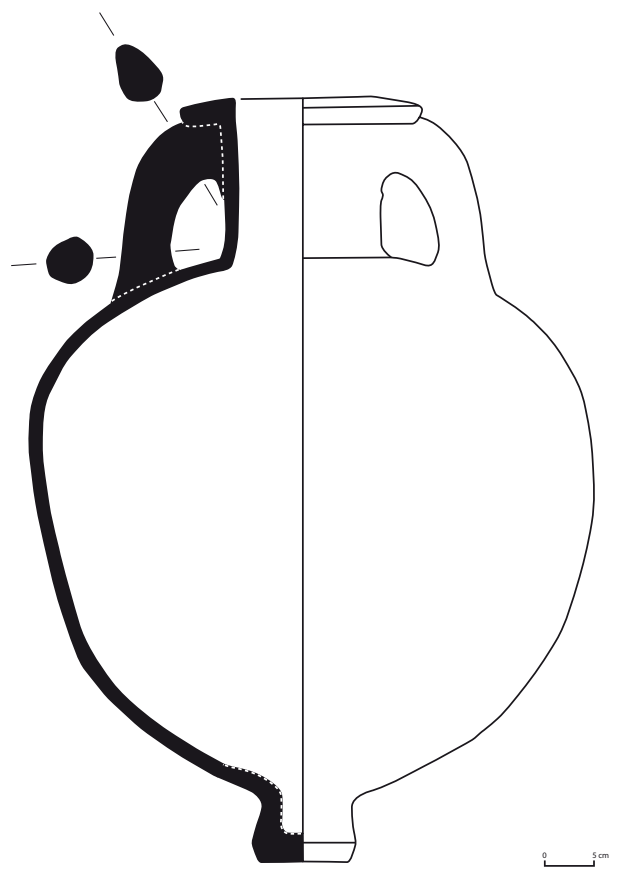

Fig. 6. Amphore de type corinthien A. Corinthe, inv. C-34-933 (d'après Koehler 1981, fig. 1.d).

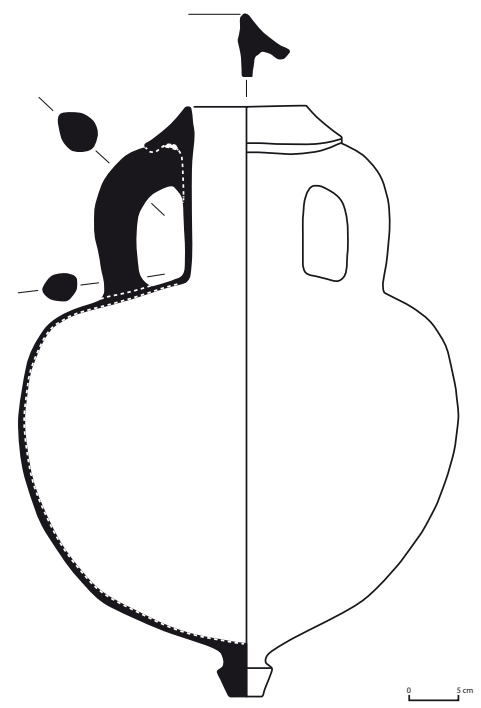

Fig. 7. Amphore de type corinthien A. Corinthe, inv. C-71-393

(d'après Koehler 1978a, fig. 1.e)
La variante A/2 de l'Agora, identifiée par M. Lawall sur la base de l'observation des lèvres, et la variante $4 \mathrm{C}$ de Camarine-Rifriscolaro, reconnue par J.-Chr. Sourisseau sur la base de la variation des pieds, sont équivalentes. Les travaux parallèles des deux auteurs s'accordent dans l'identification commune à leurs types respectifs de l'exemplaire C-37-2037 de Corinthe ${ }^{44}$.

44. Pour l'amphore C-37-2037 de Corinthe, cf. Koehler 1798 a, fig. 1.c ; Ead. 1981, pl. 98.g.
Les exemplaires de la variante $\mathrm{A} / 1$ de $\mathrm{M}$. Lawall sont surtout documentés dans des contextes clos de la fin $\mathrm{VI}^{\mathrm{e}}$ s. av. J.-C. ${ }^{45}$, ainsi que dans l'épave de la PointeLequin $1 \mathrm{~A}$, datée vers la fin du VI ${ }^{\mathrm{e}} \mathrm{s}$. av. J.-C. ${ }^{46}$, mais d'autres proviennent de contextes de l'Agora datés au plus tard vers 480 av. J.-C. ${ }^{47}$. Quelques exemplaire

45. Koehler 1979, p. 14.

46. Long, Miro, Volpe 1992, p. 227, fig. 46.2-3.

47. Voir notamment les ensembles D 15:1 et H 12:15. Sur ces dépôts, cf. Lawall 1995, p. 60, n. 14. 
présents dans d'autres contextes de l'Agora, clos vers 460-450 av. J.-C., mais contenant beaucoup d'objets plus anciens, ne peuvent être considérés comme des preuves suffisamment fiables de l'existence de cette variante au cours de la première moitié du $\mathrm{V}^{\mathrm{e}} \mathrm{s}$. av. J.-C. ${ }^{48}$.

Le début de la production de la variante $\mathrm{A} / 2$ dès la fin du $\mathrm{VI}^{\mathrm{e}}$ s. av. J.-C. est documentée par sa présence dans les mêmes contextes de l'Agora où est attestée la variante $\mathrm{A} / 1$ (dépôts $\mathrm{D}$ 15:1 et $\mathrm{H}$ 12:15) ${ }^{49}$, ainsi que dans un puits de Corinthe publié par M. Campbell ${ }^{50}$. L'existence de cette variante pendant tout le $\mathrm{V}^{\mathrm{e}} \mathrm{s}$. av. J.-C. est désormais démontrée par des attestations dans des contextes de Corinthe de la fin du siècle ${ }^{51}$ et dans cinq dépôts contemporains de l'Agora : M 17:4 ; C 18:4c ; A 20:6 ; B 13:5; B 13:6 ${ }^{52}$. Elles sont néanmoins beaucoup plus rares que les amphores de type A'.

$\mathrm{Au} \mathrm{IV}^{\mathrm{e}}$ s. av. J.-C. (fig. 7), les principales évolutions morphologiques enregistrées concernent la lèvre et le pied, alors que le profil globulaire de la panse reste inchangé tout au long du siècle, jusqu'à la disparition de la production au début du III $\mathrm{e}$ s. av. J.-C ${ }^{53}$. La lèvre, du type à marli de section triangulaire, avec un profil externe très oblique, une section plutôt massive et un fort écrasement du bord dû à l'attache haute des anses. Les anses, dont les l'attache inférieure est légèrement rentrante, gardent encore la double section due à l'écrasement de leur partie supérieure. Le pied, maintenant nettement séparé de la panse par un rétrécissement net à sa jonction avec le fond, prend la forme d'un bouton, de dimensions considérablement réduites, plein dans sa partie interne, au profil externe caréné et à la partie inférieure étroite et aplatie.

\subsubsection{Centres de production}

Le conservatisme propre aux amphores corinthiennes A, caractérisé dans le domaine morphologique par la continuité d'une tradition qui, à partir de la période géométrique, se structure à travers des changements graduels et plutôt lents jusqu'au début du $\mathrm{III}^{\mathrm{e}} \mathrm{s}$. av. J.-C., s'accorde parfaitement avec les caractéristiques technologiques de leur fabrication. On retiendra notamment

48. Voir par exemple les dépôts M 17:4 et A 18-19:1. Pour ces considérations, voir Lawall 1995, p. 60, n. 15.

49. Lawall 1995, p. 60, n. 17.

50. Campbell 1938, $\mathrm{n}^{\circ} 201$.

51. Pease 1937, p. 302-302, n 200 ; Lawall 1995, p. 61, n. 19.

52. Lawall 1995, p. 60-61, n. 18.

53. La forme globulaire des amphores corinthiennes de type A du IV $\mathrm{IV}^{\mathrm{e}}$ s. est clairement identifiable, notamment avec l'exemplaire publié par Koehler 1978b fig. 1 ; Ead. 1978a, fig. 1.e ; Ead. 1992, pl. 2.a, et n. 15, avec bibliographie relative à d'autres attestations. l'usage d'une technique de montage réservant une large part de modelage manuel associée à un tournage seulement partiel ${ }^{54}$, pendant presque toute la période de production et une persistance sur la longue durée des mêmes caractéristiques de la pâte. Les amphores corinthiennes présentent en effet une pâte bien reconnaissable qui garde des caractéristiques constantes pendant toute la période de leur production. L'analyse visuelle montre, sur la surface extérieure, une argile aux couleurs variables, avec des nuances qui vont du beige-jaune au beige-orange (MSC 7.5YR7/4-7/6), tandis que la section peut être grise (MSC 5YR6/1) avec des couches externes rougeâtres (MSC 2.5YR6/6-7.5YR7/6), entièrement rouge, ou entièrement grise. Sa matrice argileuse a une consistance particulièrement dure et très fine et présente de nombreuses inclusions de dégraissants, de dimensions petites, moyennes et grandes et aux angles vifs, principalement de couleur brun-orange (mudstone), gris (tuffite) et blanc ${ }^{55}$. Comme on l'a observé, il s'agit d'un type de pâte très proche de celle qu'on utilisait à Corinthe pour la réalisation de pithoi, de terres cuites architectoniques, ainsi que de lampes et de lécythes destinés à contenir de l'huile ${ }^{\mathbf{5 6}}$.

L'argile des amphores corinthiennes de type A a fait l'objet de nombreuses analyses physico-chimiques et pétrographiques. Dans les années 1960-70, les analyses pétrographiques et NAA menées par M. Farnsworth et décrivant la pâte des amphores corinthiennes A comme 《tempered with a sedimentary rock called mudstone ${ }^{57}$, avaient permis de situer l'origine de l'argile dans un secteur situé sur les pentes de l'Acrocorinthe et de montrer que d'autres productions céramiques de Corinthe présentaient les même caractéristiques physiques ${ }^{58}$. Plus récemment, les analyses pétrographiques de I. K. Whitbread ${ }^{59}$ ont confirmé les résultats de M. Farnsworth en ce qui concerne la relation entre la pâte des amphores corinthiennes A et l'argile de Corinthe ${ }^{60}$.

54. Sur ce point, voir Vandiver, Koehler 1986, p. 182, 187.

55. Pour les caractéristiques de la pâte des amphores corinthiennes de type A, voir essentiellement Jones 1986, p. 712-720, Whitbread 1986 , p. 97-100 et $I d$. 1995, notamment p. 268-270. Pour une description synthétique et une analyse visuelle, voir également Koehler 1992.

56. Pour des observations sur les pithoi et leur analogie avec les amphores de type A, cf. Berlingò 1995. Pour la similitude entre la pâte des amphores corinthiennes A et d'autres produits corinthiens en terre cuite, cf. Koehler 1979 p. 3-4 (pour les terres-cuites architecturales); Ead. 1981, p. 452 (pour les conteneurs à huile).

57. Farnsworth 1964, p. 224-225.

58. Farnsworth 1970, p. 10-11 ; et Farnsworth, Perlman, Asaro 1977, p. 457, 459.

59. Whitbread 1995.

60. Pour de récentes analyses pétrographiques d'amphores corinthiennes A, voir également Iceland 1988, p. 748-755., in Morter, Leonard 1998. 
En outre, l'analyse des inclusions de mudstone et la vérification de la présence de deux groupes distincts par leurs dimensions (coarse angular mudstones et mudstone breccia) a montré qu'une partie au moins de ces inclusions présentes dans la pâte des amphores corinthiennes A était spécialement ajoutée comme liant à une matrice argileuse soigneusement pétrie au cours d'un processus de fabrication particulièrement soigné et complexe ${ }^{61}$.

Les analyses pétrographiques de M. Farnsworth et I. K. Whitbread confirment donc l'origine corinthienne des amphores de type $\mathrm{A}$, déjà supposée sur la base des nombreux exemplaires restitués par les fouilles de Corinthe et surtout du lien morphologique et technologique étroit avec la tradition locale des storage jars d'époque géométrique.

Si tous ces éléments confirment bien l'attribution à Corinthe des amphores de la forme $\mathrm{A}$, il faut néanmoins signaler, en restant prudent sur leur pertinence, les résultats d'analyses NAA publiés par une équipe de l'Université de Manchester qui localisent les carrières d'argile de la plus grande partie de leurs échantillons de forme A dans l'aire de Mégalopolis ${ }^{62}$. Mieux documentées par ailleurs dans les publications archéologiques, notamment en Grande Grèce, en Sicile et en Albanie, on signalera également l'existence de productions de type A dont la morphologie ainsi que la pâte qui les composent ne correspondent pas, de toute évidence, à des fabrications corinthiennes ${ }^{63}$. Elles sont au moins pour partie issues d'ateliers grecs occidentaux, notamment de Calabre, alors que des productions indigènes sicéliotes plus rares ont également été identifiées ${ }^{64}$. Quoi qu'il en soit, toutes ces amphores non corinthiennes se distinguent maintenant assez facilement, sur la base de critères à la fois morphologiques et de l'aspect de leur pâte, des productions véritablement corinthiennes.

61. Sur ce point, voir en particulier Whitbread 1986, p. 97-100, et Id. 1995 , p. 268-270.

62. Lawall 1995, p.62-63 avec commentaires et références bibliographiques.

63. Cf. Bonghi Jovino, Donceel 1969,p. 106 et Albore Livadie 1985, p. 139 (Nola); Koehler 1979 p. 29, n. 25 (Sélinonte); Pancrazzi 1982, p. 334 (Pise) ; Albanese Procelli 1997b, p. $95-96$ (différents sites de Sicile); Vassallo 2003, p. 333-336 (Himère); Sourisseau 2002, p. 548, fig. 61, Id.2006, p. 134 (Camarine-Rifriscolaro); Mano 1971 et Whitbread 1995, p. 283 (Apollonia d'Illyrie).

64. Sourisseau 2011, p. 188-189, fig. 6, 13 et 14. Ces amphores, désignées sous l'appellation de type $1 \beta$ des productions grecques occidentales et attribuées par l'auteur à la production de Sybaris, constituent une série maintenant bien documentée par les découvertes de Camarine-Rifriscolaro. Les productions sicéliotes sont beaucoup plus rares et ne semblent pas diffusées en dehors d'un cadre régional restreint : Sourisseau 2011,p. 197-200, fig. 16.

\subsection{Le type $A^{\prime}$}

Respectant la distinction initiale de deux différentes familles d'amphores de production corinthienne, les formes $\mathrm{A}$ et $\mathrm{B}$, c'est notamment à la suite des études conduites sur le matériel du Punic Amphora Building de Corinthe que C. G. Koehler identifie une troisième production locale qu'elle considère, du fait de ses étroites affinités morphologiques et technologiques avec la forme $\mathrm{A}$, comme assimilable à la même série en la désignant sous l'appellation de type A'. Cette distinction est par ailleurs rendue nécessaire vis-à-vis de la série de type A par la mise en évidence de différences morphologiques sensibles ainsi que par l'observation d'une pâte particulière constituant ces objets. Cette pâte est perçue comme étant composée de l'argile fine et claire des productions de type $\mathrm{B}$, mais avec les mêmes inclusions de mudstone caractéristiques des amphores de type $\mathrm{A}^{65}$. Les exemplaires les plus anciens du type A' sont datés de la fin $\mathrm{du} \mathrm{VI}^{\mathrm{e}} \mathrm{s}$. av. J.-C., mais ce n'est qu'à partir du milieu du $V^{e}$ s. av. J.-C. que leur distinction par rapport au type A se dessine plus clairement. Leur production ne se substitue donc pas à celle des amphores de type A, mais se développe parallèlement jusqu'à la disparition du type A, vers la fin du IV et/ou le début du III ${ }^{\mathrm{e}} \mathrm{s}$, av. J.-C., et le remplace au $\mathrm{III}^{\mathrm{e}}$ et dans la première moitié du II ${ }^{\mathrm{e}}$ s. av. J.-C., jusqu'à la destruction de la cité en 146 av. J.-C. Pendant cette période comprise entre le début du $\mathrm{III}^{\mathrm{e}}$ et le milieu du $\mathrm{II}^{\mathrm{e}} \mathrm{s}$. av. J.-C., on évoque une augmentation relative des exportations d'amphores corinthiennes A', sur la base des cargaisons des épaves de Stentinello, près de Syracuse, et de Savelletri, près de Brindes ; c'est pourquoi C. G. Koehler considère que le type A' constituait le conteneur commercial corinthien essentiellement destiné à l'exportation ${ }^{66}$. Les contextes de la Méditerranée occidentale auraient été la destination principale de ce commerce, ce que les découvertes récentes des nécropoles de la chora de Métaponte pourraient confirmer, au moins pour le $\mathrm{V}^{\mathrm{e}} \mathrm{s}$. av J.-C. En effet la plupart des amphores attestées dans les nécropoles de Pantanello et Saldone sont attribuables à la forme corinthienne A'. Seuls deux exemplaires ont été attribués à la forme $\mathrm{A}^{67}$.

65. La définition de cette série de type A', outre Koehler 1979, p. 16-19, est exposée clairement dans Koehler 1981, notamment p. 454-457, et plus récemment dans Koehler 1992, p. 270.

66. Sur ces considérations, cf. Koehler 1979 p. 731 ; Ead. 1981, p. 454, 457 ; et, plus récemment, Ead. 1992, p. 270.

67. Exemplaires des T. 191-3 et T. 293-2 (Morter, Leonard 1998, p.734, 741 et 745). Outre ces deux exemplaires provenant de tombes, deux cols d'amphores corinthiennes A d'époque archaïque (CD 289-61 et CD 289-62) ont également été répertoriés (Ibid., p. 734, 744-745). 
On retiendra donc qu'il existe d'importantes affinités morphologiques entre les conteneurs contemporains de type $\mathrm{A}$ et de type $\mathrm{A}^{\prime}$ même si, dans la première moitié du $\mathrm{V}^{\mathrm{e}}$ s. av. J.-C., les amphores de type A' se distinguent par un profil général plus élancé et par quelques détails morphologiques. À partir du milieu du $\mathrm{V}^{\mathrm{e}} \mathrm{s}$. av. J.-C. l'aspect de la pâte des amphores de type A' se caractérise aussi par un type de pâte différent, constitué par une argile plus claire, dans les nuances de jaune et de rose, par une raréfaction des éléments de dégraissant grossiers (mudstone) et une fréquence plus élevée d'inclusions lithiques plus fines.

\subsubsection{Bilan des études}

Les premières attestations d'amphores corinthiennes A' se situent vers la fin du VI ${ }^{\text {e }}$ s. av. J.-C. ${ }^{68}$, mais ces exemplaires les plus anciens partagent encore plusieurs détails avec la production contemporaine des amphores de type $\mathrm{A}$, plus spécialement avec la variante $\mathrm{A} / 2 \mathrm{de}$ M. Lawall. La véritable différenciation morphologique par rapport à la version contemporaine de la forme $\mathrm{A}$ ne se réalise que vers le milieu du $\mathrm{V}^{\mathrm{e}} \mathrm{s}$. av. J.-C., lorsque la pâte de la série $\mathrm{A}^{\prime}$ commence à présenter des caractéristiques différentes de celles de la série A. On a aussi observé récemment qu'à partir de la même période, les dimensions des amphores de la série A' ont tendance à diminuer ${ }^{69}$.

Entre la fin du $\mathrm{VI}^{\mathrm{e}}$ et le milieu du $\mathrm{V}^{\mathrm{e}} \mathrm{s}$. av. J.-C. (fig. 8) les amphores corinthiennes A' présentent une panse assez allongée et de forme ovoïde dont on situe le diamètre maximum au niveau de l'épaule arrondie et légèrement fuyante. Le col cylindrique, plus étroit et plus haut, conserve le profil rectiligne de la forme A. Les anses présentent une section circulaire sur toute leur hauteur sans le rétrécissement médian caractérisant la forme $\mathrm{A}$ à partir du $\mathrm{V}^{\mathrm{e}} \mathrm{s}$. av. J.-C. La lèvre, du type à marli oblique, de section triangulaire, comme celle de la forme A contemporaine (variante $\mathrm{A} / 2$ de $\mathrm{M}$. Lawall), est écrasée sur l'attache supérieure des anses. Le pied, plutôt court, garde encore la forme cylindrique au profil concave et chanfreiné de la série A, mais il a un diamètre plus étroit et surtout il se présente désormais plein à l'intérieur ${ }^{70}$.

68. Koehler 1979 p. 16, n 36-38 ; Ead. 1992, p. 270.

69. Morter, Leonard 1998.

70. Pour la description de la forme du début de la série, voir Koehler 1981, p. 454-456 ; Ead. 1992, p. 270. Les amphores corinthiennes A' datées avant le milieu du $\mathrm{V}^{\mathrm{e}} \mathrm{s}$. av. J.-C. sont assez rares dans la bibliographie disponible. Pour les exemples les plus anciens cités par C. G. Koehler, de Corinthe et de Camarine (cf. Ead. 1978, p. 16, $\left.\mathrm{n}^{\circ} 36-38\right)$; pour les deux exemplaires les mieux conservés datés dans

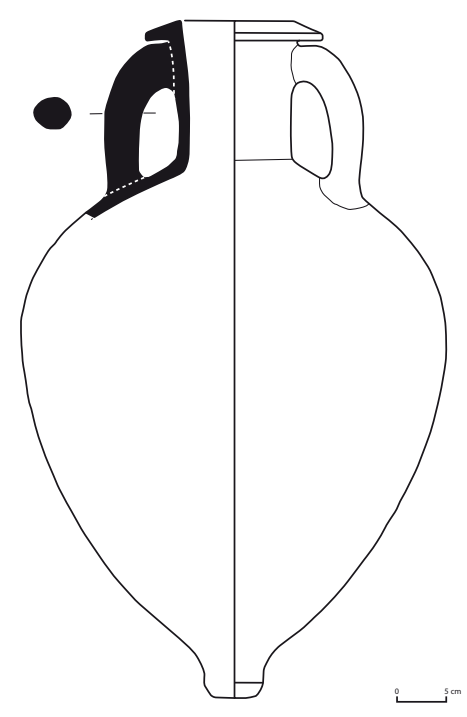

Fig. 8. Amphore de type corinthien A'. Lipari, nécropole, Tombe 1102 (d'après Cavalier 1985, fig. 4.a).

La chronologie du début de production de la série A' dans cette variante plus ancienne est située par C. G. Koehler à la fin du VI ${ }^{\mathrm{e}}$ s. av. J.-C. sur la base de la présence d'un exemplaire dans un contexte corinthien daté entre la fin du $\mathrm{VI}^{\mathrm{e}}$ et le début du $\mathrm{V}^{\mathrm{e}} \mathrm{s}$. av. J.-C. ${ }^{71}$. La fabrication et la diffusion de la forme jusqu'au milieu $\mathrm{du} \mathrm{V}^{\mathrm{e}} \mathrm{s}$. av. J.-C. est confirmée par la documentation de la première phase du Punic Amphora Building de Corinthe $^{72}$, datée du second quart du siècle, ainsi que par deux contextes de l'Agora d'Athènes (C 9:6 et B 18:15), clos avant 450 av. J.-C. ${ }^{73}$.

À partir du milieu du $\mathrm{V}^{\mathrm{e}} \mathrm{s}$. (fig. 9), la lèvre à marli oblique et section triangulaire de la série A' devient plus épaisse et plus haute. L'inclinaison du sommet de la lèvre est accentuée ainsi que l'écrasement de l'attache supérieure des anses. Tous ces éléments permettent de distinguer nettement l'évolution du type des formes plus anciennes de type A' et aussi des formes contemporaines de type A. Les anses, au profil arqué, présentent une section ronde sur toute leur extension, mais le diamètre de la partie inférieure est nettement moins important que celui de la partie supérieure. Le col cylindrique, plus haut et étroit, perd son profil droit qui devient légèrement

la première moitié du V ${ }^{\mathrm{e}} \mathrm{S}$. av. J.-C. à Lipari, cf. Cavalier 1985, cat. $\mathrm{n}^{\circ}$ 5-6, fig. 4, a-b (pour leur datation récemment revue sur la base de parallèles, voir Lawall 1995, p. 65).

71. Campbell 1938, n² 202-203; Koehler 1979 p. 16, n 37-38.

72. Koehler 1981, p. 456.

73. Lawall 1995, p. 65. 


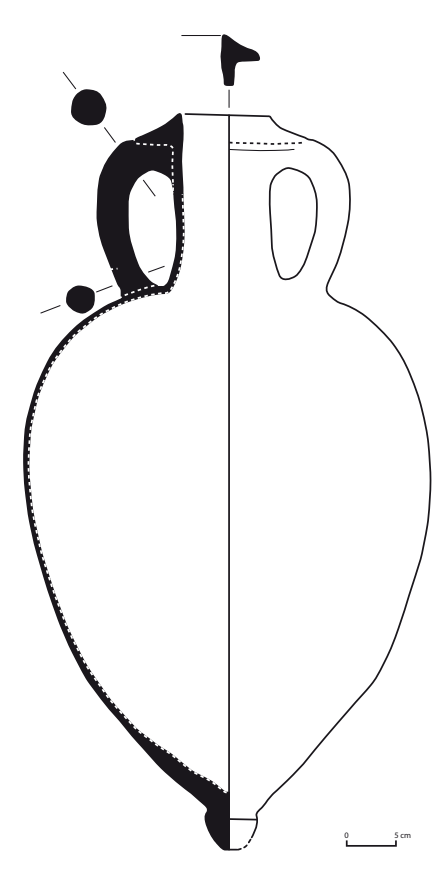

Fig. 9. Amphore de type corinthien A'. Corfou, Musée Archéologique, sans inv. (d'après Koehler 1978a, fig. 1.d).

concave. Le pied, plein et bien distinct de la panse par un resserrement à la base, présente maintenant une forme conique brève, du type en bouton ${ }^{74}$.

La datation de cette forme dans la seconde moitié du $\mathrm{V}^{\mathrm{e}} \mathrm{s}$. av. J.-C. a été établie grâce à la découverte de plusieurs exemplaires dans des contextes corinthiens datés du troisième quart du $\mathrm{V}^{\mathrm{e}} \mathrm{s}$. av. J.-C. ${ }^{75}$ et par plusieurs références à des découvertes occidentales, essentiellement funéraires, notamment la tombe 350 de Pontecagnano et plusieurs sépultures de la chora de Métaponte ${ }^{76}$. La persistance de cette forme dans le courant du $\mathrm{IV}^{\mathrm{e}} \mathrm{s}$. av. J.-C. a été proposée par C. G. Koehler sur la base d'un exemplaire hors contexte, récupéré en mer près de Corcyre ${ }^{77}$.

Les nombreuses amphores corinthiennes A' réutilisées pour les sépultures à enchytrismos ou déposées dans les tombes d'adultes des nécropoles de Pantanello et Saldone, dans la chora de Métaponte, ont permis de confirmer l'évolution générale de la forme déjà suggérée par C. G. Koehler pour le $\mathrm{V}^{\mathrm{e}} \mathrm{s}$. Toutefois, le schéma évolutif typo-chronologique, tel qu'il est présenté par

74. Pour la forme des amphores corinthiennes A' de la seconde moitié du V e s. av. J.-C., cf. Koehler 1978a, p. 236, fig. 1.d, et 3.d ; Ead. 1981, p. 456, fig. 1.b, c, pl. 99.g; Ead. 1992, pl. 2.b.

75. Pour les contextes de Corinthe, cf. Pease 1937, p. 302-303, fig. 34, n 199 ; Koehler 1981, p. 456, n. 26.

76. Di Sandro 1981b, p. 6, fig. 1 (Pontecagnano); Morter, Leonard 1998, fig. 17.3 (chora de Métaponte).

77. Koehler 1978a, fig. 1.d et fig. 3.d ; Ead. 1992, pl. 2.b.
C. G. Koehler n'est peut-être pas aussi simple et linéaire. Ainsi, la riche documentation de ces nécropoles permet également de mettre en évidence l'existence de variantes contemporaines qui associent différentes combinaisons du col, de la panse et du pied (fig. 10). L'existence de ces variantes rend donc extrêmement difficile l'établissement d'une séquence typo-chronologique plus serrée au cours du $\mathrm{V}^{\mathrm{e}} \mathrm{s}$. av. J.-C. La seule observation qu'il est possible d'ajouter est d'ordre tout à fait général et concerne la tendance à la diminution de la taille des amphores, à partir du milieu du $\mathrm{V}^{\mathrm{e}} \mathrm{s}$. av. J.-C. En effet, si les exemplaires datés de la première moitié du $\mathrm{V}^{\mathrm{e}} \mathrm{s}$. dépassent en général les $60 \mathrm{~cm}$ de hauteur et les $39 \mathrm{~cm}$ de diamètre maximum de la panse, ceux de la seconde moitié du siècle ne s'inscrivent plus que dans une série de valeurs comprises entre 52 et $72 \mathrm{~cm}$ de hauteur et entre 32 et $45 \mathrm{~cm}$ de diamètre maximum de la panse ${ }^{78}$. C'est un critère typo-chronologique tout à fait intéressant et qui semble pertinent, mais qu'il est difficile de mettre en œuvre dans le cadre d'une étude sur du matériel fragmentaire.

\subsubsection{Centres de production}

À l'exception des exemplaires du début de série qui semblent présenter la même argile et les inclusions de mudstone des amphores de type $\mathrm{A}$, la pâte des amphores corinthiennes A', à partir du milieu du $\mathrm{V}^{\mathrm{e}} \mathrm{s}$. av. J.-C., apparaît différente, plus proche de la plupart des types communs de la céramique de Corinthe. Une simple analyse visuelle permet d'observer que la couleur de l'argile sur la surface extérieure varie entre le jaune clair et le rose pâle (MSC 10YR8/2-8/3-7.5YR7/4), tandis qu'en section elle est généralement rose (MSC 5YR8/47.5YR7/4). La matrice argileuse est fine et présente des inclusions minuscules de quartz et de silex ainsi que des fragments de mudstone.

Les différents types de pâte des amphores corinthiennes $A^{\prime}$ ont été eux aussi soumis aux analyses chimiques et pétrographiques suite à l'identification du type par C. G. Koehler et ont abouti aux mêmes conclusions que pour le type $\mathrm{A}^{79}$. Ainsi, parmi les échantillons analysés par M. Farnsworth et dont l'origine corinthienne était confirmée par la composition chimique, il y avait peut-être deux exemplaires du type $\mathrm{A}^{\mathbf{8 0}}$, mais ce n'est qu'avec les analyses comparées de I. K. Whitbread qu'on a eu la certitude de l'origine

78. Morter, Leonard 1998, p. 733-734, fig. 17.1-17.3.

79. Pour les analyses effectuées sur la pâte des amphores corinthiennes A', cf. Whitbread 1995, notamment p. 270-274.

80. Farnsworth, Perlman, Asaro 1977, p. 457, 459, 463. 

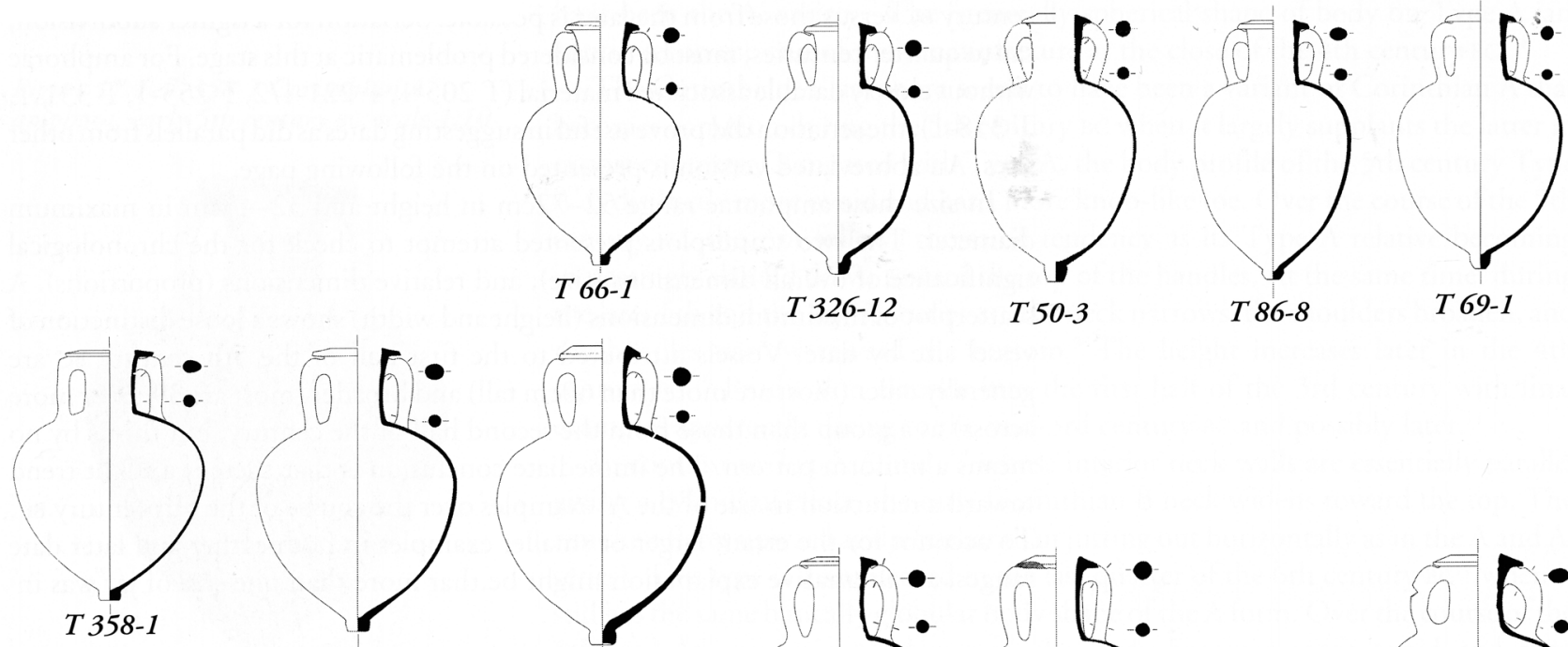

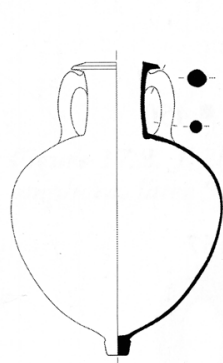

T 81-1
T266-1

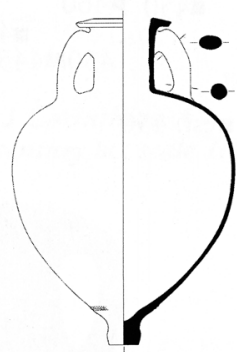

T 206-7
T 326-12

T 86-8

T 69-1

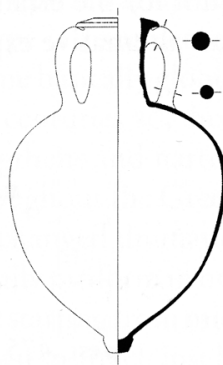

T 240-1

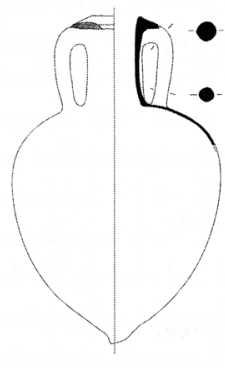

T 209-16

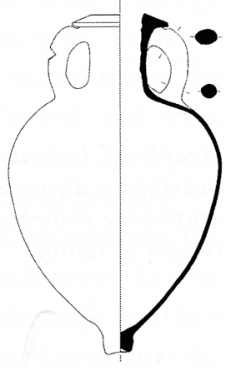

SS 24-2

ca. 490

ca. 460

ca. 450

ca. 440

ca. 430

ca. 420

ca. 400

Fig. 10. Tableau des variantes des amphores corinthiennes A' des nécropoles de la chora de Metaponto dans le courant du Ve s. av. J.-C. (échelle 1/20) (d'après Morter, Leonard 1998, fig. 17.3).

locale de la pâte caractéristique du type A'. Les analyses de I. K. Whitbread ont aussi permis d'isoler deux groupes d'échantillons qu'on peut différencier initialement sur la base de la technique de préparation de l'argile. Le premier groupe, correspond à des échantillons associés à des amphores corinthiennes A' du type le plus ancien. Ce groupe présente les mêmes caractéristiques que les échantillons du type A, notamment la même présence d'inclusions de mudstone complémentaires en plus de celles déjà naturellement contenues dans la matrice argileuse. Le second groupe correspond, quant à lui, à des échantillons d'amphores du type A' plus tardives. L'analyse de ce groupe révèle moins d'attention et de soin apportés dans la composition de la matrice argileuse, qui ne contient que les inclusions de mudstone déjà naturellement présentes.
Sur la base de ces résultats, $\mathrm{H}$. Iceland a proposé la distinction entre amphores corinthiennes type A et type A' des nécropoles de la chora de Métaponte ${ }^{81}$.

\subsection{Contenu}

L'utilisation des amphores du type A pour le transport de l'huile a été suggéré par C. G. Koehler sur la base de l'observation de la pâte, très proche voire identique de celle de lampes, lécythes et autres conteneurs à huile fabriqués à Corinthe aux $\mathrm{V}^{\mathrm{e}}$ et $\mathrm{IV}^{\mathrm{e}} \mathrm{s}$. av. J.-C. Ce type de pâte a été considéré, d'une part, apte à résister à l'érosion

81. H. Iceland, in Morter, Leonard 1998, p. 748-753. 
provoquée par ce produit sur les parois de l'amphore, et d'autre part, suffisamment imperméable à un contenu de ce type pour pouvoir se passer de tout revêtement intérieur $^{82}$. D'après les sources, dans le cas de conteneurs à huile, ce revêtement pouvait consister en cire, gomme ou bien lie d'huile d'olive ${ }^{83}$. La possibilité d'un revêtement en poix est au contraire normalement exclue dans le cas d'un contenu d'huile, car en contact avec les matières grasses la poix se dissout partiellement, ce qui modifie le goût du produit ${ }^{84}$. Des recherches récentes suggèrent toutefois que, en dépit de l'opinion générale et de ce qu'on sait par les sources, un revêtement de poix à l'intérieur des amphores pouvait être possible même dans le cas de l'huile ${ }^{85}$. Le sujet est évidemment délicat et pour un approfondissement futur il faudra aussi considérer le problème complexe de la réutilisation des amphores.

Les amphores corinthiennes A' présenteraient au contraire une pâte plus perméable, c'est pourquoi C. G. Koehler admet la possibilité qu'il y ait eu à l'origine un revêtement de résine, cire, gomme, lie d'huile d'olive ou d'un autre matériau qui ne fût pas susceptible d'en abîmer le contenu, bien qu'à sa connaissance aucune amphore de ce type ne conserve un revêtement intérieur (Koehler 1992, p. 277-278).

Une étude plus récente, portant sur de nombreuses amphores des types A et A' provenant des fouilles de Marseille-place Jules-Verne, où le matériel archéologique était conservé dans un milieu humide et anaérobie, donc optimal pour la conservation des revêtements intérieurs organiques, a apporté une contribution à la discussion sur ce thème. Sur une partie de ces amphores, J.-Chr. Sourisseau a pu observer des résidus de poix, ce qui pourrait suggérer une double utilisation de cette forme au moins dans l'intervalle chronologique auquel se rapporte le matériel en question, c'est-à-dire entre la seconde moitié du $\mathrm{VI}^{\mathrm{e}}$ et la fin du $\mathrm{V}^{\mathrm{e}} \mathrm{s}$. av. J.-C. (Sourisseau 1997, I, p. 90).

82. Koehler 1981, p. 452 ; Vandiver, Koehler 1986, p. 204-205; Koehler 1992, p. 277-278, e n. 47-48.

83. Charlin, Gassend, Lequément 1979, p. 21-22 ; et Koehler 1986, p. 52, e n. 15-16, tous les deux avec bibliographie et références aux sources.

84. La possible altération du goût de l'huile au contact avec un revêtement résineux avait été déjà supposée dans Charlin, Gassend, Lequément 1979, p. 21. Pour la réaction chimique qui se produit par le contact entre les matières grasses et la poix, cf. Heron, Pollard 1988. En alternative au vin, il semble que, du moins à l'époque romaine, la salaison de poisson aussi, ainsi que celle d'olives, pouvaient être contenues dans des amphores revêtues de poix (Charlin, Gassend, Lequément 1979, p. 22, et n. 27).

85. Pour cette possibilité, en faveur de laquelle se déclare depuis quelque temps P. Dupont (Dupont 1998, p. 182 et n. 257), voir les résultats des analyses chromatographiques en phase gazeuse effectuées sur les amphores romaines de Sagalassos (Romanus et al. 2009).
À une conclusion analogue conduiraient les observations de J.-P. Brun concernant la production de l'huile en Méditerranée dans l'Antiquité. D'après ce spécialiste, dans l'Antiquité, l'huile aurait été produite pour une consommation presque exclusivement locale et la grande diffusion d'amphores corinthiennes A dans la Méditerranée occidentale trouverait une explication uniquement si l'on admettait qu'elles étaient destinées au commerce du vin ${ }^{86}$.

\subsection{Diffusion dans la Méditerranée occidentale ${ }^{87}$}

Les amphores corinthiennes A ont connu une diffusion parmi les plus larges en Occident, dès les époques les plus anciennes. À partir de la fin du VIII e en ensuite durant tout le VII ${ }^{\mathrm{e}}$ s. av. J.-C., leur distribution capillaire en Sicile et en Grande Grèce trouve son équivalent sur les rives de la Mer Ionienne et de la Basse Adriatique. Des amphores corinthiennes qu'on peut faire remonter à ces périodes sont en effet présentes dans l'habitat de Pale (sur l'île de Céphalonie) et dans les colonies corinthiennes de Corcyre et d'Ambracie. Dans la région illyrienne méridionale et épirote, dans le territoire de l'Albanie actuelle, des amphores corinthiennes du type A attribuables à ces époques hautes sont documentées aussi bien le long de la côte à Durrës et à Treport, que dans l'arrière-pays immédiat, à Belsh, Berat et Selcë, Margëlliç ${ }^{88}$.

Dans les Pouilles, le phénomène du commerce massif corinthien d'époque archaïque, qui commence dès le début du VIII ${ }^{\mathrm{e}}$ s. av. J.-C. ${ }^{89}$, est bien documenté à la fois le long des côtes adriatiques du Salento (Oria, Vaste, Muro Leccese, Otrante, Torre dell'Orso, Rocavecchia, Cavallino, Valesio, Torre Santa Sabina, Ceglie Messapica et Egnathia) et des côtes ioniennes (Leuca, Ruffano,

86. Sur cette hypothèse on consultera toutefois la discussion critique de M. Gras (2010, p. 110-116).

87. L'inventaire qui va suivre n'a pas de prétention à l'exhaustivité. Il signale seulement les principales découvertes afin de donner au lecteur une idée schématique de l'état de la documentation connue et de permettre une évaluation tout aussi schématique de la diffusion de ces amphores en Méditerranée occidentale.

88. Pour les amphores corinthiennes A de l'habitat de Pale, sur l'île de Céphalonie, cf. d'Agostino, Soteriou 1998, p. 357, fig. 8 ; d'Agostino, Gastaldi 2002, notamment p. 169. Sur le tableau de distribution des importations corinthiennes dans le Salento et le long de la côte épirote et illyrienne aux VIII et VII ${ }^{\mathrm{e}}$ s. av. J.-C., voir D'Andria 1985, notamment p. 358-377, fig. 24, avec carte des attestations et bibliographie antérieure. Pour Margëlliç, Belsh, Berat et Selcë, voir Ceka 1983. Pour Durrës, cf. Tartari 1982, pl. I, 3 ; pour Treport $c f$. Bereti 1992, pl. I, 1-10.

89. Sur ce sujet, cf. D’Andria 1985, notamment p. 347. 
Porto Cesareo, San Pancrazio et Tarente) ${ }^{\mathbf{9 0}}$. Dans le courant des $\mathrm{VI}^{\mathrm{e}}, \mathrm{V}^{\mathrm{e}}$ et $\mathrm{IV}^{\mathrm{e}} \mathrm{s}$. av. J.-C., les importations corinthiennes dans la péninsule salentine, bien qu'elles aient diminué, ne connaissent pas d'interruptions. Des témoignages sont fournis par Vaste, avec un exemplaire de la première moitié du $\mathrm{VI}^{\mathrm{e}} \mathrm{s}$. av. J.-C. et un autre de la variante $A^{\prime}$ du milieu du $\mathrm{V}^{\mathrm{e}} \mathrm{s}$. av. J.-C. ; par Cavallino, où existe une riche documentation relative à la première moitié du $\mathrm{VI}^{\mathrm{e}}$ s. av. J.-C., par Otrante, à partir du $\mathrm{VI}^{\mathrm{e}} \mathrm{s}$. av. J.-C. avancé jusqu' au IV ${ }^{\mathrm{e}} \mathrm{s}$. av. J.-C. avec également des attestations de la variante A', et enfin par Torre San Giovanni (Ugento), avec des fragments datables dans le courant du même siècle ${ }^{91}$.

Le long de la côte centrale de l'Adriatique occidentale, le vide de la documentation, qui semble confirmer la description strabonienne d'une aire caractérisée par une présence de lieux alímenoi en opposition à une côte orientale riche en sites eulímenoi, a été récemment documentée par la découverte de quelques amphores corinthiennes A' datées de la période de transition entre la fin du $V^{\mathrm{e}}$ et le début du IV ${ }^{\mathrm{e}} \mathrm{s}$. av. J.-C. dans des contextes funéraires de Numana-Sirolo ${ }^{92}$. Il s'agit d'une donnée particulièrement significative qui laisse entrevoir la possibilité qu'une enquête thématique consacrée à l'étude des amphores dans cette zone fournirait probablement beaucoup d'éléments nouveaux.

Sur la côte orientale de l'Adriatique, les amphores corinthiennes $\mathrm{A}$ et $\mathrm{A}^{\prime}$ sont documentées à partir de l'époque tardo-archaïque à Durrës, où elles ne semblent pas être associées à d'autres fabriques jusqu'à l'époque hellénistique, et aussi à Treport ainsi que dans l'arrièrepays, à Margëlliç, où au contraire on les trouve associées à des amphores corinthiennes du type $\mathrm{B}^{93}$.

En Grande Grèce, dans les nécropoles archaïques de Siris, les amphores de transport étaient remployées pour les sépultures à enchytrismos ou comme conteneurs cinéraires et parmi celles-ci la production corinthienne de type A du VII ${ }^{\mathrm{e}}$ s. av. J.-C. est nettement prépondérante, avec un tiers des attestations. Du VII ${ }^{\mathrm{e}} \mathrm{s}$. sont également datés les exemplaires du secteur de Métaponte, aussi bien de la chora que de l'Incoronata, où les amphores

90. Sur la distribution des amphores corinthiennes A et $\mathrm{A}^{\prime}$ on renvoie en dernier à : Semeraro 1997, p. 389, fig. 297 b.

91. Berlingò 1995, p. 246-428, avec bibliographie antérieure, à laquelle il faut ajouter pour la documentation des $\mathrm{VI}^{\mathrm{e}}$ et $\mathrm{V}^{\mathrm{e}}$ siècles de Vaste, Semeraro 1990, p. 155 n $^{\circ} 249,160 n^{\circ} 261$.

92. Je fais allusion à la Tombe 64 de Numana-Sirolo, signalée récemment par M. Landolfi (1999, p. 150, n. 59), mais aussi à la Tombe 185 de la même nécropole, dont le matériel, avec celui de la Tombe 64, est exposé au Musée Archéologique National d'Ancône. 93. Pour Durrës, cf. Tartari 1982, pl. I, 1 (outre la série corinthienne, pour l'époque hellénistique, on rappellera les exemplaires de Rhodes et de Cnide); pour Treport, cf. Bereti 1992, pl. I, 1-10 ; pour Margëlliç, cf. Ceka 1986, pl. I-VI. corinthiennes de type A sont la série la mieux représentée parmi les nombreuses classes amphoriques signalées ${ }^{94}$. Dans le courant du $\mathrm{V}^{\mathrm{e}} \mathrm{s}$. av. J.-C., la documentation du territoire de Métaponte est plutôt consistante et présente surtout des amphores appartenant au type A' de la classification de C. G. Koehler, attestées ensuite jusqu'au $\mathrm{IV}^{\mathrm{e}}$ s. av. J.-C. ${ }^{95}$. Les amphores de ces espaces funéraires constituent le groupe connu le plus important en dehors de la ville même de Corinthe.

En Calabre, les attestations les plus archaïques, relatives aux VIII ${ }^{\mathrm{e}}$ et $\mathrm{VII}^{\mathrm{e}}$ siècles, viennent de Sybaris, Crotone et Caulonia dans des quartiers d'habitation, mais aussi dans les nécropoles de Métauros et de Reggio. Des amphores corinthiennes du type A du $\mathrm{VI}^{\mathrm{e}} \mathrm{s}$. av. J.-C. sont signalées à Locres Épizéphyrienne, avec une documentation qui descend ensuite (variantes A et A'), jusqu'à l'époque classique ; situation comparable à Sybaris-Thourioi et surtout à Caulonia ${ }^{96}$. Dans ce dernier site, où on trouve de nombreuses séries amphoriques, le type corinthien A est le mieux représenté pour l'époque archaïque et les attestations se poursuivent nombreuses dans le courant du $\mathrm{V}^{\mathrm{e}} \mathrm{s}$. av. J.-C. D'autres exemplaires sont connus à Héraclée, ainsi qu'à Crotone, Locres et Medma ${ }^{97}$. Au IV ${ }^{\text {e }}$ S. av. J.-C., les importations d'amphores corinthiennes A, bien qu'elles soient plutôt rares, sont néanmoins présentes à Caulonia ainsi qu'à Héraclée, en Basilicate, mais aussi en Calabre, à Sybaris, à Crotone, à Medma et à Santa Maria del Cedro, au lieu-dit Mergellina ${ }^{98}$. Au III' ${ }^{\mathrm{s}}$ s. av. J.-C., la diminution abrupte de la présence des amphores corinthiennes A dans l'habitat de Caulonia semble s'inscrire dans un phénomène plus large qui touche l'ensemble de l'aire ionienne, où l'unique attestation connue est un fragment d'Acquappesa daté de la première moitié du siècle ${ }^{99}$.

94. Pour la Siritide, cf. Berlingò 1995, avec références bibliographiques. Pour le matériel archaïque trouvé à Métaponte, cf. Lo Porto 1966 ; Id. 1992 ; D’Andria 1985, fig. 24 ; pour l'habitat de l'Incoronata, voir Scioli, Sacchi 1992 ; Cavagnera 1995 et Stea 1997.

95. Pour le matériel de $\mathrm{V}^{\mathrm{e}} \mathrm{s}$. trouvé en Basilicate, voir Berlingò 1995, p. 424-426, avec indications sur les contextes de découvertes et la bibliographie antérieure. Sur les découvertes récentes d'amphores corinthiennes A' dans la chora de Métaponte, cf. Morter, Leonard 1998. Sur l'importance de la documentation de Métaponte, cf. aussi Koehler 1979, p. 454, n. 5.

96. Berlingò 1995, p. 422-424, avec bibliographie antérieure. Sur l'ample documentation de Caulonia, voir Vandermersch 1989, p. 93, pour la période archaïque, et p. 99, pour l'époque classique.

97. Sur ces attestations, voir Vandermersch 1989.

98. Sur les importations de conteneurs à huile de Corinthe sur la côte ionienne dans le courant du IV ${ }^{\mathrm{e}}$ siècle, cf. Vandermersch 1989, p. 102-103 ; Berlingò 1995, p. 422-424. Voir également les observations sur la diffusion spécifique à cette période de la variante A' dans Koehler 1979, p. 14.

99. Vandermersch 1989, p. 108. 
Les exemplaires de Sicile couvrent un arc chronologique allant de la fin du VIII ${ }^{\mathrm{e}} \mathrm{s}$. av. J.-C. jusque vers le milieu du $\mathrm{V}^{\mathrm{e}} \mathrm{s}$. av. J.-C. ${ }^{100}$. Néanmoins, les amphores des $\mathrm{VII}^{\mathrm{e}}$ et $\mathrm{VI}^{\mathrm{e}}$ s. av. J.-C. constituent le lot le plus important, en provenance essentiellement de contextes funéraires. Mais on en connaît aussi provenant de zones d'habitat et même de contextes cultuels. C. G. Koehler avait avancé l'hypothèse d'une production locale de conteneurs de type A en se basant sur l'observation de la différence de la pâte de quelques exemplaires siciliens, notamment de Sélinonte et de Camarine ${ }^{\mathbf{1 0 1}}$. D'un contexte d'habitat, à Montagna di Ramacca, ont été signalées des amphores de type corinthienne $\mathrm{A}$ et $\mathrm{A}^{\prime}$ datables dès la seconde moitié du $\mathrm{V}^{\mathrm{e}}$ s. av. J.-C. ${ }^{102}$ De nombreux exemplaires d'amphores corinthiennes A et A' proviennent des fouilles de Lipari, dont la documentation commence dès l'époque archaïque et se poursuit au moins jusqu'au $\mathrm{V}^{\mathrm{e}} \mathrm{s}$. av. J.-C. ${ }^{103}$ Les découvertes sous-marines, particulièrement importantes pour une approche des routes commerciales, sont assez nombreuses en Sicile. On peut noter une concentration remarquable des découvertes renvoyant aux époques tardo-archaïque et classique. Il s'agit souvent de découvertes isolées, notamment à Acicastello-Acitrezza, Syracuse, Torre di Gaffe, mais aussi d'épaves de bateaux marchands, d'époques tardoarchaïque (Plemmirio ${ }^{104}$, Géla ${ }^{105}$, Camarine-Punta Braccetto ${ }^{106}$ ) et deux des épaves de Capo d'Ognina

100. Pour les amphores corinthiennes en Sicile, on consultera la synthèse de p. Pelagatti spécialement consacrée au sujet (Pelagatti 1995), ainsi que celle, plus récente, de R. M. Albanese Procelli (1997, notamment p.95-99). Nous renvoyons à ces contributions pour les indications détaillées des très nombreux sites et des contextes des découvertes avec propositions chronologiques. Plus récemment, pour Camarine, cf. Sourisseau 2002, p. 547-548, et Id. 2006 ; pour Himère, Vassallo 2003, p. 331-333, fig. 1-2 ; pour Naxos, Lentini, Savelli, Blackman 2007, p. 89, et Id. 2009, p. 102-103 ; pour Solonte, Polizzi 1997, p. 100, n 1 , fig. 6 ; pour Colle Madore, Polizzi 1999, p. 221-224, $\mathrm{n}^{\circ} 385-387$, fig. $221, \mathrm{n}^{\circ} 385,222, \mathrm{n}^{\circ} 386-387$; pour Milazzo, Tigano 2002, p. 70.

101. Koehler 1979 , p. 29, n. 25. Pour l'observation, voir également Albanese Procelli 1997, p. 95-96, n. 12, et Sourisseau 2006 et 2011. Pour des exemplaires «d'imitation corinthienne » d'Himère, cf. Vassallo 2003, p. 333-339, fig. 3-4.

102. Cf. Albanese Procelli 2003, notamment, p. 37-38.

103. Pour Lipari cf. Cavalier 1985.

104. Pour l'épave de Plemmirio, datée vers le milieu du $\mathrm{VI}^{\mathrm{e}} \mathrm{s}$. av. J.-C., cf. Wilson 1988, p. 115-116 ; Parker 1992, n 835, p. 319320, map 11 .

105. Pour l'épave archaïque de Géla, datée du premier quart du V s. av. J.-C., cf. Freschi 1991 ; Panvini 1991 ; Parker 1992, nº 441, p. 188-189, map 11 ; Panvini 1993 et Panvini 2001, notamment p. 70-72, pl. XXII.132, XXIII.133-136.

106. Sur le bateau de Punta Braccetto, dont la datation longuement débattue pourrait maintenant, à mon avis, être fixée entre la seconde moitié du $\mathrm{VI}^{\mathrm{e}} \mathrm{s}$. av. J.-C. et les débuts du $\mathrm{V}^{\mathrm{e}} \mathrm{s}$. av. J.-C., cf. Wilson 1988, p. 116 ; Parker 1992, n 915, p. 346, map 11 ; Di Stefano 1995.
(Ognina nord I et Ognina sud 3) ${ }^{\mathbf{1 0 7}}$, et d'autres datables de la deuxième moitié du $\mathrm{V}^{\mathrm{e}} \mathrm{s}$. av. J.-C., ainsi que ceux de Syracuse A-Scoglio della Galera ${ }^{108}$, de Capo d'Ognina (Ognina sud 4) ${ }^{\mathbf{1 0 9}}$ et de l'île de Filicudi-Capo Graziano ${ }^{110}$.

En Campanie aussi, les importations d'amphores corinthiennes A commencent vers la fin du VIII's. av. J.-C., en particulier à Pithécusses, dans la nécropole de San Montano et surtout dans la décharge Gosetti. On doit probablement situer avant le milieu du VII ${ }^{\mathrm{e}} \mathrm{s}$. av. J.-C. deux conteneurs corinthiens de Pontecagnano. Cette catégorie est aussi attestée par un exemplaire isolé de la seconde moitié du VI ${ }^{\mathrm{e}} \mathrm{s}$. av. J.-C. dans la nécropole de Vico Equense et par un autre exemplaire contemporain dans la nécropole de Nola pour lequel a été cependant avancée l'hypothèse d'une probable production locale. Les amphores corinthiennes A du VI ${ }^{\mathrm{e}} \mathrm{s}$. av. J.-C. de Cumes et de Vélia ont été publiées récemment ${ }^{111}$. Pour le $\mathrm{V}^{\mathrm{e}} \mathrm{s}$. av. J.-C., si l'on excepte un exemplaire de la variante A' d'une tombe de Pontecagnano daté de la seconde moitié du siècle, les attestations sont nettement concentrées à Pithécusses, où la décharge Gosetti a livré le groupe le plus important d'amphores corinthiennes A et $\mathrm{A}^{\prime}$ qu'on peut situer entre la première moitié $\mathrm{du} \mathrm{V}^{\mathrm{e}}$ et le milieu du IV ${ }^{\mathrm{e}} \mathrm{s}$. av. J.-C. ${ }^{112}$.

Les études concernant les amphores de transport dans les contextes du Latium sont encore rares. En dehors des quelques données livrées par les publications des nécropoles de Decima, Laurentina et Ficana, mais où la présence de conteneurs commerciaux archaïques est limitée au type phénicien, on attend encore un examen global des données provenant de contextes d'habitat. Malgré tout, la présence d'amphores de transport d'importation commence à être signalée dans les fouilles d'habitat à Rome, avec la publication de fragments d'amphores corinthiennes A dans le secteur de la Regia.

107. Pour ces deux épaves de Capo d'Ognina, datés de la période de transition du VI ${ }^{e}$ au V ${ }^{\text {e }}$ s. av. J.-C., voir en dernier lieu, La Fauci 2002, avec un tableau de correspondance entre le nouveau et l'ancien système de dénomination des nombreuses épaves trouvées dans la zone.

108. Pour l'épave connue sous le nom de « Syracuse A », cf. Koehler 1981, p. 17 et Parker 1992, n 1091, p. 405-406, map 11.

109. La Fauci 2002.

110. Pour l'épave de Filicudi, cf. Käpitan 1978, p. 274-275, fig. 7 ; Koehler 1979, p. 236 ; Ead. 1981, p. 29, 45 ; Parker 1992, n² 237, p. 119, map 11 .

111. Pour Cumes, Savelli 2006, p. 110-111; pour Vélia, Gassner 2003, p. 119-120.

112. Sur la documentation amphorique de Pontecagnano, Nola et Vico Equense, Di Sandro 1981, p. 5-6 et, plus récemment, Albore Livadie 1985, p. 139, avec bibliographie antérieure ; pour la nécropole de Pithécusses, Buchner, Ridgway 1993 ; pour la documentation de la décharge Gosetti, Di Sandro 1986. Informations reprises et rassemblées dans Berlingò 1995, p. 428-429. 
Signalons également la présence d'un fragment isolé d'une probable amphore corinthienne A à Ficana, daté, sur une base stratigraphique, de la première moitié du $\mathrm{VI}^{\mathrm{e}}$ s. av. J.-C. ${ }^{113}$.

En Étrurie méridionale, les importations les plus anciennes d'amphores corinthiennes remontent au deuxième quart et à la seconde moitié du VII ${ }^{\mathrm{e}} \mathrm{s}$. av. J.-C., en retard par rapport à ce qu'on observe dans les régions plus méridionales. Les objets connus proviennent de deux tombes de Caeré ainsi que de contextes d'habitat à Tarquinia. On note un accroissement de la documentation connue pour le $\mathrm{VI}^{\mathrm{e}} \mathrm{s}$. av. J.-C., avec les attestations de la nécropole de Vulci, et surtout celles des emporia de Gravisca et de Pyrgi ainsi que celles des deux centres urbains en relation de Tarquinia et de Caeré. On notera que les exemplaires de la seconde moitié du $\mathrm{VI}^{\mathrm{e}} \mathrm{s}$. apparaissent plus nombreux que ceux de la première partie du siècle. Quelques fragments d'amphores corinthiennes A, peu nombreux, datés vers la fin du $\mathrm{VI}^{\mathrm{e}}$ et le début du $\mathrm{V}^{\mathrm{e}} \mathrm{s}$. av. J.-C. proviennent aussi des fouilles de Regae (Regisvilla) ${ }^{114}$. Dans le courant du V ${ }^{\mathrm{e}}$ siècle, les attestations provenant des trois contextes emporiques subsistent, bien qu'on enregistre une diminution générale des découvertes d'amphores. Les amphores corinthiennes A ne dépassent pas, dans l'ensemble, une dizaine de fragments ${ }^{115}$. Signalons enfin un fragment d'amphore corinthienne A à la Doganella ${ }^{116}$.

En Étrurie septentrionale, de nombreux fragments d'amphores corinthiennes A et A' des $\mathrm{VI}^{\mathrm{e}}$ et $\mathrm{V}^{\mathrm{e}}$ s. av. J.-C. ont été restitués par les fouilles de Pise. Deux attestations datées de la période fin $\mathrm{VI}^{\mathrm{e}}$ - début $\mathrm{V}^{\mathrm{e}} \mathrm{s}$. av. J.-C. proviennent de San Rocchino di Massarosa. Signalons enfin un probable fragment d'époque tardo-archaïque trouvé à Piari, dans la haute vallée du Serchio ${ }^{117}$.

113. Pour les observations sur le Latium vetus et un essai de synthèse, voir Zevi 1985. Pour les amphores corinthiennes de Rome, voir Koehler 1981, p. 452 et Zevi 1985, p. 119. Pour le fragment de Ficana, cf. Zevi 1985, n. 4, avec bibliographie antérieure. Toujours provenant de Ficana, mais d'un espace funéraire, une autre amphore corinthienne A avait été signalée, mais l'attribution est aujourd'hui considérée erronée (Zevi 1985, p. 123).

114. Pour une synthèse sur les amphores corinthiennes A, cf. Berlingò 1995, p. 429-430. Pour la nécropole de Caeré, Rizzo 1990, p. 48, 55 ; pour Tarquinia, Scotti 1986, p. 169 et Scotti 1999, p. 267-268; pour Vulci, Boitani 1985 ; pour Pyrgi, Colonna 1985 ; pour Gravisca, Slaska 1985 et Fortunelli 2007, p. 269, pl. 28, G2 ; pour Regae (Regisvilla), Morselli, Tortorici 1985 ; pour Caeré, Boss 1993.

115. Cf. Slaska 1982 et Slaska 1985. La diminution observée concerne à la fois les amphores étrusques et celles d'importation.

116. Pour la Doganella, voir Perkins-Walker 1990, p. 139, fig. 40. 18 .

117. Pour Pise, on consultera les travaux d'O. Pancrazzi (1982; Ead. 1993) et les publications consacrées aux fouilles urbaines plus récentes de M. Bonamici (1989) et surtout de M. Massa (1993),
En Étrurie interne, quelques exemplaires encore inédits ont été récemment signalés dans le territoire de Chiusi, où la présence d'amphores corinthiennes A et $\mathrm{A}^{\prime}$ est également attestée dans une sépulture du milieu du $\mathrm{V}^{\mathrm{e}}$ s. av. J.-C. de Sarteano et par un fragment des débuts du même siècle à Petriolo. Un exemplaire de la seconde moitié du VII ${ }^{\mathrm{e}}$ - premier quart du $\mathrm{VI}^{\mathrm{e}}$ s. av. J.-C. provient de Murlo ${ }^{118}$.

En Ligurie, la présence de fragments d'amphores de production corinthienne est signalée à Gênes ${ }^{119}$.

À l'ouest de la péninsule italienne, les amphores corinthiennes A des époques archaïque et classique sont attestées dans la cargaison de l'épave de la Pointe Lequin $1 \mathrm{~A}$, près de Porquerolles et datée vers 515 av. J.-C., à Marseille, à Arles, à Saint-Pierre-lès-Martigues, à SaintBlaise et au Cayla de Mailhac ${ }^{120}$. En Espagne, les sites d'Emporion, Alorda Park et le gisement sous-marin d'El Cabanyal-Malvarrosa ont restitué des amphores corinthiennes A datées entre la seconde moitié du $\mathrm{VI}^{\mathrm{e}}$ et le $\mathrm{V}^{\mathrm{e}} \mathrm{s}$. av. J.-C. À Toscanos, il semble que les importations commencent déjà dans la phase initiale de production de la série ${ }^{121}$. Des amphores corinthiennes A datées du courant du IV ${ }^{\mathrm{e}}$ s. av. J.-C. sont aussi signalées dans l'épave d'El Sec, près de Majorque et de Cerro del Prado, près de Cadix ${ }^{\mathbf{1 2 2}}$.

qui confirment le cadre déjà esquissé par O. Pancrazzi. Pour San Rocchino, cf. Maggiani 1990b, p. 92-94, n 47-48, fig. 41, 44.48. Pour Piari, Ciampoltrini, Notini 1987 (non identifiée et définie comme « oll »).

118. Pour les amphores inédites du territoire de Chiusi, cf. Paolucci 2006, p. 422-423. Pour Sarteano, lieu-dit Palazzina, Tombe 13, voir Rastrelli 1997, p. 79, fig. 79, et, Minetti, Rastrelli 2001, p. 23, $\mathrm{n}^{\circ} 13.8$; pour le Petriolo et pour Murlo, Savelli sous presse, avec références bibliographiques.

119. Il s'agirait de plusieurs fragments non identifiés explicitement mais caractérisés par un examen pétrographique et la présence d'une anse caractéristique. Sur ces fragments : Milanese, Mannoni 1986, p. 130-132, fig. 8, 1-1 a (désignée de manière générique « anfora corinzia » et comparée avec une amphore corinthienne A de Civitavecchia - récupération sous-marine), et, Milanese 1987, p. 224, n 594, fig. 99, 594 (définie « anfora corinzia di tipo B »). Le profil 'coudé', signalé par les éditeurs et bien visible sur la base du dessin, pourrait laisser penser qu'il s'agit peut-être d'une amphore corinthienne A/A'.

120. Pour l'épave de la Pointe Lequin 1A, Pomey et al. 1988, p. 32-33 ; Parker 1992, p. 323, n 846, map. 5 ; Long, Pomey, Sourisseau 2002, p. 50-54 ; Long, Miro, Volpe 1992. Pour Marseille et la Provence, cf. Sourisseau 1997, I, p. 88. Pour le Cayla de Mailhac (Aude), Gailledrat, Taffanel, Taffanel 2002, p. 83, fig. 74, $\mathrm{n}^{\circ} 1$.

121. Pour Emporion, cf. Sanmartí Grego et al. 1991 et Aquilué Abadías et al. 2000, p. 329, fig. 39.1-2. Pour Alorda Park, Alaminos et al. 1991, p. 282, fig. 10, 2. Pour Toscanos, Docter 2000, fig. 7. Pour El Cabanyal-Malvarrosa, Fernández, Gomez, Ribera 1988, p. 318 , fig. 2.5 .

122. Pour le bateau d’El Sec, Cerdá 1987 ; pour Cerro del Prado, Rouillard 1991, p. 177. 
Dans l'aire méridionale du bassin de la Méditerranée, des exemplaires d'amphores corinthiennes A sont signalés à Carthage. Les plus anciens sont datés du début du VII' ${ }^{\mathrm{e}}$. av. J.-C. ${ }^{123}$.

\section{Les amphores de type corinthien B ou corcyréennes}

Dès les premières études sur les amphores «corinthiennes », il est apparu nécessaire de distinguer deux types totalement différents, à la fois par la pâte, mais également par la forme générale ${ }^{124}$. Lorsque, plus tard, les deux formes ont été désignées respectivement A et B par C. G. Koehler, des doutes ont été émis sur la production corinthienne de la forme $\mathrm{B}$, malgré la ressemblance de sa pâte avec celle des productions corinthiennes contemporaines. V. Grace a suggéré de l'attribuer à la production de Corcyre ${ }^{125}$, suivant en cela une suggestion de $\mathrm{H}$. Bulle ${ }^{126}$ qui, le premier, avait signalé à Corfou une amphore du type B avec un timbre en forme d'étoile à sept/huit branches, plutôt commun sur les amphores de cette série, et l'avait rapproché d'un type monétaire de Corcyre du IV e s. av. J.-C. V. Grace observa également que les caractéristiques morphologiques de ces conteneurs s'apparentaient de façon assez précise avec les kerkyraìous amphoreìs décrites par le Pseudo-Aristote (De mirabilis auscultationibus, 104,p 839b.8).Les études postérieures sur les amphores dites corinthiennes B se sont donc logiquement concentrées sur le problème de leur(s) lieu(x) de production et sur la difficulté que pose le fait d'attribuer à la même cité deux types amphoriques (types A et B) aux caractéristiques techniques et morphologiques si différentes. En développant la suggestion de V. Grace sur le lien entre les amphores du type B et Corcyre et en s'appuyant sur les études effectuées sur la pâte de quelques échantillons, C. G. Koehler, proposa l'hypothèse de l'existence d'une production corcyréenne parallèle à la production corinthienne ${ }^{127}$. Des découvertes plus récentes ont ensuite confirmé qu'à Corcyre, du moins entre la deuxième moitié $\mathrm{du}^{\mathrm{e}}$ et le milieu du $\mathrm{III}^{\mathrm{e}}$ s. av. J.-C., une production d'amphores du type B, documentée par la découverte de fours de potiers avec

123. Cf. Morel 1990c, p. 76-78 et Docter 2000,p. 68, fig. 6.

124. Pease 1937, n. 200-201 et Campbell 1938, p. 604-605.

125. Grace 1953 , p. 108-109. Sur la question, voir aussi les travaux de C. G. Koehler 1981, p. 452, n. 15.

126. Bulle 1934, p. 207, fig. 19.

127. Koehler 1981, p. 452, n. 14. Pour les premières analyses effectuées sur les pâtes des exemplaires de Corfou, voir Farnsworth, Perlman, Asaro 1977. leurs déchets de cuissons ${ }^{128}$, était bien attestée. Toutefois, C. G. Koehler a considéré que Corcyre ne pouvait être perçue comme le centre promoteur de la production de ces amphores, d'abord parce que les exemplaires trouvés à Corinthe sont supérieurs en nombre et couvrent un arc chronologique plus large qu'à Corcyre, mais aussi parce que dans les cargaisons d'un certain nombre d'épaves les amphores de type B sont souvent associées avec des amphores corinthiennes de type A. Sur la base de ces remarques, on a donc admis que le principal centre de production des amphores du type B était effectivement la cité de Corinthe qui, sur la base d'une sorte d'accord commercial, permettait à sa colonie sur l'île de Corfou de produire elle aussi la même forme amphorique ${ }^{129}$. Les nombreuses études sur les pâtes des amphores corinthiennes B conduites spécifiquement dans le but de déterminer leur lieu de production ont confirmé ce point de vue, car quelques-uns des échantillons examinés ont révélé des rapprochements avec l'argile de Corfou, tandis que d'autres ont été trouvés plus proches des groupes de référence de Corinthe.

Notons cependant, avec C. G. Keohler, certains points qui demeurent problématiques dans la caractérisation de la série corinthienne de type B. Ainsi, l'auteur signale prudemment la forte ressemblance morphologique des exemplaires qu'elle considère du début de la série avec des productions amphoriques, considérées comme non corinthiennes, de la Méditerranée occidentale et qui sont parfois désignées comme Ionian et/ou GraecoMassiliote ${ }^{130}$. Ces productions, caractérisées par une remarquable variété de pâtes, devaient être communes à de nombreux centres dont on ignorait, à l'époque, la localisation. Ces similitudes morphologiques associées à une diffusion essentiellement occidentale des amphores corinthiennes du type $\mathrm{B}$, a suggéré une étroite relation entre leur forme initiale et ces deux séries amphoriques de l'Occident grec. Toutefois, la nature du rapport qui les unit, ainsi que le sens de leur probable filiation, est encore très discuté ${ }^{131}$.

Enfin, pour expliquer l'existence à Corinthe de deux séries d'amphores bien distinctes morphologiquement et produites parallèlement durant une longue période, C. G. Koehler propose l'hypothèse d'une différenciation fonctionnelle : le type A, dont la pâte est proche de celle des lampes et des lécythes, était sans doute destiné au

128. Pour les fours de potiers de Corfou, voir KourkoumélisRodostamos 1988 et Preka-Alexandri 1992.

129. En dernier lieu sur ce problème, Koehler 1992.

130. Cf. Koehler 1981, p.453, pl.99, d-e (Ionian), et pl.99, f (Graeco-Massiliote).

131. Koehler 1981, p. 453-454. Sur le problème de la filiation des séries cf. infra I, 2. 
transport de l'huile, tandis que le type B, dont certains exemplaires conservent parfois des traces de résidus résineux à l'intérieur, semble plutôt réservé au transport du vin ${ }^{132}$.

La production des amphores corinthiennes de type B ne semble pas avoir commencé avant le dernier quart du VI ${ }^{\mathrm{e}} \mathrm{s}$. av. J.-C., pour se poursuivre sans interruption jusqu'au sac de la cité par le général romain Mummius en 146 av. J.-C. Dans la longue période qui marque le développement de la production, les évolutions morphologiques qui se sont évidemment produites ont permis à C. G. Koehler de proposer, la première, une classification typo-chronologique sur la base des exemplaires trouvés dans les fouilles de Corinthe, de l'Agora d'Athènes et de quelques centres de Grande Grèce et de Sicile. L'étude récente conduite par $\mathrm{M}$. Lawall ${ }^{\mathbf{1 3 3}}$ sur les amphores provenant de contextes clos de la fin $\mathrm{VI}^{\mathrm{e}}$ et du $\mathrm{V}^{\mathrm{e}}$ s. av. J.-C. de l'Agora d'Athènes, a permis de préciser, pour cette période au cour de notre travail, une définition typochronologique plus serrée et plus sûre qui complète opportunément les travaux de C. G. Koehler.

\subsection{Bilan des études}

Les exemplaires les plus anciens attribués à la production corinthienne de type B, datés par C. G. Koehler entre le dernier quart du $\mathrm{VI}^{\mathrm{e}}$ et le premier quart $\mathrm{du} \mathrm{V}^{\mathrm{e}} \mathrm{s}$. av. J.-C. ${ }^{134}$, présentent une panse à peu près piriforme, une large épaule aplatie et des parois qui se rétrécissent vers le pied (fig. 11). Le pied, qui ne se distingue pas encore nettement du fond de la panse dont il prolonge le profil, est de forme cylindrique. Le dessous est plat et le profil externe est chanfreiné. Ces détails de forme présentent de fortes analogies avec les amphores du type A contemporaines. Les anses verticales, courtes, en ruban épais et à section elliptique irrégulière, ont une courbure arrondie et relevée. Elles sont implantées, en haut, immédiatement au-dessous de la lèvre. Le col est bref, cylindrique et se distingue nettement de l'épaule. La lèvre, au profil extrêmement arrondi et épais, en bourrelet, se distingue du col par une cannelure ou par un listel en relief, tout comme celle des amphores dites « ionio-corinthiennes » contemporaines, avec lesquelles

132. Koehler 1981, p. 452 ; Ead. 1992, n. 46-48.

133. Lawall 1995 , p. $69-74$

134. La chronologie du début de série du type $B$, tout comme son origine, représente un problème particulièrement débattu par les spécialistes et sur lequel on n'a pas encore trouvé un consensus général. Pour la discussion du problème, on renvoie en particulier à Gras 1978 , p. 175 ; Koehler 1979 p. 33-34 ; Ead. 1981, p. 452-454 ; Gras 1987, p. 44 ; et, en dernier, Lawall 1995, p. 78-80.

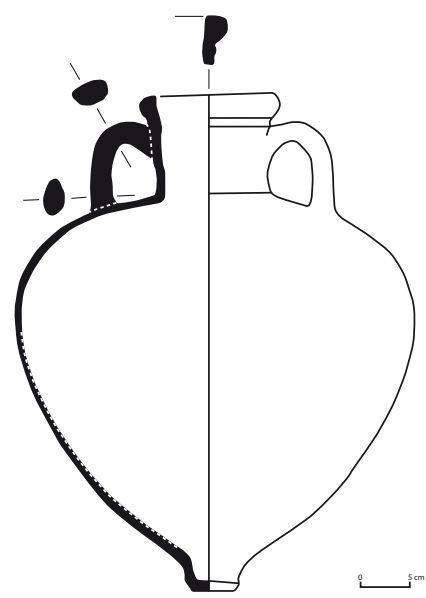

Fig. 11. Amphore de type " corinthien B archaïque ». Corinthe, inv. C-37-2042 (d'après Koehler 1978a, fig. 2.a).

ce type partage, outre la forme générale, plusieurs détails morphologiques ${ }^{135}$.

La chronologie de cette forme initiale est confirmée par la présence du type dans un contexte clos de Corinthe daté du début du $\mathrm{V}^{\mathrm{e}} \mathrm{s}$. av. J.-C. ${ }^{136}$, ainsi que dans le Stoa Gutter Well d'Athènes, dans les contextes H 12:15 et R 12:1 toujours de l'Agora, et enfin dans les fouilles du temple d'Aphaïa à égine, tous clos avant 480 av. J.-C. ${ }^{137}$. Quelques fragments isolés attribuables à ce type archaïque, trouvés dans des contextes postérieurs de Corinthe et d'Athènes, ont été considérés comme résiduels ${ }^{138}$.

Vers 480 av. J.-C., la forme corinthienne B subit une transformation radicale : la lèvre devient aplatie au sommet avec un profil extérieur rectiligne ou légèrement arrondi, en échine, toujours caractérisé par la présence d'une ou de plusieurs cannelures fines ou de listels à la jonction avec le col. C'est un caractère qu'on peut observer jusqu'à la fin du $\mathrm{IV}^{\mathrm{e}} \mathrm{s}$. av. J.-C. Le col, légèrement évasé et aux parois concaves, est bref, comme les anses, dont le profil ne diffère pas beaucoup de celui des exemplaires du début de série. La panse globulaire a une épaule arrondie et son diamètre maximum est situé à mi-hauteur. Le pied, maintenant plein, se distingue

135. Pour les anaphores corinthiennes du type B datables encore entre la fin $\mathrm{du} \mathrm{VI} \mathrm{I}^{\mathrm{e}}$ et les premières années du V $\mathrm{V}^{\mathrm{e}} \mathrm{s}$. av. J.-C. cf. : Koehler 1978a, p. 236, fig. 2, a ; Ead. 1979 p. 33-34 ; Gras 1978, p. 172,176, pl. $73, \mathrm{n}^{\circ} \mathrm{D} 15, \mathrm{pl} .74, \mathrm{n}^{\circ} \mathrm{D} 20-22$; Koehler 1981, p. 452454, pl. 99. a ; Koehler 1992, p. 274, pl. 3.a.

136. Cf. : Campbell 1938, n. 192-193.

137. Pour ces contextes, nous renvoyons respectivement à : Roberts 1986, P24126 ; Lawall 1995, p. 71, n. 68 ; Johnston 1990, p. 43-46, n. 77-81, 85-87, 91-92.

138. Cf. : Lawall 1995, p. 71-72, n. 70. 


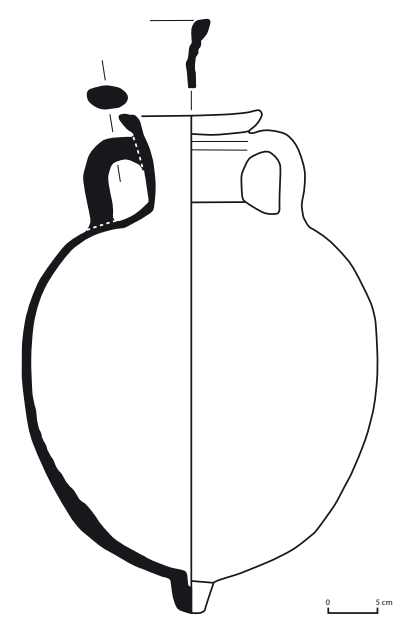

Fig. 12. Amphore de type corinthien B. Corinthe, inv. C-75-69 (d'après Koehler 1978a, fig. 2.b).

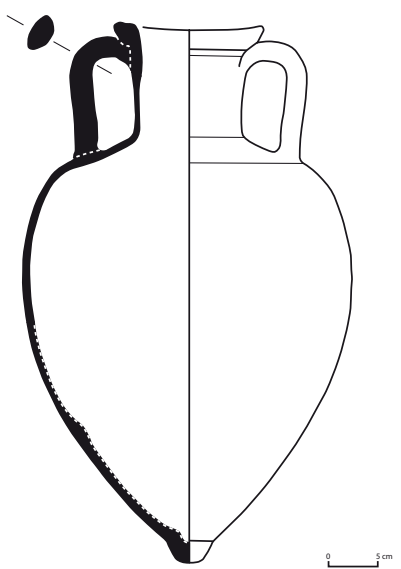

Fig. 13. Amphore de type corinthien B. Corinthe, inv. C-72-118 (d'après Koehler 1978a, fig. 2.c).

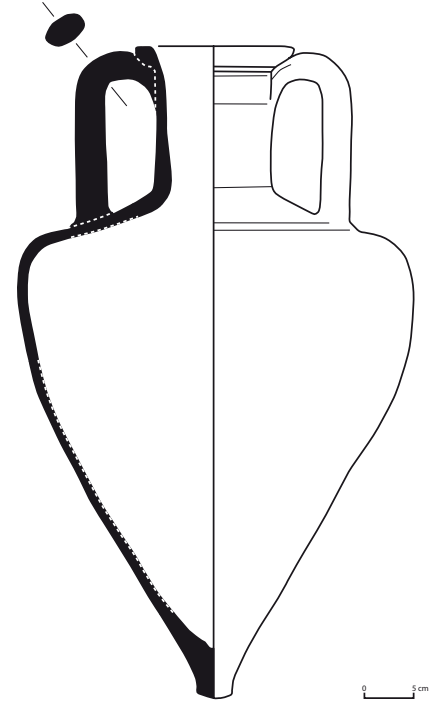

Fig. 14. Amphore de type corinthien B. Athènes, Agora, inv. P6395 (d'après Koehler 1978a, fig. 2.d).

nettement du fond arrondi, la jonction étant souvent soulignée par une cannelure ou une gorge et présente une forme tronconique brève. À l'intérieur, on note l'existence d'une cavité peu profonde (fig. 12) ${ }^{139}$.

Cette seconde forme, que C. G. Koehler situe entre 480 et 450 av. J.-C. ${ }^{140}$, d'après les plus récentes études de M. Lawall aurait connu une période de coexistence

139. Pour la forme des amphores corinthiennes B datables entre 480 et 425 av. J.-C., cf. : Gauer 1975, p. 123, fig. 9.1 ; Koehler 1979 p. 34-35 ; Koehler 1978a p.236, fig. 2.b ; Koehler 1981, p. 454, pl. 99.b-c, fig. 1.a ; Di Sandro 1986, sg. 86, p. 37, fig. 7 (se référant cependant à Koehler 1979 p. 37) ; Koehler 1992, p. 275, pl. 3.b.

140. Koehler 1979 p. 34 ; Ead. 1981, p. 454. entre la fin du $\mathrm{VI}^{\mathrm{e}}$ et le début du $\mathrm{V}^{\mathrm{e}} \mathrm{s}$. av. J.-C. avec la forme plus ancienne, les deux types étant l'un et l'autre présents dans un contexte d'Olympie daté de la fin $\mathrm{VI}^{\mathrm{e}} \mathrm{s}$. av. J.-C. ${ }^{141}$ et dans d'autres contextes de l'Agora clos avant 480 av. J.-C. ${ }^{142}$. Dans l'épave archaïque de Gela on retrouve d'ailleurs la même association ${ }^{143}$.

Ce type continue d'exister et de se développer au moins jusqu'à 425 av. J.-C., comme semblent le montrer quelques exemplaires provenant de contextes clos datés

141. Gauer 1975, pl. $7, \mathrm{n}^{\circ} 5,8$.

142. Il s'agit du contexte H 12:15 (Lawall 1995, p. 72) et du Stoa Gutter Well (Roberts 1986, p. 64-67, n 416-417, fig. 41, pl. 18)

143. Panvini 2001. 
du troisième quart du $\mathrm{V}^{\mathrm{e}} \mathrm{s}$. av. J.-C. à Olympie, à Thasos, à Athènes et à Corinthe même ${ }^{144}$.

Dans le dernier quart du $\mathrm{V}^{\mathrm{e}} \mathrm{s}$. av. J.-C., la panse devient plus élancée et présente un aspect légèrement piriforme avec une épaule brève au profil concave en haut et un fond se resserrant légèrement en descendant vers le pied. Le pied en bouton, dans la continuité du profil inférieur de la panse tout en gardant la forme tronconique pleine avec une légère cavité interne comme pour les exemplaires précédents, est plus court et aussi plus arrondi en bas. La lèvre garde la forme en échine du type précédent, bien que son profil ouvert vers l'extérieur soit plus accentué, ce qui souligne nettement la jonction anguleuse de la base du bord avec le col. La jonction bord/col est toujours soulignée par une ou plusieurs lignes en creux ou en relief. Le col s'allonge, ainsi que les anses qui accentuent aussi vers le haut leur courbure en comprimant les parois du col et le bord extérieur de la lèvre, ce qui en provoque un écrasement souvent bien marqué (fig. 13) ${ }^{145}$. Il s'ensuit une déformation de l'embouchure, qui devient elliptique ou prend la forme d'un huit.

La datation de cette forme au dernier quart du $V^{\mathrm{e}} \mathrm{s}$. av. J.-C. se fonde sur les exemplaires, nombreux, provenant de cinq contextes de l'Agora d'Athènes clos avant la fin du siècle, alors que seuls deux exemplaires isolés ont été trouvés dans deux dépôts clos avant 425 av. J.-C. ${ }^{146}$. La chronologie suggérée par les découvertes de l'Agora est confirmée par l'absence du type dans un contexte clos d'Olympie daté du troisième quart du $\mathrm{V}^{\mathrm{e}} \mathrm{s}$. av. J.-C., où en revanche la forme plus ancienne est documentée ; par contre, il est bien présent dans un autre contexte clos daté du dernier quart du $\mathrm{V}^{\mathrm{e}} \mathrm{S}$. av. J.-C. ${ }^{147}$.

La forte déformation du col, déjà bien attestée pour les exemplaires du dernier quart et la fin $d u V^{\mathrm{e}} \mathrm{s}$. av. J.-C. demeure une caractéristique de ceux du IV ${ }^{\mathrm{e}} \mathrm{s}$. av. J.-C. pour lesquels ce caractère est même accentué.

144. Pour Olympie, cf. Gauer 1975, pl. 7, n 6-7, pl.9, n 1 ; pour Thasos, cf.: Grandjean 1992, p. 549, n 22, fig. 3 ; pour Athènes, cf. : Lawall 1995, p. 73, n. 78 (contextes de l'Agora A 18-19:1, C 18:4b, F 19:4, B 13:6, B 15:1, R 13:1) ; pour Corinthe, cf. : Koehler 1981, p. $454, n^{\circ} 5-6$.

145. Pour la morphologie des exemplaires de la fin $\mathrm{V}^{\mathrm{e}}$ siècle, voir par exemple Gauer 1975, fig. 9.2 ; Koehler 1979 p. 36 ; Koehler 1978a p. 236, fig. 2.c ; Koehler 1992, pl. 3.c

146. La plupart des exemplaires de ce type proviennent des contextes A 20:6, B 13:6, O 19:4, C 19:9, B 15:1, tous datables du dernier quart du Ve s. av. J.-C. (Lawall 1995, p. 73, n. 79). Pour les deux contextes plus anciens de l'Agora (R 13:4 et E 19:5) qui ont restitué cette forme, cf. Lawall 1995, p. 73.

147. Gauer $1975, \mathrm{pl} .9, \mathrm{n}^{\circ} 1-2$ (pour le contexte du troisième quart du $\mathrm{V}^{\mathrm{e}} \mathrm{s}$. av. J.-C.), et pl. 8, $\mathrm{n}^{\circ} 14$ (pour le contexte de fin $\mathrm{V}^{\mathrm{e}} \mathrm{s}$. av. J.-C. qui a restitué la forme plus récente).
Ils se différencient néanmoins des précédents par une allure plus élancée et piriforme due à l'allongement du col et des anses, à la présence d'une ample épaule aplatie et à la disparition de tout élément de distinction entre le fond de la panse et le pivot plein qui en prolonge le profil (fig. 14) ${ }^{148}$.

Ce type, caractéristique du $\mathrm{IV}^{\mathrm{e}} \mathrm{s}$. av. J.-C. même s'il trouve son origine dans le courant du dernier quart $\mathrm{du} \mathrm{V}^{\mathrm{e}} \mathrm{s}$. av. J.-C., est bien attesté par la découverte de quelques exemplaires dans un contexte d'Olympie daté de la première moitié du $\mathrm{IV}^{\mathrm{e}} \mathrm{s}$. av. J.-C. ${ }^{149}$.

\subsection{Centres de production}

L'attribution à Corinthe du type B d'époque archaïque de la part de C. G. Koehler est particulièrement problématique. La tendance la plus récente des spécialistes est de ne pas distinguer la production corinthienne présumée de cette forme des nombreuses productions qui ont été identifiées ou qu'on a suggéré d'identifier dans la Méditerranée occidentale (cf. infra I, 2 § 1.1).

Pour ce qui concerne les amphores corinthiennes B de forme évoluée, la pâte de cette classe se distingue nettement, à l'analyse visuelle, de celle de la production parallèle d'amphores de type A. Elle se rapproche plutôt de celle utilisée dans la fabrication du type A', après le milieu $d u V^{e} s$. av. J.-C. La matrice argileuse des amphores corinthiennes B est en effet fine et caractérisée par des couleurs claires, souvent semblable à celle qui est typique des productions de céramique fine corinthienne, variant du beige au jaune (MSC 5YR8/47.5YR7/4) et englobant de rares et fines inclusions de quartz et de silex. Les inclusions de mudstone et de tuffite qu'on trouve, bien qu'en quantités et dimensions différentes, tant dans le type A que dans le type $\mathrm{A}^{\prime}{ }^{150}$ sont en revanche totalement absentes. D'après C. G. Koehler, les exemplaires produits à partir du troisième quart du $\mathrm{IV}^{\mathrm{e}} \mathrm{s}$. av. J.-C. seraient, en partie au moins, caractérisés par une argile d'une couleur plus proche de l'orange (MSC 2.5YR6/6) et par la présence en surface d'une couche d'argile semi-liquide couleur chamois, absente

148. Les exemplaires datables notamment de la seconde moitié du $\mathrm{IV}^{\mathrm{e}} \mathrm{s}$. av. J.-C. sont bien documentés et C. G. Koehler en a donné une exemplification riche et chronologiquement articulée. Cf. : Koehler 1978b fig. 2 ; Ead. 1978a, p. 236, fig. 2.d ; Ead. 1982, pl. 79. 17-19 ; Ead. 1992, pl. 4.a.

149. Gauer 1975, pl. 8, n 9-13.

150. Sur les pâtes des amphores corinthiennes de type B, les principales études sont dues à R. E. Jones et I. K. Whitbread (Jones 1986, p. 712-720 ; Whitbread 1986, p. 97-99 ; Id. 1988, p. 221-222 ; Id. 1995, notamment p. 274-278). 
sur les amphores de production plus ancienne, dont la surface était simplement lissée ${ }^{151}$.

Les pâtes des amphores corinthiennes B ont fait l'objet de nombreuses études physico-chimiques et pétrographiques dans le but de déterminer la ou plutôt les origines de cette série. Malgré les multiples analyses exécutées avec des techniques différentes, le problème des amphores corinthiennes B reste aujourd'hui encore particulièrement complexe et controversé. Si les premières analyses pétrographiques effectuées dans les années 1970 par M. Farnsworth sur des échantillons contenant des inclusions plus grossières et attribués en général à des amphores plus anciennes de la série ${ }^{152}$, ont abouties à la conclusion que la série était fabriquée avec de l'argile blanche de l'Acrocorinthe, les analyses par activation neutronique conduites plus tard par le même chercheur sur des échantillons contenant des inclusions plus fines, semblent démontrer que Corinthe et Corcyre avaient pu produire ces amphores ${ }^{153}$. Les analyses physico-chimiques plus récentes (optical emission spectroscopy et Mössbauer spectroscopy) exécutées par R. E. Jones sur les pâtes de quelques échantillons n'apportent pas de renseignements supplémentaires ou contradictoires, car elles indiquent que certains échantillons présentent de fortes similitudes chimiques avec l'échantillon de référence de Corinthe alors que d'autres sont conformes à l'échantillon de référence de Corcyre ${ }^{154}$. Les études pétrographiques de I. K. Whitbread, quant à elles, ont permis d'isoler quatre groupes sur la base du caractère plus ou moins grossier des inclusions de la pâte. Le premier groupe (Class 1) rassemble des exemplaires de la fin $\mathrm{du} \mathrm{VI}^{\mathrm{e}}$-et/ou de la première moitié $\mathrm{V}^{\mathrm{e}} \mathrm{s}$. av. J.-C. caractérisés par des inclusions plus grossières; le second (Class 2) des échantillons de la deuxième moitié du $\mathrm{V}^{\mathrm{e}} \mathrm{s}$. av. J.-C., le troisième et le quatrième (Class 3 et Class 4) des échantillons d'amphores datables entre la fin du IV et le III' ${ }^{\mathrm{e}}$ s. av. J.-C. I. K. Whitbread, tout en admettant qu'il n'y a pas de preuves pour exclure une production du type $\mathrm{B}$ à Corinthe, rappelle que beaucoup d'éléments indiquent l'existence d'un autre centre producteur et il exprime de forts doutes sur l'origine corinthienne du type B, étant donné son originalité visà-vis de la tradition morphologique et surtout technique

\section{Cf. Koehler 1992.}

152. Pour cette observation concernant le caractère plus grossier des pâtes des plus anciens exemplaires, voir en premier lieu Gauer 1975, p. 123. Pour la même opinion, plus récemment, Whitbread 1995, p. 348 et 351 .

153. Sur les premières analyses pétrographiques exécutées sur des échantillons d'amphores de type B, cf. Farnsworth 1970, p. 12-13; sur les analyses successives par activation neutronique, voir Farnsworth, Perlman, Asaro 1977, p. 455-468.

154. Cf. Jones 1986, p. 115-121, 176-189, 712-720, 739. des types $\mathrm{A}$ et $\mathrm{A}^{\prime}$ et plus généralement de la production vasculaire corinthienne. D'un point de vue technique, la fabrication des amphores de type B est caractérisée, dès l'origine, par l'usage exclusif du tour, alors que celle des types A et A' fait encore une large part au modelage, au moins jusqu' au début du $\mathrm{V}^{\mathrm{e}} \mathrm{s}$. av. J.-C. Par ailleurs, l'absence de mudstone dans la pâte des exemplaires les plus anciens de la série B, inclusions qui caractérisent la production du type $\mathrm{A}$ mais aussi les premières productions du type $A^{\prime}{ }^{155}$, apparaît également comme un élément fort de différenciation des procédés techniques.

Pour conclure, et bien que la concentration d'amphores du type B dans les fouilles de Corinthe ait poussé C. G. Koehler à attribuer leur production à la cité ${ }^{156}$, les seuls indices directs et incontestables de leur lieu de fabrication proviennent de sa colonie occidentale de Corcyre, à laquelle les sources littéraires attribuent effectivement une série amphorique. On y a trouvé des structures artisanales et des déchets de cuisson datables entre la seconde moitié du $\mathrm{V}^{\mathrm{e}}$ et le milieu du $\mathrm{III}^{\mathrm{e}}$, avec une certaine concentration dans le courant du $\mathrm{IV}^{\mathrm{e}}$ s. av. J.-C. ${ }^{157}$. L'opinion initiée par C. G. Koehler, selon laquelle Corinthe et Corcyre auraient produit des amphores de type B, sur la base d'une sorte d'accord commercial consentit par Corinthe à sa colonie ne trouve donc, à l'heure actuelle, pas de confirmation archéologique. Il est désormais exclu que ces amphores de type B aient pu constituer une production exclusive de Corinthe et même, les éléments qui poussent à douter de l'origine au moins pour partie corinthienne de la série sont nombreux.

Pour l'instant, la position la plus prudente consiste à considérer les amphores de type $\mathrm{B}$ comme une production «extra-régionale » ${ }^{158}$ fabriquée non seulement

155. Sur ces observations, voir la discussion argumentée proposée par I. Whitbread (1995, notamment p. 281-284, 344-345) ainsi que les remarques de M. Gras (1996, notamment p. 137-138). Sur les caractéristiques des deux catégories de pâtes attestées pour le type A' et leur encadrement chronologique, cf. Whitbread 1995, p. 270$273,278$.

156. D'après l'évaluation de C. G. Koehler (1992, p. 273), les amphores corinthiennes $\mathrm{B}$ trouvées à Corinthe (416 pièces inventoriées) dépasseraient en nombre tous les autres types de production, y compris celui des amphores corinthiennes de types A et A' (324 pièces inventoriées).

157. Sur les arguments en faveur d'une production corcyréenne d'amphores de type B, voir Koehler 1981; Kourkoumélis-Rodostamos 1988 ; Kourkoumélis 1992; Preka-Alexandri 1992; Whitbread 1995, p. 256, 260.

158. J'ai emprunté ces définitions (« locale », « régionale », « extrarégionale ») aux observations de $\mathrm{M}$. Lawall, avec qui j'ai eu la possibilité de discuter longuement de la structure économique des centres de production d'amphores. Pour le problème complexe de la production « extra-régionale » d'amphores corinthiennes B, voir en particulier Lawall 1995, p. 67-68, 77-80). 
à Corcyre, peut-être Corinthe, mais aussi dans d'autres centres, notamment de l'aire ionienne-adriatique. Une production de forme $\mathrm{B}$ d'époque classique et hellénistique a en effet été attribuée à Apollonia, dans l'Illyrie méridionale ${ }^{159}$. Par ailleurs, l'examen visuel des pâtes des amphores corinthiennes B de Corcyre a fait supposer à d'autres auteurs que l'atelier corcyréen n'était pas le seul et que d'autres productions étaient à rechercher peut-être en Épire (Kourkoumélis-Rodostamos 1988, p. 113). Une partie des amphores corinthiennes B d'époque classique de Butrinte est aujourd'hui attribuée à une «local production from Corfù-Butrint» (Docter sous presse). Enfin, suite à la découverte de déchets de cuisson, un atelier de la forme du $\mathrm{IV}^{\mathrm{e}} \mathrm{s}$. av. J.-C. a été récemment localisé à Pharos, en Croatie (Katić 2004).

La plupart des indices, y compris des analyses physico-chimiques ${ }^{160}$, nous amènent donc à penser que le principal secteur de production de cette forme amphorique était l'aire ionienne-adriatique.

Il faut néanmoins rappeler, pour finir, que ces amphores correspondent également à la forme MGS I de Chr. Vandermersch, selon lequel, elles pourraient avoir été produites dans le secteur du Golfe de Tarente et celui compris entre Métaponte et Sybaris/Thourioi ${ }^{161}$.

\subsection{Contenu}

Les dépôts résineux présents à l'intérieur de quelques amphores corinthiennes $\mathrm{B}$ de l'Agora d'Athènes et de Carthage ont conduit C. G. Koehler à penser que cette forme était destinée au transport du vin. D'après les sources, en effet, dans le cas des conteneurs pour le vin un traitement avec une substance résineuse était conseillé. Il permettait d'obtenir l'étanchéité du conteneur, ce qui évitait à l'oxygène d'entrer en contact avec le contenu à travers les pores des parois d'argile, activant un procès dégénératif ${ }^{162}$. Le vin produit dans l'Antiquité avait un degré alcoolique bas, ce qui facilitait l'agres-

159. Mano 1971; Desy 1982; Ceka 1986, p. 89 (pour le $\mathrm{V}^{\mathrm{e}} \mathrm{s}$. av. J.C.) ; Desy, De Paepe 1990, p. 215 (pour les IV et $\mathrm{III}^{\mathrm{e}} \mathrm{s}$. av. J.-C.) ; Whitbread 1995, p. 283.

160. Gassner 2003b, p. 183-186,213 (pour Vélia - « Randform 5 »); Docter sous presse (pour Butrinte).

161. Vandermersch 1994,p. 64. L'examen visuel de certaines de ces amphores montre qu'elles partagent parfois les mêmes pâtes que les amphores grecques occidentales archaïques (cf. infra I, 2 § 1.1) attribuées au secteur de Sybaris (Sourisseau 2011, n. 171).

162. Cf. : Koehler 1981, p. 452, et n. 16 ; Ead. 1986, p. 50-52, avec références bibliographiques et aux sources anciennes; Ead. 1992, p. 277-278, et n. 46, avec bibliographie. Pour les sources, concernant la préparation des conteneurs de vin, voir aussi Charlin, Gassend, Lequément 1979, p. 21-22. sion de la part des agents pathogènes qui provoquent la transformation du vin en vinaigre. Ce type de revêtement de l'amphore aurait aussi permis d'en aromatiser le contenu ${ }^{163}$.

On a opposé à cette idée l'observation que, d'après les sources, le vin de Corinthe était un produit de qualité médiocre (Athen., Deipnosophistae I, 30f = Alexis fr. 290 Edmonds). Cependant, la localisation d'une production de cette forme à Corcyre (Preka-Alexandri 1992) constitue un argument valide en faveur de l'utilisation de ces amphores pour transporter le vin. D'après les sources, en effet, dans les époques classique et hellénistique dans l'île de Corfou il y avait une production viticole très développée, au point de justifier l'utilisation tant de la grappe de raisin que de l'amphore sur les émissions monétaires de la fin du $\mathrm{VI}^{\mathrm{e}}$ et de la première moitié du V $\mathrm{V}^{\mathrm{e}}$ s. av. J.-C. (Kourkoumélis-Rodostamos 1988, p. 92-98, 157, $160 ;$ Id. 1992).

\subsection{Diffusion dans la Méditerranée occidentale ${ }^{164}$}

La présence d'amphores corinthiennes B est attestée dans le Salento, aussi bien sur la côte adriatique, (Oria, Cavallino et peut-être à Vaste), que sur la façade ionienne (Masseria Fano et Ruffano). Une épave datée de la seconde moitié du $\mathrm{V}^{\mathrm{e}} \mathrm{s}$. av. J.-C, en face de la côte de Gallipoli, transportait une cargaison d'amphores corinthiennes $\mathrm{B}^{\mathbf{1 6 5}}$.

Plus au nord, le long de la côte adriatique des Marches, des amphores corinthiennes $\mathrm{B}$ de fin $\mathrm{V}^{\mathrm{e}} \mathrm{s}$. av. J.-C. ont été signalées dans des contextes funéraires de Sirolo-Numana et de Camerano ${ }^{\mathbf{1 6 6}}$.

Dans l'Adriatique orientale, les amphores corinthiennes de type $\mathrm{B}$ sont documentées non seulement à Corfou, mais aussi à Margëlliç, site de l'arrière-pays albanais où, à partir du $\mathrm{V}^{\mathrm{e}} \mathrm{s}$. av. J.-C., il semble que soit

163. Pour les deux dernières observations, voir Sedda 1989, p. 126-127.

164. Sont exclues de ce catalogue non exhaustif les amphores de forme archaïque que C. G. Koehler a attribuées à la phase initiale de sa série des corinthiennes B. Ces amphores seront évoquées dans le chapitre suivant consacré aux productions grecques occidentales (cf. chap. 2.1).

165. Semeraro 1997, p. 63, $\mathrm{n}^{\circ} 95$ (Cavallino), p. 105, $\mathrm{n}^{\circ} 197$ (Masseria Fano), p. 154-156, n ${ }^{\circ} 321-322$, p. 211-212, $\mathrm{n}^{\circ}$ 697-698 (Oria), p. 271, 984,989-990 (Ruffano). M. G. Semeraro attribue aussi à la forme corinthienne $\mathrm{B} \mathrm{du} \mathrm{V}^{\mathrm{e}} \mathrm{s}$. un fragment de Vaste (Semeraro 1997, p. 302, n 1131). Pour l'épave de Gallipoli, Parker 1992, p. 186-187.

166. Il s'agit des tombes Numana-Sirolo ${ }^{\circ} 6,178$ et 185 et des tombes $n^{\circ} 90$ et 100 de Camerano (Cf. Landolfi 1999, p. 150, n. 60). Les amphores des Tombes 6 et 185 de Numana-Sirolo et 90 de Camerano sont exposées au Musée Archéologique National des Marches. 
aussi attestée la production apolloniate du type ${ }^{167}$. À Treport, sur la côte de l'Albanie, on signale des amphores corinthiennes B datables entre l'époque tardo-archaïque et le IV ${ }^{\text {e }}$ s. av. J.-C. ${ }^{168}$.

Dans l'aire ionienne, en Basilicate, quelques exemplaires d'amphores corinthiennes B sont signalés dans le territoire de Métaponte, trouvés dans des sépultures du milieu du Ve s. av. J.-C. ${ }^{169}$.

En Calabre, les amphores corinthiennes B sont présentes dans l'habitat de Caulonia, où elles sont documentées dès l'époque tardo-archaïque et jusqu'à l'époque hellénistique, et à Crotone, par quelques exemplaires du $\mathrm{IV}^{\mathrm{e}}$ et du $\mathrm{III}^{\mathrm{e}}$ s. av. J.-C. ${ }^{170}$.

En Sicile, où la documentation est plutôt consistante pour le $\mathrm{IV}^{\mathrm{e}} \mathrm{s}$. av. J.-C., un groupe d'amphores corinthiennes $\mathrm{B}$ datables du milieu et de la deuxième moitié $\mathrm{du} \mathrm{V}^{\mathrm{e}} \mathrm{s}$. av. J.-C. a été récemment signalé dans le site indigène de la Montagna di Ramacca, dans la province de Catane, et des exemplaires d'amphores corinthiennes $\mathrm{B} d u \mathrm{~V}^{\mathrm{e}} \mathrm{s}$. proviennent des fouilles de Lipari ${ }^{171}$. Enfin, des amphores corinthiennes B faisaient partie de la cargaison de l'épave de Capo Rasocolmo ${ }^{172}$ qui fit naufrage près du détroit de Messine entre 450 et 400 av. J.-C., du bateau dit « seconde épave de Géla » ${ }^{173}$ daté vers le

167. Ceka 1986, p. 89 .

168. Cf. Bereti 1992, pl. II-III.

169. Pour le secteur de Métaponte, cf. Lo Porto 1992 et Morter, Leonard 1998.

170. Pour Caulonia et Crotone, cf. Vandermersch 1989, p. 99-101, 103, 108.

171. Pour Sélinonte, Dehl-Von Kaenel 2004, p. 444 ; pour Lipari, Cavalier 1985.

172. Pour le bateau de Capo Rasocolmo, cf. Koehler 1981, p. 45 et Parker 1992, n 248, p. 122, map 11.

173. Pour les amphores corinthiennes B de la «seconde épave de Géla », découverte en 1990, Panvini 2001, p. 93-94, pl. XXXI.185186, XXXII.187-192, XXXIII.193. milieu du V ${ }^{\mathrm{e}} \mathrm{s}$. av. J.-C., de l'épave de Capo d'Ognina désignée 'Ognina sud 4' ${ }^{174}$ datée de la seconde moitié du Ve s. av. J.-C., de celle de Syracuse A-Scoglio della Galera, datée du troisième quart du $\mathrm{V}^{\mathrm{e}} \mathrm{s}$. av. J.-C. ${ }^{175}$, du bateau de même époque de l'île de Filicudi-Capo Graziano ${ }^{176}$, et enfin de l'épave de Marzamemi $\mathrm{H}^{177}$, datable entre 425 et 375 av. J.-C.

Le long de la côte tyrrhénienne, en Campanie, des exemplaires d'amphores corinthiennes B ont été signalés à Vélia et dans les nécropoles de Pontecagnano et de Vico Equense ${ }^{178}$.

En ce qui concerne l'Étrurie, la documentation relative aux amphores corinthiennes B semble être limitée à l'épave de Castiglione della Pescaia-Punta Ala, datée entre la fin $\mathrm{du} \mathrm{VI}^{\mathrm{e}}$ et la première moitié $\mathrm{du} \mathrm{V}^{\mathrm{e}} \mathrm{s}$. av.J.-C. ${ }^{179}$.

Dans les régions situées plus à l'ouest, le type corinthien B est documenté par quelques exemplaires à Marseille et dans la péninsule ibérique, où des attestations sont signalées à Cerro Macareno (près de La Rinconada), dans le secteur de Séville et dans le gisement sous-marin d'El Cabanyal-Malvarrosa, près de Valence, dans les deux cas avec une documentation de plein $V^{\text {e }}$ s. av. J.-C., et enfin dans la cargaison de l'épave d'El Sec datée vers le milieu du IV ${ }^{\mathrm{e}}$ s. av. J.-C. ${ }^{180}$.

174. Pour l'épave de Capo d'Ognina connu sous la dénomination « sud 4 », cf. : La Fauci 2002.

175. Pour l'épave dite «Siracusa A», cf. Koehler 1981, p. 17 et Parker 1992, n 1091, p. 405-406, map 11.

176. Pour l'épave de Filicudi, cf. Käpitan 1978, p. 274-275, fig. 7 ; Koehler 1979, p. 236 ; Ead. 1981, p. 29, 45 ; Parker 1992, n²37, p. 119, map 11 .

177. Pour l'épave de Marzamemi H, cf. Parker 1978, p. 629-631, pl. CXXXVIII, fig. 1-2 ; Parker 1992, n 677, p. 269, map 11.

178. Pour Vélia, Gassner 2003; pour Pontecagnano et Vico Equense, Di Sandro 1981, p. 7 et Albore Livadie 1985, p. 139 avec bibliographie.

179. Cygielman, De Tommaso 1998.

180. Pour Marseille, Sourisseau 1997, I, p.97. Pour Cerro Macareno, $c f$. Rouillard 1991,p. 177 ; pour El Cabanyal-Malvarrosa, cf. Fernández, Gomez, Ribera 1988, p. 318, fig. 2.4 ; pour l'épave «El Sec », voir Arribas et al.1987. 


\section{Chapitre 2}

\section{Les productions grecques occidentales}

$\mathrm{O}$ n situe désormais dans la partie occidentale de la Méditerranée la production de plusieurs séries amphoriques. Elles sont vraisemblablement fabriquées par plusieurs centres distincts, dans le cadre d'une « koinè occidentale » qui trouve des parallèles, au même moment dans les koinès amphoriques de l'Égée septentrionale et orientale. La chronologie du début des ces séries amphoriques grecques occidentales est traditionnellement située vers le milieu ou la seconde moitié du VI ${ }^{\mathrm{e}} \mathrm{s}$. av. J.-C., toutefois, des travaux récents suggèrent une chronologie plus haute, dans la première moitié du siècle.

Les connaissances relatives à ces productions grecques occidentales sont encore pour partie incertaines et les tentatives de synthèse récentes se sont révélées encore partielles ${ }^{181}$. En effet, si la chronologie et les grandes zones de production apparaissent plus clairement, la plupart des lieux précis de fabrication restent inconnus. Par ailleurs, on ne dispose pas encore d'une classification typologique suffisamment précise, commune et admise de tous. Enfin, on n'a pas encore trouvé un accord sur leurs dénominations, ce qui est source de grande confusion.

En Italie du nord, les formes des productions grecques occidentales attestées sont celles de l'époque archaïque et du début de l'époque classique. Il s'agit de deux séries amphoriques pour lesquelles il est difficile de choisir une terminologie assurée pour les désigner, tant les classifications typo-chronologiques comme la localisation des centres de production sont encore incertains. La terminologie traditionnelle distingue entre les amphores dites «corinthiennes B archaïques » et les amphores dites « ionio-massaliètes ». Bien qu'ils correspondent à des types morphologiques assez précis et qui trouvent des équivalents dans les travaux typologiques récents, ces deux dénominations, souvent mal interprétées ont donné lieu par le passé à de nombreuses confusions. C'est pourquoi, et par souci de clarté, nous utiliserons le cadre typologique récemment proposé par J.-Chr. Sourisseau

181. On doit à V. Gassner (2003b, p. 179-182) et à J.-Chr. Sourisseau (2011) les propositions les plus récentes relatives à la caractérisation et à l'évolution morphologique de ces séries. qui présente l'avantage de distinguer assez précisément la morphologie des deux types en question, sans préjuger de leurs lieux de productions.

\section{Les amphores du groupe « corinthien B archaïque » ou grecques occidentales de forme $1 \alpha$}

Les amphores de ce groupe présentent des caractères morphologiques généraux assez réguliers (fig. 15), bien qu'elles se distinguent les unes des autres par des variantes de formes et par leurs pâtes. D'abord attribuées à la production corinthienne par C. G. Koehler et désignées «Corinthiennes $\mathrm{B}$ », la pâte de ces amphores est néanmoins très différente de l'autre production corinthienne, dite corinthienne A. C. G. Koehler admet également que d'autres productions présentant les mêmes caractéristiques morphologiques sont possibles, notamment à Corcyre, mais aussi dans le secteur occidentale de la Méditerranée. Ainsi, elle signale l'existence d'amphores dont la forme générale et les détails

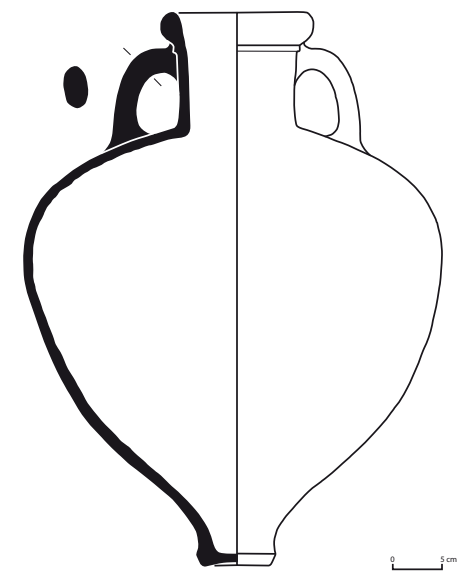

Fig. 15. Amphore du groupe " corinthien B archaïque » ou grecque occidentale de forme $1 \alpha$. Milazzo, nécropole méridionale, Tombe 15, inv. 16440 (d'après Tigano 2000, p. 69). 
de la lèvre en bourrelet gonflé renvoient à la forme d'époque archaïque du type $\mathrm{B}$, ainsi que des formes proches, et connues depuis longtemps en Italie méridionale, en Sicile et dans le sud de la France. Il s'agirait des types « often called by archaeologists there Ionian » et «sometimes called Graeco-Massaliote » (Koehler 1981, p. 453, pl. 99, d-e, et pl. 99, f) qui, par l'extrême variété des pâtes attestées dans les deux cas, semblent suggérer une pluralité de centres de production ${ }^{182}$. Alors qu'au type Graeco-Massaliote (qu'on appellera ici « ionio-massaliète » ou grecque occidentale de forme 2 cf. infra I, 2 § 2) C. G. Koehler avait reconnu quelques traits distinctifs par rapport à son type $\mathrm{B}$ archaïque de Corinthe, le type Ionian (qu'on appellera ici «corinthien B archaïque » ou grecque occidentale de type $1 \alpha$ ) fut considéré comme difficilement discernable de la production présumée corinthienne de son type $\mathrm{B}$ archaïque, tant la morphologie générale et certains traits de détail, notamment l'ensemble lèvre/col et le pied, apparaissent similaires. Ainsi, d'après C. G. Koehler, les amphores du groupe «corinthien B archaïque » sont donc «più o meno simili alle corinzie B arcaiche senza che possano identificarsi con esse » (C. G. Koehler dans Colonna 1985, p. 10 et n. 26). L'opinion de la spécialiste des amphores corinthiennes a donc imposé une tendance à distinguer un groupe corinthien $\mathrm{B}$ archaïque de production corinthienne et un autre de production grecque occidentale. Cependant, en l'état actuel des recherches, il subsiste de nombreux doutes quant à l'existence effective d'un atelier corinthien de la forme en question, dont la production semble plutôt se concentrer dans l'Occident grec $^{183}$. Compte tenu de ces difficultés, les amphores présentant cette morphologie particulière sont souvent désignées dans la bibliographie sous les appellations imprécises suivantes : «ioniennes »/ ioniche / ionian, ou « ionio-corinthiennes »/ ionio-corinzie, ou plus fréquemment « de type corinthien $\mathrm{B}$ » / di forma corinzia $B$ ou di tipo corinzio $B$, ou encore corinzie $B$. Pour éviter les confusions induites par la multiplicité de ces dénominations traditionnelles, souvent déroutantes, V. Gassner et J.-Ch. Sourisseau ont adopté respectivement les définitions Randform 1 (Gassner 2003b) et «A-MGR 1 » (Sourisseau 1993) pour désigner ce groupe. Elles sont désormais dénommées «forme $1 \alpha$ » dans les derniers travaux sur la question ${ }^{184}$. Par conséquent, on définira ici ${ }^{185}$ " corinthiennes B archaïques »

182. Pour la définition de ces deux types, voir Koehler 1981, p. 452453, pl. 99 : d, e ; Ibid., p. 453-454, pl. 99 : f.

183. Whitbread 1995, p. 278-285, pour une discussion sur le sujet.

184. Sourisseau 2011.

185. Contrairement à la terminologie choisie antérieurement d'amphores «ionio-corinthienne» (Sacchetti 2005; Ead. 2010 ; Ead. ou amphores «grecques occidentales de forme $1 \alpha$ », toutes les amphores qu'on peut rapprocher par leur forme de celles que C. G. Koehler attribue à des productions corinthiennes et qu'elle définit comme « early type B jars » ${ }^{\mathbf{1 8 6}}$, et ceci, malgré la variété observée des pâtes.

Ces amphores sont en général caractérisées par une lèvre en bourrelet gonflé, dont l'épaisseur maximale est située à mi-hauteur, un col cylindrique avec un ressaut ou, plus rarement, une cannelure au sommet, des anses en ruban épaissi, un corps globulaire, un fond cylindrique creux à l'intérieur et présentant une base plate carénée ${ }^{187}$.

Malgré ces caractéristiques communes, le groupe des amphores de «forme $1 \alpha$ » ou « corinthien B archaïque » apparaît extrêmement hétérogène. Les proportions entre les différentes parties du conteneur sont variables et une série de variantes morphologiques concernant surtout la lèvre, le col et les anses peuvent aisément être observées ${ }^{188}$, notamment si on compare les découvertes des sites qui ont livré d'abondantes attestations ${ }^{189}$. L'ensemble de ces variantes n'a pas encore fait l'objet d'un répertoire permettant d'élaborer une classification qui tienne compte non seulement de ces éléments morphologiques, mais aussi des différentes pâtes observées ainsi que des contextes stratigraphiques de découverte, qui permettrait peut-être, si ce n'est de localiser, $\mathrm{du}$ moins de distinguer les ateliers producteurs des amphores de ce groupe et les caractéristiques morphologiques propres à chacun d'entre eux. Dans cette optique, une proposition préliminaire, basée sur une campagne d'analyses des pâtes, a été initiée par V. Gassner et une étude globale des productions occidentales est actuellement en cours de la part de J.-Chr. Sourisseau ${ }^{190}$. C'est donc à ces contributions récentes que nous renvoyons pour les questions complexes concernant les productions occidentales de la «forme $1 \alpha »$ ou « corinthienne $\mathrm{B}$ archaïque » qu'on a cru pouvoir identifier jusqu'à ce jour. Les recherches en cours n'ont pas encore abouti à des conclusions définitives, ni non plus à un accord

\section{1).}

186. Koehler 1981, p. 452-454, pl. 99, a.

187. Pour certaines de ces observations relatives à la morphologie, voir Koehler 1981, p. 453 ; Slaska 1990, p. 231-232; Albanese Procelli 1997b, p. 111-113.

188. Koehler 1981, p. 453.

189. C'est le cas par exemple des sites du Salento, pour lesquels voir en dernier lieu Semeraro 1997. Pour une synthèse récente sur les présences d'amphores grecques occidentales archaïques en Grande Grèce, voir Savelli 2009, avec la liste des attestations et Sourisseau 2011.

190. Gassner 2003b ; Sourisseau 1993 et Sourisseau 2011, avec la bibliographie antérieure. 
suffisamment unanime de la part des spécialistes. En l'état actuel des recherches, des productions de ce type ont été attribuées à Sybaris (ou à un centre non identifié situé entre Crotone et Kaulonia), à Kaulonia, à Locres et à l'aire du détroit de Messine ${ }^{191}$. D'autres productions documentées en Grande Grèce restent toutefois non identifiées. On imagine que quelques-unes sont à rechercher dans d'autres centres de Calabre ${ }^{\mathbf{1 9 2}}$, mais je crois que d'autres encore, notamment celles qui sont documentées dans le secteur Nord-Adriatique, pourraient être attribuées à l'aire Adriatique méridionale. Dans cette zone doit être situé l'atelier identifié par $\mathrm{R}$. Docter à Butrinthos ${ }^{193}$ pour lequel on dispose déjà de quelques informations ${ }^{194}$, mais qui est encore inédit ${ }^{195}$. Dans le même secteur, il n'est pas sûr que la production corcyréenne de la forme corinthienne $\mathrm{B}^{196}$ puisse être datée dès le $\mathrm{VI}^{\mathrm{e}} \mathrm{s}$. av. J.-C. L'atelier de Corcyre a en effet fourni des données relatives à une production d'un type morphologique plus tardif, comprise entre la seconde moitié du $\mathrm{V}^{\mathrm{e}}$ et le $\mathrm{III}^{\mathrm{e}} \mathrm{s}$. av. J.-C. et, bien que l'hypothèse d'une fabrication locale ait été avancée pour la forme archaïque ${ }^{197}$, on ne dispose d'aucune donnée objective permettant de l'étayer. Sur ce dossier, il faudrait néanmoins vérifier si la production définie par R. Docter à Butrinthos comme « local production from Corfù-Butrint area ${ }^{\mathbf{1 9 8}}$ pourrait ou non correspondre à une production attribuable à l'atelier de Corcyre.

En ce qui concerne les questions de chronologie, relevons que les exemplaires de « forme $1 \alpha$ » ou « corinthiennes $\mathrm{B}$ archaïques » les plus anciens reconnus à Corinthe sont datés, selon C. G. Koehler, à partir de 525 av. J.-C. ${ }^{199}$ Par contre, la chronologie des attestations occidentales est plus haute : en Albanie, en Italie et en France, quelques exemplaires attribuables à ce groupe sont datables, sur une base stratigraphique, du deuxième

191. Pour la possible production de Sybaris: Sourisseau 1997, I. p. 95-96, Id. 2000, p. 140 ; Spagnolo 2002, p. 136 ; Sourisseau 2011. Voir néanmoins les réserves exprimées par V. Gassner (2003b, p. 186-188) sur cette attribution et sur la proposition de chercher l'atelier « sybarite » entre Crotone et Kaulonia. Sur l'atelier supposé de Caulonia, voir Gassner 2003b, p. 188-189. Pour Locres, voir Barra Bagnasco 1992, p. 208-211 ; Ead. 1993 ; Ead. 1995 ; Barone et al. 2002, p. 99-100 ; Spagnolo 2002, p. 37-38 ; Gassner 2003b, p. 189191. Pour la zone du détroit de Messine, Gassner 2003b, p. 193-195. 192. Gassner 2003b, p. 193-195.

193. Docter sous presse.

194. Gassner 2003b, p. 185, n. 67 ; Ead. 2003a, p. 96, n. 18

195. Je tiens à remercier C. W. Neeft qui m'a donné la possibilité de consulter l'article de R. Docter encore sous presse.

196. Preka-Alexandri 1992.

197. Kourkoumélis-Rodostamos 1988.

198. Docter sous presse.

199. Koehler 1981, p. 452. quart du VI ${ }^{\mathrm{e}}$ s. av. J.-C. au plus tard. Une datation de ce type est suggérée par les fragments provenant de contextes d'Agde, en Gaule méridionale (avant le milieu du VI ${ }^{\mathrm{e}} \mathrm{s}$. av. J.-C.), de Belsh, en Illyrie méridionale (600550 av. J.-C.), de Leuca (600-550 av. J.-C.), de Siris (avant le milieu du VI ${ }^{\mathrm{e}} \mathrm{s}$. av. J.-C.) et aussi de Pithécusses (600-550 av. J.-C.). À Marseille, on en trouve déjà dans les couches de la phase de fondation de la cité, c'est-àdire entre 600 et 580 av. J.-C. ${ }^{200}$. Enfin, dans la nécropole de Camarine, les exemplaires les plus anciens sont associés à des vases corinthiens du Corinthien Moyen, ce qui confirme cette datation haute ${ }^{201}$.

Néanmoins, la phase durant laquelle ces amphores sont le plus diffusées correspond à la seconde moitié du $\mathrm{VI}^{\mathrm{e}}$ s. av. J.-C., avec notamment des attestations dans les épaves de la Pointe Lequin 1A et de Cala Sant Vicenç. Les exemplaires les plus tardifs revoient au début du $\mathrm{V}^{\mathrm{e}} \mathrm{s}$. av. J.-C., avec en particulier l'épave de Géla ${ }^{202}$.

\subsection{Centres de production}

Différents programmes d'analyses pétrographiques et physico-chimiques ont été initiés ces dernières années dans le but de déterminer l'origine des amphores grecques occidentales archaïques. Le premier, promu par le Centre Jean Bérard, sous la direction de M. Bats, est resté iné$\mathrm{dit}^{203}$. Plus récemment, une collaboration entre l'Insitut für Klassische Archäologie de l'Université de Vienne et le Dipartimento di Discipline Storiche de l'Université de Naples «Federico II » a permis la mise en œuvre d'un vaste programme d'analyses sur les productions céramiques magno-grecques, sous la direction de V. Gassner. Des équipes des Universités de Turin (Dipartimento di Scienze Antropologiche, Archeologiche e StoricoTerritoriali, Dipartimento di Chimica Analitica et Dipartimento di Scienze Mineralogiche e Petrologiche) et de Parme (Dipartimento di Chimica) se sont occupées de la caractérisation physico-chimique et pétrographique des amphores de Locres, où, dans l'aire de la Stoa à 'U', M. Barra Bagnasco avait repéré et fouillé des ateliers

200. Pour Agde, Nickels 1990 et Nickels 1995, p. 61 ; pour Belsh, Ceka 1983,p. 209, pl. III, 4-5 ; pour Leuca, en dernier lieu, Semeraro 1997, n 172 a-b, d-f ; pour Siris, Corchia, Pancrazzi, Tagliente 1982, p. 23, pl. 13, 9-10 et Berlingò 1986; pour Pithécusses, De Caro, Gialanella 1998, p. 343 ; pour Marseille, Sourisseau 1997, I, p. 92, n. 187 et Sourisseau 2011, p. 178, fig. 7 (attribution à une production sybarite).

201. Pour Marseille et Camarine, voir en dernier lieu Sourisseau 2011

202. Sur ces découvertes, voir en dernier lieu Sourisseau 2011, avec bibliographie.

203. Pour ce programme, voir Pelagatti 1995, p. 407 et Sourisseau 2011, p. 175. 
d'amphores caractérisés par leurs déchets de cuisson. Le Servizio per i Beni Archeologici de la Soprintendenza ai Beni Culturali e Ambientali di Messina, le Dipartimento di Fisica de l'Université de Messine et le Dipartimento di Scienze Geologiche de l'Université de Catane ont enfin lancé un programme d'analyses sur les amphores découvertes en Sicile, et notamment à Messine et à Gela ${ }^{204}$.

Les aspects visuels des pâtes de la plupart des amphores de ce type et répertoriées dans notre enquête, confrontées à tout ce que j'ai pu voir en Grande Grèce et en Gaule méridionale, notamment celles qui proviennent de l'aire du delta du Pô, de Marzabotto et, en partie du moins du Forcello, ne semblent appartenir à aucune des productions mentionnées plus haut. Les pâtes des amphores de «forme $1 \alpha$ » ou «corinthiennes $\mathrm{B}$ archaïques » de l'Italie du nord peuvent en revanche être classées en deux groupes. Le premier est caractérisé par une pâte presque complètement dépourvu d'inclusions, à la surface extrêmement lisse au toucher. La consistance est généralement dure et compacte, parfois plus tendre et poussiéreuse. La couleur de l'argile est très claire, variant du jaune pâle-blanchâtre au verdâtre. Le deuxième groupe présente des argiles ayant les mêmes nuances, auxquelles s'ajoutent aussi le beige et le chamois pâle, et semble se distinguer par la présence de rares dégraissants lithiques blanchâtres (sans doute du quartz) et gris, de dimensions moyennes à grandes (0,5 mm environ). La couleur de la pâte de quelques individus provenant d'Adria présente une couleur grise. Il s'agit probablement d'un phénomène de transformation chimique intervenu après le dépôt de ces objets en milieu humide et vaseux.

Il est intéressant de constater qu'on reconnaît les deux mêmes pâtes pour les amphores dites corinthiennes $\mathrm{B}$ évoluées du $\mathrm{V}^{\mathrm{e}}$ siècle (cf. I, 1 § 2.2) trouvées dans la même zone et à Bologne. Par ailleurs, la même comparaison peut être faite concernant les amphores des deux mêmes types provenant des fouilles récentes de Phoinike dans l'Épire méridionale. D'après les analyses minéralogiques et pétrographiques exécutées sur les argiles des exemplaires de Phoinike, il s'agirait d'une fabrication «locale » ${ }^{205}$. Dans la mesure où les attestations de

204. Sur l'ensemble de ces programmes, voir le bilan proposé dans Sourisseau 2011, p. 174 et suiv.

205. Les quelques fragments d'amphores de forme «corinthienne $\mathrm{B}$ » d'époque archaïque et du $\mathrm{V}^{\mathrm{e}} \mathrm{s}$. av. J.-C. de Phoinike sont encore inédits et ont été signalés dans la thèse de Doctorat d'Erika Vecchietti. Je remercie le prof. Sandro De Maria (Université de Bologne, Département d'Archéologie), qui avec Shpresa Gjongecaj (Institut Archéologique de Tirane) dirige la fouille de Phoinike, ainsi que Erika Vecchietti et Elisa Esquilini, qui ont exécuté des analyses sur les pâtes de ces amphores, de m'avoir montré les échantillons des argiles et permis de les confronter avec ceux dont je dispose.
Phoinike sont très peu nombreuses et qu'on ne connaît pas, jusqu'à présent, de phase archaïque de la cité ${ }^{206}$, il serait peut-être plus opportun d'envisager une production régionale, plutôt que spécifiquement locale, peut-être la même que celle reconnue par R. Docter et $\mathrm{R}$. Sauer dans la documentation de la cité voisine de Butrinthos ${ }^{207}$. On peut espérer que les recherches futures pourront expliquer si, pour le matériel archaïque de ces deux établissements de l'Albanie méridionale, il s'agit des productions d'un même atelier, comme celui de Corcyre bien caractérisé pour les époques classique et hellénistique, ou bien, s'il s'agit d'un autre atelier à rechercher sur la terre ferme en face de l'île.

\subsection{Contenu}

Compte tenu de la multiplicité des centres de production potentiels, il est difficile de tenir une position univoque sur le dossier du ou des contenus de ces amphores. Toutefois, et bien que des analyses sur les possibles contenus n'aient pas été exécutées, l'opinion courante est que leur destination principale était en général vinaire. C'est en particulier la position adoptée concernant la possible production sybarite d'amphores de «forme $1 \alpha »$, d'une part parce que les sources littéraires mentionnent l'existence à Sybaris d'une production vinicole importante à l'époque archaïque, mais aussi parce que la plupart de ces amphores, lorsqu'elles sont retrouvées en contextes humides (épaves ou comblements portuaires) portent toujours un revêtement de poix bien caractérisé sur la surface interne ${ }^{208}$.

\subsection{Diffusion dans la Méditerranée occidentale}

Les amphores de «forme $1 \alpha »$ ou «corinthiennes $\mathrm{B}$ archaïques » sont très bien documentées dans les sites du Salento, notamment dans le secteur adriatique, tels Brindes, Torre Santa Sabina, Oria, Muro Tenente, Valesio, Cavallino, Rocavecchia, Torre dell'Orso, Otrante, Vaste et Leuca. On en connaît aussi plusieurs exemplaires provenant des centres du Salento de la façade ionienne, comme Masseria Fano, Torre San Giovanni et Ruffano ${ }^{209}$.

206. Pour les fouilles de Phoinike, voir De Maria, Gjongecaj 2007, avec la bibliographie précédente.

207. Docter sous presse.

208. Sourisseau 2011

209. Sur la riche documentation concernant les sites du Salento cités, voir en dernier lieu Semeraro 1997, p. 44, fig. 5 (Brindes), p. $55, n^{\circ} 25-28$, p. $61, n^{\circ} 79$ (Cavallino), p. 91-94, n' 171 a-f, 172 a-b, d-f, (Leuca), p. 105, n 198-199 (Masseria Fano), p. 129, 
Sur la côte orientale de l'Adriatique des découvertes sont signalées dans le bras de mer en face de Corfou et d'autres à Selcë, à Margëlliç, à Treport et à Belsh, en Illyrie méridionale ${ }^{\mathbf{2 1 0}}$.

Sur la côte ionienne de la Grande Grèce, des exemplaires du groupe sont attestés à Siris-Policoro, à Sybaris, à Caulonia, et à Locres ${ }^{211}$.

En Sicile ces amphores sont bien documentées à Camarine, à Naxos, à Messine, à Mylai, à Himère, à Géla ${ }^{212}$ et dans les cargaisons des épaves tardo-archaïques de Plemmirio ${ }^{213}$, de Géla ${ }^{214}$ et du Cap d'Ognina (Ognina nord I et Ognina sud 3) ${ }^{\mathbf{2 1 5}}$.

Pour la Campanie, les attestations sont également assez nombreuses, notamment à Pithécusses, à Cumes, à Nocera, à Agropoli, à Piano di Sorrento et à Fratte ${ }^{216}$. Deux exemplaires ont été récupérés grâce à une opération de récupération sous-marine et sont conservés dans la Tour de Cetara, tandis qu'un troisième, identifié à l'intérieur d'une collection privée, doit probablement être attribuée au territoire de Gragnano ${ }^{217}$. La docu-

$\mathrm{n}^{\circ}$ 253-254 (Muro Tenente), p. 154, n 320, p. 156, n 324 (Oria), p. $247, n^{\circ} 919$ a-d, p. $250, n^{\circ} 942$ a-d, 943 , p. $251, n^{\circ} 949$ (Otrante), p. $255, \mathrm{n}^{\circ} 954$ (Rocavecchia), p. 269-271, n $979-983,985-987,992-$ 993 (Ruffano), p. 277, n 1010 (Torre dell'Orso), p. 277, n' 1011 (Torre San Giovanni), p. 279, $\mathrm{n}^{\circ} 1030$ a-d (Torre Santa Sabina), p. $285, \mathrm{n}^{\circ} 1058$ (Valesio), p. $294, \mathrm{n}^{\circ} 1090$, p. $302, \mathrm{n}^{\circ} 1129,1132-$ 1122 (Vaste).

210. Pour Corfou, voir Koehler 1981, p. 453 ; pour Selcë et Belsh : Ceka 1983, p. 209, pl. III, 4-5 ; pour Margëlliç : Ceka 1986; pour Treport : Bereti 1992.

211. Pour Siris-Policoro, Spagnolo 2002, p. 34, n. 40, avec bibliographie. Pour Sybaris, Gassner 2003b, p. 187-188, avec références bibliographiques. Pour Caulonia, Vandermersch 1989, p. 94-95. Pour Locres, Barra Bagnasco 1992, n 184, p. 210-231 et Gassner 2003b, p. 190.

212. Pour Camarine, en dernier lieu, Sourisseau 2011 ; pour Naxos, Lentini, Savelli, Blackman 2009, avec bibliographie ; pour Messine, Spagnolo 1999 et Ead. 2002 ; pour Mylae, Tigano 2002, p. 69 ; pour Himère et Géla, Spagnolo 2002, p. 34, note 40, avec références bibliographiques.

213. Sur l'épave de Plemmirio, datée vers le milieu du VIe s. av. J.-C., Wilson 1988, p. 115-116 ; Parker 1992, n 835, p. 319-320, map 11.

214. Sur l'épave archaïque de Géla, datée du premier quart du V $\mathrm{V}^{\mathrm{e}} \mathrm{s}$. av. J.-C., voir : Freschi 1991 ; Panvini 1991 ; Parker 1992, n 441, p. 188-189, map 11 ; Panvini 1993 ; et en dernier lieu, Panvini 2001, notamment p. 72-73, pl. XXIV.140-141 (amphores définies respectivement comme « ionio-massaliètes » et « gréco-occidentales », mais probablement toutes les deux à rattacher au groupe « corinthien B archaïque »).

215. Sur les épaves du Cap d'Ognina, voir : La Fauci 2002, avec un tableau de correspondance entre l'ancien et le nouveau système de dénomination des nombreuses épaves trouvées dans la zone.

216. Pour Pithécusses, Di Sandro 1986, p. 34-38; pour Cumes, Savelli 2006, p. 118-120 ; pour Nocera, Albore Livadie 1985, p. 139 ; pour Agropoli, Piano di Sorrento et Fratte, on consultera le tableau de distribution des exemplaires trouvés en Campanie dans Savelli 2006, p. 118, avec bibliographie.

217. Albore Livadie 1985, p. 139. mentation de l'Étrurie est concentrée dans la zone côtière méridionale, où sont attestés quelques exemplaires dans les nécropoles et les habitats respectifs de Caeré et de Tarquinia. Des trouvailles, plus nombreuses encore, sont signalées dans les emporia de Pyrgi et de Gravisca $^{218}$. En Étrurie interne, quelques amphores de « Forme $1 \alpha$ » probables ont été signalées dans l'habitat de Petriolo-Chiusi ${ }^{219}$.

Dans le sud de la France, de nombreux exemplaires ont été récupérés à l'occasion des fouilles des sites de Marseille, Arles, Saint-Pierre-lès-Martigues, SaintBlaise, La Liquière, Agde, Le Cayla de Mailhac et Pech Maho. D'autres proviennent des cargaisons des épaves d'Antibes, de Bon Porté 1 et de la Pointe Lequin $1 \mathrm{~A}^{\mathbf{2 2 0}}$.

Quelques exemplaires sont arrivés jusqu'au site de la Heuneburg, en Allemagne, où ils se trouvent associés à des amphores plus nombreuses et relevant de la catégorie dite « ionio-massaliète » ou de « forme $2{ }^{221}$.

Plus à l'ouest, le long des côtes de l'Espagne, on connaît des exemplaires trouvés à Emporion, Illa d'en Reixac et dans l'épave de Cala Sant Vicenç 222.

Sur les côtes méridionales de la Méditerranée, les amphores de «forme 1 » ou «corinthiennes B archaïques » sont attestées à Carthage ${ }^{223}$.

\section{Les amphores du groupe « ionio-massaliète » ou grecques occidentales de forme 2}

Parfois dans les mêmes contextes où l'on enregistre la présence d'amphores relevant de la «forme $1 \alpha »$ ou « corinthien B archaïque », on trouve fréquemment des

218. Sur les nécropoles de Caeré et de Tarquinia, voir Boitani 1985, p. 25 ; sur les contextes d'habitat des deux centres, on consultera respectivement Boss 1993, p. 325-327 et Scotti 1986, p. 171 ainsi que Scotti 1999, p. 269-270 ; pour Pyrgi, Colonna 1985, p. 10 ; pour Gravisca, Slaska 1985, p. 19.

219. Savelli sous presse.

220. Pour La Liquière, Py 1990, p. 534. Pour Agde, Nickels 1990 et Nickels 1995, p. 61. Pour Le Cayla de Mailhac et Pech Maho, voir: Gailledrat, Taffanel et Taffanel 2002, p. 176, fig. 153, 1-2 et Gailledrat, Solier 2004, p. 333. Pour les autres découvertes du sud de la France : Sourisseau 1997, I, p. 91.

221. Van den Boom 1990, p. 263-266, notamment, fig. 3, 8. Plus récemment, van den Boom, Pape 2000. À propos de ces présences, voir aussi Morel 1995, p. 351, 361 et Albanese Procelli 1997b, p. 116 et n. 56. La diffusion des amphores grecques en Europe centrale est en cours d'étude dans le cadre du projet européen AGAME (Archaic and classical Greek Amphoras in north-western Mediterranean area and central Europe: diffusion, origin and contents) (FP7-PEOPLE2009-IEF) - F. Sacchetti.

222. Pour Emporion, Aquilué Abadías et al. 2000, p. 333, fig. 40 ; pour Illa d'en Reixac (Ullastret, près de Girona), Rouillard 1991, p. 173 ; pour Cala Sant Vicenç, Santos Retolaza 2008.

223. Morel 1990c, p. 75. 


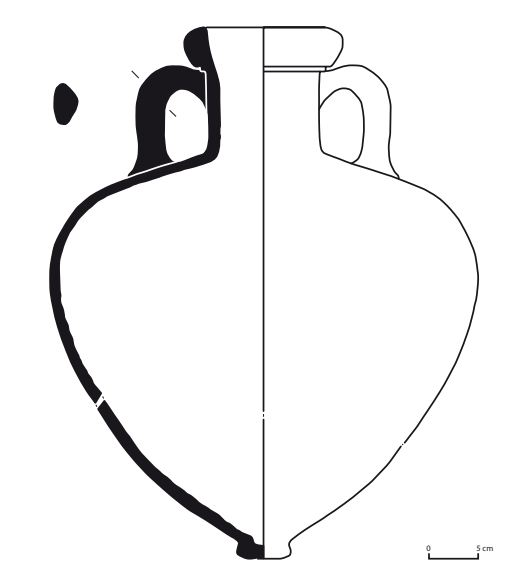

Fig. 16. Amphore du groupe « ionio-massaliète ou grecque occidentale de forme $2 »$. Milazzo, nécropole méridionale, Tombe 41, inv. 16441 (d'après Tigano 2000, p. 71).

exemplaires qu'on peut aussi attribuer au groupe des productions grecques d'Occident, plus particulièrement dans des contextes datés entre la seconde moitié du VIe et le courant du $\mathrm{V}^{\mathrm{e}} \mathrm{s}$. av. J.-C. Ces amphores présentent des affinités morphologiques fortes avec le groupe qui vient d'être décrit, bien qu'elles s'en différencient par certains détails de la lèvre, du col et du pied (fig. 16). Malgré ces différences, elles ont parfois été confondues et, comme pour le groupe précédent, elles ont aussi été identifiées de manière un peu confuse, ce que traduit la terminologie les désignant: "Ionien», « de type ionien»/ ionico, «pseudo-ionien », « ionien occidental» / $c d$. ionico, « ionio-massaliète » / ionio-massaliota, « de type massaliète »/ ionico-marsigliese, $c d$. massaliota, di tipo massaliota, «italo-grec » / massaliota, GraecoMassaliote, ou anfore tirreniche. Voici quelques-unes seulement des dénominations relevées dans la bibliographie ${ }^{224}$. Récemment, V. Gassner a choisi la définition de

224. Parmi les premiers à dénoncer le caractère ambigüe de la terminologie utilisées et à souhaiter qu'on parvienne à une plus grande clarté à ce sujet, on rappellera le rôle joué par P. Pelagatti (Pelagatti 1978 ; Pelagatti 1982, p. 722, n. 115) et, pour un véritable débat, les réflexions de M. Gras (voir notamment Gras 1987, p. 44-45, et Gras 1996). Voir également, dans le même état d'esprit, Albanese Procelli 1997b, p. 110. Malgré cela, ce groupe continue d'être souvent assimilé au groupe des amphores «corinthiennes B archaïques » par l'usage indistinct d'appellations globalisantes très imprécises (souvent les fameuses « amphores ioniennes »).
Randform $3^{225}$ et J.-Chr. Sourisseau celle de «A-MGR $2 »^{226}$ ou $\ll$ forme $2 »^{227}$.

La dénomination «ionio-massaliète ${ }^{228}$, probablement la plus fréquente, est une construction conceptuelle qui traduit à la fois la difficulté d'identification de la série, mais aussi l'idée d'un lien culturel entre l'Ionie et les établissements coloniaux occidentaux d'origine ionienne, en particulier Marseille. Le terme «massaliète» s'impose du fait de l'affinité morphologique observée entre les exemplaires relevant de ce groupe et ceux qui appartiennent à la production archaïque de Marseille, identifiée comme telle depuis longtemps. L'association avec le terme « ionien » traduit quant à lui l'idée de l'existence de prototypes grecs orientaux, en reprenant une terminologie simplificatrice désignant les amphores grecques orientale dans leur ensemble ${ }^{229}$. On rappellera qu'à ce propos, on a même formulé l'hypothèse de l'existence d'une production amphorique de Phocée, qui précisément dans les années de la chute de la cité (à partir de 540 av. J.-C.) aurait été imitée par ses colonies occidentales: Marseille et peut-être aussi Vélia, voire Aléria ${ }^{230}$. Une autre hypothèse voyait dans la production milésienne, qui présente de fortes affinités morphologiques avec les groupes occidentaux, un éventuel prototype aux productions occidentales ${ }^{231}$. Quoi qu'il en soit, jusqu'au début des années 1980, les chercheurs se sont contentés de ces distinctions simples : les amphores ioniennes et/ou ionio-massaliètes désignant donc globalement les différentes productions encore mal connues de la Grèce de l'Est et une masse hétérogène de productions grecques occidentales ${ }^{232}$.

La première tentative de définition du groupe « ionio-massaliète » est celle de M. Py, à l'occasion de la présentation de sa classification des lèvres des amphores

225. Gassner 2003b.

226. Sourisseau 1993.

227. Sourisseau 2011.

228. Sur l'origine de la définition, voir Morel 1990b, p. 283, n. 6.

229. L'idée d'une attribution du groupe à l'aire grecque orientale a été proposée par Fr. Villard (1960, p. 50).

230. Sur les indices historiques et archéologiques qui ont conduit à considérer Vélia comme une cité productrice d'amphores, voir Gras 1987, p. 44 ; Morel 1988 ; Gras 1996, p. 134.

231. Pour l'hypothèse, désormais dépassée, d'un prototype phocéen, voir Gras 1987, p. 44. Pour l'hypothèse d'un lien morpho-stylistique avec un éventuel prototype milésien, Id. 1996, p. 130-133, 137.

232. La non définition de types d'amphores grecques occidentales est très tôt apparue comme un problème, notamment dans les travaux de C. G. Koehler, à propos des amphores qualifiées de «ionian» et qui constituaient une difficulté majeure pour la définition claire de la production supposée corinthienne de type B (Koehler 1981). Remarques allant dans le même sens dans les travaux de M. Gras (Gras 1978,p.175-176 ; Id. 1987, p. 44 ; et Id. 1996, p. 130). 
massaliètes. Observant la variété des pâtes des exemplaires de la forme $1^{233}$ (la plus ancienne, correspondant à la forme Bertucchi 1), à laquelle sont associées des lèvres correspondant à ses types 1,2 et 3 , il proposa d'utiliser «provisoirement le terme ionio-massaliète pour désigner toute la série d'amphores archaïques de provenance non assurée - pour la plupart occidentales, et pour beaucoup déjà marseillaises - qui précède l'apparition des amphores dites massaliètes, c'est-à-dire celles dont la pâte très micacée correspond d'assez près à l'argile utilisée dans la suite de la production marseillaise »(Py 1980, p. 3). Exception faite donc pour tous les individus munis d'une lèvre de forme 1 dont la pâte considérablement micacée permet l'attribution à l'atelier de Marseille ${ }^{234}$, il était, selon l'auteur, préférable d'adopter la définition plus prudente de « ionio-massaliète » pour toutes les amphores dont la forme renvoie au type marseillais le plus archaïque, mais dont le centre de production, en l'absence d'analyses des argiles, reste incertain.

Dans le cadre du débat qui s'est développé à ce propos à l'occasion du congrès de Lattes consacré aux amphores de Marseille, ont été exprimés de nouveau les problèmes concernant, d'une part, l'extrême hétérogénéité du groupe et, d'autre part, les difficultés que posent l'usage de définitions globalisantes, apparemment pratiques mais ambiguës ${ }^{235}$.

Conscient de ces difficultés, on peut néanmoins considérer que la production de cette forme, en général, a commencé peu après le milieu du $\mathrm{VI}^{\mathrm{e}} \mathrm{s}$. av. J.-C., date à laquelle on fait remonter les productions de Locres et de Marseille, pour disparaitre dans le courant de la première moitié du $\mathrm{V}^{\mathrm{e}} \mathrm{s}$. av. J.-C. Pour Marseille la date qui marque la fin de la production est située vers 475 av. J.-C., lorsque la forme archaïque Bertucchi 1 est remplacée par le forme Bertucchi 2 (Bats 1990c, p. 10 ; Sourisseau 1998a, p. 139). Un terminus ante quem au début $\mathrm{du} \mathrm{V}^{\mathrm{e}}$ siècle semble être confirmé aussi pour la

233. Py 1980, p. 1-2, fig. 1. Cette forme «à panse en toupie et col cylindrique » est dite 1 car il s'agit de la forme la plus ancienne des amphores massaliètes, bien que sa caractérisation par les spécialistes soit, elle, tardive. Elle n'est pas signalée dans la première classification des amphores massaliètes de F. Benoît (Benoît 1955, p. 32-43 et Id. 1965, p. 182-185), où elle correspond cependant au type appelé «feldspathique » dont la forme n'était pas précisée. On la trouve, en revanche, dans les travaux postérieurs: Liou 1975 ; Joncheray 1976b; Wallon 1979.

234. Py 1980, p. 1-3, notamment n. 17 pour la description des pâtes identifiables comme marseillaises. Pour les pâtes des amphores de Marseille, voir aussi : Benoît 1955 ; Id. 1965, p. 182-185 ; Bats 1990a, p. 289 ; Bertucchi 1992, p. 42-43. à ce sujet, notamment pour le cas de la production archaïque, Reille-Abbas 1992, et Sourisseau 1998a. 235. Pour le débat sur les « ionio-massaliètes » voir notamment Bats 1990c, Id. 1990a, Morel 1990b et Slaska 1990. production de Locres (Barra Bagnasco 1992, p. 210, 230-231, $\left.\mathrm{n}^{\circ} 179-180\right)$, tandis que pour les autres centres de la Grande Grèce récemment évoqués on ne dispose pas de données certaines à ce propos. On notera cependant l'existence de productions non massaliètes, dites de forme $3^{236}$, qui caractérisent l'évolution de la production des centres de Grande Grèce dans le courant de la première moitié du $\mathrm{V}^{\mathrm{e}} \mathrm{s}$. av. J.-C. et qui se distinguent par une morphologie un peu plus élancée et un col plus haut. On notera également que quelques exemplaires, datés sur une base stratigraphique, pourraient aussi révéler une datation légèrement plus haute pour l'apparition de cette forme et nécessiteraient donc des vérifications. Je fais allusion à deux fragments de lèvre de Belsh, dans la chora d'Apollonia, et de Leuca, provenant de contextes de la première moitié du $\mathrm{VI}^{\mathrm{e}} \mathrm{s}$. av. J.-C., qui par leur morphologie pourraient appartenir au groupe « ionio-massaliète » ${ }^{237}$.

Dans l'attente d'une meilleure définition des productions de cette série grecque occidentale archaïque, on a choisi d'utiliser ici la définition «ionio-massaliète » ou «grecque occidentale de forme 2 » pour désigner toutes les amphores typologiquement similaires mais dont la pâte est variable (à l'unique exception de celle de Marseille fortement micacée). Elles correspondent morphologiquement au type 1 de la classification de G. Bertucchi ${ }^{238}$ (fig. 17), sont munies de lèvres de types 1 (fig. 18,1), 2 (fig. 18,2) et 3 (fig. 18,3) de la classification de M. Py ${ }^{239}$ et leur chronologie renvoie à l'intervalle

236. Récemment caractérisées par J.-Chr. Sourisseau (2011).

237. Pour Belsh, voir Ceka 1983, p. 209, pl. III, 6 ; pour Leuca, en dernier lieu, Semeraro 1997, p. 94, n 172 c, avec bibliographie.

238. Bertucchi 1992, p. 37-51.

239. Py 1980. Le type Py 1, actuellement datée entre la seconde moitié du $\mathrm{VI}^{\mathrm{e}}$ et le premier quart du $\mathrm{V}^{\mathrm{e}} \mathrm{s}$. av. J.-C., est caractérisé par le profil arrondi obtenu par un repli de la paroi du col vers l'extérieur. Cette technique entraîne la formation d'une cavité en forme de goutte plus ou moins accentuée visible en section. Immédiatement au-dessous de la lèvre, on remarque généralement un ressaut en forme de cordonnet. Ce type de lèvre est généralement défini « en bourrelet gonflé » et est le plus caractéristique du groupe «ionio-massaliète ». Le type Py 2 se distingue du type 1 par son profil moins gonflé et par un aplatissement du sommet, qui devient plus ou moins oblique vers l'extérieur. Dans le type 2 aussi on peut voir en section la cavité caractéristique et au-dessous le ressaut en forme de cordonnet. Il est caractéristique de la première moitie $\mathrm{du} \mathrm{V}$ e Le type Py 3 présente un profil allongé et assez arrondi, bien que quelques-unes de ses variantes aient le sommet aplati et oblique vers l'extérieur. On trouve ce dernier type de lèvre associé, non seulement avec des amphores de forme Bertucchi 1, mais aussi de forme Bertucchi 2 et il est attesté uniquement dans la version à pâte micacée typique de la production de Marseille. Bien que déjà présent dans des contextes du premier quart du $\mathrm{V}^{\mathrm{e}} \mathrm{s}$. av. J.-C., ses attestations sont plus fréquentes dans le courant du deuxième quart du siècle. 


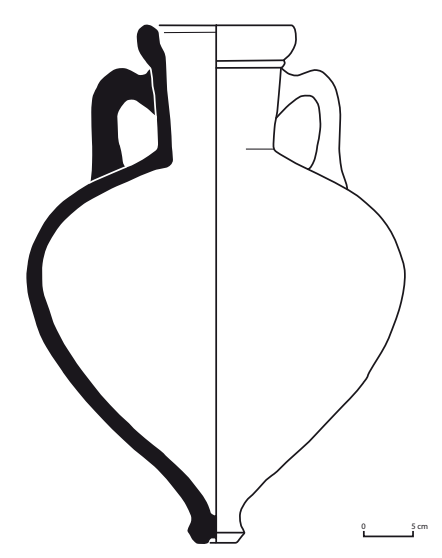

Fig. 17. Amphore massaliète archaïque (d'après Py 1980, fig. 1, forme1.a).

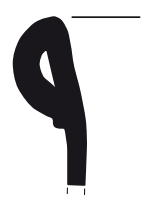

1

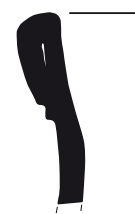

2

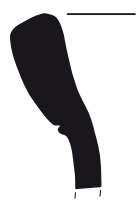

3

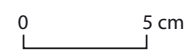

Fig. 18. Les trois premiers types de bords d'amphores massaliètes selon Py 1980, fig. 2. 1-3.

situé entre 540 et 475 av. J.-C. ${ }^{240}$. Ces amphores, bien que présentant un vaste éventail de variations morphologiques de détail, sont caractérisées par une lèvre traditionnellement définie « en bourrelet », rabattue et conservant parfois une cavité en forme de goutte visible en section, soulignée en bas par un mince ressaut, un col cylindrique, des anses en ruban épaissi, l'épaule fortement aplatie, le corps piriforme défini «en toupie», le pied cylindrique plein du type « en bouton » ou bien tronconique à la base plate ou légèrement concave ${ }^{241}$. Toutefois, nous ne disposons pas encore d'une classification typo-chronologique qui tienne compte des variantes morphologiques attribuables aux différents ateliers et de leur évolution précise ${ }^{242}$.

240. Pour la caractérisation de ce cadre et pour la concordance entre les typologies de G. Bertucchi (1990 et 1992) et de M. Py (1980), voir notamment Bats 1990c, p. 10 et Id. 1990a, p. 289-290. La même position est également exprimée par M. Slaska (1990, p. 231).

241. Pour quelques-unes de ces observations morphologiques, voir Slaska 1990, n. 20 et Albanese Procelli 1997b, p. 113.

242. Pour une présentation préliminaire des détails qui pourraient caractériser certaines productions: Gassner 2003b et Ead. 2003a. Pour une synthèse partielle de la diffusion des différentes productions

\subsection{Centres de production}

En l'état actuel des études, l'opinion généralement acceptée est que les ateliers produisant cette série d'amphores étaient nombreux en Occident : à Marseille, mais aussi dans l'aire magno-grecque et peut-être sicéliote.

Pour ce qui concerne l'atelier de Marseille, on sait aujourd'hui qu'en plus de la production caractéristique à pâte micacée, certaines amphores de cette forme et constituées de la pâte «feldspathique », ou «non micacée » décrite par F. Benoit et Fr. Villard ${ }^{243}$, ont également été produites à Marseille. La découverte ancienne d'un four, en activité entre 540 et 500 av. J.-C. à l'emplacement de l'ancienne rue Négrel, accompagnée de nombreux rebuts de cuisson caractéristiques, tant dans le secteur de la rue Négrel que dans le secteur de la Bourse ${ }^{244}$, l'a montré, ainsi que les analyses pétrographiques menées sur ces objets bien des années plus tard ${ }^{245}$. Les amphores produites lors de cette période à Marseille sont caractérisées uniquement par des bords de type 1 et 2 . Cette première production précède donc la fabrication, à partir de la fin du VI ${ }^{\mathrm{e}}$ s. av. J.-C., des mêmes amphores en pâte micacée, mais qui portent des bords non seulement de type 1 et 2 mais aussi de type Py $3^{246}$.

La question des centres de production non massaliètes se pose depuis de nombreuses années et c'est à P. Pelagatti que nous devons les premières remarques sur la diffusion importante de ces amphores de type « ionio-massaliètes » principalement dans l'aire tyrrhénienne ${ }^{247}$. Elle remarque alors leur apparition précoce et leur remarquable concentration, plus précisément sur les côtes tyrrhéniennes de Sicile, de Campanie et d'Étrurie méridionale. À partir de ces remarques, l'idée d'une production principalement tyrrhénienne des amphores du groupe « ionio-massaliète » a recueilli depuis longtemps l'accord des spécialistes ${ }^{248}$. L'état des connaissances a remarquablement progressé ces dernières années grâce aux contributions déjà mentionnées de M. Barra Bagnasco, qui a découvert un atelier de la production de

dans le cadre de la Grande Grèce, voir Savelli 2009. Sur ce point, voir également Sourisseau 2011.

243. Benoit 1965 et Villard 1960.

244. Sourisseau 1998a.

245. Reille-Abbas 1992.

246. Pour ces considérations, voir Sourisseau 1997, I, p. 28-30 et Id. 1998 a.

247. Pelagatti 1982, p. 722, n. 117.

248. Colonna 1985 ; Slaska 1990,p. 231 et n. 28 ; Albanese Procelli 1997b, p. 110-111 et n. 45-46, avec une description de l'examen visuel des pâtes attestées en Sicile et les hypothèses sur les différentes aires de production, dont une doit probablement être située dans l'espace sicéliote ou en tout cas sud tyrrhénien. 
Locres ${ }^{249}$, de V. Gassner, qui à partir du matériel de Vélia a mis en place un vaste programme d'analyses élargi à de nombreux centres de Grande Grèce, de G. Spagnolo, qui a travaillé sur le matériel de Messine et de Gela, et de J.-Chr. Sourisseau, qui a confirmé l'existence d'une production massaliète d'amphores de forme «ioniomassaliète » à pâte non micacée.

L'ensemble de ces travaux et plus particulièrement les approches archéométriques ont permis de distinguer différents ateliers de production de la forme «ioniomassaliète » qui coïncident seulement en partie avec ceux du groupe «corinthien B archaïque ». C'est le cas pour Locres, Kaulonia, et les productions non identifiées de la Calabre et de la zone du détroit de Messine. En revanche, d'autres ateliers ne semblent avoir produit que la forme « ionio-massaliète ». C'est le cas des ateliers de Vélia, de Poséidonia, d'un centre lucanien non identifié mais proche de Vélia et de Poséidonia, de la zone du golfe de Naples et de Marseille ${ }^{250}$.

\subsection{Contenu}

Compte tenu de la grande variété des centres de productions, et de la même manière que pour les amphores grecques occidentales de forme $1 \alpha$, il apparaît difficile de généraliser la destination en termes de contenus de l'ensemble du groupe. Toutefois une tendance générale semble se dégager qui considère le vin comme le produit principal. En ce qui concerne Marseille par exemple, aux nombreuses sources attestant l'importance de la production vinicole locale s'ajoutent les très nombreuses observations de dépôts de substances résineuses visibles sur les parois internes des conteneurs ${ }^{251}$. On suppose une destination analogue pour les productions amphoriques

249. Dans le secteur de la «stoa en U » de Locres : Lattanzi 1989, p. 560-561. Pour les analyses sur les pâtes et l'identification d'une production locale : Barra Bagnasco 1992, p. 208-211 ; Ead. 1993 ; Ead. 1995. Pour une description des pâtes locriennes, voir Albanese Procelli 1997b, n. 45.

250. Pour Locres : Barra Bagnasco 1992 ; Ead. 1993 ; Ead. 1995 ; Gassner 2003b, p. 189-191 ; Spagnolo 2002, p. 37-38 ; Barone et al. 2002, p. 99-100. Pour les productions de Kaulonia : Gassner 2003b, p. 188-189. Pour les productions non identifiées de la Calabre et pour celles de l'aire du détroit de Messine : Gassner 2003b, p. 193-195. Pour Vélia: Gassner 2003b, p. 195-197 et Ead. 2006, p. 497-501, fig. 7. Pour Poséidonia : Gassner 2003b, p. 197-201. Pour les centres non identifiés près de Poséidonia et de Vélia et pour l'aire du Golfe de Naples, voir respectivement : Gassner 2003b, p. 201-201 et Ibid. p. 202-203. Pour la production massaliète archaïque: Sourisseau 1998a, avec bibliographie.

251. Morel 1990b, p. 283 ; Bertucchi 1992, p. 193-210, avec références aux sources anciennes; Sourisseau 1997, I, p. 34, pour les données concernant les résidus de nature résineuse provenant des fouilles de Marseille. de Locres et, en général, pour celle de la Grande Grèce et de la Sicile ${ }^{252}$.

\subsection{Diffusion dans la Méditerranée occidentale}

Les amphores du groupe «ionio-massaliète » sont largement répandues en Occident mais il a été remarqué depuis longtemps que leur présence est particulièrement concentrée dans l'aire tyrrhénienne.

Dans l'aire ionienne-adriatique, les découvertes d'amphores appartenant au groupe «ionio-massaliète » sont beaucoup moins nombreuses que celles du groupe voisin «corinthien B archaïque ». Il faut néanmoins signaler la possible présence d'au moins un fragment à Belsh, dans la chora d'Apollonia, de deux fragments à Treport sur la côte albanaise ${ }^{253}$, et d'autres dans le Salento, notamment à Leuca et à Ruffano ${ }^{254}$.

Le long du littoral ionien de la Calabre, des amphores « ionio-massaliètes » sont signalées à Caulonia, à Locres et à Reggio de Calabre ${ }^{255}$.

La diffusion capillaire du groupe en Sicile a été soulignée par R. M. Albanese Procelli qui en signale la présence à Mylai, Himère, Palerme, Motyé, Sélinonte, Agrigente, Camarine, Mégara Hyblaea, Catane, Naxos, Lipari et dans de nombreux autres sites moins importants ${ }^{256}$. Plus récemment, sa présence a aussi été constatée à Messine ${ }^{257}$. Des amphores appartenant au groupe « ionio-massaliète » faisaient partie des cargaisons des épaves de Camarine-Punta Braccetto ${ }^{258}$, de Plemmirio ${ }^{259}$ et de Géla ${ }^{260}$.

252. Pour le contenu des amphores de Locres: Barra Bagnasco 1992, 227-228 ; Spagnolo 2002, p. 38, n. 81. Plus généralement sur ce sujet : Colonna 1985, p. 10, n. 25, avec de nombreuses références aux sources; Morel 1988 ; Vandermersch 1994, notamment p. 60, avec références bibliographiques ; Gras 1996, p. 134.

253. Pour le fragment de lèvre de Belsh, déjà mentionné, voir Ceka 1983, p. 209, pl. III, 6. Pour Treport : Bereti 1992, pl. II.29-30.

254. Du moins ceux qu'il est possible de distinguer sur la base des dessins publiés par M. G. Semeraro (1997, p. 94, n 172 c, et p. 271, $\mathrm{n}^{\circ}$ 991).

255. Pour Caulonia : Vandermersch 1989, p. 94-95 ; pour Locres : Barra Bagnasco 1992; pour Reggio de Calabre: Gassner 2003, p. 192.

256. Pour une liste détaillée des sites de Sicile et la bibliographie correspondante, voir Albanese Procelli 1997, p. 416-420.

257. Pour les amphores de Messine : Spagnolo 2002.

258. Pour Punta Braccetto: Wilson 1988, p. 116 ; Parker 1992, n 915, p. 346, map 11 ; Di Stefano 1995.

259. Pour l'épave de Plemmirio : Wilson 1988, p. 115-116 ; Parker 1992, n 835, p. 319-320, map 11.

260. Sur les amphores du groupe «ionio-massaliète » de l'épave archaïque de Géla, en dernier lieu : Panvini 2001,p. 72,pl. XXIII.138, XXIV.139. 
Ces amphores apparaissent relativement nombreuses le long de la côte tyrrhénienne méridionale avec les attestations de Vélia, Poséidonia ${ }^{261}$, Fratte, Pontecagnano, Vietri sul Mare, Vico Equense, Pompéi, Pithécusses et Cumes ${ }^{262}$.

L'Étrurie méridionale a également fourni une documentation assez importante, notamment pour la seconde moitié du VI ${ }^{e}$ s. av. J.-C. Dans le territoire de Tarquinia, un exemplaire d'amphore du groupe « ionio-massaliète » est attesté dans la nécropole, quelques-uns proviennent de l'habitat et d'autres encore, en grand nombre, de l'aire sacrée de Gravisca. En ce qui concerne le territoire de Vulci, une amphore provient de la nécropole et plusieurs fragments de son port Regae. Caeré a restitué quelques exemplaires provenant de l'habitat et plusieurs fragments du sanctuaire de Pyrgi ${ }^{263}$.

La documentation de l'Étrurie du nord est incontestablement moins riche que celle du secteur méridional, mais des exemplaires d'amphores du groupe «ioniomassaliète » ont été signalés dans les îles d'Elbe et de Capraia, à Populonia et surtout à Pise et dans son arrièrepays, comme à Fossa Nera di Porcari le long de la vallée du Serchio et à San Rocchino di Massarosa sur la côte de la Versilia ${ }^{264}$. Plus au nord, en Ligurie, le groupe est documenté dans l'habitat de Gênes ${ }^{265}$.

261. Colonna 1985 , n. 23, avec la bibliographie de référence.

262. Pour les amphores «ionio-massaliètes » en Campanie: Di Sandro 1981a ; Albore Livadie 1985, p. 139-140 ; Di Sandro 1990. Pour publication récente d'amphores « ionio-massaliètes » à Vélia et à Cumes, voir respectivement: Gassner 2003 et Savelli 2006, p. $120-121$.

263. Sur les amphores « ionio-massaliètes » en Étrurie, voir Slaska 1990, avec références bibliographiques. À cette contribution fondamentale il faut ajouter, pour Tarquinia : Scotti 1986, p. 171 et Ead. 1999, p. 271-272 ; pour Caeré, Boss 1993, p. 328-332.

264. Pour l'Île d'Elbe: Zecchini 1978, p. 103, pl. 22 ; Maggiani 1981, p. 188 et Zecchini 2001, pl. 57. 4. Pour l'Île de Capraia : Pancrazzi 1982, p. 342, fig. 3.28. Pour Populonia : Romualdi 1993, p.604-606. Pour Pise: Pancrazzi 1982, p. 338-339; Ead. 1993 ; Massa 1993, p. 370. Pour Fossa Nera di Porcari : Ciampoltrini 1994, p. 77, fig. 9.3. Pour San Rocchino di Massarosa : Maggiani 1990b, p. $92, \mathrm{n}^{\circ} 45$, fig. 42 .

265. Milanese, Mannoni 1986, fig. 8, 6 ; Milanese 1987, p. 225 , $n^{\circ} 601$, fig. 100, 601 (type Py 1). Pour les attributions à une production massaliète spécifique d'époque tardo-archaïque : Milanese 1987 , p. 225, n 599-600, fig. 100, 599-600 (type Py 2), et p. 260, $\mathrm{n}^{\circ} 762$, fig. 112, 762 (type Py 3). Sur la diffusion des amphores proprement massaliètes en Ligurie : Milanese 1990.
Dans le sud de la France les amphores du groupe « ionio-massaliète » sont présentes à Marseille, SaintPierre-lès-Martigues, Saint-Blaise, Agde, Le Cayla de Mailhac, Pech Maho, et dans les cargaisons des épaves de Bon Porté 1, du Dattier, de la Pointe Lequin 1A et de la Palud $2^{266}$.

Dans l'Europe centro-septentrionale, les amphores du groupe «ionio-massaliète », avec celles de production massaliète de forme évoluée, semblent actuellement constituer les conteneurs de transport d'origine méditerranéenne les plus diffusés. Les découvertes sont concentrées en France, le long du cours du Rhône et de la Saône, par exemple à Lyon et Bragny-sur-Saône, mais également à Vix-Mont Lassois, à Salins-Le Camp du Château et à Bourges ${ }^{267}$. Relativement nombreuses sont les attestations de la Heuneburg, en Allemagne ${ }^{268}$.

En péninsule ibérique, les découvertes d'amphores « ionio-massaliètes » concernent surtout la côte catalane, avec Emporion, La Cadira del Bisbe, Tarragone, El Puig Castellar de Santa Coloma de Gramenet, mais aussi la région de Valence, avec El Puig de la Nau, El Puig de la Misericórdia et les gisements sous-marins d'El Cabanyal-Malvarrosa et de Las Rocas de las Barbadas ${ }^{269}$.

Enfin, le long des côtes méridionales de la Méditerranée occidentale, quelques exemplaires du groupe ont été trouvés à Carthage ${ }^{270}$.

266. Sur la diffusion des amphores du groupe « ionio-massaliète » dans ce secteur, voir Nickels 1983 ; Arcelin 1990, notamment p. 199 201 ; Bats 1990b, notamment p. 210-211. Sur l'origine marseillaise de beaucoup d'exemplaires : Bats 1990a, p. 289, avec bibliographie. Sur la possibilité d'une provenance magno-grecque ou sicéliote d'une partie des amphores du groupe «ionio-massaliète » trouvées en Gaule méridionale : Morel 1995, p. 351,361. Révision récente de la documentation en Provence et des épaves de la côte méridionale de la Gaule : Sourisseau 1997, I, p. 25-26. Pour Agde : Nickels 1990. Pour le Cayla de Mailhac: Gailledrat, Taffanel et Taffanel 2002, p. 83 , fig. 74 , 11-14, p. 176, fig. 153, 4-14, et fig. 154. Pour Pech Maho : Gailledrat, Solier 2004, p. 333.

267. Pour Lyon et son territoire, pour Bragny-sur-Saône et pour le Mont Lassois, voir la bibliographie citée dans Lüscher 1996, p. 348 351 ; pour Bourges : Sourisseau, Tichit 2007, p. 261-262; pour le Camp du Château : Kimmig 1983, et Villard 1988, p. 337, fig. 2. De nombreux signalements d'amphores dites «massaliètes » d'époque archaïque devront être vérifiés. Sur ce sujet le programme européen AGAME (Archaic and classical Greek Amphoras in north-western Mediterranean area and central Europe: diffusion, origin and contents) (FP7-PEOPLE-2009-IEF) - F. Sacchetti, est en cours.

268. Koehler 981,p. 453, n. 19, avec la bibliographie antérieure. Plus récemment, pour la Heuneburg : van den Boom 1990, p. 263 266, et van den Boom, Pape 2000.

269. Pour Emporion, Aquilué Abadías et al. 2000, p. 333-334, fig. 40 ; pour le reste de la côte catalane: Alaminos et al. 1991, p. 282-283; pour El Cabanyal-Malvarrosa : Fernández, Gomez, Ribera 1988, p. 320-321, fig. 4 ; pour les autres sites du secteur de Valence : Oliver Foix, Gusi Jener 1991, p. 206 (fragments souvent attribués à une production proprement massaliète).

270. Morel 1990c, p. 74, fig. 8 et Id. 1990a, notamment, p. 270-272. 


\section{Chapitre 3}

\section{Les amphores attiques et de type attique « à la brosse »}

$\mathrm{L}$ a série la plus ancienne de conteneurs de transport de production attique est celle des amphores du type dit «SOS », dénomination qui trouve son origine dans le décor caractéristique du col qui rappelle souvent, mais pas toujours, les lettres grecques $\Sigma \mathrm{O} \Sigma^{271}$. Ces amphores doivent leur définition typologique à $\mathrm{A}$. W. Johnston et à R. E. Jones, qui ont distingué trois formes successives désignées Early, Middle et Late (Johnston, Jones 1978). Ce classement typologique a récemment fait l'objet d'une révision de la part de M. A. Rizzo, qui a identifié deux variantes du type Late (Rizzo 1990). Leur production embrasse une période assez longue, entre la fin du VIII e et le début du $\mathrm{VI}^{\mathrm{e}} \mathrm{s}$. av. J.-C. Leur succède alors la série des amphores «à la brosse » qui constituent la production attique du $\mathrm{VI}^{\mathrm{e}}$ et du début du $\mathrm{V}^{\mathrm{e}}$ s. av. J.-C.

La transition entre types SOS Late et les plus anciens exemplaires d'amphores « à la brosse » semble s'effectuer sans rupture majeure dans le courant du premier quart $\mathrm{du} \mathrm{VI}^{\mathrm{e}} \mathrm{s}$. et se caractérise essentiellement par la disparition du décor caractéristique du col. La forme générale est pratiquement la même : « only neck and lip differ ${ }^{272}$.

Les amphores «à la brosse » doivent leur nom à M. Lambrino qui a voulu désigner par ce terme une catégorie de conteneurs de transport dont elle ne connaissait pas l'origine, et qui portaient un décor appliqué sur la surface externe constitué d'un vernis brun étalé à grands coups de pinceau ${ }^{273}$. Cette définition était toutefois utilisée par M. Lambrino pour désigner à la fois les amphores attiques du type SOS et des amphores ne portant pas le décor caractéristique du col et dont le système décoratif était caractérisé par de larges bandes peintes sur la lèvre, sur l'épaule, la panse et le pied, le col restant réservé. Toutefois, les archéologues, notamment Fr. Villard,

271. Pour une illustration des types possibles de décoration du col, voir : Johnston, Jones 1978, p. 134-138.

272. Id., p. 121.

273. Lambrino 1938, p. 132-140. ont restreint la définition des amphores « à la brosse », exclusivement à cette dernière catégorie ${ }^{274}$.

L'attribution à la production athénienne de la plus grande partie des amphores de types SOS et «à la brosse» repose depuis déjà bien longtemps sur la relation des deux séries amphoriques avec la tradition morphologique attique. En effet, si les amphores du type « à la brosse » ont été très tôt rapprochées de la forme des amphores panathénaïques par E. Vanderpool, une observation analogue a été proposée par J. D. Beazley à propos du type $\mathrm{SOS}^{275}$. Des études postérieures ont désormais bien montré qu'il y avait succession entre les types «SOS » et les types «à la brosse » et que, en effet, il existait un lien morphologique fort entre les exemplaires les plus anciens du type «à la brosse » et les amphores panathénaïques qui leurs sont contemporaines, dans le courant de la première moitié du $\mathrm{VI}^{\mathrm{e}} \mathrm{s}$. av. J.-C. ${ }^{276}$ V. Grace, examinant une production d'amphores supposées attiques, de la seconde moitié du $\mathrm{V}^{\mathrm{e}}$ et du IV ${ }^{\mathrm{e}}$ s. av. J.-C., a mis en œuvre la même démarche comparative et considéré que la production tardive qu'elle étudiait constituait une phase successive de la production attique. La recherche récente l'a néanmoins contredite (cf. infra I, 5 §).

274. Johnston, Jones 1978, p. 121.

275. Vanderpool 1938, p. 378 , n 9, fig. 14 ; Beazley 1951, p. 89, n.4. L'exemplaire dont E. Vanderpool publie l'image et qu'il confronte aux amphores panathénaïques est une amphore du type «à la brosse » de forme Agora 1502. J. D. Beazley, quant à lui, établit un rapprochement entre la plus ancienne série d'amphores de transport attribuée à l'Attique (cf. Young 1939, p. 210), aujourd'hui définie « type SOS », et les amphores panathénaïques. Le spécialiste anglais mentionne aussi une "curious representation of a Panathenaicshaped amphora » sur une oenochoé proto-attique du VII ${ }^{\mathrm{e}} \mathrm{s}$. av. J.-C. de l'Agora d'Athènes (sur cet objet: Young 1938, p.417, D17, fig. 5). Le vase représenté, par sa morphologie caractéristique et la chronologie du support (qui exclut la possibilité qu'il puisse s'agir d'une amphore panathénaïque ou d'une amphore «à la brosse ») est sans aucun doute une amphore de type «SOS ». Pour l'identification de l'amphore représentée sur cette oenochoé avec une amphore de type « SOS », voir également Docter 1991, p. 48.

276. Johnston, Jones 1978, p. 121-122, 133, n. 48, suivis par Gras 1987, p. 46-47 et Docter 1991, p. 46. 
Si pour les amphores «SOS» nous disposons des classifications déjà signalées d'A. W. Johnston et R. E. Jones et de M. A. Rizzo, pour les amphores attiques «à la brosse », on ne peut s'appuyer sur une véritable typologie. Pour en établir le cadre typo-chronologique, on doit se baser sur les formes isolées des fouilles de l'Agora d'Athènes.

\section{Bilan des études}

Sur la base de la documentation de l'Agora d'Athènes, il est possible de définir deux formes d'amphore «à la brosse » correspondant respectivement aux formes Agora 1501-1503 et Agora 1502 (Sparkes, Talcott 1970, p. 192-193, 341).

La forme répertoriée sous les numéros 1501 et 1503 de l'Agora (fig. 19) correspond à une amphore à panse ovoïde et à large pied annulaire au profil tronconique fortement évasé vers le bas. Le col évasé bien distinct de l'épaule arrondie est muni d'une lèvre haute relativement épaisse. Les anses arquées à profil continu, sont attachées le long du col, sous le bord et reposent sur l'épaulement. Le décor disposé à grands coups de pinceaux de couleur brune plus ou moins diluée recouvre la lèvre, l'épaule, la panse et le pied. Seuls le col et les anses sont réservés. La datation, établie grâce aux contextes clos de l'Agora, se situe entre le premier quart du VI ${ }^{\mathrm{e}} \mathrm{s}$. av. J.-C., époque à laquelle on date l'exemplaire de type 1501 provenant d'une tombe ${ }^{277}$, et les vingt dernières années du siècle, si l'on suit la chronologie proposée pour l'exemplaire 1503 , provenant du puits P 17:1278. B. A. Sparkes et L. Talcott, justifient et relativisent cette chronologie longue en insistant sur le caractère « both valuable and durable » de ce type de récipient qui pouvait donc être réutilisé relativement longtemps. Ils considèrent même que l'amphore 1503 de l'Agora doit probablement être datée au plus tard du milieu du VI ${ }^{\mathrm{e}} \mathrm{s}$., malgré le contexte de découverte dans lequel elle était probablement en position de réutilisation tardive. C'est également l'avis de M. Lawall, qui rappelle que les amphores de ce type provenant de l'Étrurie méridionale sont toutes datables de la première moitié du $\mathrm{VI}^{\mathrm{e}} \mathrm{s}$. av. J.-C. ${ }^{279}$.

277. Sur la base de la révision critique des vases associés à l'amphore 1501, et suivant l'avis de Fr. Villard, J.-Chr. Sourisseau (1997, I, p. 101, n. 229) suggère une chronologie légèrement plus tardive pour cette tombe et donc également pour l'amphore: début du second quart du $\mathrm{VI}^{\mathrm{e}}$ s. av. J.-C.

278. Sparkes, Talcott 1970, p. 192, 341, cat. 1501, 1503, pl. 64.

279. Lawall 1995, p. 39, n. 22. Pour les amphores du type Agora 1501/1503 des contextes funéraires de l'étrurie méridionale, Rizzo 1990, p. 72, fig. 99-100, 367.

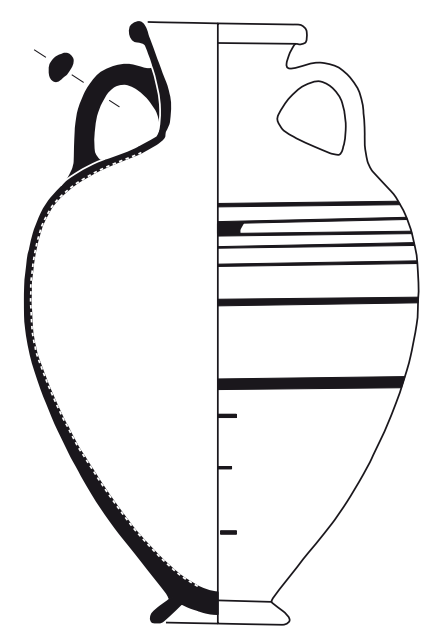

Fig. 19. Amphore de type « à la brosse » de forme Agora 1501 et 1503. Cerveteri, nécropole de la Bufolareccia, Tombe 999 (d'après Rizzo 1990 fig. 367).

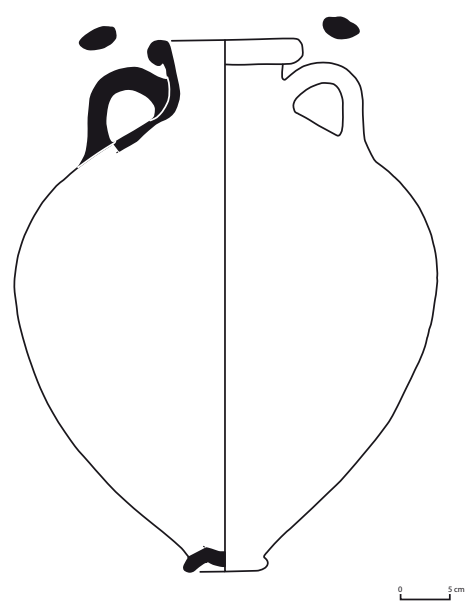

Fig. 20. Amphore de type « à la brosse » de forme Agora 1502. Vulci, Tombe Radicetti, 18.XI.73 (d'après Rizzo 1990 fig. 368 ).

La forme Agora 1502 (fig. 20) constitue une évolution morphologique significative par rapport à la forme précédente. Elle est caractérisée par une lèvre épaissie plus basse et saillante ${ }^{280}$, un col plus bref au profil concave, une épaule plus élargie, une panse ovoïde ou sphérique. Le pied, toujours en anneau, a un diamètre et une hau-

280. Une certaine variabilité dans la forme et dans l'épaisseur des lèvres a été observée au cours de l'étude des exemplaires d'Athènes (Lawall 1995, p. 35, n. 6-7, fig. 3-4, avec références bibliographiques). 
teur remarquablement inférieurs. Le système décoratif et la technique des gros coups de pinceau ne diffèrent pas fondamentalement de ceux de la forme Agora 1501 et 1503 , même si dans ce type plus récent on remarque parfois une plus grande régularité et une meilleure définition des bandes horizontales ${ }^{281}$. La datation de l'exemplaire 1502 est situé entre 575 et 535 av. J.-C. ${ }^{282}$ En fait, la production de cette forme pourrait avoir commencé vers le milieu du $\mathrm{VI}^{\mathrm{e}} \mathrm{s}$. av. J.-C., date avant laquelle on peut en effet situer les exemplaires de types 1501 et 1503 provenant de nombreux contextes et notamment des tombes de l'Étrurie méridionale ${ }^{283}$. La forme Agora 1502 représente en effet l'étape successive du développement de la série « à la brosse » comme le montrent les contextes de découvertes de l'Acropole d'Athènes des exemplaires du même type, tous datés entre la seconde moitié du $\mathrm{VI}^{\mathrm{e}}$ s. et 480 av. J.-C., ce qui en suggère une continuité d'utilisation au moins jusqu'au début du $\mathrm{V}^{\mathrm{e}} \mathrm{s}$. av. J.-C. ${ }^{284}$. L'étude systématique des amphores provenant des contextes de l'Agora d'Athènes fournit une confirmation de ce cadre chronologique ${ }^{285}$. En outre, la découverte de 16 exemplaires provenant de 5 dépôts de l'Agora, clos vers le milieu du $\mathrm{V}^{\mathrm{e}} \mathrm{s}$. av. J.-C. et qui contenaient des objets datables pour la plupart du second quart du siècle a suggéré, de manière tout à fait hypothétique, la continuité de production de cette forme jusqu'à cette date postérieure à la destruction perse ${ }^{286}$. Dans les exemplaires de l'Agora, de nombreuses variantes ont été enregistrées qui sont surtout liées à la forme des lèvres ainsi qu'à l'épaisseur et aux types de profils des anses. Cependant, la multiplicité des associations qu'on peut observer entre ces variantes n'a pas permis de parvenir à l'élaboration d'une typo-chronologie détaillée de la forme Agora 1502, mais suggère que les exemplaires les plus tardifs du type, datables du courant de la première moitié $\mathrm{du} \mathrm{V}^{\mathrm{e}} \mathrm{s}$. av. J.-C., présentent une lèvre moins épaisse et un col plus haut par rapport aux exemplaires datables encore entre la fin du $\mathrm{VI}^{\mathrm{e}} \mathrm{s}$. et le début du $\mathrm{V}^{\mathrm{e}} \mathrm{s}$. av. J.-C. ${ }^{287}$.

281. Sur cette observation, voir : Lawall 1995, p. 35, n. 4.

282. Sparkes, Talcott 1970, p. 192, 341, cat. 1502, pl. 64.

283. Sur cette datation, voir : Lawall 1995, p. 36, n. 10, avec bibliographie des contextes de référence, et Sourisseau 1997, p. 103. Pour les amphores «à la brosse » du type Agora 1502 des tombes de l'étrurie méridionale : Rizzo 1990, p. 18, 23, 100, fig. 13, 181, 368.

284. Sparkes, Talcott 1970, p. 193, n. 36. Pour les exemplaires à la brosse de l'Acropole : Broneer 1938, p. 184-185, fig. 21 et Roebuck 1940 , p. 249,257 , cat. 337 , fig. 61.

285. Lawall 1995, p.36-37, avec références aux exemplaires datables de contextes d'Athènes et de Corinthe et bibliographie.

286. Lawall 1995, p. 37, n. 15-17, avec références précises aux contextes de provenance.

287. Lawall 1995, p. 35-36, 38, fig. 1, 3-4.

\section{Centres de production}

Les analyses archéométriques (OES analysis) effectuées sur les pâtes de 91 amphores SOS et de 7 amphores «à la brosse » par A. W. Johnston et R. E. Jones ont permis de confirmer l'origine attique de la plupart des exemplaires examinés. Cependant, pour ce qui concerne les amphores SOS, on a pu identifier aussi une production euboïco-chalcidienne et une hypothèse a été avancée selon laquelle des imitations de cette forme auraient été produites, bien qu'en moindre mesure, dans d'autres centres, notamment dans la colonie eubéenne de Pithécusses ${ }^{288}$.

Dans le cas spécifique des amphores "à la brosse », cinq échantillons examinés par R. E. Jones ont révélé des affinités dans la composition chimique avec la céramique fine attique, tandis que les deux autres seraient de provenance incertaine ${ }^{289}$. Dans la plupart des cas, les amphores «à la brosse » seraient donc de production attique. Dans l'attente de recherches ultérieures et sur la base de l'observation visuelle de différents types de pâtes, plusieurs spécialistes préfèrent toutefois éviter d'attribuer les amphores « à la brosse » à la production attique sur la seule base des affinités morphologiques évoquées et du nombre limité d'analyses physico-chimiques.

\section{Contenu}

Des ateliers pour la production d'amphores commerciales étaient implantés en Attique dès le VIII ${ }^{\mathrm{e}}$ s. av. J.-C. et l'on suppose traditionnellement que ces amphores étaient destinées au transport de l'huile.

L'hypothèse a été avancée dans un premier temps sur la base de la vocation agricole historique de cette région pour l'oléiculture (Vallet 1962), mais des arguments ultérieurs se sont ajoutés à l'appui de cette hypothèse en rapport avec la morphologie des amphores attribuées à la production attique (Johnston, Jones 1978). Les éléments considérés ont été d'une part le détail de la forme de la lèvre des amphores SOS, semblable à celui des lécythes attiques, et d'autre part, la relation morphologique

288. Johnston, Jones 1978, p. 111-112, 125-128 ; Jones 1986, p. 706712 ; Buchner, Ridgway 1993, pl.210, 476; Albanese Procelli 1997b, p. 99-100, avec références bibliographiques.

289. Johnston, Jones 1978, p. 121-128 (notamment p. 122 pour les amphores « à la brosse »). Pour d'autres analyses sur les pâtes des amphores de cette série : Dupont 1982,p. 193. 
générale existant entre les amphores de type SOS et «à la brosse » avec les amphores panathénaïques.

Cependant, quelques aspects de type iconographique pourraient aussi justifier leur emploi pour le commerce du vin ${ }^{290}$. À ce propos, les exemples les plus significatifs sont constitués par la scène du vase François de Vulci, daté vers 570 av. J.-C., sur lequel Dionysos porte une amphore SOS (Stewart 1988, p. 57, fig. 4,2), et par la représentation du même type d'amphore sur une oenochoé proto-attique d'Athènes datée du troisième quart du VII' s. av. J.-C. (Young 1938, p. 417, 418, 425, fig. 5). Sur la base de ces éléments et d'autres aussi, R. F. Docter a avancé l'hypothèse que les amphores SOS, nées sans doute comme des conteneurs à huile à la fin du VIII $\mathrm{s}$. av. J.-C., aient été aussi utilisées comme conteneurs à vin. Ce double emploi pourrait avoir eu lieu entre la deuxième moitié du VII' s. av. J.-C., datation de l'oenochoé proto-attique mentionnée, et la réforme agraire de Solon qui vers 590-580 av. J.-C a interdit l'exportation de produits attiques autres que l'huile ${ }^{291}$.

En revanche, les amphores « à la brosse » de fabrication attique, dont la production commence exactement à l'époque des réformes de Solon, ou peu après, devraient être exclusivement des conteneurs à huile (Gras 1987, p. 46). Cette hypothèse repose cependant sur une position de principe qui n'est étayée sur aucune analyse de contenus. Par ailleurs, elle ne préjuge pas du problème des marchandises transportées dans les amphores du type «à la brosse » de production non attique.

\section{Diffusion dans la Méditerranée occidentale}

Même si elles sont bien documentées en Occident, la circulation commerciale en Occident des amphores attiques «à la brosse » apparaît plus limitée que celle du type SOS.

Pour ce qui concerne la Grande Grèce, on en trouve à Caulonia, à Cumes, à San Nazzaro d'Avellino, à Poséidonia et dans le Salento ${ }^{292}$. En Sicile, où leur

290. Pour une vaste discussion sur ce sujet et la bibliographie complète sur les différentes hypothèses et le détail des arguments : Docter 1991.

291. Docter 1991, p. 48. Pour une position critique vis-à-vis de cette hypothèse, voir M. Gras 2010,p. 111.

292. Pour Caulonia: Vandermersch 1989, p. 97, n. 487, fig. 64. Pour Camarine : Pelagatti 2006 et Sourisseau 2006. Pour Cumes : Di Sandro 1981b, p. 4-5 ; Albore Livadie 1985, p. 138, n 1 ; Savelli 2006, p. 107-109. Pour San Nazzaro : Albore Livadie 1985, p. 138 , diffusion est assez vaste, on en connaît aussi bien des exemplaires considérés de production attique, que des produits d'imitation dont la provenance n'a pas été identifiée. On les trouve notamment à Himère, Camarine, Monte San Mauro, Messine, Colle Madore, Naxos ; des exemplaires de ce type sont aussi attestés dans l'épave de Géla ${ }^{293}$. Dans la Mer Tyrrhénienne centrale et septentrionale, des amphores « à la brosse » sont documentées en Étrurie méridionale, à Caeré, Vulci, Pyrgi et Regae, ainsi qu'en Étrurie du nord et vers l'intérieur, notamment à Pise, Chiusi, Chianciano Terme, Sarteano et Corciano $^{294}$.

La diffusion du type en Occident arrive jusqu'en Provence, où l'on en trouve un nombre assez important à Marseille, quelques exemplaires dans l'épave de la Pointe Lequin A1, et des fragments isolés sur d'autres sites provençaux, tels Arles, Saint-Pierre-lès-Martigues, Saint Blaise, les Baou de Saint-Marcel, le Mont-Garou et la Courtine à Ollioules ${ }^{295}$.

En Espagne, les amphores «à la brosse » sont présentes à Emporion et on en signale aussi dans le secteur de Valence ${ }^{296}$. $\mathrm{n}^{\circ}$ 2. Pour Poséidonia: Greco, Theodorescu 1987, p. 139, n 655, 788b, fig. 15. Pour le Salento : Semeraro 1997, p. 55, cat. 30, fig. 13 ; p. 58 , cat. 54 , fig. 15 ; p. 156 , cat. 323.

293. Sur la diffusion du type en Sicile : Albanese Procelli 1997b, p. 103. En compléments, pour Colle Madore : Polizzi 1999, p. 224 n³ 392-395, fig. 222, n 392-395 ; pour l'épave de Géla: Panvini 2001, p. 73-74 ; pour Messine : Spagnolo 2002, p. 32 ; pour Himère : Vassallo 2003, p. 341, n 21, fig. 5, 21 ; pour Camarine : Sourisseau 2002, p. 548 , et $I d$. 2006, p. 133 et fig. 1 ; pour Naxos : Lentini, Savelli, Blackman 2007, p. 98, et Id. 2009, p. 103, fig. 9-10.

294. Pour Caeré et Vulci : Rizzo 1990, p. 72, fig. 99-100, 367 ; pour Pyrgi : Colonna 1985 ; pour Gravisca : Slaska 1985 ; pour Regae : Morselli, Tortorici 1985 ; pour Pise : Pancrazzi 1982, p. 336, fig. 2,

13. Pour l'Étrurie interne: Martelli, Nasorri 2000, p.96, fig. 11 (Tombe de l'Inscription de Poggio Renzo - Chiusi) ; Paolucci 2006, p. 421-423 (deux exemplaires inédits de Chianciano et de Sarteano); Bruschetti 1993,p. 11, 59-60, fig. 11 (Corciano).

295. Sourisseau 1997, I, p. 105, 115 ; Id. 2000, p. 140-141.

296. Voir respectivement: Aquilué Abadías et al. 2000, p. 333, fig. 40, 4 et Fernández, Gomez, Ribera 1988, p. 318. 


\section{Chapitre 4}

\section{Les productions amphoriques de l'Égée septentrionale}

L a région de l'Égée septentrionale est surtout connue pour les productions amphoriques de la cité de Mendé et de l'île de Thasos. Depuis longtemps identifiés sur la base des timbres, les deux ateliers présentent d'importantes analogies au point de vue morphologique. Par conséquent, la distinction entre les deux séries n'est pas facile, notamment en ce qui concerne la production moins connue des époques tardoarchaïque et classique. La difficulté est d'autant plus importante qu'on sait aujourd'hui qu'il existe d'autres productions, issues d'ateliers situés hors de Mendé et de Thasos, et qui présentent des caractéristiques morphologiques également très proches.

Entre l'époque tardo-archaïque et la période classique, dans l'aire de l'Egée septentrionale, devait donc exister une sorte de « koinè nord-égéenne » qui, de manière analogue à ce qui se manifeste au même moment dans le cadre d'une «koinè sud-ionienne" (cf. infra I, 5 § 3), se caractérise par la production de modèles amphoriques similaires issus de plusieurs ateliers dispersés dans une région assez vaste correspondant à l'aire de la péninsule Chalcidique, comprise entre le cours du fleuve Axios à l'ouest et l'île de Thasos à l'est. Parmi les localités actives aux époques tardo-archaïque et classique dans cette production d'amphores de type « régional », en plus de Mendé et de Thasos, qui sont placés aux deux extrémités de cet espace, il faut citer par exemple Acanthos, comme en témoignent des timbres amphoriques incluant les lettres $A K A N$ imprimées sur des amphores du groupe, ainsi que la découverte de fours de potiers ${ }^{297}$. Cependant, à en juger par l'hétérogénéité des pâtes et l'abondance de variantes attestées par rapport aux modèles qu'on pourrait définir comme standards, beaucoup d'autres ateliers nord-égéens attendent encore une identification précise.

297. Pour cet atelier, encore mal connu, repéré sur la base des timbres, voir en premier lieu : Rhomiopoulou 1986. La notice de la découverte de fours à Acanthos (Garlan 1989, p. 480, et n. 11 ; Grandjean 1992, p. 578, et n. 29) laisse supposer que la cité était le siège de la production des amphores timbrées attribuées dans un premier temps à Amphipolis (Nikolaïdou-Patera 1986).

\section{La production amphorique attribuée à Mendé}

Malgré l'état encore insuffisant des recherches sur les amphores commerciales fabriquées à Mendé, les documentations d'Athènes, de Corinthe et de l'épave de Porticello, en Calabre, ont cependant permis de distinguer quelques variantes typologiques datées dans un arc chronologique allant du dernier quart $\mathrm{du}^{\mathrm{VI}^{\mathrm{e}}}$ au $\mathrm{IV}^{\mathrm{e}} \mathrm{s}$. av. J.-C. ${ }^{298}$.

Nous devons l'identification des amphores de Mendé à V. Grace qui, entre la fin des années 1940 et le début des années 1950, a attribué à la cité de la péninsule Chalcidique une anse avec un timbre représentant Dionysos, le bras tendu, tenant sans doute un canthare, et assis à l'envers sur un âne ${ }^{299}$. Cette représentation fut associée par la spécialiste à une série de monnaies de Mendé du troisième quart du $\mathrm{V}^{\mathrm{e}} \mathrm{s}$. av. J.-C. et cette chronologie s'accordait bien avec la datation du dépôt $\mathrm{R}$ 13:1 de l'Agora d'Athènes, clos vers 400 av. J.-C., d'où provenait ce fragment timbré ayant le numéro d'inventaire SS6917. Cette anse timbrée constitua pour V. Grace le point de départ qui lui permit ensuite d'attribuer à Mendé d'autres fragments de plus grandes dimensions et de définir enfin les caractéristiques générales de la morphologie et de la pâte des amphores produites par cette cité vers la fin du $\mathrm{V}^{\mathrm{e}} \mathrm{s}$. av. J.-C. ${ }^{300}$. Récemment, M. Lawall, ayant accès au matériel amphorique des dépôts de l'Agora, a pu établir une classification typo-chronologique de cette série pour le $\mathrm{V}^{\mathrm{e}} \mathrm{s}$. av. J.-C. Le point de départ de ce classement repose sur les exemplaires timbrés plus récents. Grâce à l'association des caractéristiques de leurs pâtes avec les principaux traits morphologiques identifiés par V. Grace comme des éléments caractérisant la production de Mendé, M. Lawall a pu retracer l'évolution

298. Pour les exemplaires d'Athènes, voir: Grace 1953, p. 106 107, n 161-162, fig. 5, pl. 40 et Ead. 1961, fig. 43 (on trouve la même amphore dans Biers 1980, p. 235). Pour la documentation de Corinthe : Williams 1978, p. 19, fig. 5. Pour les amphores de Mendé de l'épave de Porticello : Eiseman 1973, p. 13-15.

299. Pour la première identification du type voir: Grace 1949, p. 182, 186, pl. 20, 1 ; Grace 1953, p. 106-107, notes 161-162.

300. Grace $1953, \mathrm{n}^{\circ} 161$. 
morphologique de la série à partir du début du $\mathrm{V}^{\mathrm{e}} \mathrm{s}$. av. J.-C. Il s'agit donc d'un processus de classification qui, partant de l'identification des exemplaires les plus récents et dont le lieu de fabrication était garanti par les timbres, a construit à rebours l'identification des exemplaires plus anciens susceptibles d'appartenir au même atelier de production et dépourvus de timbres.

\subsection{Bilan des études}

Les plus anciens exemplaires des amphores de Mendé sont datés de la fin du $\mathrm{VI}^{\mathrm{e}} \mathrm{s}$. av. J.-C. Ces exemplaires, qui n'ont pas encore fait l'objet d'une description et d'un encadrement chronologique précis à l'exception de quelques exemplaires publiés ${ }^{301}$, présentent des caractéristiques qui les distinguent sans aucun doute des exemplaires $d u \mathrm{~V}^{\mathrm{e}} \mathrm{s}$. av. J.-C., notamment la forme du pied. Alors que dans l'évolution suivante de la série le pied présente une forme «en bobine », dans les exemplaires datables avant la fin du $\mathrm{VI}^{\mathrm{e}}$ siècle il est court et évasé, de forme tronconique et sa base en anneau a une circonférence modérée ${ }^{302}$. La panse de ces premiers exemplaires est ovoïde, le col cylindrique au profil légèrement concave et les anses à section biconvexe présentent des impressions digitales caractéristiques à leur base. La lèvre, de petites dimensions et épaissie, est légèrement ouverte et se distingue du col par un ou deux ressauts. L'épaississement peut être observé vers l'intérieur ou bien vers l'extérieur; dans ce deuxième cas, il est dû au profil latéral oblique de la lèvre qui détermine une section triangulaire (fig. 21) ${ }^{303}$.

$\mathrm{Au}$ début du $\mathrm{V}^{\mathrm{e}} \mathrm{s}$. av. J.-C., la morphologie des amphores de la série présente des modifications qui semblent rester stables jusqu' au milieu du siècle (fig. 22). Les exemplaires appartenant à cette première variante $\mathrm{du} \mathrm{V}^{\mathrm{e}}$ siècle, Early Mendean Variant selon la définition de M. Lawall, ont une lèvre courte, plutôt épaissie et anguleuse à l'extérieur qui est définie wedge-shaped. Sa morphologie est assez irrégulière, de même que la

301. à ce propos, il faut signaler l'importante contribution de S. Ju. Monachov sur les amphores de la Mer Noire (Monachov 1999b), où la forme la plus ancienne a été largement diffusée. Plus récemment et fondamentale pour les repères chronologiques fournis, voir la publication de S. G. Schmid (2001).

302. Je fais allusion, par exemple, aux deux amphores du Forcello (De Marinis 1996, p. 334, fig. 7-9 ; Cf. infra cat. FO39-FO40), datées par R. De Marinis entre la fin du VI et le tout début du V ${ }^{\mathrm{e}} \mathrm{s}$. av. J.-C. sur la base de la comparaison avec deux exemplaires du puits Q12:3 de l'Agora d'Athènes (Grace 1961, fig. 35), antérieur à la destruction perse de 480 av. J.-C. et contenant de la céramique attique datée entre 520 et 490 av. J.-C. (Thompson 1855, p. 62).

303. Exemplaires nord-égéens du début de la série et provenant de contextes datés sur une base stratigraphique, dans : Schmid 2001. manière dont elle se joint au col, qui varie d'un individu à l'autre. Les anses, en ruban épaissi, sont assez caractéristiques. Leurs attaches présentent souvent des bavures d'argile, conséquence sans aucun doute de l'opération de jonction de l'anse au col et à l'épaule. Des impressions digitales caractérisent en général les attaches inférieures. La panse, de forme ovoïde, présente des bandes fines horizontales peintes dans la moitié inférieure. Le pied, défini disc-shaped toe, est tronconique, de hauteur modérée et présente un plan d'appui discoïde légèrement concave au milieu et au profil latéral évasé ${ }^{304}$. La chronologie de cette forme se base, pour le premier quart $\mathrm{du} \mathrm{V}^{\mathrm{e}}$ siècle, sur un unique fragment trouvé dans le dépôt D 15:1 de l'Agora et sur un second exemplaire, lui aussi fragmentaire, d'Égine, tandis que pour le second quart du siècle, les attestations d'amphores de Mendé provenant de contextes clos de l'Agora d'Athènes deviennent relativement plus consistantes : dépôts $\mathrm{F}$ 19:4, N 7:3 et C 18:4b, tous clos avant 440 av. J.-C. C'est évidemment grâce à ces exemplaires, bien qu'ils soient encore peu nombreux, qu'il a été possible de suggérer une description de la forme des amphores de Mendé de la première moitié du Ve s. av. J.-C. ${ }^{305}$.

L'étape suivante de l'évolution de la série, définie Middle Mendean Variant, occupe le troisième quart du $\mathrm{V}^{\mathrm{e}}$ siècle (fig. 23), lorsque les attestations d'amphores de Mendé deviennent nettement plus nombreuses, probablement en liaison avec un accroissement de la production ainsi que de ses exportations ${ }^{306}$. Les amphores comprises dans cet arc chronologique se distinguent des précédentes surtout par le type du pied allongé, en forme d'une courte bobine, ou short stem-toe, évasé vers le bas et au fond ombiliqué. La lèvre, plus amincie, présente encore la forme "en coin », aiguisée en haut et légèrement ouverte ; à la base il est possible d'observer une cannelure ou un ressaut. La panse conserve la forme ovoïde des exemplaires de la première moitié du siècle et présente parfois, comme ces derniers, des bandes peintes. Les anses ne présentent pas non plus de variations remarquables et conservent les caractéristiques de la bavure d'argile et des impressions digitales à la base

304. Pour ces caractéristiques morphologiques, voir l'exemplaire de Vico Equense publié par N. Di Sandro (1981b, p. 10-11, fig. 3.4) et daté ensuite par le même auteur du milieu du V ${ }^{\mathrm{e}}$ s. av. J.-C. (Di Sandro 1986, p. 82) sur la base des analogies relevées avec les exemplaires athéniens publiés par V. Grace et provenant du dépôt N 7:3 (Grace 1953, p. 106-107, n $\left.{ }^{\circ} 161-162\right)$. L'exemplaire est reproduit aussi dans Bonghi Jovino 1982, p. 83, pl. 45.3, 122.4.

305. Pour le fragment le plus ancien des contextes de l'Agora, voir : Lawall 1995, p. 120, et n. 12 ; pour l'exemplaire du temple d'Aphaïa à égine : Johnston 1990, n 135 ; pour les dépôts de l'Agora clos en 440 av. J.-C. et qui ont livré des amphores de Mendé : Grace 1953, p. 106-107, n 161-162 et Lawall 1995, p. 120 et n. 15.

306. Sur ces considérations, voir Lawall 1995, p. 120-121. 

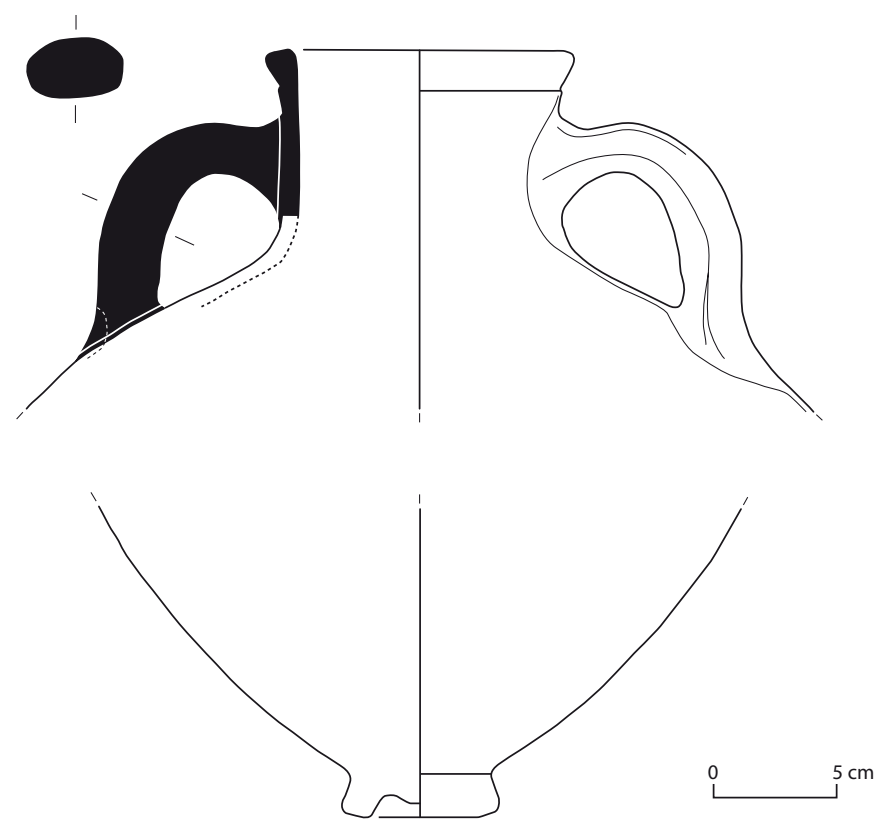

Fig. 21. Amphore nord-égéenne. Athènes, Céramique, sépulture (d'après Schmid 2001, pl. 4).
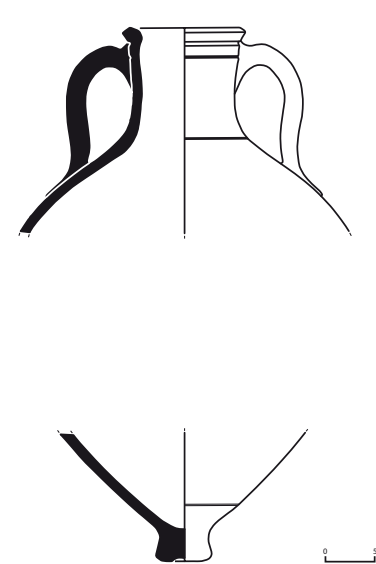

Fig. 22. Amphore nord-égéenne (Mendé)

Athènes, Agora, inv. P21987

(d'après Grace 1953, fig. 5.161).

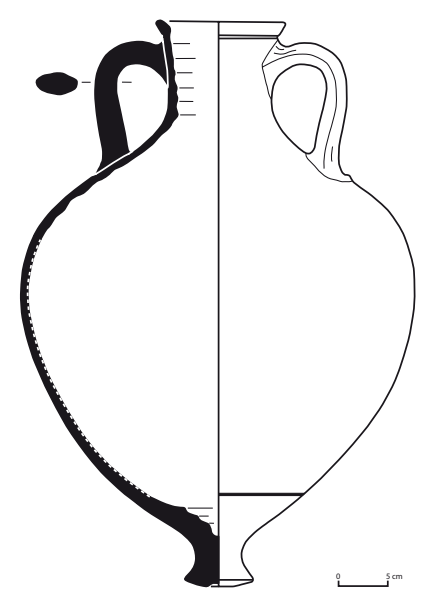

Fig. 23. Amphore nord-égéenne (Mendé). Athènes, Agora, inv. P23777 (d'après Papadopoulos, Paspalas 1999, fig. 2). 
des anses. La datation au troisième quart du siècle des exemplaires de cette forme est confirmée non seulement par des exemplaires de la Mer Noire, mais aussi par des attestations des dépôts M 17:7 et R 13:4 de l'Agora d'Athènes, tous deux clos vers 425 av. J.-C. et dont le second comprenait cinq exemplaires entiers ${ }^{307}$.

Dans le dernierquart du $\mathrm{V}^{\mathrm{e}} \mathrm{s}$. av.J.-C., on remarque une évolution morphologique de la série de Mendé (fig. 24). Les amphores datables de cette période, Late Mendean Variant, commencent à présenter une forme plus élancée et anguleuse qui caractérise clairement l'évolution qui va s'accomplir au $\mathrm{IV}^{\mathrm{e}} \mathrm{s}$. av. J.-C. La lèvre conserve, bien qu'avec des variations, la forme générale en coin. Le col, toujours cylindrique et au profil concave, s'allonge et se resserre légèrement, alors que l'épaulement devient plus aplati et se distingue aussi bien du col que de la panse, donnant ainsi au corps de l'amphore un profil piriforme. Les anses en ruban épaissi s'amincissent et s'allongent légèrement à cause de l'allongement du col mais conservent les caractéristiques tant des impressions digitales aux jonctions inférieures que de l'implantation irrégulière sur l'épaule. Le détail le plus carcatéristique de cette forme de fin $\mathrm{V}^{\mathrm{e}} \mathrm{s}$. av. J.-C. reste de toute façon le pied en bobine, évasé vers le bas et au fond ombiliqué, mais désormais plus allongé et formant un profil continu avec le fond de la panse. La datation au dernier quart $\mathrm{du} \mathrm{V}^{\mathrm{e}} \mathrm{s}$. av. J.-C. de cette forme est proposée sur la base de la documentation de la Mer Noire et de contextes de l'Agora d'Athènes clos en 410 (Dépôt $S$ 16:1), ou au plus tard en 390 av. J.-C. (Dépôt Q 15:2) ${ }^{308}$.

Au passage entre le $\mathrm{V}^{\mathrm{e}}$ et le $\mathrm{IV}^{\mathrm{e}} \mathrm{s}$. av. J.-C., la morphologie générale des amphores de Mendé change de manière évidente : le corps de l'amphore, nettement élancé, prend une forme biconique et assume les caractéristiques du haut col tronconique, des anses surélevées et convergentes, de l'épaule aplatie et au profil concave et enfin du pied en bobine, caractéristiques bien connues pour les exemplaires de l'épave de Porticello (fig. 25) ${ }^{309}$.

307. Pour les amphores de la Mer Noire : Brašinskij 1976, p. 68, 72, fig. 1-2; pour l'Agora d'Athènes : Lawall 1995, p. 122 et notes 19 et 21 .

308. Pour la Mer Noire : Brašinskij 1976, p. 70, fig. 3. Sur la documentation de l'Agora: Lawall 1995, p. 122-124. Des amphores de cette forme proviennent également de l'épave d'Alonnesos (Mantzouka 2004, pl. 36-37).

309. Pour l'épave de Porticello et la description de amphores de Mendé, dites Type 1C, voir: Eiseman 1973, p. 14, n 4-5, fig. 1, Type 1c: $n^{\circ}$. 4, et Eiseman, Ridgway 1987, notamment p. 39-40, où la proposition chronologique avancée oscille entre la fin du $\mathrm{V}^{\mathrm{e}}$ et le début du IV e s. av. J.-C. Au contraire, D. Gill (1987) propose pour l'épave une datation vers 425 av. J.-C., qui cependant ne s'accorde pas avec la proposition typo-chronologique de C. J. Eiseman et de M. Lawall.

\subsection{Centres de production}

On l'a vu, les caractéristiques morphologiques générales des amphores attribuées à Mendé sont communes à plusieurs autres amphores de production nord-égéenne. Ce qui différencie de la production spécifique de la cité de Mendé est donc surtout le type de pâte. La matrice argileuse des amphores de Mendé a une couleur plus ou moins intense, avec des nuances variables du beige au rouge-chamois, à l'orange, mais virant parfois au rougeâtre. La pâte extrêmement grossière et micacée, souvent caractérisée par des inclusions de quartz de dimensions considérables, apparaît à une première analyse visuelle comme l'un des éléments les plus distinctifs de la production de Mendé dans le cadre de la koinè nord-égéenne.

La pâte des amphores de Mendé a fait depuis longtemps l'objet d'un programme d'analyses pétrographiques. Sur une base documentaire d'un peu plus de vingt échantillons datés entre la seconde moitié du $\mathrm{V}^{\mathrm{e}}$ et le début du IV ${ }^{\mathrm{e}} \mathrm{s}$. av. J.-C. et provenant des fouilles de Corinthe, I. K. Whitbread a pu identifier deux groupes qui se différencient entre eux par la texture de la matrice argileuse, mais non par le type d'inclusions dans la pâte. Alors que le Group 1 de I. K. Whitbread présente une argile assez dépurée et micacée, peut-être tamisée et pétrie, avec du sable ayant la fonction de liant. Le Group 2 présente au contraire une argile moins dépurée et, même si dans la pâte de ce second groupe était présent un liant, celui-ci est plus difficilement identifiable à cause d'une argile plus boueuse ${ }^{310}$. L'attribution à Mendé de la production est enfin confirmée par des analyses effectuées sur des échantillons d'argiles provenant de la zone nord et sud de Mendé. Ces analyses ont en effet montré les analogies entre la texture de ces échantillons d'argiles et celle des échantillons d'amphores du Group 2, et leur composition minéralogique est compatible avec celle de l'un et de l'autre groupe. D'après I. K. Whitbread, il n'est pas possible d'associer ces deux groupes distincts à des phases chronologiques différentes de la production. Toutefois les observations visuelles, suivies par les analyses des lames minces de trois échantillons que M. Lawall a pu effectuer sur des exemplaires de contextes bien datés de l'Agora d'Athènes pourraient suggérer l'emploi d'une pâte plus fine et moins homogène pour les amphores datées avant 440 av. J.-C., plus dure et moins homogène au contraire pour les amphores postérieures à cette date ${ }^{311}$.

310. Whitbread 1995, p. 198-209, notamment, p. 200-203.

311. Pour les résultats de ces lames minces et les observations de type chronologique qui en sont la conséquence, voir Lawall 1995, p. 119 et n. 11 . 


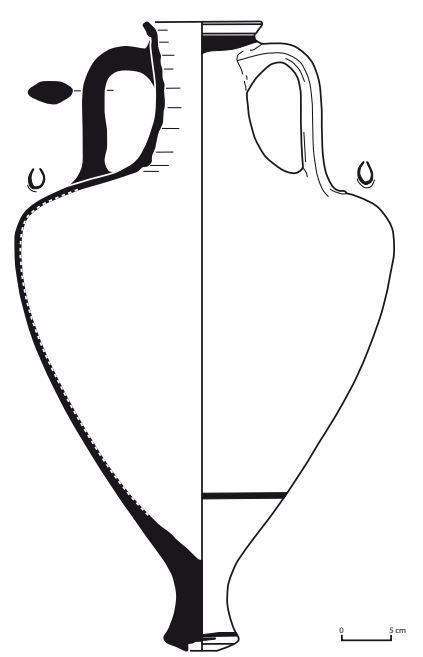

Fig. 24. Amphore nord-égéenne (Mendé). Athènes, Agora, inv. P23683 (d'après Papadopoulos, Paspalas 1999, fig. 4.
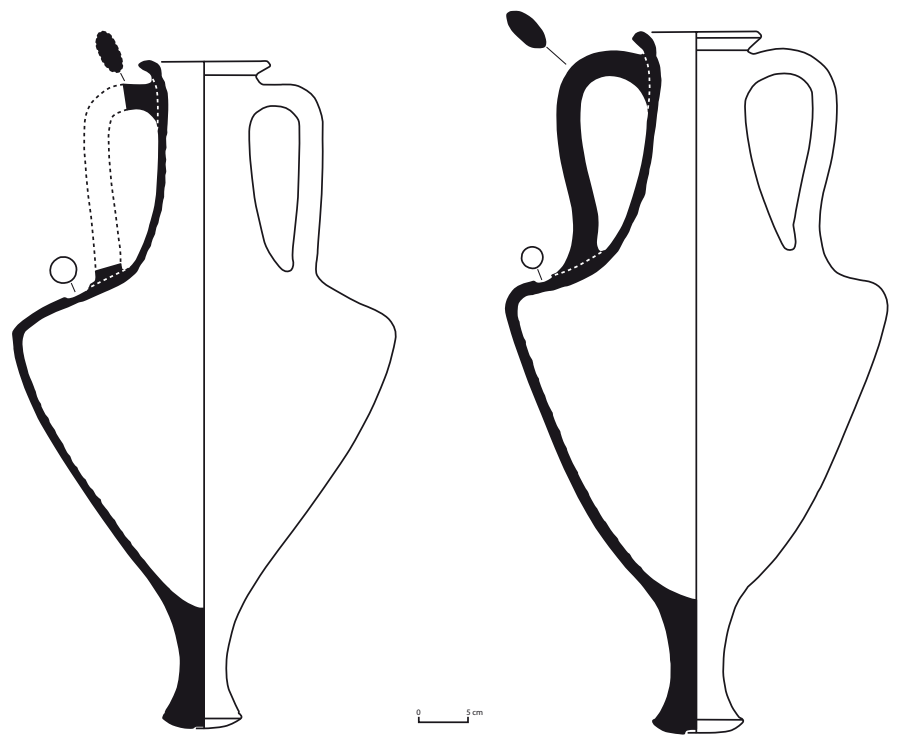

Fig. 25. Amphores nord-égéenne (Mendé). Épaves de Porticello (d'après Eiseman 1973, fig. 1).

\section{La production amphorique attribuée à Thasos}

À l'exception de quelques études consacrées aux timbres amphoriques, et uniquement à ceux qui ne sont pas antérieurs à la fin du $\mathrm{V}^{\mathrm{e}} \mathrm{s}$. av. J.-C., les amphores de Thasos attendent encore de faire l'objet d'une étude typologique d'ensemble ${ }^{312}$. Toutefois, on dispose de travaux partiels parmi lesquels celui de A. M. et A. Bon, qui ont proposé une classification des amphores de Thasos en distinguant trois types principaux : le premier daté entre la fin du $\mathrm{V}^{\mathrm{e}}$ et le III ${ }^{\mathrm{e}} \mathrm{s}$. av. J.-C., le second entre la deuxième moitié du $\mathrm{IV}^{\mathrm{e}}$ et le début du $\mathrm{III}^{\mathrm{e}} \mathrm{s}$. av. J.-C. et un troisième situé au III ${ }^{\mathrm{e}}$ s. av. J.-C. Y. Grandjean a isolé plus récemment un quatrième type associé à des contextes de fin $\mathrm{V}^{\mathrm{e}}$ et du début $\mathrm{IV}^{\mathrm{e}} \mathrm{s}$. av. J.-C. et, grâce aux recherches conduites par l'École française d'Athènes dans le quartier de la Porte du Silène à Thasos ${ }^{313}$, nous disposons également d'une documentation qui couvre la

312. La première étude sur les timbres amphoriques de Thasos est due à A. M. et A. Bon (Bon 1957). Plus récemment, le sujet a également été traité par V. Grace (Grace 1985) et surtout par Y. Garlan (en dernier: Garlan 1999, avec bibliographie précédente). Pour la définition d'une typologie des amphores de Thasos, il y a quelques années encore, on devait s'appuyer sur la classification établie par A. M. et A. Bon, dans le cadre de leur étude sur les timbres amphoriques (Bon 1957, p. 13-26). Pour une synthèse, voir aussi Whitbread 1995, p. 165-167.

313. Grandjean 1992. fourchette chronologique du début de la série, entre la fin $\mathrm{du} \mathrm{VI}^{\mathrm{e}}$ et le $\mathrm{V}^{\mathrm{e}} \mathrm{s}$. av. J.-C. Bien que les fouilles de l'École française d'Athènes aient comblé une grave lacune dans la documentation, une véritable sériation typologique à l'intérieur du $\mathrm{V}^{\mathrm{e}} \mathrm{s}$. av. J.-C. n'a pas encore été proposée et l'on doit à M. Lawall une tentative d'identification de quelques types thasiens appartenant au $\mathrm{V}^{\mathrm{e}} \mathrm{s}$. av. J.-C. et leur encadrement chronologique sur la base des association des contextes clos de l'Agora d'Athènes ${ }^{314}$.

\subsection{Bilan des études}

Les recherches récemment conduites à Thasos, dans le quartier de la Porte du Silène, ont permis d'identifier et de définir le type amphorique du début de la série thasienne, qui peut être encadré entre la fin du $\mathrm{VI}^{\mathrm{e}}$ et le début du $\mathrm{V}^{\mathrm{e}} \mathrm{s}$. av. J.-C. Il s'agit du type défini doublebanded rim type ${ }^{315}$, qui doit son nom à la morphologie de la lèvre, à section quadrangulaire, sur laquelle on trouve deux listels horizontaux, en relief et de hauteur égale mais dont celui du dessus est toujours plus saillant que celui de dessous (fig. 26). Les amphores de ce type sont caractérisées par un col cylindrique au profil variable,

314. Lawall 1995, p. 129-148.

315. Garlan 1988, p. 168, n 75/2081; Johnston 1991, p. 363-365, pl. 96.1 ; Grandjean 1992, p. 581-583, n² 2, 7, 10, 16-18, 26, 28, 61-62. 


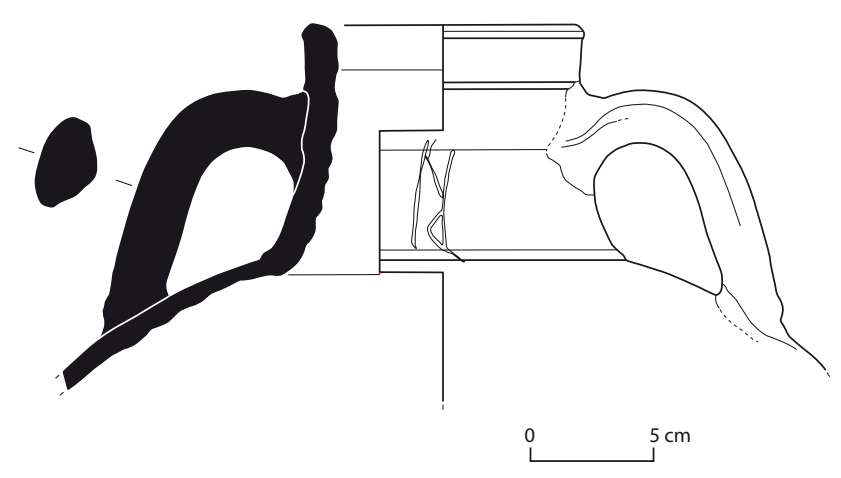

Fig. 26. Amphore nord-égéenne (Thasos). Thasos, quartier de la Porte du Silène, inv. 75/3511 (d'après Grandjean 1992, fig. 1. 2).

mais en tout cas toujours plutôt court, une épaule arrondie et une panse de forme dans l'ensemble globulaire ou ovoïde. Le pied de hauteur variable a une forme en bouton (fig. 27). Les anses de ces amphores tardo-archaïques de production thasienne seraient caractérisées non seulement par une impression digitale à la base, mais aussi par une gorge moulurée verticale au centre sur la surface externe. Alors que les impressions digitales semblent communes à toutes les productions amphoriques de la koinè nord-égéenne entre la fin du $\mathrm{VI}^{\mathrm{e}}$ et le $\mathrm{V}^{\mathrm{e}} \mathrm{s}$. av. J.-C., la gorge sur l'anse pourrait être propre à la production de l'île de Thasos de la fin $\mathrm{VI}^{\mathrm{e}}$ s. av. J.-C. ${ }^{316}$ L'encadrement chronologique de ce type exclusivement sur la base de la documentation des fouilles du quartier de la Porte du Silène présente néanmoins quelques difficultés. En effet, les contextes de provenance des amphores présentent un terminus post quem situé entre la fin $\mathrm{du} \mathrm{V}^{\mathrm{e}}$ et le $\mathrm{III}^{\mathrm{e}} \mathrm{s}$. av. J.-C., bien que le matériel trouvé à l'intérieur soit majoritairement daté avant la première moitié du $\mathrm{V}^{\mathrm{e}} \mathrm{s}$. av. J.-C. Par ailleurs, certains contextes de l'Agora d'Athènes révèlent au contraire pour ce type une chronologie plus précise, située entre la fin du $\mathrm{VI}^{\mathrm{e}}$ et le début du $\mathrm{V}^{\mathrm{e}} \mathrm{s}$. av. J.-C., puisqu'il est présent fréquemment dans des dépôts datés avant 480 av. J.-C. ${ }^{317}$ Les amphores de ce type de la porte du Silène à Thasos sont donc probablement en position résiduelle.

M. Lawall, s'appuyant aussi sur l'analyse de la pâte de quelques échantillons de l'Agora d'Athènes, a pu isoler pour le $\mathrm{V}^{\mathrm{e}} \mathrm{s}$. av. J.-C. deux types d'amphores : le

316. Sur cette proposition fondée sur la base de la documentation de Géla (Spagnolo 2003, pl. IV.2), d'égine (Johnston 1990, n 131-132, 177, fig. 12) et de l'île même de Thasos (Grandjean 1992, fig. 1.2, 4.26, 9.63.), voir : Spagnolo 2003, p. 624 e n. 96.

317. à ce propos, voir : Lawall 1995, p. 133-134.

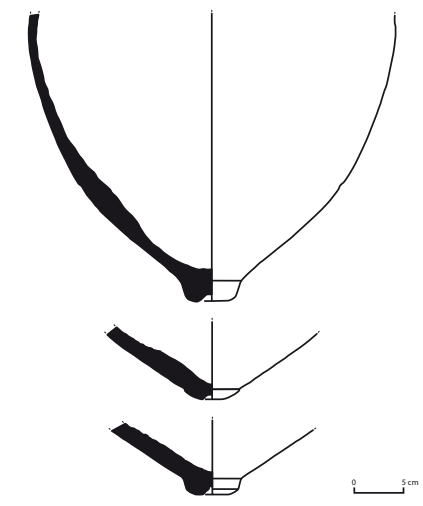

Fig. 27. Amphore nord-égéenne (Thasos). Thasos, quartier de la Porte du Silène, inv. 77/3510, 77/1098, 77/1328 (d'après Grandjean 1992, fig. 3. 16-18).

ring-toe type et le stem-toe-type, qu'il attribue à la production de Thasos. Ce faisant, il comble une importante lacune dans nos connaissances relatives à la production thasienne du plein $\mathrm{V}^{\mathrm{e}} \mathrm{s}$. av. J.-C., entre les amphores tardo-archaïques caractérisées par Y. Grandjean et A W. Johnston et celles de la fin $\mathrm{V}^{\mathrm{e}}$ et du $\mathrm{IV}^{\mathrm{e}} \mathrm{s}$. av. J.-C., identifiées par A. M. Bon et A. Bon et dont les variantes ont été postérieurement décrites par Y. Grandjean.

Les amphores définies ring-toe type présentent une lèvre en forme de coin se distinguant nettement d'un col court, dont la hauteur est presque complètement occupée par les attaches supérieures des anses. Le corps de ces amphores, à épaule arrondie et panse se rétrécissant en bas, est de forme ovoïde. Le pied, d'où le type prend son nom, est court, en anneau au profil évasé et caractérisé par une protubérance centrale de la base de forme conique (fig. 28). Les légères variations dans la forme de la lèvre, dans l'inclinaison des anses et dans le profil même du pied qu'on peut observer dans les exemplaires attribuables à ce type amphorique ne semblent pas constituer des variantes chronologiques, même si on connaît une variante caractérisée par le pied en disque avec une dépression semi-circulaire de la base. Le type, bien représenté par un exemplaire entier du Céramique d'Athènes daté vers 500 av. J.-C., peut être attribué à la première moitié du $\mathrm{V}^{\mathrm{e}} \mathrm{s}$. av. J.-C., et peut-être plus précisément au premier quart du siècle, étant donné sa présence dans des dépôts de l'Agora d'Athènes clos avant 480 av. J.-C. et sa représentation moins importante dans des contextes datés entre 460 et 440 av. J.-C. ${ }^{318}$.

318. à propos de la définition morphologique et de l'encadrement chronologique de ce premier type du $\mathrm{V}^{\mathrm{e}}$ s. av. J.-C., voir : Lawall 1995, p. 140-141. 


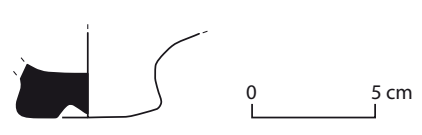

Fig. 28. Amphore nord-égéenne (Thasos?).

Athènes, Agora, E 14:5

(d'après Lawall 1995, fig. 51).

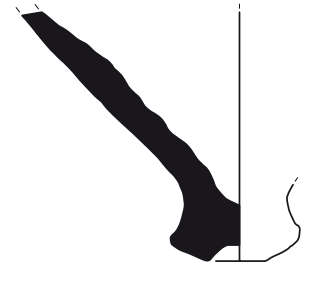

Fig. 29. Amphore nord-égéenne (Thasos ?). Athènes, Agora, R 13:1 (d'après Lawall 1995, fig. 52).

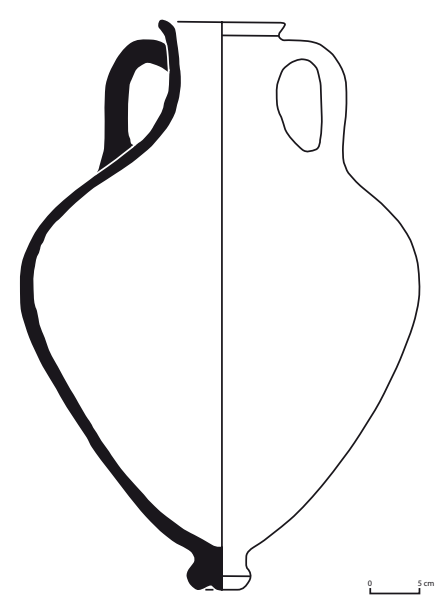

Fig. 31. Amphore nord-égéenne (Thasos) (d'après Garlan 1988, fig. 11).

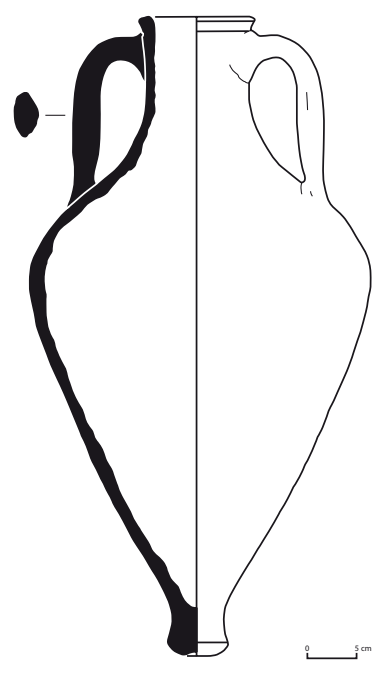

Fig. 32. Amphore nord-égéenne (Thasos). Thasos, quartier de la Porte du Silène, inv. 73/3766

(d'après Grandjean 1992, fig. 11.73).
Le type stem-toe type se décompose en deux variantes morphologiques. La première, de forme plus massive, est en un conteneur assez semblable aux amphores de Mendé du troisième quart du $\mathrm{V}^{\mathrm{e}} \mathrm{s}$. av. J.-C. (type Middle Mendean Variant), caractérisé par la lèvre en forme de coin, le corps piriforme plutôt élargi dans sa partie supérieure et le pied court en bobine évasé vers le bas et avec une base en ombilic (fig. 29). On peut la rapprocher au type Pithoid Thasian décrit d'abord par I. B. Brašinskij et ensuite par Y. Garlan (fig. 31) ${ }^{319}$. La seconde variante correspond à une amphore à la forme générale plus élan-

319. Brašinskij 1980, pl. 8 ; Garlan 1988, fig. 11. cée, mais avec une lèvre toujours en forme de coin et au pied en bobine plus haut qui présente une concavité de base dont le profil prolonge de manière plus graduelle celui du fond de la panse (fig. 30). On peut la rapprocher du type Unstamped Thasian de I. B. Zeest ${ }^{320}$, dont on connaît différents exemplaires provenant des fouilles de la Porte du Silène (fig. 32). Ce type, sous ses deux variantes, peut être encadré d'un point de vue chronologique dans la seconde moitié du $\mathrm{V}^{\mathrm{e}} \mathrm{s}$. av. J.-C. Les premiers exemplaires sont en effet attestés dans des contextes du milieu du $\mathrm{V}^{\mathrm{e}} \mathrm{s}$. av. J.-C., comme le dépôt 

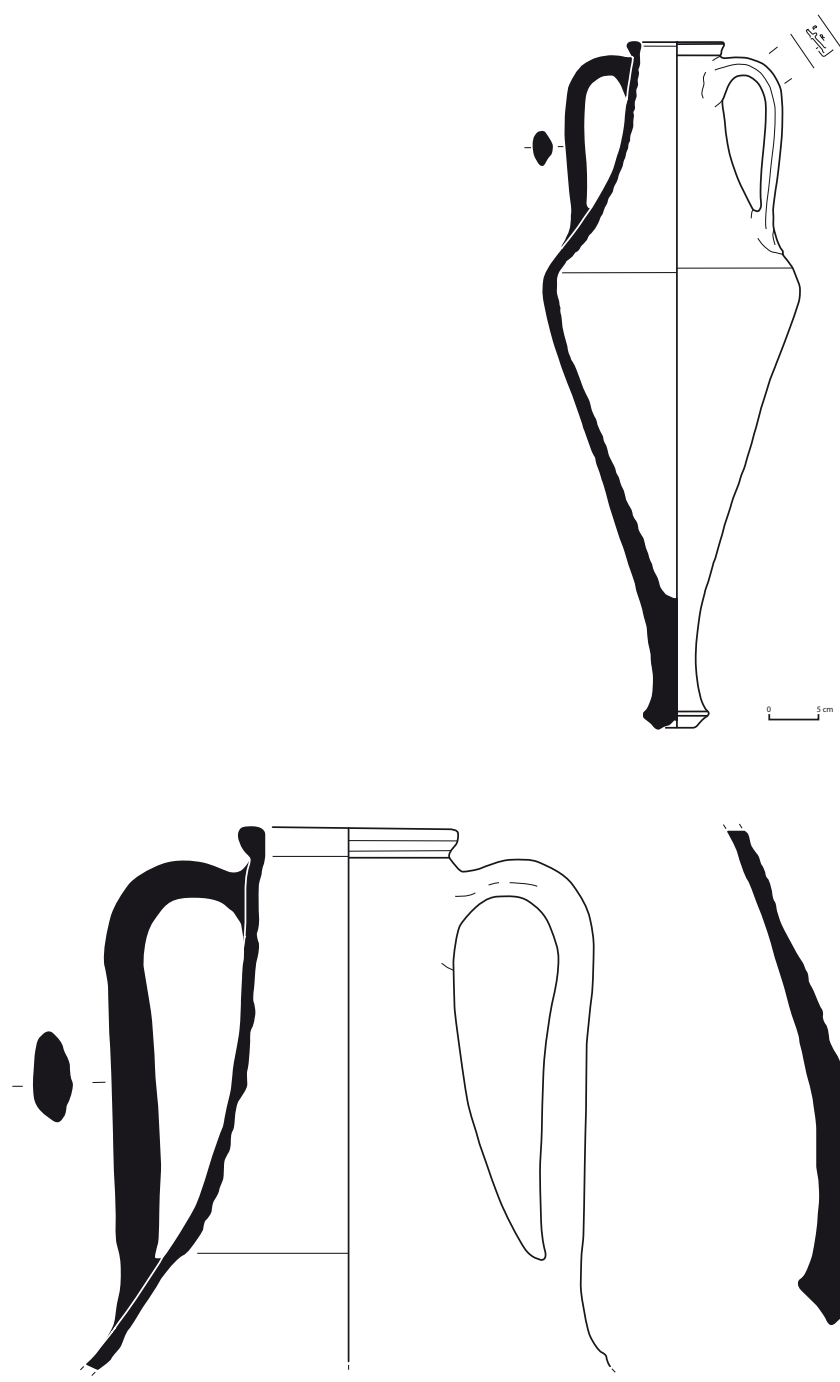

Fig. 33. Amphore nord-égéenne (Thasos).

Thasos, quartier de la Porte du Silène, inv. $79 / \mathrm{S} 80$

(d'après Grandjean 1992, fig. 7.47.
Fig. 34. Amphore nord-égéenne (Thasos). Thasos, Agora, Puits public, inv. 84.264.94, 84.170.38 (d'après Blondè, Muller, Mulliez 1991, fig. 3.16 et 3.19).
N 7:3 de l'Agora d'Athènes clos vers 440 av. J.-C., mais leur présence devient de plus en plus fréquente dans les contextes de la seconde moitié du siècle, tant de l'Agora que du Céramique d'Athènes, d'où proviennent en particulier deux exemplaires entiers de la première variante décrite ${ }^{321}$. Les deux variantes du type stem-toe de production probablement thasienne pourraient constituer les prototypes ou du moins les modèles des deux types thasiens de la fin $\mathrm{V}^{\mathrm{e}}$ et du $\mathrm{IV}^{\mathrm{e}} \mathrm{s}$. av. J.-C. Plus précisément, du type Unstamped Thasian pourrait dériver le biconical type de la fin du $\mathrm{V}^{\mathrm{e}} \mathrm{s}$. av. J.-C., tandis que la variante Pithoid Thasian pourrait être considérée comme le type précurseur des amphores top-shaped type

321. à propos du second type d'amphore probablement thasienne du $\mathrm{V}^{\mathrm{e}}$ s. av. J.-C., isolé par M. Lawall, voir : Lawall 1995, p. 137-138, 141-143, fig. 52-53, avec bibliographie. du $\mathrm{IV}^{\mathrm{e}}$ s. av. J.-C. Donc, les types amphoriques considérés de manière hypothétique comme thasiens de la première et de la seconde moitié $d u \mathrm{~V}^{\mathrm{e}} \mathrm{s}$. av. J.-C. par M. Lawall, et déjà identifiés en partie par I. B. Brašinskij et I. B. Zeest, constituent bien un lien morphologique entre les amphores sûrement thasiennes de la fin du $\mathrm{VI}^{\mathrm{e}} \mathrm{s}$. av. J.-C. et celles de la fin du $\mathrm{V}^{\mathrm{e}}$ et du $\mathrm{IV}^{\mathrm{e}} \mathrm{s}$. av. J.-C.

Les amphores de Thasos datables entre la fin du $\mathrm{V}^{\mathrm{e}}$ et le début du $\mathrm{IV}^{\mathrm{e}} \mathrm{s}$. av. J.-C., qu'on peut attribuer au premier type de la classification de A. M. et A. Bon, sont caractérisées par un profil très élancé et effilé qui leur confère un aspect biconique (fig. 33). La lèvre, petite et ouverte, présente une section triangulaire. Le col, très long, est de forme cylindrique, même s'il devient légèrement évasé vers l'épaule. Les longues anses à section biconvexe présentent des attaches inférieures légèrement enfoncées par rapport aux attaches supérieures et 
sont marquées par des impressions digitales à la base. L'épaule est très aplatie et carénée. Le pied extrêmement long présente une forme cylindrique évasée vers le fond, ce qui le fait ressembler au pied du type en bobine typique des amphores de Mendé. à la différence de ces dernières, cependant, la base se présente concave en-dessous, avec une profonde et large dépression centrale en forme de cône ${ }^{322}$ (fig. 34). Il est intéressant d'observer que l'image de cette classe d'amphores thasiennes, dont la production continue jusqu' au III ${ }^{\mathrm{e}} \mathrm{s}$. av. J.-C., apparaît souvent sur les émissions monétaires et sur les timbres, surtout sur ceux de Krini, où l'amphore est représentée au-dessus d'un tour ${ }^{323}$. Il s'agit du type amphorique récemment défini biconical type par M. Lawall qui, grâce aux associations des contextes clos de l'Agora d'Athènes, a pu en établir un encadrement chronologique plus précis au dernier quart du $\mathrm{V}^{\mathrm{e}} \mathrm{s}$. av. J.-C., s'appuyant sur le fait que les premiers rares fragments de cette forme, ainsi que l'amphore complète P4752 du

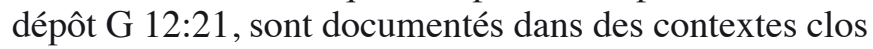
entre 410 et 400 av. J.-C. ${ }^{324}$.

\subsection{Centres de production}

A. M. et A. Bon remarquaient déjà en leur temps qu' «il n'y a pas une argile thasienne, mais toute une série ${ }^{325}$. Ainsi, les pâtes varient du type avec une argile de couleur rouge intense et des inclusions importantes, au type avec une argile rosée ou orange et des dégraissants de dimensions moyennes, jusqu'au type avec une argile couleur ocre clair et des inclusions fines. Il faut toutefois considérer que malgré ces fortes différences d'aspects, un trait commun semble être l'abondante présence d'écailles de mica, parfois de dimensions considérables et une structure interne généralement de couleur grisâtre ${ }^{326}$.

La diversité des aspects de pâtes a été mise en relation avec la dispersion des nombreux ateliers de l'île, situés non seulement dans et aux abords de la cité de Thasos mais aussi le long de la côte méridionale et orientale de l'île. Une quinzaine d'ateliers ont en effet été identifiés, dont quatre seulement ont fait l'objet d'une véritable publication, parmi lesquels un seul,

322. Type I de A.M. et A. Bon (1957, p. 16-19, fig. 2-3,5.a). Pour ce type voir également la synthèse de I. K. Whitbread (1995, p. 165-166.).

323. Bon 1957, p. 16, note 3-4, fig. 1.

324. Pour les autres dépôts de l'Agora dans lesquels on trouve des fragments d'amphores thasiennes biconical type, voir : Lawall 1995, p. 135 e n. 85 .

325. Bon 1957, p. 14.

326. Pour cette observation voir aussi: Grace 1961, p. 24 et De Marinis 1988, n. 22. celui du site de Keramidi sur la côte méridionale de l'île, a livré du matériel du $\mathrm{V}^{\mathrm{e}} \mathrm{s}$. av. J.-C., tandis que les autres fournissent une documentation relative au $\mathrm{IV}^{\mathrm{e}} \mathrm{s}$. av. J.-C. ${ }^{327}$ I. K. Whitbread, qui a exécuté des analyses pétrographiques sur un nombre important d'échantillons thasiens, estime que la diversité des pâtes attestées, n'est en fait, en grande partie, qu'une illusion visuelle. Ainsi, si l'activité de plusieurs ateliers est une donnée qui doit être prise en considération pour expliquer l'extrême hétérogénéité des pâtes, les récentes recherches de I. K. Whitbread ont cependant montré, tant à l'analyse visuelle qu'à travers l'étude des lames minces, qu'elles se répartissent uniquement en deux groupes. Au Group 1 d'I. Whitbread correspondent des argiles allant du rouge clair-orange au jaune pâle (de 2.5 YR 6/6 à 10 YR 7/5), avec des inclusions de petites dimensions de consistance dure, soumises à un procédé de cuisson très soigné. Au contraire, le Group 2 rassemble des argiles de couleur brune (de 2.5 YR 6/6 à 2.5 YR 6/4), avec des inclusions plus grossières et dont la cuisson a été moins soignée ${ }^{328}$. Indépendamment de la distinction de ces deux groupes, dont le plus grossier, le Group 2, a été principalement attribué à l'atelier repéré à Kalonero, sur la côte orientale de l'île ${ }^{329}$, tandis que la localisation des ateliers des amphores du Group 1 ne peut être mieux précisée, les analyses effectuées ont en tout cas assuré l'origine thasienne, non seulement des amphores du $\mathrm{IV}^{\mathrm{e}} \mathrm{s}$. av. J.-C. attribuées à l'île dans un premier temps uniquement sur la base des timbres, mais aussi de celles du V ${ }^{\mathrm{e}} \mathrm{s}$. av. J.-C. considérées du point de vue morphologique comme la série archaïque de ces amphores timbrées.

\section{Les productions amphoriques des centres nord-égéens non identifiés}

En dehors des ateliers thasiens et de Mendé qui viennent d'être évoqués, il reste à envisager d'autres productions de fabrication nord-égéenne dont il est encore aujourd'hui difficile de préciser la ou les origines et qui sont généralement désignées par des dénominations conventionnelles parfois confuses.

327. Pour les ateliers amphoriques de Thasos, voir: Garlan 1986 ; Jones 1986, p. 280-286 ; Garlan 1979. Pour le site de Keramidi en particulier: Garlan 1986, p. 229-230.

328. Les analyses sur quelques échantillons d'amphores de Thasos ont conduit à l'identification de deux classes de pâtes qui présentent en tout cas des analogies de base (Whitbread 1995, p. 167-176). Pour les analyses sur les amphores thasiennes voir également la contribution de R. E. Jones dans : Recherches sur les amphores, p. 279-285. 329. Pour l'atelier de Kalonero, voir en particulier: Garlan 1986, p. 221-225. 
Parmi celles-ci, deux séries amphoriques de classification difficile ont été attribuées, du moins pour partie, à la production nord-égéenne. Il s'agit des séries désignées dans la bibliographie spécialisée sous les dénominations Zeest's Samian type, ou pseudo-Samian, voire Zeest's Protothasian type, dont les ateliers de fabrication devaient être nombreux et non circonscrits à l'aire nord-égéenne.

Au même cadre géographique sont également attribuées quelques séries qui présentent des affinités stylistiques avec les productions de Mendé et de Thasos, bien qu'elles s'en distinguent par des détails morphologiques et par leur pâte, connues sous la dénomination de Zeest's Thasian Circle ${ }^{330}$.

\section{Zeest's Samian et Zeest's Protothasian}

Les Zeest's Samian, ou pseudo-Samian, et les plus petites Zeest's Protothasian consistent en deux séries diffusées surtout en Mer Noire et qui semblent appartenir à une même série du fait des détails de la lèvre en échine, parfois à profil suspendu en gouttière et de leur pied annulaire bas multicaréné. P. Dupont les a considérées comme deux étapes évolutives du même type dont le développement pourrait se situer respectivement entre la seconde moitié du VI ${ }^{\mathrm{e}}$ et le début du V ${ }^{\mathrm{e}} \mathrm{s}$. av. J.-C. et la fin du VI ${ }^{\mathrm{e}}$ et le milieu du $\mathrm{V}^{\mathrm{e}} \mathrm{s}$. av. J.-C. ${ }^{331}$. La production des Zeest's Protothasian a été attribuée par I. B. Zeest à l'aire nord-égéenne sur la base de l'observation de la pâte et du profil particulier du pied, mis en relation avec celui des amphores de Thasos du $\mathrm{IV}^{\mathrm{e}} \mathrm{s}$. av. J.-C. ${ }^{332}$. La fabrication nord-égéenne de cette série semble confirmée par quelques découvertes de Thasos et surtout d'Abdère, colonie de Téos ${ }^{333}$. La production des Zeest's Samian a été dans un premier temps attribuée à Samos par I. B. Zeest sur la base de la pâte ${ }^{334}$. Reprenant à son compte l'hypothèse, V. Grace a considéré les Zeest's Samian comme une étape de la production amphorique de Samos, située dans la période du passage entre le $\mathrm{VI}^{\mathrm{e}}$ et le $\mathrm{V}^{\mathrm{e}} \mathrm{s}$. av. J.-C. ${ }^{335} \mathrm{~V}$. V. Ruban a, en revanche plus

330. Tandis que la définition pseudo-Samian a été utilisée pour la première fois à la fin des années soixante (Lordkipanidze 1968, p. 40), les dénominations précédentes et encore aujourd'hui utilisées Zeest's Samian type, Zeest's Protothasian type, Zeest's Thasian Circle sont dues à I. B. Zeest (Zeest 1960, p. 70, 79-80, pl. I, 3, VI, 15).

331. Pour les séries Zeest's Samian et Protothasian on renvoie à Dupont 1998, p. 178-186, avec bibliographie ; Id. 1999, p. 153-157 ; Id. 2000 , p. 58 ; Id. 2007, p. 621-623.

332. Zeest 1960, p. 79-80, pl. VI, 15 .

333. Peristeri-Otatzi 1986, p. 496, fig. 1-14 ; Dupont 1998, p. 182 , n. 248-249.

334. Zeest 1960, p. 70, pl. I, 3.

335. Grace 1971,p. 73, fig. 2, 4, pl. 15, 3 . récemment, attribué les Zeest's Samian et les Zeest's Protothasian à des ateliers de Milet ${ }^{336}$, où par ailleurs ces formes semblent particulièrement bien attestées ${ }^{337}$. L'origine et la plus grande partie de la production des Zeest's Samian ont été plus récemment encore réattribuées par P. Dupont au cadre nord-égéen sur la base des analogies morphologiques entre ces amphores et les Zeest's Protothasian ${ }^{338}$.

Dans la mesure où les deux séries sont désormais considérées comme des étapes évolutives de la même forme, les définitions de Zeest's Samian et Zeest's Protothasian sont souvent utilisées indifféremment par les chercheurs, avec une préférence pour la seconde dénomination qui en évoque l'origine nord-égéenne ${ }^{339}$. Cependant, la variabilité des pâtes et de la morphologie des lèvres et des pieds a suggéré l'hypothèse que ces deux séries étaient fabriquées par plusieurs ateliers ${ }^{340}$. C'est pourquoi P. Dupont n'a pas exclu la possibilité d'une production en partie partagée entre l'aire nordégéenne et celle de Samos et de Milet, dans le cadre de laquelle la caractérisation des variantes locales est loin d'être clairement définie et reste aujourd'hui une source de confusion. Par ailleurs et comme le fait remarquer P. Dupont, le fait que les productions de $\mathrm{V}^{\mathrm{e}} \mathrm{s}$. av. J.-C. $\mathrm{du}$ « groupe Samos-Milet » partage des caractéristiques communes avec les amphores identifiées par I. B. Zeest complique la question, surtout en présence de formes non complètes ${ }^{341}$. V. Gassner, s'appuyant sur les résultats d'analyses effectuées sur les pâtes, a récemment suggéré d'attribuer certains exemplaires se rapprochant de ces formes à une production régionale ionienne, qu'elle identifie avec la zone comprise entre l'aire milésienne et la péninsule de Karaburun ${ }^{342}$. M. Kerschner a, quant à lui, proposé de localiser l'un des ateliers à Éphèse ou dans le secteur du golfe d'Ephèse ${ }^{343}$. L'existence d'ateliers grecs-orientaux pour la fabrication d'amphores proches de celles identifiées par I. B. Zeest a également été proposée, suite à la découverte d'un exemplaire du $\mathrm{V}^{\mathrm{e}}$ siècle portant un timbre qu'on a voulu attribuer à la cité

336. Ruban 1991.

337. Pour cette observation et pour les problèmes d'interprétation qu'elle a suscités, cf. : Dupont 1998, p. 183 ; et Id. 2007, p. 622. Le haut pourcentage de fragments appartenant à des amphores Zeest's Samian et Protothasian des fouilles récentes de Milet-Kalabaktepe a été confirmé par l'étude d'A. Naso, d'après laquelle au moins une partie de ces fragments serait de production locale (Naso 2005, p. 77, n. 24).

338. Dupont 1998, p. 178-186.

339. Dupont 2007 ; Monachov 2003 b.

340. Dupont 1998, p. 183.

341. Dupont 2007, p. 621-623.

342. Gassner 2003b, p. 123-129 ; Ead. 2003a, p. 96-97 ; Ead. 2005 , p. 37 .

343. Kerschner, Mommsen 2005, p. 125-126. 
d'Érythrées ${ }^{344}$. À la suite de cette trouvaille, P. Dupont est revenu sur le sujet et bien qu'il n'abandonne pas la " piste pontique ", avec une attention particulière pour Thasos et à Maronée, il suggère maintenant une " piste nord-ionienne », en considérant en particulier les cas de Téos et de Chios ${ }^{345}$.

Pour toutes ces raisons, ces séries seront encore mentionnées dans le chapitre consacré aux productions grecques orientales (cf. infra I, 5 § 3).

\section{Zeest's Thasian Circle et les productions nord- égéennes non identifiées du $V^{\mathrm{e}} \mathrm{s}$. av. J.-C.}

La définition d'amphores du Thasian Circle a été créée par I. B. Zeest ${ }^{346}$ et a été employée couramment pour classer une série de types amphoriques d'époque classique qui, tout en présentant des analogies avec les amphores de Thasos du $\mathrm{V}^{\mathrm{e}} \mathrm{s}$. av. J.-C., ne correspondaient parfaitement à aucun des types standards reconnus à l'époque. Actuellement, l'opinion courante est que ces amphores doivent être attribuées à de nombreux ateliers situés bien au-delà de l'aire thasienne et qu'il faudrait rechercher notamment dans la péninsule de Chalcidique, sans exclure la possibilité de productions dans les sites de l'Égée septentrionale déjà connus pour la fabrication d'une série amphorique propre, tels que Mendé, Thasos et Acanthos.

P. Dupont a eu le mérite de réattribuer au cercle de Thasos de I. B. Zeest quelques formes amphoriques d'époque tardo-archaïque et d'avoir distingué parmi ces formes trois types différents (A, B et C) en fonction de leur morphologie générale ${ }^{347}$. En ce qui concerne le $\mathrm{V}^{\mathrm{e}} \mathrm{s}$. av. J.-C., un cadre typo-chronologique pour quelquesunes des amphores produites par ces centres encore inconnus de l'Égée septentrionale a été proposé par M. Lawall sur la base du matériel de l'Agora d'Athènes. Cette proposition permet d'encadrer ces exemplaires fragmentaires dont l'attribution à tel ou tel atelier de Mendé, Thasos ou Acanthos serait hasardeuse. Il montre également comment la morphologie de l'ensemble des amphores de ce groupe générique, qu'elles proviennent d'un atelier connu ou non, s'inscrit dans une évolution homogène, cohérente bien que parallèle. Il illustre ainsi et surtout appuie fortement l'hypothèse de l'existence d'un style et d'une tradition amphorique nord-égéenne qu'on peut assimiler à tout point de vue à une véritable «production régionale » à propos de laquelle il est permis de parler de koinè nord-égéenne.

344. Carlson 2003, p. 584-587.

345. Dupont 2010.

346. Zeest 1960,p. 81-82.

347. Dupont 1998, p. 186-190, pl. 23, 13.

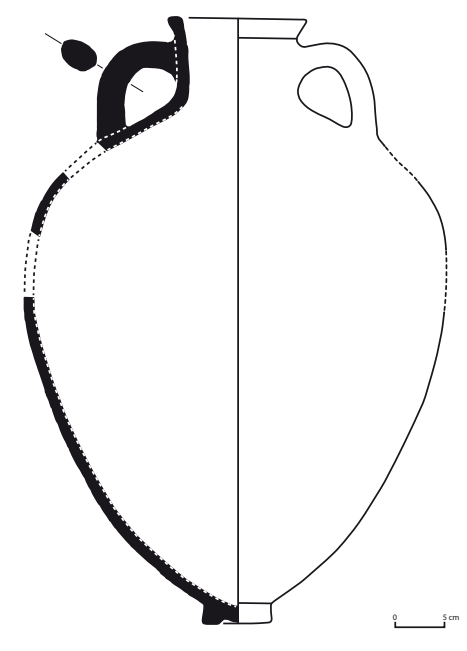

Fig. 35. Amphore nord-égéenne. Athènes, Agora, inv. P24888 (d'après Roberts 1986, fig. 43.426).

Le matériel des contextes de l'Agora d'Athènes a permis à M. Lawall de distinguer trois types successifs à partir de l'évolution de trois formes de pieds : ring-toe type, disc-toe type, conical-base type.

Les amphores du ring-toe type (fig. 35) sont caractérisées par un pied bas de forme tronconique ayant une base en anneau avec une cavité centrale. La panse présente une forme ovoïde, le col plutôt court a un profil cylindrique ou tronconique, la lèvre est en forme de coin et les anses de section elliptique présentent des impressions digitales à la base. La chronologie de cette forme peut se situer entre la fin du $\mathrm{VI}^{\mathrm{e}}$ et le premier quart du $\mathrm{V}^{\mathrm{e}} \mathrm{s}$. av. J.-C., étant présente dans quelques contextes de l'Agora clos vers 480 a. J.-C. et dans d'autres contextes contemporains du Céramique d'Athènes ${ }^{348}$.

La forme disc-toe type (fig. 36) se distingue par un pied massif au profil cylindrique ou légèrement tronconique et une base discoïde légèrement convexe avec une dépression semi-circulaire centrale. Le col et les anses de ce type amphorique sont plus longs que dans la forme précédente. Les anses conservent la présence des impressions digitales à la base, vers leur jonction avec l'épaule. La lèvre est toujours en forme de coin mais se présente souvent plus massive, arrondie à l'extérieur, avec un marli supérieur plus incliné vers l'extérieur. Le type est attesté dans des contextes athéniens, tant de l'Agora que du Céramique, datés entre 480 et 440 av. J.-C., mais quelques exemplaires plus anciens proviennent

348. Lawall 1995, p. 157-158, n. 178-181, fig. 56-57, avec références bibliographiques, et, p. 162-164, fig. 64-65. 


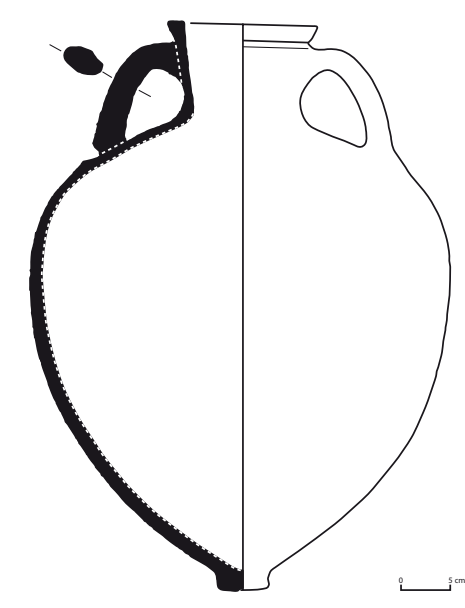

Fig. 36. Amphore nord-égéenne. Athènes, Agora, inv. P24893 (d'après Roberts 1986, fig. 43.424).

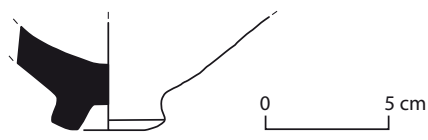

Fig. 37. Amphore nord-égéenne. Athènes, Agora, R 12:1 (d'après Lawall 1995, fig. 62).

de contextes antérieurs à 480 av. J.-C., comme l'exemplaire P24893 provenant du Stoa Gutter Well étudié par S. Roberts et clos vers 480 av. J.-C. ${ }^{349}$.

Les amphores définies conical-base type (fig. 37) doivent leur nom au pied tronconique plus haut que celui du type ring-toe mais encore caractérisé par une base en anneau. À la différence du type ring-toe, le pied du conical-base présente en outre un profil légèrement caréné tandis qu'il est dépourvu de convexité centrale à la base. La panse des amphores de ce type est de forme ovoïde, mais au profil plus élancé par rapport aux deux types précédents. La forme de la lèvre est variable : soit un profil en coin proche des celui des amphores tardo-archaïque du type ring-toe, soit une forme au profil plus arrondi caractéristique du type disc-toe plus récent. La chronologie proposée pour cette forme est incertaine et comprise

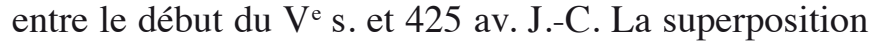
chronologique de cette forme avec les deux types précédents explique probablement la présence de deux formes de lèvres associées et leurs analogies respectives avec

349. À ce propos, voir : Lawall 1995, p. 158-159 et notes 184-190. Pour l'amphore P24893 : Roberts 1986, p. 69, n 424, fig. 43. les deux autres types du $\mathrm{V}^{\mathrm{e}} \mathrm{s}$. av. J.-C. L'ample arc chronologique du type conical-base est suggéré sur la base des exemplaires fragmentaires trouvés aussi bien dans le contexte R 12:1 de l'Agora clos en 480 av. J.-C., que dans d'autres également de l'Agora et du Céramique d'Athènes clos entre le milieu et le troisième quart du $\mathrm{V}^{\mathrm{e}}$ s. av. J.-C. ${ }^{350}$.

\section{Contenu}

L'île de Thasos est souvent citée dans les sources pour la haute qualité de son vin, mis souvent au niveaux des vins de Chios. La cité de Mendé fut également productrice d'un vin considéré comme de très haute qualité et pour le commerce duquel Athénée signale la réalisation d'une forme amphorique particulière ${ }^{351}$. Cela autorise à supposer que le contenu principal des amphores produites dans l'aire nord-égéenne était le vin. L'hypothèse pourrait être confirmée par la présence de résidu résineux observé à l'intérieur des amphores attribuées à ces productions et provenant de l'Agora d'Athènes ${ }^{352}$. La même observation a pu être faite à l'intérieur du fond d'une amphore de fabrication nord-égéenne provenant de Marzabotto et signalée dans ce volume. Il nous faut cependant rappeler que neuf des dix amphores de Mendé de l'épave de Tektaş Burnu avaient été remplies de poix, tandis que la dixième contenait de la viande de bœuf ${ }^{353}$. Il est toutefois probable qu'on soit, dans ce cas précis en face d'une utilisation secondaire de ces amphores, dans le cadre d'un commerce de petit cabotage.

\section{Diffusion dans la Méditerranée occidentale}

Les amphores qu'on peut rattacher à la koinè nordégéenne, très répandues en Mer Egée et surtout en Mer Noire, semblent au contraire avoir connu en Occident une diffusion plus limitée. On les trouve néanmoins ponctuellement en Méditerranée occidentale, principalement dans l'aire ionienne et tyrrhénienne, et plus rarement le long du littoral adriatique.

En ce qui concerne l'Adriatique, un vide documentaire dans le secteur le plus méridional a été récemment comblé par la découverte d'une amphore de Thasos

350. Lawall 1995, p. 159-160, n. 192-194, fig. 62.

351. Pour les sources sur le vin de Thasos, voir le riche dossier proposé par Fr. Salviat (Salviat 1986) ; pour la qualité du vin de Mendé : Biers 1980, p. 235 ; Whitbread 1995, p. 199 et Papadopoulos, Paspalas 1999, avec renvois aux sources littéraires. Pour la péninsule de Chalcidique en général, voir également : Salviat 1990.

352. Roberts 1986, p. 68-70.

353. Carlson 2003, p. 589-590. 
trouvée dans la Tombe 69 de la nécropole de NumanaSirolo et d'une amphore de Mendé datable peut-être de la période de transition du $\mathrm{V}^{\mathrm{e}}$ au $\mathrm{IV}^{\mathrm{e}} \mathrm{s}$. av. J.-C. et faisant partie du mobilier de la Tombe 117 de la même nécropole ${ }^{354}$. La découverte de ces amphores de transport dans cette nécropole, ainsi que dans celle de Camerano, commence à combler un vide d'attestations amphoriques qui soulevait quelque perplexité, au regard de l'abondance de la céramique attique et d'autres biens de prestige en provenance de Mer Égée et de l'Orient qui sont arrivés jusqu'à la région picénienne. L'attestation dans l'aire de l'Adriatique centrale d'amphores qu'on peut attribuer à la koiné nord-égéenne me paraît particulièrement significative en considération des découvertes de l'Italie du nord, où la documentation relative à ces productions s'est révélée plutôt riche si on la compare avec la rareté des attestations occidentales en général.

Dans la Calabre ionienne, dans un secteur proche du Détroit, il faut noter la présence du bateau marchand de Porticello qui transportait, entre autres produits, des amphores de Mendé et de Thasos datées entre la fin du $\mathrm{V}^{\mathrm{e}}$ et le début du $\mathrm{VI}^{\mathrm{e}} \mathrm{s}$. av. J.-C. Les amphores nordégéennes sont documentées par ailleurs dans l'habitat de Caulonia. On connaît aussi un fragment d'anse avec un timbre de Thasos provenant de l'habitat de Tiriolo ${ }^{355}$.

En ce qui concerne la Sicile, on peut signaler les exemplaires anciens, datables de la seconde moitié ou de la fin du VI ${ }^{\mathrm{e}}$ siècle et identifiés comme des productions nord-égéennes d'Himère et de Camarine ${ }^{356}$.

354. Landolfi 1999, p. 150, n. 61-62.

355. Pour l'épave de Porticello: Eiseman 1973 ; Eiseman 1975 ; Eiseman 1979 ; Eiseman, Ridgway 1987 ; Empereur, Garlan 1987, p. 108 ; Gill 1987 ; Parker 1992, n 879, p. 332-333, map 11. Pour Caulonia: Vandermersch 1989, p. 108-109, avec bibliographie antérieure.

356. Pour Himère: Vassallo 2003, p. 343-346, fig. 7 ; Pour Camarine : Sourisseau 2002, p. 548, et Id. 2006, p. 133 et fig. 1.
Correspondant plutôt à des productions $\mathrm{du} \mathrm{V}^{\mathrm{e}} \mathrm{s}$. av. J.-C., on retiendra les attestations de Géla (identifiées comme des productions de Mendé ou du moins nord-égéennes), de l'habitat indigène de Montagna di Ramacca, à Colle Madore, de Lipari et de Naxos, dont les exemplaires sont datés du courant du $\mathrm{V}^{\mathrm{e}} \mathrm{s}$. av. J.-C. ${ }^{357}$

Quelques exemplaires considérés de fabrique nordégéenne sont signalés en Campanie, à Vico Equense, à Cumes, à Pithécusses et à Vélia ${ }^{358}$.

En Étrurie, l'unique attestation d'amphores nordégéennes vient de Pise, où quelques fragments appartenant probablement à une production de Mendé de la fin $\mathrm{du} \mathrm{V}^{\mathrm{e}} \mathrm{s}$. av. J.-C. ont été récupérés dans les fouilles de la Piazza dei Cavalieri ${ }^{359}$. Je suis cependant de l'avis qu'au moins une des amphores récupérées dans le port de Civitavecchia et classée de manière hypothétique comme samienne, pourrait être attribuée à une production de Mendé de la seconde moitié du $\mathrm{V}^{\mathrm{e}} \mathrm{s}$. av. J.-C. ${ }^{360}$.

À l'est de la péninsule italienne, les amphores de production nord-égéenne sont plutôt rares. En Gaule méridionale, quelques exemplaires sont signalés à Marseille, à Arles, à Saint-Pierre-lès-Martigues et dans l'épave de la Pointe Lequin 1A. En péninsule ibérique, un fragment d'époque tardo-archaïque, attribué au «Cercle de Thasos » de I. B. Zeest, a été découvert à Emporion, sur la côte catalane. Enfin, d'autres exemplaires de production nord-égéenne du milieu du $\mathrm{IV}^{\mathrm{e}} \mathrm{s}$. av. J.-C. faisaient partie de la cargaison du bateau marchand d'El Sec ${ }^{361}$.

357. Pour le cas de Géla : Spagnolo 2003 ; pour le site de Montagna di Ramacca: Albanese Procelli 2003, p. 40 ; pour Colle Madore: Polizzi 1999, p. 225, n 401-402, fig. 222, n 401-402 ; pour Lipari : Cavalier 1985, p. 65, n 61, fig. 16.f ; pour Naxos : Lentini, Savelli, Blackman 2007, p. 89 et Id. 2009, p. 102-103, fig. 16.

358. Pour Vico Equense: Di Sandro 1981, p. 10-11; pour Pithécusses : Di Sandro 1986, p. 82-84 ; pour Vélia : Gassner 2003, p. 120-121.

359. Pancrazzi 1982, p. 338, fig. 2.21.

360. Sur le matériel provenant du port de Civitavecchia, en partie attribué à une épave et en partie récupéré par des filets de pêche, voir : Sonno 1997, p. 78, 81, fig. 19.

361. Pour la Provence : Sourisseau 1997, I, p. 157. Pour Emporion : Aquilué Abadías et al. 2000, p. 333, fig. 40.3. Pour l'épave d'El Sec : Cerdá 1987 et Cabrera, Rouillard 2003. 


\section{Chapitre 5}

\section{Les productions amphoriques de l'Égée orientale}

$\mathrm{L}$ 'aire de l'Égée orientale, qui comprend à la fois les côtes de l'Asie Mineure et les îles en face de cette même côte, a été, dès l'époque archaïque et jusqu'à l'époque hellénistique, un foyer de production important de différentes formes d'amphores exportées très tôt vers l'Occident.

Parmi les productions grecques orientales attestées en Italie du nord, on trouve à la fois des séries amphoriques bien caractérisées et qui ont fait l'objet d'études approfondies (Chios et Clazomènes), mais aussi des séries dont l'identification et la définition sont plus difficiles à établir. La difficulté principale pour ces séries réside dans un phénomène de production qui dépasse le simple caractère local. Nous sommes donc confrontés à des productions qualifiées de « régionales » ou même « extra-régionales », suivant en cela les définitions proposées par M. Lawall, et qui sont partagées par de multiples centres de production. C'est le cas notamment des groupes connus respectivement sous les noms de « Samos-Milet » et de « Solokha I».

La définition précise de ces deux « groupes » demeure cependant partielle et, à y regarder de près, il règne encore une certaine confusion, notamment en ce qui concerne leur développement diachronique d'ensemble. L'hétérogénéité à l'intérieur de chacun des groupes est évidente, tant d'un point de vue morphologique que des pâtes. Le phénomène doit probablement être mis au compte de la multiplicité des ateliers contemporains, parfois localisés dans des zones très éloignées les unes des autres, et qui en adaptant localement un prototype commun, ont introduit des variantes de détails, probablement issus de la tradition vasculaire locale.

\section{La production amphorique attribuée à Chios}

La production d'amphores à Chios est un phénomène ancien qui n'a pas connu d'interruption depuis le $\mathrm{VII}^{\mathrm{e}} \mathrm{s}$. jusqu'au I ${ }^{\text {er }}$ s. av. J.-C. L'importance du commerce lié aux amphores dans le cadre de l'économie et de l'histoire de l'île est attestée notamment par la présence de l'amphore comme emblème de Chios sur ses émissions monétaires jusqu'au III' $\mathrm{e}$. av. J.-C. ${ }^{362}$.

L'attribution à Chios de la forme d'époque tardoarchaïque et classique à col "bombé », ainsi que de son évolution vers le type à col droit du dernier quart du $V^{e}$ s. av. J.-C., a d'abord été mise en évidence par V. Grace sur la base de la représentation de ces deux types de conteneurs sur deux émissions monétaires de l'île datées du Ve s. av. J.-C. Dans le cas de la forme à col bombé, l'attribution a été confirmée ultérieurement par les analyses archéométriques et par la présence de l'effigie de l'amphore sur une gemme attribuée au tailleur Dexamenos de Chios ${ }^{363}$.

Le tableau d'ensemble du développement morphologique de la production chiote peut être considéré comme bien défini, aussi bien pour l'époque archaïque, sur laquelle se sont concentrées les recherches de P. Dupont, que pour la production du Ve s. av. J.-C., qui a bénéficié d'importants points d'ancrages typochronologiques grâce aux exemplaires livrés par les contextes du Céramique et de l'Agora d'Athènes, étudiés respectivement par U. Knigge et M. Lawall ${ }^{364}$. À ces fonds fondamentaux, il faut également ajouter les découvertes de Chios même et de ses alentours ${ }^{365}$,

362. Grace 1953,p. 105 ; Grace, Savvatianou-Petropoulakou 1970 ; Whitbread 1995, p. 135.

363. En ce qui concerne les monnaies, il s'agit respectivement d'une émission avec un sphinx assis devant une amphore à col bombé (Grace 1934, p. 296 et Ead. 1953, p. 105) et d'une autre sur laquelle on a reconnu la représentation de la forme la plus évoluée à col droit (Grace 1979b, fig. 45). Pour la gemme de Dexamenos représentant une amphore du type later bulgy, voir : Boardman 1970, p. 194-197, pl. 470 ; Grace 1979a, p. 120-121, pl. XXXV, 6.

364. Sur la production d'époque archaïque, voir: Dupont 1998, p. 146-151, fig. 23.1-23.2. Sur les amphores chiotes de la nécropole du Céramique : Knigge 1976, notamment, p. 23-24. U. Knigge a identifié pour le $\mathrm{V}^{\mathrm{e}}$ siècle trois variantes qu'elle a définies $\mathrm{C} / 1, \mathrm{C} / 2$ et $\mathrm{C} / 3$. La même terminologie a été reprise dans l'étude de M. Lawall des exemplaires de l'Agora, à partir de laquelle il a établi la chronologie des variantes du V ${ }^{\mathrm{e}}$ s. av. J.-C. : Lawall 1995, p. 88-115.

365. Sur la zone suburbaine de Kophinà : Anderson 1954 ; pour Emporio : Boardman 1967 ; pour la zone du petit port de Limnia, sur la côte occidentale de l'île : Dupont 1982,p. 198 ; pour les amphores récupérées dans le bras de mer compris entre Chios et la côte anatolienne : Grace 1953, p. 105. 
les contextes funéraires du secteur de Elizavetovskoe, sur la Mer Noire, ainsi que les épaves de la Pointe Lequin 1A, de Cala Sant Vicenç et de Géla ${ }^{366}$.

\subsection{Bilan des études}

Les types les plus anciens, que P. Dupont date de la seconde moitié du VII ${ }^{\mathrm{s}} \mathrm{s}$. av. J.-C., sont caractérisés par une panse large et ovoïde, un col court cylindrique à lèvre épaissie, des anses à section elliptique et un court et large pied tronconique creux à la base, en forme d'anneau. Compte tenu de la présence d'un engobe blanc-crème sur l'ensemble de la surface externe du vase, le type est généralement qualifié white slipped. Sur cet engobe sont réalisées des décors peints de couleurs sombres, généralement brun ou rouge. Le décor consiste en d'amples bandes horizontales, réalisées sur la lèvre, à la base du col et sur la panse : trois bandes rapprochées sur l'épaule et deux plus éloignées sur la partie inférieure de l'amphore. Toujours sur l'épaule, au-dessus des trois bandes supérieures, un décor caractéristique en forme de « $\mathrm{S}$ » couché est généralement apposé. Une bande peinte forme un anneau autours des jonctions supérieures et inférieures des anses, qui portent également une bande peinte verticale sur leur surface externe. Cette bande verticale, relativement longue, rejoint généralement les bandes horizontales placées dans la partie inférieure de la panse ${ }^{367}$. La définition des types anciens de Chios et leur chronologie est cependant discutée. Pour M. Slaska, par exemple, les exemplaires qui ouvrent la série au milieu du VII ${ }^{\mathrm{e}} \mathrm{s}$. av. J.-C. présenteraient un engobe transparent mince, de la même couleur que l'argile ou un peu plus clair, alors que les types white slipped n'apparaîtraient pas avant la fin du VII ${ }^{\mathrm{e}} \mathrm{s}$. av. J.-C. pour continuer dans le courant de la première moitié du siècle suivant. Ces amphores, surtout les exemplaires fragmentaires, seraient donc difficiles à distinguer de la série contemporaine de Clazomènes qui, bien qu'elle présente le

366. Pour la Mer Noire : Brašinskij 1980 et Brašinskij, Marčenko 1984 ; pour l'épave de la Pointe Lequin 1A : Long, Pomey, Sourisseau 2002, p. 50-54, avec bibliographie précédente ; pour l'épave de Cala Sant Vicenç : Nieto, Santos 2008 ; pour l'épave de Géla, voir en dernier lieu : Panvini 2001.

367. Pour le début de la série, voir: Slaska 1986, p. 55-56; sur la morphologie de ce type initial, voir : Dupont 1998, p. 146-148, n. 37, fig. 23.1, a-e, et dernièrement la synthèse de R. De Marinis (1999, p. 263-265, fig. 7. 1), avec références bibliographiques aux exemplaires de Thasos, Rhodes, Milazzo, Caere et Athènes qui fournissent des repères chronologiques ponctuels pour la forme. R. F. Docter signale cependant quelques fragments d'amphores chiotes de Toscanos qui pourraient être datés dans le courant du deuxième quart du VII' s. av. J.-C. (Docter 2000, p. 74 e fig. 4-5). même système décoratif du type chiote white slipped, est dépourvue de l'engobe blanc ${ }^{368}$.

L'évolution morphologique de la série, dans le courant de la première moitié et du troisième quart du $\mathrm{VI}^{\mathrm{e}} \mathrm{s}$. av. J.-C., se caractérise par l'apparition d'une forme plus élancée, avec l'épaule nettement fuyante. La panse présente alors son diamètre maximal environ à mi-hauteur, et le pied tronconique a un diamètre réduit. C'est à sa morphologie fusiforme que le type doit la dénomination de bobbin type par laquelle cette forme est généralement connue dans la bibliographie. L'engobe blanchâtre et un décor de couleur brun-rouge analogue à celui du type précédent white slipped est toujours présent ${ }^{369}$.

Entre la fin du second et le troisième quart du $\mathrm{VI}^{\mathrm{e}} \mathrm{s}$. av. J.-C., on assiste à la production concomitante de deux types d'amphores de production chiote. En effet, alors que la série white slipped semble encore en cours de production, une nouvelle forme chiote apparaît, connue sous le nom de type «Lambrino A1 » ou Zeest's funnel-necked (fig. 38 a-b), dont la production est attestée jusqu'en 510 av. J.-C., lorsqu'elle évolue vers le type «Lambrino A2 » (fig. 38 c et fig. 40, première à gauche) documenté entre 510 et 490 av. J.-C. Les formes «Lambrino A » ne présentent plus la forme fusiforme typique de la première moitié du siècle, mais un profil plutôt piriforme, une lèvre en bourrelet légèrement épaissie et un col cylindrique se distinguant nettement de l'épaule. Le col peut être haut et légèrement évasé ou bien bref et au profil rectiligne. Le pied, plus haut qu'auparavant mais également plus étroit, peut être défini de forme cylindrique creuse à base annulaire. L'engobe blanc-crème n'est presque plus présent et le décor est plus simple que celui des types précédents, se limitant à une bande de peinture sur la lèvre, à une ou deux lignes horizontales minces tracées sur l'épaule et à une ligne courant verticalement le long des anses ${ }^{370}$. Un élément important pour la datation du type «Lambrino A » est

368. Pour la définition du type chiote sans engobe qui précéderait la fabrication des types à engobe, voir: Slaska 1986, p. 55-56. Sur le problème de la série similaire de Clazomènes, voir récemment : Sezgin 2004, p. 170-172.

369. Sur le type: Dupont 1998, fig. 23.1, f-h ; de Marinis 1999, p. 265-266, fig. 7.2, avec références bibliographiques concernant les nombreuses trouvailles d'amphores de cette forme depuis les côtes de l'Asie Mineure jusqu'à la Provence. Le bobbin type présente des bandes plus fines et un «S» couché de forme plus allongée par rapport au type white slip.

370. Sur l'évolution morphologique des productions des trente dernières années du $\mathrm{VI}^{\mathrm{e}} \mathrm{s}$. av. J.-C., correspondant aux types Lambrino A1 et A2 (Lambrino 1938, p. 110-112, fig. 71-72), voir également : Dupont 1998, p. 148-149, fig. 23.2, a-c. Sur ce sujet, voir en dernier lieu : de Marinis 1999, p. 266, fig. 7.3, avec renvois bibliographiques aux exemplaires constituant une référence chronologique pour le type. 

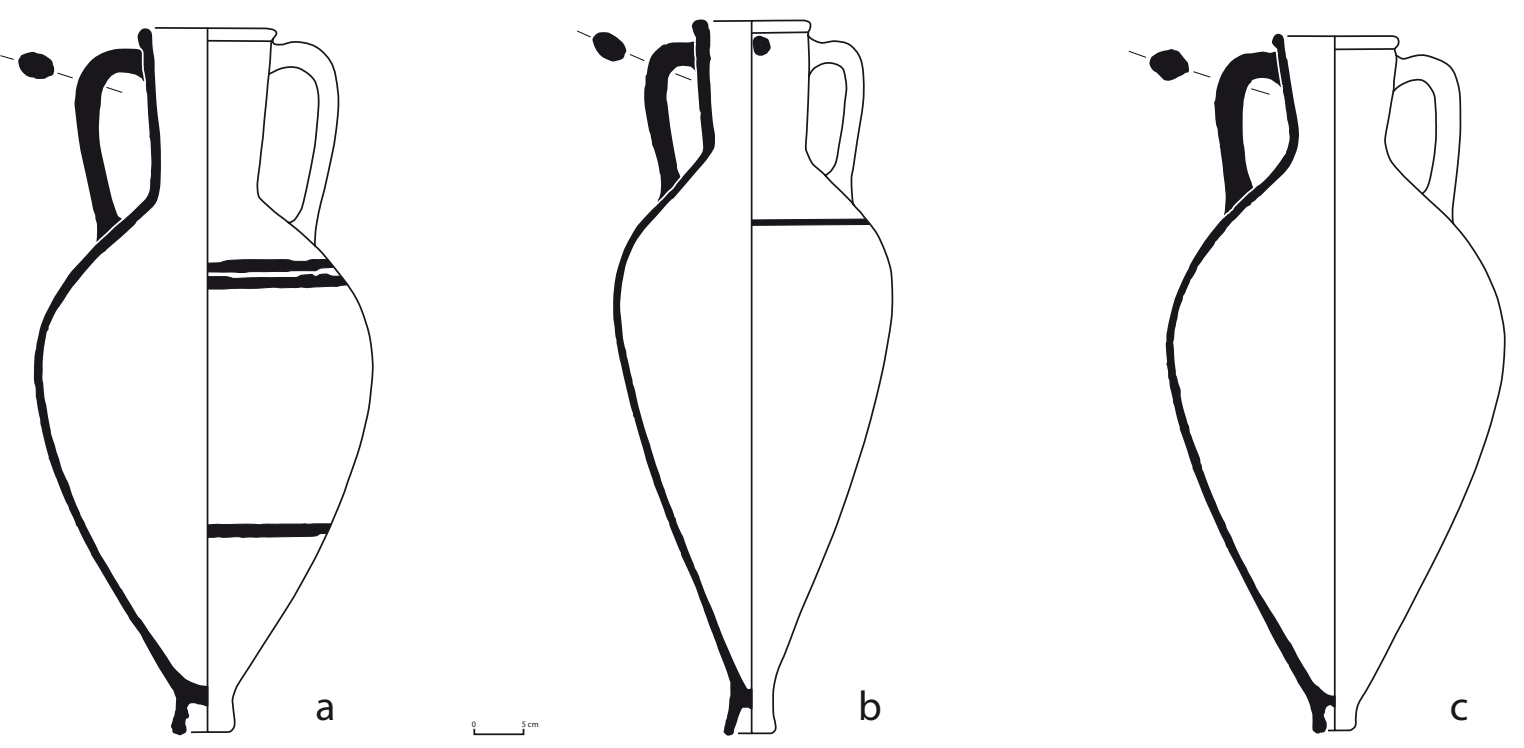

Fig. 38. Amphores de Chios de la deuxième moitie du Vle s. av. J.-C. : types « Lambrino A » (d'après Dupont 1998, fig. 23.2.a-c).

constitué par la présence d'exemplaires de cette forme dans les épaves de la Pointe Lequin 1A et de Cala Sant Vicenç, dont les naufrages sont datés respectivement vers 515 et 510 av. J.-C. ${ }^{371}$.

Durant la période de transition entre le $\mathrm{VI}^{\mathrm{e}}$ et le $\mathrm{V}^{\mathrm{e}} \mathrm{s}$. av. J.-C., les amphores de la série adoptent une forme marquée par le gonflement du col, qui détermine le passage entre les types «Lambrino A » et les types appelés swollen-necked (Dupont 1998; de Marinis 1999) ou bulging-neck types (Lawall 1995), caractéristiques de la fin du $\mathrm{VI}^{\mathrm{e}}$ et des premiers trois quarts du $\mathrm{V}^{\mathrm{e}} \mathrm{s}$. av. J.-C.

Entre la fin $\mathrm{du} \mathrm{VI}^{\mathrm{e}}$ et les trois premiers quarts du $\mathrm{V}^{\mathrm{e}} \mathrm{s}$. av. J.-C., d'après la classification récente de R. de Marinis, on peut distinguer quatre variantes d'amphores swollen-necked assimilables au sous-type dit early bulgy et une au sous-type later bulgy. Aux quatre variantes du type swollen-necked/early bulgy de R. de Marinis correspondent grosso modo les deux premières variantes du type à col bombé, désignées $\mathrm{C} / 1$ et $\mathrm{C} / 2$ dans la classification proposée par U. Knigge pour les amphores du Céramique d'Athènes ${ }^{372}$ et récemment reprise par M. Lawall, qui à travers l'étude des fonds de l'Agora a pu en préciser le cadre chronologique ${ }^{373}$. Au sous-type connu sous le nom de later bulgy correspond enfin la variante $\mathrm{C} / 3$ de $\mathrm{U}$. Knigge.

371. Long, Miro, Volpe 1992.

372. Knigge 1976, p. 23-24.

373. Lawall 1995, p. 89-92.
La série des amphores chiotes à col bombé commence donc à la fin du VI' $\mathrm{s}$. av. J.-C. et continue d'exister tout en évoluant jusque dans le courant $\mathrm{du} \mathrm{V}^{\mathrm{e}} \mathrm{s}$. av. J.-C. avancé. La variante la plus ancienne du type d'après la sériation de R. de Marinis est datable autour du passage entre le $\mathrm{VI}^{\mathrm{e}}$ et le $\mathrm{V}^{\mathrm{e}} \mathrm{s}$. av. J.-C. sur la base de quelques exemplaires trouvés dans des contextes d'époque tardoarchaïque de l'espace égéen telle la zone suburbaine de Kophinà à Chios, le temple d'Aphaïa à Égine et en Mer Noire, notamment Elizavetovskoe et Histria ${ }^{374}$. Cette variante archaïque présente un panse ovoïde assez large, une lèvre légèrement épaissie, un col bref au gonflement encore peu marqué et un pied de forme presque cylindrique, creux et à la base en anneau. Les exemplaires de cette forme présentent de la peinture sur la lèvre, un décor peint de deux lignes minces horizontales sur l'épaule, une sur le bas de la panse et parfois des petits cercles sur le col ou sur l'épaule ${ }^{375}$ (fig. 39 a).

L'étape évolutive suivante de la sériation de R. de Marinis doit être située entre la fin $\mathrm{du} \mathrm{VI}^{\mathrm{e}}$ s. et 480 av. J.-C. et je crois qu'elle pourrait correspondre à la

374. Pour Chios: Anderson 1954, p. 139, n, 51, fig. 8.51 ; pour égine : Johnston 1990, p. 38-40, fig. 1-2.8, 36 ; pour Elizavetovskoe : Brašinskij 1984, pl. VII, 3 et VIII ; pour Histria : Condurachi 1954, fig. 166-167 et Id. 1966, pl. 52, n 368-369. Pour d'autres références bibliographiques aux types chiotes à col bombé les plus anciens : Lawall 1995, p. 89, note 5 et p. 96, note 26.

375. De Marinis 1999, p. 266, fig. 7.4 et notes 71-74, avec références bibliographiques relatives aux découvertes d'amphores de ce type ; Lawall 1995, p. 89-90, fig. 19, 23-25. 


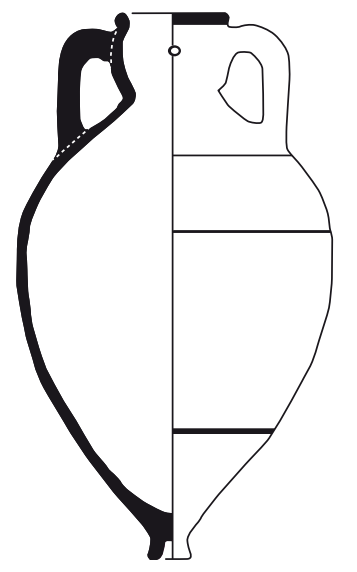

a
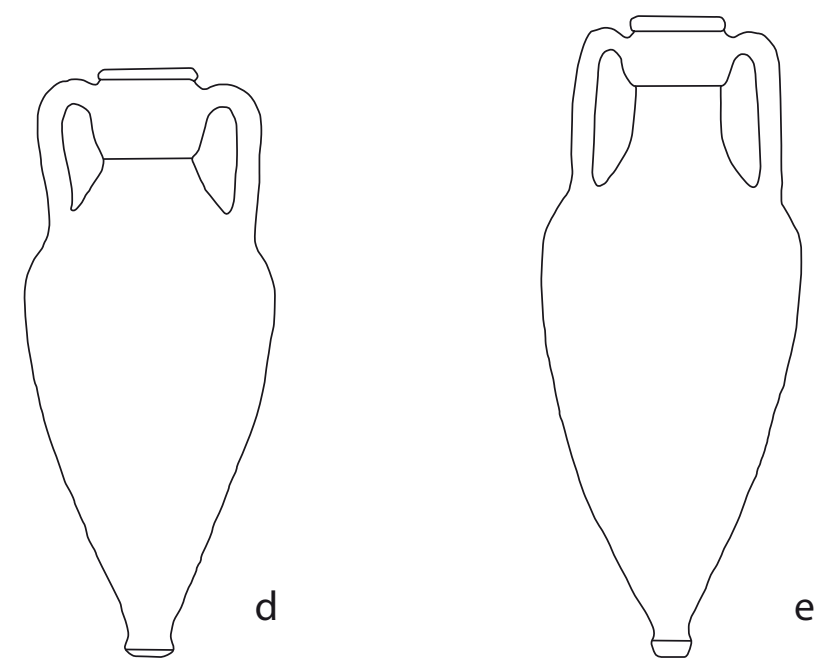

variante C/1 di U. Knigge (fig. 39 b et fig. 40, deuxième à gauche). La chronologie de cette variante, déjà bien établie sur la base de la documentation du puits Q 12:3 de l'Agora, daté entre 520 et 490 av. J.-C., ainsi que de la cargaison de l'épave de Gela, datée entre 500 et 480 av. J.-C., a été confirmée par l'étude de différents autres dépôts de l'Agora d'Athènes ${ }^{376}$. Les amphores chiotes

376. Knigge 1976, p. 23-24 ; Dupont 1998, fig. 23.2, d ; de Marinis 1999, p. 266-267, fig. 7.5. Pour la chronologie de la variante C/1 sur la base de la documentation de l'Agora, ainsi que sur la base d'autres contextes (principalement ceux du Céramique d'Athènes) : Lawall 1995, p. 96-97 et note 35. Pour le matériel du puits Q 12:3 de l'Agora, daté entre 520 et 490 av. J.-C. : Roberts 1986, notamment p. 67, n 419-420, fig. 42 ; pour l'épave de Géla : Fiorentini 1990, p. 27-28, pl. XII. 1, 3, XIV.1-6 et Panvini 1997, p. 137-138, fig. 8. L'épave, dans laquelle on trouve de la céramique attique datable entre 500 et 480 av. J.-C., transportait une cargaison d'amphores parmi lesquelles les productions chiotes toutes attribuables à cette variante du type early bulgy, représentaient environ $60 \%$ des amphores.

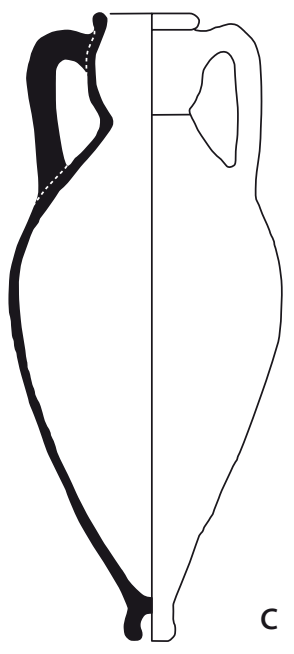

Fig. 39. Tableau typochronologique des amphores de Chios au Ve s. av. J.-C. (d'après de Marinis 1999,

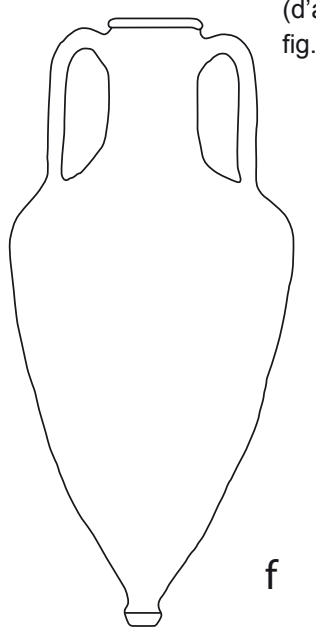

livrées par ces contextes sont caractérisées par un profil plus sensiblement piriforme qu'auparavant, dû à un épaulement plus marqué et plus aplati. Les anses, de section elliptique à circulaire, sont brèves et rentrantes dans la partie inférieure ${ }^{377}$. Le pied, de forme cylindrique plus marquée, présente en général une profonde cavité centrale et une base en anneau légèrement épaissi au profil rentrant en bas. On peut cependant affirmer, sur la base de l'observation de nombreux exemplaires, que tant le profil du pied que la profondeur et la forme de sa cavité sont assez variables ${ }^{378}$. Le décor peint est limité à la lèvre, à de simples et fines lignes horizontales qui peuvent être situées au-dessous de la lèvre ou sur

377. Lawall 1995, p. 90, fig. 19, 23-25 (variante C/1).

378. Sur ces observations : Lawall 1995, p. 90 et note 9, avec références bibliographiques à des exemplaires contemporains dont les pieds présentent des formes différentes. 

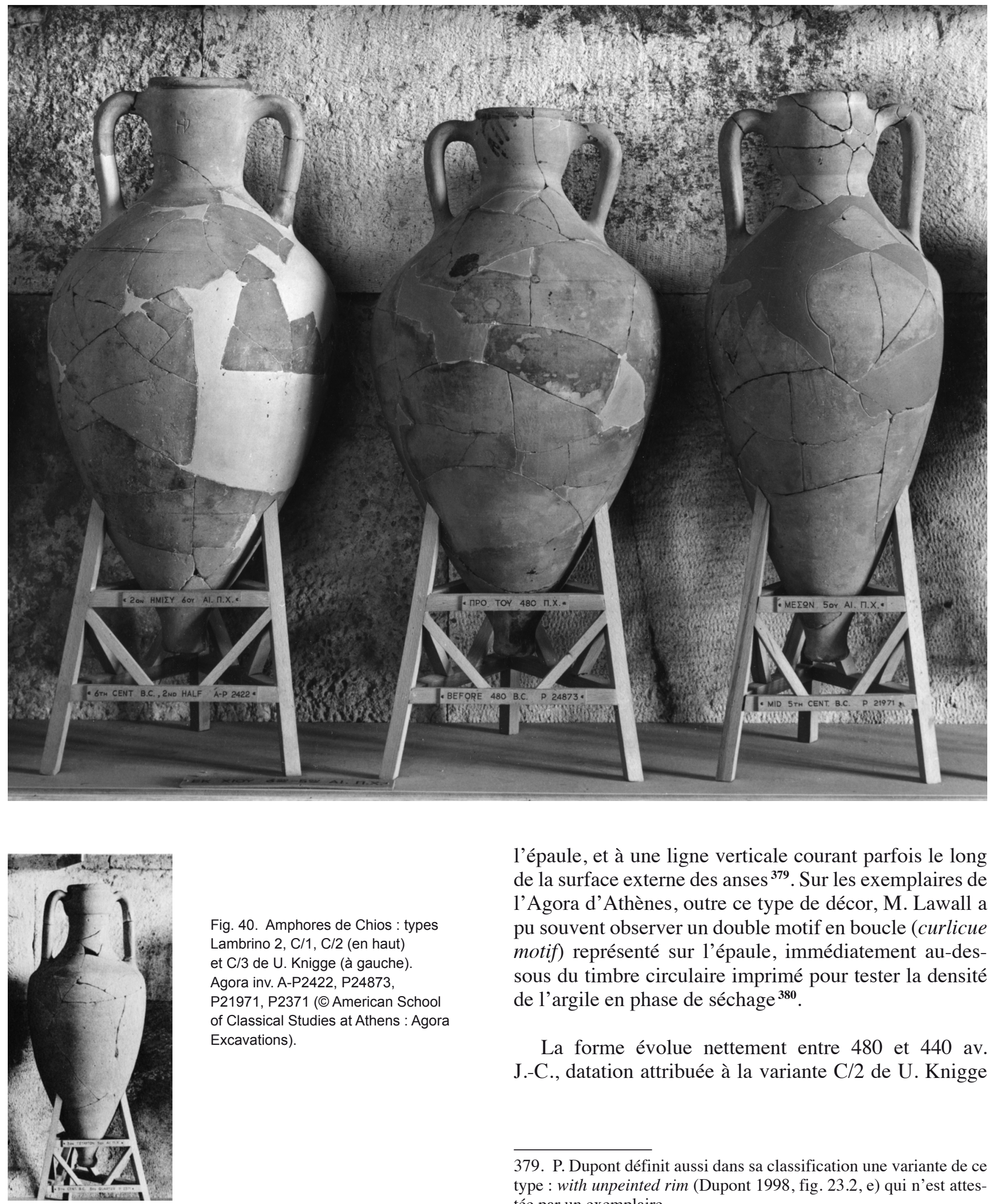

l'épaule, et à une ligne verticale courant parfois le long de la surface externe des anses ${ }^{379}$. Sur les exemplaires de l'Agora d'Athènes, outre ce type de décor, M. Lawall a pu souvent observer un double motif en boucle (curlicue motif) représenté sur l'épaule, immédiatement au-dessous du timbre circulaire imprimé pour tester la densité de l'argile en phase de séchage ${ }^{380}$.

La forme évolue nettement entre 480 et 440 av. J.-C., datation attribuée à la variante $\mathrm{C} / 2$ de U. Knigge

379. P. Dupont définit aussi dans sa classification une variante de ce type : with unpeinted rim (Dupont 1998, fig. 23.2, e) qui n'est attestée par un exemplaire.

380. Lawall 1995, p. 90 (variante C/1). Pour une interprétation du timbre circulaire, voir : Eiseman, Ridgway 1987, p. 41-42 et, plus récemment : Carlson 2003, p. 586. 
correspondant à la troisième variante de $\mathrm{R}$. de Marinis ${ }^{\mathbf{3 8 1}}$ (fig. 39 c et fig. 40, troisième à gauche). La première partie de la période, entre 480 et 450 av. J.-C., est illustrée par des exemplaires d'Elizavetovskoe, et située dans le temps sur la base de la découverte d'une amphore complète dans le colmatage du puits H 6:5 de l'Agora, lui-même daté de 470-460 av. J.-C. Plusieurs autres exemplaires provenant de dépôts plus récents, dont le

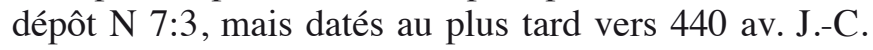
sont également connus ${ }^{382}$. Il s'agit d'un conteneur qui, bien qu'il ne présente pas d'importantes variations morphologiques avec les exemplaires précédents, est caractérisé par une forme générale visiblement plus allongée et par un profil plus fuselé. Les anses, implantées sur une épaule fuyante plus que dans les types précédents, sont plus longues et plus recourbées dans la partie supérieure et moins rentrantes dans la section inférieure, car elles descendent vers l'épaule en se tenant plus proches du $\mathrm{col}^{383}$. Le pied est encore du type cylindrique creux à l'intérieur avec une base en anneau et une profonde cavité à la base. Le profil du pied serait, selon R. de Marinis, plus rentrant dans sa partie inférieure que pour la variante précédente. Cette remarque ne semble pas recouper complètement les observations de M. Lawall sur le matériel de l'Agora qui considère ce détail morphologique comme non déterminant en matière chronologique ${ }^{384}$.

La quatrième et dernière variante du type early bugly identifiée par R. de Marinis se situerait vers 450 av. J.-C., datation établie sur la base de l'amphore P21971 du contexte N 7:3 de l'Agora d'Athènes, daté entre 460 et 440 av. J.-C., et de quelques exemplaires d'Histria ${ }^{385}$. Les conteneurs appartenant à cette variante ne présenteraient pas de différences substantielles par rapport à la variante précédente, si ce n'est le profil du pied, toujours de type cylindrique creux à l'intérieur, mais légèrement concave et non rentrant et présentant même une carène à l'extérieur, à proximité de la base

381. Pour le type : Knigge 1976, p. 23-24 ; Dupont 1998, fig. 23.2, f; de Marinis 1999, p. 267, fig. 7.6 ; Lawall 1995, p. 90-91, fig. 20, 26-27, 31 (variante $\mathrm{C} / 2$ ).

382. Sur la définition de cette troisième variante, voir : de Marinis 1999, p. 267, fig. 7.6. Pour les exemplaires de la Mer Noire: Brašinskij 1980, p. 15-16, pl. 1-3, et Id. 1984, pl. VII, 5-6 e VIII, 4. L'amphore complète P 27727 du contexte H 6:5 de l'Agora est inédite. Pour celle-ci et pour le reste de la documentation de l'Agora : Lawall 1995, p. 97-98, note 37.

383. Pour une description des anses de cette forme, voir : Lawall 1995, p. 90, fig. 20 (variante C/2).

384. Lawall 1995, p. 91, fig. 31

385. Sur l'amphore P21971 de l'Agora : Grace 1953,p. 104, n 150 , et Ead. 1961, fig. 44 (seconde à partir de droite); pour les exemplaires d'Histria, : Condurachi 1954, p. 369, fig. 100-111, 163. en anneau ${ }^{386}$ (fig. 39 d). Cette quatrième variante de la proposition typologique de R. de Marinis, absente dans la typologie de U. Knigge et de M. Lawall ${ }^{387}$, s'avère assez difficile à caractériser au regard des éléments qui définissent la troisième. Le même R. de Marinis a d'ailleurs remarqué que «le due varietà databili al secondo quarto e alla metà del V secolo sono difficilmente distinguibili quando si conservano solamente frammenti del collo, delle anse, della spalla (...) ${ }^{388}$. Quoi qu'il en soit, au regard de la rareté des exemplaires documentés pour cette nouvelle variante décrite par R. de Marinis, de l'absence de modifications morphologiques significatives, d'une chronologie qui semble se confondre avec celle de la variante précédente et des doutes émis par $\mathrm{M}$. Lawall sur le caractère non pertinent en termes chronologiques des variations morphologiques des pieds, je crois qu'il est plus prudent d'éviter une fragmentation excessive du cadre typo-chronologique des amphores de Chios au $\mathrm{V}^{\mathrm{e}} \mathrm{s}$. av. J.-C. La présence à Chios de plusieurs ateliers parallèles et contemporains pourrait expliquer la raison pour laquelle on trouve documentés des exemplaires qui présentent de légères variations par rapport à un modèle général par ailleurs assez bien défini, sans que ces variations puissent toutefois justifier la création d'une variante en tant que telle.

Au troisième quart du $\mathrm{V}^{\mathrm{e}} \mathrm{s}$. av. J.-C. est daté le soustype de la série swollen-necked désigné later bulgy et correspondant à la variante $\mathrm{C} / 3 \mathrm{de} \mathrm{U}$. Knigge (fig. 39 e et fig. 40, quatrième à gauche). Le caractère distinctif le plus significatif de cette variante concerne la forme du col. Celui-ci est articulé en deux parties placées l'une sur l'autre: dans la partie supérieure le profil bombé conserve donc la rondeur caractéristique des types swollen-necked qui se sont affirmés à partir de la fin du $\mathrm{VI}^{\mathrm{e}}$ s. av. J.-C. ; la partie inférieure est de forme cylindrique s'ouvrant largement vers le bas, avec un profil dans la continuité de l'épaulement qui adopte une allure concave. La morphologie générale du pied est modifiée par rapport au sous-type précédent early bulgy et se caractérise maintenant par une forme cylindrique grossie. La base, en anneau, présente une cavité centrale de profondeur modérée mais de toute façon susceptible de variations tout à fait secondaires ${ }^{389}$. Le passage du sous-

386. De Marinis 1999, p. 267-268, fig. 7. 7.

387. M. Lawall utilise la même amphore P21971 comme illustration du type C/2 (Lawall 1995, p. 90, n. 11, fig. 20).

388. De Marinis 1999, p. 268.

389. Sur la définition du sous-type later bulgy, voir : de Marinis 1999, p. 268-269, fig. 7. 8, avec références aux attestations du type à Histria : Condurachi 1966, p. 161, pl. 86, 89, à Elizavetovskoe : Brašinskij 1980, p. 107, n 6-12, pl. 1, 8, 11 ; Id. 1984, pl. I, 3, VII,

7 ; VIII, 5-6, à égine et Marathon. Pour le type, correspondant à la 
type early bulgy aux sous-types later bulgy est illustré par sa découverte dans le remblai le plus récent du Punic Amphora Building de Corinthe, dont le plus ancien niveau ne contenait que des sous-types early bulgy. La chronologie est confirmée par l'attestation des premiers exemplaires de cette forme dans le contexte $\mathrm{N}$ 7:3 de l'Agora d'Athènes, clos vers 440 av. J.-C., et des derniers dans les dépôts M 17:7 et R 13:14, l'un et l'autre clos en 425 av. J.-C. ${ }^{390}$.

Dans le courant du dernier quart du $\mathrm{V}^{\mathrm{e}} \mathrm{s}$. av. J.-C., la série swollen-necked disparait lorsque le sous-type later bulgy est remplacé par un conteneur dont l'originalité la plus évidente est l'abandon du caractère bombé du col. Les nouvelles amphores « à col droit », ou straight-neck type (fig. 39 f et fig. 41), ont un col cylindrique au profil rectiligne et une épaule plus haute et plus aplatie que dans la série précédente. La forme du pied subit également une modification car sa terminaison perd la forme cylindrique pour adopter une forme tronconique avec base en anneau et cavité centrale. On trouve parfois à la base des anses un timbre représentant un sphinx et une amphore du type ancien à col bombé. ${ }^{391}$ La chronologie de cette forme est établie une fois de plus par l'étude des contextes de l'Agora d'Athènes. Ici, les exemplaires les plus anciens proviennent du dépôt $\mathrm{R} 13: 4$, clos en 425 av. J.-C., et cinq des huit contextes datés du dernier quart $\mathrm{du} \mathrm{V}^{\mathrm{e}} \mathrm{s}$. av. J.-C. et contenant des amphores chiotes ont exclusivement livré le type à col droit ${ }^{392}$. C'est à partir de ce type à col cylindrique, qui reste inchangé jusqu'au début du $\mathrm{IV}^{\mathrm{e}} \mathrm{s}$. av. J.-C., que se développeront ensuite les formes des amphores chiotes du $\mathrm{IV}^{\mathrm{e}} \mathrm{s}$. av. J.-C. et des siècles suivants, caractérisées par des cols de plus en plus longs, des épaulements de plus en plus aplatis, et des pieds de plus en plus hauts ${ }^{393}$.

On a longtemps estimé que l'introduction des amphores chiotes du type later bulgy, ainsi que leur remplacement par le type straight-neck, ancrés dans un cadre chronologique bien défini, était liés à un événement historico-économique bien précis, c'est-à-dire

variante C/3 d'U. Knigge et M. Lawall, voir : Knigge 1976, p. 23-24 ; Lawall 1995, p. 91, fig. 22, 28-30.

390. Pour la chronologie du sous-type later bulgy, ou variante C.3 : Lawall 1995, p. 99-102, avec références aux dépôts cités et discussion.

391. Sur ce type : Lawall 1995, p. 91-92, fig. 22, 33-36 ; de Marinis 1999, p. 269 , fig. 7.9.

392. Sur la chronologie de cette phase fondamentale, voir : Lawall 1995, p. 102-103 et note 66.

393. Grace 1961, fig. 45-47.

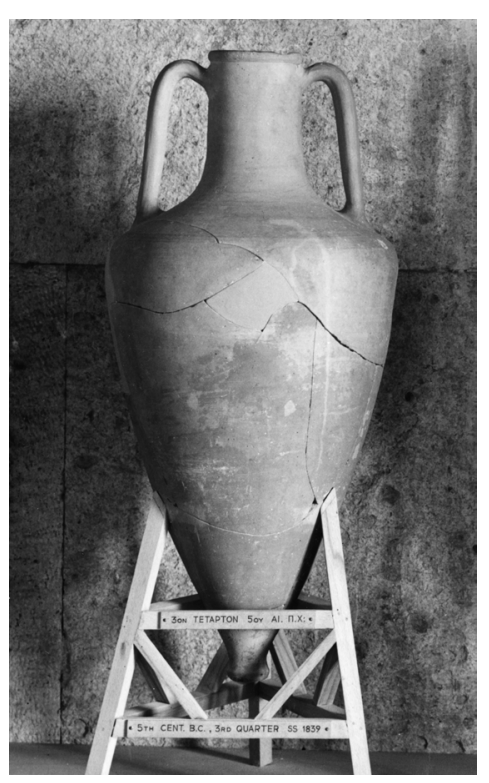

Fig. 41. Amphore de Chios de type straight neck. Agora inv. SS 1839 (๔ American Schoolof Classical Studies at Athens : Agora Excavations).

la promulgation du décret de Klearchos de 449-448 av. J.-C. ${ }^{394}$ Ce décret impose les nouveaux standards des poids et des mesures athéniens auxquels tous les membres de la ligue de Délos devaient se conformer. Chios devait donc s'aligner sur les nouveaux standards athéniens et selon V. Grace, ce règlement trouve une illustration dans les changements de capacité des amphores. Ainsi elle observe que le sous-type later bulgy était de 8 choes chiotes environ, ce qui correspondait à 7 choes attiques, alors que les amphores du sous-type early bulgy ne pouvaient contenir que 7 choes chiotes. Les amphores du type later bulgy auraient donc, selon V. Grace, un terminus post quem de type historique, fixé en 449-448 av. J.-C., qui est aussi confirmé par les données archéologiques. L'accroissement de la capacité des conteneurs du nouveau type later bulgy a dû impliquer une augmentation du prix unitaire des amphores de vin de Chios, ayant, à son avis, pour conséquence une chute des ventes non seulement à cause du prix mais aussi du fait que le marché aurait eu des difficultés à accepter ces conteneurs qui, malgré leurs plus grandes dimensions, conservaient la forme ancienne au col bombé. D'après la spécialiste américaine, ce fut pour résoudre ce problème de perte de garantie auprès des acheteurs que dans le dernier quart du $\mathrm{V}^{\mathrm{e}} \mathrm{s}$. av. J.-C. aurait été introduite la nouvelle forme à col droit. Ce nouveau modèle, qui se distingue nettement du «style » auparavant reconnu comme originaire de Chios, a dû recevoir au début

394. Grace, Savvatianou-Petropoulakou 1970, p. 359-360 ; Grace 1979a, p.121-122. 
une véritable marque de garantie par l'apposition d'un timbre où, à côté d'un sphinx, apparaissait une amphore du type ancien à col bombé.

Toutefois, des doutes sur la relation existant entre les deux événements ont été successivement exprimés par V. Grace elle même et par M. B. Wallace ${ }^{395}$. La datation incertaine du décret (449 av. J.-C. ou peut-être 425 av. J.-C.), ainsi que de la relation difficile à établir entre cette décision politique et un réel changement de la capacité des amphores de Chios ${ }^{396}$, apparaissent comme des difficultés majeures. Il subsiste par ailleurs des problèmes métrologiques complexes concernant tant le système attique lui-même, ses rapports avec le système chiote ${ }^{397}$, que la correspondance entre ces systèmes et notre système métrique décimal ${ }^{398}$. Il faut rappeler enfin que les études conduites sur un certain nombre d'exemplaires complets ont montré l'extrême variabilité de la capacité des amphores chiotes à l'intérieur d'un même type ${ }^{399}$.

\subsection{Centres de production}

L'attribution à l'île de Chios de la série amphorique qu'on vient de décrire a été confirmée par un programme d'analyses effectuées sur la pâte. Si les analyses chimiques mises en œuvre par P. Dupont avaient déjà donné des résultats positifs en ce sens ${ }^{400}$, les analyses pétrographiques qui les ont suivies, effectuées par I. K. Whitbread ${ }^{401}$, ont confirmé non seulement le résultat précédent, mais ont aussi permis de distinguer deux groupes pour le $\mathrm{V}^{\mathrm{e}} \mathrm{s}$. av. J.-C.

Le premier groupe renvoie aux amphores de type swollen-necked / early bulgy. Il est constitué d'une matrice argileuse de couleur brun-rougeâtre, avec des dégraissants de différentes dimensions incluant des fragments de quartz, de feldspaths gris, de calcaire blanc et de particules minérales de couleur brun-rouge foncé. La pâte présente de très nombreuses vacuoles, ce qui semble être un élément caractéristique de ce groupe.

Le second groupe renvoie quant à lui aux exemplaires les plus récents, c'est-à-dire les amphores du type later bulgy et celles à col droit. Il est constitué de matrices

395. Grace 1979a,p. 121-122 ; Wallace 1986, p. 88.

396. Pour une discussion sur la datation controversée du décret et son éventuelle relation avec le changement présumé de la capacité des amphores chiotes de la seconde moitié du $\mathrm{V}^{\mathrm{e}} \mathrm{s}$. av. J.-C., voir : Lawall 2000, p. 4-7 et notes 5-8, avec références bibliographiques. 397. En dernier lieu : Lawall 2000, p. 22, n. 1, avec bibliographie. 398. De Marinis 1999, p. 271-272, avec bibliographie.

399. Brashiskij 1984, p. 170-175

400. Dupont 1982, p. 198 ; Id. 1983, p. 24, 30-31.

401. Whitbread 1995, p. 138-144. argileuses de couleur variable, du brun clair au jaunerougeâtre, avec une section interne allant du gris rosé au brun clair. Les dégraissants présents dans ces pâtes, moins nombreux et de plus petites dimensions, incluent des fragments de quartz et de feldspaths gris clair et blancs, des fragments de calcaire de couleur jaune pâle et de rares écailles de mica argenté ${ }^{402}$.

Les différences d'aspects de pâtes qui affectent les productions chiotes d'époque archaïques et celles d'époque classique est un phénomène qui a déjà été remarqué par simple analyse visuelle par I. K. Anderson et ensuite par p. Dupont ${ }^{403}$. Cette dernière distinction au sein des productions du $\mathrm{V}^{\mathrm{e}} \mathrm{s}$. av. J.-C., mise en évidence par I. K. Whitbread, grâce aux résultats d'analyses pétrographiques, montre toutefois que les amphores du groupe 2, c'est-à-dire celles de la seconde moitié du $\mathrm{V}^{\mathrm{e}} \mathrm{s}$. av. J.-C., ont une composition proche de celle des amphores archaïques à engobe.

La variabilité visuelle des pâtes correspondant globalement aux étapes importantes de l'évolution morphologique de la série, ainsi que la réapparition à la fin $d u \mathrm{~V}^{\mathrm{e}} \mathrm{S}$. av. J.-C. d'une pâte utilisée à l'époque archaïque, autorisent à penser que l'organisation de la production d'amphores de Chios relevait d'un nombre assez important d'ateliers distincts, actifs dans la fabrication de formes différentes. Toutefois, il apparaît difficile de considérer qu'à une forme particulière et donc à un arc chronologique spécifique corresponde strictement un type de pâte. En revanche leur coexistence, au moins partielle, pourrait expliquer la présence des variantes qui viennent d'être évoquées et qu'on peut observer au cours de tout le $\mathrm{V}^{\mathrm{e}} \mathrm{s}$. av. J.-C. ${ }^{404}$. Par ailleurs, la proximité à la fois géographique et géologique de l'île avec le territoire d'Érythrée, situé en face sur le continent, suggère de ne pas exclure l'hypothèse de fabrications d'amphores de type chiote sur la terre ferme, où d'autres productions céramiques chiotes étaient imitées ${ }^{405}$.

\subsection{Contenu}

De nombreuses sources littéraires célèbrent la renommée du vin de Chios, considéré comme un des plus réputés dans l'Antiquité. Comme nous en informe

402. Les recherches sur les pâtes des amphores de Chios, initiées par p. Dupont et R. E. Jones (Jones 1986, p. 740), ont été récemment complétées par l'importante contribution de I. K. Whitbread, auquel nous renvoyons aussi pour une synthèse des résultats obtenus par les deux auteurs précédents (Whitbread 1995, p. 138-144). à ce propos, voir aussi : Lawall 1995, p. 93-96 et de Marinis 1999, p. 263.

403. Anderson 1954, p. 169 et Dupont 1982, p. 98.

404. Lawall 1995, p. 95-103.

405. Dupont 1998, p. 151. 
Plutarque, à l'époque de Socrate une amphore de ce vin était payée à Athènes cent drachmes, c'est-à-dire l'équivalent de cent jours de salaire d'un ouvrier spécialisé ${ }^{406}$.

Un autre document sur l'importance de la viticulture pour l'économie de l'île est l'effigie de la grappe de raisin sur une émission monétaire d'époque classique, sur laquelle elle est associée à une amphore clairement identifiée comme une amphore de Chios ${ }^{407}$.

Enfin, l'observation de restes de poix sur des exemplaires d'Athènes ${ }^{408}$, ainsi que la récente relecture de quelques graffiti commerciaux sur des amphores fragmentaires de forme straight-neck ${ }^{409}$ semblent confirmer la vocation vinaire de ces conteneurs.

\subsection{Diffusion dans la Méditerranée occidentale}

Les premiers exemplaires d'amphores chiotes sont arrivés en Occident dès le début de leur production. Des amphores chiotes du type white slipped sont en effet attestées en Sicile, où elles ont été trouvées dans plusieurs sépultures de Mylai, Camarine, Himère et Naxos, ainsi que dans des contextes d'habitat ou cultuels à Himère, Mégara Hyblaea, Monte San Mauro et Naxos ${ }^{410}$. Le type est également documenté en Campanie, à Cumes, à Pithécusses, à Calatia et à Vélia, ainsi qu'en Basilicate, à Siris ${ }^{411}$. En Étrurie méridionale, notamment à Gravisca et dans les nécropoles de Caeré et de Vulci, les amphores produites à Chios représentent l'une des séries les plus abondamment attestées avec celles de Clazomènes et de Samos ${ }^{412}$. Il s'agit aussi bien d'exemplaires sans engobe blanc que du type white slipped, datables entre le milieu $\mathrm{du} \mathrm{VII}^{\mathrm{e}}$ et le milieu du VI ${ }^{\mathrm{e}} \mathrm{s}$. av. J.-C., période de leur plus ample diffusion ${ }^{413}$. D'autres exemplaires de pleine époque archaïque, datés d'avant le milieu du $\mathrm{VI}^{\mathrm{e}} \mathrm{s}$. av. J.-C., sont documentés dans l'arrière-pays albanais, à Margëlliç, mais aussi le long des côtes françaises, à Marseille, à Saint-Blaise, aux Baou de Saint-Marcel, à L'Arquet, à Tamaris, à Château-Virant, et enfin en

406. Pour une synthèse sur les sources qui mentionnent les qualités du vin de Chios : Grace 1934, p. 296.

407. Mattingly 1981, pl. I, b.

408. Roberts 1986, p. 66-67.

409. Lawall 2000, p. 18, 32, $\mathrm{n}^{\circ} 20-21$.

410. Sur la diffusion du type en Sicile: Albanese Procelli 1997b, p. 105-106. Plus récemment, pour Himère : Vassallo 2003, p. 342, $\mathrm{n}^{\circ} 23$, fig. 6,23 .

411. Pour Cumes : Savelli 2006, p. 112-113; pour Pithécusses : Di Sandro 1986, p. 53-54 ; pour Calatia : Albore Livadie 1985, p. 139 ; pour Vélia : Gassner 2003b, p. 122-123 ; pour Siris : Berlingò 1986. 412. Pour l'Étrurie méridionale, voir en particulier : Boitani 1985 ; Rizzo 1990 et Slaska 1986, p. 55-56, avec la bibliographie antérieure. 413. Sur la diffusion générale du type archaïque: Slaska 1986, p. 55-57, n. 7, avec références bibliographiques.
Espagne, à Toscanos et peut-être à Huelva ${ }^{414}$. Dans l'aire méridionale du bassin méditerranéen occidental, la présence d'amphores chiotes est signalée à Carthage, où les attestations commencent dans la phase du début de la production $^{415}$.

Le cadre de la diffusion des types plus récents d'amphores chiotes, de production postérieure au milieu du $\mathrm{VI}^{\mathrm{e}} \mathrm{s}$. av. J.-C., se présentait, encore récemment, difficile à préciser. La redéfinition d'une cadre typo-chronologique plus précis et l'avancée des recherches de terrain permettent actuellement de compléter, au moins partiellement, l'inventaire concernant la Méditerranée occidentale et la péninsule italienne en particulier.

En Grande Grèce, la présence d'amphores chiotes récentes est signalée surtout à Caulonia. Les exemplaires les plus anciens sont datés, sur la base de la stratigraphie, entre la seconde moitié du VI ${ }^{\mathrm{e}}$ et le premier tiers du V ${ }^{\mathrm{e}} \mathrm{s}$. av. J.-C. À la même phase chronologique tardo-archaïque appartiennent quelques exemplaires trouvés le long de la côte ionienne de la péninsule italienne, tels des fragments provenant de Sybaris et surtout de Locres ; sur le littoral tyrrhénien méridional d'autres témoignages sont également fournis par Medma et par la nécropole de Métauros. Du plein $\mathrm{V}^{\mathrm{e}} \mathrm{s}$. av. J.-C. sont datés deux pieds trouvés dans l'habitat de Caulonia, quelques fragments de Vélia récemment publiés, et d'autres, nombreux, de Locres qui descendent jusqu'au IV ${ }^{\mathrm{e}} \mathrm{s}$. av. J.-C. ${ }^{416}$

Des amphores de Chios datées du VIe à la fin du $\mathrm{V}^{\mathrm{e}} \mathrm{s}$. av. J.-C. proviennent de nombreux sites de Sicile. Les découvertes concernent des contextes d'habitats, comme à Géla, Mégara Hyblaea, Monte Saraceno, Montagna di Ramacca, Naxos et Sélinonte, ainsi que des contextes funéraires, comme à Camarine, Himère, Leontinoï, Mylai, Monte San Mauro et Naxos ${ }^{417}$. D'un intérêt particulier est la découverte de nombreux exemplaires du

414. Pour Margëlliç : Ceka 1986, p. 89. Sur la documentation de Marseille et de la Provence en général, voir: Sourisseau 1997, I, p. 134. Pour Toscanos : Docter 2000, p. 72-74, n 18-25, fig. 10, g-n. Pour Huelva : Rouillard 1991, p. 177.

415. Docter 2000, p. 72-74, n 12-17, fig. 10, a-f ; Id. 2007, p. 660. 416. Vandermersch 1989,p. 94 (pour les exemplaires archaïques) et p. 101-102 (pour ceux du Ve s. av. J.-C.). Sur les amphores chiotes archaïques de Métauros: Sabbione 1981a; Sabbione 1981b; Sabbione 1987. Pour Locres: Barra Bagnasco 1992. Pour Vélia: Gassner 2003b, p. 122-123.

417. Albanese Procelli 1997b, p. 106, avec détails et références bibliographiques. Sur le site de la Montagna di Ramacca, en dernier lieu : Albanese Procelli 2003, notamment p. 39 ; pour Naxos, voir dernièrement : Lentini, Savelli, Blackman 2007, p. 89, et Id. 2009, p. 102-103, fig. 17 ; pour Camarine : Sourisseau 2002, p. 548, et Id. 2006, p. 133 et fig. 1. 
type early bulgy dans la cargaison de l'épave archaïque de Géla, datée du début du $\mathrm{V}^{\mathrm{e}} \mathrm{s}$. av. J.-C. ${ }^{418}$.

Pour le Latium, on peut signaler la présence d'une amphore chiote à Rome, provenant des stratigraphies de l'habitat archaïque ${ }^{419}$.

En Étrurie méridionale, à partir du milieu du $\mathrm{VI}^{\mathrm{e}} \mathrm{s}$. av. J.-C., on ne connaît aucune attestation de la présence d'amphores chiotes provenant des nécropoles, mais on en trouve dans les contextes d'habitat. Gravisca a fourni des attestations des types du $\mathrm{V}^{\mathrm{e}} \mathrm{s}$. av. J.-C. et quelques exemplaires tant archaïques que $\mathrm{du} \mathrm{V}^{\mathrm{e}} \mathrm{s}$. av. J.-C. sont maintenant attestés dans le secteur de Tarquinia ${ }^{\mathbf{4 2 0}}$. Quelques exemplaires fragmentaires appartenant à des amphores chiotes datables du courant du VIe s. av. J.-C. sont documentés dans les habitats de Caeré, où il existe peut-être aussi d'autres attestations de plein $\mathrm{V}^{\mathrm{e}} \mathrm{s}$. av. J.-C., et de Regae, où les fragments trouvés ont été datés entre la fin du VI et le milieu du V ${ }^{\mathrm{e}}$ s. av. J.-C. ${ }^{421}$

Dans le secteur nord-tyrrhénien, la présence d'amphores chiotes est signalée dans l'habitat de Pise, où leur apparition peut être située dans la deuxième moitié $\mathrm{du} \mathrm{VI}^{\mathrm{e}}$ s. av. J.-C. Toutefois, quelques fragments attribuables au type à col bombé témoignent de la continuité de leur importation dans le courant $\mathrm{du} \mathrm{V}^{\mathrm{e}} \mathrm{s}$. av. J.-C. ${ }^{422}$ Un groupe d'amphores chiotes faisait partie de la cargaison de l'épave A de Punta Ala, près de Castiglione della Pescaia, datée entre la fin $\mathrm{du}^{\mathrm{VI}} \mathrm{C}^{\mathrm{e}} \mathrm{s}$. et la première moitié du $\mathrm{V}^{\mathrm{e}} \mathrm{s}$. av. J.-C. ${ }^{423}$ Plus au nord, trois exemplaires

418. Sur les amphores chiotes de l'épave archaïque de Géla: Freschi 1991; Panvini 1991; Parker 1992, n 441, p. 188-189, map 11 ; Panvini 1993 ; Id. 1997 et Id. 2001, p. 64-70, pl. XVII.97102, XVIII.103-108, XIX.109-114, XX.115-120, XXI.121-126, XXII.127.

419. Zevi 1985, notamment p. 119, n. 5, avec bibliographie antérieure.

420. Pour Gravisca : Slaska 1978, p. 228 et Ead. 1985, p. 19 ; pour Tarquinia : Scotti 1986, p. 171 et Ead. 1999, p. 272-273.

421. Boss 1993, p. 325 (fragments datables dans le courant du VI ${ }^{\mathrm{e}} \mathrm{s}$. av. J.-C.). Le pied L38, que M. Boss attribue de manière dubitative à un atelier samien ou chiote (Ibid., p. 325-326, L38, fig. 522.L38), doit plutôt être attribué, à mon avis, à une production chiote de la première moitié $\mathrm{du} \mathrm{V}^{\mathrm{e}} \mathrm{s}$. av. J.-C.

422. Pancrazzi 1982, p. 336, 341, fig. 2.17-18. O. Pancrazzi a attribué à une production de Chios deux types dont le premier me semble plutôt devoir être assigné à une production de Samos-Milet, comme l'avait déjà proposé A. Maggiani (1990b, p. 94), du moins pour un fragment de pied. En l'absence d'une publication exhaustive du matériel trouvé à Pise, je crois qu'il est plus prudent de situer le début des importations d'amphores chiotes vers une période qui n'est pas antérieure au début du $\mathrm{V}^{\mathrm{e}} \mathrm{s}$. av. J.-C. En faveur du caractère archaïque des premières importations d'amphores chiotes à Pise, voir néanmoins : Bonamici 1996, p. 22.

423. Cygielman, De Tommaso 1998, p. 93-101 (épave A). fragmentaires du type chiote à col bombé ont été trouvés lors des fouilles de l'habitat de Gênes ${ }^{424}$.

Le long du littoral français, les découvertes concernent principalement l'épave de la Pointe Lequin $1 \mathrm{~A}^{425}$, datée vers 515 av. J.-C. ainsi que des attestations, surtout à Marseille, mais aussi à Arles, Saint-Pierre-lèsMartigues, Saint-Blaise et Mont-Garou ${ }^{426}$.

En ce qui concerne l'Espagne, signalons les attestations de l'épave de Cala Sant Vicenç, datée vers 510 av. J.-C., les découvertes d'Emporion et celles du gisement sous-marin d'El Cabanyal-Malvarrosa. Enfin, et pour une période un peu plus tardive, on doit signaler la présence d'amphores chiotes à col droit dans la cargaison du bateau marchand d'El Sec, qui a fait naufrage près de Palma de Majorque et qui transportait de nombreux exemplaires datés du milieu du $\mathrm{IV}^{\mathrm{e}} \mathrm{s}$. av. J.-C. ${ }^{427}$.

\section{La production amphorique attribuée à Clazomènes}

Le premier à suggérer d'attribuer à Clazomènes l'une des productions amphoriques grecques orientales d'époque archaïque a été P. Dupont. Sa proposition, qui s'appuie sur les résultats des analyses des argiles effectuées sur un vaste échantillonnage, a été ultérieurement confirmée par la découverte de déchets de cuisson dans les niveaux archaïques de la cité même de Clazomènes ${ }^{428}$. Il a ainsi attribué à ce site nord-ionien la fabrication d'une série amphorique à bandes peintes, caractérisée par une lèvre épaissie du type en bourrelet, un col bref cylindrique ou se resserrant légèrement vers le bas, une panse ovoïde et un pied cylindrique haut et large à base en anneau et de profil concave. Cette série, décrite la première fois par M. F. Lambrino ${ }^{429}$, a ensuite été classée par I. B. Zeest dans la catégorie dite with broad bands ${ }^{430}$. On trouve dans la bibliographie ce

424. Milanese, Mannoni 1986, p. 134, fig. 8, 4-5 et Milanese 1987, p. 196, n 446, fig. 91, 446, e p. 224, n 595, fig. 100, 595.

425. Sur l'épave de la Pointe Lequin 1A: Pomey et al. 1988 , p. 32-35 ; Long, Miro, Volpe 1992, p. 199-243 ; Parker 1992, n 846, p. 323, map 5 ; Gras 1996, p. 168-169 ; Long, Pomey, Sourisseau 2002, p. 50-54.

426. Pour la Provence : Sourisseau 1997,p. 134.

427. Sur l'épave de Cala Sant Vicenç: Nieto, Santos 2008 ; sur Emporion : Sanmartì Grego et al. 1991 et Aquilué Abadías et al. 2000, p. 330, fig. 39.6-7. Pour El Cabanyal-Malvarrosa, voir: Fernández, Gomez, Ribera 1988, p. 318, fig. 2.3. Pour le bateau d'El Sec : Arribas et al. 1987.

428. Sur les analyses archéométriques des pâtes: Dupont 1982, p. 194 ; Id. 1983, p. 25-26, 31, fig. 18. Sur les déchets de cuisson de Clazomènes : Doğer 1986, p. 467.

429. Lambrino 1938, n. 1, p. 114-118, fig. 76-80, 87, 89.1, 90 b.

430. Zeest 1960, n. 2, p. 69-72, pl. I.1, II.6 a-b. 
type d'amphores désignées comme telle ou de manière plus générique comme "grecque orientale » ${ }^{431}$. Une œnochoé grecque orientale, récemment publiée ${ }^{432}$ et attribuée à une production nord-ionienne définie Mixed Technique Style ${ }^{433}$, présente un décor constitué d'une frise d'amphores dont la forme peut être assimilée à celle des conteneurs attribués à Clazomènes. Ce vase, qui pour le moment reste isolé, est un élément supplémentaire permettant de confirmer l'attribution de cette série d'amphores au secteur de Clazomènes.

D'après E. Doğer, se basant sur l'observation d'éléments morphologiques et décoratifs, la production amphorique de Clazomènes a pu être commune, durant certaines périodes anciennes, avec celle de sa voisine Chios ${ }^{434}$. En effet, les amphores à décor de bandes et de « $\mathrm{S}$ » couchés sur l'épaule, caractéristiques de l'époque archaïque, ont généralement été considérées par le passé comme spécifiquement chiotes ${ }^{435}$. Les trouvailles récentes de Clazomènes ont néanmoins confirmé que la cité avait bien produit, durant l'époque archaïque ancienne (VII ${ }^{\mathrm{e}}$ s. av. J.-C.), une série avec un système décoratif analogue ${ }^{436}$. À partir de la fin du VII ${ }^{\text {e }}$ s. av. J.-C., les nombreuses variantes qu'on peut observer à l'intérieur de la série clazoménienne ont laissé imaginer l'existence de plusieurs ateliers, dans lesquels étaient fabriquées des amphores de formes similaires et avec le même décor mais qui se différenciaient uniquement par quelques détails morphologiques, et qui pouvaient être situés dans différents centres de la région nordionienne ${ }^{437}$. Parmi ceux-ci, outre Clazomènes et les éventuels ateliers chiotes différents de ceux qui produisaient la série chiote plus connue, on a proposé comme centre producteur les cités de Téos, Colophon, Erythrée et peut-être Smyrne, notamment pour la production initiale de la série ${ }^{438}$.

Les exemplaires publiés de la série clazoménienne, trouvés principalement en Mer Noire, avaient déjà permis à $\mathrm{P}$. Dupont de distinguer des variantes morphologiques, définies A, B, C, D, E. Seul le type A

431. Doğer 1986, p. 464-465, n. 7-13 et Lawall 1995, p. 48, n. 2, l'un et l'autre avec références bibliographiques.

432. CVA, Russie, XVII, p. 10, pl. 4, M485.

433. Sur cette catégorie de vases : Cook, Dupont 1998, p. 52, n. 44. 434. Doğer 1986, p. 462 ; Sezgin 2004, p. 169, n. 7, avec références bibliographiques.

435. Dupont 1998, p. 151 ; Sezgin 2004, p. 169, 171, n. 14.

436 Doğer 1986, p. 461-462, fig. 1-2 ; Sezgin 2004, p. 170-173, fig. 1-2, 5 (Group I et Group II).

437. Dupont 1998, p. 154.

438. L'hypothèse de localisation d'un des ateliers de production à Téos est fondée sur l'observation croisée de données archéologiques et archéométriques (Dupont 1982, p. 200-201 ; Id. 1983, p. 26). Pour les autres centres proposés comme de possibles lieux de production : Sezgin 2004, p. 172, et n. 8, 18. permettait de suivre un développement morphologique entre la fin du VII et le début du V ${ }^{\mathrm{e}}$ s. av. J.-C. (Dupont 1998, p. 151-155, fig. 23.3).

Les découvertes effectuées dans le cadre des fouilles de Clazomènes archaïque, et notamment celles de conteneurs entiers utilisés pour les sépultures d'enfants à enchytrismos dans les nécropoles de Yildiztepe et d'Akpinar, ont récemment permis à Y. Sezgin de proposer une autre classification préliminaire des amphores clazoméniennes. Celles-ci ont été ainsi subdivisées en six groupes ${ }^{439}$.

\subsection{Bilan des études}

Le début de la production des amphores de Clazomènes a été situé par E. Doğer et P. Dupont ${ }^{440}$ vers la fin du VII ${ }^{\mathrm{e}}$ s. av. J.-C. R.F. Docter a récemment proposé de remonter la date de démarrage de la production sur la base d'un fragment trouvé dans un contexte de Toscanos daté entre 675 et 640 av. J.-C. ${ }^{441}$ Une proposition chronologique analogue, entre 650 et 620 av. J.-C., a été proposée par Y. Sezgin ${ }^{442}$ qui attribue à cette période son Group I.

Le Group I définit par Y. Sezgin est le moins homogène de la série. Il regroupe quatre types amphoriques qui se distinguent par leur morphologie et leur décor. Les caractéristiques communes sont le col court, l'épaulement large et arrondi, ainsi que la panse ovoïde se rétrécissant de manière prononcée vers le pied, dont le diamètre est très ample et le profil extérieur légèrement concave. Les variations morphologiques les plus marquées se manifestent au niveau de la lèvre, qui peut être épaissie ou bien du type triangulaire. On peut également remarquer une certaine diversité des décors, notamment des combinaisons de bandes peintes en marron. Elles se trouvent sur la lèvre, sur le col, le long des anses,

439. Sezgin 2004. On remarquera que la classification typologique des amphores clazoméniennes proposée par Y. Sezgin est uniquement basée sur les exemplaires qu'il a lui-même directement analysés et provenant des fouilles de la cité. La chronologie de la série s'appuie par contre sur des exemplaires provenant de contextes différents.

440. Cf. : Doğer 1986, p. 462 ; Dupont 1998, p. 152, fig. 23.3a.

441. Docter 2000 , p. 75 , fig. 11, b, qui propose d'établir un parallèle entre ce fragment et un exemplaire de la nécropole de Pithécusses (Tombe $n^{\circ} 397$ ) daté, sur la base du contexte, de la fin du VIII' $\mathrm{s}$. av. J.-C. L'exemplaire de Pithécusses, considéré de production «protochiote » par G. Buchner et D. Ridgway (1993, p. 429, pl. 211, 227), a été attribué à une production clazoménienne par $\mathrm{A}$. W. Johnston (Docter 2000, p. 75, avec références bibliographiques).

442. Cette datation a été proposée par Y. Sezgin sur la base d'exemplaires provenant de contextes différents, tels Chypre (nécropole de Salamine), Histria, Kommos, Carthage et Cerveteri (Sezgin 2004, p. 172, n. 25-30, avec références bibliographiques). 
sur l'épaule et sur la partie supérieure de la panse. On trouve parfois sur l'épaule le motif décoratif en « $\mathrm{S}$ » couché que la série de Clazomènes partage avec celle de Chios. Cependant, alors que dans la production chiote ce motif survit jusqu'au milieu ou vers le troisième quart du VI ${ }^{\mathrm{e}}$ s. av. J.-C., dans celle de Clazomènes il semble limité à la seconde moitié du VII ${ }^{\mathrm{e}}$ s. av. J.-C. ${ }^{443}$ Ce qui permettrait de distinguer les deux séries, hormis la pâte, serait selon Sezgin l'absence de white slip dans la production de Clazomènes ${ }^{444}$.

Le second groupe de la série clazoménienne (Group II) est daté entre 630 et 600 av. J.-C. Il est caractérisé par une lèvre en bourrelet, un col cylindrique, une épaule arrondie et fuyante, une panse ovoïde, un pied large et haut au profil concave. Le décor, qui dans quelques cas seulement conserve la présence du « $\mathrm{S}$ » couché sur l'épaule, consiste principalement en des bandes horizontales situées à la hauteur de la lèvre, de l'épaule et de la panse. Sur quelques exemplaires, ce décor linéaire se place aussi à la base du col et des anses. Le long des ces mêmes anses, une bande verticale se prolongeant jusqu'à l'intersection avec la bande horizontale inférieure peut même être observée. C'est à partir des amphores attribuées à ce groupe que le décor à double bande sur l'épaule devient une caractéristique de la série $^{445}$. à la même phase chronologique est assigné un exemplaire sans décor qui semble pour l'instant constituer un unicum ${ }^{446}$.

Le troisième groupe identifié par Y. Sezgin (Group III), daté entre 630 et 600/590 av. J.-C., est contemporain du groupe précédent, dont il standardise certaines caractéristiques. Il s'agit d'amphores à la lèvre épaissie, au profil en forme de bec, au col évasé, à la panse ovoïde et au pied ample creux à l'intérieur et au profil concave. Le motif du « $\mathrm{S} »$ réalisé horizontalement sur

443. Sur le décor de la série de Chios : Dupont 1982, p. 194-196 ; Id. 1998, p. 146-148, fig. 23.3. Sur le décor de la série de Clazomènes : Sezgin 2004, p. 171, n. 13, p. $178, \mathrm{n}^{\circ} 5$, fig. 5 .

444. Sezgin 2004, p. 170, n. 10. À propos du problème d'une hypothétique production chiote archaïque sans engobe blanc, cf. supra I, 5 § 1.1.

445. Sezgin 2004, p. 172-173. Les exemplaires de référence pour cette proposition de datation dans les trente dernières années du $\mathrm{VII}^{\mathrm{e}}$ s. av. J.-C. proviennent de Salamine de Chypre, de la Mer Noire et de tombes à enchytrismos de la nécropole d'Akpinar, à Clazomènes (Ibid., n. 35-39, avec références bibliographiques).

446. Doğer 1986, p. 468, fig. 13 (type 5A); Dupont 1998, p. 155 , n. 82 , fig. $23.3 \mathrm{~h}$ (type E). p. Dupont a proposé l'hypothèse que cet exemplaire sans décor avait pu servir au transport de l'huile d'olive, à la différence de la plupart des amphores clazoméniennes qui pouvaient en revanche servir au transport du vin. Compte tenu du caractère isolé de l'attestation, je suis d'avis qu'on ne peut exclure que la peinture originale de cet exemplaire se soit effacée. E. Doğer signale d'ailleurs que la lèvre conserve des traces de peinture. l'épaule semble avoir complètement disparu du système décoratif ${ }^{447}$.

Entre 600 et 575 av. J.-C. est situé le Group IV (fig. 42), qui regroupe des conteneurs dont la morphologie générale est plus élancée par rapport aux formes précédentes ${ }^{448}$. Le décor consiste en une bande sur la lèvre, deux bandes rapprochées sur l'épaule, une autre au milieu de la panse et une dernière dans sa partie inférieure. Une dernière bande verticale est placée sur les anses et rejoint la bande horizontale inférieure de la panse. L'attache inférieure des anses est soulignée par une cercle peint.

On date entre 575 et 550 av. J.-C. le Group V(fig. 43), que Y. Sezgin a décrit en s'appuyant sur de rares exemplaires fragmentaires. Sur la base de l'unique amphore entière attribuable à ce groupe, la forme a été distinguée de celle du groupe suivant par le profil de la lèvre, moins ouverte, et par sa morphologie générale plus trapue. En ce qui concerne le décor, la double bande sur l'épaule demeure inchangée ${ }^{449}$.

Entre 550 et $530 / 20$ av. J.-C., on remarque à Clazomènes un vide documentaire concernant les amphores de transport, qui a été interprété par Y. Sezgin comme la conséquence de l'invasion de la cité par les Perses en 547 av. J.-C. Des amphores clazoméniennes datables du troisième quart du $\mathrm{VI}^{\mathrm{e}} \mathrm{s}$. av. J.-C. sont cependant connues dans d'autres contextes de la Méditerranée et de la Mer Noire et ont permis à P. Dupont de caractériser, pour cette phase, dans le cadre de son Type A (fig. 44), un conteneur de forme plus élancée, au col plus haut, au corps ovoïde avec un profil plus sinueux et au pied cylindrique se distinguant plus nettement du fond de la panse ${ }^{450}$.

C'est dans le dernier quart du VI' s. av. J.-C. que s'inscrit le Group VI de Y. Sezgin. D'après l'auteur, durant cette phase, la production des amphores de Clazomènes connait une forte standardisation (fig. 45). Sur la base de la documentation de la nécropole de Yildiztepe et des déchets de cuisson du quartier artisanal de la cité, il distingue deux variantes principales à l'intérieur de ce groupe. La première renvoie à un type de conteneur de forme plutôt trapue et au pied bref évasé. La seconde à des amphores plutôt fuselées à haut pied cylindrique

447. Sezgin 2004, p. 174-175. Des exemplaires attribuables à ce groupe ont été largement exportés. C'est sur les contextes de leur découverte, ainsi que sur quelques contextes de Clazomènes même, que s'appuie la datation proposée (Ibid., p. 174, n. 41-49).

448. Sur les données chronologiques dont on dispose à Clazomènes et dans d'autres sites pour la datation du groupe, voir : Sezgin 2004, p. $174-175$, n. 50, 52-57, avec renvois bibliographiques.

449. Sezgin 2004, p. 175-176.

450. Dupont 1998, p. 154, fig. 23.3c. 

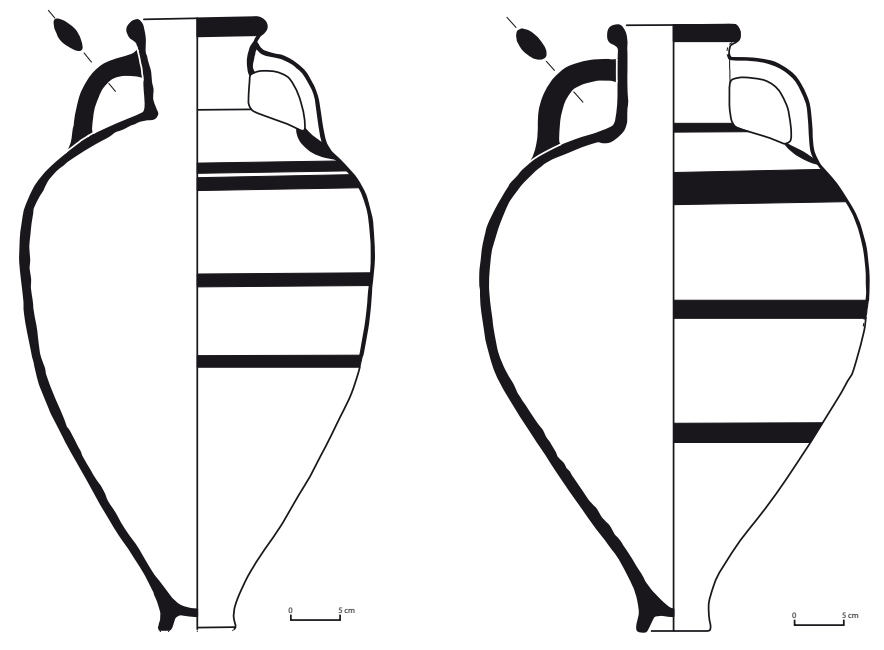

Fig. 42. Amphores de Clazomènes. Group IV de Sezgin 2004, fig. 10-11.

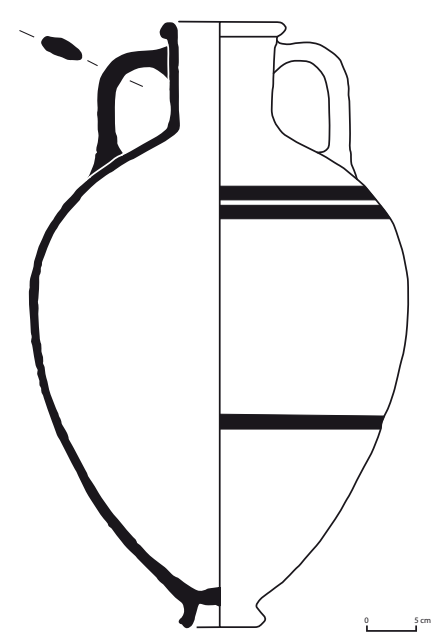

Fig. 44. Amphore de Clazomènes. Type A de Dupont 1998, fig. 23.3.c.

avec une profonde cavité à la base. Dans les deux cas, le décor ne présente pas de variations par rapport au groupe précédent ${ }^{451}$.

Rien ne nous permet d'être sûr qu'après la destruction de Clazomènes par les Perses en 494 av. J.-C. et la fin de la révolte ionienne, les ateliers de production des amphores soient restés actifs dans la cité. $\mathrm{Vu}$

451. Sezgin 2004, p. 176-178, fig. 13-14. Pour la chronologie du groupe, Ibid. n. 89-99, avec bibliographie.

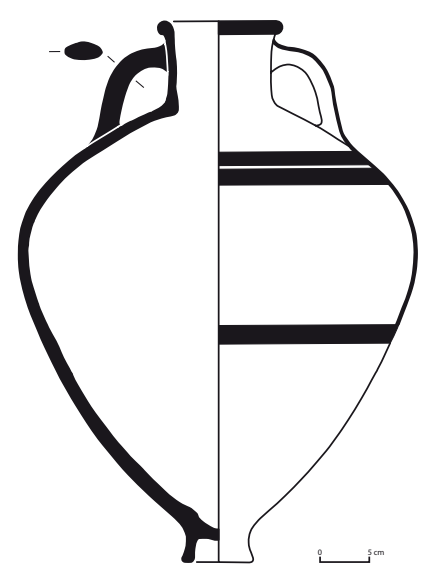

Fig. 43. Amphore de Clazomènes. Group V de Sezgin 2004, fig. 12.
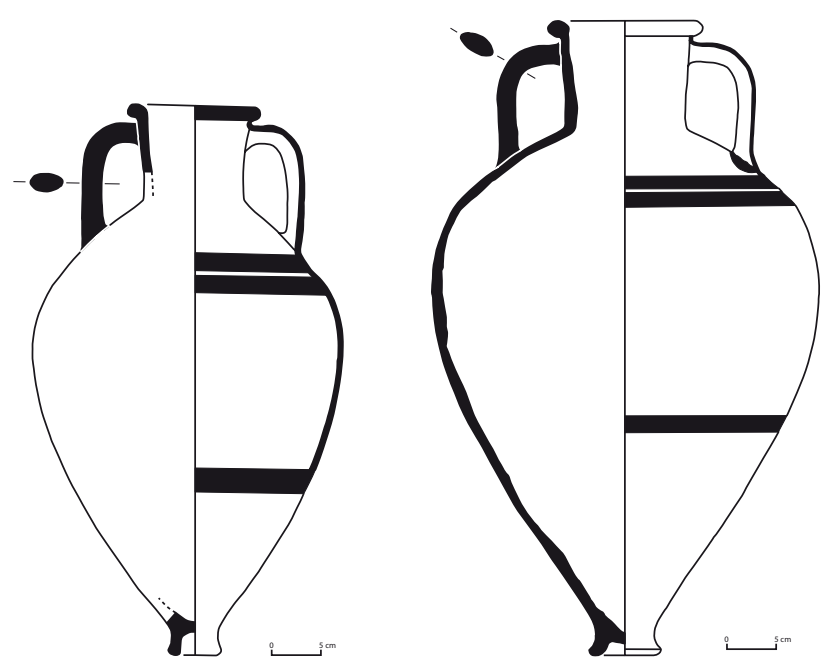

Fig. 45. Amphores de Clazomènes. Group VI de Sezgin 2004, fig. 13-14.

l'absence de trouvailles d'amphores dans les contextes de Clazomènes postérieurs à cette date, Y. Sezgin suggère qu'il faut envisager une interruption de la production d'amphores ${ }^{452}$. P. Dupont a cependant observé que des découvertes effectuées dans des secteurs d'importation pourraient témoigner d'une continuation de la production, du moins au début du $\mathrm{V}^{\mathrm{e}}$ siècle $^{453}$. L'étude

452. Sezgin 2004, p. 177.

453. Dupont 1982,p. 201,n.47-48, avec références bibliographiques. 
systématique des amphores de l'Agora d'Athènes par M. Lawall a confirmé que des exemplaires attribuables à la série clazoménienne sont présents dans six dépôts clos avant 480 av. J.-C. et dans un dépôt clos avant le milieu du siècle ${ }^{454}$. Enfin, le fait qu'on puisse montrer que dans le courant du IV ${ }^{\mathrm{e}} \mathrm{s}$. av. J.-C. les ateliers amphoriques de Clazomènes étaient en activité, produisant les types 6 A-B de Doğer, ainsi que le type «à lèvre champignon » dans des fours datés du troisième quart du $\mathrm{IV}^{\mathrm{e}} \mathrm{s}$. av. J.-C., suggère une continuité de la production pendant tout le $\mathrm{V}^{\mathrm{e}}$ s. av. J.-C. ${ }^{455}$.

\subsection{Centres de production}

L'attribution à Clazomènes de la série décrite ci-dessus se fonde sur plusieurs types de données archéologiques, parmi lesquelles on retiendra la fréquence très élevée de ces formes dans les fouilles de la cité et la découverte de déchets de cuisson sur le site d'un quartier artisanal ${ }^{456}$. Cependant, la preuve la plus solide de cette attribution réside dans les résultats de la vaste campagne d'analyses archéométriques XRF conduite par P. Dupont et qui a abouti à l'identification d'un important et unique groupe chimique dont seuls se distinguent les échantillons prélevés à Téos. Toutefois, ceux-ci se différencient de manière significative par des variantes morphologiques, notamment par la position des anses ${ }^{457}$. La découverte à Clazomènes de nombreux fours de potiers destinés à la production d'amphores peut dès lors être considérée comme une confirmation de cette attribution ${ }^{458}$.

La pâte des amphores de Clazomènes se présente sous la forme d'une argile assez fine, vacuolaire et micacée, avec des inclusions de mica de grandes dimensions ou de cristaux de quartz. La couleur de la surface varie du rouge clair au rosé, mais la section est habituellement grise ou couleur mauve ${ }^{459}$.

\subsection{Contenu}

Les sources littéraires ne fournissent pas beaucoup d'informations sur les activités agricoles de Clazomènes à l'époque archaïque, mais les données disponibles concernant les périodes postérieures indiquent aussi bien une production vinicole (Pline, Nat. Hist., XIV,

454. Lawall 1995, p. 51, n. 14.

455. Doğer 1986, p. 469, fig. 15-18.

456. Doğer 1986, p. 466-467, fig. 1.

457. Dupont 1982, p. 200-201; Id. 1983, p. 25.

458. Dupont 1982, p. 200 ; Id. 1983, p. 26.

459. Doğer 1986, p. 466 ; Johnston 1990, p. 42.
74) qu'une production d'huile d'olive (Pseudo-Aristote, Economique, II, 2, 16) qui pouvaient être destinées à l'exportation.

L'utilisation de ces amphores pour le transport du vin de Clazomènes, coupé avec de l'eau de mer, a été suggérée par les résidus résineux trouvés sur la surface interne d'un certain nombre d'exemplaires d'époque archaïque $^{460}$. La présence de poix à l'intérieur d'une amphore de transport pourrait cependant suggérer de ne pas exclure l'emploi du conteneur pour d'autres types de marchandises ${ }^{461}$. La production d'huile de la cité a été confirmée par l'archéologie grâce à la découverte d'une installation artisanale datée du VI ${ }^{\mathrm{e}}$ s. av. J.-C. ${ }^{462}$.

\subsection{Diffusion dans la Méditerranée occidentale}

Les exemplaires les plus anciens de la série ont été trouvés dans la cité même de Clazomènes, mais il existe des attestations provenant des colonies de la Mer Noire et des côtes de la Méditerranée ${ }^{463}$. Pendant toute la période de la production amphorique de Clazomènes, du moins jusqu'à la révolte ionienne, c'est la Mer Noire qui constitue la zone de plus grande diffusion des amphores de Clazomènes. Dans les sites les plus importants de la région, comme Istros et Olbia, cette production représente entre 20 et $30 \%$ des importations amphoriques. Cependant, après l'attribution de la production à la cité nord-ionienne, d'autres exemplaires provenant de différentes régions de la Méditerranée ont été publiés.

En ce qui concerne la Méditerranée orientale, outre les exemplaires d'Asie Mineure et de Chypre, des amphores clazoméniennes sont signalées dans toute l'aire égéenne, à Corinthe, Athènes, Égine, Théra et en Crète, ainsi que sur le delta du Nil, plus précisément à Tanis et à Abusir ${ }^{464}$.

En Méditerranée occidentale, plusieurs exemplaires ont été découverts en Sicile, à Himère, à Messine, dans

460 Roberts 1986, p. 66 (Athènes) et Sourisseau 1997, I, p. 121 (Marseille).

461. Pour la possible utilisation de la poix comme isolant pour les conteneurs à huile, voir : Dupont 1998, p. 182 et n. 257 et Romanus et al. 2009. Pour le transport de marchandises autres que le vin et l'huile dans des amphores enduites avec de la poix, voir: Charlin, Gassend, Lequément 1979, p. 22 et n. 27.

462. Dupont 1998 , p. 155 , n. $80-81$, avec bibliographie; Sezgin 2004, p. 178, n. 100-104.

463. Sur la diffusion des exemplaires du début de série (Group I) : Sezgin 2004, p. 172, avec la liste des sites et les références bibliographiques.

464. Sur les présences d'amphores clazoméniennes en Méditerranée orientale de la deuxième moitié du VII ${ }^{e}$ à la fin du $\mathrm{VI}^{\mathrm{e}} \mathrm{s}$. av. J.-C., voir : Sezgin 2004 
la nécropole de Camarine, à Naxos et dans l'épave de Gela ${ }^{465}$.

En Grande Grèce, les amphores de Clazomènes sont documentées dans le Salento, à Cavallino, Vaste, Masseria Fano et Ruffano, ainsi qu'à Siris, à l'Incoronata de Métaponte et à Cumes ${ }^{\mathbf{4 6}}$.

D'autres exemplaires sont connus en Étrurie tyrrhénienne, en particulier à Gravisca, à Vulci, à Cerveteri et, plus au nord, dans l'épave du Giglio et à San Rocchino di Massarosa ${ }^{467}$.

La côte méridionale de la France fournit une ample documentation provenant de Marseille et d'autres sites, comme Arles, Saint-Pierre-lès-Martigues, Saint-Blaise et Pech Maho. Quelques exemplaires se trouvaient dans les épaves de Bon Porté 1, du Dattier et de la Pointe Lequin $1 \mathrm{~A}^{468}$.

En péninsule Ibérique, des exemplaires ont été signalés à Emporion, Toscanos et Malaga ${ }^{469}$. Enfin, sur la côte de la Méditerranée sud-occidentale, un fragment d'amphore provenant des fouilles de Carthage a été attribué avec prudence à la production de Clazomènes ${ }^{470}$.

\section{Les amphores de type samien, milésien et du groupe « Samos-Milet »}

À l'île égéenne de Samos et à la cité de Milet, située en face sur la côte anatolienne, sont respectivement assignées deux célèbres productions amphoriques d'époque archaïque. Cependant les analyses récemment menées sur les pâtes des amphores habituellement attribuées à

465. Pour les sites de Sicile, voir : Vassallo 1999, p. 346-347, Id. 2003 , p. 346-347, ${ }^{\circ} 30$, fig. 8, 30, et Id. 2003, p. 346-347, fig. 8, $\mathrm{n}^{\circ} 30$; Spagnolo 2002, p. 33 ; Sourisseau 2002, p. 548, et Id. 2006, p. 133 et fig. 1 ; Albanese Procelli 1997a ; Panvini 2001, p. 74, tav. XXV, 146 .

466. Pour le Salento: Semeraro 1997, p. 61, n 80-81 (Cavallino), p. 105-106, n 201 (Masseria Fano), p. 271-272, n 994 (Ruffano), p. 305, n 1162 (Vaste) ; pour Siris : Berlingò 1993, p. 11, fig. 21 ; pour l'Incoronata : Cavagnera 1995, p. 46, fig. 41-42 ; pour Cumes : Savelli 2006, p. 113, tav. 25.

467. Pour Gravisca: Slaska 1978, p.229-230, pl. 100, fig. 30 ; pour Vulci : Rizzo 1990, p. 104-109; pour Cerveteri : Ibid., p. 22. Dans ces derniers cas il s'agit de deux exemplaires identifiés comme chiotes mais qui d'après Y. Sezgin devraient être attribués à la série de Clazomènes en raison de l'absence de l'engobe blanc qui caractérise la production contemporaine et similaire de Chios (Sezgin 2004, p. 172, n. 30). Pour l'épave du Giglio : Bound 1985 ; pour San Rocchino di Massarosa : Maggiani 1990b, p. 94, n 49-50, fig. 44. 468. Pour Marseille, voir : Sourisseau 1997, I, p. 120 et plus récemment, Sourisseau 2000, p. 141, fig. 2, 145, pl. 3 a-3 b, 146, pl. 4 a-4 b. Pour Pech Maho, voir : Gailledrat, Solier 2004, p. 333, fig. 273, 2. 469. Pour Emporion: Aquilué Abadías et al. 2000, p. 329-330, fig. 39, 3-5. Pour Toscanos : Docter 2000, p. 76, fig. 11 a, n² 26 . Pour Malaga : Cisneros Garcia et al. 2000, p. 196, fig. 9, 11.

470. Docter 2000, p. 74, fig. 2, 6 ; Id. 2007, p. 660-661.
Samos et à Milet suggèrent que des multiples centres de production de l'Égée orientale étaient impliqués dans la production d'amphores de «type samien » et de «type milésien » dès l'époque archaïque. Aux périodes archaïque tardive et classique, on sait même que Samos, Milet et probablement d'autres centres grecs orientaux, se partagent la fabrication de formes amphoriques communes, ou en tout cas dérivant d'un modèle commun. Celui-ci est généralement désigné sous le nom d'amphores du groupe « Samos-Milet».

Des analyses chimiques et pétrographiques effectuées sur les pâtes de ces amphores ont confirmé le rôle que Samos et la chora de Milet ont joué dans la fabrication de ces formes. Toutefois, certains éléments indiquent aussi la possibilité que d'autres centres, nombreux mais dont on ignore encore aujourd'hui la localisation, étaient impliqués dans cette fabrication. Compte tenu des nombreuses variantes morphologiques attestées et d'une certaine diversité des pâtes documentées, le groupe Samos-Milet apparaît comme une production de type « régional » parfois qualifiée de « South Ionian amphoric koine ${ }^{471}$.

L'usage de matrices monétaires samiennes dans le timbrage de certaines amphores de la série a abouti, dans un premier temps, à l'attribution de cette production amphorique à Samos et c'est à V. Grace que l'on doit ces premiers travaux de synthèse. Depuis l'œuvre pionnière de V. Grace, les travaux de P. Dupont et de M. Lawall ont largement contribué à éclaircir une situation relativement complexe ${ }^{472}$. sur la série amphorique de Samos, P. Dupont a proposé une distinction interne, entre une production samienne proprement dite et une production milésienne ${ }^{473}$. Cette distinction fondée sur la morphologie et corroborée par les analyses sur les pâtes, ne concerne pas la forme générale de l'amphore mais plutôt certains éléments de détail, dont le plus important est la morphologie des lèvres : plus hautes et plus minces pour la série milésienne (high lip), plus épaisses pour les productions samiennes (thickened rim). Par ailleurs,

471. Dupont 1998, p. 177.

472. Pour une synthèse pionnière sur les amphores samiennes, voir : Grace 1971. Plus récemment, voir les contributions suivantes : Slaska 1978 ; Mattingly 1981 ; Dupont 1982 ; Dupont 1983 ; Johnston 1993. En particulier Dupont 1982 et Id. 1983 pour certaines corrections des attributions de V. Grace. Sur les amphores de Samos d'époque archaïque, on dispose aujourd'hui de la synthèse de p. Dupont (1998, p. 164-169, fig. 23.6, 23.9, a-c). Sur les productions plus récentes : Lawall 1995, p. 176-195.

473. Dupont 1982, p. 203-206 ; Id. 1983, p. 27-28 ; Id. 1998, p. $164-$ 169 , pour la production de Samos, et p. 170-177, pour la production de Milet. 
soulignons aussi d'autres caractéristiques spécifiques de la production milésienne : les anses qui peuvent être aussi bifides ou trifides, le listel que l'on observe sous la lèvre jusqu'à la fin du $\mathrm{VI}^{\mathrm{e}} \mathrm{s}$. av. J.-C. et la cannelure ou encore le ressaut marqué à la base du col, ce dernier étant d'ailleurs tout à fait analogue à celui des amphores peintes du style de Fikellura ${ }^{474}$.

Tout en reconnaissant que ces détails formels sont spécifiques à la production milésienne, P. Dupont luimême admet pourtant que la caractérisation des deux ateliers n'est pas sans poser de problèmes, surtout pour les époques tardo-archaïque et classique. En effet, à partir de la fin du VI ${ }^{\mathrm{e}} \mathrm{s}$. av. J.-C., on assiste à une multiplication des variantes morphologiques des lèvres, que l'on pourrait qualifier d'intermédiaires par rapport à celles reconnues comme typiques de l'une ou de l'autre production, de sorte que - selon P. Dupont - dans le cadre de la production milésienne non seulement sont attestées des formes très diverses, mais on trouve aussi des lèvres épaissies, en tous points semblables à celles de l'atelier de Samos ${ }^{475}$.

La difficulté liée à la caractérisation des deux ateliers que nous connaissons dans le cadre de cette koinè sud-ionienne, associée à l'impossibilité d'identifier les autres centres qui devaient être sûrement impliqués dans la production de cette série amphorique régionale, conduit à une certaine prudence tant dans la distinction trop nette entre les deux fabriques, que dans l'attribution trop sûre en faveur de l'une ou de l'autre localité, notamment pour les périodes archaïque tardive et classique. C'est pourquoi, nous ne maintiendrons la distinction entre les fabriques de Samos et de Milet, que pour les amphores produites avant la fin de la seconde moitié du $\mathrm{VI}^{\mathrm{e}}$ s. av. J.-C., lorsque les caractères morphologiques des deux ateliers sont encore identifiables. En revanche, en ce qui concerne toutes les amphores de la série sudionienne, datées entre la fin du $\mathrm{VI}^{\mathrm{e}}$ et la fin du $\mathrm{V}^{\mathrm{e}} \mathrm{s}$. av. J.-C., nous avons choisi l'appellation communément utilisée de groupe de Samos-Milet.

La localisation récente, dans la cité nord-ionienne d'Érythrées, d'un atelier qui produisait différentes séries d'amphores aux formes proches de celles de la zone sudionienne donne la mesure de la complexité du système productif et commercial de l'aire grecque orientale au cours du $\mathrm{V}^{\mathrm{e}} \mathrm{s}$. av. J.-C.

474. A propos des détails qui permettent de distinguer la série milésienne de celle de Samos, voir en dernier lieu : Dupont 1998, notamment p. 174 et 176 . En ce qui concerne les analogies entre les amphores milésiennes et les amphores peintes du style de Fikellura, voir : Dupont 1983, p. 28, 34, fig. 19 et Id. 1998, p. 174 et n.190.

475. À ce sujet, voir : Dupont 1998,p. 176-177, et surtout l'exemple de la fig. 23.8, i.

\subsection{Bilan des études}

En ce qui concerne les amphores samiennes, P. Dupont a proposé quelques modifications au classement de V. Grace, dont la plus évidente est, comme nous l'avons déjà souligné, la distinction entre la production de Samos et celle de Milet. Mais d'autres modifications ont été introduites successivement dans le classement général des formes attribuées à Samos. Or, si les découvertes les plus récentes ont en effet confirmé la validité substantielle de la reconstitution de V. Grace pour la première moitié du $\mathrm{VI}^{\mathrm{e}}$ av. J.-C. ${ }^{476}$, elles ont également souligné la nécessité d'apporter quelques modifications à la définition des types de la seconde moitié du siècle ${ }^{477}$.

\section{Les amphores de type samien}

Pour ce qui est de la production de Samos, les exemplaires les plus anciens qu'on peut lui attribuer sont traditionnellement situés, du point de vue chronologique, dans la période comprise entre la fin du $\mathrm{VII}^{e}$ et le milieu environ du $\mathrm{VI}^{\mathrm{e}} \mathrm{s}$. av. J.-C. Néanmoins, au vu des données stratigraphiques, des fragments d'amphores samiennes de Carthage ont été datés dès le troisième quart du VIII et jusqu'à la fin $\mathrm{VII}^{\mathrm{e}} \mathrm{s}$. av. J.-C. Cette datation haute est par ailleurs confirmée par leur présence dans des contextes clos de Toscanos. En se fondant sur les découvertes provenant de ces deux établissements, R. F. Docter a proposé de remonter la date du début de la production amphorique de Samos ${ }^{478}$.

Parmi les plus anciens produits dont on connaît la forme complète, on distingue deux séries : la première dite massive echinus rim type (fig. 46 a-d) et la seconde pear-shaped type ${ }^{479}$ (fig. 46 e-g). Ces deux types, différents mais néanmoins contemporains, ont en commun une épaisse lèvre en échine, (moins prononcée dans le second type), un col court, tronconique, plus ou moins évasé mais nettement distinct de l'épaule grâce à une sorte de ressaut. Les anses présentent un profil coudé et une section biconvexe. Le pied bas et creux est de forme tronconique. Ce qui varie, en revanche, d'une série à l'autre, c'est la forme de la panse : ovoïde à l'épaule arrondie dans le cas du massive echinus rim type, piriforme avec une épaule fuyante ou aplatie dans celui du pear-shaped type.

476. Dupont 1998, p. 164-165.

477. Dupont 1998, p. 168.

478. Docter 2000, p. 69 et fig. 1 ; Id. 2007, p. 658-659.

479. Sur la première série, voir: Dupont 1998, p. 165, fig. 23.6, a-d ; sur la seconde série, Ibid., p. 165, fig. 23.6, e-g, avec références bibliographiques aux exemplaires qui fournissent des éléments chronologiques utiles à l'encadrement des deux types dans la première moitié du $\mathrm{VI}^{\mathrm{e}}$ s. av. J.-C. 


$$
\begin{aligned}
& D \\
& 0 \\
& 0 \\
& 0
\end{aligned}
$$


Dans la seconde moitié du VI ${ }^{\mathrm{e}} \mathrm{s}$. av. J.-C. la forme générale du conteneur commence à devenir graduellement plus élancée annonçant la morphologie des types du $V^{e}$ s. av. J.-C. La panse, de forme ovoïde, trouve sa largeur maximale au milieu de la panse et l'épaule s'abaisse graduellement et devient plus fuyante par rapport aux formes précédentes. Cette mutation dans la forme de l'épaulement, sur lequel sont implantées les attaches inférieures des anses, entraîne l'allongement de celles-ci qui adoptent une forme coudée légèrement relevée, et dont l'attache supérieure est située non pas immédiatement sous la lèvre, mais à la base du col comme dans les exemplaires plus anciens. Le col demeure plutôt court et la lèvre s'épaissit mais ne présente plus de manière accentuée la forme « en échine ». Une variation plus nette par rapport aux exemplaires de la première moitié du $\mathrm{VI}^{\mathrm{e}}$ av. J.-C. se manifeste dans la forme plus massive du pied toujours tronconique, mais avec un profil caréné et une base en anneau légèrement épaissi replié vers intérieur ${ }^{\mathbf{4 8 0}}$ (fig. 48 b). La chronologie de cette forme est encadrée par quelques exemplaires de l'épave de la pointe Lequin 1A, datée vers 515 av. J.-C. et par le Stoa Gutter Well de l'Agora d'Athènes, daté entre 520 et 480 av. J.-C. ${ }^{481}$.

\section{Les amphores de type milésien}

Sur la base de la documentation de la Mer Noire, de l'Égée méridionale et de la Grande Grèce, les exemplaires les plus anciens de la production archaïque de Milet sont, eux aussi, traditionnellement datés à partir de la fin du VII ${ }^{\mathrm{e}} \mathrm{s}$. av. J.-C. Toutefois, une étude récente d'A. Naso, fondée sur un contexte clos issu des fouilles récentes de Milet, propose de remonter la chronologie du début de la série à la fin du VIII ${ }^{\mathrm{e}} \mathrm{s}$. av. J.-C. Malheureusement, le mobilier daté du VII ${ }^{\mathrm{e}}$ s. av. J.-C. provenant de ce contexte, est beaucoup trop fragmentaire pour permettre la reconstitution de formes complètes ${ }^{\mathbf{4 8 2}}$.

Les amphores les plus anciennes des ateliers de Milet, dont on connaît la forme complète, sont donc celles qui, jusqu'à présent, sont datées de la fin du VII ${ }^{\mathrm{e}} \mathrm{s}$. av. J.-C. (fig. 47 a). Elles présentent une panse ovoïde (d'où leur nom ovoid belly early type), un pied court creux,

480. Dupont 1998, fig. 23.9, b.

481. Pour l'épave de la Pointe Lequin 1A : Long, Pomey, Sourisseau 2002, p. 50-54 ; pour le contexte de l'Agora d'Athènes: Roberts 1986, n 435 (P24883), 439 (P24901), fig. 44, pl. 19.

482. Concernant la diffusion des exemplaires les plus anciens reconnus comme milésiens, voir : Dupont 1998, p. 174 et n. 196-199, avec références bibliographiques. Sur la proposition de remonter la chronologie du début de la série : Naso 2005, notamment p. 75, auquel on renvoie également pour les observations et la morphologie particulière (des lèvres notamment) de ces toutes premières attestations, hélas fragmentaires. de forme tronconique et des anses coudées de section biconvexe. Leur particularité réside dans le col, plutôt court et légèrement évasé, bien distinct de l'épaule grâce à une cannelure. Il existe également des cannelures soulignant la lèvre amincie et concave à l'intérieur, du type couramment appelé «en amande » ${ }^{483}$.

Entre le deuxième et le troisième quart du $\mathrm{VI}^{\mathrm{e}} \mathrm{s}$. av. J.-C, une nouvelle forme se répand, dite ovoid belly middle type (fig. 47 b), moins élancée que la précédente, à cause du diamètre de la panse qui se dilate ainsi que du col qui s'élargit et, avec un profil légèrement convexe, prend un aspect renflé. Dans cette série également, des cannelures distinguent le col de l'épaule et de la lèvre en amande. Les anses, de section biconvexe, sont coudées. Le pied court est creux et de forme tronconique ${ }^{484}$.

Dans la seconde moitié du VI ${ }^{\mathrm{e}}$ av. J.-C., en dehors de l'évolution du type ovoid belly, d'autres types d'amphores milésiennes sont documentés, dont la morphologie générale plus fuselée que dans les précédentes annonce les formes du $\mathrm{V}^{\mathrm{e}}$ siècle av. J.-C. La datation de ces types dans la seconde moitié du VI ${ }^{\mathrm{e}} \mathrm{s}$. av. J.-C. est suggérée par quelques découvertes effectuées en Mer Noire et en Méditerranée ${ }^{485}$.

Le type appelé ovoid belly later type (fig. 47 e) est l'évolution évidente du type précédent ovoid belly middle type auquel il s'apparente tant par la forme de la panse que par celle du col. Ce dernier, de forme tronconique, se distinguant de l'épaule ainsi que de la lèvre « en amande » par une cannelure, apparaît légèrement renflé en raison de son profil convexe. Le pied court est creux et de forme tronconique ${ }^{486}$.

Toujours au troisième quart du $\mathrm{VI}^{\mathrm{e}} \mathrm{s}$. av. J.-C. peut être attribué le type broad belly later type (fig. 47 c), un conteneur à la panse ovoïde plus haute et plus large que le type ovoid belly et caractérisé par de légères carènes vers le milieu et vers le fond de la panse. Le pied est très bas, plutôt large, creux et de forme tronconique. Le col tronconique est plutôt étroit par rapport à la panse, dont il se distingue par une cannelure. On relève d'autres cannelures au-dessous de la lèvre ${ }^{487}$.

483. Dupont 1998, p. 174, fig. 23.7, a.

484. Dupont 1998, p. 174, fig. 23.7, b.

485. Pour la bibliographie relative à la diffusion de ces types et à leur chronologie, voir : Dupont 1998, p. 174-175.

486. Dupont 1998, p. 174-175, fig. 23.7, e.

487. Dupont 1998, p. 174, fig. 23.7, c. Selon P. Dupont, la fonction des cannelures qui apparaissent, dans la deuxième moitié du $\mathrm{VI}^{\mathrm{e}} \mathrm{s}$. av. J.-C., sur tous les types de la série serait de contenir l'écoulement accidentel de l'huile contenue dans les amphores (Dupont 1998, p. 175 , fig. 23.8 , c-e). 

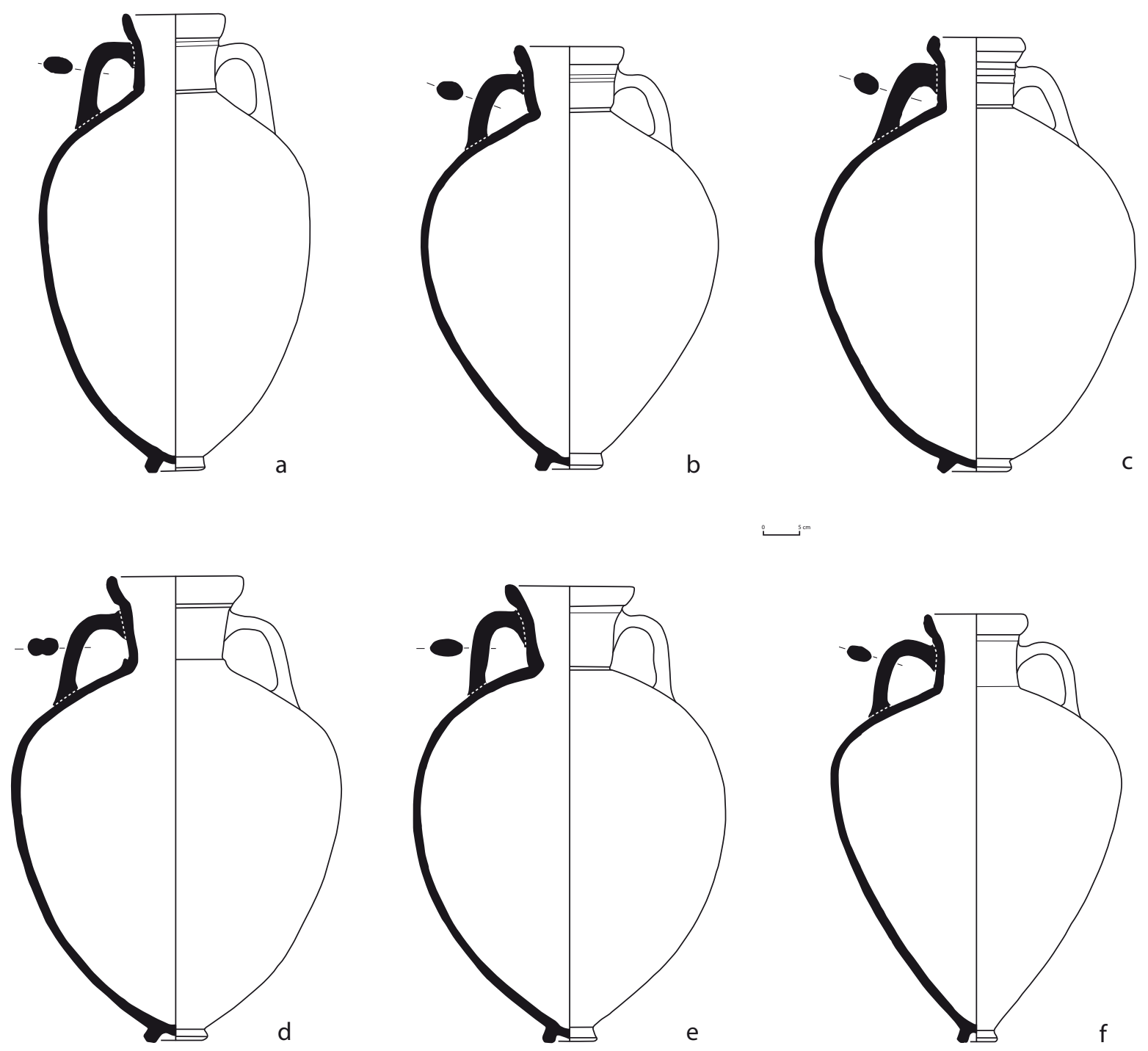

Fig. 47. Amphores de type milésien archaïque (d'après Dupont 1998, fig. 23.7).

Dans la seconde moitié du VI ${ }^{\mathrm{e}}$ s. av. J.-C., on rencontre un autre type encore, appelé ogival belly later type (fig. 47 d) du fait de la forme ovoïde de la panse et de l'épaule aplatie qui rapprochent son profil de celui des amphores du style de Fikellura. Le col est évasé et nettement distinct de l'épaulement grâce à un ressaut situé au niveau de l'épaule. La lèvre « en amande » nettement ouverte se distingue du col par un listel. Le pied est bas, creux et de forme tronconique. Les anses peuvent également être du type bifide ${ }^{488}$.

Le dernier type datable de la seconde moitié du $\mathrm{VI}^{\mathrm{e}} \mathrm{s}$. av. J.-C. est le conical belly later type (fig. $\mathbf{4 7}$ f), un conteneur au corps piriforme et à l'épaule très aplatie.
Son pied creux, de forme tronconique, est bas et de petit diamètre. Le col est bien distinct de l'épaule ainsi que de la lèvre très ouverte « en amande » ${ }^{489}$.

\section{Les amphores du groupe «Samos-Milet »}

À partir de la fin du VIe et pendant tout le $\mathrm{V}^{\mathrm{e}} \mathrm{s}$. av. J.-C., il semble très difficile, en s'appuyant uniquement sur l'aspect morphologique, de distinguer les amphores produites à Samos de celle de celles fabriquées à Milet. En effet, dans le cadre de la koinè sud-ionienne, on produit pendant cette période des conteneurs commerciaux à la morphologie plus fuselée qu'auparavant et dont l'évolution, commune et parallèle, ne présente de différences 
que pour certains détails (fig. 48) ${ }^{490}$. C'est à cette série amphorique des époques tardo-archaïque et classique que renvoie la définition du groupe Samos-Milet.

Les variantes que l'on observe dans les détails morphologiques de cette série permettent de déterminer, pour le $\mathrm{V}^{\mathrm{e}}$ s. av. J.-C., trois types contemporains que M. Lawall a désignés $S / 1, S / 2$ et $S / 3$, et dont il a retracé l'évolution avec rigueur grâce à l'étude comparée des associations des contextes clos de l'Agora d'Athènes ${ }^{491}$.

Le type $\mathrm{S} / 1$, le plus commun des trois, est décrit comme un conteneur à panse arrondie, muni d'une lourde lèvre en bourrelet épaissie à l'extérieur et arrondie. On note également un ressaut à la base du col. À l'exception de ces trois caractéristiques générales, les autres parties de ce conteneur présentent, entre la fin du VI ${ }^{e}$ et le cours $\mathrm{du} \mathrm{V}^{\mathrm{e}} \mathrm{s}$. av. J.-C., une évolution morphologique dont on peut suivre ponctuellement les étapes.

Entre la fin du $\mathrm{VI}^{\mathrm{e}}$ s. et 480 av. J.-C. (fig. 47-50), le ressaut qui se trouve sur le col du type $\mathrm{S} / 1$ est situé au niveau de l'épaulement. L'épaulement est large et la panse se resserrant vers le bas, donnent à l'amphore une morphologie piriforme. Le pied est souvent bas, de petit diamètre, de forme tronconique avec une base annulaire et peut présenter un profil plus ou moins caréné et facetté (fig. 51) ${ }^{492}$. Toutefois, dans les contextes de l'Agora clos en 480 av. J.-C., ont été aussi trouvés, bien que rarement, des pieds de forme à tendance cylindrique, épaissis et légèrement creux en bas (fig. 52), premières manifestations de la morphologie qui caractérise les pieds de la l'évolution du type $\mathrm{S} / 1$. Sa chronologie comprise entre la fin du VI e s. et 480 av. J.-C. est donc fondée par les exemplaires de certains dépôts de l'Agora, datés avant 480 av. J.-C., mais aussi par les découvertes du sanctuaire d'Aphaïa à Égine ${ }^{493}$.

Entre 480 et 450 av. J.-C. (fig. 53), le col du type S/1 s'allonge et le ressaut caractéristique est placé plus haut que pour la forme précédente. L'ensemble de la morphologie subit également un allongement qui confère à ce type un aspect plus élancé dit «en bobine ». Le

490. À ce sujet, voir : Dupont 1998, notamment p. 176-177.

491. À propos de la description des formes du V $\mathrm{V}^{\mathrm{e}}$ s. av. J.-C., voir : Dupont 1998, p. 165, 168-169, fig. 23.9, c, pour la production concernant Samos, et p. 176, fig. 23.9, d-g, pour celle de Milet ; pour les amphores du groupe Samos-Milet de l'Agora d'Athènes : Lawall 1995, p. 177-185.

492. M. Lawall fait précisément observer l'analogie existant entre ce type de pied annelé et celui des amphores contemporaines du Nord de l'Égée (Lawall 1995, p. 179 et n. 14).

493. En ce qui concerne les exemplaires d'Égine, voir: Johnston 1990 , p. 47-49, fig. 7, 9-10 ; pour les contextes de l'Agora : Lawall 1995 , p. 178 et n.12. pied est désormais cylindrique, épaissi, creux à la base, avec un étranglement net au niveau de la jonction panse/ pied (fig. 54, en haut). La chronologie est fondée sur des contextes de l'Agora clos vers le milieu du V ${ }^{\mathrm{e}}$ s. av. J.-C. et confirmée, par exemple, par l'exemplaire de la tombe 73 de Fratte ${ }^{494}$.

Entre 450 et 400 av. J.-C. (fig. 55), la dernière variante du type $\mathrm{S} / 1$ se caractérise par un allongement général de la forme bien qu'elle garde dans le détail les caractéristiques du stade précédent : panse en bobine, col long marqué au milieu par un ressaut qui est l'élément distinctif de ce type (fig. 56) et enfin pied cylindrique épaissi, plus ou moins creux à la base et avec un étranglement supérieur net à la jonction panse/pied (fig. 54, en bas). C'est surtout grâce aux exemplaires de l'Agora d'Athènes, où de nombreux dépôts clos avant 410 av. J.-C. ont livré des exemplaires fragmentaires de cette forme ${ }^{495}$, que l'on a pu établir la chronologie de ce dernier stade évolutif ${ }^{496}$.

Le type $S / 2$, se distingue du précédent par trois détails : la panse moins arrondie et plus anguleuse; la lèvre plus anguleux, bien qu'encore épaisse ; l'absence du ressaut sur le col. Malgré ces différences avec le type $\mathrm{S} / 1$, on perçoit, en analysant l'évolution du type $\mathrm{S} / 2$ au cours de la deuxième moitié du $\mathrm{V}^{\mathrm{e}} \mathrm{s}$. av. J.-C., une tendance à l'uniformisation des deux types $\mathrm{S} / 1$ et $\mathrm{S} / 2$, qui en rend la distinction très difficile.

Entre la fin du VI ${ }^{\mathrm{e}}$ siècle et 480 av. J.-C. (fig. 57), outre les détails mentionnés ci-dessus, le type $\mathrm{S} / 2$ se caractérise par un profil à l'épaule large et aplatie qui se rétrécit dans la partie inférieure de la panse, comme le montrent un exemplaire complet du Céramique d'Athènes, un col milésien et d'autres fragments provenant des contextes H 12:15 e D 15:1 de l'Agora, tous clos en 480 av. J.-C. ${ }^{497}$.

Entre 480 et 450 av. J.-C. (fig. 58), étant donné la rareté et l'état fragmentaire des exemplaires retrouvés, l'évolution morphologique du type $\mathrm{S} / 2$ est plus

494. Concernant l'amphore de la tombe 73 de Fratte qui était associée à des objets importés du milieu du $\mathrm{V}^{\mathrm{e}} \mathrm{s}$. av. J.-C., voir Di Sandro 1981b, fig. 3 ; Sur les dépôts de l'Agora: Lawall 1995, p. 180 et n.20.

495 Pour ces contextes d'Athènes, voir : Lawall 1995, p. 181 et n.26.

496. A titre documentaire, on peut signaler que c'est à partir de ces exemplaires de l'Agora, notamment les fragments P27530 et P27531 du dépôt $\mathrm{S}$ 16:1, que V. Grace avait établi le type de la fin du $\mathrm{V}^{\mathrm{e}} \mathrm{s}$. av. J.-C. qu'elle attribuait à Samos : Grace 1971, fig. 3.3.

497. Pour l'amphore du Céramique : Knigge 1976, p. 101 et n. 50 , pl. 49.3 ; pour Milet : Voigtländer 1983, p. 70, fig. 28.172 , et peutêtre aussi fig. 27.176 ; pour l'Agora : Lawall 1995, p. 182 et n. 30. 

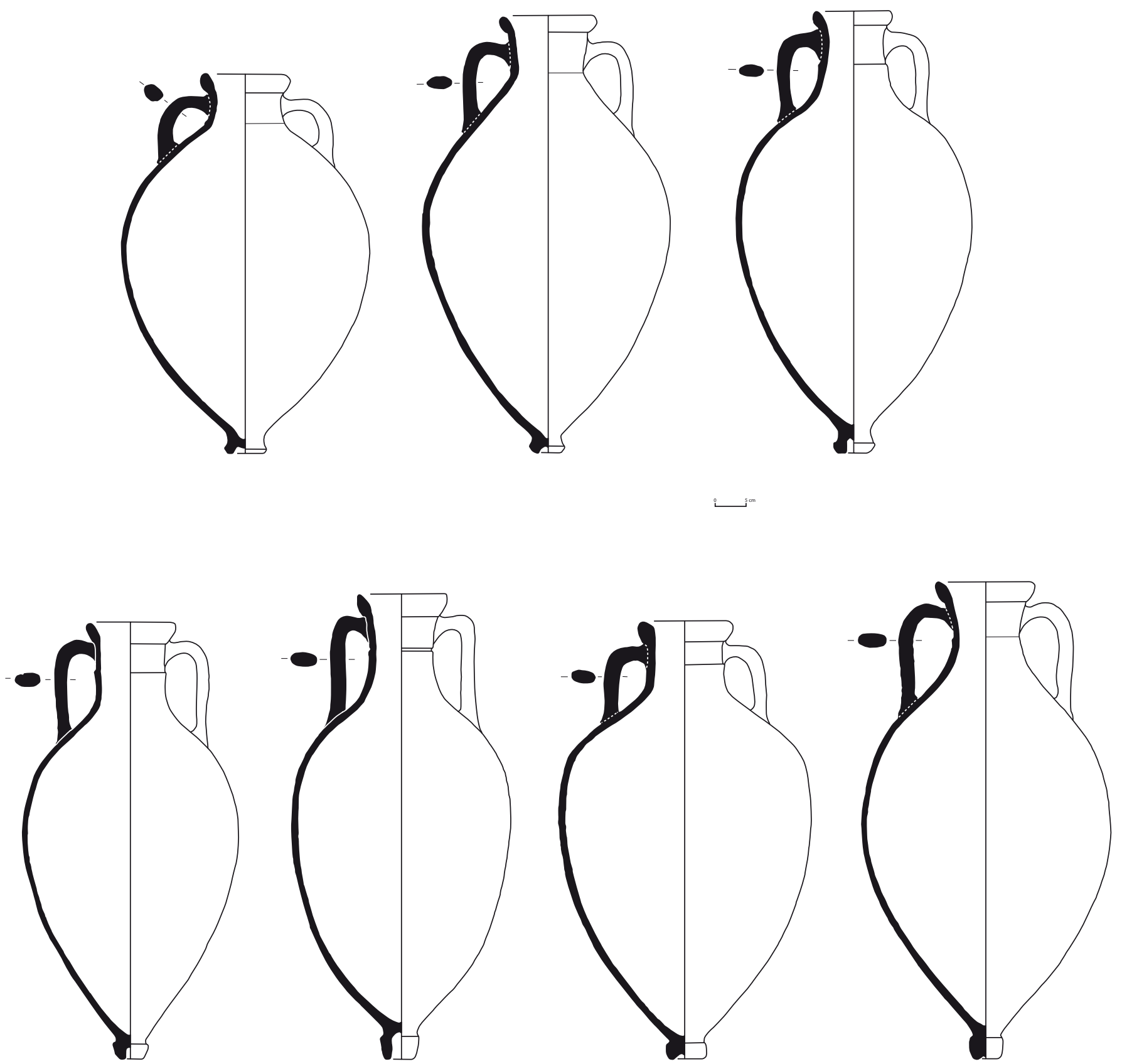

Fig. 48. Amphores du groupe « Samos-Milet » (d'après Dupont 1998, fig. 23.9).

malaisée à déterminer. Toutefois, un exemplaire restitué par V. Grace à partir de l'amphore fragmentaire P21986, du contexte N 7:3 de l'Agora, clos en 440 av. J.-C., permet d'illustrer le type. Il s'agit d'une amphore munie d'un col cylindrique sans ressaut, avec une lèvre épaissie vers l'extérieur et anguleux. La restitution proposée évoque une profil muni d'un épaulement large et plat, mais avec une panse plus renflée dans sa partie inférieure par rapport aux exemplaires du stade précédent et par un pied bas, cylindrique, trapu, se rétrécissant à la jonction avec le fond de la vasque et présentant une profonde cavité à la base ${ }^{498}$.

Dans la deuxième moitié du $\mathrm{V}^{\mathrm{e}} \mathrm{s}$. av. J.-C. (fig. 59), l'évolution du type $\mathrm{S} / 2$ est plus facile à caractériser, et, comme nous l'avons déjà indiqué, elle s'aligne sur celle du type $\mathrm{S} / 1$. La forme de la panse est en effet plus

498. En ce qui concerne cette proposition, voir Grace 1953,p. 109 et n. 167, pl. 40, fig. 5, et Ead. 1971, p. 76, 109, fig. 3.1. 


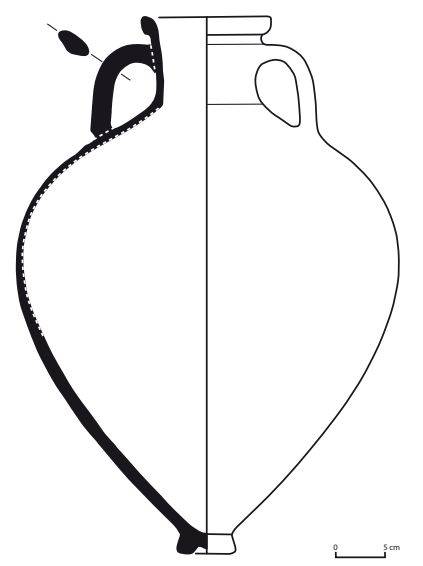

Fig. 49. Amphore du groupe "Samos-Milet», Type S/1 de M. Lawall. Athènes, Agora, inv. P24870 (d'après Roberts 1986, fig. 41.413).

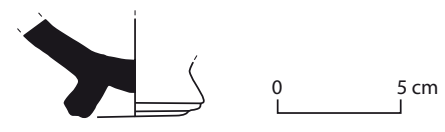

Fig. 51. Fond d'amphore du groupe «Samos-Milet », Type S/1 de M. Lawall. Athènes, Agora, R 12:1 (d'après Lawall 1995, fig. 78).

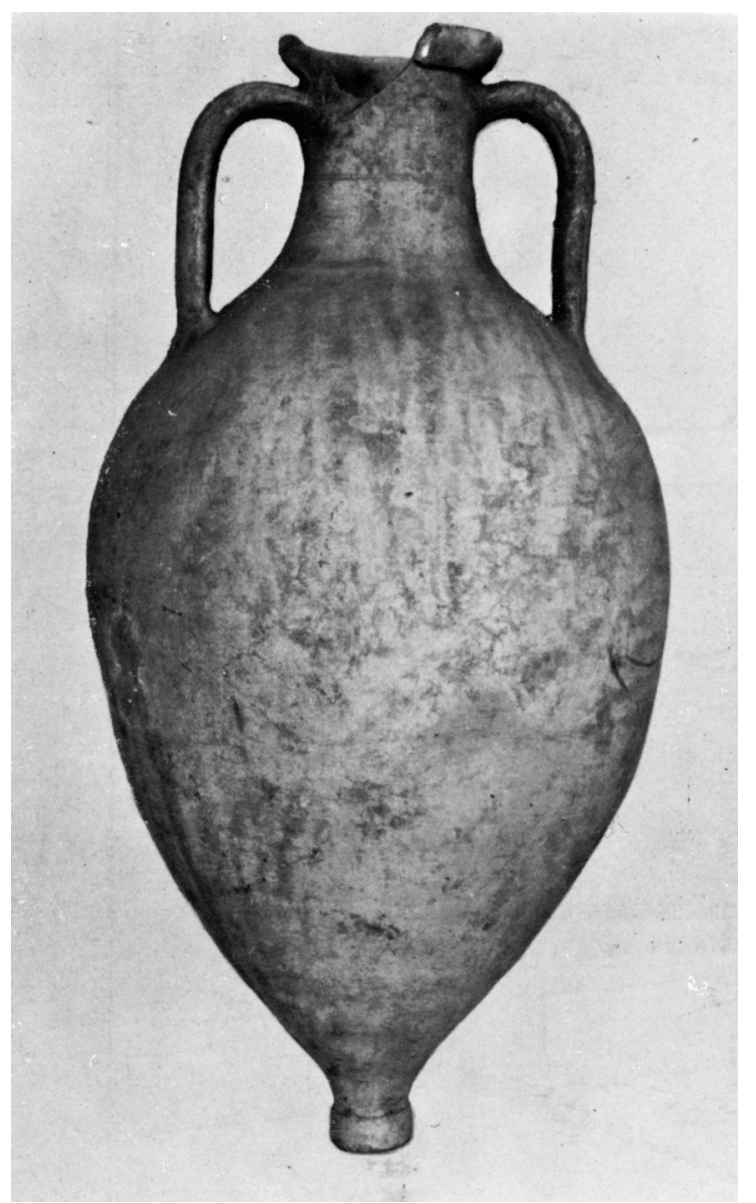

Fig. 53. Amphore du groupe « Samos-Milet », Type S/1 de M. Lawall. Fratte, Tomba 73 (d'après Di Sandro 1981b, fig. 3.3).

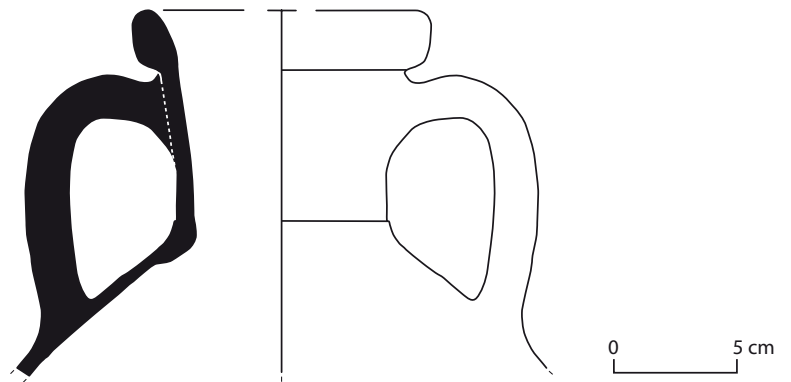

Fig. 50. Col d'amphore du groupe « Samos-Milet », Type S/1 de M. Lawall. Athènes, Agora, E 14:5 (d'après Lawall 1995, fig. 77, en haut).
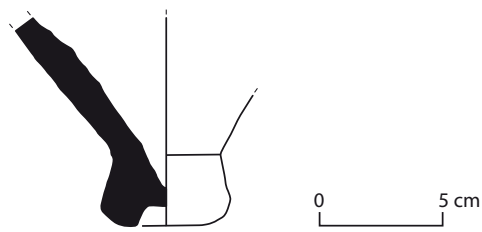

Fig. 52. Fond d'amphore du groupe « Samos-Milet », Type S/1 de M. Lawall. Athènes, Agora, E 14:5 (d'après Lawall 1995, fig. 77, en bas).

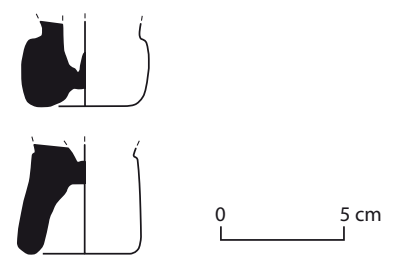

Fig. 54. Fonds d'amphores du groupe "Samos-Milet », Type S/1 de M. Lawall. Athènes, Agora, F 19:4 (en haut) et B 15:1 (en bas) (d'après Lawall 1995, fig. 75).

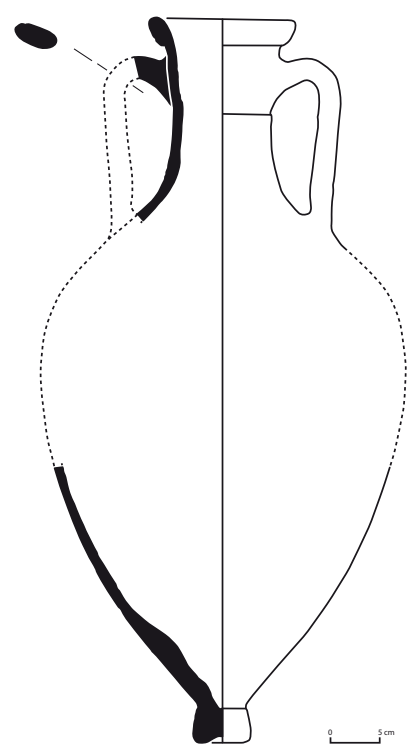

Fig. 55. Amphore du groupe "Samos-Milet », Type S/1 de M. Lawall. Athènes, Agora, S 16:1, inv. P27530/P27531 (d'après Grace 1971, fig. 3.3). 


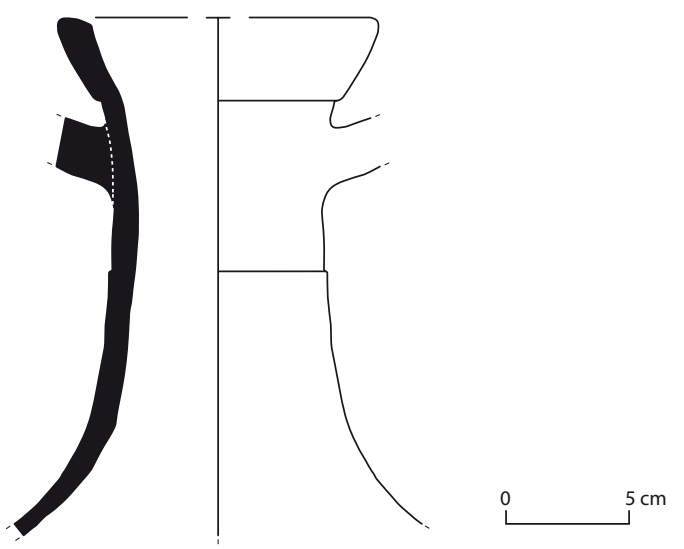

Fig. 56. Col d'amphore du groupe « Samos-Milet », Type S/1 de M. Lawall. Athènes, Agora, C 19:9 (d'après Lawall 1995, fig. 74).

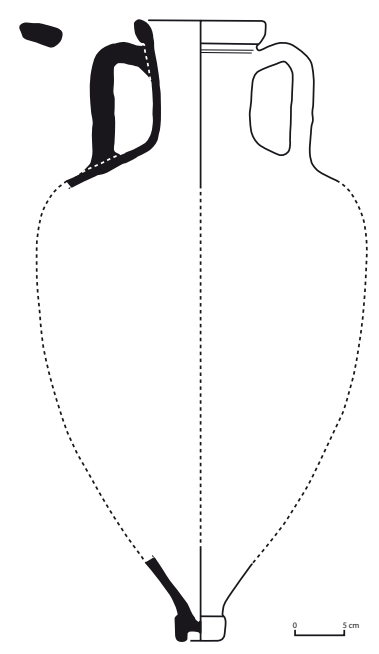

Fig. 58. Amphore du groupe « Samos-Milet », Type S/2 de M. Lawall. Athènes, Agora, N 7:3. inv. P21984a (col) et P21984b (fond) (d'après Grace 1971, fig. 3.1).

arrondie du fait d'un épaulement plus fuyant. Le col, toujours sans ressaut, s'allonge et est surmonté d'une lèvre plus basse. Enfin, le pied est encore de type cylindrique, étranglé dans sa partie supérieure et très creux dans sa partie inférieure. La chronologie est établie avec certitude grâce à de nombreux exemplaires retrouvés dans des contextes de l'Agora d'Athènes clos entre 425 et 410 av. J.-C., parmi lesquels l'amphore complète P18988, provenant du contexte C 19:9 clos en 410 av. J.-C. ${ }^{499}$

En revanche, en raison de la forme plus ventrue de sa panse, du ressaut sur le col et de la morphologie de son pied en anneau au profil émoussé, le type $S / 3$ (fig. 60-61) présente beaucoup plus d'analogies avec

499. Au sujet de ce dépôt de l'Agora et sur les autres ayant restitué des exemplaires du type $\mathrm{S} / 2$ de la seconde moitié du $\mathrm{V}^{\mathrm{e}}$ s. av. J.-C. : Lawall 1995, p. 183.

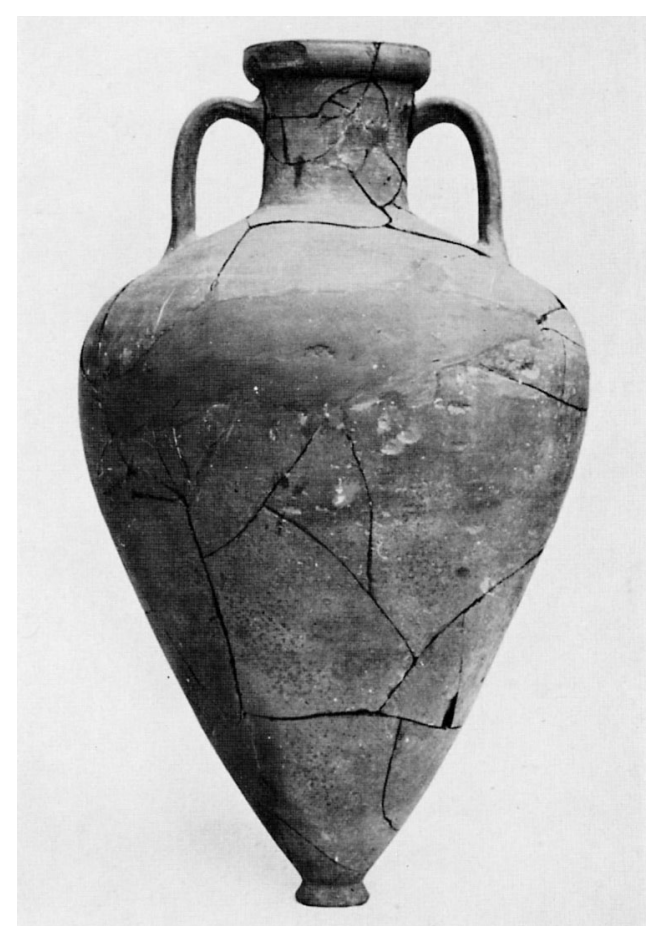

Fig. 57. Amphore du groupe "Samos-Milet », Type S/2 de M. Lawall. Athènes, Céramique (d'après Knigge 1976, pl. 49.3).

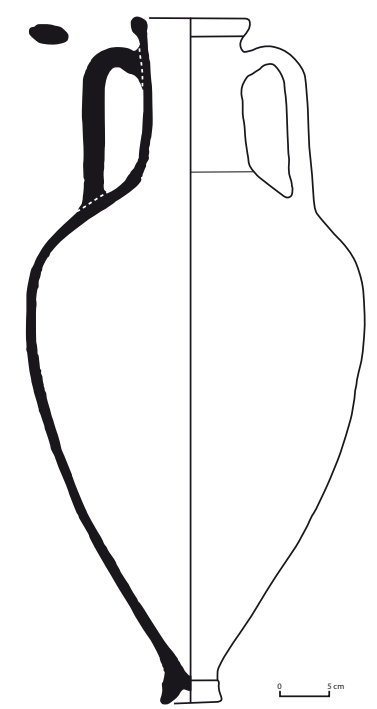

Fig. 59. Amphore du groupe "Samos-Milet », Type S/2 de M. Lawall. Athènes, Agora, C 19:9, inv. P18988 (d'après Grace 1971, fig. 3.2).

le type $S / 1$. Il s'en distingue néanmoins par sa lèvre en quart de cercle, plus haute, plus fine et plus ouverte. Le type $S / 3$ est documenté aussi bien dans une version à anses en ruban épaissi, que dans celle à anses bifides, ou encore dans une version hybride caractérisée par une anse bifide et l'autre en ruban épaissi. Si les deux autres types sont parfaitement contemporains, la synchronie du troisième n'est attestée que pendant la brève période 


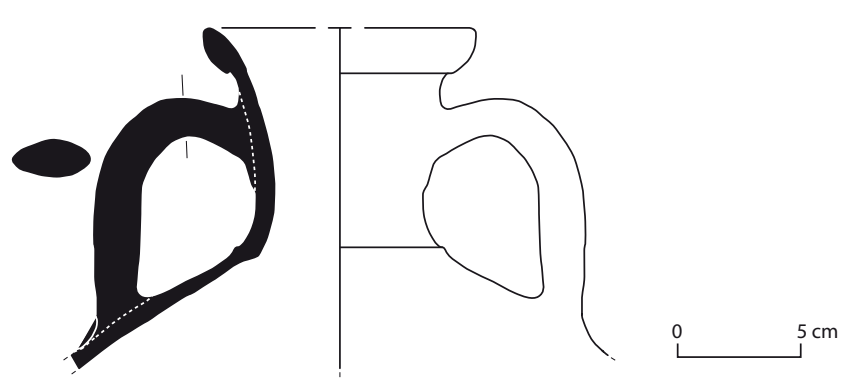

Fig. 60. Col d'amphore du groupe «Samos-Milet », Type S/3 de M. Lawall. Athènes, Agora, E 14:5 (d'après Lawall 1995, fig. 76).

comprise entre la fin du $\mathrm{VI}^{\mathrm{e}}$ et le premier quart du $\mathrm{V}^{\mathrm{e}} \mathrm{s}$. av. J.-C. ${ }^{500}$. La plupart des exemplaires répertoriées de ce type s'inscrivent dans des contextes dont la datation ne dépasse pas la fin du VI ${ }^{\mathrm{e}}$ s. av. J.-C. ${ }^{501}$ Cependant, des exemplaires documentés par des contextes clos en 480 av. J.-C. de Milet, du sanctuaire d'Aphaïa à Égine et de l'Agora d'Athènes, témoignent de sa persistance jusqu'au début du $\mathrm{V}^{\mathrm{e}} \mathrm{s}$. av. J.-C. ${ }^{502}$ En revanche, sa présence est résiduelle dans les dépôts de l'Agora clos après 480 av. J.-C. ${ }^{503}$

La continuité de la production du groupe SamosMilet au cours du IV ${ }^{\mathrm{e}} \mathrm{s}$. av. J.-C. a depuis longtemps été mise en relation avec la série controversée des amphores dites à mushroom rim du $\mathrm{IV}^{\mathrm{e}} \mathrm{s}$. av. J.-C., elles-mêmes associées aux amphores de la seconde moitié du $\mathrm{V}^{\mathrm{e}} \mathrm{s}$. av. J.-C. à lèvre de section triangulaire, dites « attiques » par V. Grace. Ces amphores, dont un débat scientifique récent a confirmé la relation partielle avec Samos, tout en excluant une production attique, sont aujourd'hui conventionnellement désignées par les spécialistes sous l'appellation de groupe « Solokha I » (cf. infra I, 5 § 4). Comme l'attestent les caractéristiques de certaines pâtes et surtout la présence de timbres identifiés par V. Grace comme samiens sur des exemplaires de ce groupe hétérogène, la production de cette forme devait aussi compter, parmi ses nombreux ateliers, une fabrique samienne.

500. Lawall 1995, p. 178, 184-185, fig. 76, 79.

501. Ruban 1991 ; Voigtländer 1986, $\mathrm{n}^{\circ}$ 99, 659-660, fig. 30.

502. Pour Milet : Voigtländer $1983, \mathrm{n}^{\circ} 182$; pour Égine : Johnston 1990, n¹07-109. Pour les sites de l'Agora - Stoa Gutter Well: Roberts 1986, p. 70-72, n438, 443, fig. 44, pl. 19 ; dépôts H 12:15 ; E 14:5 ; R 12:1 (tous clos en 480 av. J.-C.) : Lawall 1995, p. 184-185 et n. 42 .

503. À ce sujet, voir le raisonnement de M. Lawall (1995, p. 185, et n. 43).

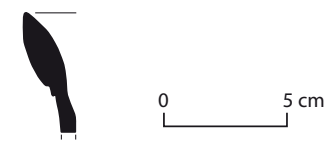

Fig. 61. Lèvre d'amphore du groupe "Samos-Milet », Type S/3 de M. Lawall. Athènes, Agora, Rectangular Rock Cut Shaft (d'après Lawall 1995, fig. 79).

\section{Érythrées}

L'une des 195 amphores constituant la cargaison de l'épave de Tektaş Burnu, dont la forme a d'abord été définie Zeest's Samian ou pseudo-Samian ${ }^{504}$, porte un timbre avec le monogramme $E P Y$ imprimé sur le col. Ce timbre, comparé aux émissions monétaires, laisse présumer que la cité nord-ionienne d'Érythrées a été le centre producteur d'une forme proche de la série en question, attribuée habituellement avec prudence à des ateliers grecs orientaux ou nord égéens (cf. supra I, 4 § 3). Cette découverte pourrait corroborer les résultats des analyses archéométriques effectuées sur des exemplaires de la série Zeest's Samian trouvés en Mer Noire et qui ont mis en évidence l'existence de quatre groupes chimiques que P. Dupont attribue respectivement à la Thrace, à Milet, à Samos et à Chios. Comme l'a suggéré D. Carlson, étant donné la proximité géologique de Chios et d'Érythrées, déjà soulignée par P. Dupont, il est possible que le groupe identifié comme chiote par P. Dupont soit en réalité attribuable à la cité de la péninsule de Çeşme, située sur la côte d'en face ${ }^{\mathbf{5 0 5}}$.

La forme des amphores de l'épave de Tektaş Burnu (fig. 62), dont la cargaison est datée du troisième quart du $\mathrm{V}^{\mathrm{e}} \mathrm{s}$. av. J.-C., se caractérise par une grosse lèvre en quart de cercle, un col court cylindrique et une panse ovoïde. Le pied, de type cylindrique épaissi et creux à l'intérieur, présente une certaine variabilité dans l'articulation de son profil ${ }^{506}$. Cette morphologie générale, définie « archaïsante » par rapport à la date qu'on peut en donner sur une base archéologique, rapproche cette

504. Carlson 2003, p. 583-585.

505. Sur l'épave de Tektaş Burnu: Carlson 2003, notamment p. 583-587, fig. 5 et 8, pour l'amphore pseudo-Samian ou Zeest's Samian avec timbre EPY. À propos des analyses de P. Dupont sur les amphores Zeest's Samian: Ibid. n. 29. Sur la proximité géologique de Chios et du territoire d'Érythrées situé en face : Dupont 1998, p. 151. Sur la production amphorique d'Érythrées, voir en dernier lieu : Carlson, Lawall 2007. Malgré les découvertes de Tektaş Burnu, P. Dupont a réaffirmé sa conviction qu'un des ateliers producteurs de cette forme doit être cherché à Chios ou à Téos plutôt qu'à Érythrées (Dupont 2010, p. 40, 43).

506. À ce propos: Carlson 2003, p. 583-584 et Carlson, Lawall 2007, p. 34. 


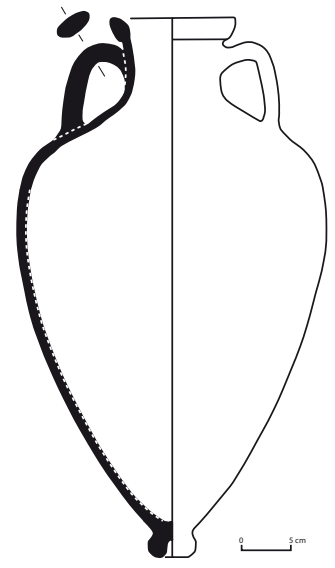

Fig. 62. Amphore du groupe "Samos-Milet », attribuée à Érythrées. Épave de Tektaş Burnu (d'après Carlson, Lawall 2007, fig. 2).

production nord-ionienne d'époque classique de la tradition sud-ionienne d'époque archaïque ${ }^{507}$.

En effet, même si dans sa description des amphores du groupe Samos-Milet provenant de l'Agora d'Athènes, M. Lawall ne fait pas spécifiquement allusion aux formes problématiques du Zeest's Samian type, les caractéristiques du pied en anneau, caréné et émoussé qu'il décrit dans certains cas pour le premier stade d'évolution de son type $\mathrm{S} / 1$ (entre la fin du $\mathrm{VI}^{\mathrm{e}}$ siècle et 480 av. J.-C.), ne sont pas très éloignées de celles que l'on observe dans ces formes. De la même manière, je crois que l'on peut associer à ces mêmes formes aussi bien la lèvre du type $\mathrm{S} / 1$ que celle du type $\mathrm{S} / 2$ (variantes archaïques), l'une et l'autre à section quadrangulaire.

En traçant les grandes lignes d'une première classification typologique de la production amphorique d'Érythrées pour la période allant du milieu du $\mathrm{V}^{\mathrm{e}}$ au $\mathrm{II}^{\mathrm{e}}$ s. av. J.-C., D. Carlson et M. Lawall ont proposé d'attribuer à cette ville la fabrication d'une forme très proche du type Lawall $\mathrm{S} / 1$, de la seconde moitié du $\mathrm{V}^{\mathrm{e}}$ s. av. J.-C. et dont elle est contemporaine. Une des amphores de l'épave de Tektaş Burnu et au moins trois autres exemplaires de l'Agora d'Athènes, de Makri et de Gordion pourraient être associés à cette « minor branch of Eritrean production », ce qui montrerait que la cité d'Érythrées a produit différentes formes contemporaines et ayant toutes un lien avec la tradition sud-ionienne ${ }^{\mathbf{5 0 8}}$. Ce lien pourrait trouver confirmation dans le développement ultérieur de la série. En effet, au $\mathrm{IV}^{\mathrm{e}} \mathrm{s}$. av. J.-C., différents timbres certifient l'attribution à Érythrées d'amphores du type mushroom rim $^{509}$.

507. Carlson, Lawall 2007, p. 33-34, fig. 2.

508. Carlson, Lawall 2007, p. 34, n. 19-20, fig. 3.

509. Carlson, Lawall 2007, p. 34-35, fig. 4-5.
Tous ces indices s'associent donc pour suggérer qu'il existe une koinè amphorique $\mathrm{du} \mathrm{V}^{\mathrm{e}} \mathrm{s}$. av. J.-C. à laquelle participe probablement l'Ionie dans son ensemble et dont les nombreuses variantes morphologiques ainsi que les ateliers de production ne sont pas encore très bien définis.

\subsection{Centres de production}

À l'analyse visuelle, la pâte des amphores de Samos se caractérise par une matrice argileuse souvent de couleur rouge ou rougeâtre intense, parfois chamois rosé ou encore gris orangé et, dans certains cas, sa structure interne est d'un ton brun. La pâte, fine et de consistance compacte, contient de menues écailles de mica argenté visibles surtout en surface et de très rares petites inclusions composées de fragments de roche brun foncé et de calcaire jaunâtre.

Toujours selon une analyse exclusivement autoptique, les amphores de Milet se caractérisent par des pâtes plutôt fines mais plus granuleuses et surtout plus micacées que celles des productions samiennes, et par des matrices argileuses de couleur orange ou orange rosé ${ }^{510}$. Certains considèrent que la concentration de mica doré, visible non seulement en surface mais également en section, est l'élément qui distingue les amphores produites à Milet de celles produites à Samos. Cependant, P. Dupont signale l'existence d'exemplaires attribuables à la fabrique milésienne qui ne contiennent aucune trace de mica ${ }^{511}$.

Malgré les critères distinctifs (tant dans la forme que dans la pâte) traditionnellement rappelés pour les deux séries d'époque archaïque de Samos et de Milet, leur distinction ainsi que leur attribution aux deux ateliers, sont en fait beaucoup plus complexes que ce qu'on pouvait imaginer il y a encore quelques années. De récentes analyses archéométriques (NAA) effectuées sur des amphores de «type milésien » d'époque archaïque ont révélé l'existence d'au moins trois groupes dont un seulement ( Herkunftsgruppe A») peut être attribué avec certitude à Milet ${ }^{512}$. On ne peut donc plus exclure que pour ces productions d'époque archaïque, il faille envisager une participation régionale à la production de la même forme de la part de différents ateliers. Dans le même sens semblent aussi aller les observations faites à

510. À propos des observations relatives aux pâtes des amphores de Milet : Dupont 1982, p. 205, n. 77 ; Slaska 1986, p. 60 ; de Marinis 1988, p. 217 ; Id. 1996, p. 335.

511. Dupont 1998, p. 174 ; Id. 2000, p. 58.

512. Kerschner, Mommsen 2005 (analyses exécutées sur du matériel provenant des fouilles d'Éphèse). 
l'occasion de la récente publication de l'épave de Pabuç Burnu. Ainsi, de sa cargaison, datée du second quart du $\mathrm{VI}^{\mathrm{e}}$ s. av. J.-C., on trouve des amphores dont la forme est semblable à celles traditionnellement attribuées à Samos ou a Milet. Cependant, certaines d'entre celles-ci présentent une pâte qui, à l'analyse visuelle, semblent devoir être attribuées à Halicarnasse. ${ }^{513}$

Les pâtes des amphores de la série Samos-Milet ont fait l'objet de nombreuses analyses. Rappelons, tout d'abord, les premières recherches de p. Dupont qui ont constitué le fondement même de sa proposition de distinction des deux ateliers, grâce à l'identification chimique d'un groupe pertinent à Milet. Nous disposons aujourd'hui d'une contribution plus récente: les analyses pétrographiques d'I. Whitbread sur la production de Samos. Ces dernières ne se sont pas révélées concluantes car, selon lui, il n'y a pas lieu de distinguer plusieurs groupes à partir des échantillons qu'il a analysés. Ceci pourrait être dû soit à une production issue du même centre, soit à une ressemblance géologique entre l'île Samos et le continent (Milet), raison qui pourrait ne pas permettre de différencier avec précision les deux fabriques ${ }^{514}$.

Bien que ces résultats ne soient pas vraiment satisfaisants et qu'il soit souhaitable d'approfondir les recherches, M. Lawall a récemment proposé quelques observations intéressantes au sujet des centres de production du groupe Samos-Milet et de la possibilité concrète d'attribuer les différents types à des ateliers bien distincts. En ce qui concerne les formes du $\mathrm{V}^{\mathrm{e}} \mathrm{s}$. av. J.-C., M. Lawall observe qu'en croisant certains résultats obtenus par p. Dupont et I. Whitbread, on peut émettre l'hypothèse d'une attribution samienne des types $S / 1$ et $S / 2$. Ceux-ci sont similaires, du point de vue de la morphologie, entre eux et avec la forme archaïque qu'I. Whitbread a analysée et qu'il considère, ainsi que le type $S / 2$, compatible avec la géologie de Samos. En revanche, il attribuerait à Milet le type $\mathrm{S} / 3$, que P. Dupont a inclus dans le groupe chimique milésien et qui est nettement distinct, chimiquement parlant, du type $S / 1^{515}$. Cette probable relation entre les trois types du $V^{\mathrm{e}}$ siècle et les deux centres de Samos et de Milet est d'autant plus fondée si l'on prend en considération certains éléments de nature archéologique et historique. Les deux types, $\mathrm{S} / 1$ et $\mathrm{S} / 2$, hypothétiquement attribués à Samos, présentent non seulement une évolution commune et synchronique, que l'on peut suivre

513. Greene, Lawall, Polzer 2008, notamment p. 688, n. 20.

514. En ce qui concerne les analyses chimiques, voir notamment : Dupont 1982, p. 204, 206-208 ; Id. 1983, p. 27 ; Id. 1986, p. 60-62, 68. Sur les analyses pétrographiques: Whitbread 1995, p. 124-127. 515. Lawall 1995, p. 187-188. grâce aux exemplaires trouvés dans des contextes dont la datation est comprise entre la fin du $\mathrm{VI}^{\mathrm{e}}$ et la fin $\mathrm{du} \mathrm{V}^{\mathrm{e}}$ s. av. J.-C., mais aussi une tendance à l'uniformisation morphologique dans la seconde moitié du $\mathrm{V}^{\mathrm{e}} \mathrm{s}$. av. J.-C. Les attestations du type $\mathrm{S} / 3$, en revanche, se limitent à la charnière des $\mathrm{VI}^{\mathrm{e}}-\mathrm{V}^{\mathrm{e}} \mathrm{s}$. av. J.-C., sans que l'on puisse discerner un développement postérieur au cours du $\mathrm{V}^{\mathrm{e}}$ siècle. Enfin, à l'aspect archéologique s'ajoutent les éléments historiques concernant ces deux centres : on ne peut pas ne pas faire le lien entre la disparition du type $S / 3$ dans le deuxième quart du $\mathrm{V}^{\mathrm{e}} \mathrm{s}$. av. J.-C. et la destruction de Milet par les Perses qu'Hérodote (VI, 21-22) situe en 494 av. J.-C. Que ce soient les sources historiques, qui rappellent la présence des troupes milésiennes à Mycale en 479 ou la documentation archéologique de Milet relative aux niveaux postérieurs au sac des Perses ${ }^{516}$, elles révèlent comment, après un laps de temps assez court, compris entre 494 et 479 av. J.-C., la cité a dû réactiver ses relations politico-économiques avec les zones limitrophes et avec l'Attique. Selon M. Lawall, la reprise de l'activité économique a signifié une relance des ateliers amphoriques qui ont dû s'adapter aux modèles samiens à bord épaissi, après l'interruption de la fabrication du type $S / 3$ à lèvre amincie. Cette hypothèse pourrait expliquer le nombre des fabriques qui ont produit le type $\mathrm{S} / 1$ à lèvre épaissie, dans la deuxième moitié du $\mathrm{V}^{\mathrm{e}} \mathrm{s}$. av. J.-C. En tout état de cause, au-delà de la distinction entre la production de Samos et celle de Milet, la grande diversité des pâtes pour la production de la même forme, au cours du $V^{e}$ s. av. J.-C., fait penser à l'existence de plusieurs ateliers que l'on doit rechercher non seulement à Samos et à Milet, mais aussi, comme nous l'avions évoqué précédemment, dans toute la Carie. Toute la zone côtière de la péninsule anatolienne située en face de Samos a dû en effet produire des amphores de type régional dont les différences morphologiques locales, bien identifiables entre la fin du VII et la fin du VI ${ }^{\mathrm{e}} \mathrm{s}$. av. J.-C., se sont progressivement atténuées tout au long du siècle suivant pour confluer dans une koinè amphorique sud-ionienne pour laquelle il est difficile de préciser la localisation des ateliers.

La production d'Érythrées serait caractérisée par une pâte « reddish yellow (5 YR 6/6) to pale brown (7.5 YR 6/6) over a bluish-gray or dark red core, with a moderate scatter of silt-sized black and more numerous white inclusions, and little to no mica ${ }^{517}$.

516. Voigtländer 1983, p. 85-101, avec référence aux importations attiques.

517. Carlson, Lawall 2007, p. 33. 


\subsection{Contenu}

\section{Les amphores de type samien}

Dès les premières recherches, on a considéré que la production amphorique de Samos était dévolue au stockage et au transport de l'huile d'olive dont la réputation est connue dans l'Antiquité par différentes sources littéraires. Signalons notamment le papyrus $n^{\circ} 59015$ de Zénon, daté de 259-258 av. J.-C., qui mentionne des conteneurs samiens et milésiens pour l'huile. Dans le même ordre d'idée, V. Grace a mis l'accent sur le lien existant entre l'amphore (du groupe dit aujourd'hui Samos-Milet) et le rameau d'olivier relevé sur certaines monnaies de l'île ${ }^{518}$.

Cependant, les sources littéraires antiques mentionnent également une production vinicole samienne, fort peu prisée d'ailleurs, du moins à l'époque romaine. C'est l'argument mis en avant par P. Dupont qui insiste sur le binôme vin samien/coupes ioniennes également samiennes ${ }^{519}$. La découverte de résidus de nature résineuse dans deux amphores attribuées à Samos a rouvert le débat en ce sens, et ouvre la possibilité effective que ces amphores ont pu être utilisées peut-être avant tout pour le commerce de l'huile, mais également pour celui du vin ${ }^{520}$.

\section{Les amphores de type milésien}

Il en va de même pour les amphores de Milet qui sont considérées comme principalement liée au transport de l'huile issue d'une production oléicole dont l'existence semble bien documentée dans toute la Carie. Les sources littéraires (Aristote, Politique, I, 4, 5) et papyrologiques (papyrus de Zénon $\mathrm{n}^{\circ}$ 59015) confirmeraient cette destination. Ajoutons que l'interprétation fonctionnelle que propose $\mathrm{p}$. Dupont du ressaut en collerette placé sur le col de certaines variantes d'amphores de production milésienne - éviter l'épandage de l'huile - pourrait aller dans ce sens ${ }^{521}$. Néanmoins, étant donné la localisation d'une production de coupes ioniennes à Milet, le même chercheur n'exclut pas qu'une partie des amphores milésiennes aient été destinées au transport du vin.

518. Au sujet des sources littéraires, papyrologiques et numismatiques qui soutiennent la thèse que les amphores de Samos étaient destinées au transport de l'huile : Grace 1971, p. 79-82.

519. Sur la possibilité que du vin ait également été transporté dans ces amphores, voir : Dupont 1982, p. 206, et n. 86-87; Shipley 1987, p. 16-17, 61 et Dupont 2002.

520. Roberts 1986, p. 46, 65 n412-413.

521. Sur l'hypothèse que les amphores milésiennes étaient des conteneurs à huile, voir: Grace 1971, p. 82 ; Dupont 1982, p. 206, n. 83 ; Johnston 1990, p. 61 ; Whitbread 1995, p. 122 ; Albanese Procelli 1997b, p. 105 ; Dupont 1998, p. 175 et n. 208-209, avec bibliographie et références aux sources.
Récemment, A. Naso a proposé qu'elles aient pu également servir pour le commerce des olives, des herbes médicinales comme l'hellébore, ainsi que des produits liés à l'industrie de la laine, ressources très réputées de l'économie milésienne ${ }^{522}$.

\section{Les amphores du groupe 'Samos-Milet'}

Compte tenu des difficultés que pose la distinction entre les ateliers de production des amphores du groupe Samos-Milet, le problème des marchandises que ces dernières contenaient s'en trouve encore évidemment compliqué. Néanmoins, la localisation générale de ces ateliers dans l'Ionie méridionale suggère que la problématique touchant les contenus des productions plus archaïques de Samos et Milet est la équivalente à celle concernant ces amphores. L'effigie d'une amphore de ce groupe associée à un rameau d'olivier sur des émissions monétaires samiennes tendrait à confirmer que, en partie du moins, ces amphores contenaient de l'huile.

La multiplicité des lieux de productions laisse toutefois ouverte la question d'une pluralité des contenus différents.

\section{Érythrées}

Au moins un des exemplaires découverts dans l'épave de Tektaş Burnu et attribué à la production d'Érythrées, a été employé pour le stockage de viande bovine ${ }^{523}$. Nous ne disposons pas d'éléments suffisants pour établir s'il s'agissait d'un usage primaire ou secondaire du conteneur.

\subsection{Diffusion dans la Méditerranée occidentale}

Les formes les plus anciennes des séries amphoriques de Samos et de Milet sont assez répandues en Italie où nous connaissons de nombreux exemplaires en Étrurie, notamment à Caeré, Tarquinia et Vulci, dans les habitats du Petriolo et de Murlo, dans l'épave du Giglio-Campese et dans la seconde épave du Giglio ${ }^{524}$. Elles sont aussi diffusées en Sicile, en particulier dans la nécropole de Camarine qui en a restitué de nom-

522. En ce qui concerne le vin, voir: Dupont 1982, p. 206 ; pour les autres produits, voir: Naso 2005, p.78-79, avec références bibliographiques.

523. Carlson 2003, p. 589-590

524. Pour les nécropoles de l'Étrurie méridionale: Boitani 1985, p. 25. Pour le Petriolo: Savelli sous presse. Pour Murlo: Nielsen, Phillips 1997, p. 93, fig. 21-23. Pour l'épave archaïque du Giglio : Bound, Vallintine 1983; Bound 1985 ; Abbate Edlmann, Giachi 1989 ; Rendini 1988 ; Parker 1992, n 451,p. 192, map 7 ; Cristofani 1995. Pour la « seconde épave du Giglio » : Rendini 1988, p. 193, fig. 2. 
breux exemplaires, mais aussi de Butera, Géla, Himère, Monte San Mauro, Naxos, Mégara Hyblaea et Mylae ${ }^{525}$. Des exemplaires qui pourraient être samiens, ou plus génériquement grecs orientaux, datés entre le $\mathrm{VII}^{\mathrm{e}}$ et le milieu du VI ${ }^{\text {e }}$ s. av. J.-C., sont signalés également de l'autre côté du détroit, à Métauros, sur le littoral tyrrhénien de la Calabre ${ }^{526}$. Dans le secteur ionien, en Basilicate, des amphores milésiennes datées du VII ${ }^{\mathrm{e}} \mathrm{s}$. av. J.-C. ont été découvertes dans l'habitat de l'Incoronata de Métaponte et dans la nécropole de Siris ${ }^{527}$. En Campanie, on a trouvé des amphores milésiennes et samiennes à Cumes, Pithécusses, Capoue, Nuceria et Fratte $^{528}$. En continuant vers l'Occident, les deux séries sont attestées en Provence, à Marseille, à Saint-Blaise et à Tamaris, mais aussi en Espagne, probablement à Huelva et à Toscanos ${ }^{529}$. Sur les côtes méridionales de la Méditerranée, les types archaïques de Milet et surtout de Samos sont documentés à Carthage ${ }^{530}$.

En ce qui concerne le groupe, plus problématique, de Samos-Milet, d'époque tardo-archaïque et classique, il est documenté dans le Salento à travers quelques exemplaires trouvés à Oria, Cavallino, Soleto, Vaste et Masseria Fano ${ }^{531}$. En Sicile, la présence de ce groupe est attestée par un petit nombre d'exemplaires pourtant distribués sur de nombreux sites, comme Catane, Himère, Camarine, Géla, Monte Saraceno, Motyé, RamaccaMontagna, Sélinonte, Naxos et peut-être Colle Madore

525. Pour la Sicile, voir la synthèse de R. M. Albanese Procelli (Albanese Procelli 1997, p. 107-108). Plus récemment pour Himère : Vassallo 2003, p. 342-343, fig. 6, $\mathrm{n}^{\circ} 24-26$; pour Camarine : Sourisseau 2002 p. 548, et Id. 2006, p. 133 et fig. 1.

526. Pour Métauros : Sabbione 1981a ; Id. 1981b ; Id. 1987 (également pour la documentation samienne de Mylai).

527. Pour l'Incoronata de Métaponte, en dernier lieu : Stea 2000. Pour Siris : Berlingò 1986.

528. Pour Cumes : Savelli 2006, p. 113-114; pour Pithécusses : Di Sandro 1986, p. 69-76, 121 ; pour Capoue et Fratte : Di Sandro 1981, p. 7-8.

529. Pour la Provence: Sourisseau 1997, I, p. 143. Pour Huelva : Rouillard 1991, p. 177. Pour Toscanos: Docter 2000, p. 69-70, $\mathrm{n}^{\circ}$ 4-6, fig. 8, d-f (amphores de Samos) et p. 76, $\mathrm{n}^{\circ} 28$, fig. 11, c (amphores de Milet).

530. Docter 2000, p. 69, n 1-3, fig. 8, a-c (amphores de Samos) et p. 76, n. 26, avec bibliographie antérieure (amphore peut-être de Milet). En dernier lieu, voir aussi : Docter 2007, p. 658-659 (pour les amphores de Samos) et p. 661 (pour l'amphore attribuée de façon hypothétique à une fabrique milésienne).

531. Pour les découvertes d'amphores dans le Salento, voir: Semeraro 1997, p.392-393 (définies comme des amphores samiennes), avec renvois aux fiches du catalogue. Le fragment d'Oria (Semeraro 1997, p. 212, n 699) pourrait être attribué à une forme milésienne d'époque archaïque. D'après M. G. Semeraro, un fragment d'Otrante pourrait aussi être attribué à une production samienne (Semeraro 1997, p. 247, n 920). et Morgantina ${ }^{532}$. Quelques exemplaires complets proviennent de la nécropole de Lentini et de l'épave de Géla, qui a livré aussi un exemplaire fragmentaire ${ }^{533}$. Il faut signaler, à Camarine et à Géla, la présence de la forme Zeest's Samian.

Le long de la côte tyrrhénienne méridionale, quelques amphores du groupe Samos-Milet datables de la fin du $\mathrm{VI}^{\mathrm{e}}$ et $\mathrm{du} \mathrm{V}^{\mathrm{e}} \mathrm{s}$. av. J.-C. ont été récemment signalées à Vélia. Toujours en Campanie, un autre exemplaire a été trouvé dans la nécropole de Fratte et un fragment du type Zeest's Samian, déjà documenté à Vélia, provient des fortifications de Cumes ${ }^{534}$.

En Étrurie méridionale on en connaît des exemplaires provenant des fouilles urbaines de Caeré et de l'emporion di Pyrgi ${ }^{535}$. D'autres attestations sont signalées dans l'habitat de Tarquinia, où les fragments contextualisés remontent à la fin du $\mathrm{VI}^{\mathrm{e}} \mathrm{s}$. av. J.-C., et surtout dans l'aire sacrée de Gravisca, dont la documentation couvre également le $\mathrm{V}^{\mathrm{e}} \mathrm{s}$. av. J.-C. Dans l'arc chronologique compris entre la fin du $\mathrm{VI}^{\mathrm{e}}$ et la première moitié $d u \mathrm{~V}^{\mathrm{e}} \mathrm{s}$. av. J.-C. sont situées enfin les nombreuses attestations de Regae et l'unique amphore du groupe Samos-Milet trouvée à la Doganella ${ }^{536}$.

Dans l'Étrurie interne, plusieurs amphores de ce groupe ont été découvertes à Chiusi, à Chianciano Terme, à Sarteano et à Camporsevoli ${ }^{537}$.

532. Pour la distribution en Sicile, voir: Albanese Procelli 1997, p. 107, avec références bibliographiques. Plus récemment, pour le site de la Montagna di Ramacca: Albanese Procelli 2003, p. 39. Pour Sélinonte: Mertens et al. 2004, p. 444-445. Pour Naxos: Lentini, Savelli, Blackman 2007, p. 89, et Id. 2009, p. 102-103, fig. 15. Pour Colle Madore: Polizzi 1999, p. 225, n 398-400, fig. 221 , $\mathrm{n}^{\circ} 398,222, \mathrm{n}^{\circ}$ 400. Pour Himère : Vassallo 2003, p. 342-343, fig. 6 , $\mathrm{n}^{\circ}$ 24-26. Pour Camarine : Sourisseau 2002, p. 548 et Id. 2006, fig. 1 (aussi forme Zeest's Samian). Pour Géla : Spagnolo 2003, p. 627-628 (forme Zeest's Samian).

533. Panvini 2001, notamment p. 72, 75, pl. XXIII.137 (définie comme samienne), pl. XXV.147 (amphore samienne fragmentaire) et pl. XXV.149 (définie comme milésienne).

534 Pour Vélia : Gassner 2003,p. 121-122 ; pour Fratte : Di Sandro 1981, p.7-8; Albore Livadie 1985, p.138-139; pour Cumes: Savelli 2006, pp. 115-116.

535 Pour l'habitat de Caeré : Boss 1993, p. 325-326 ; pour Pyrgi : Colonna 1985, p. 10.

536. Pour Tarquinia: Scotti 1999, p. 273-274; pour Gravisca : Slaska 1985, p. 19-20 et Fortunelli 2007, p. 268-269, pl. 28, G1 ; pour Regae: Morselli, Tortorici 1985, p. 33-34; pour la Doganella: Perkins, Walker 1990, fig. 40. 19 (définie comme « ionio-massaliète »).

537. Pour les amphores du groupe Samos-Milet de Chianciano Terme : Paolucci, Rastrelli 1999, p. 28, n 2.23-2.24, fig. 2.23-2.24 (définies respectivement comme samienne et chiote), p. 28 et p. 72, $\mathrm{n}^{\circ} 21.8$, fig. 21.8 (Tombes $\mathrm{n}^{\circ} 2$ et 21 de la nécropole de la Pedata et de la nécropole des Morelli), et Paolucci 1992, p. 80, n 124-125 (de la Via della Libertà); pour Sarteano, lieu-dit Palazzina, Tombe 13 : Rastrelli 1997, p. 79 et Minetti, Rastrelli 2001, p. 22, n 13.7 ; pour les exemplaires de Chiusi, lieu-dit Paccianese et lieu-dit San 
En Étrurie septentrionale, le centre qui a restitué la plus abondante documentation est l'habitat de Pise, mais un fragment du début du $\mathrm{V}^{\mathrm{e}} \mathrm{s}$. av. J.-C. a également été trouvé à Fossa Nera di porcari, le long de la vallée du Serchio, et un autre à San Rocchino di Massarosa ${ }^{538}$. Des amphores du groupe Samos-Milet faisaient partie de la cargaison du bateau marchand qui fit naufrage à Punta Ala, près de Castiglione della Pescaia ${ }^{539}$. Plus au nord, deux exemplaires du groupe Samos-Milet proviennent des fouilles de l'habitat de Gênes ${ }^{540}$.

Des amphores attribuées au même groupe ont été aussi trouvées dans le sud de la France. On en connaît à Marseille, à Arles, à Saint-Pierre-lès-Martigues, à SaintBaise et dans la cargaison de l'épave de la Pointe Lequin $1 \mathrm{~A}^{541}$. D'autres attestations occidentales viennent enfin d'Emporion, en Espagne, et de Carthage, en Tunisie ${ }^{542}$.

\section{Les amphores du groupe « Solokha I »}

Deux formes amphoriques d'époque classique, généralement désignées types «Solokha I» et «Solokha II $»^{543}$, doivent leur dénomination au kourgane scythe éponyme découvert près de Velikaja Znamenka, sur la rive gauche du fleuve Dniepr, sur le territoire de l'Ukraine actuelle. La tombe latérale du kourgane, fouillée par I. N. Veselovski en 1913 et aujourd'hui datée du premier quart du $\mathrm{IV}^{\mathrm{e}} \mathrm{s}$. av. J.-C., a livré trois exemplaires

Vincenzo : Rastrelli 1993, p. 125, pl. XII ; pour les exemplaires inédits signalés dans le cadre de Musées et collections, voir : Rastrelli 1993, p. 125 n. 52 et Paolucci, Rastrelli 1999, p. 28, n 2.23 ; pour un exemplaire de la Tombe de l'Inscription de Poggio Renzo : Martelli, Nasorri 2000, p. 98 . Pour une liste récente des attestations provenant du territoire de Chiusi avec signalement d'exemplaires inédits : Paolucci 2006, p. 422-424.

538. Pour Pise, voir principalement : Pancrazzi 1982, p. 336, 341, fig. 2.14-16 et fig. 2.19-20 (ces derniers exemplaires, attribués par O. Pancrazzi à son « tipo 2 » de Chios, appartiennent plus probablement au groupe Samos-Milet), Bruni 1993, p. 290 et Massa 1993, p. 349351. Pour Fossa Nera di Porcari : Ciampoltrini 1994, p. 77. Pour San Rocchino : Maggiani 1990b, p. 94, $n^{\circ}$ 51, fig. 44.51.

539. Pour Punta Ala (épave A) : Cygielman, De Tommaso 1998, p. $93-101$.

540. Milanese, Mannoni 1986, p. 133, fig. 8, 2 et Milanese 1987, p. $224, n^{\circ} 596$, fig. 100,596 .

541. Pour les sites de Provence: Sourisseau 1997, I, p. 143. Pour l'épave de la Pointe Lequin 1A : Pomey et al. 1988, p. 32-35 ; Long, Miro, Volpe 1992, p. 199-243 ; Parker 1992, n 846, p. 323, map 5; Gras 1996, p. 168-169.

542. Pour Emporion : Sanmartí Grego et al. 1991 et Aquilué Abadías et al. 2000, p. 330-333, fig. 40.1-2. Pour Carthage: Morel 1990c, p. 75 , fig. $8.13-15$.

543. Zeest 1960,p. 91-92, pl. XIV-XVI, 32. de la forme I (fig. 63) ${ }^{544}$ et huit de la forme II ${ }^{545}$. Il s'agit donc de deux formes distinctes, dont la dénomination n'a bien entendu aucun rapport avec leur lieu de production, mais renvoie au lieu de la découverte des ces premiers exemplaires.

Les amphores dites « Solokha I », qui nous intéressent plus particulièrement dans le cadre de cette étude, constituent un groupe d'amphores de type " extra-régional », car les différentes pâtes utilisées dans la fabrication de la même forme indiquent que la production est partagée par de nombreux centres distincts. La plupart d'entre eux n'ont pas encore été identifiés avec précision. Il semble néanmoins très probable que la fabrication de cette série, doive être située dans l'aire de l'Égée orientale et méridionale. Ainsi, les principaux centres supposés et souvent évoqués seraient Clazomènes, Samos, Cos, Cnide et Rhodes. Toutefois nous manquons de données claires, notamment pour les périodes les plus anciennes de la production, en particulier la seconde moitié du $\mathrm{V}^{\mathrm{e}} \mathrm{s}$. av. J.-C.

Les amphores du groupe «Solokha I » ont d'abord été identifiées par V. Grace comme attiques. C'est en examinant quelques exemplaires de l'Agora d'Athènes, qu'elle a construit l'hypothèse d'une origine attique de cette production dont la genèse serait située dans le courant du $\mathrm{V}^{\mathrm{e}} \mathrm{S}$. av. J.-C. ${ }^{546} \mathrm{La}$ forme aurait ensuite été adoptée par divers ateliers de l'Égée orientale, notamment à Samos, dans le courant du siècle suivant ${ }^{547}$.

544. Zeest 1960, pl. XV, 32b ; Mancevič 1987, p. 50, n 31.1, et fig. a p. 51 .

545. Sur les premières fouilles du tumulus de Solokha, qui se sont déroulées entre 1912 et 1913, voir : Farmakovskiy 1914. Les travaux postérieurs ont fait l'objet de rapports préliminaires (pour lesquels la bibliographie est commodément rassemblée dans DoulgériIntzessiloglou, Garlan 1990, n. 33). Il faut attendre la publication posthume des études qu'A. Mancevič a consacré au sujet (Mancevič 1987) pour disposer d'une documentation à peu près complète. La chambre centrale (violée) et la chambre latérale (intacte) qui contenait les onze amphores ont fait l'objet de propositions de datation dès la première publication. Ainsi B.V. Farmakovskiy considérait que la chambre centrale était la plus ancienne et devait être située entre le milieu du $\mathrm{V}^{\mathrm{e}}$ et le milieu du IV ${ }^{\mathrm{e}} \mathrm{s}$. av. J.-C., alors que la chambre latérale, plus récente, pouvait être datée de la seconde moitié du IV s. av. J.-C. Ces propositions chronologiques ont été révisées, notamment par Brašinskij (1965) et Mancevič (1987) qui considèrent que les deux sépultures seraient à peu près contemporaines ou qu'elles se succèdent dans le temps dans un intervalle assez court, situé entre la fin $\mathrm{du} \mathrm{V}^{\mathrm{e}}$ (tombe centrale) et le premier quart du IV ${ }^{\mathrm{e}} \mathrm{s}$. av. J.-C. (tombe latérale).

546. Grace 1953.

547. Grace 1953, p. 101-102, n 147, pl. 39, n 147 ; Ead. 1971, p. $74,78-79$ et note 68 . Sur l'impact des travaux de V. Grace sur ces amphores, voir par exemple, pour le cas des études conduites sur du matériel provenant d'Italie : de Marinis 1988 ; Desantis 1989 ; Maggiani, Paribeni 1990 ; Cattaneo Cassano 1995 ; Cattaneo, De Marinis 1996. Sur le matériel publié de l'Égée et de la Mer Noire, voir Lawall 1995, p. 223-224, n. 23. 


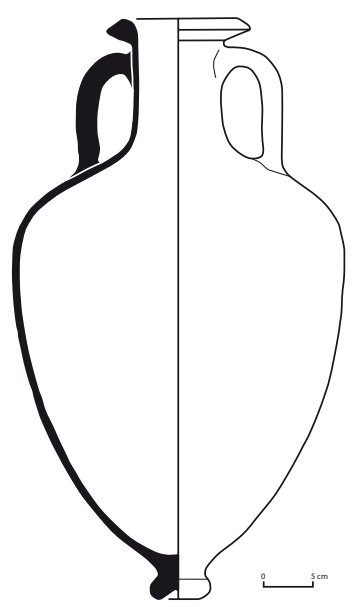

Fig. 63. Amphore du groupe Solokha I. Kurgan de Solokha (d'après Doulgéri-Intzessiloglou, Garlan 1990, fig. 35.a).

La forme aurait ensuite graduellement évoluée vers des types à lèvre plus lourdes de section triangulaire, jusqu'à aboutir à la forme de type "mushroom rim ». Pour construire son schéma évolutif, V. Grace met en relation, sur la base de leur morphologie, plusieurs amphores des dépôts de l'Agora, notamment les exemplaires P21970 (du contexte N 7:3), P16526 et P9429. Elle mobilise également l'amphore P12760 du contexte G 11:3, qui, avec sa lèvre courte, aurait constitué le point de départ possible de la série au début du $\mathrm{V}^{\mathrm{e}} \mathrm{s}$. av. J.-C. Le problème est qu'on sait aujourd'hui, grâce aux travaux de M. Lawall, que cette amphore doit plus probablement être identifiée comme une production nord-égéenne ${ }^{548}$. La série définie par V. Grace apparaît donc construite à partir d'éléments hétérogènes, ce qui a introduit de nombreuses confusions dans les déterminations proposées dans les publications de matériel dans l'ensemble du bassin méditerranéen pendant de très nombreuses années.

Pour les amphores de la forme Solokha I on manque donc, aujourd'hui encore, non seulement de publications de synthèse sur leur évolution typo-chronologique, mais aussi d'un accord de la part du monde scientifique sur leur dénomination et leur aire de production. C'est donc sur la base des rares publications d'amphores de la forme Solokha I et des résultats des nouvelles études récemment effectuées sur les exemplaires de l'Agora d'Athènes qu'on en propose ci-dessous une

548. Grace 1953, p. 101-103. Pour une nouvelle attribution de l'amphore P12760 aux productions de l'Égée septentrionale, voir : Lawall 1995, p. 223-224 et n. 23, avec une analyse historiographique précise et pertinente des conséquences de cette confusion introduite par V. Grace dans l'identification des séries de forme Solokha I et de production nord-égéenne. description morphologique et une synthèse de l'évolution typo-chronologique.

\subsection{Bilan des études}

La production des amphores du groupe « Solohka I » a dû probablement commencer vers le milieu du $\mathrm{V}^{\mathrm{e}} \mathrm{s}$. av. J.-C. pour ne disparaitre que dans le courant du $\mathrm{IV}^{\mathrm{e}} \mathrm{s}$. av. J.-C. Les exemplaires de la seconde moitié du $\mathrm{V}^{\mathrm{e}} \mathrm{s}$. av. J.-C., qui nous intéressent ici, sont caractérisés par une forme globalement ovoïde, une épaule arrondie et une panse qui se rétrécit légèrement dans la partie inférieure. Le col, plutôt étroit, est de forme cylindrique au profil rectiligne. La lèvre, du type à section triangulaire, est particulièrement caractéristique de cette série. Les anses, en ruban épaissi, retombent verticalement de la moitié supérieure du col sur l'épaule, où une impression digitale caractéristique marque la base externe de l'attache inférieure. Le pied, de forme tronconique, est massif. Sa base en anneau épaissi présente une profonde cavité semi-circulaire qui, surtout pour les exemplaires les plus anciens, est caractérisée par la présence fréquente d'un revêtement sableux ou, plus rarement, par une petite protubérance centrale ${ }^{549}$. Cette forme du pied, qui rappelle un gros anneau de préhension, est un élément fortement caractéristique du type ${ }^{550}$.

Le début de la série peut être situé vers le milieu du $\mathrm{V}^{\mathrm{e}} \mathrm{s}$. av. J.-C. sur la base des premiers exemplaires d'un contexte de l'Agora d'Athènes clos avant 440 av. J.-C. En effet, bien qu'un fragment de lèvre soit signalé dans un dépôt plus ancien (C 8:4b), clos vers 450 av. J.-C. mais non exempt de contaminations, c'est du contexte N 7:3, clos autour de 440 av. J.-C., que provient de manière fiable le lot le plus ancien d'amphores de forme «Solokha I ${ }^{551}$. Il comprend plusieurs fragments ainsi que l'amphore complète P21970 (fig. 64) examinée et signalée par V. Grace en son temps. Ces exemplaires de début de série présentent la lèvre typique à section triangulaire caractérisant le groupe pendant toute la période

549. Sur ces observations, voir Lawall 1995, p. 220-221 et notes 5, 8-9, fig. 93. La présence du revêtement sableux sous le pied, aussi que la caractéristique de la protubérance centrale sont presque systématiques sur les exemplaires de la seconde moitié du Ve s. av. J.-C. En revanche, ces caractéristiques de détail sont observées de manière moins systématique sur les exemplaires attribués à la période de transition entre le $\mathrm{V}^{\mathrm{e}}$ et le $\mathrm{IV}^{\mathrm{e}} \mathrm{s}$. av. J.-C.

550. La morphologie générale du pied des amphores de type «Solokha I » rappelle de très près le pied d'autres types, notamment du groupe Samos-Milet, mais il est possible d'observer que le type « Solokha I » s'en distingue par de plus grandes dimensions (le diamètre est d'environ $5-6 \mathrm{~cm}$ ) et par la concavité marquée du creux semi-circulaire de base.

551. Lawall 1995, p. 220, note 4, fig. 88, 93. 


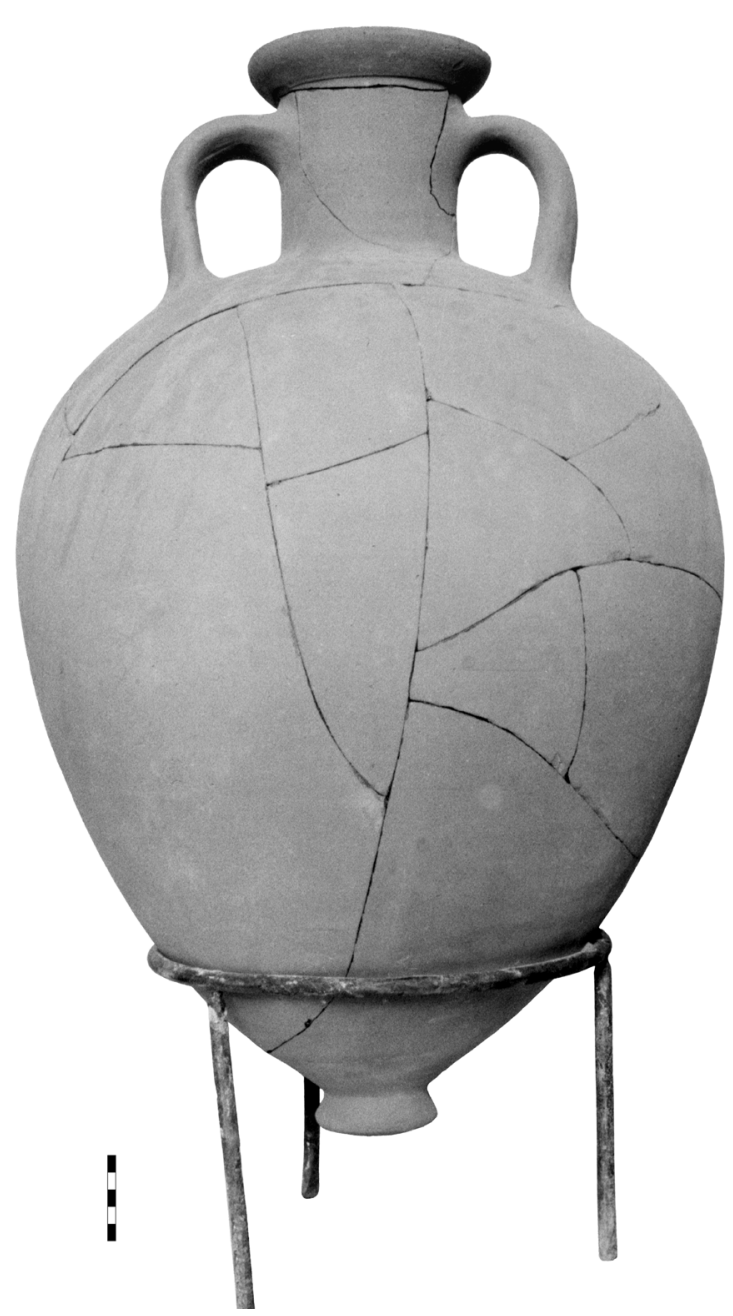

Fig. 64. Amphore du groupe Solokha I. Athènes, Agora, N 7:3, inv. P21970 (@ American Schoolof Classical Studies at Athens : Agora Excavations).

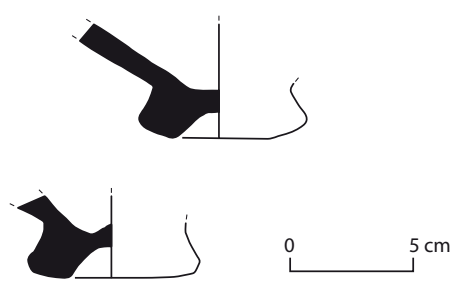

Fig. 65. Fonds d'amphores du groupe Solokha I. Athènes, Agora, N 7:3 (d'après Lawall 1995, fig. 93).

de son évolution. Toutefois, les dimensions sont encore réduites et la surface supérieure est plate et horizontale ce qui apparait comme un caractère d'ancienneté. Le pied est de forme tronconique (fig. 65).

D'autres contextes de l'Agora clos autour de 425 av. J.-C. et de 410 av. J.-C., période durant laquelle les attestations sont de plus en plus nombreuses, ont livré des exemplaires fragmentaires ainsi qu'un exemplaire complet (P27418 ; fig. 66) issu du contexte S 16:1 (clos vers 410 av. J.-C.) ${ }^{552}$. Ces derniers exemplaires présentent les mêmes caractéristiques que l'amphore P21970 (fig. 64), mais avec des lèvres plus massives munies d'un marli supérieur légèrement oblique vers l'extérieur. Le profil des lèvres documentées dans cette seconde moitié du $\mathrm{V}^{\mathrm{e}} \mathrm{s}$. av. J.-C. par ailleurs variable, peut présenter différents degrés d'inclinaison et un angle rentrant à la jonction du col plus ou moins marqué. Ce qui caractérise en général leur morphologie est l'obliquité encore modérée de la surface supérieure, dont l'ampleur est toujours moindre par rapport à la surface inférieure (fig. 67). Le pied est de type tronconique.

La période de transition entre le $\mathrm{V}^{\mathrm{e}}$ et le $\mathrm{IV}^{\mathrm{e}} \mathrm{s}$. av. J.-C. marque une évolution morphologique qui touche essentiellement la partie supérieure des amphores du groupe « Solokha I ». Si l'on met en rapport l'exemplaire complet P24211 (fig. 68) du dépôt H 13:4, clos vers 390 av. J.-C., avec ceux qui proviennent de contextes clos deux décennies auparavant, on voit bien que le col et les anses sont plus longs, l'épaulement plus ample et aplati et la lèvre plus pendante en raison de l'obliquité plus marquée de la surface supérieure du bord. Le pied, toujours de forme tronconique, peut présenter un profil latéral concave ${ }^{553}$.

Le développement de la forme se poursuit au moins jusqu'au troisième quart du $\mathrm{IV}^{\mathrm{e}} \mathrm{s}$. av. J.-C., mais sans qu'il soit possible de définir, à l'heure actuelle, un rythme d'évolution typo-chronologique précis. Les exemplaires $\mathrm{du} \mathrm{IV}^{\mathrm{e}}$ siècle présentent des formes plus fluides par l'absence d'accidents, mais on peut identifier des variantes aussi bien dans les détails des lèvres et des pieds que dans le profil de la panse ${ }^{554}$. En général ils sont caractérisés par une lèvre à section triangulaire qui devient de plus en plus pendante, comme dans les amphores du tumulus de Solokha. Les épaves d'El Sec (première moitié du IV ${ }^{\mathrm{e}} \mathrm{s}$. av. J.-C.) et de Chypre-Mazotos (troisième quart du IV ${ }^{\mathrm{e}} \mathrm{s}$. av. J.-C.) transportaient de nombreuses amphores dont la morphologie est analogue à celle des exemplaires du tumulus de Solokha ${ }^{555}$; elles suggèrent que l'inversion des proportions entre les deux faces inférieure et supérieure de la lèvre, qui finit ainsi par prendre une forme définie aussi « en col de cygne » et qui semble

552. Lawall 1995 , p. 220, notes 6, fig. 89, 91-92.

553. Lawall 1995, p. 220-221, notes 7-9, fig. 90.

554. Sur le développement général de la forme « Solokha I » au IV $^{\text {e }}$ s. av. J.-C., voir Lawall 1995, p. 221-222 et note 17-18, avec références bibliographiques.

555. Pour El Sec, Cerdá 1987 (« type El Sec N1-3 »); pour Chypre-Mazotos, Demesticha 2011. 


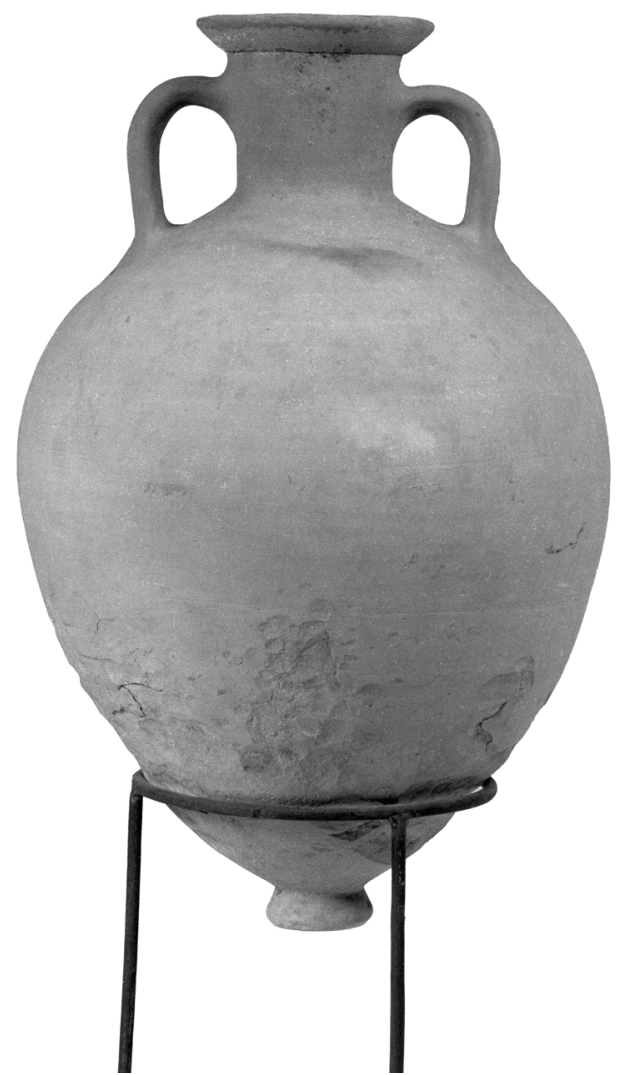

Fig. 66. Amphore du groupe Solokha I. Athènes, Agora, S $16: 1$, inv. P27418 (@ American Schoolof Classical Studies at Athens : Agora Excavations).

inaugurer le début de la série dite à mushroom rim, ou à « lèvre champignon », se produit avant le milieu du $\mathrm{IV}^{\mathrm{e}}$ S. av. J.-C. ${ }^{556}$.

\subsection{Centres de production}

Traitant des amphores de l'Agora d'Athènes qu'elle définissait comme attiques, V. Grace ${ }^{557}$ en a décrit la pâte comme étant plutôt fine, à l'argile claire, variant du rose au chamois, mais parfois grise en section et dépourvue de quantités remarquables d'écailles de mica, ce qui, à son avis, la distinguait nettement des productions samiennes. C'est cette description qui a servi de base aux attributions successives à la production supposée attique de plusieurs exemplaires de forme « Solokha I »

556. Ce n'est pas par hasard que les amphores dites « attiques» $\mathrm{du} \mathrm{V}^{\mathrm{e}} \mathrm{s}$. av. J.-C. ont parfois été considérées comme « proto-grécoitaliques », c'est-à-dire correspondant au prototype du type le plus ancien (type A1) d'amphore gréco-italique (Lyding Will 1982, p. 341, n. 4). Sur ce thème, voir également Lawall 2005, p. 33 et note 14.

557. Grace 1953, p. 101. Sur la distinction entre productions supposées attiques et samiennes : Ead.1971,p. 74, 78.
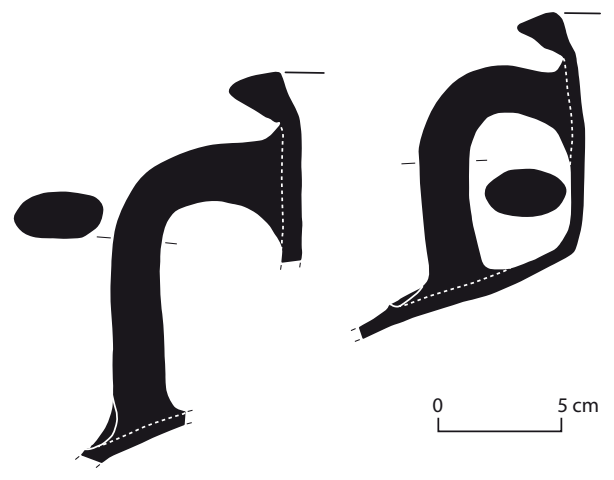

Fig. 67. Cols d'amphores du groupe Solokha I. Athènes, Agora, R 13:1 (à gauche) et B 13:6 (à droite) (d'après Lawall 1995, fig. 91-92).

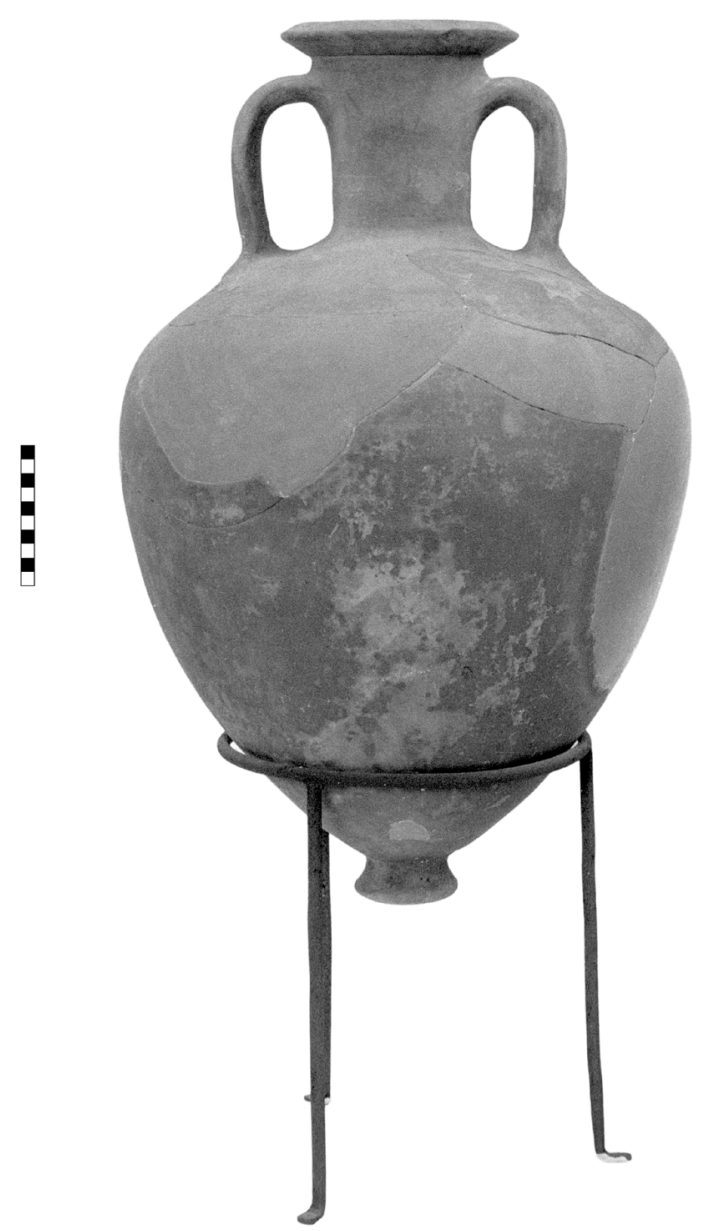

Fig. 68. Amphore du groupe Solokha I. Athènes, Agora, H $13: 4$, inv. P24211 (@ American Schoolof Classical Studies at Athens : Agora Excavations) 
provenant de contextes de l'Égée, de la Mer Noire et de la Méditerranée occidentale ${ }^{558}$. Cependant, l'examen visuel des pâtes d'un plus ample échantillonnage de la forme "Solokha I » montre son extrême variabilité et la distinction très claire de ces séries par rapport aux amphores de production samienne ou milésienne. L'éventail chromatique des matrices argileuses est vaste, des tonalités claires du beige, du chamois et du rose à celles, intenses, de l'orange et du rouge. La consistance et la sensation au toucher de la surface, lisse et compacte ou bien poudreuse et vacuolaire est également très variable. La même observation s'applique aussi aux types et à la densité des inclusions, à commencer par la présence plus ou moins fréquente de fines écailles de mica ${ }^{559}$.

En dépit de l'homogénéité des formes, une telle variabilité des pâtes doit correspondre à une multiplicité de centres producteurs, même si la plupart d'entre eux n'ont pas encore été localisés et si nos connaissances relatives aux débuts de la production, et donc à l'origine de la forme, restent incertaines. Les centres indiqués au cours des années comme des lieux de fabrication du groupe « Solokha I » sont nombreux et leur proposition de localisation s'appuie sur des arguments divers.

L'hypothèse la plus répandue, et celle qui a rencontré la plus large audience de la part du monde scientifique, est celle de V. Grace qui, comme on l'a déjà dit, estime que la forme $\mathrm{du} \mathrm{V}^{\mathrm{e}}$ siècle doit être attribuée au milieu

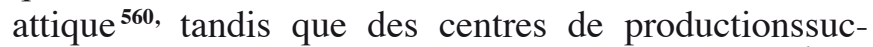
cessifs, du IV e siècle, se seraient installés dans l'Égée orientale, notamment à Samos, probablement après 365 av. J.-C. par l'intermédiaire des clérouques athéniens ${ }^{561}$. Le problème est que la théorie de $\mathrm{V}$. Grace reposait au moins pour partie sur l'identification comme attique de l'amphore P12760 de l'Agora aujourd'hui considérée comme issue d'un atelier nord-égéen.

L'hypothèse d'une production nord-égéenne de la forme «Solokha I » a été proposée par A. Mancevič ${ }^{562}$

558. Pour la Méditerranée occidentale, voir par exemple de Marinis 1988, p. 218 et Maggiani, Paribeni 1990.

559. Ces observations sont fondées, non seulement sur les amphores de forme « Solokha I » de l'Italie septentrionale, mais aussi sur de nombreux exemplaires, notamment ceux de l'Agora d'Athènes que j'ai pu examiner grâce à la disponibilité de l'École Américaine. Pour une description précise des quatre types de pâte en fonction desquels il a été possible de classer les exemplaires de l'Agora, voir : Lawall 1995 , p. 222. Il est à remarquer que la pâte $n^{\circ} 3$ est décrite comme « quite similar to the fabric of some Samian-Milesian examples». Des recherches personnelles en cours sur le matériel de Milet tendent à confirmer cette hypothèse.

560. Grace 1953, p. 101-102, n 147.

561. Grace 1971, p. 74, 78.

562. Mancevič 1987, notamment p. 123. S. sur la base de la comparaison entre une amphore représentée sur un type monétaire frappé à Toronè (dans la péninsule Sithonia) entre la fin du $\mathrm{VI}^{\mathrm{e}}$ et le début du $\mathrm{V}^{\mathrm{e}}$ s. av. J.-C.et la forme caractéristique des amphores Solokha I. L'hypothèse apparaît néanmoins discutable. D'une part la série monétaire identifiée semble un peu trop ancienne vis-à-vis des amphores évoquées, quant au rapprochement morphologique entre les deux types de documents il apparait plutôt improbable. Si l'hypothèse de la production nord-égéenne ne convainc pas totalement la plupart des spécialistes ${ }^{563}$, les nombreuses propositions qui se sont orientées vers l'Égée orientale et méridionale sont en général plus largement acceptées ${ }^{\mathbf{5 6 4}}$

I. Zeest a suggéré comme centres de production des amphores du groupe «Solokha I » les îles de Rhodes et de Cos. Cette hypothèse tire son origine de l'analogie morphologique présumée du type «Solokha I » avec une forme amphorique plus récente, le type «UstLabinskaja », aux anses bifides, dont la production est attestée aussi bien à Rhodes qu'à Cos. Le type «UstLabinskaja », selon I. Zeest, pourrait être interprété comme une évolution de la forme « Solokha I » aux III et $\mathrm{II}^{\mathrm{e}} \mathrm{s}$. av. J.-C. et il pourrait aussi être mis en rapportavec les amphores mushroom rimmed de la Méditerranée occidentale, définies ensuite comme gréco-italiques ${ }^{565}$.

Sur cette base, la proposition de I. Zeest de localiser un atelier pour la production d'amphores du groupe Solokha I à Cos a été accueillie favorablement même par I. Brašinskij ${ }^{566}$, qui admettait pourtant l'existence de plusieurs centres producteurs pour le groupe « Solokha I ». A. Avram a même suggéré que Cos avait aussi produit un type d'amphores sans anses bifides, et donc plus proche encore de la forme «Solokha I », dont quelques exemplaires timbrés seraient documentés à Histria ${ }^{567 .} \mathrm{La}$ relation entre Cos et les amphores du groupe « Solokha I », qui récemment encore était exclusivement fondée sur ces analogies morphologiques constatables, a récemment reçu une importante confirmation archéologique.

563. Voir à ce propos : Brašinskij 1984, p. 43-45 ; DoulgériIntzessiloglou, Garlan 1990, p. 388 ; Lawall 1995, p. 225-226.

564. Empereur, Picon 1986, p. 112.

565. Zeest 1960, p. 91-94, tav. 14-16. Quant aux observations concernant les liens présumés entre les deux formes amphoriques et sur l'opportunité même de considérer le type Ust-Labinskaja comme un type en soi plutôt qu'une simple évolution de la forme « Solokha I », voir : Lawall 1995, p. 226 et n. 32. Pour une brève discussion sur le rapport entre le type Ust-Labinskaja, la forme «Solokha I » et les amphores gréco-italiques, voir : Lyding Will 1982, p. 341, n. 4 et Lawall 1995, p. 226 et n. 33. Pour une analyse spécifique du problème des amphores gréco-italiques et de leurs dérivations de la tradition locale plutôt que d'une tradition égéenne, voir : Vandermersch 1994 et plus récemment Cibecchini 2007.

566. Brašinskij 1984, p. 43-45.

567. Avram 1989. 
En 1991 en effet, dans le site de l'ancienne Halasarna, l'un des six dèmes de Cos, a été fouillé un atelier d'amphores dont la pâte, d'après une analyse visuelle, a été reconnue par Ch. Kantzia comme locale ${ }^{568}$. Des trois types d'amphores qu'il a été possible de distinguer et dont la typologie précise est en cours d'élaboration, les types Cos I et Cos II présentent des affinités morphologiques remarquables avec la forme «Solokha I » du $\mathrm{IV}^{\mathrm{e}}$ s. av. J.-C., à commencer par la forme de la lèvre à section triangulaire. Le type Cos I, qui présente la particularité d'être muni d'anses bifides rappelant la tradition tardo-archaïque de Milet, a été daté de la première moitié du $\mathrm{IV}^{\mathrm{e}} \mathrm{s}$. av. J.-C. en raison de la ressemblance morphologique de son pied avec celui des amphores contemporaines du groupe « Solokha $1 »$. Le type Cos II, qui se distingue du type I surtout par l'absence des anses bifides et par son pied en bobine, a été mis en parallèle avec un exemplaire trouvé dans la péninsule de Koroni, sur la côte orientale de l'Attique ${ }^{569}$, et daté, du fait de cette ressemblance, de la première moitié du $\mathrm{III}^{\mathrm{e}} \mathrm{s}$. av. J.-C. ${ }^{570}$. Plus globalement, l'activité de cet atelier de Cos a été datée à partir de la première moitié $d u \mathrm{IV}^{\mathrm{e}} \mathrm{s}$. av. J.-C. Il pourrait toutefois avoir été actif dès le courant du $\mathrm{V}^{\mathrm{e}}$ S. av. J.-C.

En ce qui concerne l'hypothèse de la production rhodienne, elle a été corroborée par la publication de la part de V. Grace d'un fonds d'amphores de type mushroomrimmed portant des timbres rhodiens ${ }^{571}$. Mais la théorie de V. Grace selon laquelle les amphores qu'elle qualifiait d'attiques étaient aussi produites, dans la seconde moitié du IV ${ }^{\mathrm{e}}$ s. av. J.-C., dans l'Égée orientale, n'était pas limitée à l'île de Rhodes. Elle évoquait également une production à Samos, sur la base de deux amphores du groupe « Solokha I » trouvées à Samos et datées de la seconde moitié du IV ${ }^{\mathrm{e}} \mathrm{s}$. av. J.-C., qui portaient deux timbres que V. Grace a reconnus comme originaires de l'île. Il s'agit d'un monogramme et d'une tête de lion qui trouvent de bonnes comparaisons avec un type monétaire samien contemporain ${ }^{572}$. Sur cette base, l'hypothèse samienne a donc été très largement suivie ${ }^{573}$, du moins

568. Sur la fouille de l'atelier de l'île de Cos, voir. : Kantzia 1994, p. 332-335, fig. 5 (pour le type Cos I), et p. 335-337, fig. 6 (pour le type Cos II). Pour une synthèse récente et une esquisse de typologie des trois formes amphoriques produites à Cos, mais uniquement sur la base de la morphologie du pied, voir : Georgopoulos 2004.

569. Vanderpool, Mccredie, Steinberg 1962, p. 33, n 6, pl. 19.

570. Certains éléments archéologiques en contexte, mais inédits de Cos semblent suggérer une chronologie plus haute. Sur ce point, voir la notice préliminaire : Georgopoulos 2004, n. 30.

571. Grace 1963, p. 322-323, fig. 1, n 1-3, 5 .

572. Grace 1971, notamment p. 56-57, 62-67, 74 et n. 57, 78, pl.12.10, 13.53.

573. Cf. : Brašinskij 1984, p. 45 ; Cerdá 1987, p. 447, n. 1; Cerdá 1989, p. 65, 88; Blondé, Muller, Mulliez 1991. pour le $\mathrm{IV}^{\mathrm{e}} \mathrm{s}$. av. J.-C. Le problème reste néanmoins ouvert pour les périodes plus anciennes, dans la mesure où une éventuelle production samienne de ce type dans la seconde moitié du $\mathrm{V}^{\mathrm{e}} \mathrm{s}$. av. J.-C. ne s'appuie que sur la découverte, dans des contextes de l'Agora datés de la fin du $\mathrm{V}^{\mathrm{e}} \mathrm{s}$. av. J.-C., de quelques exemplaires dont la pâte est très proche de celle des amphores dont la production est certainement samienne ${ }^{574}$.

On a aussi attribué à la cité de Cnide une production d'amphores de type "Solokha I », d'abord sur la base de quelques exemplaires pourvus d'un timbre représentant la proue d'un bateau reconnu comme cnidien ${ }^{575}$. L'hypothèse a ensuite été confirmée par la découverte, dans la péninsule cnidienne, de fours de potiers avec leurs déchets de cuisson caractéristiques de la forme en question. Par ailleurs, des analyses pétrographiques effectuées sur les pâtes des exemplaires portant le timbre représentant la proue d'un bateau ont confirmé une origine cnidienne ${ }^{576 .}$

D'autres fours du IV e s. av. J.-C. et ayant produit des amphores de forme « Solokha I » ont également été découverts à Clazomènes. Les exemplaires identifiés, définis comme « amphores à lèvre champignon » et considérés par E. Doğer comme une imitation des productions « attique » ou « samienne », avec une allusion évidente aux travaux de V. Grace, doivent très probablement être identifiés comme des amphores de forme «Solokha I », datées, sur la base du contexte, du troisième quart du $\mathrm{IV}^{\mathrm{e}} \mathrm{s}$. av. J.-C. ${ }^{577}$

$\mathrm{Si}$, dans le but d'identifier les lieux de production du groupe « Solokha I », la plupart des spécialistes ont orienté leurs recherches vers l'aire égéenne orientale ou méridionale, une autre hypothèse situe en revanche un éventuel atelier du groupe « Solokha I » dans les Sporades septentrionales. D'après A. Doulgéri-Intzessiloglou et Y. Garlan, l'île de Skopélos (l'ancienne Péparéthos) aurait pu fabriquer des amphores attribuables à ce groupe. Toutefois, si des amphores du groupe « Solokha I » caractéristiques des productions du $\mathrm{IV}^{\mathrm{e}} \mathrm{s}$. av. J.-C. (amphores dites « Péparéthos II ») ont bien été découvertes sur place, l'atelier connu sur l'île renvoie essentiellement à la production d'amphores de type "Solokha II » (dites amphores «Péparéthos I») et peut-être seulement, et à partir d'une documentation très restreinte, à

574. Il s'agit d'exemplaires provenant des contextes de l'Agora B 13:5, A-B 21-22:1 pour lesquels on renvoie à : Lawall 1995, notamment p. 222, 228 et n. 45, 229 et n. 51 .

575. Börker 1986, p. 473-478.

576. Sur l'attribution à Cnide de la forme, voir Monachov 2003a, p. 101-104, fig. 71, 1 (type Cnide I ou Elizavetovski). Sur les fours de potiers de Cnide, voir Tuna et al. 1991, p. 39-43 ; sur les analyses pétrographiques, voir en dernier lieu : Whitbread 1995, p. 107-116.

577. Doğer 1986, p. 469-471, type 7, fig. 18. 
une éventuelle production de types « Solokha I ». Cette hypothèse est défendue avec force par ses partisans s'appuyant sur la découverte de quelques fragments du type I dans les ateliers de Skopélos, identifiés avec certitude comme producteurs du type II ${ }^{578}$. On observera, cependant, que malgré une indéniable ressemblance entre les amphores de type « Péparéthos II » et celles du groupe « Solokha I », l'analogie morphologique n'apparait pas aussi nette que nécessaire ${ }^{579}$. C'est pourquoi nous resterons prudente vis-à-vis de cette hypothèse.

La ressemblance morphologique de la lèvre de la forme du $\mathrm{IV}^{\mathrm{e}}$ siècle avec celle d'éventuelles productions contemporaines de Grande Grèce, bien différentes dans le développement du col et de la partie inférieure du conteneur, a enfin suggéré une provenance magno-grecque ou sicilienne des amphores trouvées dans un contexte d'Emporion daté du IV ${ }^{\mathrm{e}}$ s. av. J.-C. ${ }^{580}$. L'hypothèse apparaît encore néanmoins fragile, du moins insuffisamment documentée.

Pour conclure, la variété des pâtes dans lesquelles la forme a été réalisée dès l'origine de la série, associée aux témoignages archéologiques nombreux concernant les productions du $\mathrm{IV}^{\mathrm{e}} \mathrm{s}$. av. J.-C., autorisent à penser que déjà, dès le $\mathrm{V}^{\mathrm{e}} \mathrm{S}$. av. J.-C., les centres de production pouvaient être divers. Ceux qui sont actuellement identifiés, pour la forme du $\mathrm{V}^{\mathrm{e}} \mathrm{s}$. av. J.-C., sont situés dans l'aire de l'Égée orientale. Une production localisée entre Éphèse, Samos et Milet a été suggérée par les résultats des analyses effectuées sur les pâtes d'exemplaires du $\mathrm{V}^{\mathrm{e}}$ s. av. J.-C. de Vélia et un atelier milésien produisant cette forme est actuellement en cours d'étude ${ }^{581}$.

578. Sur l'hypothèse relative à l'attribution à Skopélos d'une production d'amphores du groupe «Solokha I », voir : DoulgériIntzessiloglou, Garlan 1990, notamment p. 386-388, fig. 35 ; Les analyses chimiques menées par M. Picon (1990) semblent montrer que les amphores du groupe «Solokha II » découvertes entre 1912 et 1913 dans le kourgane éponyme proviennent si ce n'est des ateliers de Skopélos, du moins du secteur des Sporades septentrionales. En revanche, aucune indication claire n'est donnée concernant le groupe des éventuelles productions locales de forme supposée « Solokha I ». 579. L'assimilation de la forme « Péraréthos II » au type «Solokha I » est néanmoins acceptée par certains (Georgopoulos 2004, p. 131), peut-être parce qu'il permet de soutenir l'hypothèse attrayante qui voudrait que toutes les amphores du kourgane de Solokha aient été fabriquées à Skopélos (Doulgéri-Intzessiloglou, Garlan 1990, p. 388). La démonstration définitive de cette hypothèse reste, de notre point de vue, encore à faire.

580. Sanmartí Grego et al. 1995, p. 33-35, fig. 6-9, attribuées à une production magno-grecque en raison de la ressemblance morphologique avec les productions magno-grecques du $\mathrm{IV}^{\mathrm{e}}$ siècle, objets de l'étude de Chr. Vandermersch (1986a et 1986b), notamment les productions locriennes du $\mathrm{IV}^{\mathrm{e}} \mathrm{s}$. av. J.-C. identifiée par M. Barra Bagnasco (1989a et 1989b).

581. Sur les analyses de pâtes des amphores «Solokha I » de Vélia, voir : Gassner 2005, p. 40-42, fig. 3 (« Scherbentyp A 23 »). Une
Par ailleurs, une des amphores de l'épave de Kyrenia à Chypre (type X), qui d'après les indications de V. Grace pourrait correspondre à la phase évoluée du $\mathrm{IV}^{\mathrm{e}}$ siècle de la forme en question, soumise à des analyses pétrographiques par I. K. Whitbread, s'est révélée compatible avec des amphores samiennes de l'Agora d'Athènes et de l'Héraion de $\operatorname{Samos}^{582}$.

Le type «Solokha I » constitue donc une forme amphorique de type « extra-régional » dont la production était vraisemblablement partagée par plusieurs ateliers situés dans une aire assez vaste mais essentiellement en milieu égéen oriental et méridional. Ces ateliers menaient, au même moment et de manière parallèle, leurs activités de production et d'exportation d'un type de conteneur ayant la même morphologie, et ceci, peutêtre en vertu d'une sorte d'accord commercial ${ }^{583}$.

\subsection{Contenu}

En ce qui concerne le type de marchandise contenu dans ces amphores, on ne dispose pas, à l'heure actuelle, de données objectives bien établies. L'hypothèse que ces amphores aient pu être utilisées indifféremment pour le transport du vin et de l'huile a été avancée, car sur la paroi interne de quelques fragments d'amphores du groupe «Solokha I », et non de toutes, il a été possible d'observer des traces d'un revêtement blanc dont la fonction aurait été d'assurer l'étanchéité du conteneur ${ }^{584}$. Cependant, la nature de ce résidu et sa fonction précise n'a été confirmée par aucune analyse de laboratoire. L'observation de la présence de résidus résineux nous amène à la même conclusion. On n'a pu en observer que rarement, et il faut rappeler que ce type de revêtement est très périssable. Toutefois, certains indices épigraphiques ponctuels plaident pour un usage si ce n'est systématique, du moins occasionnel de cette forme d'amphore pour le transport de l'huile ${ }^{585}$.

production de la forme « Solokha I » à Milet est en cours d'étude de ma part dont l'attribution à un atelier milésien a été confirmée par des analyses NAA effectuées par H. Mommsen (Archaeometry Group, Universität Bonn).

582. Sur les amphores de l'épave de Kyrenia : Grace 1971, p. 78-79 et n. 68. Pour les résultats des analyses réalisées sur la pâte, Whitbread 1995, p. 127-130.

583. En faveur de l'existence d'une sorte de koinè des productions amphoriques du IV ${ }^{\text {e }}$ s. av. J.-C. dans cette région, avant l'établissement d'un type spécifique pour chaque cité, voir les commentaires de J.-Y. Empereur et M. Picon sur la localisation des fours pour la production de la forme «mushroom-rimmed » (Empereur, Picon 1986, notamment p. 112).

584. Grace 1953, p. 102.

585. Sur cette hypothèse, voir Lawall 2000, p. 18, 46-47, $\mathrm{n}^{\circ}$ 54, p. $51, \mathrm{n}^{\circ} 66$. 
Il est donc difficile d'établir une tendance générale dont la définition est par ailleurs rendue difficile du fait de la pluralité des centres de production.

\subsection{Diffusion dans la Méditerranée occidentale des amphores du groupe « Solokha I »}

Les amphores du groupe « Solokha I » ont connu une vaste diffusion en Mer Égée et surtout en Mer Noire ${ }^{586}$. Leur présence en Méditerranée occidentale, en revanche, est apparemment très faible et concentrée presque exclusivement dans l'aire géographique qui fait l'objet de cette étude. En dehors de ces exemplaires, tous datés dans le courant de la seconde moitié du $\mathrm{V}^{\mathrm{e}} \mathrm{s}$. av. J.-C., seuls quelques autres fragments contemporains sont connus à ce jour, provenant de la côte tyrrhénienne de la péninsule italienne : à Vélia, en Campanie, à Casa Baldi de Pozzi-Seravezza, à Versilia, et peut-être à Gênes, en Ligurie $^{587}$. On doit probablement attribuer au IV ${ }^{\mathrm{e}}$ s. av. J.-C. une amphore provenant d'une récupération sousmarine près des côtes de l'Étrurie méridionale ${ }^{588}$.

586. Pour une synthèse sur les découvertes de la Mer Noire : Doulgéri-Intzessiloglou, Garlan 1990, p. 386, avec références bibliographiques. Plus récemment, voir Monachov 2003.

587. Pour Vélia, voir en dernier lieu : Gassner 2005, p. 40-41, avec la bibliographie antérieure. Pour l'exemplaire fragmentaire de Casa Baldi di Pozzi, voir Maggiani, Paribeni 1990, p. 145, $\mathrm{n}^{\circ} 25$, fig. 78.25. Pour le pied de Gênes, attribué à une production samienne : Milanese, Mannoni 1986, p. 133, fig. 8, 3.

588. Gianfrotta 1981, p. 80, n. 64, fig. 34 (définie comme gréco-italique et comparée à une amphore publiée par A. Mancevič).
Certaines amphores appartenant à la cargaison de l'épave d'El Sec, datée du milieu du IV ${ }^{\mathrm{e}}$ s. av. J.-C., pourraient être à mon avis attribuées à ce groupe ${ }^{589}$. À la même série, on peut aussi rattacher quelques amphores trouvées dans des contextes du $\mathrm{IV}^{\mathrm{e}} \mathrm{s}$. av. J.-C. d'Emporion ${ }^{590}$.

Il est difficile d'établir avec certitude si la faible quantité et la carte de diffusion des exemplaires trouvés en Méditerranée occidentale reflète un état effectif des découvertes ou plutôt une carence des recherches. Il est vrai que les possibilités de confusions sont nombreuses, notamment dans les contextes du IV ${ }^{\mathrm{e}}$ s. av. J.-C., avec certaines productions « magno-grecques » ou « grécoitaliques anciennes ${ }^{\mathbf{5 9 1}}$ et que les fouilleurs contournent souvent la difficulté en renonçant à prendre parti dans un débat qui, à l'échelle d'un site et de quelques objets, est évidemment marginal. L'examen de la documentation de l'Italie du nord a d'ailleurs montré que les amphores du groupe «Solokha I », en particulier celles de la seconde moitié du $\mathrm{V}^{\mathrm{e}} \mathrm{s}$. av. J.-C., souffrait d'un fort déficit d'identification et qu'il fallait donc rétablir la part effective de la diffusion de ces productions à un niveau supérieur à ce qu'on imaginait auparavant.
589. Cerdá 1987. Je crois qu'on pourrait attribuer, du moins en partie, au groupe « Solokha I » les amphores «type El Sec N ». Après les avoir définies dans un premier temps comme samiennes, suivant les travaux de V. Grace (1971), D. Cerdá les a ensuite classées en trois groupes distincts : «type El Sec N1 », «type El Sec $\mathrm{N}_{2}$ », « type El $\operatorname{Sec} \mathrm{N}_{3}$ ", après une confrontation avec la spécialiste américaine, qui admettait la possibilité d'une attribution à Samos uniquement pour le groupe $\mathrm{N}_{1}$ (Cerdá 1987, p. 447, n. 1).

590. Sanmartí Grego et al. 1995, p. 33-35, fig. 6-9, attribuées à une production magno-grecque. Pour la diffusion en Espagne des exemplaires susceptibles d'appartenir à cette forme : Sanmartĺ Grego et al. 1995 , p. 35

591. Cibecchini 2007, p. 51, avec un tableau de concordance entre les nombreuses dénominations. 
Deuxième Partie

\section{LES AMPHORES GRECQUES DU NORD DE L'ITALIE}

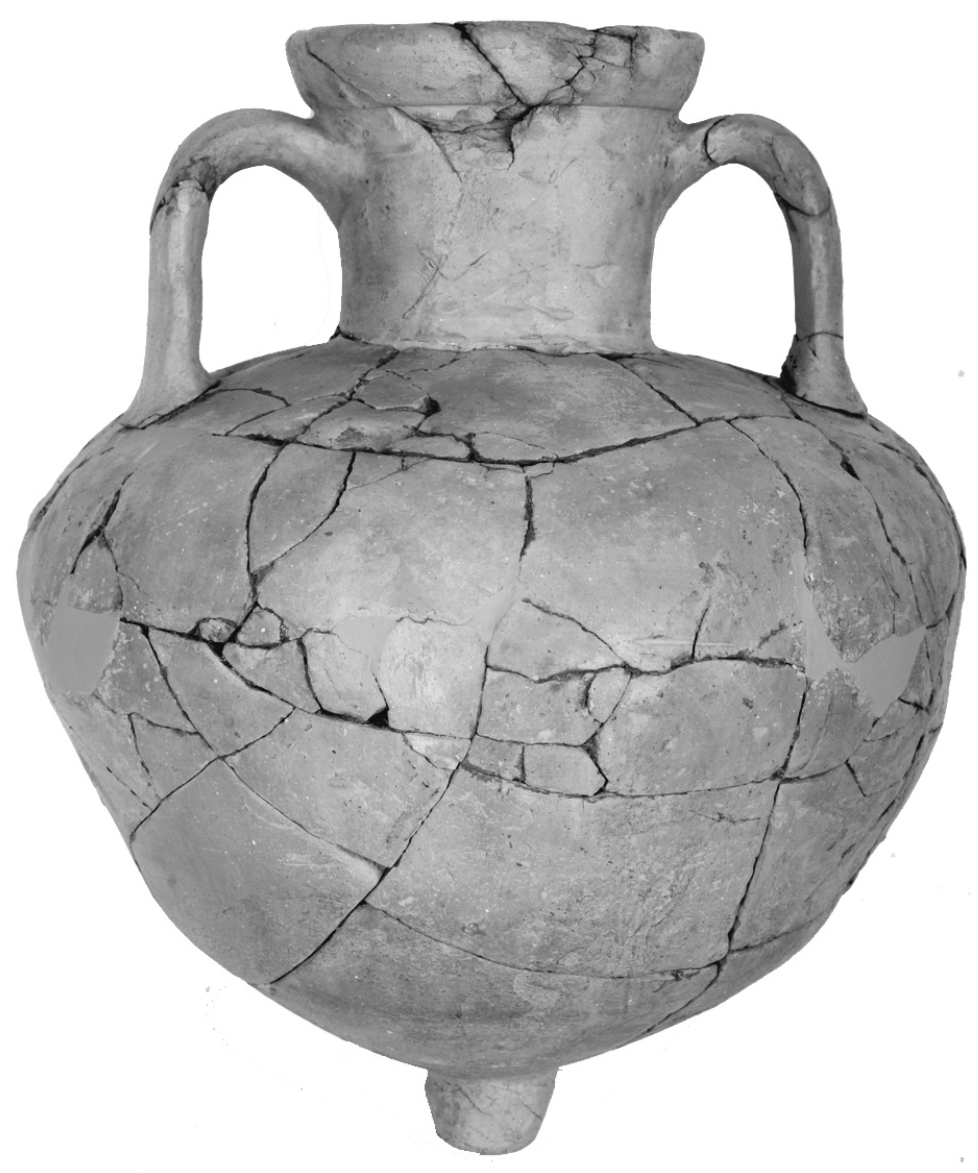



$\mathrm{L}$ a documentation qui a servi de base à l'étude des amphores de l'Italie septentrionale (fig. 69) est constituée pour l'essentiel par du matériel inédit, ou du matériel publié qu'il a été possible de réexaminer intégralement. Dans deux cas seulement, Spina et Forcello, on s'appuie exclusivement sur la documentation écrite.

Dans les chapitres de cette seconde partie, le matériel de l'Italie du nord est présenté selon les sites de découverte. Au début de chaque chapitre, une brève introduction présente l'état des recherches sur le site et sur le matériel qu'il a restitué, et notamment sur le matériel amphorique. L'étude analytique du matériel a été consignée dans un catalogue. L'analyse des données fait l'objet de la conclusion de chaque chapitre.

Un examen global des données ainsi obtenues et réélaborées est enfin proposé dans les observations conclusives dans la tentative de retracer le cadre historico-économique de la région comprise entre les Apennins et les Alpes pendant la période du plus grand développement de l'Étrurie padane et des plus nombreux contacts commerciaux avec la Grèce.

Chaque fiche de catalogue (dont on présente un index complet dans le tableau 1) est identifiée par un sigle constitué de deux lettres, correspondant à l'abréviation du nom du site de la découverte, et d'un chiffre, indiquant le numéro d'ordre de présentation des exemplaires à l'intérieur de chaque site.
Dans le catalogue, les objets sont regroupés par aire de production et, là où c'est possible, par type ${ }^{592}$. À l'intérieur de chaque regroupement par aire de production et par type, on a choisi un critère de présentation suivant l'ordre chronologique. Les objets dont il n'est pas possible d'identifier la production ou de déterminer la chronologie viennent à la suite des différents regroupements.

Sur chaque fiche de catalogue sont reportées les données relatives à chaque exemplaire, entier ou fragmentaire, dont on peut identifier des parties du vase morphologiquement révélatrices, ainsi que la lèvre, le col, les anses ou le pied. Dans les cas d'exemplaires fragmentaires, qui dans des contextes le plus souvent d'habitat (c'est le cas des sites examinés) représentent évidemment la totalité des exemplaires trouvés, chaque fiche concerne un ou plusieurs fragments qui n'ont été associés que dans le cas où la présence de fractures correspondantes permette de les attribuer à un seul individu. Lorsque l'appartenance à un même exemplaire n'est pas prouvée par la présence de parties coïncidentes, mais qu'elle est seulement supposée sur la base de la morphologie et/ou de la pâte, on a préféré d'établir des fiches distinctes. Dans ces cas, l'éventualité d'une appartenance à un seul individu a été à chaque fois signalée.

Le nombre des fiches du catalogue ne correspond donc pas au nombre d'exemplaires, dont les quantités minimale et maximale ont été calculées sur la base des lignes directrices en vigueur dans le cadre de l'étude et de la classification de matériel céramique ${ }^{593}$ (tableau 2).

592. Puisqu'il n'existe dans ce secteur, à l'heure actuelle, ni des critères typologiques uniques de référence ni un langage commun à tous les spécialistes, on renvoie, pour les définitions utilisées, à la première partie du volume.

593. Cf. Quantification des céramiques. 


\begin{tabular}{|c|c|c|}
\hline Cat. & Type & Chronologie \\
\hline AD1 & Corinthienne A' & 450-400 av. J.-C. \\
\hline AD2 & Corinthienne A' & Vers 450 av. J.-C. \\
\hline AD3 & Corinthienne B & 500-480 av. J.-C. \\
\hline AD4 & Corinthienne B & 500-480 av. J.-C. \\
\hline AD5 & Corinthienne B & 500-480 av. J.-C. \\
\hline AD6 & Corinthienne B & 480-460 av. J.-C. \\
\hline AD7 & Corinthienne B & 450-400 av. J.-C. \\
\hline AD8 & Gréco-occidentale « Forme $1 \alpha »$ & 550-490 av. J.-C. \\
\hline AD9 & Gréco-occidentale «Forme $1 \alpha »$ & 550-490 av. J.-C. \\
\hline AD10 & Gréco-occidentale « Forme $1 \alpha »$ & 550-490 av. J.-C. \\
\hline AD11 & Gréco-occidentale « Forme $1 \alpha »$ & 550-490 av. J.-C. \\
\hline AD12 & Nord-égéenne & 525-500 av. J.-C. \\
\hline AD13 & Nord-égéenne & 525-500 av. J.-C. \\
\hline AD14 & Chiote Swollen-necked, Early bulgy, variante C/2 Knigge-Lawall & 475-450 av. J.-C. \\
\hline AD15 & Samos-Milet, S/1 ou S/3 Lawall & 525-480 av. J.-C. \\
\hline AD16 & Samos-Milet, S/1 ou S/3 Lawall & 525-480 av. J.-C. \\
\hline SB1 & Corinthienne B & 500-425 av. J.-C. \\
\hline SB2 & Corinthienne B & 500-425 av. J.-C. \\
\hline SB3 & Corinthienne B & 500-425 av. J.-C. \\
\hline SB4 & Corinthienne B & Ve s. av. J.-C. \\
\hline SB5 & Corinthienne B & Non définissable \\
\hline SB6 & Corinthienne B & Non définissable \\
\hline SB7 & Corinthienne B & Non définissable \\
\hline SB8 & Gréco-occidentale « Forme $1 \alpha »$ ou corinthienne B & Non définissable \\
\hline SB9 & Gréco-occidentale « Forme $1 \alpha »$ ou corinthienne B & Non définissable \\
\hline SB10 & Gréco-occidentale «Forme $1 \alpha »$ ou corinthienne B & Non définissable \\
\hline SB11 & Gréco-occidentale « Forme $1 \alpha »$ & 575/550-490 av. J.-C. \\
\hline SB12 & Gréco-occidentale « Forme $1 \alpha »$ & 575/550-490 av. J.-C. \\
\hline SB13 & Gréco-occidentale « Forme $1 \alpha »$ & 575/550-490 av. J.-C. \\
\hline SB14 & Gréco-occidentale « Forme $1 \alpha »$ & 575/550-490 av. J.-C. \\
\hline SB15 & Gréco-occidentale «Forme $1 \alpha »$ & 575/550-490 av. J.-C. \\
\hline SB16 & Nord-égéenne & 525-480 av. J.-C. \\
\hline SB17 & Nord- égéenne, Disc-toe Lawall & 500-440 av. J.-C. \\
\hline SB18 & Nord- égéenne & Non définissable \\
\hline SB19 & Nord- égéenne & Non définissable \\
\hline SB20 & Nord- égéenne & Non définissable \\
\hline SB21 & Nord- égéenne & Non définissable \\
\hline SB22 & Chiote, Lambrino A ou Swollen-necked, Early bulgy, variante C/1 Knigge-Lawall & 550-475 av. J.-C. \\
\hline SB23 & Chiote, Swollen-necked & 500-425 av. J.-C. \\
\hline SB24 & Clazoménienne & 575/550-500 av. J.-C. \\
\hline SB25 & Milésienne & 575/550-525 av. J.-C. \\
\hline SB26 & Milésienne & 575/550-525 av. J.-C. \\
\hline
\end{tabular}




\begin{tabular}{|c|c|c|}
\hline Cat. & Type & Chronologie \\
\hline SB27 & Milésienne & 575/550-525 av. J.-C. \\
\hline SB28 & Milésienne & 575/550-525 av. J.-C. \\
\hline SB29 & Samos-Milet, S/1 Lawall & 525-480 av. J.-C. \\
\hline SB30 & Samos-Milet, S/3 Lawall & 525-480 av. J.-C. \\
\hline SB31 & Samos-Milet, S/3 Lawall & 525-480 av. J.-C. \\
\hline SB32 & Samos-Milet, S/1 Lawall & 450-400 av. J.-C. \\
\hline SB33 & Samos-Milet, S/1 ou S/3 Lawall & Non définissable \\
\hline SB34 & Solokha I & 450-400 av. J.-C. \\
\hline SB35 & Solokha I & 450-400 av. J.-C. \\
\hline $\mathrm{SC} 1$ & Gréco-occidentale «Forme $1 \alpha »$ & 510-490 av. J.-C. \\
\hline $\mathrm{SC} 2$ & Gréco-occidentale « Forme $1 \alpha »$ & 510-490 av. J.-C. \\
\hline $\mathrm{SC} 3$ & Gréco-occidentale «Forme $1 \alpha »$ & 510-490 av. J.-C. \\
\hline $\mathrm{SC} 4$ & Gréco-occidentale «Forme $1 \alpha »$ & 510-490 av. J.-C. \\
\hline SC5 & Gréco-occidentale « Forme $1 \alpha »$ & 510-490 av. J.-C. \\
\hline SC6 & Gréco-occidentale « Forme $1 \alpha »$ & 510-490 av. J.-C. \\
\hline $\mathrm{SC} 7$ & Gréco-occidentale « Forme $1 \alpha »$ & 510-490 av. J.-C. \\
\hline SC8 & Gréco-occidentale « Forme $1 \alpha »$ & 510-490 av. J.-C. \\
\hline SC9 & «À la brosse », forme Agora 1502 & 510-480 av. J.-C. \\
\hline $\mathrm{SC} 10$ & « À la brosse », forme Agora 1502 & 510-480 av. J.-C. \\
\hline SC11 & Non définissable & Non définissable \\
\hline LB1 & Corinthienne A & 500-450 av. J.-C. \\
\hline LB2 & Corinthienne B & Vers 450 av. J.-C. \\
\hline SP1 & Corinthienne A' & 500-450 av. J.-C. \\
\hline SP2 & Corinthienne B & 500-450 av. J.-C. \\
\hline SP3 & Corinthienne B & 500-450 av. J.-C. \\
\hline SP4 & Corinthienne B & 480-450 av. J.-C. \\
\hline SP5 & Corinthienne B & 480-425 av. J.-C. \\
\hline SP6 & Corinthienne B & 480-425 av. J.-C. \\
\hline SP7 & Corinthienne B & 480-425 av. J.-C. \\
\hline SP8 & Corinthienne B & 480-425 av. J.-C. \\
\hline SP9 & Corinthienne B & 480-425 av. J.-C. \\
\hline SP10 & Corinthienne B & 480-425 av. J.-C. \\
\hline SP11 & Corinthienne B & 480-425 av. J.-C. \\
\hline SP12 & Corinthienne B & 480-400 av. J.-C. \\
\hline SP13 & Corinthienne B & 425-400 av. J.-C. \\
\hline SP14 & Corinthienne B & 425-400 av. J.-C. \\
\hline SP15 & Gréco-occidentale « Forme $1 \alpha »$ & 550-490 av. J.-C. \\
\hline SP16 & Gréco-occidentale «Forme $1 \alpha »$ & 550-490 av. J.-C. \\
\hline SP17 & Gréco-occidentale « Forme $1 \alpha »$ & 550-490 av. J.-C. \\
\hline SP18 & Gréco-occidentale « Forme $1 \alpha »$ & 550-490 av. J.-C. \\
\hline SP19 & Gréco-occidentale « Forme $2 »$ & 540-500 av. J.-C. \\
\hline SP20 & Gréco-occidentale « Forme $2 »$ & 540-500 av. J.-C. \\
\hline
\end{tabular}




\begin{tabular}{|c|c|c|}
\hline Cat. & Type & Chronologie \\
\hline SP21 & Nord-égéenne, Middle Mendean variant Lawall & 450-425 av. J.-C. \\
\hline SP22 & Nord-égéenne, Middle Mendean variant Lawall & 450-425 av. J.-C. \\
\hline SP23 & Nord-égéenne, Middle Mendean variant Lawall & 450-425 av. J.-C. \\
\hline SP24 & Nord-égéenne, Middle Mendean variant Lawall & 450-425 av. J.-C. \\
\hline SP25 & Nord-égéenne, Ring-toe Lawall & 525-480 av. J.-C. \\
\hline SP26 & Nord-égéenne & 500-450 av. J.-C. \\
\hline SP27 & Nord-égéenne & 480-450 av. J.-C. \\
\hline SP28 & Nord-égéenne, Disc-toe Lawall & 480-440 av. J.-C. \\
\hline SP29 & Nord-égéenne & 450-400 av. J.-C. \\
\hline SP30 & Chiote, Swollen-necked, Early bulgy, variante C/1 ou C/2 Knigge-Lawall & 500-450 av. J.-C. \\
\hline SP31 & Chiote (?) & Non définissable \\
\hline SP32 & Chiote & Non définissable. \\
\hline SP33 & Samos-Milet, S/1 Lawall & 525-480 av. J.-C. \\
\hline SP34 & Solokha I & 410-390 av. J.-C. \\
\hline SP35 & Solokha I (?) & Non définissable \\
\hline SP36 & Solokha I & 410-350 av. J.-C. \\
\hline SP37 & Solokha I & 450-400 av. J.-C. \\
\hline SP38 & Solokha I & Vers 450 av. J.-C. \\
\hline SP39 & Solokha I & Vers 450 av. J.-C. \\
\hline SP40 & Solokha I & 400-350 av. J.-C. \\
\hline FO1 & Corinthienne A & Vers le milieu du Ve s. av. J.-C. (?) \\
\hline $\mathrm{FO} 2$ & Corinthienne A' & Milieu-deuxième moitié du Ve s. av. J.-C. (?) \\
\hline FO3 & Corinthienne A & 450-400 av. J.-C. (?) \\
\hline FO4 & Corinthienne A' & 450-400 av. J.-C. (?) \\
\hline FO5 & Corinthienne A' & 450-400 av. J.-C. \\
\hline FO6 & Corinthienne A' & 450-400 av. J.-C. (?) \\
\hline FO7 & Corinthienne A' & V sec. av. J.-C. \\
\hline FO8 & Corinthienne A & Non définissable \\
\hline FO9 & Corinthienne A' & Non définissable \\
\hline FO10 & Corinthienne A' & Non définissable \\
\hline FO11 & Corinthienne B & 525-480 av. J.-C. \\
\hline FO12 & Corinthienne B & Débuts du Ve s. av. J.-C. \\
\hline FO13 & Corinthienne B & Débuts du Ve s. av. J.-C. \\
\hline FO14 & Corinthienne B & 490-480 av. J.-C. \\
\hline FO15 & Corinthienne B & Ve s. av. J.-C. \\
\hline FO16 & Corinthienne B & Vers 450 av. J.-C. \\
\hline FO17 & Corinthienne B & Vers 450 av. J.-C. \\
\hline FO18 & Corinthienne B & 425-400 av. J.-C. \\
\hline FO19 & Corinthienne B & Non définissable \\
\hline FO20 & Corinthienne B & Non définissable \\
\hline FO21 & Corinthienne B & Non définissable \\
\hline FO22 & Gréco-occidentale « Forme $1 \alpha »$ & 530-520 av. J.-C. \\
\hline FO23 & Gréco-occidentale « Forme $1 \alpha »$ & 520-500 av. J.-C. \\
\hline
\end{tabular}




\begin{tabular}{|c|c|c|}
\hline Cat. & Type & Chronologie \\
\hline $\mathrm{FO} 24$ & Gréco-occidentale « Forme $1 \alpha »$ & 520-500 av. J.-C. \\
\hline $\mathrm{FO} 25$ & Gréco-occidentale « Forme $2 »$ & 540-520 av. J.-C. \\
\hline FO26 & Gréco-occidentale « Forme $2 »$ & 525-500 av. J.-C. \\
\hline FO27 & Gréco-occidentale « Forme $3 »(?)$ & 475-450 av. J.-C. \\
\hline FO28 & Nord-égéenne, Early Meandean Variant Lawall & 525-480 av. J.-C. \\
\hline FO29 & Nord-égéenne, Middle Mendean variant Lawall & $450-425$ av. J.-C. \\
\hline FO30 & Nord-égéenne & 450-400 av. J.-C. \\
\hline FO31 & Nord-égéenne, Late Mendean variant Lawall & 425-400 av. J.-C. \\
\hline FO32 & Nord-égéenne, Late Mendean variant Lawall & 425-400 av. J.-C. \\
\hline FO33 & Nord-égéenne, Late Mendean variant Lawall & 425-400 av. J.-C. \\
\hline FO34 & Nord-égéenne & Non définissable \\
\hline FO35 & Nord-égéenne & Non définissable \\
\hline FO36 & Nord-égéenne & Non définissable \\
\hline FO37 & Nord-égéenne & Non définissable \\
\hline FO38 & Nord-égéenne & Non définissable \\
\hline FO39 & Nord-égéenne, Ring-toe Lawall & 520-490 av. J.-C. \\
\hline FO40 & Nord-égéenne, Ring-toe Lawall & 520-490 av. J.-C. \\
\hline FO41 & Nord-égéenne, Ring-toe Lawall & 520-490 av. J.-C. \\
\hline FO42 & Nord-égéenne, Ring-toe Lawall & 520-490 av. J.-C. \\
\hline FO43 & Nord-égéenne, Ring-toe Lawall & 520-490 av. J.-C. \\
\hline FO44 & Nord-égéenne o greco-orientale (?) & Vers le milieu du V V s. av. J.-C. \\
\hline FO45 & Nord-égéenne & Vers 475 av. J.-C. \\
\hline FO46 & Nord-égéenne & Non définissable \\
\hline FO47 & Chiote, Lambrino A 2 & 510-490 av. J.-C. \\
\hline FO48 & Chiote, Swollen-necked, Early bulgy, variante C/1 Knigge-Lawall & 500-475 av. J.-C. \\
\hline FO49 & Chiote, Swollen-necked, Early bulgy, variante C/1 Knigge-Lawall & 500-475 av. J.-C. \\
\hline FO50 & Chiote, Swollen-necked, Early bulgy, variante C/1 Knigge-Lawall & 500-475 av. J.-C. \\
\hline FO51 & Chiote, Swollen-necked, Early bulgy, variante C/1 ou C/2 Knigge-Lawall & 500-450 av. J.-C. \\
\hline FO52 & Chiote, Swollen-necked, Early bulgy, variante C/2 Knigge-Lawall & 475-450 av. J.-C. \\
\hline FO53 & Chiote, Swollen-necked, Early bulgy, variante C/2 Knigge-Lawall & 475-450 av. J.-C. \\
\hline FO54 & Chiote, Swollen-necked, Early bulgy, variante C/2 Knigge-Lawall & 475-450 av. J.-C. \\
\hline FO55 & Chiote, Swollen-necked, Early bulgy, variante C/2 Knigge-Lawall & 475-450 av. J.-C. \\
\hline FO56 & Chiote, Swollen-necked, Later bulgy, variante C/3 Knigge-Lawall o straight-neck & 450-400 av. J.-C. \\
\hline FO57 & Samos-Milet & Fin VI'-débuts $\mathrm{V}^{\mathrm{e}}$ s. av. J.-C. \\
\hline FO58 & Samos-Milet, S/2 Lawall & 480-450 av. J.-C. \\
\hline FO59 & Samos-Milet, S/2 Lawall & 480-450 av. J.-C. \\
\hline FO60 & Samos-Milet, S/1 Lawall & 480-450 av. J.-C. \\
\hline FO61 & Samos-Milet, S/1 Lawall & 480-450 av. J.-C. \\
\hline FO62 & Samos-Milet, S/1 Lawall & 480-450 av. J.-C. \\
\hline FO63 & Samos-Milet, S/1 Lawall & 480-450 av. J.-C. \\
\hline FO64 & Samos-Milet, S/1 Lawall & 450-400 av. J.-C. \\
\hline FO65 & Samos-Milet, S/1 Lawall & 450-400 av. J.-C. \\
\hline FO66 & Samos-Milet & 450-400 av. J.-C. \\
\hline FO67 & Samos-Milet & 450-400 av. J.-C. \\
\hline
\end{tabular}




\begin{tabular}{|c|c|c|}
\hline Cat. & Type & Chronologie \\
\hline FO68 & Samos-Milet, S/1 Lawall & 410-380 av. J.-C. \\
\hline FO69 & Solokha I & 450-400 av. J.-C. \\
\hline FO70 & Solokha I & 450-400 av. J.-C. \\
\hline FO71 & Solokha I & 450-400 av. J.-C. \\
\hline FO72 & Solokha I & 450-400 av. J.-C. \\
\hline FO73 & Solokha I & 450-400 av. J.-C. \\
\hline FO74 & Solokha I & 450-400 av. J.-C. \\
\hline FO75 & Solokha I & 450-400 av. J.-C. \\
\hline FO76 & Solokha I & 450-400 av. J.-C. \\
\hline FO77 & Solokha I & 450-400 av. J.-C. \\
\hline FO78 & « À la brosse », forme Agora 1502 & Fin VIe-début Ve s. av. J.-C. \\
\hline FO79 & «À la brosse » & Fin VI'-début Ve s. av. J.-C. (?) \\
\hline FE1 & Corinthienne $\mathrm{A} / \mathrm{A}^{\prime}$ & $\mathrm{V}^{\mathrm{e}}$ s. av. J.-C. \\
\hline FE2 & Corinthienne $\mathrm{A} / \mathrm{A}^{\prime}$ & Non définissable \\
\hline FE3 & Corinthienne B & 480-400 av. J.-C. \\
\hline FE4 & Nord-égéenne & Fin Ve s. av. J.-C. \\
\hline FE5 & Nord-égéenne & Fin Ve-début IVe s. av. J.-C. \\
\hline FE6 & Samos-Milet & 525-480 av. J.-C. \\
\hline FE7 & Solokha I & 450-400 av. J.-C. \\
\hline FE8 & Solokha I & 450-400 av. J.-C. \\
\hline FE9 & Solokha I & 450-400 av. J.-C. \\
\hline FE10 & Solokha I & 410-390 av. J.-C. \\
\hline FE11 & Solokha I & 410-390 av. J.-C. \\
\hline FE12 & Solokha I & 450-400 av. J.-C. \\
\hline FE13 & Solokha I & 450-400 av. J.-C. \\
\hline FE14 & Solokha I & 450-400 av. J.-C. \\
\hline FE15 & Solokha I & 450-400 av. J.-C. \\
\hline FE16 & Solokha I & Non définissable \\
\hline MA1 & Corinthienne A & 580-540 av. J.-C. \\
\hline MA2 & Corinthienne A & Milieu VIe-début Ve sec. av. J.-C. \\
\hline MA3 & Corinthienne A & Milieu VI'-début Ve sec. av. J.-C. \\
\hline MA4 & Corinthienne A & Milieu VI'-début Ve sec. av. J.-C. \\
\hline MA5 & Corinthienne A & Fin VIe-début $\mathrm{V}^{\mathrm{e}}$ s. av. J.-C. \\
\hline MA6 & Corinthienne A & Fin $\mathrm{VI}^{\mathrm{e}}-\mathrm{V}^{\mathrm{e}}$ s. av. J.-C. \\
\hline MA7 & Corinthienne A & Fin $\mathrm{VI}^{\mathrm{e}}-\mathrm{V}^{\mathrm{e}} \mathrm{s}$. av. J.-C. \\
\hline MA8 & Corinthienne A & Fin $\mathrm{VI}^{\mathrm{e}}-\mathrm{V}^{\mathrm{e}}$ s. av. J.-C. \\
\hline MA9 & Corinthienne A & Fin $\mathrm{VI}^{\mathrm{e}}-\mathrm{V}^{\mathrm{e}}$ s. av. J.-C. \\
\hline MA10 & Corinthienne A' & Fin $\mathrm{VI}^{\mathrm{e}}-\mathrm{V}^{\mathrm{e}}$ s. av. J.-C. \\
\hline MA11 & Corinthienne A' & Fin $\mathrm{VI}^{\mathrm{e}}-\mathrm{V}^{\mathrm{e}} \mathrm{s}$ s. av. J.-C. \\
\hline MA12 & Corinthienne A' & Fin $\mathrm{VI}^{\mathrm{e}}-\mathrm{V}^{\mathrm{e}}$ s. av. J.-C. \\
\hline MA13 & Corinthienne A' & Fin $\mathrm{VI}^{\mathrm{e}}-\mathrm{V}^{\mathrm{e}}$ s. av. J.-C. \\
\hline MA14 & Corinthienne A' & Fin $\mathrm{VI}^{\mathrm{e}}-\mathrm{V}^{\mathrm{e}} \mathrm{s}$. av. J.-C. \\
\hline MA15 & Corinthienne $A^{\prime}$ & Fin $\mathrm{VI}^{\mathrm{e}}-\mathrm{V}^{\mathrm{e}} \mathrm{s}$ s. av. J.-C. \\
\hline
\end{tabular}




\begin{tabular}{|c|c|c|}
\hline Cat. & Type & Chronologie \\
\hline MA16 & Corinthienne A' & Fin $\mathrm{VI}^{\mathrm{e}}-\mathrm{V}^{\mathrm{e}}$ s. av. J.-C. \\
\hline MA17 & Corinthienne A' & Fin $\mathrm{VI}^{\mathrm{e}}-\mathrm{V}^{\mathrm{e}} \mathrm{s}$. av. J.-C. \\
\hline MA18 & Corinthienne A' & Fin $\mathrm{VI}^{\mathrm{e}}-\mathrm{V}^{\mathrm{e}} \mathrm{s}$. av. J.-C. \\
\hline MA19 & Corinthienne A' & Milieu Ve-début IVe s. av. J.-C. \\
\hline MA20 & Corinthienne A' & Milieu Ve-début IVe s. av. J.-C. \\
\hline MA21 & Corinthienne A' & Milieu Ve-début IVe s. av. J.-C. \\
\hline MA22 & Corinthienne A' & Milieu Ve-début IVe s. av. J.-C. \\
\hline MA23 & Corinthienne A' & Milieu Ve-début IVe s. av. J.-C. \\
\hline MA24 & Corinthienne A & Fin Ve-début IV $\mathrm{V}^{\mathrm{e}}$ s. av. J.-C. \\
\hline MA25 & Corinthienne A & Non définissable \\
\hline MA26 & Corinthienne A & Non définissable \\
\hline MA27 & Corinthienne A & Non définissable \\
\hline MA28 & Corinthienne A & Non définissable \\
\hline MA29 & Corinthienne A' & Non définissable \\
\hline MA30 & Corinthienne A' & Non définissable \\
\hline MA31 & Corinthienne A' & Non définissable \\
\hline MA32 & Corinthienne A' & Non définissable \\
\hline MA33 & Corinthienne B & 480-425 av. J.-C. \\
\hline MA34 & Corinthienne B & 480-425 av. J.-C. \\
\hline MA35 & Corinthienne B & 425-400 av. J.-C. \\
\hline MA36 & Corinthienne B & 425-400 av. J.-C. \\
\hline MA37 & Gréco-occidentale « Forme $2 »$ & Milieu VI'-débuts Ve s. av. J.-C. \\
\hline MA38 & Nord-égéenne, Ring-toe Lawall & 525-480 av. J.-C. \\
\hline MA39 & Nord-égéenne, Ring-toe Lawall & 525-480 av. J.-C. \\
\hline MA40 & Nord-égéenne & 525-480 av. J.-C. \\
\hline MA41 & Nord-égéenne & Fin $\mathrm{VI}^{\mathrm{e}}$-début $\mathrm{V}^{\mathrm{e}} \mathrm{s}$. av. J.-C. \\
\hline MA42 & Nord-égéenne & Fin $\mathrm{VI}^{\mathrm{e}}$-début $\mathrm{V}^{\mathrm{e}} \mathrm{s}$. av. J.-C. \\
\hline MA43 & Nord-égéenne, Disc-toe Lawall & 480-440 av. J.-C. \\
\hline MA44 & Nord-égéenne & 475-450 av. J.-C. \\
\hline MA45 & Nord-égéenne, Middle Mendean variant Lawall & 450-425 av. J.-C. \\
\hline MA46 & Nord-égéenne, Stem-toe, variant 2 Lawall & 450-400 av. J.-C. \\
\hline MA47 & Nord-égéenne, Late Mendean variant Lawall & 425-400 av. J.-C. \\
\hline MA48 & Nord-égéenne, Late Mendean variant Lawall & 425-400 av. J.-C. \\
\hline MA49 & Nord-égéenne, Late Mendean variant Lawall & 425-400 av. J.-C. \\
\hline MA50 & Nord-égéenne, Late Mendean variant Lawall & 425-400 av. J.-C. \\
\hline MA51 & Nord-égéenne, Biconical Lawall & 425-400 av. J.-C. \\
\hline MA52 & Nord-égéenne & Non définissable \\
\hline MA53 & Nord-égéenne & Non définissable \\
\hline MA54 & Nord-égéenne & Non définissable \\
\hline MA55 & Nord-égéenne & Non définissable \\
\hline MA56 & Nord-égéenne & Non définissable \\
\hline MA57 & Nord-égéenne & Non définissable \\
\hline MA58 & Nord-égéenne & Non définissable \\
\hline
\end{tabular}




\begin{tabular}{|c|c|c|}
\hline Cat. & Type & Chronologie \\
\hline MA59 & Chiote, Swollen-necked, Early bulgy, variante C/1 ou C/2 Knigge-Lawall & 500-450 av. J.-C. \\
\hline MA60 & Chiote, Swollen-necked, Early bulgy, variante C/1 ou C/2 Knigge-Lawall & 500-450 av. J.-C. \\
\hline MA61 & Chiote, Swollen-necked, Later bulgy, variante C/3 Knigge-Lawall & 450-425 av. J.-C. \\
\hline MA62 & Chiote, Swollen-necked, Later bulgy, variante C/3 Knigge-Lawall ou Straight-neck & 450-400 av. J.-C. \\
\hline MA63 & Chiote, Swollen-necked & 500-425 av. J.-C. \\
\hline MA64 & Chiote, Swollen-necked & 500-425 av. J.-C. \\
\hline MA65 & Chiote & Non définissable \\
\hline MA66 & Samos-Milet, S/2 Lawall & 525-480 av. J.-C. \\
\hline MA67 & Samos-Milet, S/1 Lawall & 525-480 av. J.-C. \\
\hline MA68 & Samos-Milet, S/1 ou S/3 Lawall & 525-480 av. J.-C. \\
\hline MA69 & Samos-Milet, S/1 Lawall & 525-450 av. J.-C. \\
\hline MA70 & Samos-Milet, S/1 Lawall & 525-450 av. J.-C. \\
\hline MA71 & Samos-Milet, S/2 Lawall & 525-450 av. J.-C. \\
\hline MA72 & Samos-Milet, S/2 ou S/3 Lawall & 525-450 av. J.-C. \\
\hline MA73 & Samos-Milet, S/2 Lawall & 525-450 av. J.-C. \\
\hline MA74 & Samos-Milet, S/2 Lawall & 525-450 av. J.-C. \\
\hline MA75 & Samos-Milet, S/1 Lawall & 525-450 av. J.-C. \\
\hline MA76 & Samos-Milet, S/1 Lawall & 480-450 av. J.-C. \\
\hline MA77 & Samos-Milet, S/1 Lawall & 480-400 av. J.-C. \\
\hline MA78 & Samos-Milet, S/1 Lawall & 480-400 av. J.-C. \\
\hline MA79 & Samos-Milet, S/1 Lawall & 450-400 av. J.-C. \\
\hline MA80 & Samos-Milet, S/1 Lawall & 450-400 av. J.-C. \\
\hline MA81 & Samos-Milet, S/1 Lawall & 450-400 av. J.-C. \\
\hline MA82 & Samos-Milet, S/1 Lawall & 450-400 av. J.-C. \\
\hline MA83 & Samos-Milet (?) & Non définissable \\
\hline MA84 & Samos-Milet (?) & Non définissable \\
\hline MA85 & Samos-Milet (?) & Non définissable \\
\hline MA86 & Samos-Milet (?) & Non définissable \\
\hline MA87 & Samos-Milet (?) & Non définissable \\
\hline MA88 & Samos-Milet (?) & Non définissable \\
\hline MA89 & Gréco-orientale & Non définissable \\
\hline MA90 & Gréco-orientale & Non définissable \\
\hline MA91 & Gréco-orientale & Non définissable \\
\hline MA92 & Gréco-orientale & Non définissable \\
\hline MA93 & Solokha I & Vers 450 av. J.-C. \\
\hline MA94 & Solokha I & Vers 450 av. J.-C. \\
\hline MA95 & Solokha I & 450-400 av. J.-C. \\
\hline MA96 & Solokha I & 450-400 av. J.-C. \\
\hline MA97 & Solokha I & 450-400 av. J.-C. \\
\hline MA98 & Solokha I & 410-390 av. J.-C. \\
\hline MA99 & Solokha I & 450-400 av. J.-C. \\
\hline MA100 & Samos-Milet & fin $\mathrm{VI}^{\mathrm{e}}$-début $\mathrm{V}^{\mathrm{e}}$ s. av. J.-C. \\
\hline MA101 & Solokha I & 450-400 av. J.-C. \\
\hline
\end{tabular}




\begin{tabular}{|c|c|c|}
\hline Cat. & Type & Chronologie \\
\hline MA102 & Solokha I & 450-400 av. J.-C. \\
\hline MA103 & Solokha I & 450-400 av. J.-C. \\
\hline MA104 & Solokha I & 450-400 av. J.-C. \\
\hline MA105 & Solokha I & 450-400 av. J.-C. \\
\hline MA106 & Solokha I & 450-400 av. J.-C. \\
\hline MA107 & Solokha I & Non définissable \\
\hline MA108 & Solokha I & Non définissable \\
\hline MA109 & Solokha I & Non définissable \\
\hline MA110 & Solokha I & Non définissable \\
\hline MA111 & Solokha I & Non définissable \\
\hline MA112 & Non définissable & Non définissable \\
\hline MA113 & Non définissable & Fin $\mathrm{VI}^{\mathrm{e}}$ s. av. J.-C. \\
\hline MA114 & Corinthienne A/A' & 450-375 av. J.-C. \\
\hline MA115 & Corinthienne A/A' & 450-375 av. J.-C. \\
\hline MA116 & Corinthienne B & 480-400 av. J.-C. \\
\hline MA117 & Gréco-occidentale « Forme $1 \alpha »$ & 550-490 av. J.-C. \\
\hline MA118 & Gréco-occidentale « Forme $2 »$ & 540-500 av. J.-C. \\
\hline MA119 & Nord-égéenne & 525-500 av. J.-C. \\
\hline MA120 & Nord-égéenne & 525-500 av. J.-C. \\
\hline MA121 & Nord-égéenne, Stem toe, variant 2 Lawall & 450-400 av. J.-C. \\
\hline MA122 & Nord-égéenne, Biconical Lawall & 425-400 av. J.-C. \\
\hline MA123 & Nord-égéenne, Biconical Lawall & 425-400 av. J.-C. \\
\hline MA124 & Nord-égéenne & Non définissable \\
\hline MA125 & Nord-égéenne & 525-480 av. J.-C. \\
\hline MA126 & Nord-égéenne, double-banded rim & Fin $\mathrm{VI}^{\mathrm{e}}$ s. av. J.-C. \\
\hline MA127 & Nord-égéenne, Stem-toe, variante 2 Lawall & Deuxième moitié du V $\mathrm{V}^{\mathrm{e}} \mathrm{s}$. av. J.-C. \\
\hline MA128 & Samos-Milet, S/1 Lawall & 525-480 av. J.-C. \\
\hline MA129 & Samos-Milet, S/2 Lawall & 480-450 av. J.-C. \\
\hline MA130 & Samos-Milet & 525-480 av. J.-C. \\
\hline MA131 & Samos-Milet & 525-450 av. J.-C. \\
\hline MA132 & Samos-Milet & 525-450 av. J.-C. \\
\hline MA133 & Samos-Milet & 525-450 av. J.-C. \\
\hline MA134 & Samos-Milet, S/2 Lawall & 525-450 av. J.-C. \\
\hline MA135 & Samos-Milet, S/2 Lawall & 525-450 av. J.-C. \\
\hline MA136 & Samos-Milet, S/2 Lawall & 525-450 av. J.-C. \\
\hline MA137 & Samos-Milet, S/2 Lawall & 525-450 av. J.-C. \\
\hline MA138 & Samos-Milet, S/1 Lawall & 450-400 av. J.-C. \\
\hline MA139 & Solokha I & 450-400 av. J.-C. \\
\hline MA140 & Solokha I & 450-400 av. J.-C. \\
\hline MA141 & Solokha I & 450-400 av. J.-C. \\
\hline MA142 & Samos-Milet, S/1 ou S/2 Lawall & Non définissable \\
\hline MA143 & Samos-Milet & Non définissable \\
\hline
\end{tabular}

Tableau 1. Tableau de synthèse des fiches du catalogue indiquant le numéro d'identification de chaque fiche, l'aire de production, le type et la chronologie. 


\begin{tabular}{|l|c|c|c|c|}
\hline \multicolumn{1}{|c|}{ Site } & Code Catalogue & $\begin{array}{c}\text { Total Fiches } \\
\text { Catalogue }\end{array}$ & $\begin{array}{c}\text { Nombre minimum } \\
\text { d'individus }\end{array}$ & $\begin{array}{c}\text { Nombre maximum } \\
\text { d'individus }\end{array}$ \\
\hline Adria & AD & 16 & 15 & 16 \\
\hline San Basilio & SB & 35 & 35 & 11 \\
\hline San Cassiano & SC & 11 & 10 & 2 \\
\hline Le Balone & LB & 2 & 40 & 40 \\
\hline Spina & SP & 40 & 75 & 79 \\
\hline Forcello & FO & 79 & 16 & 143 \\
\hline Bologna/Felsina & FE & 16 & 131 & Total maximum : 342 \\
\hline Marzabotto & MA & 143 & Total minimum $: 324$ & \\
\hline
\end{tabular}

Tableau 2. Tableau indiquant le total des fiches du catalogue et le nombre relatif minimum et maximum d'exemplaires. 


\section{Chapitre 1}

\section{Le littoral et la chora d'Adria}

$\mathrm{L}$ es recherches effectuées au cours des vingt dernières années ont contribué de manière importante au développement de nos connaissances sur les phases des époques archaïque et classique d'Adria, dont le rôle stratégique dans le cadre de l'Adriatique ressort clairement de la racine commune des termes Adria-as et Adrias-ou utilisés par les Grecs pour définir respectivement la cité et la mer qui lui faisait face.

Une série d'interventions de fouilles programmées et quelques découvertes occasionnelles ont aussi permis d'identifier quelques sites proches de la cité d'Adria et sans doute reliés à celle-ci. Parmi ces centres, trois, San Basilio di Ariano Polesine, San Cassiano di Crespino et Le Balone di Rovigo, ont restitué des amphores commerciales grecques (fig. 70).

\section{Adria (prov. de Rovigo) : les nécropoles et l'habitat (fig. 69)}

\subsection{Bilan des études}

Adria a été l'un des centres les plus anciens et les plus importants des gros trafics commerciaux qui ont envahi le territoire padan dès la première moitié $\mathrm{du} \mathrm{VI}^{\mathrm{e}} \mathrm{s}$. av. J.-C.

La fondation d'Adria remonte au début du $\mathrm{VI}^{\mathrm{e}} \mathrm{s}$. av. J.-C., et en tout cas bien avant celle de Spina, non seulement en raison de la précocité de ses premiers contacts commerciaux avec l'aire daunienne (Bonomi 2003, p. 140 n. 1), datables vers la fin du VII ${ }^{\mathrm{e}}$ et le début du

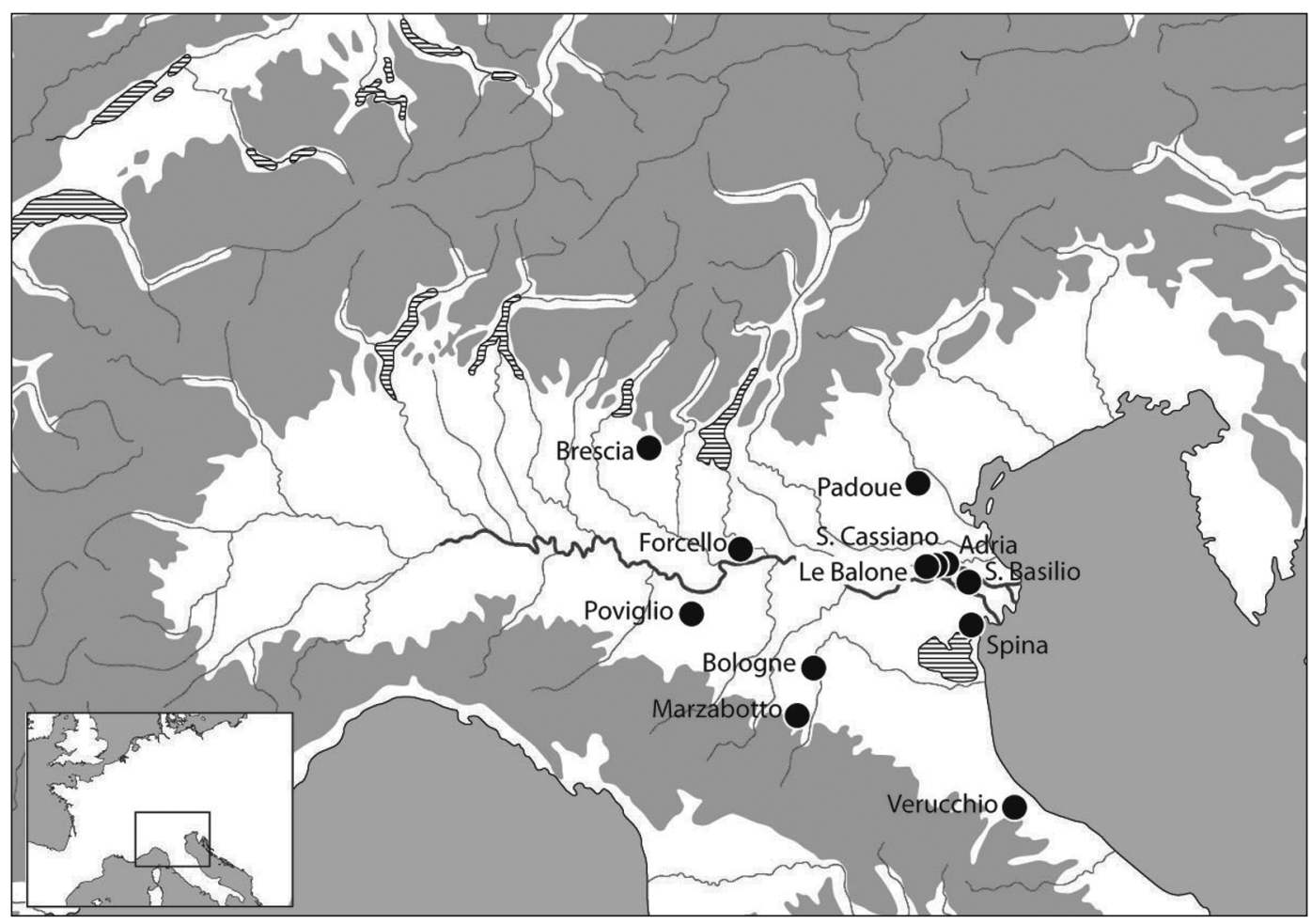

Fig. 69. Carte du Nord de l'Italie. Emplacement des sites concernés par les échanges d'amphores commerciales grecques dans le secteur compris entre les Apennins et les Alpes (F. Sacchetti). 


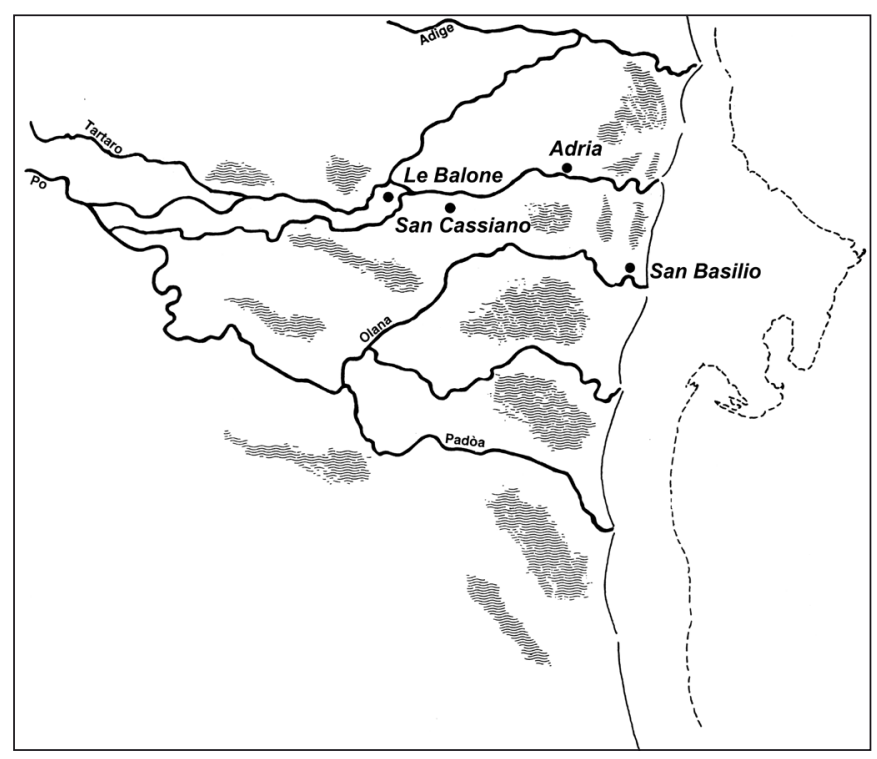

Fig. 70. Carte du delta septentrional du Pô à l'époque protohistorique (en ligne continue le littoral fossile ; en pointillé le littoral actuel). Emplacement des sites qui ont livré des amphores grecques (DAO F. Sacchetti d'après Peretto 1994, fig. 5).

$\mathrm{VI}^{\mathrm{e}} \mathrm{s}$. av. J.-C., et avec le monde grec, dont sont témoins la céramique du corinthien moyen datée de 580-570 av. J.-C. (Bonomi 1991, pl. I, 5-6 ; Ead. 2000a, p. 122) et la céramique attique contemporaine (Bonomi 1991, pl. 35, 1 ; Ead. 1997, p. 32, fig. 20 ; Ead. 2000a, p. 122123) - donc antérieures d'un demi siècle environ à la documentation de Spina - mais aussi par le fait que ce fut Adria et non pas Spina qui donna son nom au secteur septentrional de la mer sur laquelle donnaient les deux cités portuaires ${ }^{594}$.

Le site choisi se trouvait dans une zone particulièrement stratégique du point de vue de l'activité commerciale. La cité, située dans un territoire marécageux et sujet aux divagations du Pô (le Pô d'Adria des sources littéraires), se trouvait à l'époque à 14 kilomètres seulement de la mer et pouvait en même temps disposer des matières premières, des ressources agricoles et de l'élevage offertes par son riche arrière-pays.

D'après les données archéologiques, et en particulier épigraphiques, l'aire d'Adria devait être habitée par des Vénètes et fréquentée dans un but commercial par des

594. Sur la question complexe de la plus ancienne présence grecque dans l'Adriatique septentrionale et du rapport entre Adria et Spina, dont les grandes lignes sont présentées dans la contribution toujours fondamentale de G. Colonna (Colonna 1974), on renvoie à la récente mise à jour du même spécialiste (Id. 2003). Pour une synthèse et une mise à jour des plus anciennes importations grecques d'Adria, cf. Bonomi 2000a. Pour les premières importations grecques de Spina, datées entre 540 et 520 av. J.-C., cf., en dernier, Bruni 2004, notamment p. 84.
Grecs et des Étrusques, qui s’intégrèrent bientôt au tissu social de la cité. Quant aux Grecs, que les inscriptions ont révélé être des Éginètes, on discute encore s'ils étaient organisés en une véritable apoikia, plus précisément celle qui d'après Strabon (VIII, 6, 16) fut envoyée par Égine dans la deuxième moitié du $\mathrm{VI}^{\mathrm{e}} \mathrm{s}$. av. J.-C. ${ }^{595}$, ou bien s'ils s'étaient simplement insérés dans un emporion déjà actif et préexistant (Braccesi 1977, p. 128-133). En ce qui concerne les Étrusques, il s'agissait probablement de peuples de l'Étrurie méridionale intérieure, en particulier de Volsinii ${ }^{596}$, dont la présence est attestée par les données archéologiques à partir du milieu du $\mathrm{VI}^{\mathrm{e}} \mathrm{s}$. av. J.-C. et qui furent peut-être remplacés seulement dans le courant du Ve s. av. J.-C. par les Étrusques padans ${ }^{597}$. La conséquence de la situation favorable de l'emporion fluvial d'Adria fut l'accroissement démographique et le développement économique de tout le territoire avoisinant. On peut situer vers le dernier quart du $\mathrm{VI}^{\mathrm{e}} \mathrm{s}$. av. J.-C. la transformation d'Adria d'emporion ( «Adria I ») en polis («Adria II ») ${ }^{598}$. L'un des indices les plus évidents du nouveau statut politico-administratif du site est l'éclosion dans son arrière-pays de plusieurs habitats dont témoignent le plus souvent des trouvailles sporadiques, à Taglio di Po, Borsea, Contarina et Gavello ${ }^{599}$, et quelquefois une documentation plus consistante dans les cas de l'emporion de San Basilio, du complexe d'habitations de San Cassiano et de la petite nécropole, avec la zone afférente d'habitat, de Le Balone.

595. La présence à Adria de Grecs, et plus particulièrement d'Éginètes, est clairement attestée par la documentation épigraphique (Colonna 1974 ; Guarducci 1978), peut-être dans une véritable apoikia, dont la présence dans le territoire des « Umbri » est documentée également par les sources littéraires (Colonna 1974, p. 10-13; Id. 2003).

596. Pour les composantes ethno-linguistiques d'Adria, identifiées grâce à la documentation épigraphique, on renvoie aux contributions de Colonna et Guarducci citées à la note précédente et, en dernier lieu, à Maggiani 2002, notamment, p. 57-58, avec d'amples références bibliographiques, et Colonna 2003, p. 166-168, avec bibliographie. Pour les récentes acquisitions sur les phases de l'habitat d'Adria et sur ses composantes culturelles, Bonomi, Camerin, Tamassia 2003, notamment p. 207, avec références bibliographiques.

597. C'est vers le milieu du VI' $\mathrm{s}$. av. J.-C. que l'on fait remonter les premières attestations de bucchero à Adria et c'est entre 520 et 460 av. J.-C. que l'on situe les inscriptions étrusques, volsiniennes, documentées dans la cité (cf. bibliographie citée à la note précédente). Pour les données épigraphiques et archéologiques concernant l'introduction d'Adria dans les itinéraires commerciaux étrusco-padans, on renvoie respectivement à Maggiani 2002, p. 58 et n. 25, et à Bonomi 2003.

598. Pour les définitions politico-institutionnelles «Adria emporion » et "Adria polis », cf. en dernier Colonna 2003 ; pour les définitions archéologiques chronologiquement correspondantes «Adria I » et « Adria II », cf. Donati, Parrini 1999.

599. Pour la documentation sporadique relative à ces localités du territoire d'Adria cf. De Min, Iacopazzi 1986, p. 171-172 ; et en dernier, Peretto, Vallicelli, Wiel-Marin 2003, avec bibliographie. 
Les nécropoles découvertes autour de la ville d'Adria sont presque exclusivement datées de l'époque hellénistique et elles ont donc livré principalement des amphores des $\mathrm{IV}^{\mathrm{e}}$, $\mathrm{III}^{\mathrm{e}}$ et $\mathrm{II}^{\mathrm{e}}$ s. av. J.-C. qui ont été intégralement publiées par A. Toniolo (Toniolo 2000). Les sépultures les plus anciennes, datables de la fin du $\mathrm{VI}^{\mathrm{e}}$ et du $\mathrm{V}^{\mathrm{e}} \mathrm{s}$. av. J.-C., découvertes occasionnellement dans les différents groupes de nécropoles, ne contenaient pas d'amphores. On ne connaissait qu'une amphore seulement d'Adria appartenant à l'époque tardo-archaïque et provenant probablement d'une des nécropoles, vu qu'elle était conservée intégralement. L'exemplaire, conservé aujourd'hui dans le Musée Archéologique National d'Adria, a été découvert au XIX ${ }^{\mathrm{e}}$ siècle et intégré dans la collection de la famille Bocchi ; sur le contexte de sa découverte on ne dispose d'aucune information (Cattaneo Cassano 1995 ; Cattaneo 1996, p. 319-321). Ce n'est que vers le milieu des années 1980 que la fouille conduite dans la nécropole de Cà Cima (Bonomi 1995 ; Ead. 1997 ; Bonomi, Camerin, Tamassia 2002), avec des sépultures datables à partir au moins du milieu $\mathrm{du} \mathrm{VI}^{\mathrm{e}}$ s. av. J.-C. ${ }^{600}$, a permis de mettre au jour trois tombes $(\mathrm{T} .2,11,17)$ dont les mobiliers contenaient des amphores commerciales, dont l'étude m'a été confiée. En ce qui concerne l'habitat d'Adria, il est très peu connu d'un point de vue archéologique, car l'implantation ancienne s'étend au-dessous du secteur sud-ouest du centre actuel, ce qui rend évidemment impossible une fouille extensive qui, comme tout le laisse imaginer, pourrait restituer une quantité importante de matériel amphorique. Les recherches de Via San Francesco, conduites dans les années 1980, ont en effet livré des fragments d'amphores archéologiquement contextualisés et publiés rapidement par les auteurs de la fouille (Bonomi, Camerin, Tamassia 2003). En outre, la fouille de Via Ex Riformati, réalisée en 2004, a restitué un peu moins de deux cents fragments amphoriques, qu'on peut attribuer à une quinzaine d'individus, que j'ai pu repérer et étudier à la demande de $\mathrm{S}$. Bonomi, responsable de l'enquête archéologique. Bien que les contextes et le matériel susceptible de dater cette fouille, complètement inédite, n'aient pas encore été étudiés, j'ai pu disposer, grâce à la générosité de $\mathrm{S}$. Bonomi, de renseignements préliminaires relatifs à la datation des niveaux les plus anciens du secteur d'habitat, autour de 550 av. J.-C.

Aucune amphore commerciale ne semble provenir des fouilles du XIX ${ }^{\mathrm{e}}$ siècle de Francesco Antonio Bocchi dans l'aire des Jardins Publics (Donati, Parrini 1999).

600. On fait remonter vers le milieu du $\mathrm{VI}^{\mathrm{e}}$ s. av. J.-C. la datation d'un aryballe globulaire de production corinthienne provenant d'une des tombes plus anciennes (Bonomi 2000a, p. 123 et n. 15, avec bibliographie).
Les amphores d'Adria présentées ci-dessous, quatre individus de la nécropole et douze de l'habitat, sont indiquées par le sigle «AD ». Elles sont publiées dans Sacchetti 2012, avec la même dénomination.

\subsection{Catalogue}

\section{AMPHORES DU TYPE CORINTHIEN A'}

\section{$\mathrm{AD} 1$ (pl. I)}

Année de la fouille / trouvaille : 2004.

Secteur : Via Ex Riformati.

Sporadique.

État de conservation : embouchure, conservée pour la moitié de sa circonférence, et partie du col portant la trace de la jonction supérieure d'une anse. H. max. cons. : 6,9. Lèvre à marli, oblique, à section trapézoïdale. H. : 4,1 ; épaiss. max. : 3,4 ; diam. int./ext. restituable embouchure : 12 / 19. H. max. cons. col : 2,8; diam. min./max. restituable : 14 / 14,4.

Surface ext. et int. : gris clair $(2.5 \mathrm{Y} 7 / 2)^{601}$.

Fracture : gris (2.5Y6/1).

Inclusions : inclusions gris-brun de petites, moyennes et grandes dimensions à fréquence haute ; inclusions blanches de petites et moyennes dimensions à fréquence moyenne-basse ; et inclusions noires de petites dimensions a fréquence moyenne-basse.

Observations et comparaisons : l'obliquité accentuée de la lèvre, qui s'écrase lourdement sur les anses, est une caractéristique morphologique qui distingue les amphores de production corinthienne A' entre la seconde moitié du Ve et le IV s. av. J.-C. Pour ce type, Koehler 1978a, fig. 1.d-e. La couleur de la pâte virant au gris, bien qu'elle soit documentée pour cette classe, pourrait être due à des phénomènes post-dépositionnels. En effet, la boue gris-bleuâtre dans laquelle elle baignait a modifié la couleur d'une grande partie des objets céramiques découverts.

Chronologie : 450-400 av. J.-C.

\section{AD2 (pl. I)}

Année de la fouille / trouvaille : 1994.

Secteur : Via S. Francesco - Secteur C.

US : 266 .

État de conservation : fragment de pied avec une partie du fond de la panse. H. max. cons. : 9,5. Pied en bouton, au profil caréné, arrondi en bas. H. : 2,9 ; diam. min./ $\max .: 2 / 5,4$.

Surface ext. et int., fracture : beige-rose.

601. Munsell Soil Colour Charts, Baltimore 1975. 
Inclusions : petites inclusions lithiques et inclusions grossières.

Engobe : engobe jaune clair.

Observations et comparaisons : attribuée par les éditeurs à la production corinthienne A' et datée autour du milieu du $\mathrm{V}^{\mathrm{e}}$ s. av. J.-C. sur la base de la stratigraphie et après confrontation morphologique, tout à fait ponctuelle, avec Koehler 1981, fig. 1.b.

Chronologie : vers 450 av. J.-C.

Bibliographie : Bonomi, Camerin, Tamassia 2003, p. $205, \mathrm{n}^{\circ} 37$, fig. 3,2 .

\section{AMPHORES GRÉCO-OCCIDENTALES}

\section{AD3 (fig. 71 ; pl. II)}

Collection Bocchi.

Inventaire $\mathrm{n}^{\circ}$ : IG 20570.

Type : corinthienne B.

État de conservation : entière. H. totale : 38 ; diam. emb. : 14 ; diam. max. panse : 35 . Lèvre en échine, légèrement ouverte. Col cylindrique, légèrement concave, avec une cannelure profonde située un peu au-dessous de la lèvre. Anses obliques ouvertes, en ruban épaissi, à section elliptique. Épaule aplatie. Corps piriforme. Pied en bouton creux à l'intérieur, au profil caréné et plat en bas.

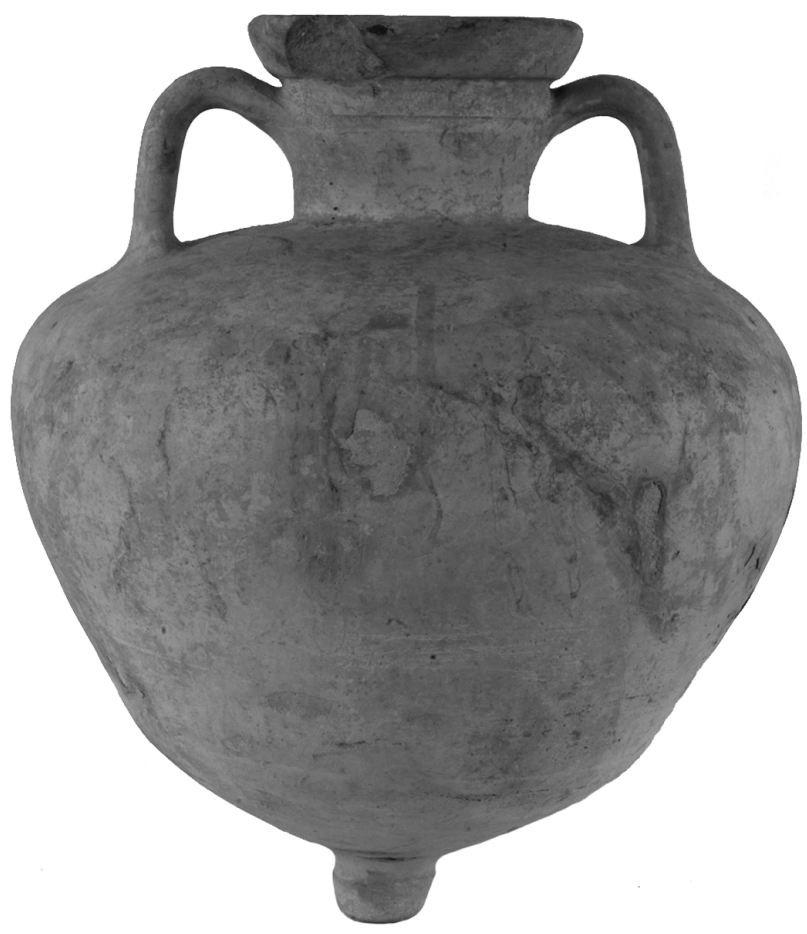

Fig. 71. Adria, amphore de type corinthien B de la Collezione Bocchi. Cat. AD3 (@ Soprintendenza per i Beni Archeologici del Veneto).
Surface ext. et int. : jaune pâle (2.5Y8/2).

Fracture : jaune (2.5Y8/4).

Inclusions : argile épurée avec inclusions blanches et brunes de petites dimensions à fréquence basse. Mica presque complètement absent.

Observations et comparaisons : attribuée par M.C. Cattaneo à la production corinthienne $\mathrm{B}$ et datée de la période de passage du VI ${ }^{\mathrm{e}}$ au Ve $\mathrm{s}$. av. J.-C. Pour ce type, on renvoie à Koehler 1978a, fig. 2.b ; 1981, fig. 1.a, pl. 99.c ; daté dans un premier temps du deuxième quart $d u V^{e} s$. av. J.-C., une chronologie plus ample a été cependant proposée plus récemment, couvrant aussi une période de superposition avec le type plus archaïque de la série, entre la fin du VI et les débuts du Ve s. av. J.-C. (Gauer 1975, pl. 7, n 5, 8 ; Roberts 1986, p. 64-67, $\mathrm{n}^{\circ} 416-417$, fig. 41, pl. 18 ; Lawall 1995, p. 72). L'amphore, conservée au Musée National d'Adria, a été trouvée au XIX ${ }^{\mathrm{e}}$ siècle et intégrée à la collection de la famille Bocchi. Le lieu et le contexte de la découverte sont inconnus. On sait que la trouvaille a eu lieu génériquement dans la «zone d'Adria » et l'intégrité de l'exemplaire nous suggère qu'elle provient d'un contexte funéraire. Ses dimensions réduites indiquent qu'il s'agit d'un exemplaire fractionnaire.

Chronologie : 500-480 av. J.-C.

Bibliographie : Cattaneo Cassano 1995, p. 384, pl. V.1 ; Cattaneo 1996, p. 320, fig. 2 ; Toniolo 2000, p. 175, fig. 409-410.

\section{AD4 (fig. 72 ; pl. II)}

Année de la fouille / trouvaille : 1994.

Secteur : Cà Cima.

Structure : Tombe 11.

Inventaire $\mathrm{n}^{\circ}$ : IG 9653.

Type : corinthienne B.

État de conservation : entière, reconstituée à partir de nombreux fragments. $\mathrm{H}$. tot. : 48,8. Lèvre en échine. H. : 2 ; épaiss. max. : 1,6 ; diam. int./ext. embouchure : $12,8 / 13,8$. Col cylindrique, avec double rainure audessous de la lèvre. H. : 6,4 ; diam. min./max. : 10,8. Anses obliques, légèrement ouvertes, en ruban épaissi, à section elliptique. H. : 8,4 ; épaiss. max. : 3,4 x 2. Épaule aplatie. Larg. : 15. Corps piriforme, au profil légèrement caréné dans la moitié inférieure. H. : 37 ; diam. max. : 39,2 . Pied en bouton, creux à l'intérieur et plat en bas. H. : 3,2 ; diam. min./max. : 2 / 4,4.

Surface ext. : orange rosé (10R6/4-10R6/6-5YR7/4).

Surface int. et fracture : orange (10R6/4-10R6/6).

Inclusions : argile très épurée avec inclusions blanches, grises et brunes de petites dimensions à fréquence basse. En surface, de rares écailles de mica argenté très fines. Engobe : engobe crème. 

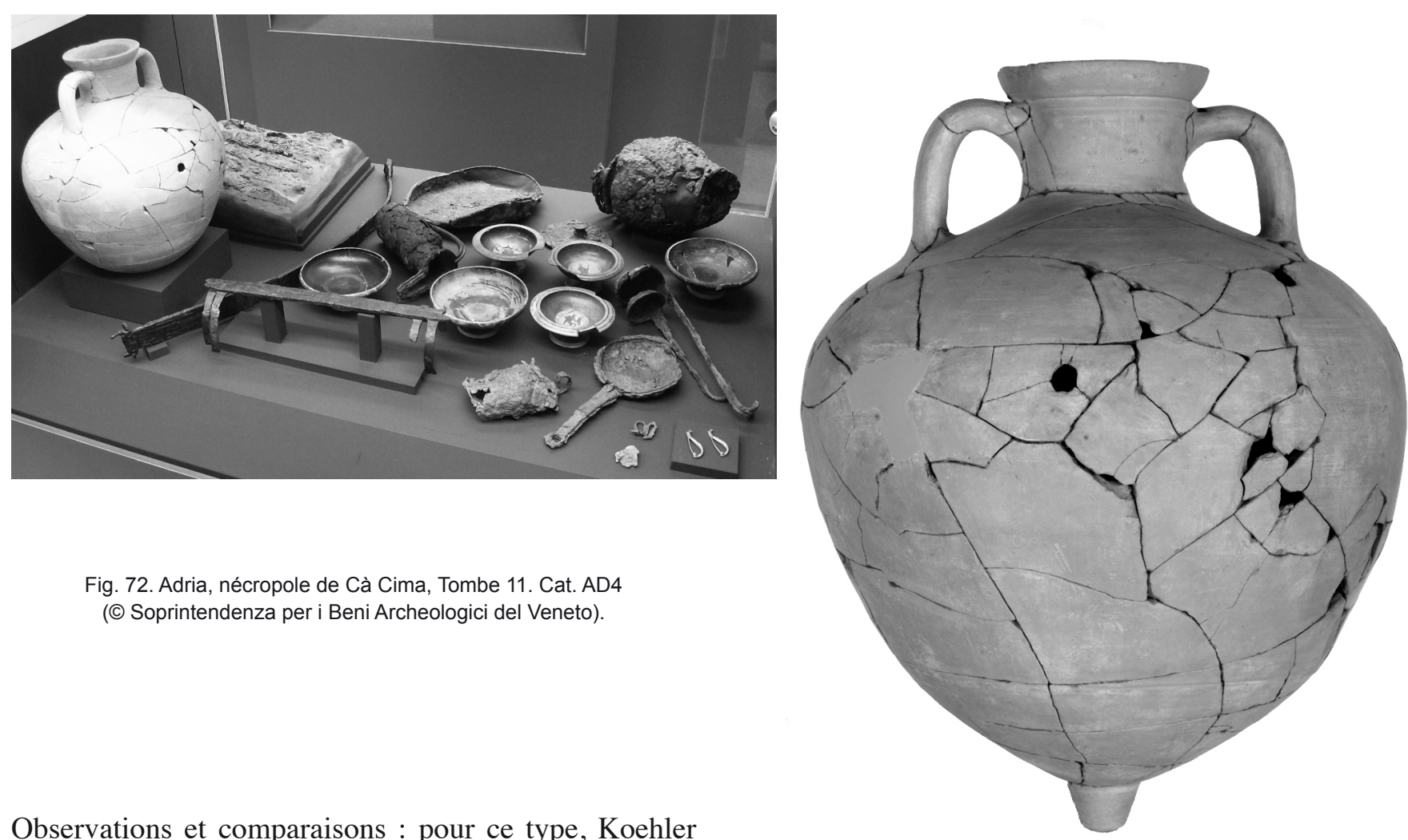

Observations et comparaisons : pour ce type, Koehler 1978a, fig. 2.b, et Ead. 1981, fig. 1.a, pl. 99.c ; traditionnellement daté du deuxième quart du $\mathrm{V}^{\mathrm{e}} \mathrm{s}$. av. J.-C., remonté plus récemment autour de 500 av. J.-C. (Lawall 1995, p. 72, sur la base de Gauer 1975, pl. 7, n 5, 8 et Roberts 1986, p. 64-67, $\mathrm{n}^{\circ}$ 416-417, fig. 41, pl. 18). Une datation dans les vingt premières années du $\mathrm{V}^{\mathrm{e}} \mathrm{s}$. av. J.-C., suggérée par la forme archaïsante des anses, encore brèves et non surélevées, et du pied, en bouton encore court, est confirmée par l'ensemble du mobilier de la tombe 11 de la nécropole de Cà Cima (Bonomi, Camerin, Tamassia 2002, p. 5-6).

Chronologie : 500-480 av. J.-C.

\section{AD5}

Année de la fouille / trouvaille : 1995.

Secteur : Cà Cima.

Structure : Tombe 2.

Inventaire $\mathrm{n}^{\circ}$ : IG AD 8916.

Type : corinthienne B.

État de conservation : moitié inférieure d'amphore, reconstituée à partir de fragments. Pied en bouton. H. : 2,5 ; diam. min./max. : 3 .

Surface ext., int. et fracture : beige clair.

Observations et comparaisons : pour le type, voir la fiche précédente. Sur la base du mobilier de la tombe 2 de Cà Cima (Bonomi, Camerin, Tamassia 2002, p. 11), la datation de cet exemplaire peut être circonscrite aux vingt premières années du $\mathrm{V}^{\mathrm{e}} \mathrm{s}$. av. J.-C.

Chronologie : 500-480 av. J.-C.

AD6 (fig. $\mathbf{7 3}$; pl. II)

Année de la fouille / trouvaille : 1994.

Secteur : Cà Cima.

Structure : Tombe 17.

Inventaire $\mathrm{n}^{\circ}$ : IG 9939.

Type : corinthienne B.

État de conservation : entière, reconstituée à partir de nombreux fragments. H. tot. : 36,6. Lèvre en amande, ouverte. H. : 2,6 ; épaiss. max. : 1,4 ; diam. int./ext. embouchure : 13,6 / 13,8. Col évasé, une cannelure audessous de la lèvre. H. : 7,4 ; diam. min./max. : 10 / 11,6. Anses obliques, légèrement ouvertes en ruban épaissi, à section elliptique. H. : 8,8; épaiss. max. : 3,2 x 1,6. Épaule aplatie. Larg. : 10. Corps piriforme, au profil légèrement caréné dans la moitié inférieure. $H$. : 23,2 ; diam. max. : 30,8. Pied en bouton, légèrement creux à l'intérieur et plat en bas. H. : 3,2; diam. min./max. : 3 / 4,8 .

Surface ext., int. et fracture : jaune-beige (10YR7/4). Inclusions : inclusions blanches, grises et brunes de dimensions petites-moyennes, à fréquence moyenne. Des vacuoles sont visibles en surface. Mica presque complètement absent.

Observations et comparaisons : pour le type, on renvoie à cat. $\mathrm{AD} 4 \mathrm{.} \mathrm{Vu}$ ses dimensions réduites, l'amphore constitue un exemplaire fractionnaire. Les amphores fractionnaires sont plus difficilement datables sur une base morphologique, mais une datation entre le premier 


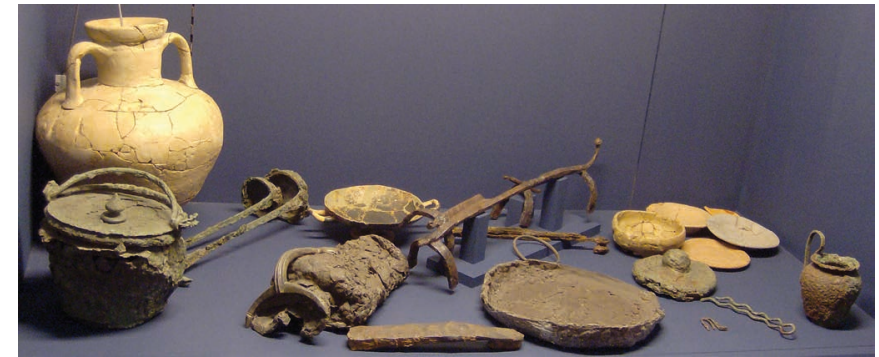

Fig. 73. Adria, nécropole de Cà Cima, Tombe 17. Cat. AD6 (๑) Soprintendenza per i Beni Archeologici del Veneto).

et le deuxième quart du $\mathrm{V}^{\mathrm{e}} \mathrm{s}$. av. J.-C. est dans ce cas corroborée par le reste du mobilier (Bonomi, Camerin, Tamassia 2002, p. 11).

Chronologie : 480-460 av. J.-C.

\section{AD7 (pl. I)}

Année de la fouille / trouvaille : 1994.

Secteur : Via S. Francesco - Secteur C.

US : 266.

Type : corinthienne B.

État de conservation : fragment de lèvre avec une petite partie du col. H. max. cons. : 4,6. Lèvre en échine, légèrement ouverte. H. : 2,6 ; épaiss. max. : 1,9 ; diam. int./ ext. restituable embouchure : 11,6/15.

Surface ext., int. et fracture : gris.

Inclusions : inclusions très fines.

Engobe : engobe jaunâtre.

Observations et comparaisons : attribuée par les éditeurs à la production corinthienne $\mathrm{B}$ et datée de la seconde moitié du Ve s. av. J.-C. sur la base de la stratigraphie. Pour le type, Koehler 1978a, fig. 2.b-c, et Ead. 1981, fig. 1.a, pl. 99.c, datables entre 480 et 400 av. J.-C.

Chronologie : 450-400 av. J.-C.

Bibliographie : Bonomi, Camerin, Tamassia 2003, p. $205, n^{\circ} 36$, fig. 3,1 .

\section{AD8 (fig. 74 ; pl. I)}

Année de la fouille / trouvaille : 2004.

Secteur : Via Ex Riformati - Secteur 3.

US : 8/9.

Type : « forme $1 \alpha »$.

État de conservation : fond complet avec la jonction de la partie inférieure de la panse. H. max. cons. : 4,3. Fond cylindrique caréné. H. : 3,2 ; diam. min./max. : 4,9 / 6,4. Surface ext. et int. : gris (2.5Y6/1-GLEY1 4/1).

Fracture : gris clair-beige (2.5Y6/2).

Inclusions : argile épurée, avec des inclusions grises de petites dimensions à fréquence basse et des inclusions

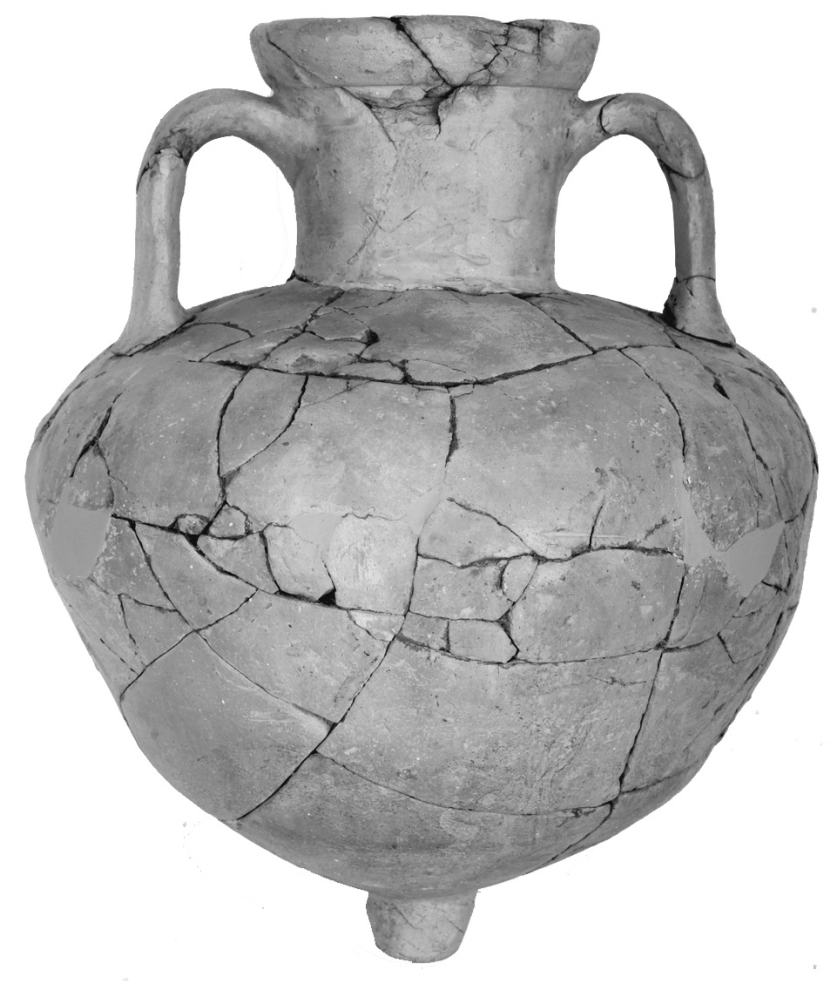

blanches de petites dimensions à fréquence très basse. Tant en surface qu'en fracture, du mica fin argenté à fréquence basse.

Appareil épigraphique : sur le fond extérieur, un graffiti au trait peu profond et assez régulier tracé après la cuisson avec une pointe fine. La marque consiste de deux chi (de l'alphabet étrusque ou vénétique) en combinaison, disposés à angle droit, dont l'un fait face à un signe en forme de « ramo secco » (Sacchetti 2011, cat. ${ }^{\circ} 1$, fig. 2, pl. I. 1).

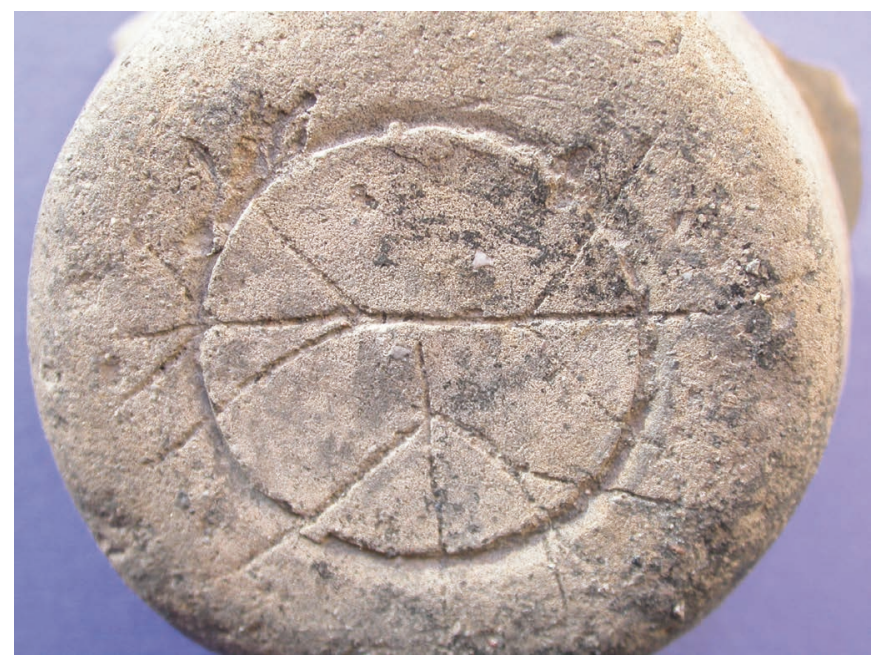

Fig. 74. Adria, via Ex Riformati, fond d'amphore gréco-occidentale de forme « $1 \alpha$ » avec graffito. Cat. AD8 (photo F. Sacchetti). 
Observations et comparaisons : pour la forme, Panvini 2001, fig. 46, 5 (de l'épave de la Pointe Lequin) ; Semeraro 1997, fig. 5 (de Brindes), 48, 171b et 171d (de Leuca), fig. 228, 985 et 986 (de Ruffano) ; Tigano 2002, p. 69 (de Mylai), toutes datées de la seconde moitié ou du dernier quart du $\mathrm{VI}^{\mathrm{e}}$ s. av. J.-C.

Chronologie : 550-490 av. J.-C.

Bibliographie : Sacchetti 2011, cat. $n^{\circ}$ 1, fig. 2, pl. I. 1.

\section{AD9 (pl. I)}

Année de la fouille / trouvaille : 2004.

Secteur : Via Ex Riformati.

US : 150 .

Type : « forme $1 \alpha »$.

État de conservation : fond complet avec une partie inférieure de la panse. H. max. cons. : 10,2. H. max. cons. panse : 7,2. Fond cylindrique caréné. H. : 3 ; diam. min./ max. : 6,5/ 7 .

Surface ext., int. et fracture : beige (10YR6/3).

Inclusions : argile épurée avec inclusions brunes, grises et blanchâtres de petites dimensions, à fréquence basse, distribuées de manière uniforme. Écailles de mica argenté de petites dimensions, à fréquence très basse. Vacuoles, dont quelques-unes même de dimensions considérables, visibles aussi bien en surface qu'en fracture.

Appareil épigraphique : sur le fond extérieur, deux traits parallèles incisés avant la cuisson à l'aide d'une pointe moyennement épaisse et avec un sillon profond et régulier (Sacchetti 2011, cat. n 2, fig. 3, pl. I. 2).

Observations et comparaisons : pour la forme, on renvoie à la fiche précédente.

Chronologie : 550-490 av. J.-C.

Bibliographie : Sacchetti 2011, cat. n² 2, fig. 3, pl. I. 2.

\section{$\mathrm{AD} 10$ (pl. I)}

Année de la fouille / trouvaille : 2004.

Secteur : Via Ex Riformati.

US : 72.

Type : « forme $1 \alpha »$.

État de conservation : fragment de fond, manquant de la base, avec la jonction à la panse. H. max. cons. : 5,6. Fond cylindrique caréné. H. max. cons. : 1, 7 ; diam. min./max. restituable : 6,1 / 6,5.

Surface ext., int. et fracture : gris clair (2.5Y7/2-2.5Y5/1). Inclusions : argile épurée avec inclusions blanchâtres et grises de petites et moyennes dimensions, à fréquence très basse, distribuées d'une manière non uniforme. Mica argenté très fin à fréquence très basse.

Observations et comparaisons : malgré le mauvais état de conservation, la forme du fond permet de l'attribuer sans difficulté à la « forme $1 \alpha »$. Cf. Koehler 1978a, fig. 2.a ; Sourisseau 2011.

Chronologie : 550-490 av. J.-C.
AD11 (pl. I)

Année de la fouille / trouvaille : 2004.

Secteur: Via Ex Riformati.

US : 72 .

Type : « forme $1 \alpha »$.

État de conservation : fragment de l'embouchure avec un très court segment du col qui présente la trace de la jonction supérieure d'une anse. H. max. cons. : 5,2. Lèvre en bourrelet gonflé, légèrement ouverte. $\mathrm{H}$. : 3,5 ; épaiss. max. : 2,5 ; diam. int./ext. restituable embouchure : $11,6 / 15,4$. Col avec une cannelure fine immédiatement au-dessous de la lèvre. H. max. cons. : 1,7 ; diam. min./ max. restituable : 11,5 / 11,9.

Surface ext. et int. : jaune clair-verdâtre $(2.5 \mathrm{Y} 7 / 3)$.

Fracture : jaune très pâle $(2.5 \mathrm{Y} 8 / 2-5 \mathrm{Y} 7 / 2)$.

Inclusions : argile épurée avec inclusions blanchâtres et grises de petites dimensions à fréquence basse, distribuées de manière assez uniforme. Écailles de mica argenté de petites dimensions, à fréquence très basse. De nombreuses vacuoles, visibles aussi bien en surface qu'en fracture, de dimensions parfois considérables.

Observations et comparaisons : pour la forme Semeraro 1997, $\mathrm{n}^{\circ} 253$ (de Muro Tenente), $\mathrm{n}^{\circ} 1011$ (de Torre San Giovanni) et, en particulier, $n^{\circ}$ 919a (d'Otrante), toutes datées dans la seconde moitié du VI' $\mathrm{s}$. av. J.-C.

Chronologie : 550-490 av. J.-C.

\section{AMPHORES DE L'ÉGÉE SEPTENTRIONALE}

\section{$\mathrm{AD} 12$ (pl. I)}

Année de la fouille / trouvaille : 2004.

Secteur : Via Ex Riformati.

US : Tranchée E-O Sud US 43.

Type : nord-égéenne.

État de conservation : départ supérieur d'une anse avec jonction au col. Dimensions max. fr. : 6,4 x 5,9. Anses en ruban épaissi, à section elliptique, avec une arête centrale. H. max. cons. : 3,5 ; épaiss. max. : 4,1 x 2,1.

Surface ext. et int. : brun (7.5YR5/3-7.5YR4/2).

Fracture : brun-orange (5YR5/4-5YR5/6).

Inclusions : argile très épurée, avec inclusions blanchâtres et noires de petites dimensions à fréquence basse, visibles surtout en section. En surface et en fracture, écailles de mica argenté de dimensions petites et moyennes à fréquence moyenne-haute. En section, des vacuoles.

Observations et comparaisons : pour la forme, Johnston 1990, $\mathrm{n}^{\circ}$ 131-132, 177, fig. 12, et Grandjean 1992, fig. 1.2, 4.26, 9.63. D'après la pâte céramique, l'anse en question peut être attribuée à une production thasienne et le détail morphologique de l'arête centrale suggère une datation vers la fin du $\mathrm{VI}^{\mathrm{e}} \mathrm{s}$. av. J.-C. Pour le type, Spagnolo 2003, p. 624 et n. 96, pl. III. 2. Tant en 
surface qu'en fracture l'exemplaire présente par endroit des taches bleues probablement dues à des phénomènes survenus après la déposition. De nombreux fragments céramiques issus de la fouille présentent en effet une couleur de la pâte virant au gris bleuté imputable à la boue dans laquelle ils ont été trouvés. Le fragment a dû aussi probablement être exposé à une très haute température qui a complètement modifié sa couleur en surface. Chronologie : 525-500 av. J.-C.

\section{$\mathrm{AD} 13$ (pl. I)}

Année de la fouille / trouvaille : 2004.

Secteur : Via Ex Riformati.

US : 72 .

Type : nord-égéenne.

État de conservation : segment central d'une anse, près de sa courbure. H. max. cons. : 7,4. Anses en ruban épaissi, à section elliptique, avec une arête centrale. H. max. cons. : 7,4 ; épaiss. max. : 3,3 x 2,1.

Surface ext. et int. : brun-noirâtre (GLEY1 4/1).

Fracture : brun-orange (5YR5/6).

Inclusions : argile épurée avec des inclusions blanches de petites dimensions, à très basse fréquence, distribuées de manière uniforme, et de très rares inclusions de couleur brun-grisâtre (chamotte). En surface et en fracture, écailles de mica argenté de petites dimensions à fréquence moyenne-haute.

Observations et comparaisons : voir la fiche précédente. Chronologie : 525-500 av. J.-C.

\section{AMPHORES DE L'ÉGÉE ORIENTALE}

\section{AD14 (pl. I)}

Année de la fouille / trouvaille : 1994.

Secteur : Via S. Francesco - Secteur C.

US : 266 B.

Type : chiote, Swollen-necked, Early bulgy. Variante : C/2 Knigge-Lawall.

État de conservation : fragment de lèvre et col. H. max. cons. : 9,5. Lèvre en cordon, ouverte. H. : 1,3 ; épaiss. max. 1,4 ; diam. int./ext. restituable embouchure : 8 / 9,5. Col bombé. H. max. cons. : 7,4 ; diam. min./max. : $8,6 / 10$.

Surface ext., int. et fracture : chamois rosé.

Inclusions : de rares inclusions blanches et du mica fin. Observations et comparaisons : attribuée par les éditeurs à une production chiote. Pour le type, Lawall 1995, p. 90-91, fig. 20, 26-27 ; Dupont 1998, fig. 23, 2. f ; de Marinis 1999, p. 267, fig. 7.6, datable du deuxième quart du Ve s. av. J.-C.

Chronologie : 475-450 av. J.-C.

Bibliographie : Bonomi, Camerin, Tamassia 2003, p. $205, \mathrm{n}^{\circ} 55$, fig. 3,7 .
AD15 (pl. I)

Année de la fouille / trouvaille : 2004.

Secteur: Via Ex Riformati.

US : $500 \mathrm{~B}$.

Type : Samos-Milet, S/1 ou S/3 Lawall.

État de conservation : anse entière. H. max. cons. : 13. Anses verticales en ruban épaissi, à section elliptique. H. : 12,5 ; épaiss. max. : 3,5 x 2,3.

Surface ext. et int. : beige (7.5YR7/3-7.5YR6/4).

Fracture : beige-orange (2.5YR6/4).

Inclusions : argile épurée avec inclusions brun-orange, grises et blanches de petites dimensions, à fréquence moyenne-basse, distribuées de manière uniforme. En surface, des vacuoles et de très fines écailles de mica doré à fréquence très basse.

Observations et comparaisons : pour le type, Dupont 1998, fig. 23.9.b. Sur la base des caractéristiques morphologiques et de la pâte on propose d'attribuer l'anse à une amphore gréco-orientale, notamment du groupe Samos-Milet. Une datation entre la fin $\mathrm{du} \mathrm{VI}^{\mathrm{e}}$ et les débuts du $V^{e} s$. av. J.-C. est non seulement suggérée par l'anse brève, mais aussi corroborée par le contexte de la découverte. L'affinité de la pâte de l'anse en question avec celle du col fragmentaire objet de la fiche suivante, à laquelle on renvoie, rend vraisemblable l'appartenance des deux fragments à un seul individu.

Chronologie : 525-480 av. J.-C.

$\mathrm{AD} 16$ (pl. I)

Année de la fouille / trouvaille : 2004.

Secteur: Via Ex Riformati.

US : $500 \mathrm{~B}$.

Type : Samos-Milet, S/1 ou S/3 Lawall.

État de conservation : fragment de col et deux fragments de paroi, non reconstituables mais appartenant peut-être au même individu. H. max. cons. : 7. Col cylindrique, avec un léger ressaut en correspondance de la jonction à l'épaulement. H. max. cons. : 4,4; diam. min./max. restituable : 9,6.

Surface ext. et int. : beige (7.5YR7/3).

Fracture : beige-orange (2.5YR6/4-7.5YR6/6).

Inclusions : argile épurée avec inclusions brun-orange, grises et blanches de petites dimensions, à fréquence moyenne-basse, distribuées de manière uniforme. En surface, des vacuoles et de très fines écailles de mica doré à fréquence très basse.

Observations et comparaisons : le détail morphologique du listel à la jonction entre le col et l'épaule, ainsi que les caractéristiques de la pâte, suggèrent l'attribution du fragment à la production du groupe Samos-Milet de la fin $\mathrm{d} \mathrm{VI}^{\mathrm{e}} \mathrm{s}$. av. J.-C. La chronologie proposée est basée sur le contexte stratigrafique de la fouille et sur la comparaison avec les variantes archaïques des types $\mathrm{S} / 1$ 

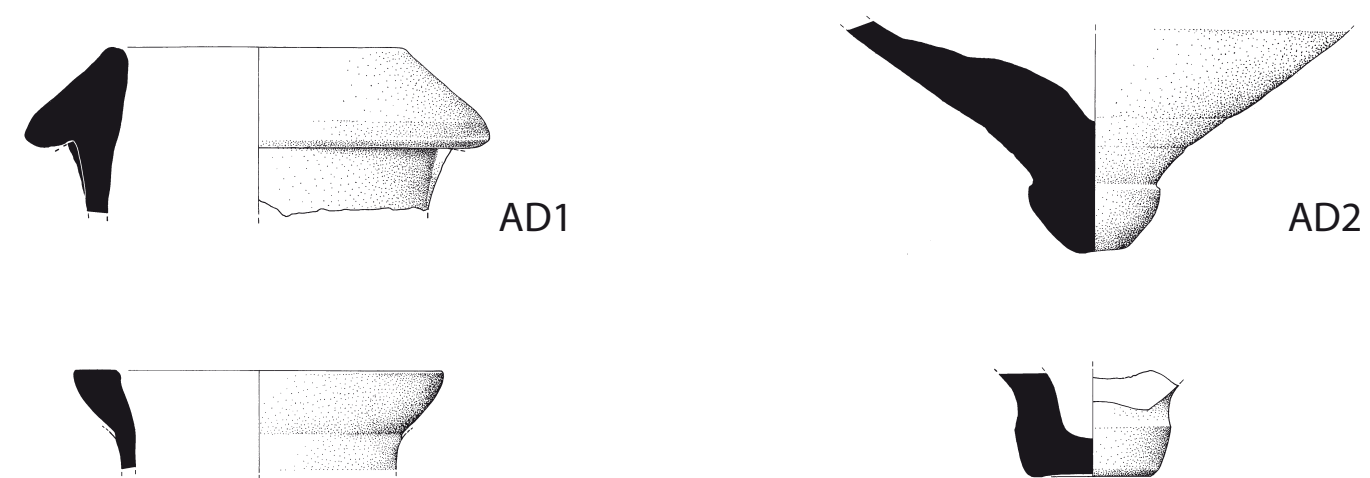

AD7
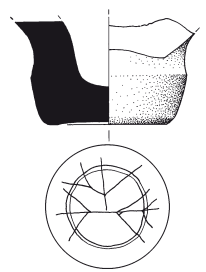

AD8
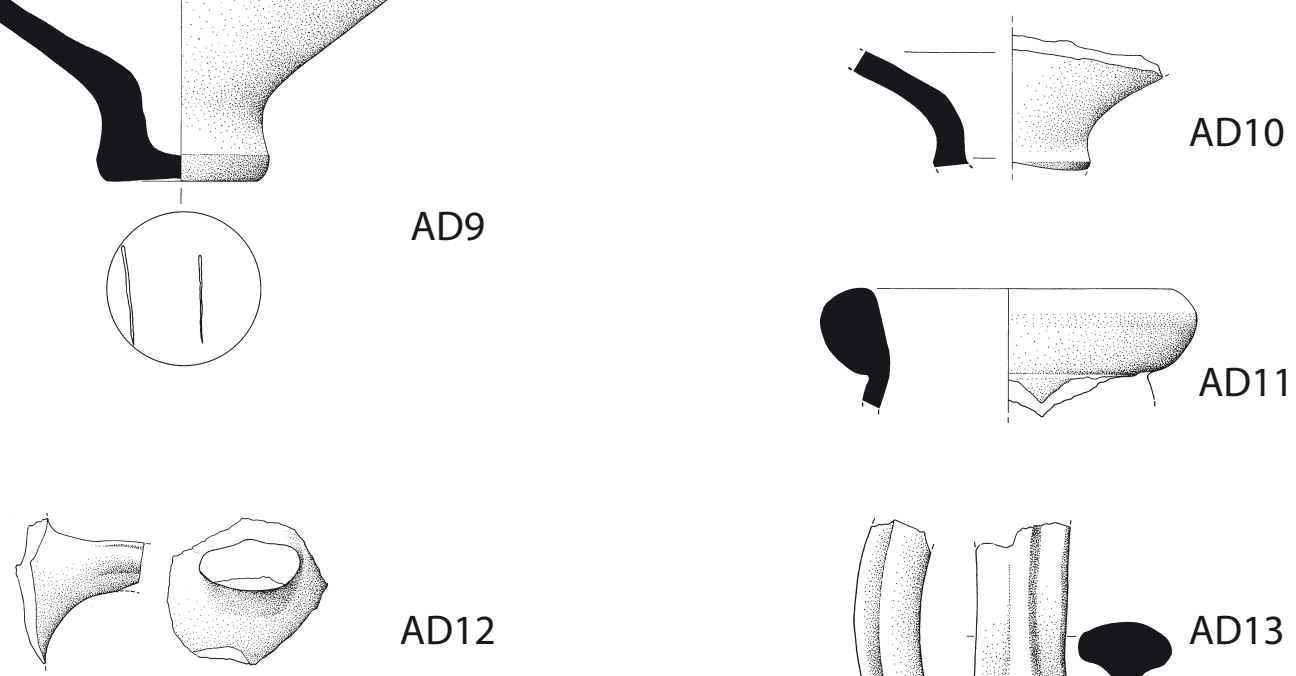

AD12
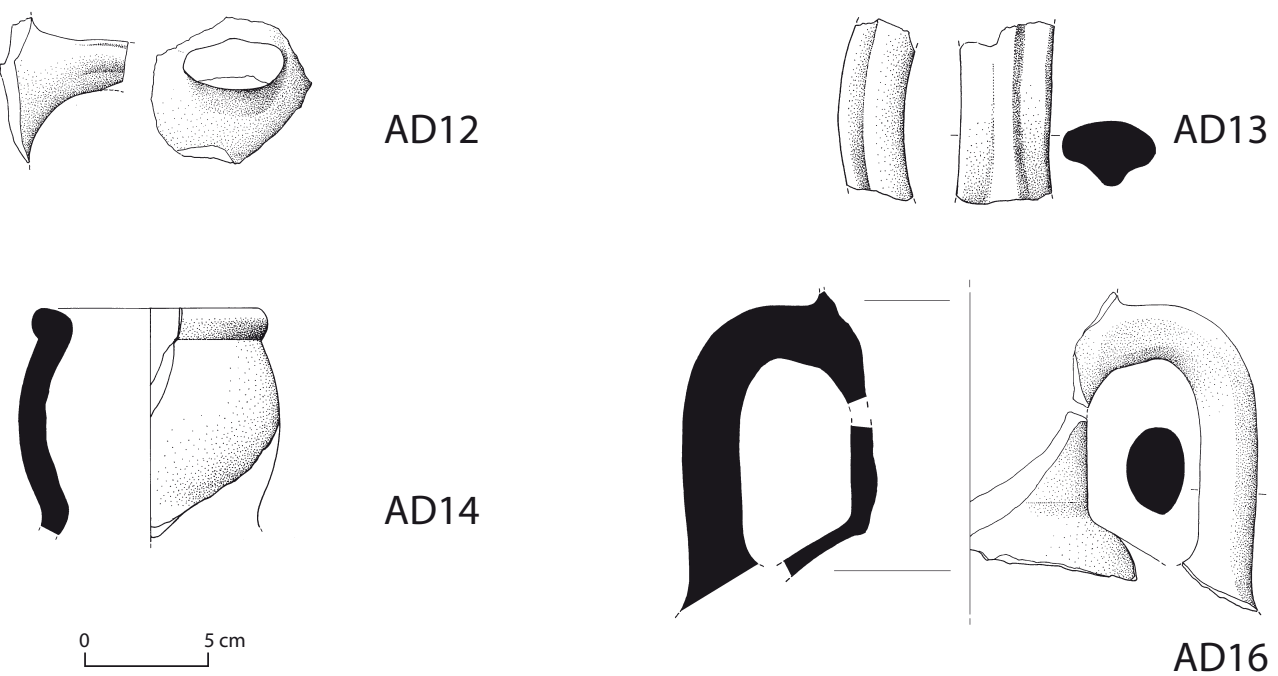

AD14
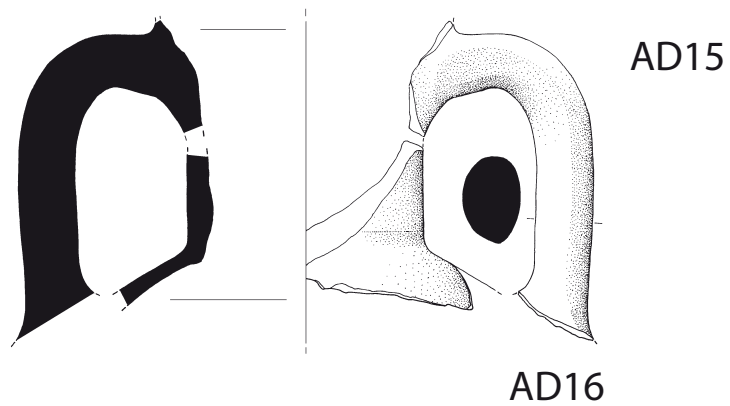

PI. I. Adria. 


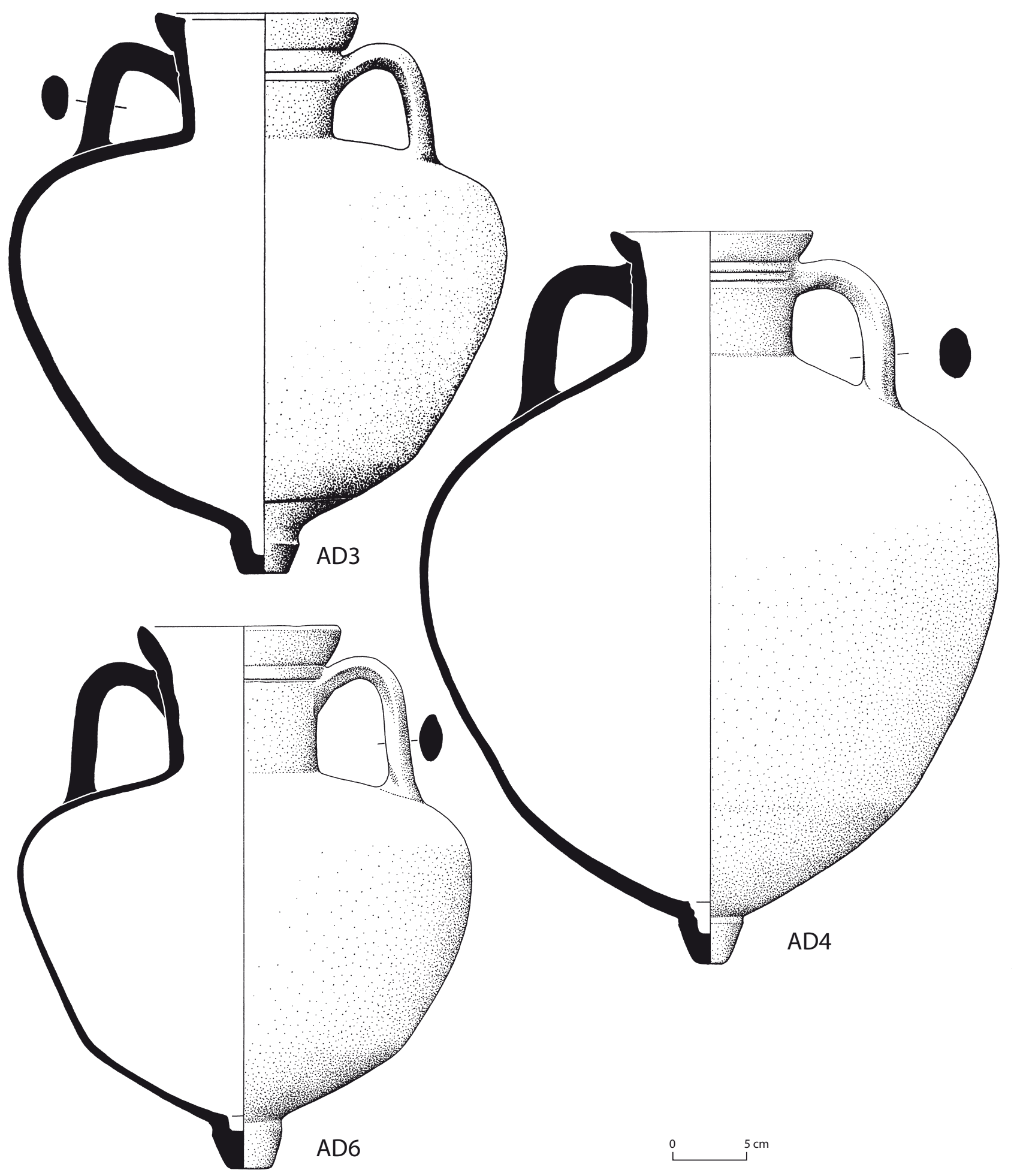

PI. II. Adria 
et $\mathrm{S} / 3$ de M. Lawall (pour lesquelles, respectivement, Roberts 1986, pl. 17.413, et Lawall 1995, fig. 76), l'une et l'autre caractérisées par la présence d'un listel en correspondance du passage du col à l'épaulement. La lèvre de l'amphore n'étant pas conservée, il n'est pas possible d'indiquer de manière précise l'appartenance de l'exemplare d'Adria à l'un ou à l'autre type. Le fragment est probablement attribuable au même individu que celui de la fiche précédente.

Chronologie : 525-480 av. J.-C.

\section{San Basilio di Ariano Polesine (Prov. de Rovigo) : l'habitat (fig. 69)}

\subsection{Bilan des études}

L'habitat de la bande côtière d'Adria qui fut considéré, dès les premières découvertes, comme le plus important «centre satellite » de la cité, a été repéré à $20 \mathrm{~km}$ environ au sud, au lieu-dit San Basilio, dans la commune d'Ariano Polesine (fig. 75). Le centre a été découvert au début des années quatre-vingt du siècle dernier dans le secteur occidental d'une dune marine appartenant au littoral fossile d'époque étrusque, à quelques centaines de mètres de la rive septentrionale du Pô de Goro, dans cette ancienne aire du delta lagunaire aujourd'hui connu comme l' « Île d'Ariano ». L'habitat se trouvait donc à cette époque près de la mer, sur la rive septentrionale d'un paléo-lit du Pô qu'on peut identifier d'une manière quasi certaine avec l'ancien Pô d'Ariano

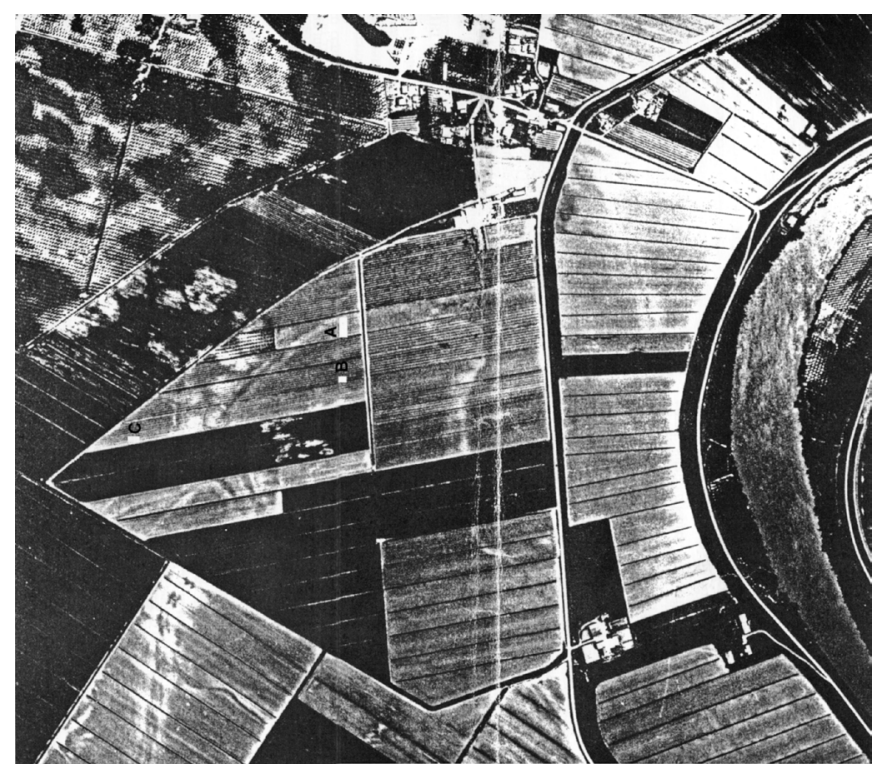

Fig. 75. Ariano Polesine, lieu-dit San Basilio, terrain Forzello, photo aérienne (d'après Salzani, Vitali 2003, fig. 1). et qui dans certains trajets est de nouveau parcouru aujourd'hui par l'actuel Pô de Goro ${ }^{602}$. Les fouilles, effectuées à plusieurs reprises et avec des sondages limités dans le « Podere Forzello », ont permis de déterminer l'existence de plusieurs phases d'implantation. Celles-ci peuvent être attribuées à des réfections successives des structures d'habitation à la suite d'exondations fluviales répétées et datables de la période comprise entre le premier ou le deuxième quart du $\mathrm{VI}^{\mathrm{e}}$ et la fin du $V^{\mathrm{e}} \mathrm{s}$. av. J.-C. ${ }^{603}$. L'ancienneté des premiers témoignages du site, constitués de céramique daunienne (Bonomi 2003, p. 140, n. 1) et de céramique étrusco-corinthienne de production de Vulci datée autour de 600 av. J.-C. ${ }^{604}$, de kotylai corinthiennes datables à partir de 580-570 av. $\mathrm{J}$.-C. ${ }^{605}$ et de céramique paléovénète à dater vers le milieu du VI ${ }^{\mathrm{e}} \mathrm{s}$. av. J.-C. ${ }^{606}$, a souligné sa contemporanéité avec Adria, en suggérant que San Basilio, plus proche de la mer qu'Adria, ait été le premier avant-poste grec dans l'aire du delta. Cette éventualité a été suggérée comme une hypothèse de travail, sans pour autant écarter la possibilité qu'Adria elle-même ait structuré « cet avantposte stratégique sur la mer » (Salzani, Vitali 1991, p. 419). Une hypothèse fondée sur le matériel archéologique, composé surtout de céramique étrusco-padane et bucchero mais aussi de céramique vénète et d'importations grecques, suggère qu'à San Basilio, tout comme dans la voisine Adria, se serait instaurée une cohabitation de groupes ethniquement hétérogènes constitués de Vénètes, Grecs et Étrusques. L'implantation, située entre Adria et Spina, qui a livré du matériel d'importation grecque la rapprochant beaucoup d'Adria, mais également beaucoup d'objets en bucchero la reliant au territoire étrusque, aurait gravité selon quelques-uns autour du centre d'Adria ${ }^{607}$, tandis que, selon d'autres, elle aurait constitué un avant-poste du territoire étruscopadan dans le cadre d'un projet général de contrôle et d'endiguement de la présence grecque qui s'élargissait de plus en plus au nord du territoire contrôlé par les

602. L'étude géomorphologique de l'aire de l' « Isola di Ariano », où surgit l'habitat, est due à R. Peretto (en dernier, Peretto 1999).

603. Les premiers sondages dans l'aire de l'habitat archaïque, conduits par M. De Min en 1983 (De Min 1984, p. 822 ; De Min, Iacopazzi 1986 ; De Min 1988), ont été suivis par d'autres interventions de fouille, entre 1987 et 1989, sous la direction de L. Salzani et D. Vitali (Salzani, Vitali 1988 ; Id. 1991 ; Id. 2003). Après une période d'abandon, qui a suivi les dernières décennies du Ve s. av. J.-C. (Salzani, Vitali 2003, p. 118), l'aire fut occupée par un vaste habitat d'époque romaine. 604. Bruni 1994 ; Bonomi 2000a, p. 122 et n. 9.

605. Salzani, Vitali 1991, p. 418, fig. 13 ; Bonomi 2000a, p. 122 et n. 10-12 ; Salzani, Vitali 2003, p. 118, n. 8, fig. 17.

606. De Min, Iacopazzi 1986, p. 173-174; De Min 1988, p. 85.

607. Salzani, Vitali 1988 ; Id. 1991 ; Cattaneo 1996,p. 323 ; Salzani, Vitali 2003 ; Colonna 2003 
Étrusques ${ }^{608}$. En considérant que le bucchero est maintenant bien attesté même dans les phases archaïques d'Adria, où d'ailleurs il semble appartenir, en partie au moins, à une production locale (Donati, Parrini 1999, p. 590 et n. 82, 609-612), je penche plutôt pour l'hypothèse que l'habitat de San Basilio se rattache à l'influence de cette cité, plus proche du point de vue géographique et plus similaire par sa civilisation que ne l'était le territoire étrusque gravitant autour de Bologne. Le fait que le matériel le plus récent de la première phase de l'habitat ne va pas au-delà des dernières décennies du $\mathrm{V}^{\mathrm{e}} \mathrm{s}$. av. J.-C. pourrait être expliqué par le nouveau rôle prédominant rempli par Adria, comme semblerait le montrer la nouvelle documentation relative à la céramique attique, dans le courant du IVe s. av. J.-C. (Bonomi 2000a ; WielMarin 2005).

La présence d'amphores commerciales grecques parmi le matériel d'importation découvert à l'occasion de la fouille de l'habitat de San Basilio a été rapportée par Cattaneo ${ }^{609}$, à qui fut confiée l'étude des amphores provenant des premières recherches archéologiques conduites par M. De Min.

Quant aux fouilles qui ont suivi dans cette zone sous la direction de L. Salzani et D. Vitali, j'ai été chargée de l'étude du matériel amphorique dégagé. L'analyse du matériel provenant des contextes de cette fouille susceptibles de fournir une datation, et dont des notices préliminaires ont été déjà publiées, n'est pas encore complétée, mais le terminus post quem des importations du monde grec est assuré par deux kotylai assignées respectivement au Corinthien Moyen et au Corinthien Tardif ${ }^{610}$. Dans l'attente de la conclusion l'étude et de la publication des contextes, le deuxième quart du $\mathrm{VI}^{\mathrm{e}} \mathrm{s}$. av. J.-C. constitue donc le terme chronologique le plus haut qui sera ici pris en considération relativement à des productions datables dans un arc chronologique plus ample. Sur l'indication de S. Bonomi, à l'époque Directrice du Musée Archéologique National d'Adria (dans les réserves duquel est conservé le matériel de la fouille de San Basilio), et avec l'accord de M. De Min, qui a conduit les premières recherches archéologiques dans la zone, j'ai pu aussi prendre vision du matériel de ces premières fouilles.

L'habitat de San Basilio a restitué 400 fragments environ d'amphores grecques, appartenant à une quarantaine

608. Sassatelli 1993, p. 185.

609. Pour les amphores publiées de San Basilio, cf. Cattaneo Cassano 1995 ; Cattaneo 1996, p. 323-326.

610. Il s'agit des deux objets attribués d'abord par J. C. Szylàgyi à la seconde moitié du Corinthien Moyen (à ce sujet, cf. les contributions de L. Salzani et D. Vitali citées dans le texte), dont l'un a été assigné par S. Bonomi de préférence au Corinthien Tardif I (Bonomi 2000a, p. 122 et n. 11 avec comparaisons). d'individus qui sont catalogués ci-dessous avc le sigle « SB ». Les fragments sont publiés dans Sacchetti 2012 avec la même numérotation.

\subsection{Catalogue}

\section{AMPHORES GRÉCO-OCCIDENTALES}

\section{SB1 (pl. III)}

Année de la fouille / trouvaille : 1989.

Secteur : « Podere Forzello », Sondage 2.

US : 9. 10.11 .

Type : corinthienne B.

État de conservation : partie de lèvre et col avec la jonction supérieure d'une anse. H. max. cons. : 9,9. Lèvre en échine. H. : 2,2 ; épaiss. max. : 2,1 ; diam. int./ext. restituable embouchure : $11,8 / 15,9$. Col cylindrique. H. max. cons. : 6. Diam. min./max. restituable : 12,6. Anses en ruban épaissi, à section elliptique. H. max. cons. : 3 ; épaiss. max. : 2,2 x 4.

Surface ext., int. et fracture : beige clair (10YR8/2).

Inclusions : inclusions grisâtres et blanches de petites dimensions, à fréquence basse, et inclusions blanches, de dimensions moyennes-petites, à fréquence basse.

Engobe : traces d'engobe gris.

Observations et comparaisons : l'emplacement des anses, qui ne sont pas encore écrasées contre la lèvre en échine, suggère un encadrement chronologique entre le premier et le troisième quart du $\mathrm{V}^{\mathrm{e}} \mathrm{s}$. av. J.-C. Pour le type, Koehler 1978a, fig. 2.b, et Ead. 1981, pl. 99.c, traditionnellement daté du deuxième quart du $\mathrm{V}^{\mathrm{e}} \mathrm{s}$. av. J.-C. Pour la possibilité d'une chronologie plus ample du type, Lawall 1995, p. 70, 72-73.

Chronologie : 500-425 av. J.-C.

\section{SB2 (pl. III)}

Année de la fouille / trouvaille : 1989.

Secteur : «Podere Forzello », Sondage 2.

US $: 1$

Type : corinthienne B.

État de conservation : fragment de lèvre et col. H. max. cons. : 6,5. Lèvre en échine, légèrement ouverte. $H$. : 3,5 ; épaiss. max. : 1,8 ; larg. max. cons. embouchure : 10,7. Col ayant une cannelure légèrement au-dessous de la lèvre. H. max. cons. : 3 ; larg. max. cons. : 9,4.

Surface ext., int. et fracture : blanc-verdâtre.

Inclusions : inclusions de petites dimensions blanchâtres et brunes à fréquence très basse. Mica argenté très fin à fréquence basse.

Observations et comparaisons : voir la fiche précédente. Chronologie : 500-425 av. J.-C.

Bibliographie : Salzani, Vitali 2003, fig. 18 (pour le dessin). 


\section{SB3 (pl. III)}

Année de la fouille / trouvaille : 1989.

Secteur : « Podere Forzello », Sondage 2.

US : 9. 10. 11.

Type : corinthienne B.

État de conservation : fond avec une partie du pied, manquant de la base. H. max. cons. : 6,5. Pied cylindrique creux, au profil moulé. H. max. cons. : 1,8; diam. min./ max. restituable : 5,1 / 5,5.

Surface ext., int. et fracture : beige clair (10YR8/2).

Inclusions : inclusions grisâtres et blanches de petites dimensions, à fréquence basse, et inclusions blanches, de dimensions moyennes-petites, à fréquence basse.

Engobe : traces d'engobe gris.

Observations et comparaisons : attribuable à la production corinthienne $\mathrm{B}$ et datable entre le premier et le troisième quart du $\mathrm{V}^{\mathrm{e}} \mathrm{s}$. av. J.-C. sur la base du type, pour lequel, Koehler 1978a, fig. 2.b, et Lawall 1995, p. 70, 72-73.

Chronologie : 500-425 av. J.-C.

\section{SB4 (pl. III)}

Secteur : « Podere Forzello ».

Inventaire $\mathrm{n}^{\circ}:$ IG 44663.

Type : corinthienne B.

État de conservation : fragment de lèvre. Dimensions max. cons. : 7,5 x 4. Lèvre en échine, ouverte, avec une fine cannelure de base. H. : 3 ; épaiss. max. : 2,2 ; diam. int./ext. restituable embouchure : 12,6 / 16,5.

Surface ext. et int. : beige (10YR7/3).

Surface int. : beige (10YR7/3).

Fracture : beige rosé (7.5YR7/3).

Inclusions : inclusions blanchâtres, grises et brunes de petites dimensions, à fréquence haute, distribuées de manière assez uniforme et inclusions blanches et orange de dimensions petites et moyennes, à fréquence bassse, distribuées de manière non homogène. Rares écailles de mica argenté de petites dimensions.

Observations et comparaisons : attribué par M.C. Cattaneo à la production corinthienne du type B. Pour le type de lèvre, Koehler 1978a, fig. 2.b-c ; et Koehler 1981, fig. 1.a, pl. 99.c., datables entre le deuxième et le dernier quart du $\mathrm{V}^{\mathrm{e}} \mathrm{s}$. av. J.-C. mais avec des attestations datables aussi de la période de transition $\mathrm{du} \mathrm{VI}^{\mathrm{e}}$ au $\mathrm{V}^{\mathrm{e}} \mathrm{s}$. av. J.-C. (Lawall 1995, p. 72).

Chronologie : Ve s. av. J.-C.

Bibliographie : Cattaneo Cassano 1995, p. 383, pl. VI, 2 ; Cattaneo 1996, p. 323, fig. 3, 2.

\section{SB5 (pl. III)}

Année de la fouille / trouvaille : 1989.

Secteur : «Podere Forzello », Sondage 2.

US : 1 .
Type : corinthienne B.

État de conservation : partie de fond, manquant de la base. H. max. cons. : 3,1 ; larg. max. cons. 5 .

Surface ext. : beige rosé (5YR7/3-10YR8/2).

Surface int. et fracture : jaune clair (10YR8/3).

Inclusions : inclusions noirâtres, de petites dimensions, à fréquence moyenne-haute ; inclusions blanches de dimensions petites et moyennes, à fréquence basse et rares inclusions brunes de dimensions petites et moyennes.

Observations et comparaisons : attribuable par son profil et sa pâte à un exemplaire de forme corinthienne B . L'absence de la base du fond empêche d'en préciser la datation.

Chronologie : non définissable.

\section{SB6 (pl. III)}

Année de la fouille / trouvaille : 1989.

Secteur : « Podere Forzello », Sondage 2.

US $: 1$.

Type : corinthienne B.

État de conservation : partie du fond de la panse jusqu'à la jonction du pied.

Surface ext. : jaune clair (2.5YR8/2).

Surface int. et fracture : jaune verdâtre (2.5YR7/2).

Inclusions : inclusions grisâtres et blanches de dimensions moyennes-petites, à fréquence basse. Surface légèrement vacuolaire.

Observations et comparaisons : attribuée par M.C. Cattaneo à la production corinthienne du type B. L'absence d'éléments morphologiques conservés susceptibles de suggérer une datation ne permet pas de fixer une date pour cet exemplaire.

Chronologie : non définissable.

Bibliographie : Cattaneo Cassano 1995, p. 383, pl. VI, 3 ; Cattaneo 1996, p. 323, fig. 3, 3.

\section{SB7 (pl. III)}

Année de la fouille / trouvaille : 1989.

Secteur : «Podere Forzello », Sondage 2.

US : 9. 10. 11.

Type : corinthienne B.

État de conservation : anse presque complète, reconstituée à partir de quatre fragments et conservant sa jonction supérieure et une partie de la jonction avec l'épaule. H. max. cons. : 9,7. Anses en ruban épaissi, à section elliptique. Épaiss. max. : 2 x 3,5.

Surface ext., int. et fracture : jaune clair (2.5Y8/2).

Inclusions : inclusions grisâtres et blanches de petites dimensions, à fréquence basse, et inclusions blanches, de dimensions moyennes-petites, à fréquence basse.

Engobe : traces d'engobe gris. 
Observations et comparaisons : attribuable à une amphore corinthienne du type B par sa morphologie et sa pâte, sans aucune possibilité d'en préciser la chronologie. Chronologie : non définissable.

\section{SB8 (pl. III)}

Année de la fouille / trouvaille : 1989.

Secteur : «Podere Forzello », Sondage 2. US : 9. 10. 11 .

Type : « forme $1 \alpha$ » ou corinthienne B.

État de conservation : segment inférieur d'une anse avec la jonction de l'épaule. H. max. cons. : 5 . Anses en ruban épaissi, à section elliptique. H. max. cons. : 4,5 ; épaiss. $\max .: 2,2 \times 4,1$.

Surface ext., int. et fracture : jaune clair (2.5Y8/2).

Inclusions : inclusions grisâtres et blanches de petites dimensions, à fréquence basse, et inclusions blanches, de dimensions moyennes-petites, à fréquence basse.

Observations et comparaisons : attribuable à une amphore de production occidentale, sans aucune possibilité d'en préciser la chronologie.

Chronologie : non définissable.

\section{SB9 (pl. III)}

Année de la fouille / trouvaille : 1983.

Secteur : «Podere Forzello ».

Inventaire $\mathrm{n}^{\circ}$ : IG 45618.

Type : «forme $1 \alpha »$ ou corinthienne B.

État de conservation : court segment d'épaule reconstitué à partir de trois fragments. Dimensions max. cons. : $9,5 \times 4,6$.

Surface ext. et int. : jaune pâle-verdâtre (5Y8/2).

Inclusions : argile épurée avec inclusions blanchâtres, brunes et grises de petites dimensions, à fréquence basse, distribuées de manière uniforme. Rarissimes écailles fines de mica argenté.

Appareil épigraphique : graffito alphanumérique tracé après cuisson avec une pointe de grandes dimensions. Le graffito apparait incomplet mais il semble possible d'y lire, sur la gauche, un lien alphabétique réalisé avec un trait peu profond et régulier (bien que délabré par endroits) et sur la droite une barre verticale incisée plus en profondeur (Sacchetti 2010, p. 47-49, cat. n 6, fig. 2, pl. II. 6, III. 6).

Observations et comparaisons : par le type de la pâte le fragment en question pourrait appartenir à un exemplaire de « forme $1 \alpha$ » ou bien à une amphore corinthienne du type $\mathrm{B}$, mais l'absence d'éléments morphologiques conservés susceptibles d'indiquer une datation impose évidemment la prudence quant à son encadrement typo-chronologique. Chronologie : non définissable.

Bibliographie : Sacchetti 2010, p. 47-49, cat. $n^{\circ}$ 6, fig. 2, pl. II. 6, III. 6.

\section{SB10 (pl. III)}

Année de la fouille / trouvaille : 1989.

Secteur : «Podere Forzello », Sondage 2.

US : 1

Type : « forme $1 \alpha »$ ou corinthienne B.

État de conservation : fragment de col avec jonction de l'épaule. H. max. cons. : 3, 6. Col cylindrique. H. max. cons. : 2,3 ; larg. max. cons. 3,1 .

Surface ext. : beige rosé (5YR7/3-10YR8/2).

Surface int. : beige (10YR8/2).

Fracture : jaune clair (10YR8/3).

Inclusions : inclusions noirâtres, de petites dimensions, à fréquence moyenne-haute ; inclusions blanches de dimensions petites et moyennes, à fréquence basse ; et rares inclusions brunes de dimensions petites et moyennes.

Observations et comparaisons : attribuable par sa pâte à un exemplaire de production occidentale.

Chronologie : non définissable.

\section{SB11 (pl. III)}

Année de la fouille / trouvaille : 1989.

Secteur : «Podere Forzello».

US : 31 .

Type : « forme $1 \alpha »$.

État de conservation : partie de lèvre et de col avec traces de la jonction supérieure d'une anse. H. max. cons. : 5,3. Lèvre en bourrelet gonflé. H. : 2,6 ; épaiss. max. : 2 ; diam. int./ext. restituable : 11,2 x 14,8. Col qui présente une ample cannelure immédiatement au-dessous de la lèvre.

Surface ext., int. et fracture : gris-jaunâtre (2.5YR7/2). Inclusions : inclusions noirâtres, brunes et blanches, de dimensions petites et moyennes, à fréquence moyenne-basse.

Observations et comparaisons : la morphologie de la lèvre, du type en bourrelet gonflé, et la présence d'une cannelure au-dessous, permettent de la situer à l'intérieur du groupe de «forme $1 \alpha$ ».

Pour le type, Koehler 1978a, fig. 2.a et Sourisseau 2011 ; pour la forme, assez proche, Semeraro 1997, fig. 208.949, d'Otrante.

Chronologie : 575/550-490 av. J.-C.

\section{SB12 (pl. III)}

Lieu de découverte : San Basilio, Ariano Polesine (Ro). Année de la fouille / trouvaille : 1989.

Secteur : « Podere Forzello ».

US : 31 .

Type : « forme $1 \alpha »$.

État de conservation : fragment de lèvre. H. max. cons. : 3,9. Lèvre en bourrelet gonflé. H. : 3 ; épaiss. max. : 1,8 ; diam. int./ext. restituable embouchure : 12 x 15,2. 
Surface ext.,int.et fracture : gris-verdâtre (5Y6/1-5Y6/2). Inclusions : inclusions noirâtres, brunes et blanches, de dimensions petites et moyennes, à fréquence moyenne. Observations et comparaisons : sa morphologie indique son appartenance à la production du groupe de « forme $1 \alpha »$. Pour le type, Koehler 1978a, fig. 2.a ; pour la forme, Semeraro 1997, fig. 98.320, d'Oria.

Chronologie : 575/550-490 av. J.-C.

\section{SB13 (pl. IV)}

Année de la fouille / trouvaille : 1983.

Secteur : «Podere Forzello », Sondage 1.

Type : « forme $1 \alpha »$.

État de conservation : amphore commerciale dont la partie supérieure est conservée, de la lèvre jusqu'à une partie de l'épaule, reconstituée à partir de trois fragments. H. max. cons. : 14. Lèvre en bourrelet gonflé, ouverte. H. : 3 , 1 ; épaiss. max. : 2,2 ; diam. int./ext. embouchure : 12 / 15,2. Col cylindrique, légèrement évasé, avec un haut ressaut immédiatement au-dessous de la lèvre et un fin ressaut à la base. H. : 6,8; diam. min./max. : 11,9. Anses verticales, en ruban épaissi, à section elliptique. H. : 9 ; épaiss. max. : 2,3 x 3,7. Épaule aplatie. Larg. max. cons. 9 .

Surface ext. : beige rosé (5YR7/3-10YR8/2).

Surface int. : beige (10YR8/2).

Fracture : jaune clair (10YR8/3).

Inclusions : inclusions noirâtres, de petites dimensions, à fréquence moyenne-haute ; inclusions blanches de dimensions petites et moyennes, à fréquence basse, et rares inclusions brunes de dimensions petites et moyennes.

Observations et comparaisons : attribuée par M.C. Cattaneo au groupe «corinthien B archaïque ». Pour le type, Koehler 1978a, fig. 2.a ; pour la forme de la lèvre, spécifiquement, Semeraro 1997, fig. 208. 942a (d'Otrante, datée sur base stratigraphique de la fin $\mathrm{du}^{\mathrm{VI}}{ }^{\mathrm{e}}$ s. av. J.-C.) ; Ceka 1986, pl. III, 5 (de Belsh, datable sur la base du contexte de la première moitié du $\mathrm{VI}^{\mathrm{e}} \mathrm{s}$. av. J.-C.). Dans le cadre de l'Italie septentrionale on signale son analogie morphologique avec un exemplaire de Marzabotto (Sacchetti 2005, cat. MA41).

Chronologie : 575/550-490 av. J.-C.

Bibliographie : Cattaneo Cassano 1995, p. 385, pl. VI, 5 ; Cattaneo 1996, p. 324, fig. 3, 5.

\section{SB14 (pl. IV)}

Année de la fouille / trouvaille : 1989.

Secteur : « Podere Forzello », Saggio 2.

US : 9 .

Type : « forme $1 \alpha$ ».

État de conservation : anse complète. H. max. cons. : 12, 3. Anses en ruban épaissi, à section elliptique. H. : 12, 3 ; épaiss. max. : $2,3 \times 4$.
Surface ext., int. et fracture : gris-jaunâtre (2.5YR8/2-2.5YR7/1).

Inclusions : inclusions noirâtres, brunes et blanches, de dimensions petites et moyennes, à fréquence moyenne-basse.

Observations et comparaisons : sa morphologie et sa pâte indiquent une probable appartenance à une amphore de « forme $1 \alpha$ ».

Chronologie : 575/550-490 av. J.-C.

SB15 (pl. IV)

Secteur : «Podere Forzello ».

US : 34 .

Type : « forme $1 \alpha »$.

État de conservation : partie de fond, reconstituée à partir de deux fragments. H. max. cons. : 5,1. Fond cylindrique au profil caréné. H. : 2,5; diam. min./max. restituable : 5,1 / 7 . Surface ext. et int. : chamois-gris (10YR6/2-10YR6/3). Fracture : beige-gris (10YR7/4).

Inclusions : inclusions noires et brunes à fréquence moyenne, de petites dimensions, et rares inclusions blanches de grandes dimensions. Mica argenté à fréquence basse distribué sur toute la surface.

Appareil épigrafique : sur le fond extérieur, au centre, signe circulaire incisé avant la cuisson à l'aide d'une pointe fine et avec un trait peu profond et régulier (Sacchetti 2010, p. 46-47, cat. n 5, pl. II. 5, III. 5).

Observations et comparaisons : attribuable à une amphore de «forme $1 \alpha$ ». Pour le type, Koehler 1978a, fig. 2.a et Sourisseau 2011 ; pour la forme, Slaska 1990, pl. 9.

Chronologie : 575/550-490 av. J.-C.

Bibliographie : Sacchetti 2010, p. 46-47, cat. n 5, pl. II. 5, III. 5.

\section{AMPHORES DE L'ÉGÉE SEPTENTRIONALE}

\section{SB16 (pl. IV)}

Année de la fouille / trouvaille : 1989.

Secteur : « Podere Forzello », Saggio 2.

US $: 1$.

Type : nord-égéenne.

État de conservation : partie de lèvre et col, complet jusqu'à la jonction de l'épaule, avec des traces de la jonction supérieure d'une anse. H. max. cons. : 8,4. Lèvre en coin, ouverte, avec un léger ressaut de base. H. : 1,7 ; épaiss. max. : 1,5; larg. max. cons. embouchure : 8,5. Col tronconique. H. : 6,5; larg. max. cons. : 10,7.

Surface ext. et int. : chamois (2.5YR6/4).

Fracture : mauve (2.5YR6/3).

Inclusions : inclusions de petites dimensions blanches et noirâtres à fréquence moyenne-basse. Mica fin, argenté, à fréquence basse.

Engobe : traces d'engobe blanchâtre. 
Appareil épigraphique : sur le col, entre les anses, trait vertical brun-noirâtre peint avant la cuisson. Le dipinto est coupé en bas par la fracture horizontale du col de l'amphore, en correspondance de la jonction de l'épaule, et immédiatement à sa gauche est située la fracture verticale (Sacchetti 2010, p. 49-50, cat. ${ }^{\circ}$ 7, fig. 3, pl. II. 7, III. 7).

Observations et comparaisons : la lèvre du type en coin renvoie sans aucun doute à la production nord-égéenne et le col, encore court, permet de circonscrire la datation entre la fin du $\mathrm{VI}^{\mathrm{e}}$ et la première moitié du $\mathrm{V}^{\mathrm{e}} \mathrm{s}$. av. J.-C. Pour le type de lèvre, on renvoie en général aux exemplaires de Roberts 1986, fig. 43, du dépôt Q 12:3 de l'Agora datable entre 520 et 480 av. J.-C. Pour le détail de la forme ouverte de la lèvre et du fin ressaut de base, cf. plus spécifiquement Schmid 2001, p. 146, 152, n 4 , pl. 4, Kat. Nr. 4, datable du début du V $\mathrm{V}^{\mathrm{s}} \mathrm{s}$. Pour la forme, Monachov 1999b, fig. 18. 1-2, de la fosse n 69 d'Olbia, datables sur la base du contexte et de comparaisons entre 490 et 470 av. J.-C. ; et Schmid 2001, Kat. Nr. 3-7, pl. 3-7, datables entre la fin du $\mathrm{VI}^{\mathrm{e}}$ et la première moitié du Ve s. av. J.-C.

Chronologie : 525-480 av. J.-C.

Bibliographie : Salzani, Vitali 2003, fig. 18 (pour dessin); Sacchetti 2010, p. 49-50, cat. n 7, fig. 3, pl. II. 7, III. 7.

\section{SB17 (pl. IV)}

Année de la fouille / trouvaille : 1987.

Secteur : « Podere Forzello ».

US : 9.

Type : nord-égéenne, Disc-toe Lawall.

État de conservation : pied avec partie du fond de la panse. H. max. cons. : 5,1. Pied tronconique plein, à base légèrement convexe et ombiliquée. H. : 2,4 ; diam. min./ max. restituable : 5,2 / 5,4.

Surface ext. : chamois (10YR6/3-10YR6/4).

Surface int. : rougeâtre (2.5YR6/6).

Fracture : mauve (2.5YR5/4).

Inclusions : inclusions noirâtres de petites dimensions, à fréquence moyenne-haute, et inclusions blanches de dimensions petites et moyennes, à fréquence basse. Mica argenté, fin, à fréquence basse.

Observations et comparaisons : attribuable à la production nord-égéenne et plus précisément au type disc-toe de M. Lawall, sans qu'il soit possible d'indiquer son lieu de production. Pour le type, Lawall 1995, p. 158-159, fig. 66, datable dans le courant de la première moitié du $\mathrm{V}^{\mathrm{e}}$ s. av. J.-C. ; pour la forme, Spagnolo 2003, pl. II.4, de production thasienne et datable déjà à partir de la fin $\mathrm{du} \mathrm{VI}^{\mathrm{e}}$ s. av. J.-C. Pour la possibilité d'une datation de la forme dès la prériode de transition du $\mathrm{VI}^{\mathrm{e}}$ au $\mathrm{V}^{\mathrm{e}} \mathrm{s}$. av. J.-C., Lawall 1995, p. 132-134, 158-159.

Chronologie : 500-440 av. J.-C.

\section{SB18 (pl. IV)}

Année de la fouille / trouvaille : 1983.

Secteur : " Podere Forzello », Sondage dans la fosse à l'ouest de la plantation de pêchers.

Type : nord-égéenne.

État de conservation : partie inférieure d'une anse. H. max. cons. : 8,6. Anses en ruban épaissi, à section elliptique, avec impression digitale à la base. H. max. cons. : 8,6 ; épaiss. max. : 2 x 3,2.

Surface ext. et int. : rose-gris (5YR7/1).

Fracture : mauve (2.5YR6/2).

Inclusions : inclusions noires de petites dimensions, à fréquence haute, et inclusions blanches et orange, de dimensions petites et moyennes, à fréquence moyenne. Observations et comparaisons : le type de la pâte et la présence d'une impression digitale à la base de l'anse indiquent son appartenance à la production nord-égéenne. Pour le type de l'anse on renvoie en général à Blondé, Muller, Mulliez 1991 ; Grandjean 1992 ; Schmid 2001.

Chronologie : non définissable.

\section{SB19 (pl. IV)}

Année de la fouille / trouvaille : 1987.

Secteur : « Podere Forzello ».

$\mathrm{I}^{\text {er }}$ niveau à cote -20.

Type : nord-égéenne.

État de conservation : départ inférieur d'une anse avec la jonction de l'épaule. H. max. cons. : 10,2. Anses en ruban épaissi, à section elliptique, avec impression digitale à la base. H. max. cons.10,2 : épaiss. max. : 2,1 x 3,1 . Surface ext. : beige rosé (2.5YR7/6).

Surface int. et fracture : orange rosé (2.5YR6/4).

Inclusions : inclusions brunes, blanches et noirâtres de petites dimensions, à fréquence moyenne. Écailles de mica argenté de petites dimensions, à fréquence moyenne.

Observations et comparaisons : voir la fiche précédente. Chronologie : non définissable.

\section{SB20 (pl. IV)}

Année de la fouille / trouvaille : 1989.

Secteur : «Podere Forzello », Sondage 2 (approfondissement pompe).

US : 9. 10.11.

Type : nord-égéenne.

État de conservation : secteur supérieur d'une anse avec une partie de la jonction du col. H. max. cons. : 4,6 . Anses en ruban épaissi, à section elliptique. H. max. cons. : 4,6 ; épaiss. max. : $2,2 \times 3,4$.

Surface ext. et int. : mauve (5YR6/3).

Fracture : brune (5YR5/4).

Inclusions : inclusions blanches de dimensions petites et moyennes, à fréquence moyenne-basse, et inclusions 
noirâtres de petites dimensions, à fréquence basse. Rares écailles de mica argenté.

Engobe : engobe blanchâtre.

Observations et comparaisons : on suggère l'attribution à une fabrique nord-égéenne, en raison non seulement du type de la pâte, mais aussi du caractéristique écrasement latéral de l'anse. Pour le type, cf. cat. SB18. Chronologie : non définissable.

\section{SB21 (pl. IV)}

Année de la fouille / trouvaille : 1983.

Secteur : «Podere Forzello », Sondage 1.

Type : nord-égéenne.

État de conservation : fragment de lèvre. H. max. cons. : 3,2. Lèvre en coin, avec une fine cannelure à la base. H. : 2,4 ; épaiss. max. 1,4; diam. int./ext. restituable embouchure : 13,2 / 16.

Surface ext. : chamois (2.5YR6/4).

Surface int. : mauve (2.5YR6/3).

Fracture : mauve intense (2.5YR5/3).

Inclusions : inclusions blanches de dimensions petites et moyennes, à fréquence moyenne-haute, et inclusions noirâtres de petites dimensions, à fréquence moyenne. Mica argenté et doré très fin, à fréquence basse.

Observations et comparaisons : le fragment, assigné d'une manière dubitative par M.C. Cattaneo à une fabrique milésienne, est attribuable à la production nord-égéenne de la fin du $\mathrm{VI}^{\mathrm{e}}$ ou du Ve s. av. J.-C. Pour la forme, Roberts 1986, fig. 43 (notamment, pour la fine cannelure à la base de la lèvre, l'exemplaire $n^{\circ} 424$ ), du dépôt Q 12:3 de l'Agora datable entre 520 et 480 av. J.-C. Les dimensions réduites du fragment suggèrent cependant d'être prudents sur une excessive limitation chronologique, car des lèvres analogues à celle en question caractérisent la production nord-égéenne de tout le $\mathrm{V}^{\mathrm{e}} \mathrm{s}$. av. J.-C., comme le montre l'ample documentation publiée dans : Blondé, Muller, Mulliez 1991 ; Grandjean 1992 ; Schmid 2001. Chronologie : non définissable.

Bibliographie : Cattaneo Cassano 1995, p. 387, pl. VI, 4 ; Cattaneo 1996, p. 324, fig. 3, 4.

\section{AMPHORES DE L'ÉGÉE ORIENTALE}

\section{SB22 (pl. V)}

Année de la fouille / trouvaille : 1989.

Secteur : «Podere Forzello », Sondage 2.

US : 7/7bis.

Type : chiote, Lambrino A ou swollen-necked, early bulgy, variante $\mathrm{C} / 1$ Knigge-Lawall.

État de conservation : fragment d'anse. H. max. cons. : 4,5 ; épaiss. max. cons. : 2,6 x 1,5.

Surface : chamois-beige (7.5YR6/4).

Fracture : chamois rosé (5YR6/4).
Inclusions : inclusions grisâtres et blanches de dimensions petites et moyennes, à fréquence moyenne-basse.

Observations et comparaisons : les caractéristiques de la pâte et la présence d'une fine ligne rouge qui parcourt l'anse sur toute sa longueur, suggèrent l'appartenance à une amphore chiote. Ce détail décoratif, ainsi que l'absence de white slip permettent de l'attribuer soit aux types Lambrino A1/A2 (Lambrino 1938, p. 110-112, fig. 71-72 ; Dupont 1998, p. 148-149, fig. 23.2, a-c.), datables de la seconde moitié du VI s. av. J.-C., soit à la variante plus archaïque (C1 Knigge-Lawall) du type swollen-necked, early bulgy (cf. Knigge 1976, p. 23-24 ; Lawall 1995, p. 89-90 ; Dupont 1998, fig. 23.2, d), datable du premier quart du $\mathrm{V}^{\mathrm{e}} \mathrm{s}$. av. J.-C.

Chronologie : 550-475 av. J.-C.

\section{SB23 (pl. V)}

Année de la fouille / trouvaille : 1989.

Secteur : « Podere Forzello », Sondage 2.

US : 1

Type : chiote, swollen-necked.

État de conservation : fragment de pied. H. max. cons. : 2 . Pied cylindrique creux, avec base en anneau épaissi. H. max. cons. : 2 ; diam. min./max. restituable : 4,6 / 5 . Surface ext. et int. : beige (7.5YR7/3).

Fracture : orange (5YR7/3-5YR7/4).

Inclusions : inclusions noirâtres, de petites dimensions, à fréquence haute, inclusions blanches de dimensions petites et moyennes, à fréquence moyenne. Rares écailles minces de mica argenté.

Observations et comparaisons : sa forme et sa pâte témoignent de son attribution à la production de Chios. La morphologie de sa base d'appui, épaissie et repliée vers l'intérieur suggèrent son appartenance aux formes swollen-necked et par conséquent une datation entre le premier et le troisième quart du $\mathrm{V}^{\mathrm{e}} \mathrm{s}$. av. J.-C.

Chronologie : 500-425 av. J.-C.

\section{SB24 (pl. V)}

Année de la fouille / trouvaille : 1989.

Secteur : «Podere Forzello », Sondage 2.

US : 10 .

Type : clazoménienne.

État de conservation : partie de l'épaulement avec la jonction inférieure d'une anse. Épaule fuyante. Larg. max. cons. 11,3. Anses en ruban épaissi. H. max. cons. : 3,5 ; épaiss. max. cons. : 1 x 2,5.

Surface ext. et int. : beige rosé (5YR7/4-10YR7/4).

Fracture : brun-mauve (2.5YR5/4-2.5YR5/6).

Inclusions : inclusions noirâtres et brunes de petites dimensions, à fréquence basse, et inclusions blanches, de dimensions petites et moyennes, à fréquence basse. Mica argenté à fréquence basse. 
Engobe : traces d'engobe blanchâtre à l'intérieur.

Vernis : vernis brun-rouge étalé à grands coups de pinceau sur l'épaule, horizontalement, et sur l'anse, verticalement. La bande en vernis brun-rouge parcourant l'anse verticalement se prolongeait sans doute en bas, le long du corps de l'amphore, en dépassant la bande de vernis horizontale qui décorait l'épaule.

Observations et comparaisons : sa pâte et sa décoration indiquent son appartenance à la production de Clazomènes, mais, vu l'absence d'éléments morphologiques conservés susceptibles de fournir une datation, on ne peut qu'indiquer une collocation générique dans le cadre du VI ${ }^{\mathrm{e}}$ s. av. J.-C. (cf. en dernier, Sezgin 2004, avec bibliographie antérieure), sans cependant remonter avant 575 av. J.-C. en raison du contexte de la découverte.

Chronologie : 575/550-500 av. J.-C.

\section{SB25 (pl. V)}

Année de la fouille / trouvaille : 1989.

Secteur : « Podere Forzello».

US : 31 .

Type : milésienne.

État de conservation : fragment de lèvre avec une petite partie du col. H. max. cons. : 4. Lèvre en amande avec un ressaut de base. H. : 2,2 ; épaiss. max. : 0,9; larg. max. cons. embouchure : 2,5 .

Surface ext. et int. : beige (7.5YR8/3).

Fracture : chamois (7.5YR6/4).

Inclusions : inclusions noirâtres et blanches de petites dimensions et à fréquence très basse. Écailles de mica doré, de dimensions moyennes et petites, à fréquence basse.

Observations et comparaisons : la morphologie de la lèvre, à section en amande et avec un ressaut en dessous, ainsi que son épaisseur particulièrement mince, attestent son attribution à la production milésienne d'époque archaïque. Cette morphologie est caractéristique tant des formes de la seconde moitié du VII ${ }^{\mathrm{e}}$ s. av. J.-C. (Naso 2005, fig. 2, cat. 4), que des formes datables de la première moitié ou au plus tard du troisième quart du $\mathrm{VI}^{\mathrm{e}} \mathrm{s}$. av. J.-C. (Dupont 1998, fig. 23.8, e). La chronologie du site de la découverte fixe pour cet exemplaire un terminus post quem à 575 av. J.-C.

Chronologie : 575/550-525 av. J.-C.

\section{SB26 (fig. 76 ; pl. V)}

Secteur : «Podere Forzello ».

US : 40 .

Type : milésienne.

État de conservation : fragment de lèvre. H. max. cons. : 5. Lèvre en amande soulignée par un ressaut prononcé à la base. H. : 2 ; épaiss. max. : 0,8 ; diam. int./ext. restituable embouchure : $15 / 15,4$.

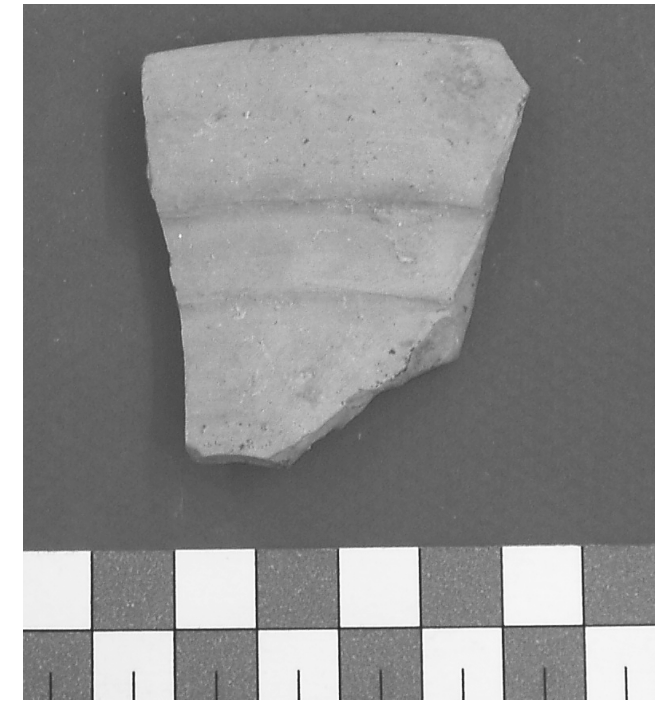

Fig. 76. Ariano Polesine, lieu-dit San Basilio, lèvre d'amphore de type milésien archaïque. Cat. SB26 (photo F. Sacchetti).

Surface ext. et int. : beige-chamois (7.5YR7/4).

Fracture : chamois-mauve (7.5YR6/2).

Inclusions : inclusions noirâtres, de dimensions petites et moyennes, à fréquence moyenne, et inclusions blanches, de dimensions petites et moyennes, à fréquence basse. Écailles de mica doré de petites dimensions, à fréquence moyenne.

Observations et comparaisons : pour la morphologie et l'encadrement chronologique, on renvoie à la fiche précédente.

Chronologie : 575/550-525 av. J.-C.

\section{SB27 (pl. V)}

Année de la fouille / trouvaille : 1989.

Secteur : « Podere Forzello».

US : 35 .

Type : milésienne.

État de conservation : fragment de lèvre avec un petit segment du col. H. max. cons. : 4,8. Lèvre en amande soulignée par un ressaut à la base. H. : 2 ; épaiss. max. : 0,9 ; larg. max. cons. embouchure : 3,3 .

Surface ext. et int. : beige (7.5YR8/3).

Fracture : chamois (7.5YR6/4).

Inclusions : inclusions noires et blanches de petites dimensions et à fréquence très basse. Écailles de mica doré de dimensions petites et moyennes, à fréquence basse.

Observations et comparaisons : voir la fiche SB25.

Chronologie : 575/550-525 av. J.-C.

SB28 (pl. V)

Année de la fouille / trouvaille : 1988.

Secteur : «Podere Forzello ».

US : 56 . 
Type : milésienne.

État de conservation : lèvre, ébréchée, avec un court segment du col. H. max. cons. : 5,5. Lèvre en amande soulignée par un ressaut à la base. H. : 1,5 ; épaiss. max. 1 ; larg. max. cons. embouchure : 1,5.

Surface ext., int. et fracture : beige (10YR7/4).

Inclusions : inclusions noires et blanches de petites dimensions, à fréquence basse. Écailles de mica doré de dimensions petites et moyennes, à fréquence moyenne-basse.

Observations et comparaisons : voir la fiche SB25.

Chronologie : 575/550-525 av. J.-C.

\section{SB29 (pl. V)}

Secteur : « Podere Forzello ».

Type : Samos-Milet, S/1 Lawall.

État de conservation : fragment de lèvre avec un court segment du col. Dimensions : non repérées. Lèvre en cordon, arrondie à l'intérieur et à l'extérieur, avec un renfoncement à la base. Col cylindrique qui présente un ressaut au-dessous de la lèvre.

Observations et comparaisons : non identifiée par M.C. Cattaneo qui en publie le dessin. La morphologie de la lèvre suggère l'attribution au groupe Samos-Milet et en particulier aux types dits Zeest's Samian. Cette forme présente une certaine analogie avec le type $\mathrm{S} / 1$ di M. Lawall et notamment, par la ligne incisée au-dessous de la lèvre, avec sa version la plus archaïque, datable entre la fin du VI ${ }^{\mathrm{e}}$ s. av. J.-C. et 480 av. J.-C. (Lawall 1995, p. 178-179 ; Roberts 1986, pl. 17.413).

Chronologie : 525-480 av. J.-C.

Bibliographie : Cattaneo Cassano 1995, pl. VI, 6 (pour le dessin) ; Cattaneo 1996, fig. 3, 6 (pour le dessin).

\section{SB30 (pl. V)}

Année de la fouille / trouvaille : 1988.

Secteur : «Podere Forzello».

US : 56.

Type : Samos-Milet, S/3 Lawall.

État de conservation : fragment de lèvre. H. max. cons. : 4,8. Lèvre en amande avec une arête marquée à la base. H. : 3,1 ; épaiss. max. : 1,1 ; diam. int./ext. restituable embouchure : 17 / 17, 2.

Surface ext. : chamois (7.5YR6/4).

Surface int. : orange (7.5YR6/6).

Fracture : gris (7.5YR6/1-7.5YR6/2).

Inclusions : inclusions noirâtres et brunes de dimensions petites et moyennes, à fréquence moyenne-basse, et rares inclusions blanches de dimensions moyennes. Écailles de mica argenté et doré de dimensions moyennes et petites, à fréquence moyenne-haute.

Observations et comparaisons : par la forme de la lèvre, haute, fine et ouverte, elle est attribuable à la variante sans ressaut de base du type $\mathrm{S} / 3$ de $\mathrm{M}$. Lawall, exclusivement attesté dans des contextes d'époque archaïque tardive. Pour le type, Lawall 1995, p. 178, 184-185, fig. 76 ; pour la forme, Monachov 2003a, pl. 17.3.

Chronologie : 525-480 av. J.-C.

\section{SB31 (pl. V)}

Année de la fouille / trouvaille : 1989.

Secteur : «Podere Forzello », Sondage 2.

US : 15 .

Type : Samos-Milet, S/3 Lawall.

État de conservation : partie de lèvre et de col avec une anse complète. Reconstituée à partir de deux fragments. H. max. cons. : 11,8. Lèvre en amande, légèrement ouverte. H. : 2,5 ; épaiss. max. : 1,4 ; larg. max. cons. embouchure : 11. Col cylindrique, au profil légèrement concave. H. max. cons. : 4,8; larg. max. cons. 7,3. Anses verticales, en ruban épaissi, à section elliptique. H. max. cons. 8,5 ; épaiss. max. : 3,4 x 1,8 .

Surface ext., int. et fracture : chamois clair (7.5YR7/4). Inclusions : inclusions noirâtres et blanches de petites dimensions, à fréquence basse. Mica argenté fin, à fréquence basse.

Observations et comparaisons : par la forme de la lèvre, l'exemplaire peut être attribué au type $\mathrm{S} / 3$ de la classification de M. Lawall (Lawall 1995, p. 178, 184-185, fig. 76, 79).

Chronologie : 525-480 av. J.-C.

Bibliographie : Salzani, Vitali 2003, fig. 18 (pour le dessin).

\section{SB32 (pl. V)}

Année de la fouille / trouvaille : 1989.

Secteur : «Podere Forzello », Sondage 2.

US : 7 .

Type : Samos-Milet, S/1 Lawall.

État de conservation : pied et partie du fond de la panse, reconstitué à partir de deux fragments. Deux autres fragments de la panse, non reconstituables, appartiennent sans doute au même individu. H. max. cons. : 10,2. Pied cylindrique plein, épaissi, à base ombiliquée. H. : 3,1 ; diam. min./max. : 4,6 / 5,6.

Surface ext. et fracture : gris-mauve (7.5YR6/2).

Surface int. : mauve (5YR6/3).

Inclusions : inclusions de petites dimensions blanchâtres et noirâtres, à fréquence très basse. Écailles de mica argenté de petites et moyennes dimensions, à fréquence moyenne.

Observations et comparaisons : pour le type nous renvoyons à un fragment de l'Agora (dépôt F 19:4) datable encore du milieu du $\mathrm{V}^{\mathrm{e}} \mathrm{s}$. av. J.-C., sur base stratigraphique (Lawall 1995, fig. 75), et au fond d'un exemplaire lui aussi de l'Agora (P27531 du dépôt S 16:1) mais datable du dernier quart du Ve s. av. J.-C. (Grace 1971, p. 76, fig. 3.3 ; Lawall 1995, fig. 73). Pour la forme, Dupont 1998, fig. 23.9, g. La morphologie du pied en 

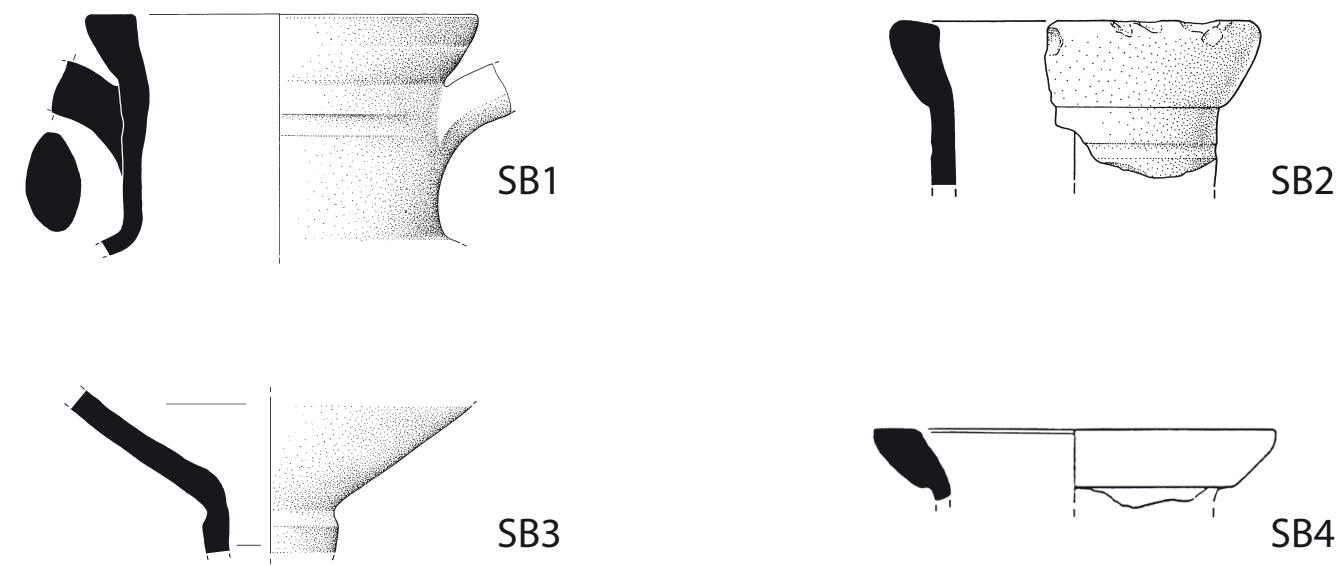

SB5
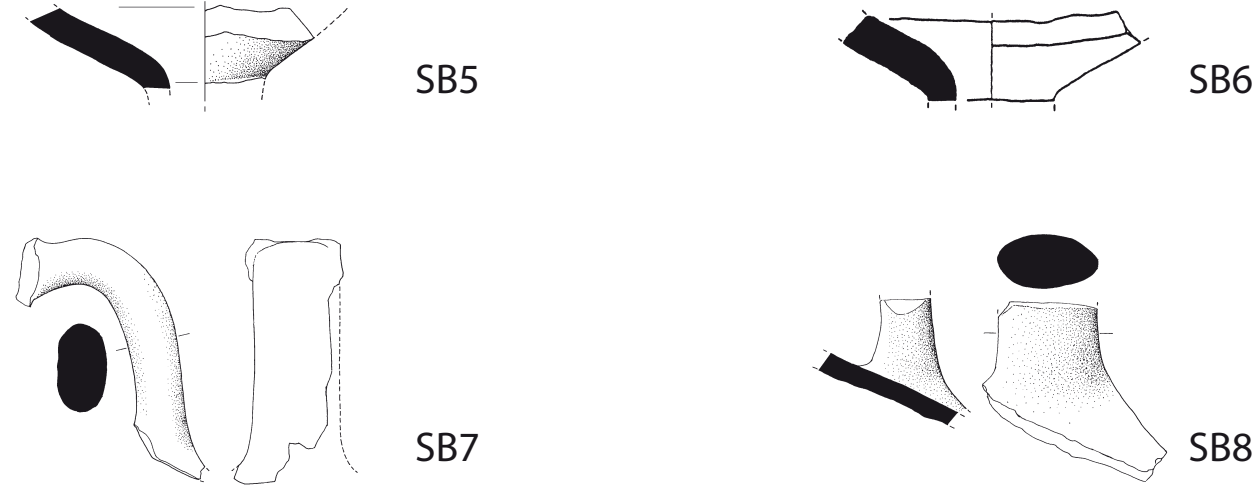

SB7
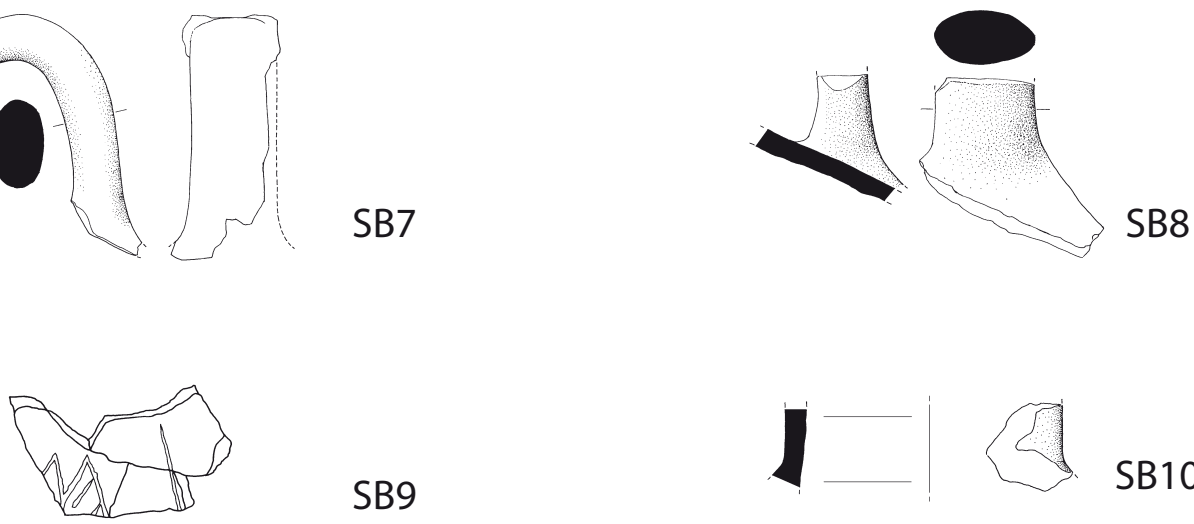

SB9
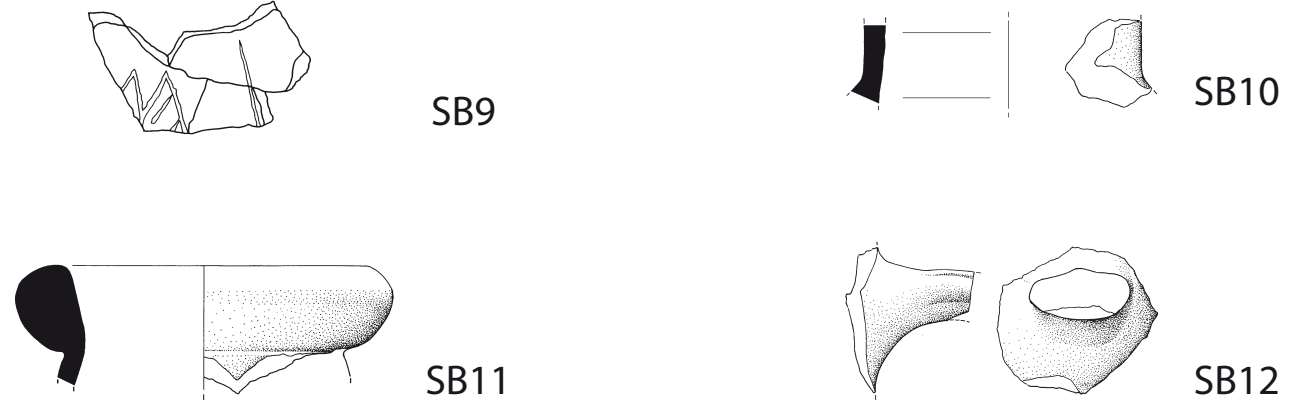

SB12

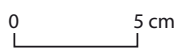

PI. III. San Basilio. 

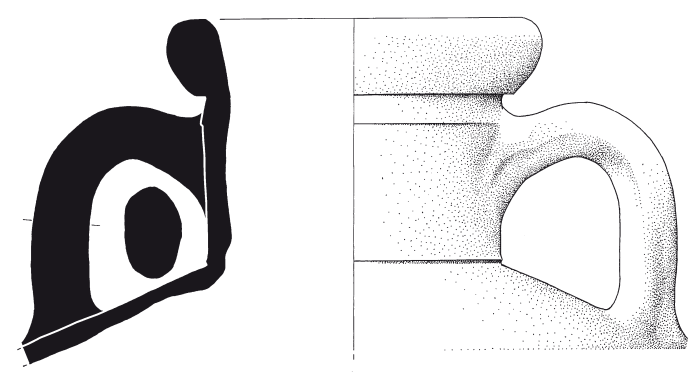

SB13
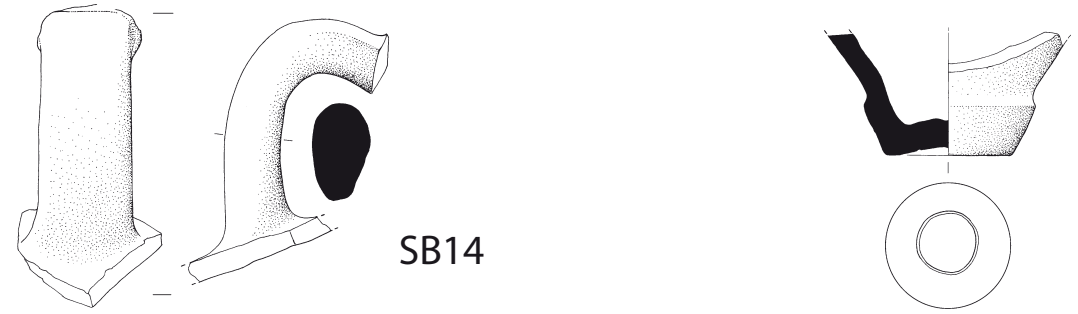

SB15
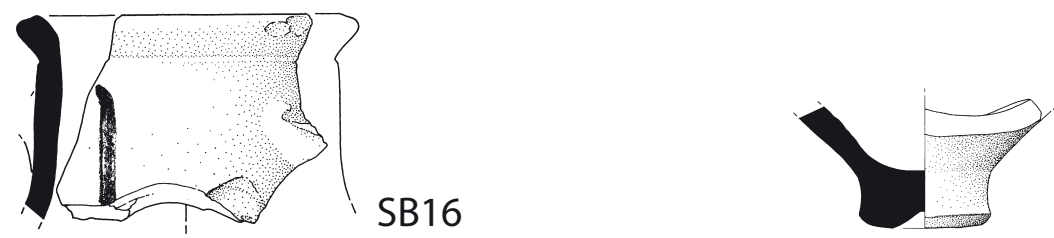

SB17
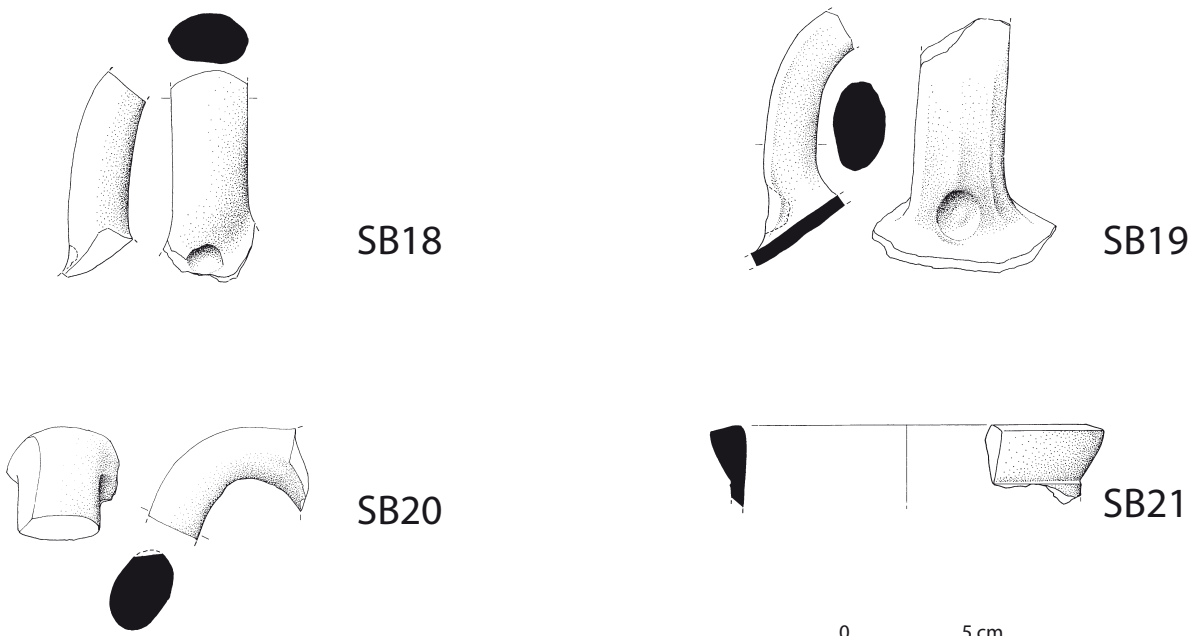

SB20 

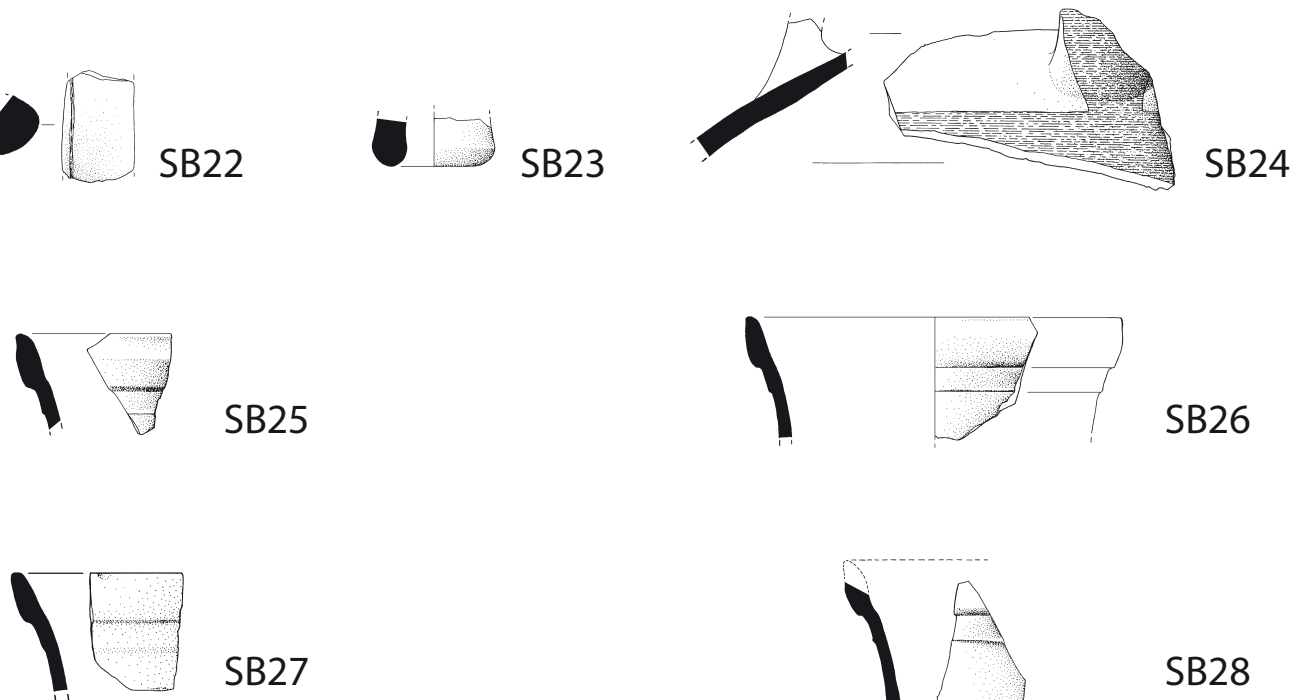

SB27

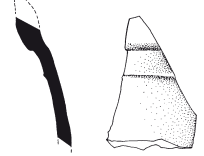

SB28

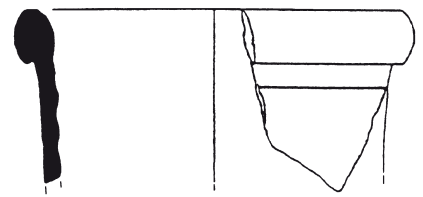

SB29

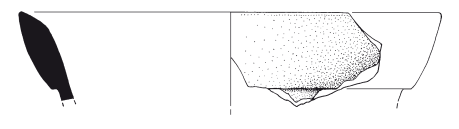

SB30
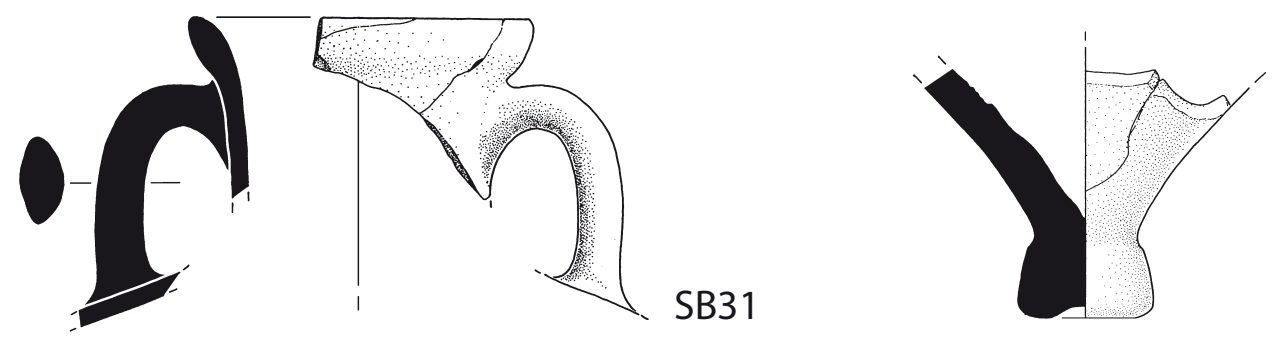

SB32
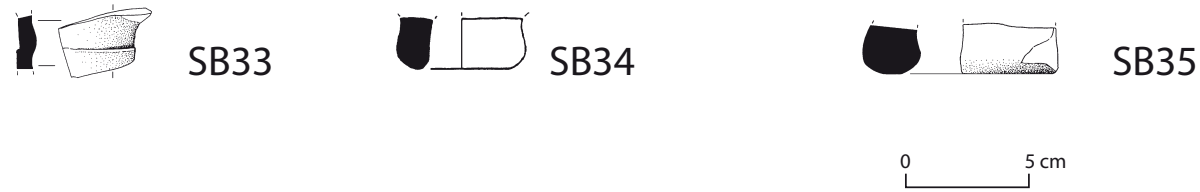

PI. V. San Basilio. 
question ne permet donc pas de l'encadrer avec précision dans le contexte de la variante plus récente du type $\mathrm{S} / 1$ de M. Lawall.

Chronologie : 450-400 av. J.-C.

Bibliographie : Salzani, Vitali 2003, fig. 18 (pour le dessin).

\section{SB33 (pl. V)}

Année de la fouille / trouvaille : 1989.

Secteur : « Podere Forzello ».

US : 7 .

Type : Samos-Milet, S/1 ou S/3 Lawall.

État de conservation : fragment de col. Dimensions max. cons. : 2,3 x 3,2. Col avec un fin ressaut. H. max. cons. : 3,2 ; larg. max. cons. 2,3.

Surface ext., int. et fracture : chamois (7.5YR6/4).

Inclusions : inclusions noirâtres et blanches de petites dimensions, à basse fréquence. Écailles de mica doré, de petites dimensions, à basse fréquence.

Observations et comparaisons : malgré les dimensions réduites du fragment, le ressaut conservé autorise sa probable attribution au groupe Samos-Milet, sans qu'il soit pour autant possible de distinguer entre les types $\mathrm{S} / 1$ et $\mathrm{S} / 3$ de $\mathrm{M}$. Lawall, l'un et l'autre caractérisés par ce détail morphologique (Lawall 1995, p. 177-185). L'absence d'éléments morphologiques conservés susceptibles d'indiquer une datation empêche de préciser un encadrement chronologique.

Chronologie : non définissable.

\section{SB34 (pl. V)}

Année de la fouille / trouvaille : 1989.

Secteur : «Podere Forzello », Sondage 2.

US : 1 .

Type : Solokha I.

État de conservation : fragment de pied. Pied tronconique creux, à base annulaire.

Surface ext. et int. : beige rosé (7.5YR8/3).

Fracture : orange-mauve (5YR7/6-5YR6/4).

Inclusions : inclusions blanches et grises de petites dimensions, à fréquence moyenne, et inclusions brunes de petites dimensions, à basse fréquence.

Observations et comparaisons : définie «attique » par M.C. Cattaneo. Pour le type, datable dans le courant de la seconde moitié du Ve s. av. J.-C., Grace 1953, p. 101102 ; Grace 1971, p. 72 ; et, en dernier, Lawall 1995, p. 220-221, fig. 93.

Chronologie : 450-400 av. J.-C.

Bibliographie : Cattaneo Cassano 1995, p. 388, pl. VI, 7 ; Cattaneo 1996, p. 326, fig. 3,7.

\section{SB35 (pl. V)}

Année de la fouille / trouvaille : 1989.

Secteur : « Podere Forzello », Sondage 2.
US : 1 .

Type : Solokha I.

État de conservation : fragment de pied. H. max. cons. :

2. Pied en anneau épaissi. H. max. cons. : 2 ; larg. max. cons. 3,9.

Surface ext. : beige rosé (5YR7/3-10YR8/2).

Surface int. : beige (10YR8/2).

Fracture : jaune clair (10YR8/3).

Inclusions : inclusions noirâtres, de petites dimensions, à fréquence moyenne-haute, inclusions blanches de petites et moyennes dimensions, à basse fréquence, et rares inclusions brunes de petites et moyennes dimensions.

Observations et comparaisons : attribuable par la morphologie à une amphore du groupe Solokha I de la seconde moitié du Ve s. av. J.-C. Pour le type, Lawall 1995, p. 220-221, fig. 93.

Chronologie : 450-400 av. J.-C.

\section{San Cassiano di Crespino (Rovigo) : l'habitat (fig. 69)}

\subsection{Bilan des études}

Dans la commune de Crespino (prov. de Rovigo), au lieu-dit San Cassiano, une enquête extensive a été conduite sur une petite implantation de la chora d'Adria qui présente des caractères étrusques marqués aussi bien par la récurrence de la céramique étrusco-padane (face à l'absence de classes typiquement vénètes) que par les données épigraphiques ${ }^{611}$.

Le site, situé aujourd'hui à $15 \mathrm{~km}$ au sud-ouest d'Adria, le long de la rive gauche du Pô, devait être établi, à l'époque étrusque, à $6 \mathrm{~km}$ environ au sud de la branche septentrionale du Pô, le Pô d'Adria des sources anciennes, actuellement correspondant au Canalbianco.

L'aire, objet de recherches de la part d'une équipe de l'Université de Pavie sous la direction de M. Harari, a restitué les vestiges d'un complexe d'édifices de planimétrie et de typologie différentes, mais caractérisés par la même orientation N-S et par leur intégration dans un projet intentionnel et cohérent qui prévoyait aussi un système de canalisation des eaux à travers des fosses parallèles orientées par rapport aux édifices (fig. 77).

Au cours des neuf campagnes menées sur le site de San Cassiano (1995-1998 ; 2000-2004) ont été isolés 332 fragments provenant peut-être d'amphores, dont l'étude m'a été confiée. On peut maintenant prendre

611. Pour les recerches archéologicques conduites à San Cassiano, cf. Harari 1999 ; Id. 2002 ; Id. 2004a ; Id. 2004b. 


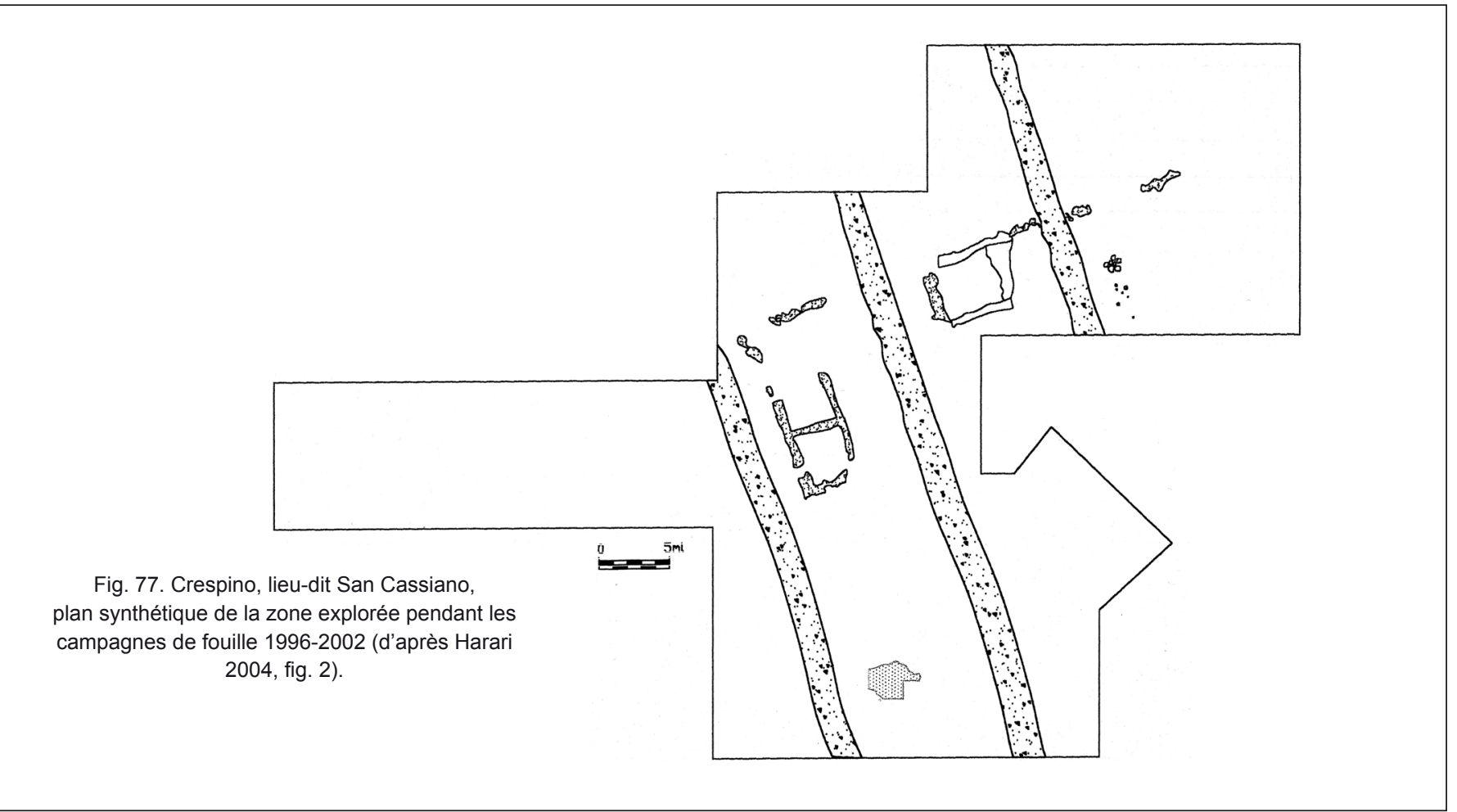

en compte 316 fragments appartenant à des amphores commerciales grecques, essentiellement des fragments de parois, mais aussi 11 fragments de formes : lèvre, col, anses, pied/fond. Il est bien évident que c'est exclusivement sur la base de ces fragments qu'il est possible d'obtenir des indications fiables quant à la provenance ou au type des conteneurs, à leur fourchette chronologique et au nombre même d'individus attestés à San Cassiano.

Les secteurs de l'aire fouillée ayant livré des amphores appartiennent pour la plupart à la première phase du grand oikos occidental (217) objet de recherches entre 1995 et 1998, dans les sondages profonds dénommés êta, kappa et iota. D'autres, moins nombreux, proviennent de la zone examinée entre 2000 et 2004 à l'est du deuxième canal de bonification ou de drainage. On a mis au jour ici un second complexe (41), compris entre le deuxième et le troisième canal, où les amphores ont été trouvées principalement dans les sondages phi et sigma et dans ce cas aussi elles doivent être attribuées à la première phase de l'édifice. En ce qui concerne la chronologie de cette phase, les données disponibles à l'heure actuelle suggèrent de la situer entre la fin du $\mathrm{VI}^{\mathrm{e}}$ et les débuts du Ve s. (Harari 1999, p. 629 ; Id. 2004a, p. 27).

Les seuls individus qu'il a été possible de distinguer parmi les fragments amphoriques de San Cassiano sont identifiés dans les fiches qui suivent par le sigle « $\mathrm{SC} »$. Ils sont publiés dans Sacchetti 2012 avec la même numérotation.

\subsection{Catalogue}

\section{AMPHORES GRÉCO-OCCIDENTALES (forme $1 \alpha)$}

\section{SC1 (pl. VI)}

Année de la fouille / trouvaille : 1997.

Secteur : sondage Êta.

Structure : au nord du grand oikos.

US : 4056.

Inventaire $n^{\circ}: 4056 / 3$.

État de conservation : partie de fond, reconstitué à partir de deux fragments. H. max. cons. : 3. Fond cylindrique au profil inférieurement caréné. H. : 2 ; diam. min./max. restituable : 5 / 5,3.

Surface ext. : gris-beige (2.5Y7/2-2.5Y6/2).

Surface int. et fracture : gris $(2.5 \mathrm{Y} 6 / 2)$.

Inclusions : inclusions blanchâtres de dimensions petites et moyennes, à fréquence moyenne, et inclusions grises et brunes de petites dimensions, à fréquence moyenne. Mica argenté fin, à basse fréquence. Quelques vacuoles. Observations et comparaisons : pour le type, Koehler 1978a, fig. 2.a.

Chronologie : 510-490 av. J.-C.

\section{SC2 (pl. VI)}

Année de la fouille / trouvaille : 2002.

Secteur : tranchée 2002.

US : 8001.

Inventaire $n^{\circ}: 8001 / 28$. 
État de conservation : fond, presque complet et reconstitué à partir de trois fragments, et fragment de paroi probablement relatif au même individu. H. max. cons. : 4. Fond cylindrique caréné. H. : 2 ; diam. min./ max. : 7 / 7,8.

Surface ext. : blanchâtre (5Y8/1-5Y8/2).

Surface int. et fracture : gris-beige (2.5Y8/2-2.5Y7/2). Inclusions : pâte épurée avec inclusions blanchâtres de dimensions petites et moyennes, à fréquence basse, et inclusions orange, de petites dimensions, à fréquence basse. Mica fin argenté à fréquence très basse.

Observations et comparaisons : pour le type, Koehler 1981, p. 452-453, pl. 99.a, d-e ; pour la forme, Semeraro 1997, fig. 48.171d, de Leuca, datée du dernier quart du $\mathrm{VI}^{\mathrm{e}}$ s. av. J.-C. ; Tigano 2002, p. 69, de la nécropole de Mylai, datée sur la base du mobilier de la seconde moitié du VI' s. av. J.-C. ; et Long, Miro, Volpe 1992, fig. 46.5, de l'épave de la Pointe Lequin, datable sur la base de la cargaison au dernier quart du $\mathrm{VI}^{\mathrm{e}} \mathrm{s}$. av. J.-C.

Chronologie : 510-490 av. J.-C.

\section{SC3 (pl. VI)}

Année de la fouille / trouvaille : 2002.

Secteur : tranchée 2002.

US : 8001.

Inventaire $n^{\circ}: 8001 / 27$.

État de conservation : anse presque complète. H. max. cons. : 13,7. Anses verticales, en ruban épaissi, à section elliptique, avec deux cannelures fines et irrégulières sur la face extérieure. H. max. cons.13,7 ; épaiss. max. : 5 x 2,5. Surface ext., int. et fracture : blanchâtre (5Y8/1-5Y8/2). Inclusions : pâte épurée, avec inclusions blanches de petites et moyennes dimensions, à basse fréquence, et inclusions orange, de petites dimensions, à basse fréquence. Mica fin argenté à fréquence très basse.

Observations et comparaisons : l'anse, vu le type de la pâte, fait probablement partie du même individu de la fiche précédente. La définition du type et la chronologie proposées s'appuient donc sur cette hypothèse.

Chronologie : 510-490 av. J.-C.

\section{SC4 (pl. VI)}

Année de la fouille / trouvaille : 1998.

Secteur : sondage Kappa.

Structure : grand oikos, pièce nord.

US : 5401 .

Inventaire $n^{\circ}: 5401 / 22$.

État de conservation : fragment de lèvre, à la surface supérieure lacunaire, et 27 fragments de paroi non reconstituables mais appartenant sans doute au même individu. H. max. cons. : 3. Lèvre en bourrelet gonflé, qui présente une très fine cannelure à la jonction avec le col. H. max. cons. : 2,4 ; épaiss. max. : 2,2 ; diam. int./ ext. restituable embouchure : 10,6 / 15 .

Surface ext. et fracture : gris clair (2.5Y7/2-2.5Y6/2).

Surface int. : gris-brun (2.5Y5/2).

Inclusions : inclusions blanchâtres, grises et brunes, de dimensions moyennes, à fréquence moyenne-haute. Mica argenté à fréquence très basse.

Observations et comparaisons : pour la forme, Semeraro 1997, fig. 208. 919a-d, d'Otrante.

Chronologie : 510-490 av. J.-C.

\section{SC5 (pl. VI)}

Année de la fouille / trouvaille : 1998.

Secteur : sondage Êta.

Structure : au nord du grand oikos.

US : 5012 .

Inventaire $\mathrm{n}^{\circ}: 5012 / 10$.

État de conservation : fragment de lèvre, à la surface supérieure lacunaire. H. max. cons. : 3,7. Lèvre en bourrelet gonflé, légèrement ouverte. H. max. cons. : 2,3 ; épaiss. max. : 2,1 ; diam. int./ext. restituable embouchure : 10,8 / 15 .

Surface ext., int. et fracture : beige clair (2.5Y8/2).

Inclusions : pâte épurée, avec inclusions grises et orange, de petites dimensions, à fréquence très basse. Mica argenté fin, à fréquence moyenne. Vacuoles.

Observations et comparaisons : pour la forme, Semeraro 1997, fig. 208. 919a-d, d'Otrante.

Chronologie : 510-490 av. J.-C.

\section{SC6 (pl. VI)}

Année de la fouille / trouvaille : 2003.

Secteur: Sondage Phi.

Structure : II ${ }^{\mathrm{e}}$ oikos, $\mathrm{I}^{\mathrm{ère}}$ phase.

US : 9406.

Inventaire $\mathrm{n}^{\circ}: 9406 / 10$.

État de conservation : partie de lèvre, reconstituée à partir trois fragments. H. max. cons. : 3,2. Lèvre en bourrelet gonflé. H. : 2,2 ; épaiss. max. : 2,3 ; diam. int./ ext. restituable embouchure : $11 / 14,9$.

Surface ext. et int. : beige clair $(2.5 \mathrm{Y} 8 / 2)$.

Fracture : beige-gris $(2.5 \mathrm{Y} 7 / 2)$.

Inclusions : pâte épurée, avec inclusions grises et brunes de petites dimensions, à fréquence très basse. Mica fin argenté à basse fréquence. Vacuoles, de petites dimensions mais nombreuses.

Observations et comparaisons : pour la forme, plutôt similaires, Semeraro 1997, fig. 208.942b, 943, dont la première est datée entre la fin du $\mathrm{VI}^{\mathrm{e}}$ et le début du Ve $\mathrm{s}$. av. J.-C. Chronologie : 510-490 av. J.-C.

\section{SC7 (pl. VI)}

Année de la fouille / trouvaille : 2003.

Secteur: sondage Phi. 
Structure : $\mathrm{II}^{\mathrm{e}}$ oikos, $\mathrm{I}^{\text {ère }}$ fase.

US : 9403.

Inventaire $\mathrm{n}^{\circ}: 9403 / 5-7$.

État de conservation : fragment de lèvre, lacunaire en correspondance de la surface supérieure, et deux fragments de paroi, non reconstituables mais probablement appartenant au même individu. H. max. cons. : 3. Lèvre en bourrelet gonflé. H. max. cons. : 2,2 ; épaiss. max. : 2, 3 ; diam. int./ext. restituable embouchure : 11,2 / 15. Surface ext. et int. : beige clair $(2.5 \mathrm{Y} 8 / 2)$.

Fracture : beige-gris $(2.5 \mathrm{Y} 7 / 2)$.

Inclusions : pâte épurée, avec inclusions grises et brunes de petites dimensions, à fréquence très basse. Mica fin argenté à basse fréquence. De nombreuses vacuoles de petites dimensions.

Observations et comparaisons : pour la forme, on renvoie à la fiche précédente.

Chronologie : 510-490 av. J.-C.

\section{SC8}

Année de la fouille / trouvaille : 1996.

Secteur : sondage Êta.

Structure : au nord du grand oikos.

US : 3034 .

Inventaire $n^{\circ}: 3034$ / 90 .

État de conservation : partie de fond, manquant du plan d'appui, et trois fragments de paroi non reconstituables mais probablement appartenant au même individu. H. max. cons. : 3,2. Fond cylindrique caréné. H. max. cons. : 2,5 ; diam. min./max. restituable : $5 / 5,8$.

Surface ext. : gris-beige (2.5Y7/2-2.5Y6/2).

Surface int. : grisâtre $(2.5 \mathrm{Y} 6 / 2-2.5 \mathrm{Y} 4 / 1)$.

Fracture : gris $(2.5 \mathrm{Y} 6 / 2)$.

Inclusions : inclusions blanchâtres de dimensions petites et moyennes, à fréquence moyenne, et inclusions grises et brunes de petites dimensions, à fréquence moyenne. Mica argenté fin, à basse fréquence. Quelques vacuoles. Observations et comparaisons : pour le type, Koehler 1981, p. 452-453, pl. 99.a, d-e.

Chronologie : 510-490 av. J.-C.

\section{AMPHORES DU TYPE À LA BROSSE (Agora 1502)}

\section{SC9 (fig. 78 ; pl. VI)}

Année de la fouille / trouvaille : 1998.

Secteur : sondage Kappa.

Structure : grand oikos, pièce nord.

US : 5404 .

Inventaire $n^{\circ}: 5404$ / 8 .

État de conservation : fragment de lèvre. H. max. cons. : 3,5 . Lèvre épaissie et saillante. H. : 2,5 ; épaiss. max. 1,8 ; diam. int./ext. restituable embouchure : $11 / 13$.

Surface ext. et int. : beige rosé clair (10YR8/2-10YR7/3).

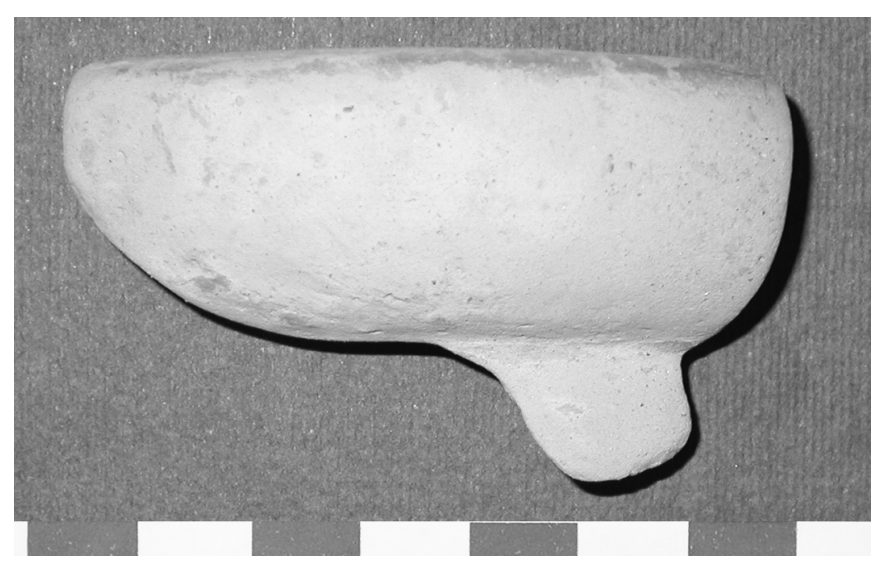

Fig. 78. Crespino, lieu-dit San Cassiano, lèvre d'amphore "à la brosse ». Cat. SC9 (photo F. Sacchetti).

Fracture : beige rosé (7.5YR7/4).

Inclusions : argile épurée avec de rares inclusions orange (chamotte) de petites dimensions. Mica fin argenté et doré à basse fréquence. Quelques vacuoles.

Observations et comparaisons : pour le type, Sparkes, Talcott 1970, p. 192, 341, cat. 1502, pl. 64, datable entre le milieu du VIe et 480 av. J.-C. Par sa morphologie on peut la comparer à un exemplaire du Forcello de Bagnolo San Vito daté, sur base stratigraphique, de la période de transition du VI ${ }^{\mathrm{e}}$ au V $\mathrm{V}^{\mathrm{e}} \mathrm{s}$. av. J.-C. (de Marinis 2007, p. 166, fig. 112, 1). Le fragment de San Cassiano présente une abrasion marquée de la surface (ce qui est le cas d'une grande partie du matériel céramique provenant du site) et ne conserve que de faibles traces de la peinture originaire dans la partie supérieure de la lèvre. Chronologie : 510-480 av. J.-C.

SC10

Année de la fouille / trouvaille : 1997.

Secteur : sondage Iota.

Struttura : grand oikos, pièce sud.

US : 4417.

Inventaire $n^{\circ}: 4417$ / 74 .

État de conservation : partie de col gardant des traces de la jonction inférieure d'une anse. Dimensions max. fr. : 11 x 5,2 . Col probablement cylindrique. H. max. cons. : 5,2 ; diam. min./max. restituable : 10 .

Surface ext. et int. : beige rosé clair (10YR8/2-10YR7/3). Fracture : beige rosé (7.5YR7/4).

Inclusions : pâte épurée, presque complètement dépourvue d'inclusions et contenant du mica argenté et doré.

Observations et comparaisons : l'attribution au type à la brosse et la chronologie sont suggérées par la possible appartenance du fragment au même exemplaire que celui de la fiche précédente, à laquelle on renvoie pour les observations et pour les comparaisons.

Chronologie : 510-480 av. J.-C. 

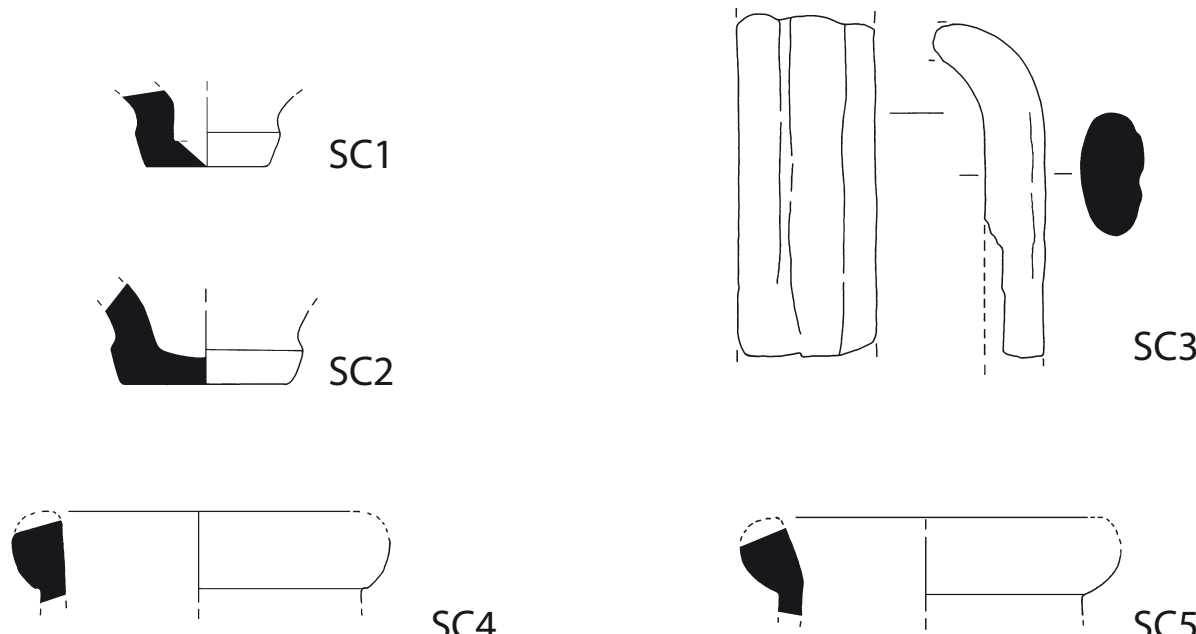

SC4
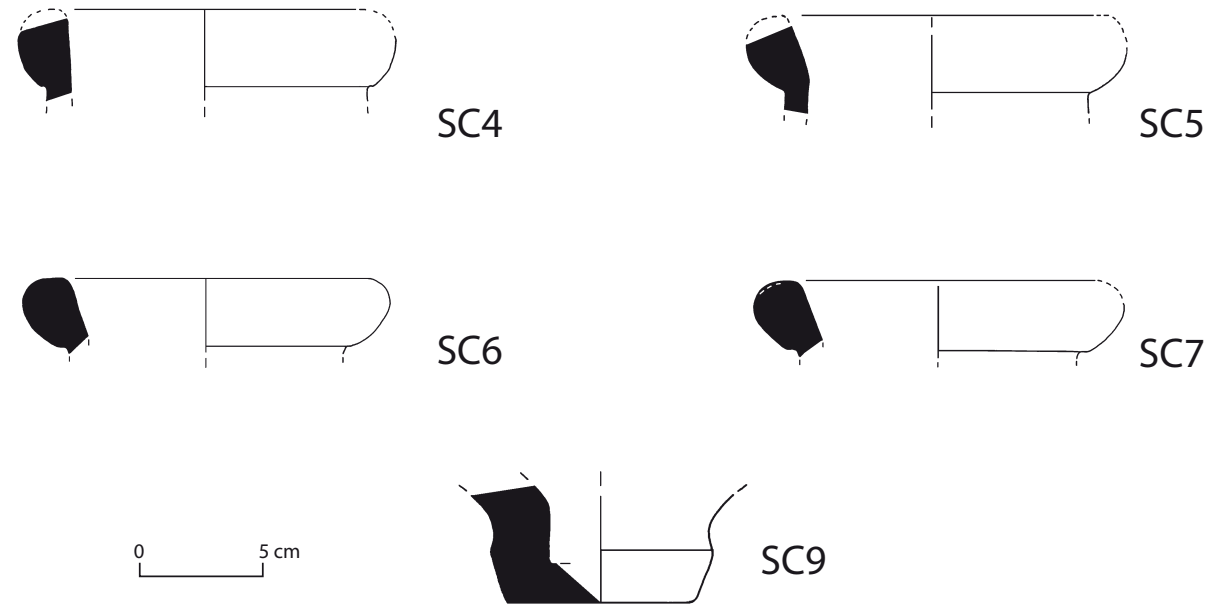

PI. VI. San Cassiano.

AMPHORES DE PRODUCTION NON IDENTIFIÉE

\section{SC11}

Année de la fouille / trouvaille : 2002.

Secteur : sondage Sigma.

Structure : $\mathrm{II}^{\mathrm{e}}$ oikos, $\mathrm{I}^{\mathrm{ere}}$ phase.

US : 8009 .

Inventaire $n^{\circ}: 8009 / 13$.

État de conservation : fragment de l'attache inférieure d'une anse.

Surface ext., int. et fracture : beige clair (2.5Y8/2).

Inclusions : inclusions brunes, de petites dimensions, à basse fréquence. Mica fin argenté à basse fréquence. Vacuoles.

Observations et comparaisons : le mauvais état de conservation du fragment ne permet pas de formuler un encadrement typologique. Sa provenance des niveaux de la première phase du site suggère une chronologie autour de la période de transition du $\mathrm{VI}^{\mathrm{e}}$ au $\mathrm{V}^{\mathrm{e}} \mathrm{s}$. av. J.-C., mais l'absence d'éléments morphologiques caractérisants nous oblige à le considérer de datation incertaine.

Chronologie : non déterminable.

\section{Le Balone de Rovigo : \\ la nécropole et l'habitat (fig. 69)}

\subsection{Bilan des études}

La découverte d'un site archéologique au lieu-dit Le Balone, dans le territoire méridional de la commune de Rovigo, eut lieu en 1985 à l'occasion de travaux publics effectués à proximité de la voie rapide Transpolesana ${ }^{612}$. L'intervention dans cette aire a consisté en deux campagnes de sondages, en 1978 et en 1988, mais aucune intervention extensive n'a encore été faite. La Surintendance Archéologique de la région de la Vénétie et le Musée « dei Grandi Fiumi » de Rovigo ont pu vérifier, à ces occasions-là, la présence d'une aire probablement destinée aux habitations et d'une aire réservée à la nécropole.

Seules quatre tombes ont été mises au jour. Le secteur principal de la nécropole semble en effet se trouver juste

612. Alberti 1986, p. 317-324. 

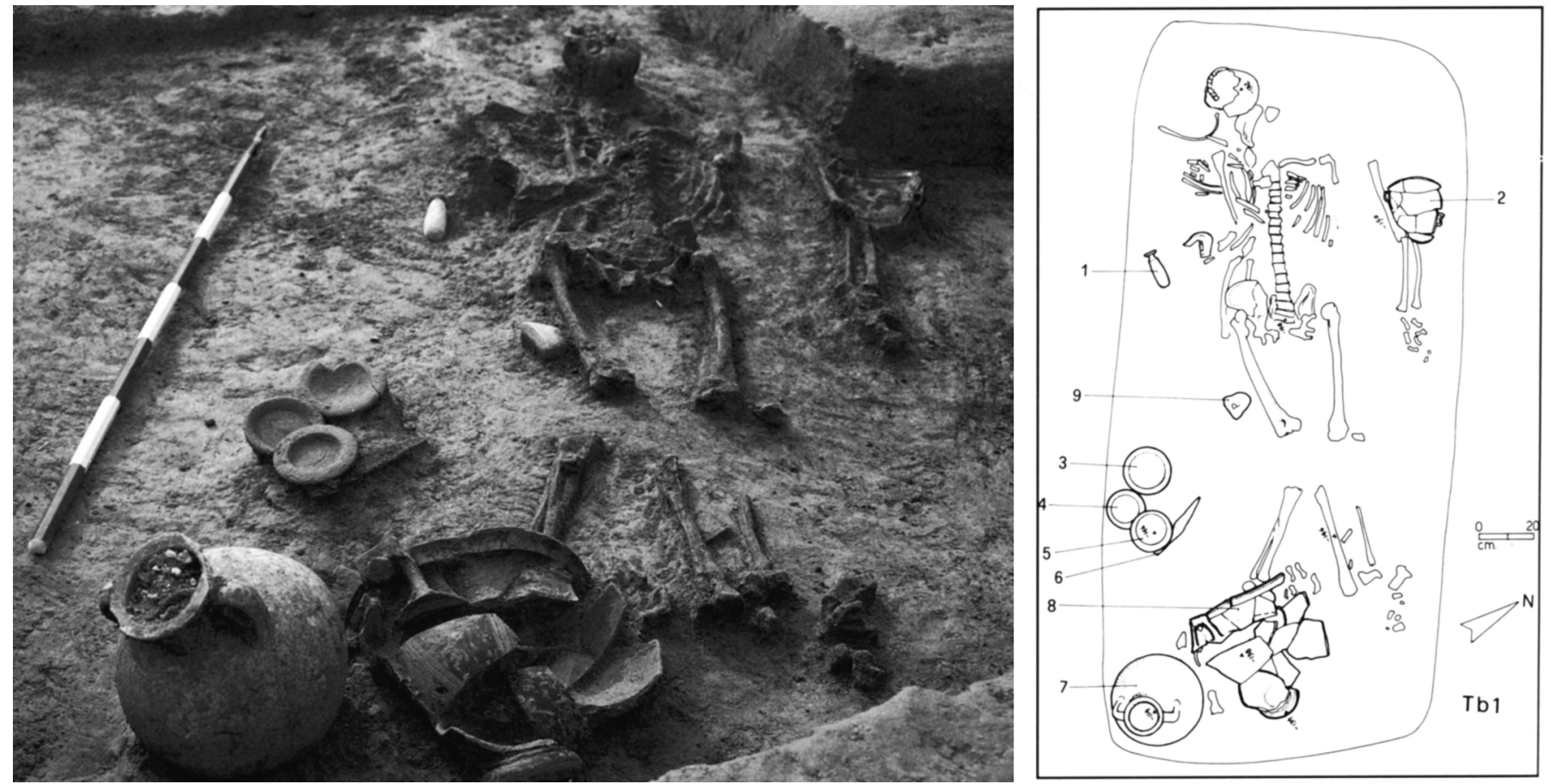

Fig. 79. Rovigo, lieu-dit Le Balone, Tombe 1 (D'après R. Peretto éd, Balone. Insediamento etrusco presso un ramo del Po, Padova 1994, fig. 26-27).

au-dessous du tracé de la voie rapide Transpolesana ${ }^{613}$. La typologie funéraire, consistant en inhumations dans des fosses ou des caisses en bois, comme les mobiliers funéraires, comprenant un service complet de vases pour le banquet et pour le symposium, présentent des analogies étroites avec les tombes de Spina et de Bologne. De plus, sur le pied d'un bol faisant partie d'un des mobiliers on voit un graffito indiquant un gentilice à terminaison en - alu, forme typique des gentilices étrusco-padans ${ }^{614}$. Plutôt qu'à des Paléovénètes, tous ces éléments suggèrent l'attribution de la nécropole et de l'habitat associé à des populations étrusques, dont la présence dans l'arrière-pays d'Adria dans le courant du $V^{e}$ s. av. J.-C. est aussi documentée par d'autres centres probablement installés le long d'un trajet conduisant aux sites étrusco-padans du territoire de Mantoue ${ }^{615}$.

La présence de l'habitat est confirmée par une série de structures, chenaux et fosses de différentes formes et dimensions comblées de matériel céramique et de restes d'animaux, dont l'interprétation n'est pas encore claire. Le matériel trouvé rappelle de très près les typologies des formes céramiques documentées dans les contextes

613. Pour la fouille et la documentation de la nécropole on renvoie à Salzani 1991 et $I d .1994$.

614. Marinetti 1994.

615. Pour la navigabilité endolagunaire de l'aire septentrionale du delta du Pô, cf. Peretto 1999.

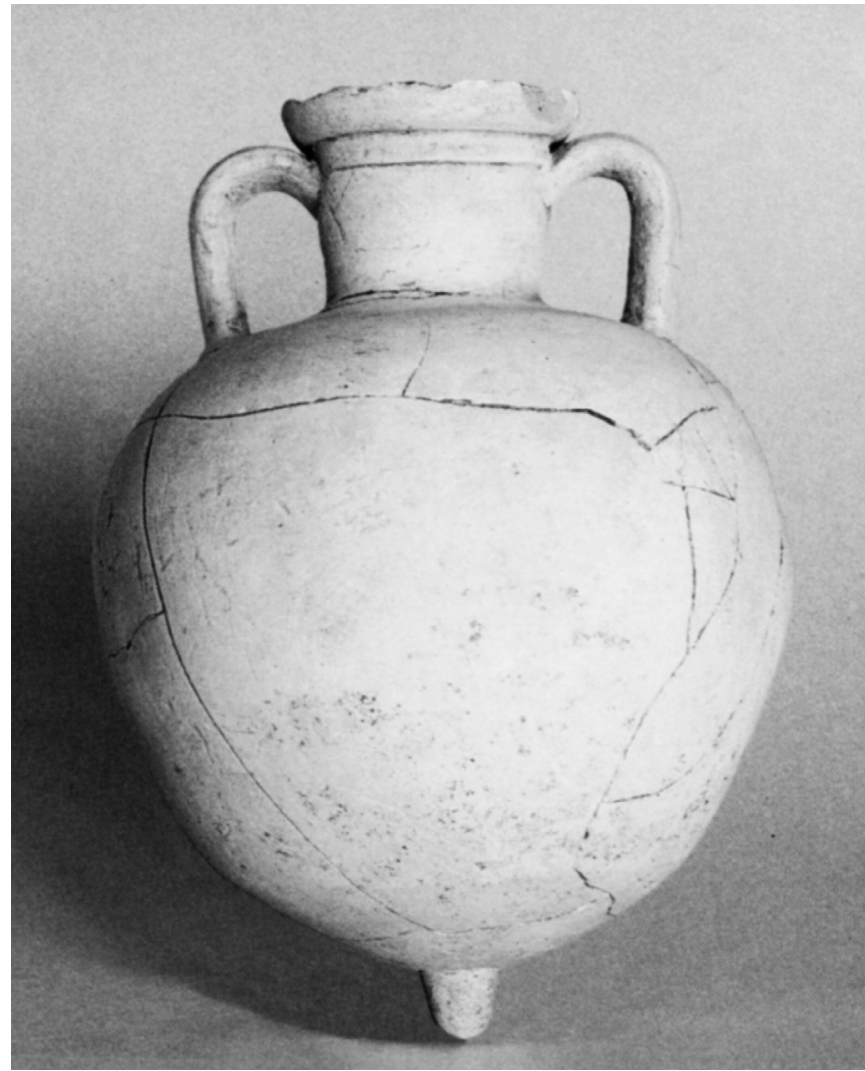

Fig. 80. Rovigo, lieu-dit Le Balone, amphore de type corinthien B de la Tombe 1 (d'après Balone. Insediamento etrusco presso un ramo del Po, Padova, 1994, fig. 50). 

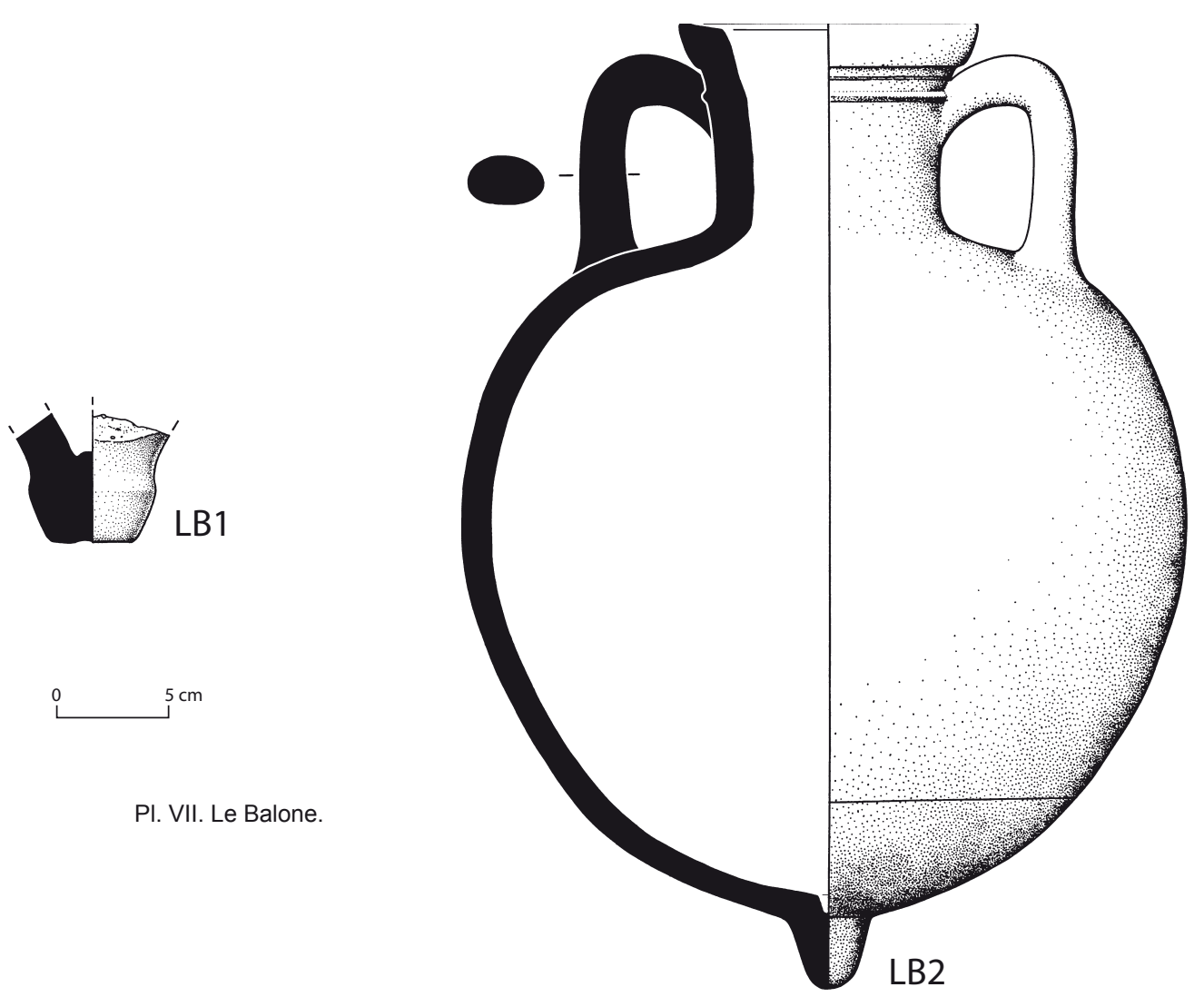

étrusco-padans du Ve s. av. J.-C., tandis qu'on remarque l'absence totale de matériel clairement identifiable comme paléo-vénète ${ }^{\mathbf{6 1 6}}$.

Quant aux amphores commerciales, un exemplaire fragmentaire vient de la zone de l'habitat et une amphore entière faisait partie du mobilier de la Tombe 1 de la petite nécropole (fig. 79 et 80 ). Les amphores de Le Balone sont indiquées dans le catalogue qui suit par le sigle « LB ». Elles sont publiées dans Sacchetti 2012 avec la même numérotation.

\subsection{Catalogue}

\section{AMPHORE CORINTHIENNE A}

\section{LB1 (pl. VII)}

Secteur C.

État de conservation : pied complet. Dimensions : non repérées. Pied en bouton, au profil caréné, à base plate.

616. Pour la documentation de fouille relative au repérage du quartier d'habitation on renvoie à Salzani, Peretto, Zerbinati 1988 et à Salzani 1994.
Observations et comparaisons : attribué par L. Salzani à une amphore corinthienne du type A. Pour le type, Cavalier 1985, fig.4.a, datable de la première moitié du $\mathrm{V}^{\mathrm{e}}$ s. av. J.-C. Pour la forme, Di Sandro 1986, pl. 6, sg. 69. Chronologie : 500-450 av. J.-C.

Bibliographie : Salzani, Peretto, Zerbinati 1988, p. 32, fig. 4. 12 ; Salzani 1994, fig. 25, 22.

\section{AMPHORE GRÉCO-OCCIDENTALE («corinthienne $B »)$ (fig. 80)}

\section{LB2 (pl. VII)}

Structure : Tombe 1.

État de conservation : entière. H. tot. : 42. Lèvre en échine, légèrement ouverte. Col cylindrique, légèrement évasé, avec une profonde cannelure immédiatement audessous de la lèvre. Anses verticales, en ruban épaissi, à section elliptique. Épaule arrondie. Corps globulaire avec une rayure sur le fond de la panse. Pied en bouton, arrondi en bas, assez allongé.

Surface ext., int. et fracture : jaune clair (2.5Y8/3).

Inclusions : inclusions fines blanches, rougeâtres et noires et probablement quelques fragments de silex.

Engobe : quelques traces d'engobe dans la partie inférieure.

Observations et comparaisons : attribuée par R. de 
Marinis à la production corinthienne B et datée vers le milieu du Ve s. av. J.-C. sur la base du mobilier. Pour le type, Koehler 1978a, fig. 2.b, et Ead. 1981, fig. 1, a, pl. 99.c, daté entre le second et le troisième quart du $\mathrm{V}^{\mathrm{e}} \mathrm{s}$. av. J.-C.

Chronologie : milieu du Ve s. av. J.-C.

Bibliographie : Salzani 1991 ; de Marinis 1994, fig. 73 ; Salzani 1994, fig. 28, 7 et 50 ; de Marinis 1996, p. 320.

\section{Analyse des données (graphiques 1-4)}

Les sites de la bande côtière et de la chora d'Adria ont jusqu'ici restitué dans l'ensemble plusieurs centaines de fragments amphoriques, correspondant à un nombre minimum/maximum de 62/64 individus, calculé sur la base du protocole de Lattes. De ceux-ci, 35 proviennent de San Basilio, 15/16 d'Adria, 10/11 de San Cassiano et 2 de la petite nécropole et de l'aire d'habitat de Le Balone.

\section{Adria}

Le site d'Adria (cf. supra II, $1 \S 1$ ), qui encore il y a quelques années n'avait restitué aucune attestation amphorique, si l'on excepte la découverte au $\mathrm{XIX}^{\mathrm{e}} \mathrm{s}$. de l'exemplaire entier de la collection Bocchi, a récemment fourni d'appréciables confirmations archéologiques de la présence prévisible d'amphores commerciales grecques. Trois amphores entières, ou presque entièrement reconstituables, faisaient partie des mobiliers de la nécropole de Cà Cima (cat. AD4-AD6), et deux-cents autres fragments d'amphores ont été trouvés lors des fouilles des quartiers d'habitation de Via San Francesco et de Via Ex Riformati. Ces recherches récentes permettent à l'heure actuelle de disposer pour Adria d'une quinzaine d'individus.

Malgré les récentes acquisitions, le matériel amphorique trouvé à Adria est encore peu abondant par rapport aux potentialités d'un site portuaire qui joua un rôle historique et économique déterminant dans le cadre des plus anciens contacts des régions de l'Adriatique septentrionale avec la Grèce. Cette faible quantité dépend de la surface fouillée, très limitée, pour les niveaux d'habitat $\mathrm{du} \mathrm{VI}^{\mathrm{e}}$ et $\mathrm{V}^{\mathrm{e}}$ s. av. J.-C. La documentation aujourd'hui disponible permet de toute façon de fournir un premier tableau que nous considérerons comme représentatif des productions amphoriques parvenues à Adria et de la date de leur arrivée.

Le début des importations d'amphores se situe autour du milieu du $\mathrm{VI}^{\mathrm{e}} \mathrm{s}$. av. J.-C., pratiquement en correspondance avec les données déjà connues sur les premières

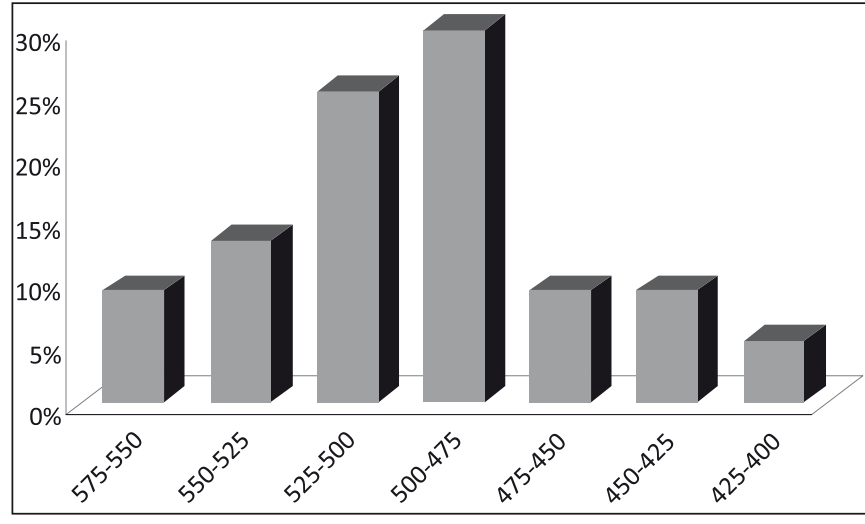

Graphique 1. Bande côtière et chora d'Adria : tendance des importations d'amphores grecques dans la période considérée (deuxième-troisième quarts du $\mathrm{Vl}^{\mathrm{e}}$-fin du $\mathrm{V}^{\mathrm{e}} \mathrm{s}$. av. J.-C.).

importations de céramique grecque figurée, qui remontent au deuxième quart du $\mathrm{VI}^{\mathrm{e}} \mathrm{s}$. av. J.-C. et qui sont de production corinthienne (ou peut-être corcyréenne) et attique ${ }^{617}$. Pour la seconde moitié du même siècle et le premier quart du suivant on assiste à une concentration de découvertes. En face de cette augmentation des présences d'amphores, on remarque également le développement consistant et constant des importations attiques pour la seconde moitié du $\mathrm{VI}^{\mathrm{e}}$ et le premier quart $\mathrm{du} \mathrm{V}^{\mathrm{e}} \mathrm{s}$. av. J.-C. Cette époque de relations commerciales complexes avec la Grèce, phase d'hégémonie d'Adria dans l'aire de la Haute-Adriatique, est documentée aussi par la présence isolée de céramiques décorées corinthennes, gréco-orientales et laconiennes ${ }^{618}$. Ensuite, la diminution des présences amphoriques vers les décennies centrales du $V^{e}$ s. av. J.-C. semble correspondre également à une diminution des importations attiques à partir du milieu du siècle, bien que des découvertes récentes en aient augmenté le nombre ${ }^{619}$.

Ces données ne sont pas surprenantes pour la période ancienne (à partir du troisième quart du $\mathrm{VI}^{\mathrm{e}}$ ), au vu de ce que nous savons de l'ancienneté des importations égéennes dans la partie Nord du delta. Pour la seconde période (à partir de la fin $\mathrm{du} \mathrm{VI}^{\mathrm{e}} \mathrm{s}$.), on peut avancer deux

617. Pour la céramique corinthienne : Bonomi 1991, pl. I, 5-6 ; Ead. 2000a, p. 122. Pour les premières importations de céramique attique : Bonomi 1991, pl. 35, 1 ; Ead. 1997, p. 32, fig. 20 ; Ead. 2000a, p. 122-123). Pour la céramique attique d'Adria on renvoie aussi à Bonomi 1988 ; Ead. 2000 b ; Vallicelli 2004 ; et Wiel-Marin 2005.

618. Bonomi 1988, p. 67, 81-82.

619. Pour une synthèse sur la tendance des importations de céramique figurée grecque à Adria, voir Boardman 1979 et Bonomi 1988, p. 67. Plus récemment, cf. : Giudice 2004, fig. 14-20 ; et WielMarin 2005, p. 89. Pour quelques observations sur les céramiques attiques du $\mathrm{V}^{\mathrm{e}} \mathrm{s}$. provenant des fouilles de l'habitat d'Adria, cf. aussi les considérations de Bonomi 2003, p. 142 et Bonomi, Camerin, Tamassia 2003, p. 207. 
Graphique 2. Bande côtière et chora d'Adria : données quantitatives des importations des différents groupes d'amphores pendant toute la période examinée (deuxième-troisième quarts du $\mathrm{Vl}^{\mathrm{e}}$-fin du $\mathrm{V}^{\mathrm{e}} \mathrm{s}$. av. J.-C.)

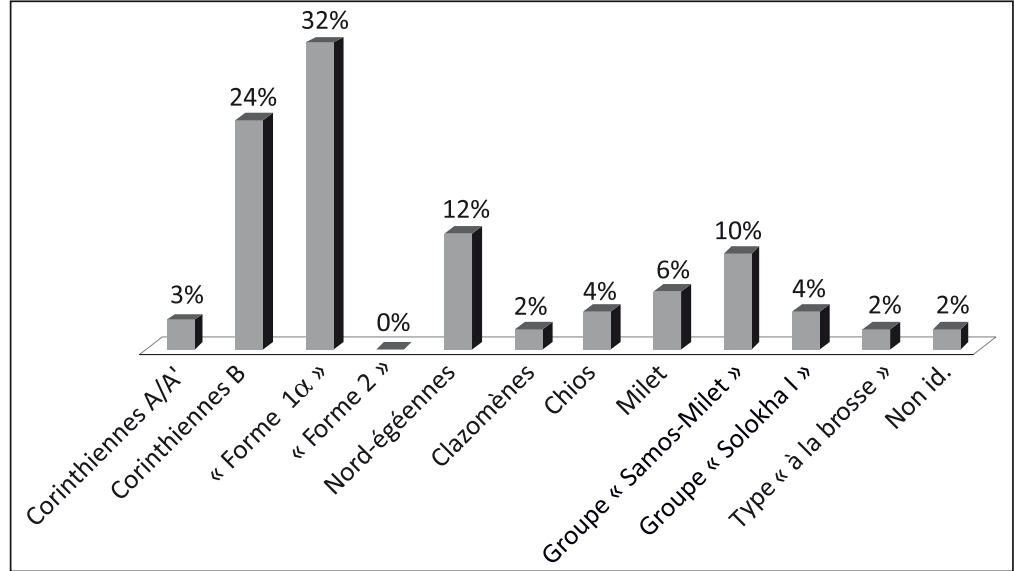

explications : soit c'est la conséquence de l'hégémonie d'Adria dans la haute Adriatique du dernier quart du $\mathrm{VI}^{\mathrm{e}}$ au premier quart du $\mathrm{V}^{\mathrm{e}} \mathrm{s}$. av. J.-C., comme l'ont bien décrit notamment S. Bonomi et G. Colonna, soit il faut y voir l'effet de l'état incomplet de nos connaissances sur l'habitat et sur les nécropoles d'Adria au Ve s. av. J.-C. ${ }^{620}$, phase pour laquelle on a récemment souligné la continuité de la prospérité économique et du développement culturel de la cité (Bonomi 2003, p. 142) ${ }^{\mathbf{6 2 1}}$.

Les productions amphoriques les plus fréquemment attestées sont les gréco-occidentales archaïques, et en particulier la «forme $1 \alpha$, mais dans le dernier quart du $\mathrm{VI}^{\mathrm{e}}$ s. av. J.-C. sont aussi documentées les productions nord-égéennes, ainsi que le groupe Samos-Milet.

$\mathrm{Au} \mathrm{V}$ s. av. J.-C., la production pour laquelle on enregistre le maximum de présences est celle des amphores corinthiennes du type B. Jusqu'au milieu du $V^{\mathrm{e}} \mathrm{s}$. av. J.-C. sont aussi documentés les ateliers gréco-orientaux, avec le groupe Samos-Milet et avec la production chiote respectivement dans le premier et dans le deuxième quart du siècle. Ce n'est qu'après le milieu du Ve s. av. J.-C. qu'on enregistre les rares amphores corinthiennes A'.

\section{Le littoral d'Adria : San Basilio}

Le matériel amphorique du site de San Basilio comprend quatre-cents fragments environ appartenant

620. Sur le floruit d'Adria : Colonna 1974 ; Bonomi 1988 ; et Colonna 2003, notamment p. 61. Sur les lacunes dans nos connaissances sur la phase du VI et du Ve s. av. J.-C. d'Adria, récemment comblées par quelques découvertes dans l'habitat (Bonomi, Camerin, Tamassia 2003) e dans les nécropoles (Id. 2002), Bonomi 1988, p. 67.

621. Par exemple, pour la phase problématique du IV s. av. J.-C., les plus récentes recherches sur les céramiques attiques à figures rouges des nécropoles et de l'habitat d'Adria (cf. respectivement : Bonomi 2000a ; Wiel-Marin 2005) ont augmenté le nombre d'attestations connues en modifiant ainsi la tendance des importations précédemment connue pour cette phase historique. au moins à trente-cinq individus (cf. supra II, 1 § 2). Ceux-ci proviennent d'une surface de fouille de $103 \mathrm{~m}^{2}$ à laquelle il faut ajouter deux tranchées de 7 x $2 \mathrm{~m}$ et $8,9 \times 2 \mathrm{~m}$.

Le début des importations amphoriques est daté entre le second et le troisième quart du $\mathrm{VI}^{\mathrm{e}} \mathrm{s}$. av. J.-C., pratiquement en correspondance avec les données fournies par les présences les plus anciennes de la céramique corinthienne. Dans le dernier quart du siècle on peut remarquer sa nette augmentation, en concomitance avec le début des importations attiques, qui semblent à l'heure actuelle continuer jusqu'aux débuts ou au milieu du $\mathrm{V}^{\mathrm{e}} \mathrm{s}$. av J.-C. ${ }^{622}$. Après une diminution dans le premier quart $\mathrm{du} \mathrm{V} \mathrm{V}^{\mathrm{e}}$ s. av. J.-C., elles deviennent constantes jusque vers la fin du siècle.

Les premières attestations datables avec une certaine précision, c'est-à-dire pas avant le deuxième quart du $\mathrm{VI}^{\mathrm{e}}$ à cause du terminus post quem fourni par la céramique corinthien moyen et pas au-delà du troisième quart du siècle pour des raisons de typologie, sont de production gréco-orientale et de type milésien de pleine tradition archaïque. Il s'agit de quatre lèvres de même forme dont une (cat. SB28) provient de la même unité

622. Sur les importations grecques les plus anciennes de San Basilio : Salzani, Vitali 1991, p. 418, fig. 13 ; Bonomi 2000a, p. 122 et n. 10-12 ; Salzani, Vitali 2003, p. 118, n. 8, fig. 17. On peut dater autour de 600 av. J.-C. une kotyle étrusco-corinthienne que S. Bruni considère de production de Vulci (Bruni 1994 et Id. 1998, n. 24). Si pour le matériel corinthien, comme on le verra mieux ci-après, l'hypothèse d'une provenance à travers l'Adriatique est désormais largement acceptée, ce produit etrusco-corinthien constitue un indice de l'intérêt porté par l'Étrurie propre à la région du delta du Pô (Bonomi 2000a, p. 122). D’après S. Bruni, le vase serait arrivé dans l'aire de la Haute Adriatique grâce à la médiation de Bologne (Bruni 1994, p. 189) : pour G. Colonna, il serait au contraire arrivé via Volsinii-Verucchio (Colonna 2003, p. 158). Outre cette kotyle il faut aussi rappeler l'oenochoé trilobée récemment assignée par S. Bonomi à la même production (Bonomi 2000a, p. 122 et n. 9). La céramique attique, en cours d'étude, semble datable entre les dernières décennies du $\mathrm{VI}^{\mathrm{e}}$ et les débuts ou le milieu du V $\mathrm{V}^{\mathrm{e}} \mathrm{s}$. av. J.-C. (Salzani, Vitali 1991, p. 417 ; Id. 2003, p. 118). 

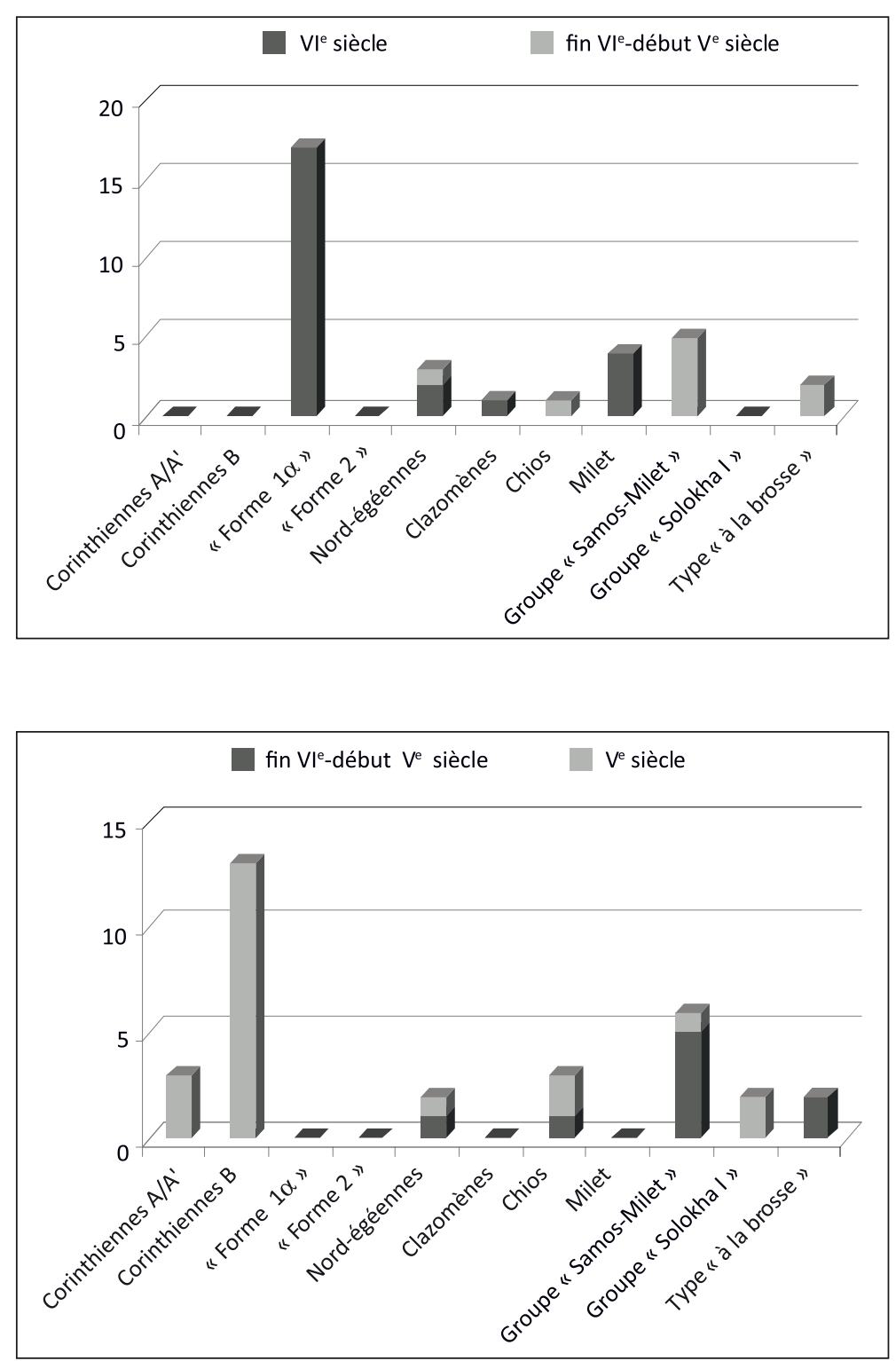

Graphique 3. Bande côtière et chora d'Adria : données quantitatives des importations des différents groupes d'amphores entre les deuxième-troisième quart du $\mathrm{Vl}^{\mathrm{e}}$ et les débuts du Ve s. av. J.-C.
Graphique 4. Bande côtière et chora d'Adria : données quantitatives des importations des différents groupes d'amphores entre la fin du $\mathrm{Vl}^{\mathrm{e}}$ et la fin du Ve s. av. J.-C. stratigraphique (US 56) qui a livré un fragment de kotyle corinthien moyen considéré comme le plus ancien du site ${ }^{623}$. Au plan quantitatif, dans le courant du $\mathrm{VI}^{\mathrm{e}}$ s., les productions les plus documentées sont les grecques d'Occident, plus précisément la «forme $1 \alpha$ ». Les amphores de cette forme sont datables dans le courant du $\mathrm{VI}^{\mathrm{e}}$ s. av. J.-C. sans qu'il y ait une possibilité de préciser ultérieurement la chronologie sur une base exclusivement morphologique. La datation des exemplaires de San Basilio, pour lesquels on ne dispose pas encore des données chronologiques de tous les contextes, ne pourra pas remonter avant le deuxième quart du siècle en raison du terminus post quem mentionné plus haut

623. Salzani, Vitali 1991, p. 418, fig. 13 (en bas) ; Bonomi 2000a, p. 122 ; Salzani, Vitali 2003 , p. 118 , n. 8, fig. 17 (le second de la colonne de droite). (importations du Corinthien moyen). Les productions gréco-occidentales sont numériquement suivies par les gréco-orientales, parmi lesquelles se distingue par son unicité dans tout le territoire de l'Italie septentrionale un exemplaire de Clazomènes, et enfin par les nordégéennes. Au cours du $\mathrm{V}^{\mathrm{e}} \mathrm{s}$. av. J.-C., les importations les plus nombreuses sont les corinthiennes $\mathrm{B}$, et donc une fois de plus les fabriques occidentales qui, comme au siècle précédent, sont suivies par celles de la Grèce de l'Est et de l'Égée septentrionale.

\section{La chora d'Adria : San Cassiano et Le Balone}

Dans la chora d'Adria le complexe de San Cassiano a livré plus de trois-cents fragments amphoriques (cf. supra II, 1 § 3). Ils représentent un pourcentage 
assez élevé de l'ensemble des importations grecques trouvées sur le site ${ }^{624}$, et les fragments caractéristiques appartiennent à une dizaine d'individus.

Les types identifiables dans un matériel amphorique, malheureusement très fragmenté et fortement abrasé en surface, sont tous attribuables par leur typologie à l'époque archaïque et proviennent, d'après la stratigraphie, de la phase initiale du site ${ }^{625}$, correspondant au moment de la mise en œuvre des fondations trachytiques des structures et actuellement datée entre la fin du $\mathrm{VI}^{\mathrm{e}}$ et les débuts du Ve s. av. J.-C.

La production la mieux documentée est celle des amphores occidentales de « forme $1 \alpha$ ». La chronologie de ce groupe est comprise entre les débuts du $\mathrm{VI}^{\mathrm{e}} \mathrm{s}$. av. J.-C. et 490 av. J.-C., mais la datation des exemplaires de San Cassiano, en raison de la stratigraphie, ne pourra pas remonter avant la dernière décennie du $\mathrm{VI}^{\mathrm{e}} \mathrm{s}$. av. J.-C.
Deux fragments, appartenant peut-être au même individu, sont attribuables à la forme Agora 1502 du type « à la brosse », probablement mais non sûrement de production attique. Le type est datable entre le milieu du $\mathrm{VI}^{\mathrm{e}} \mathrm{s}$. et 480 av. J.-C., mais, pour des raisons stratigraphiques, dans ce cas aussi le vase n'est pas antérieur aux dernières années du $\mathrm{VI}^{\mathrm{e}}$ s. av. J.-C., trouvant son parallèle dans les deux autres uniques exemplaires d'amphores «à la brosse » actuellement connus de l'aire padane, et plus exactement du Forcello de Mantoue ${ }^{\mathbf{6 2 6}}$.

Un peu plus à l'ouest de San Cassiano, la petite nécropole de Le Balone et la zone d'habitat lui étant reliée qu'il a été possible d'explorer (cf. infra II, 1 § 4), ont livré uniquement deux amphores commerciales (respectivement cat. LB2 et LB1). Les deux exemplaires sont attribuables au type A et au type B de Corinthe et peuvent être situés respectivement dans la première moitié et vers le milieu du Ve $\mathrm{s}$. av. J.-C.
624. Cf. à ce propos les graphiques relatifs au matériel du site de San Cassiano di Crespino, pour lequel on renvoie en dernier à Harari 2004a, fig. 4-5.

625. Je dois les informations sur les contextes de San Cassiano à la gentille collaboration de l'équipe de l'Université de Pavie dirigée par le Prof. Maurizio Harari qui a conduit les fouilles du site (dont la publication est en préparation).
626. Cf. de Marinis 2007, p. 166, fig. 112, 1-2, pour lequel cf. cat. FO78 e FO79). 



\section{Chapitre 2}

\section{Spina : les nécropoles de Valle Trebba et de Valle Pega et l'habitat de Valle Lepri di Comacchio (prov. de Ferrare)}

\section{Bilan des études}

La découverte du site de Spina (fig. 69) est liée aux travaux d'assainissement hydraulique et d'aménagement agricole des bassins lagunaires ou 'valli' de Comacchio (fig. 81), entrepris par l'État italien après la première guerre mondiale. Le site fut localisé en 1922, lors de la découverte des premières tombes de la nécropole septentrionale de la cité, à Valle Trebba ${ }^{627}$, l'une des 'valli' septentrionales de Comacchio. Les fouilles, qui se déroulèrent principalement entre 1922 et 1935 sous la direction d'A. Negrioli d'abord puis sous celle de S. Aurigemma, permirent de découvrir 1213 tombes et d'ouvrir le Musée Archéologique National de Spina à Ferrare $^{628}$. En 1962, s'ajouta la découverte de 198 nouvelles tombes dans la même nécropole ; parallèlement, les fouilles menées dans l'autre nécropole de Spina, située au sud de Valle Pega, où, dès 1954, s'était portée l'attention des fouilleurs, P. E. Arias puis N. Alfieri, avaient mis au jour 2650 tombes ${ }^{629}$.

Les tombes des nécropoles de Spina, dont on déplore encore aujourd'hui l'absence d'une publication d'ensemble, sont célèbres pour la richesse des objets d'importation, tant d'Étrurie que de Grèce ${ }^{630}$. On dénombre plus de cent cinquante amphores commerciales provenant

627. Pour les nécropoles de Spina : Negrioli 1924, p. 279-322 ; Id. 1927, p. 143-198 ; Aurigemma 1936 ; Id. 1960 ; Id. 1965 ; De Luca De Marco 1979, p. 571-600 ; Berti 1993, p. 33-45 ; Camerin 1993, p. 267-272 ; Desantis 1993, p. 157-181 ; Guzzo 1993, p. 219-229 ; Parrini 1993,p. 273-286 ; Sassatelli 1993, p. 179, 199 ; Desantis 1996, p. 346-372.

628. Sur les campagnes de fouilles des années 1924 et 1925, voir Negrioli 1924, p. 279-322 et Negrioli 1927, p. 143-198; pour les interventions successives, voir Aurigemma 1936.

629. On trouvera une synthèse des fouilles de la nécropole de Valle Pega et des autres interventions réalisées par N. Alfieri au cours des mêmes années sur ce site, dans Alfieri 1993, p. 17-18, n. 39, avec bibliographie précédente.

630. Pour le mobilier publié, $c f$. Aurigemma 1960 ; Aurigemma 1965. On trouvera quelques informations générales sur le mobilier des tombes de Spina, avec références spécifiques aux rares tombes publiées, dans Camerin 1993, p. 267-272 et Parrini 1993, p. 273286. Les produits des échanges entre Grecs et Étrusques documentés à Spina et sur tout le territoire de la plaine du Pô ont été minutieusement analysés par G. Sassatelli (Sassatelli 1993, p. 189-205). des 4061 tombes des nécropoles de Valle Trebba et de Valle Pega. La plus grande partie des amphores publiées appartient à des mobiliers datables entre le $\mathrm{IV}^{\mathrm{e}}$ et le $\mathrm{III}^{\mathrm{e}} \mathrm{s}$. av. J.-C., tandis que huit seulement (dont six venant de la nécropole de Valle Trebba et deux de celle de Valle Pega) se rapportent à la période comprise entre le $\mathrm{V}^{\mathrm{e}}$ et le début du IV s. av. J.-C.

À partir de 1956, fut entreprise la prospection de l'habitat ${ }^{631}$ qui fut partiellement localisé et ultérieurement fouillé grâce à N. Alfieri et aux recherches géomorphologiques de S. Patitucci Uggeri et de G. Uggeri (fig. 82). Dès le départ, il fut évident que les recherches devaient se concentrer sur les terres qui étaient émergées à l'époque antique, en d'autres termes, soit les dunes fluvio-maritimes des littoraux préromains qui avaient déjà servi de fil conducteur pour le repérage des divers noyaux de la nécropole de Valle Pega, soit les tertres fluviaux, tous deux décelables sur les photographies aériennes contemporaines de l'assainissement hydraulique des 'valli' et précédant la bonification agraire. Ce fut un tertre fluvial, constitué d'un bras-mort antique du delta correspondant au Padovetere (ou Padiverio) dont parlent les sources médiévales - le fleuve Spinete des sources antiques qui retint l'attention pour la recherche de l'habitat ${ }^{632}$. Les fouilles de 1956 ont reporté à la lumière sur le bord oriental de Valle Lepri un système de fondation de palafittes, témoignage d'une installation humaine du IVe $\mathrm{s}$. av. J.-C., ainsi qu'un canal rectiligne d'une quinzaine de mètres de largeur, provenant du Padovetere et dirigé vers l'est, que l'on interpréta comme un ouvrage hydraulique réalisé à l'époque étrusque pour faciliter les liaisons entre la ville et la côte. Le centre habité principal, décou-

631. Pour l'habitat de Spina : Alfieri 1959a, p. 31 ; Id. 1959b, p. 89-102 ; Uggeri, Uggeri Patitucci 1974, p. 69-97 ; Alfieri 1981, p. 3-11 ; Berti 1985 ; Alfieri 1987, p. 177-179 ; Id. 1988, p. 283-288; Desantis 1989, p. 103-127 ; Uggeri 1991 ; Alfieri 1993, p. 3-18 ; Braccesi, Coppola 1993, p. 71-79 ; Colonna 1993, p. 131-143 ; Malnati 1993, p. 145-177 ; Patitucci Uggeri, Uggeri 1993, p. 21-31; Sassatelli 1993, p. 179-217 ; Torelli 1993, p. 53-69.

632. Pour les recherches géomorphologiques qui ont conduit à la localisation de l'habitat de Spina, voir Alfieri 1993, p. 18 et Patitucci Uggeri, Uggeri 1993, p. 21-22. 


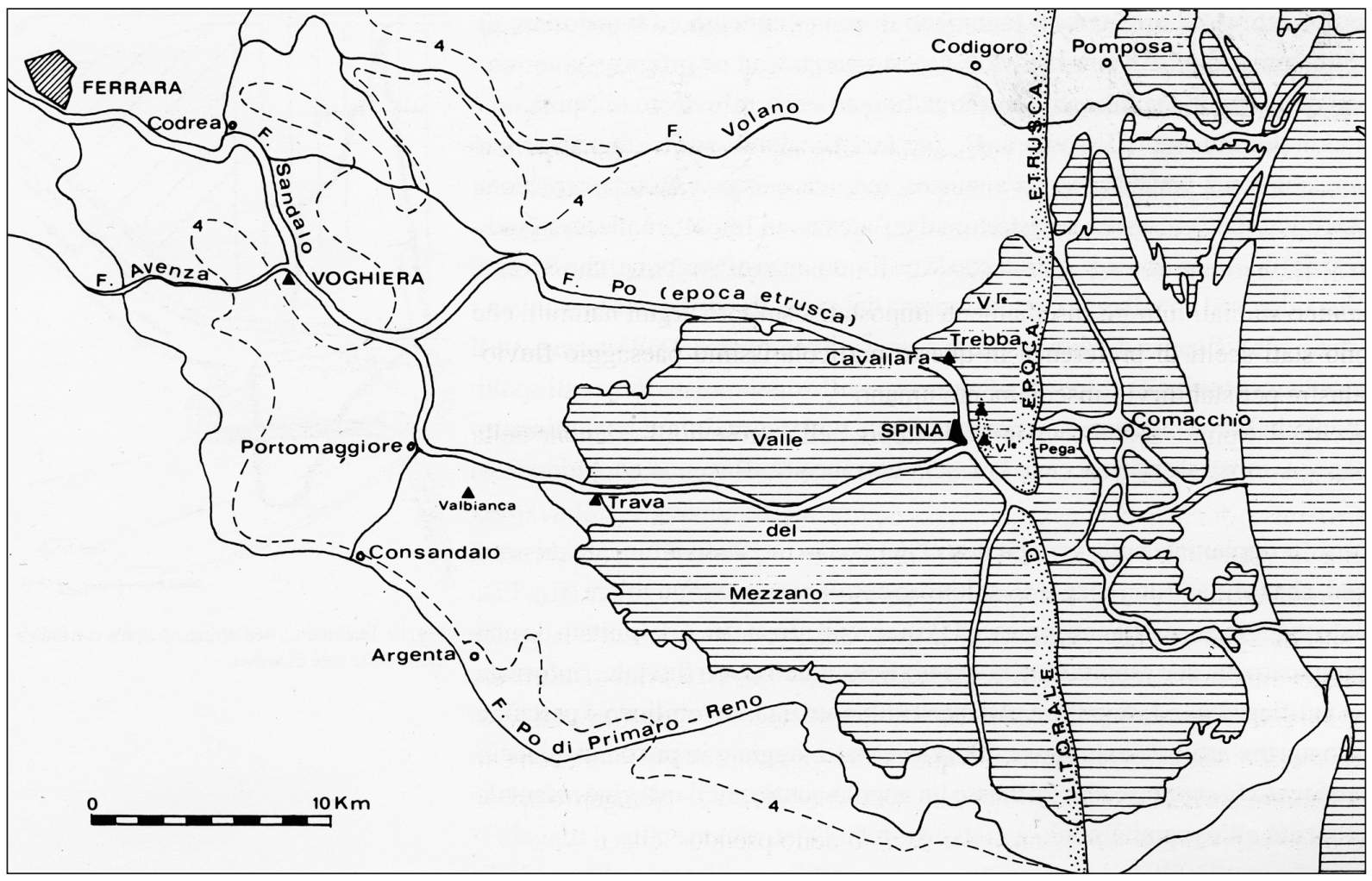

Fig. 81. Carte hydrographique de la zone de Spina à l'époque protohistorique (d'après Patitucci Uggeri, Uggeri 1993, fig. 16).

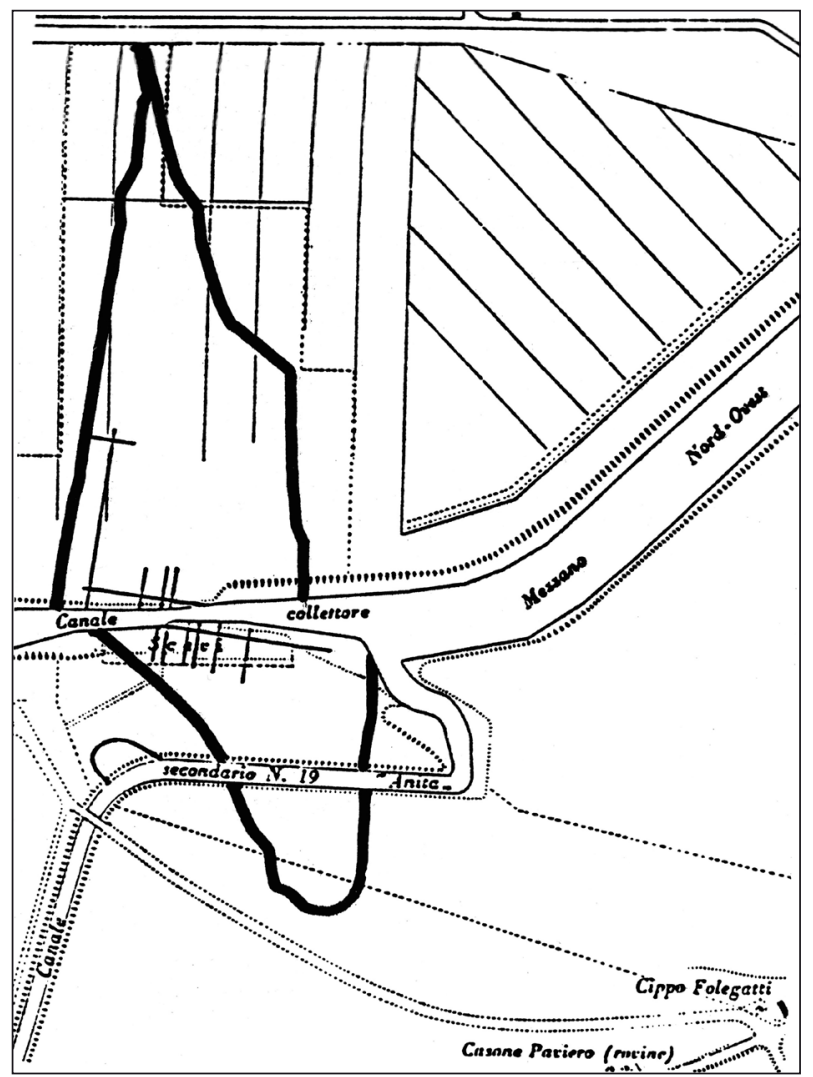

Fig. 82. Plan de l'habitat de Spina avec l'emplacement des zones fouillées (d'après Patitucci Uggeri, Uggeri 1993, fig. 17). vert plus tard grâce aux travaux d'assainissement de l'ensemble de la Valle du Mezzano, fut décelé à l'angle nord-oriental de cette 'valle' et se situait dans la Valle Lepri.

La cité ne fut donc pas construite sur la bande de dunes côtières d'époque étrusque, incontestablement trop exposées, mais sur une ligne de tertres dont la position, plus en arrière, était nettement plus favorable. En effet, le site se dressait au confluent d'un cours d'eau secondaire provenant du sud-ouest et du bras du Pô de Spina (ou Padovetere) cité par les sources antiques, pour contrôler à la fois la mer qui était à environ $3,5 \mathrm{~km}$ (les vingt stades du Pseudo-Scylax) et les deux voies fluviales qui permettaient la liaison respectivement avec la région des Apennins au sud-ouest, et avec la plaine du Pô au nordouest. Selon l'interprétation de G. Colonna, la fondation de Spina vers la fin du VI ${ }^{e}$ s. av. J.-C., lorsque Athènes, concurrençant sa rivale Égine, commença également à s'intéresser à la Haute Adriatique, doit être considérée comme une réponse des Étrusques de la plaine du Pô à la présence grecque de plus en plus massive et organisée sur les côtes de la Plaine Padane, au-delà du territoire qu'ils contrôlaient. Les Étrusques auraient donc fondé Spina pour « s'emparer des commerces du Caput Adriae » (Colonna 1974, p. 21), c'est-à-dire de la Haute 
Adriatique, et pour ne pas perdre ce rôle d'intermédiaires commerciaux entre le monde grec d'une part et celui des Celtes transalpins de l'autre, rôle qu'ils détenaient désormais depuis longtemps et qui dérivait étroitement du contrôle du territoire même de la Plaine Padane ${ }^{633}$. La chronologie des premières importations de céramique grecque de Spina, de production attique à figures noires et datées entre 540 et 520 av. J.-C. (Berti 1985, p. 194 ; Lippolis 2000, p. 111 ; Bruni 2004, p. 84), a plus récemment suggéré de situer la fondation de la ville autour de 540 av. J.-C. (Malnati, Manfredi 1991, p. 155).

S'agissant des amphores commerciales, S. De Luca De Marco a étudié celles des nécropoles dont elle a publié quatre-vingt-quinze exemplaires entiers sur plus de cent cinquante retrouvés (De Luca De Marco 1979, p. 571, n. 1). Sur ces quatre-vingt-quinze exemplaires, quatre seulement sont datables avant le tournant du $\mathrm{V}^{\mathrm{e}}$ au $\mathrm{IV}^{\mathrm{e}} \mathrm{s}$. av. J.-C. Plus récemment, P. Desantis (Desantis 1993 ; Ead. 1996) publie deux autres amphores du Ve s. av. J.-C.provenant de la nécropole de Valle Trebba.

Les amphores archaïques tardives et classiques provenant des fouilles de l'habitat sont plus nombreuses et leur étude a été confiée à P. Desantis (Desantis 1989).

Une quarantaine d'amphores déjà publiées sont prises ici en considération pour leur éventuel encadrement chronologique entre la deuxième moitié $\mathrm{du} \mathrm{VI}^{\mathrm{e}}$ et le début

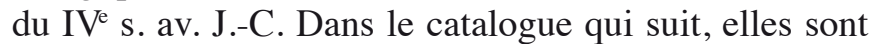
identifiée par le sigle 'SP'.

\section{Catalogue}

\section{AMPHORE CORINTHIENNE DE TYPE A'}

\section{SP1 (pl. VIII)}

Lieu de découverte : Valle Lepri, Comacchio.

Inventaire $\mathrm{n}^{\circ} 55000$.

État de conservation : partie de la lèvre, du col ainsi que la moitié supérieure d'une anse reconstituées à partir de deux fragments. H. max. cons. : 11. Lèvre à marli, oblique, à section trapézoïdale. Diam. restituable embouchure : 16. Col évasé. Anses obliques légèrement rentrantes, « a bastoncello », à section circulaire.

Surface ext., int. et fracture : beige rosé (7.5YR7/4). Inclusions : inclusions blanches, rouges et grises, dont quelques-unes de grandes dimensions.

Observations et comparaisons : attribuée à la production corinthienne A' par P. Desantis, et datée du milieu du $\mathrm{V}^{\mathrm{e}} \mathrm{s}$. av. J.-C. Une datation dans le courant de la première moitié du Ve s. av. J.-C. est suggérée par la position des

633. Voir à ce propos, en dernier, Harari 2003, notamment p. 21 et n. 20, avec références bibliographiques.

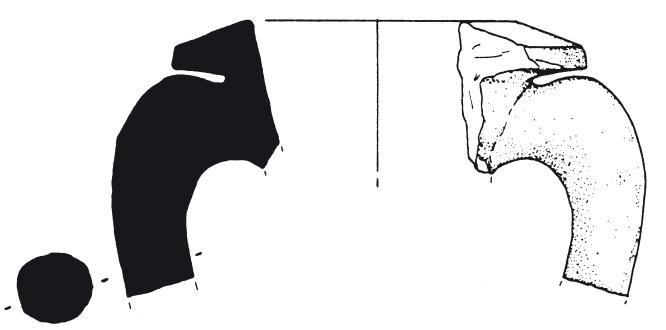

SP1

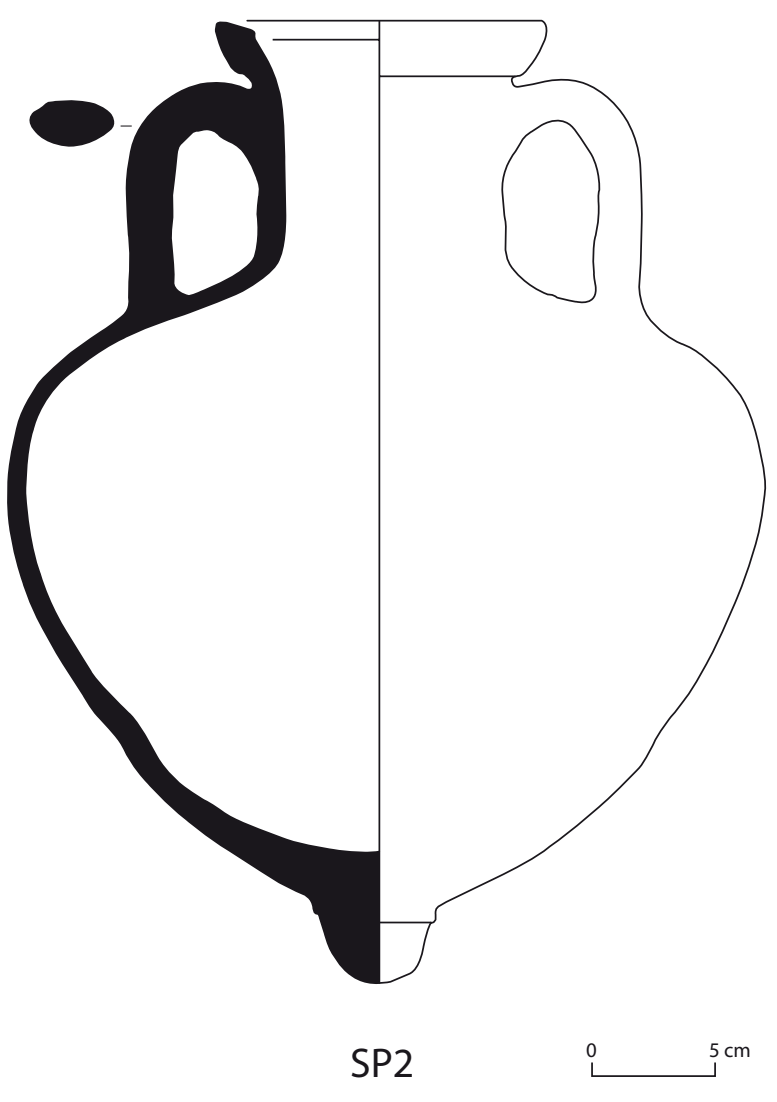

PI. VIII. Spina.

anses, dont la courbure n'est pas encore écrasée par la lèvre. Pour le type, Morter, Leonard 1998, fig. 17. 3 (variantes datables avant 450 av. J.-C.). Pour la forme, Cavalier 1985, fig. 4.a-b (datables de la première moitié $\mathrm{du} \mathrm{V} \mathrm{V}^{\mathrm{e}} \mathrm{s}$. av. J.-C. sur la base de parallèles avec des exemplaires de l'Agora d'Athènes, pour lesquels cf. Lawall 1995 , p. 65 et n. 42).

Chronologie : 500-450 av. J.-C.

Bibliographie : Desantis 1989, p. 103-104, 117, $\mathrm{n}^{\circ} 1$, pl. I, 1 . 


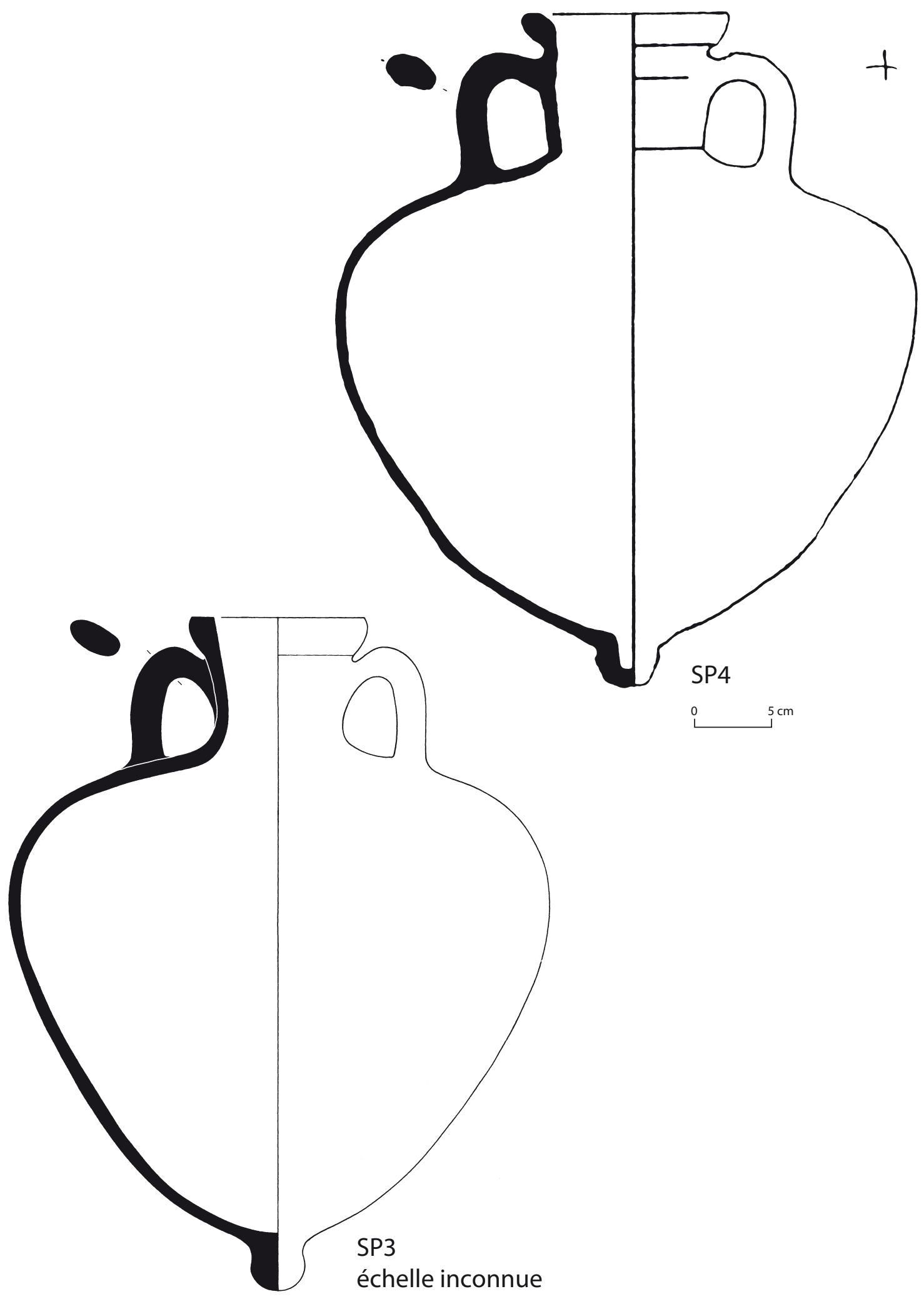

PI. IX. Spina. 


\section{AMPHORES GRÉCO-OCCIDENTALES}

\section{SP2 (pl. VIII)}

Lieu de découverte : Valle Trebba, Comacchio. Inventaire $n^{\circ} 45049$.

Structure : Tombe 603.

Type : corinthienne B.

État de conservation : entière. H. : 40,2 ; diam. embouchure : 13,7. Lèvre en échine. Col évasé, légèrement concave, avec une cannelure au-dessous de la lèvre. Anses verticales, en ruban épaissi, à section elliptique. Épaule aplatie. Corps piriforme, panse légèrement carénée. Pied en bouton, arrondi en bas.

Observations et comparaisons : attribuée par S. De Luca De Marco à la production corinthienne du type $\mathrm{B}$ et datée vers le milieu du $\mathrm{V}^{\mathrm{e}} \mathrm{s}$. av. J.-C. sur la base de confrontations avec des exemplaires athéniens (Grace 1953, n. 3, p. 108-109, n 164-166). Pour le type, Koehler 1978a, fig. 2.b, et $E a d$. 1981, fig. 1.a, pl. 99.b-c, datable entre la fin $\mathrm{du} \mathrm{VI} \mathrm{C}^{\mathrm{e}}$ et le troisième quart du $\mathrm{V}^{\mathrm{e}} \mathrm{s}$. av. J.-C. (Lawall 1995, p. 72). Une datation dans la première moitié du $V^{e}$ s. av. J.-C. a été proposée pour l'amphore en question par D. Baldoni, suggérée par le mobilier, qui comprenait de la céramique attique datable du premier et du deuxième quart du $\mathrm{V}^{\mathrm{e}} \mathrm{s}$. av. J.-C.

Chronologie : 500-450 av. J.-C.

Bibliographie : De Luca De Marco 1979, p. 572, pl. I, $\mathrm{n}^{\circ} 1$; Baldoni 1984, p. 48-49, fig. 19.

\section{SP3 (pl. IX)}

Lieu de découverte : Valle Trebba, Comacchio.

Sporadique.

Type : corinthienne B.

État de conservation : amphore commerciale entière. Lèvre en échine. Col évasé, légèrement concave, avec une cannelure au-dessous de la lèvre. Anses verticales, en ruban épaissi, à section elliptique. Épaule aplatie. Corps piriforme, à panse légèrement carénée. Pied en bouton, arrondi en bas et légèrement épaissi.

Observations et comparaisons : attribuée par S. De Luca De Marco à la production corinthienne du type $\mathrm{B}$ et datée vers le milieu du V $\mathrm{V}^{\mathrm{e}} \mathrm{s}$. av. J.-C. sur la base de parallèles avec des exemplaires athéniens (Grace 1953, n. 3, p. 108-109, n 164-166). Pour le type nous renvoyons à la fiche précédente. Une datation dans la première moitié $\mathrm{du} \mathrm{V}^{\mathrm{e}} \mathrm{s}$. av. J.-C. est aussi suggérée par les confrontations avec des exemplaires provenant de contextes funéraires d'Adria (cat. AD3 e AD6).

Chronologie : 500-450 av. J.-.C.

Bibliographie : De Luca De Marco 1979, p. 572, pl. I, 2.

SP4 (pl. IX)

Lieu de découverte : Valle Trebba, Comacchio.
Inventaire $\mathrm{n}^{\circ} 45085$.

Structure : Tombe 267.

Type : corinthienne B.

État de conservation : entière. Dimensions : non repérées. Lèvre en échine. Col légèrement évasé et concave, avec une cannelure au niveau de la jonction des anses. Anses verticales, en ruban épaissi. Épaule aplatie. Corps piriforme, à panse carénée dans sa partie inférieure. Pied en bouton, au profil caréné, arrondi en bas et creux à l'intérieur.

Appareil épigraphique : «signe en forme de croix » orthogonal (Sacchetti 2010, p. 38-41, n 1, pl. I, 1).

Observations et comparaisons : attribuée par P. Desantis à la production corinthienne $\mathrm{B}$ et datée avant le milieu $\mathrm{du} \mathrm{V}^{\mathrm{e}}$ s. av. J.-C. sur la base de confrontations et du contexte. Pour le type, voir cat. SP2. Le contexte de la découverte de l'exemplaire (enregistré comme sporadique mais attribué par P. Desantis à laTombe 267 de Valle Trebba) comprend de la céramique attique datée du deuxième quart du Ve s. av. J.-C. (Patitucci 1971, p. 6, pl. 5, 5).

Chronologie : 480-450 av. J.-C.

Bibliographie : Desantis 1993, p. 159, note 19-20, fig. 1, $\mathrm{n}^{\circ} 2$; Ead.1996, p. 353, n. 7, fig. 1, 2 ; Sacchetti 2010, p. $38-41, \mathrm{n}^{\circ} 1$, pl. I, 1 .

\section{SP5 (pl. X)}

Lieu de découverte : Valle Lepri, Comacchio

Inventaire $\mathrm{n}^{\circ} 55033$.

Type : corinthienne B.

État de conservation : amphore commerciale, conservée pour sa partie supérieure, de la lèvre jusqu'à une partie de l'épaulement, avec le segment supérieur des anses, reconstituée à partir de nombreux fragments. H. max. cons. : 11,5. Lèvre en échine, ouverte. Diam. embouchure : 12. Col cylindrique, avec une cannelure au niveau de la jonction supérieure des anses. Anses en ruban épaissi, à section elliptique.

Surface ext., int. et fracture : jaune pâle à reflets verdâtres (10YR8/2).

Inclusions : pâte riche en inclusions de petites dimensions. Engobe : engobe jaune pâle, poudreux au toucher.

Observations et comparaisons : attribuée par P. Desantis à la production corinthienne du type $\mathrm{B}$ et datée du milieu $\mathrm{du} \mathrm{V}{ }^{\mathrm{e}}$ s. av. J.-C. Pour le type, datable entre la fin du VI et le troisième quart du Ve s. av. J.-C., cf. cat. SP2 ; pour la forme, Di Sandro 1981b, p. 7, fig. 1, 3.2.

Chronologie : 480-425 av. J.-C.

Bibliographie : Desantis 1989, p. 104, 117, n³ 3, pl. I, 3.

\section{SP6 (pl. X)}

Lieu de découverte : Valle Lepri, Comacchio.

Inventaire $n^{\circ} 55032$. 


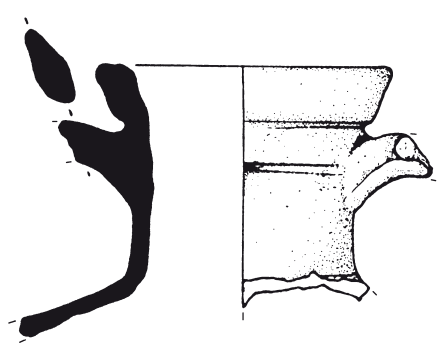

SP5

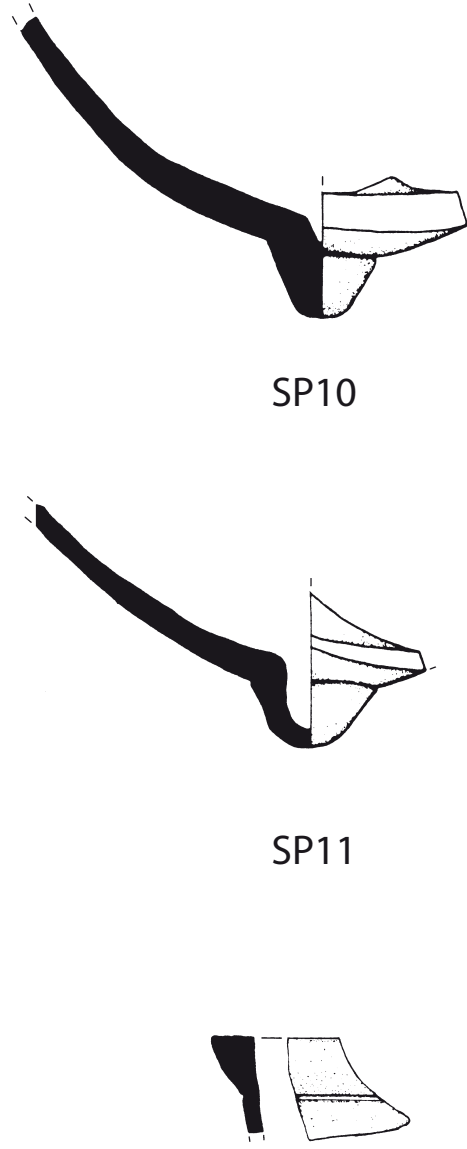

SP12

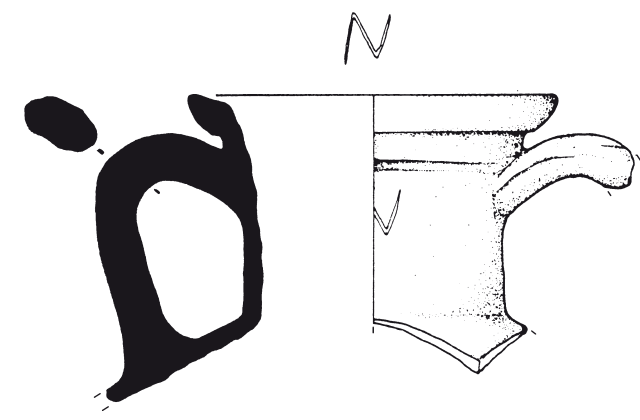

SP6

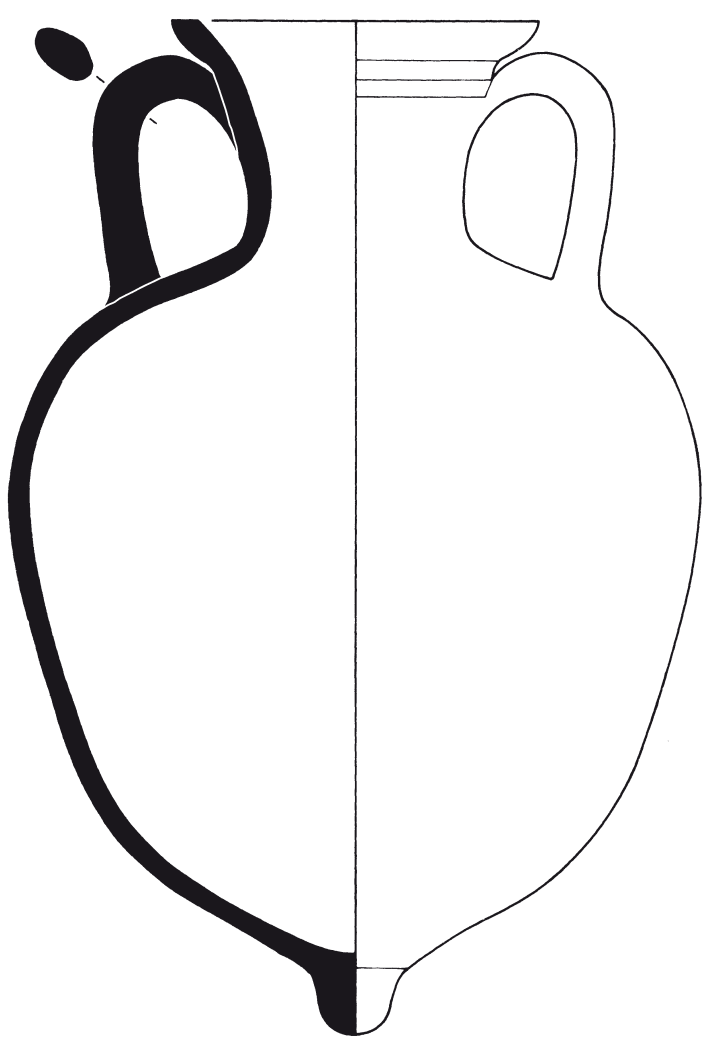

SP13

échelle inconnue 
Type : corinthienne B.

État de conservation : amphore commerciale, conservée pour sa partie supérieure, de la lèvre jusqu'à une partie de l'épaulement, y compris les anses, l'une fragmentaire. H. max. cons.: 13. Lèvre en échine, ouverte, se terminant par un secteur aplati et ouvert. Diam. embouchure : 15 . Col cylindrique, qui présente une cannelure au niveau de la jonction supérieure des anses. Anses obliques rentrantes, en ruban épaissi, à section elliptique.

Surface ext. : jaune pâle à reflets verdâtres (10YR8/2). Surface int. : jaune pâle à reflets verdâtres (10YR8/2). Fracture : jaune pâle à reflets verdâtres (10YR8/2). Inclusions : pâte riche en inclusions de petites dimensions. Engobe : engobe jaune pâle, poudreux au toucher.

Appareil épigraphique : sur le col, entre les anses, la lettre ny (Sacchetti 2010, p. 41-44, n 2, pl. II, 2).

Observations et comparaisons : voir la fiche précédente. Chronologie : 480-425 av. J.-C.

Bibliographie : Desantis 1989, p. 104, 117, n 4, pl. I, 4.

\section{SP7}

Lieu de découverte : Valle Lepri, Comacchio.

Inventaire $\mathrm{n}^{\circ} 55035$.

Type : corinthienne B.

État de conservation : partie de la lèvre et du col reconstituée à partir de deux fragments. H. max. cons. : 10 . Lèvre en échine. Diam. restituable embouchure : 12 . Col avec une cannelure au-dessous de la lèvre et à la jonction de l'épaule.

Surface ext., int. et fracture : jaune pâle à reflets verdâtres (10YR8/2).

Inclusions : pâte riche en inclusions de petites dimensions. Engobe : engobe jaune pâle, poudreux au toucher.

Observations et comparaisons : on renvoie à cat. SP5.

Chronologie : 480-425 av. J.-C.

Bibliographie : Desantis 1989, p. 104, 117, n 5 .

\section{SP8}

Lieu de découverte : Valle Lepri, Comacchio.

Inventaire $\mathrm{n}^{\circ} 55036$.

Type : corinthienne B.

État de conservation : fragment de lèvre avec un court segment du col. H. max. cons. : 4,5. Lèvre en échine. Diam. restituable embouchure : 14. Col avec double cannelure immédiatement au-dessous de la lèvre.

Surface ext., int. et fracture : jaune pâle à reflets verdâtres (10YR8/2).

Inclusions : pâte riche en inclusions de petites dimensions.

Engobe : engobe jaune pâle, poudreux au toucher.

Observations et comparaisons : on renvoie à cat. SP5.

Chronologie : 480-425 av. J.-C.

Bibliographie : Desantis 1989, p. 104, 117-118, nº 6.
SP9

Lieu de découverte : Valle Lepri, Comacchio.

Inventaire $\mathrm{n}^{\circ} 55038$.

Type : corinthienne B.

État de conservation : fragment de lèvre et col avec un segment d'une anse. H. max. cons. : 10. Lèvre en échine. Diam. restituable embouchure : 14. Col avec une cannelure immédiatement au-dessous de la lèvre. Anses en ruban épaissi.

Surface ext., int. et fracture : jaune pâle à reflets verdâtres (10YR8/2).

Inclusions : pâte riche en inclusions de petites dimensions. Engobe : engobe jaune pâle, poudreux au toucher.

Observations et comparaisons : on renvoie à cat. SP5.

Chronologie : 480-425 av. J.-C.

Bibliographie : Desantis 1989, p. 104, 118, nº 7.

\section{SP10 (pl. X)}

Lieu de découverte : Valle Lepri, Comacchio.

Inventaire $\mathrm{n}^{\circ} 55045$.

Type : corinthienne B.

État de conservation : deux fragments du pied et du fond de la panse. H. max. cons. : 10. Corps vraisemblablement globulaire. Pied en bouton, légèrement creux à l'intérieur, qui présente une encoche à la jonction de la panse. Diam. max. restituable : 5 .

Surface ext., int. et fracture : jaune-verdâtre (10YR8/2). Inclusions : inclusions de petites dimensions à fréquence haute.

Engobe : engobe jaune pâle, poudreux au toucher.

Observations et comparaisons : attribué par P. Desantis à la production corinthienne $\mathrm{B}$. La morphologie du pied, du type tronconique encore bref, et du fond de la panse, ample et arrondi, suggèrent une chronologie entre le second et le troisième quart du $\mathrm{V}^{\mathrm{e}} \mathrm{s}$. av. J.-C. Pour le type, Koehler 1978a, fig. 2.b ; Ead. 1981, fig. 1.a, pl. 99.c. ; Lawal 1995, p. 72.

Chronologie : 480-425 av. J.-C.

Bibliographie : Desantis 1989, p. 118, n ${ }^{\circ}$, pl. II, 1.

\section{SP11 (pl. X)}

Lieu de découverte : Valle Lepri, Comacchio.

Inventaire $\mathrm{n}^{\circ} 55043$.

Type : corinthienne B.

État de conservation : pied et une partie du fond de la panse. H. max. cons. : 10. Corps vraisemblablement globulaire. Pied en bouton, creux à l'intérieur, avec une encoche à la jonction de la panse. Diam. max. restituable : 4,3.

Surface ext., int. et fracture : beige rosé (7.5YR8/4).

Inclusions : inclusions grises, de dimensions moyennes à basse fréquence.

Engobe : engobe rosé, poudreux au toucher. 


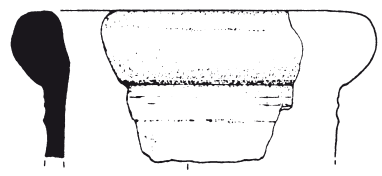

SP15
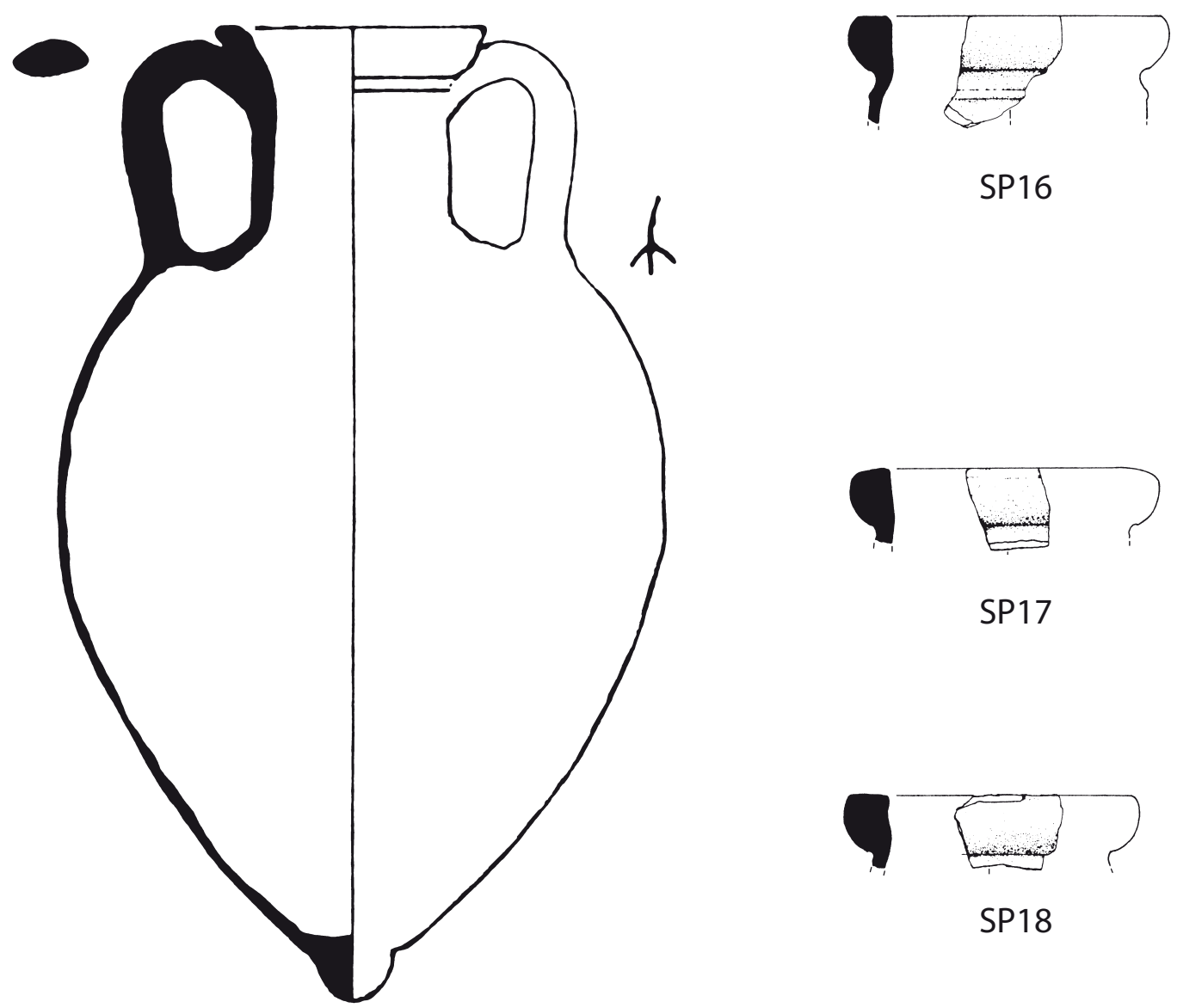

SP16

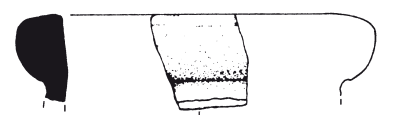

SP17

SP14
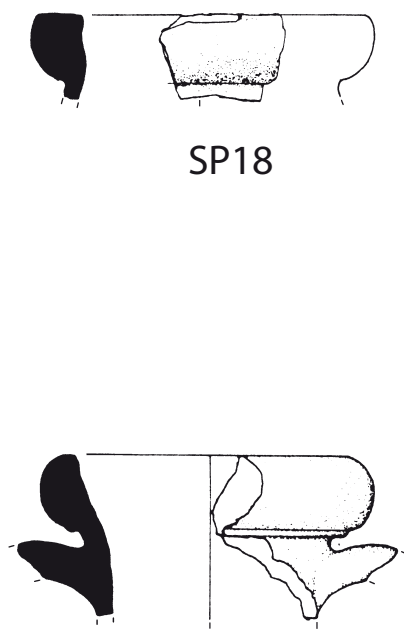

SP19 
Observations et comparaisons : voir la fiche précédente. Chronologie : 480-425 av. J.-C.

Bibliographie: Desantis 1989, p. 118, n 9, pl. II, 2.

\section{SP12 (pl. X)}

Lieu de découverte : Valle Lepri, Comacchio.

Inventaire $\mathrm{n}^{\circ} 55081$.

Type : corinthienne B.

État de conservation : fragment de lèvre avec un bref segment du col. Dimensions max. fr. : 3,9 x 4,3. Lèvre en échine, avec une fine cannelure à la base.

Surface ext., int. et fracture : blanchâtre (10YR8/2).

Inclusions : pâte sableuse.

Engobe : engobe de consistance farineuse au toucher.

Observations et comparaisons : P. Desantis propose d'assigner cet exemplaire à la production des amphores massaliètes, et plus exactement au type Py 6 (Py 1980, p. 14-15, fig. 9). Cependant, dans la description de la pâte, les grosses écailles de mica typiques de cette production ne sont pas mentionnées et la morphologie de cette lèvre semblerait rappeler les amphores de production corinthienne type B du Ve s. av. J.-C. Pour le type, Koehler 1978a, fig. 2.b-c.

Chronologie : 480-400 av. J.-C.

Bibliographie : Desantis 1989, p. 113, 120, n 40, pl. VII, 6.

\section{SP13 (pl. X)}

Lieu de découverte : Valle Trebba, Comacchio.

Sporadique.

Type : corinthienne B.

État de conservation : entière. Dimensions : non repérées. Lèvre en échine, ouverte. Col évasé, légèrement concave, avec double cannelure au-dessous de la lèvre. Épaule arrondie. Corps ovoïde, à panse légèrement carénée. Anses obliques rentrantes, en ruban épaissi, à section elliptique. Pied en bouton, arrondi en bas.

Observations et comparaisons : définie par S. De Luca De Marco comme corinthienne B et située chronologiquement entre la fin $\mathrm{du} \mathrm{V}^{\mathrm{e}}$ et la première moitié du $\mathrm{IV}^{\mathrm{e}}$ s. av. J.-C. sur la base de la confrontation avec Grace 1953, n. 3, n 107, pl. 34, datée du dernier quart du $\mathrm{V}^{\mathrm{e}} \mathrm{s}$. av. J.-C. et, d'après la spécialiste qui a publié l'amphore de Spina, aux caractères morphologiques plus archaïsants par rapport à l'exemplaire en question. Toutefois, la forme ovoïde du corps, non encore élancé, ainsi que la morphologie du pied, non encore fuselé jusqu'à prendre une forme véritablement pointue, suggèrent de ne pas baisser la datation au-delà de la fin du $\mathrm{V}^{\mathrm{e}} \mathrm{s}$. av. J.-C. Pour le type, Koehler 1978a, fig. 2.c ; et Lawall 1995, fig. 17, du dépôt 19:9 de l'Agora fermé vers 410 av. J.-C.

Chronologie : 425-400 av. J.-C.

Bibliographie : De Luca De Marco 1979, p. 572-573, pl. I, 3 .

\section{SP14 (pl. XI)}

Lieu de découverte : Valle Trebba, Comacchio.

Inventaire $\mathrm{n}^{\circ} 45974$.

Sporadique.

Type : corinthienne B.

État de conservation : entière. Dimensions : non repérées. Lèvre du type en échine. Col cylindrique. Épaule arrondie. Corps ovoïde allongé. Anses en ruban épaissi, obliques et rentrantes. Pied en bouton arrondi.

Appareil épigraphique : marque en forme de trident (Sacchetti 2010, p. 44-45, n 3, pl. I, 3).

Observations et comparaisons : attribuée par P. Desantis à la production corinthienne $\mathrm{B}$ et datée entre 420 et 390 av. J.-C. sur la base de confrontations avec Koehler 1979, p. 36,185, pl. $39, n^{\circ} 233-237$, et pl. $40, n^{\circ} 239$. Pour le type, Koehler 1978a, fig. 2.c., datable du dernier quart du Ve s. av. J.-C.

Chronologie : 425-400 av. J.-C.

Bibliographie : Desantis 1993, p. 159, notes 22-23, fig. 1, n 3 ; Ead. 1996, fig. 1, 3 ; Sacchetti 2010, p. 44-45, $\mathrm{n}^{\circ} 3, \mathrm{pl}$. I, 3 .

\section{SP15 (pl. XI)}

Lieu de découverte : Valle Lepri, Comacchio.

Inventaire $\mathrm{n}^{\circ} 55054$.

Type : « forme $1 \alpha »$.

État de conservation : fragment de lèvre et col. H. max. cons. : 5,8. Lèvre en bourrelet gonflé. Diam. restituable embouchure : 14,4. Col cylindrique, avec une rainure au-dessous de la lèvre.

Surface ext., int. et fracture : gris clair (10YR8/3).

Inclusions : pâte sableuse riche en écailles de mica de petites dimensions.

Observations et comparaisons : P. Desantis propose d'attribuer cet exemplaire à la production des amphores massaliètes, et plus exactement au type Py 6 (pour lequel cf. Py 1980, p. 14-15, fig. 9).

Sur la base du dessin publié on propose sa possible attribution au groupe « corinthien B archaïque » Pour le type, Koehler 1981, pl. 99, a. Les amphores de ce groupe sont datables entre les débuts du $\mathrm{VI}^{\mathrm{e}}$ et les débuts du $\mathrm{V}^{\mathrm{e}} \mathrm{s}$. av. J.-C. Étant donné la chronologie du site de Spina, la limite chronologique la plus haute de cet exemplaire ne pourra remonter avant le milieu du $\mathrm{VI}^{\mathrm{e}} \mathrm{s}$. av. J.-C.

Chronologie : 550-490 av. J.-C.

Bibliographie : Desantis 1989,p. 113, 120, n 36, pl. VII, 2.

\section{SP16 (pl. XI)}

Lieu de découverte : Valle Lepri, Comacchio.

Inventaire $\mathrm{n}^{\circ} 55055$.

Type : « forme $1 \alpha »$.

État de conservation : fragment de lèvre et col. H. max. cons. : 5 . Lèvre en bourrelet gonflé, aplatie au-dessus. Diam. 
restituable embouchure : 15 . Col cylindrique ayant une profonde cannelure immédiatement au-dessous de la lèvre.

Surface ext., int. et fracture : gris clair (10YR6/2).

Inclusions : pâte sableuse riche en écailles de mica de dimensions petites et moyennes.

Observations et comparaisons : voir la fiche précédente. Chronologie : 550-490 av. J.-C.

Bibliographie : Desantis 1989, p. 113, 120, n 37, pl. VII, 3.

\section{SP17 (pl. XI)}

Lieu de découverte : Valle Lepri, Comacchio.

Inventaire $\mathrm{n}^{\circ} 55079$.

Type : « forme $1 \alpha »$.

État de conservation : fragment de lèvre avec un court segment du col. H. max. cons. : 3,8. Lèvre en bourrelet gonflé. Diam. restituable embouchure : 14,5. Col avec une cannelure immédiatement au-dessous de la lèvre.

Surface ext., int. et fracture : gris (10YR7/4).

Inclusions : pâte riche en inclusions et écailles de mica. Observations et comparaisons : voir cat. SP15.

Chronologie : 550-490 av. J.-C.

Bibliographie : Desantis 1989,p. 113, 120, n 38, pl. VII, 4.

\section{SP18 (pl. XI)}

Lieu de découverte : Valle Lepri, Comacchio.

Inventaire $\mathrm{n}^{\circ} 55082$.

Type : « forme $1 \alpha »$.

État de conservation : fragment de lèvre avec un court segment du col. H. max. cons. : 3,5. Lèvre en bourrelet gonflé, aplatie au-dessus. Diam. restituable embouchure : 14. Col avec une cannelure au-dessous de la lèvre.

Surface ext., int. et fracture : grisâtre (10YR7/2).

Inclusions : pâte riche en inclusions et écailles de mica. Observations et comparaisons : on renvoie à cat. SP15. Chronologie : 550-490 av. J.-C.

Bibliographie : Desantis 1989,p. 113, 120, n 39, pl. VII, 5.

\section{SP19 (pl. XI)}

Lieu de découverte : Valle Lepri, Comacchio.

Inventaire $\mathrm{n}^{\circ} 55052$.

Type : « forme 2 ».

État de conservation : fragment de lèvre et col avec la jonction d'une anse. H. max. cons. : 6,7. Lèvre en bourrelet gonflé, ouverte, avec un léger ressaut à la base. Diam. restituable embouchure : 13,5 .

Surface ext., int. et fracture : rosée (7.5YR8/4).

Inclusions : pâte à consistance compacte avec de nombreuses écailles de mica de petites dimensions.

Engobe : engobe clair.

Observations et comparaisons : attribuée par P. Desantis au type Py 3 de la production massaliète et datée vers le milieu du Ve s. av. J.-C. Sur la base du dessin publié, je proposerais son appartenance au type Py 1 (Py 1980, p. 5-6, fig. 3.1, 3), datable entre la seconde moitié du VI ${ }^{\mathrm{e}}$ et le premier quart du $\mathrm{V}^{\mathrm{e}} \mathrm{s}$. av. J.-C. (Sourisseau 1997, I, p. 29-30).

Chronologie : 550-475 av. J.-C.

Bibliographie : Desantis 1989,p. 113, 120, n 34, pl.VII, 1.

SP20

Lieu de découverte : Valle Lepri, Comacchio.

Inventaire $\mathrm{n}^{\circ} 55053$.

Type : « forme 2 ».

État de conservation : fragment de lèvre et col avec la jonction d'une anse. H. max. cons. : 4. Lèvre en bourrelet gonflé, avec un fin ressaut à la base. Diam. restituable embouchure : 12. Anses en ruban épaissi, à section elliptique.

Surface ext., int. et fracture : brun rosé (5YR6/6).

Inclusions : pâte à consistance compacte avec de nombreuses écailles de mica.

Observations et comparaisons : attribuée par P. Desantis au type Py 3 de la production massaliète et datée vers le milieu du Ve s. av. J.-C. Le dessin du fragment n'a pas été publié mais il est défini par P. Desantis comme semblable en tous points à celui de la fiche précédente, à laquelle on renvoie pour les considérations de caractère typologique et chronologique.

Chronologie : 550-475 av. J.-C.

Bibliographie : Desantis 1989, p. 113, 120, nº 35.

\section{AMPHORES DE L'ÉGÉE SEPTENTRIONALE}

\section{SP21 (pl. XII)}

Lieu de découverte : Valle Lepri, Comacchio.

Inventaire $\mathrm{n}^{\circ} 55024$.

Type : Middle Mendean Variant Lawall.

État de conservation : pied complet. H. max. cons. : 5,7. Pied en bobine, bref, avec base d'appui convexe et ombiliquée. h. : 5,7. Diam. min./max. : 7,2.

Surface ext., int. et fracture : rosée (7.5YR8/4).

Inclusions : mica.

Engobe : engobe blanchâtre.

Observations et comparaisons : attribuée par P. Desantis à la production de Mendé et datée du troisième quart du $\mathrm{V}^{\mathrm{e}}$ s. av. J.-C. après confrontation avec Anderson 1954, p. 155, fig. 10, 274 f. Pour le type, Lawall 1995, fig. 38. Chronologie : $450-425$ a.C.

Bibliographie: Desantis 1989, p. 110, 120, n³ 30, pl. V, 4.

\section{SP22 (pl. XII)}

Lieu de découverte : Valle Lepri, Comacchio.

Inventaire $\mathrm{n}^{\circ} 55016$.

Type : Middle Mendean Variant Lawall.

État de conservation : partie supérieure d'amphore, de la lèvre à l'épaulement. H. max. cons. : 22. Lèvre en coin, 


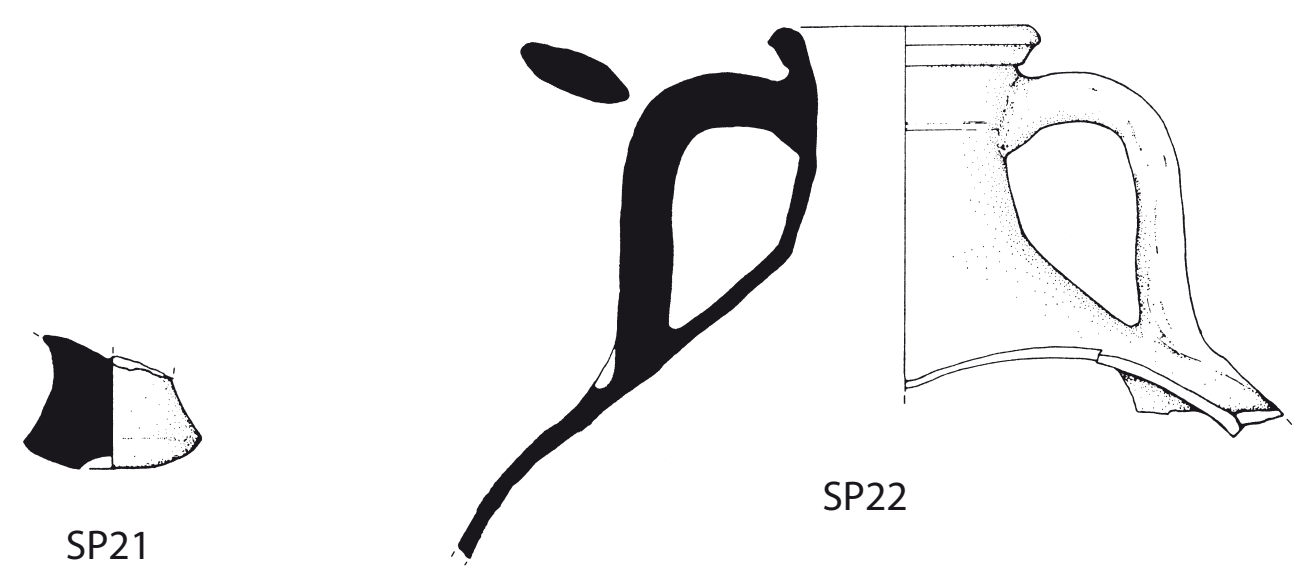

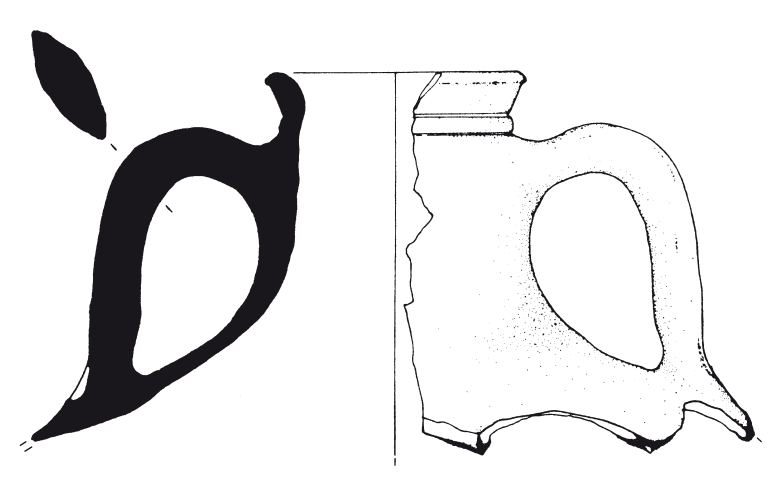

SP23

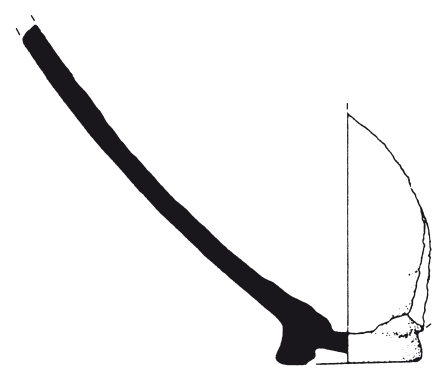

SP25

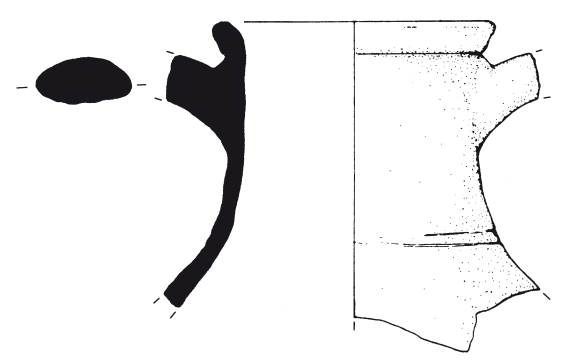

SP24

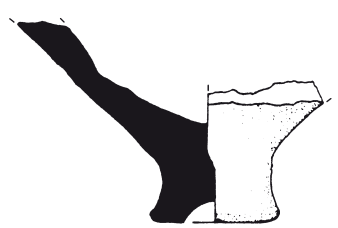

SP26

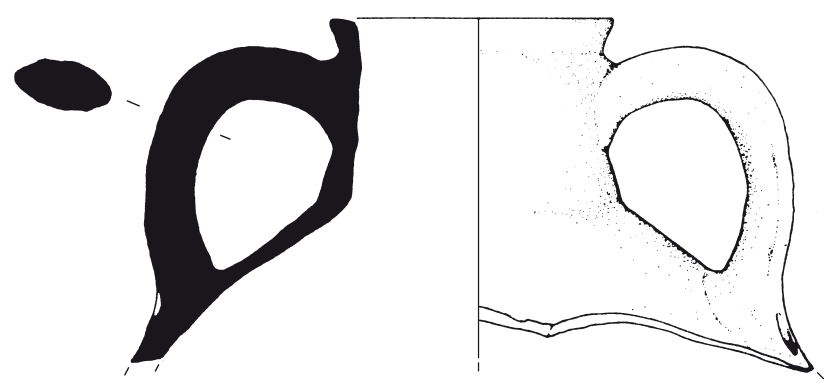

SP27

PI. XII. Spina. 
ouverte, avec la surface extérieure moulurée. Diam. embouchure : 11. Col tronconique, légèrement concave. Anses verticales, en ruban épaissi, à section elliptique, avec des impressions digitales au niveau de la jonction inférieure.

Surface ext., int. et fracture : rosée (5YR6/6).

Inclusions : argile riche en mica.

Engobe : engobe blanchâtre, poudreux au toucher.

Observations et comparaisons : voir la fiche précédente. Chronologie : 450-425 av. J.-C.

Bibliographie : Desantis 1989, p. 110, 119, n² 27, pl. V, 2.

\section{SP23 (pl. XII)}

Lieu de découverte : Valle Lepri, Comacchio.

Inventaire $\mathrm{n}^{\circ} 55017$.

Type : Middle Mendean Variant Lawall.

État de conservation : amphore conservée de la lèvre à l'épaulement. H. max. cons. : 15,5. Lèvre en coin, ouverte, à la surface extérieure moulurée. Diam. embouchure : 10,6. Col tronconique au profil concave. Anses verticales, en ruban épaissi, à section elliptique, avec des impressions digitales au niveau de la jonction inférieure. Ėpaule fuyante.

Surface ext., int. et fracture : rosée (5YR7/4).

Inclusions : argile avec mica.

Engobe : engobe blanchâtre, poudreux au toucher.

Observations et comparaisons : on renvoie à cat. SP21 . Chronologie : 450-425 av. J.-C.

Bibliographie : Desantis 1989, p. 110, 119, n² 28, pl. V, 1 .

\section{SP24 (pl. XII)}

Lieu de découverte : Valle Lepri, Comacchio. Inventaire $\mathrm{n}^{\circ} 55019$.

Type : Middle Mendean Variant Lawall.

État de conservation : partie supérieure d'une amphore, de la lèvre à la jonction du col et de l'épaule, avec les anses incomplètes. H. max. cons. : 13,5. Lèvre en coin, légèrement ouverte. Diam. embouchure : 11,5. Col de forme tronconique, au profil légèrement concave. Anses en ruban épaissi, à section elliptique.

Surface ext. : rougeâtre (5YR6/8).

Surface int. : rougeâtre (5YR6/8).

Fracture : rougeâtre (5YR6/8).

Inclusions : argile riche en mica et avec des vacuoles.

Observations et comparaisons : on renvoie à cat. SP21.

Chronologie : 450-425 av. J.-C.

Bibliographie : Desantis 1989, p. 110, 119-120, n 29, pl. V, 3.

\section{SP25 (pl. XII)}

Lieu de découverte : Valle Lepri, Comacchio.

Inventaire $\mathrm{n}^{\circ} 55023$.

Type : Ring-toe Lawall.
État de conservation : pied avec un morceau de la partie inférieure du corps. H. max. cons.: 14,5. Pied tronconique creux, à base annulaire. Diam. min./max. restituable : 6 .

Surface ext. et int. : rosée (7.5YR8/4).

Fracture : gris.

Inclusions : mica fin.

Engobe : engobe beige en surface.

Observations et comparaisons : attribué par P. Desantis à la production samienne du $\mathrm{V}^{\mathrm{e}} \mathrm{s}$. sur la base de Grace 1971, p. 72. Vu l'apport des recherches plus récentes, on suggère son appartenance à la production nord-égéenne d'époque tardo-archaïque. Pour le type, Lawall 1995, fig. 51 ; pour la forme, Schmid 2001, p. 148, n. 22, pl. 4, datable entre la fin du $\mathrm{VI}^{\mathrm{e}}$ et les débuts du V $\mathrm{V}^{\mathrm{e}} \mathrm{s}$. av. J.-C. Chronologie : 525-480 av. J.-C

Bibliographie : Desantis 1989, p. 107-110, 119, n 25, pl. IV, 6 .

\section{SP26 (pl. XII)}

Lieu de découverte : Valle Lepri, Comacchio.

Inventaire $\mathrm{n}^{\circ} 55026$.

État de conservation : pied et fond de la panse. H. max. cons. : 8. Pied tronconique à profil concave et base d'appui en anneau. Diam. min./max. : 5,3.

Surface ext., int. et fracture : rosée (5YR7/4).

Inclusions : inclusions blanches et inclusions noires de petites dimensions.

Engobe : traces d'engobe.

Observations et comparaisons : définie par P. Desantis «attique », selon l'attribution bien connue de V. Grace, et datée de la fin du V $\mathrm{V}^{\mathrm{e}} \mathrm{s}$. av. J.-C. La morphologie de ce pied pourrait plutôt suggérer une production nordégéenne de la première moitié du $\mathrm{V}^{\mathrm{e}} \mathrm{s}$. av. J.-C., lorsque le pied court en anneau de la première production évolue vers la forme en bobine courte. Ce stade, qu'on peut rapprocher de la «early mendean variant » de la production de Mendé (Lawall 1995, fig. 37), est datable de la première moitié du $\mathrm{V}^{\mathrm{e}} \mathrm{s}$. av. J.-C. Pour la forme, Schmid 2001, p.148, 156, n ${ }^{\circ} 6$, pl. 6, n 6 , datable de 460-450 av. J.-C.

Chronologie : 500-450 av. J.-C.

Bibliographie : Desantis 1989, p. 110, 120, n 33 , pl. VI, 3.

\section{SP27 (pl. XII)}

Lieu de découverte : Valle Lepri, Comacchio.

Inventaire $\mathrm{n}^{\circ} 55018$.

État de conservation : partie supérieure d'une amphore, de la lèvre à l'épaulement. H. max. cons. : 14,5. Lèvre en coin. Diam. embouchure : 12. Col cylindrique. Anses circulaires, en ruban épaissi, à section elliptique, avec impression digitale au niveau de la jonction inférieure. Surface ext., int. et fracture : rougeâtre (5YR6/6). 
Inclusions : pâte à consistance sableuse, avec de petites inclusions blanches, grises et micacées.

Engobe : restes d'engobe blanchâtre.

Observations et comparaisons : P. Desantis propose d'attribuer l'exemplaire à la production chiote de la fin du $\mathrm{V}^{\mathrm{e}}$ s. av. J.-C., sur la base de la confrontation avec l'exemplaire de la Tombe 642 de Valle Trebba (cf. fiche suivante) et avec Grace 1961, fig. 35, où apparaissent plusieurs amphores datées de la période de la destruction persane. D'après les dessins publiés des deux amphores de Spina on suggère son attribution à la production nord-égéenne. Vu la hauteur encore modérée du col, on propose une datation dans le deuxième quart du $\mathrm{V}^{\mathrm{e}} \mathrm{s}$. av. J.-C. Pour le type, Grace 1953, fig. 5.161 ; et Lawall 1995, fig. 60-61. L'impression digitale à la base des anses est un trait caractéristique des productions amphoriques nord-égéennes de la fin du $\mathrm{VI}^{\mathrm{e}}$ et du $\mathrm{V}^{\mathrm{e}} \mathrm{s}$. av. J.-C. (Blondé, Muller, Mulliez 1991 ; Grandjean 1992 ; Schmid 2001).

Chronologie : 480-450 av. J.-C.

Bibliographie : Desantis 1989, p. 107, 118-119, n 18, pl. III, 3 .

\section{SP28 (pl. XIII)}

Lieu de découverte : Valle Trebba, Comacchio.

Structure : Tombe 642.

Inventaire $\mathrm{n}^{\circ} 45011$.

Type : Disc-toe Lawall.

État de conservation : entière. Dimensions : non repérés. Lèvre en coin, amincie et ouverte. Col tronconique, légèrement concave. Anses verticales, en ruban épaissi. Épaule fuyante. Corps ovoïde. Pied tronconique plein, à base légèrement convexe et ombiliquée.

Appareil épigraphique : impression circulaire sur un côté du col et marque alpha sur l'autre (Sacchetti 2010, p. 45-46, n 4, pl. I, 4).

Observations et comparaisons : S. De Luca De Marco et P. Desantis attribuent l'exemplaire à la production chiote. Vu sa morphologie, on propose maintenant son attribution à la production nord-égéenne. Pour le type, Lawall 1995, fig. 58, 66, respectivement du dépôt F 19:4 de l'Agora, fermé vers 450 av. J.-C., et du dépôt Q 12:3, datable entre 520 et 480 av. J.-C. ; Roberts 1986, fig. 43, $\mathrm{n}^{\circ} 424$; Schmid 2001, p. 148, 156, n 6, pl. 6, 6, daté de 460-450 av. J.-C. Une datation vers le milieu du Ve s. av. J.-C. est corroborée aussi par la présence à l'intérieur du mobilier d'une oenochoé attique à figures rouges que Beazley attribue au Peintre de Hasselmann (Arv2, p. $\left.1138, n^{\circ} 46\right)$.

Chronologie : 480-440 av. J.-C.

Bibliographie : De Luca De Marco 1979, p. 575, pl. III, $\mathrm{n}^{\circ} 8$; Desantis 1993 , p. 158, fig. $1, \mathrm{n}^{\circ} 1$; Ead. 1996 , p. 350, fig. 1, 1 ; Sacchetti 2010, p. 45-46, n 4, pl. I, 4.

\section{SP29 (pl. XIII)}

Lieu de découverte : Valle Lepri, Comacchio.

Inventaire $\mathrm{n}^{\circ} 55015$.

État de conservation : partie supérieure, de la lèvre à l'épaulement. H. max.cons.: 20,5. Lèvre en cordon. Diam. embouchure : 12,5. Col cylindrique. Anses circulaires, en ruban épaissi, à section elliptique, avec impression digitale au niveau de la jonction inférieure. Épaule fuyante.

Surface ext., int. et fracture : rouge (2.5YR6/8).

Inclusions : pâte à consistance sableuse, avec de très fines inclusions blanches et grises et de mica.

Engobe : restes d'engobe blanchâtre.

Observations et comparaisons : P. Desantis attribue l'amphore à la production chiote du troisième quart du Ve s. av. J.-C. par confrontation avec Grace 1934, p. 303304, fig. 1.1-2 (la première assignée à Chios, la seconde non classifiée) et avec Grace 1961, fig. 44-45 (attribuées l'une et l'autre à Chios). On suggère une possible attribution de l'exemplaire à la production nord-égéenne de la seconde moitié du Ve s. av. J.-C. Pour la forme, Grandjean 1992, fig. 11.71. À cette production renvoie également le détail de l'impression digitale à la jonction inférieure des anses (Blondé, Muller, Mulliez 1991 ; Grandjean 1992 ; Schmid 2001).

Chronologie : 450-400 av. J.-C.

Bibliographie : Desantis 1989, p. 107, 119, n 19, pl. III, 4.

\section{AMPHORES DE L'ÉGÉE ORIENTALE}

\section{SP30 (pl. XIII)}

Lieu de découverte : Valle Lepri, Comacchio.

Inventaire $\mathrm{n}^{\circ} 55028$.

Type : chiote, Swollen-necked, Early bulgy, variante C/1 ou C/2 Knigge-Lawall.

État de conservation : pied complet. H. max. cons. : 7,6. Pied cylindrique creux, à base en anneau épaissi et légèrement replié vers l'intérieur. Diam. min./max. : 5,2.

Surface ext., int. et fracture : rosée (7.5YR7/6).

Inclusions : inclusions blanches, grisâtres, rougeâtre et mica.

Engobe : engobe clair en surface.

Observations et comparaisons : P. Desantis attribue le pied à la production chiote de la fin $\mathrm{du} \mathrm{VI}^{\mathrm{e}}$-début du $\mathrm{V}^{\mathrm{e}} \mathrm{s}$. sur la base de confrontations avec des exemplaires provenant de contextes de l'Agora (Roberts 1986, p. 67, fig. $\left.42, n^{\circ} 419-421\right)$. Au vu des plus récentes études sur la classification des amphores chiotes, on propose d'attribuer le pied au type de production chiote connu sous le nom de swollen-necked, early bulgy, datable entre le début et le milieu du Ve s. av. J.-C. Pour le type, Lawall 1995, fig. 31, sans qu'il soit possible de distinguer entre les variantes C/1 et C/2 ; Dupont 1998, fig. 23.2, d-f ; et de Marinis 1999, p. 266-268, fig. 7. 4-7. 


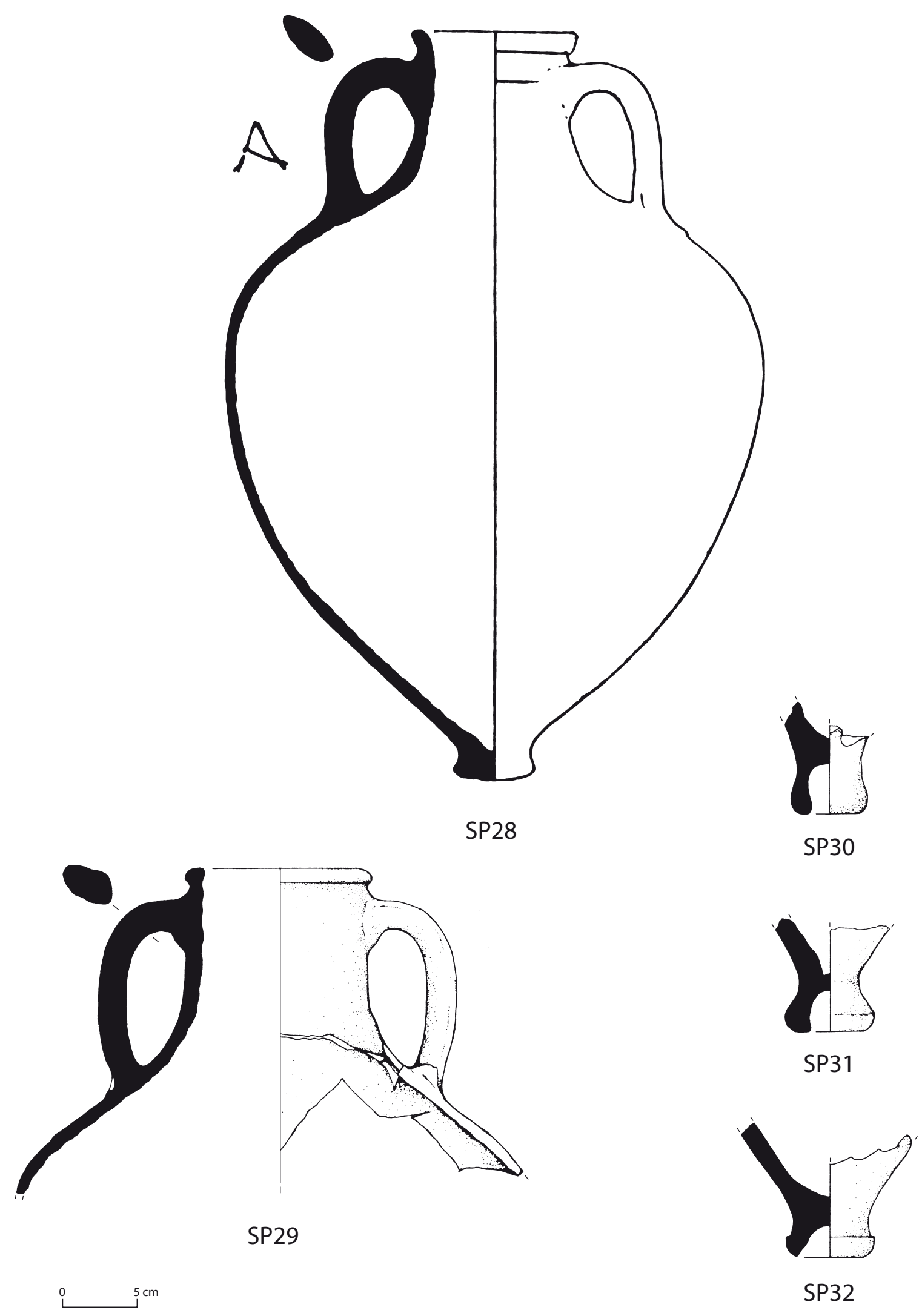

PI. XIII. Spina. 

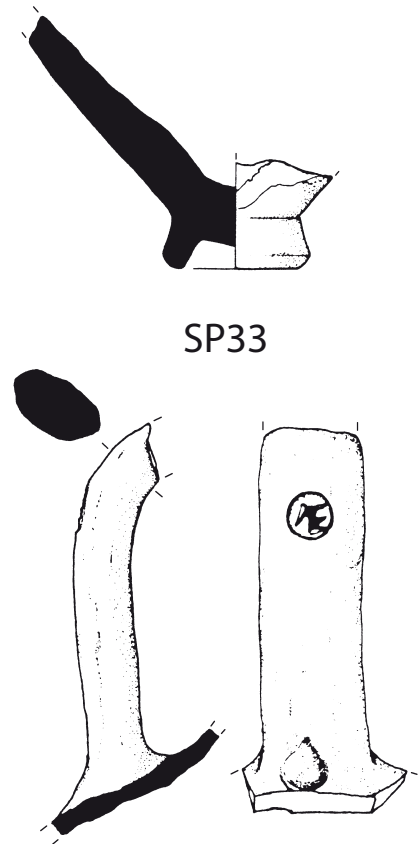

SP35
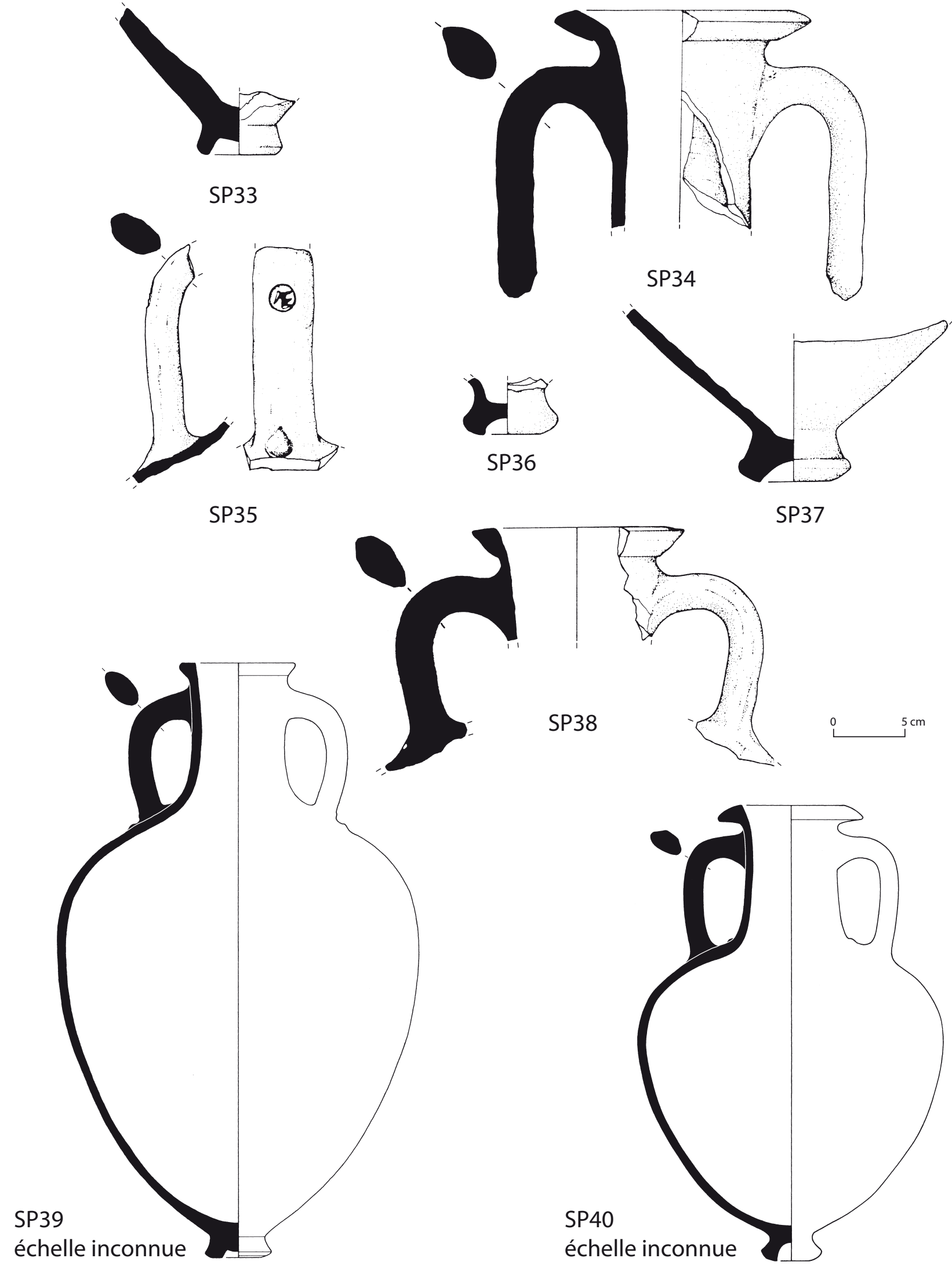

PI. XIV. Spina. 
Chronologie : 500-450 av. J.-C.

Bibliographie : Desantis 1989, p. 107, 119, $\mathrm{n}^{\circ}$ 20, pl. IV, 1 .

\section{SP31 (pl. XIII)}

Lieu de découverte : Valle Lepri, Comacchio.

Inventaire $\mathrm{n}^{\circ} 55025$.

Type : chiote (?).

État de conservation : pied et partie du fond de la panse. H. max. cons. : 7,8. Pied tronconique creux, à base en anneau légèrement épaissi. Diam. min./max. : 6.

Surface ext. et int. : chamois rosé (10YR7/3).

Fracture : gris.

Inclusions : inclusions blanches, grisâtres et mica.

Engobe : engobe beige.

Observations et comparaisons : attribuée par P. Desantis à la production chiote de la fin $\mathrm{VI}^{\mathrm{e}}$-début $\mathrm{V}^{\mathrm{e}} \mathrm{s}$. av. J.-C. sur la base de confrontations avec des exemplaires de l'Agora d'Athènes (Roberts 1986, p. 67, fig. 42, nº 419-421). On observera, en regardant le dessin publié, qu'une forme aussi évasée du pied s'écarte de la norme dans le cadre de la production de Chios.

Chronologie : non déterminable.

Bibliographie : Desantis 1989, p. 107, 119, n 21, pl. IV, 2.

\section{SP32 (pl. XIII)}

Lieu de découverte : Valle Lepri, Comacchio.

Inventaire $\mathrm{n}^{\circ} 55027$.

Type : chiote.

État de conservation : pied avec une petite partie du fond de la panse. H. max. cons. : 9. Pied tronconique creux, à base annulaire. Diam. min./max. : 6 .

Surface ext. et int. : chamois rosé (5YR7/4).

Fracture : gris.

Inclusions : inclusions blanches et grisâtres, de petites dimensions. Mica.

Engobe : restes d'engobe blanchâtre.

Observations et comparaisons : classée par P. Desantis comme chiote et encadrée dans le IV ${ }^{\mathrm{e}} \mathrm{s}$. sur la base de confrontations avec des exemplaires de Chios (Anderson 1954, p. 169, fig. 9. 54g). La morphologie du pied peut renvoyer tant aux formes de la première moitié du $\mathrm{IV}^{\mathrm{e}} \mathrm{s}$. av. J.-C. (Cerdá 1987, fig. 124, 623), qu'à celles de la seconde moitié du Ve s. av. J.-C. (de Marinis 1999, fig. 7, 8-9 ; Monachov 2003a, pl. 7-8).

Chronologie : non déterminable.

Bibliographie : Desantis 1989, p. 107, 119, n²3, pl. IV, 5.

\section{SP33 (pl. XIV)}

Lieu de découverte : Valle Lepri, Comacchio.

Inventaire $\mathrm{n}^{\circ} 55030$.

Type : Samos-Milet, S/1 Lawall.
État de conservation : pied avec un morceau de la partie inférieure du corps. H. max. cons. : 10,5. Pied tronconique creux, à base en anneau caréné. Diam. min./max. : 6 .

Surface ext. et int. : rouge foncé (2.5YR6/8).

Fracture : gris.

Inclusions : mica à fréquence haute.

Observations et comparaisons : attribué par P. Desantis à la production samienne du $\mathrm{V}^{\mathrm{e}} \mathrm{s}$. sur la base de la description de V. Grace (Grace 1971, p. 72). La morphologie du pied, du type tronconique creux à base annulaire, autorise maintenant d'encadrer l'amphore de Spina dans la forme tardo-archaïque du type $\mathrm{S} / 1$ de la récente classification de M. Lawall. Pour la forme, cf. Roberts 1986, fig.4. 413 .

Chronologie : 525-480 av. J.-C.

Bibliographie : Desantis 1989, p. 107-110, 119, n 26, pl. IV, 7.

\section{SP34 (pl. XIV)}

Lieu de découverte : Valle Lepri, Comacchio.

Inventaire $\mathrm{n}^{\circ} 55005$.

Type : Solokha I.

État de conservation : fragment de lèvre, col et anse privée de la jonction inférieure. H. max. fr. : 20,5. Lèvre à marli oblique, à section triangulaire, légèrement ouverte. Diam. restituable embouchure : 18. Col évasé. Anses en ruban épaissi, à section elliptique.

Surface ext., int. et fracture : rouge (5YR6/8).

Inclusions : inclusions blanches et noires de petites dimensions.

Engobe : restes d'engobe superficiel farineux au toucher. Observations et comparaisons : classée par P. Desantis comme amphore 'attique' sur la base de V. Grace (Grace 1953, p. 101-102) et datée de la fin du IV $\mathrm{s}$. av. J.-C. L'exemplaire, maintenant attribuable au type Solokha I, peut être daté entre la fin du $\mathrm{V}^{\mathrm{e}}$ et les débuts du IV ${ }^{\mathrm{e}} \mathrm{s}$. av. J.-C. à cause de l'inclinaison de la lèvre et des proportions du col et des anses. Pour le type, Lawall 1995, p. 220-221, fig. 90, du dépôt H 13:4 de l'Agora d'Athènes, fermé vers 390 av. J.-C.

Chronologie : 410-390 av. J.-C.

Bibliographie : Desantis 1989, p. 110, 120, $\mathrm{n}^{\circ} 32$, pl. VI, 2.

\section{SP35 (pl. XIV)}

Lieu de découverte : Valle Lepri, Comacchio.

Inventaire $\mathrm{n}^{\circ} 55046$.

Type : Solokha I (?).

État de conservation : anse presque complète, sans la jonction supérieure. H. max. cons. : 16. Anse verticale, en ruban épaissi, à section elliptique, avec une impression digitale au niveau de la jonction inférieure.

Surface ext., int. et fracture : rosée (7.5YR8/4). 
Inclusions : inclusions blanches et noirâtres de petites dimensions.

Engobe : restes d'engobe rosé poudreux au toucher.

Observations et comparaisons : attribuée par P. Desantis à une amphore corinthienne B d'époque hellénistique sur la base de la confrontation qu'elle a proposée pour le type de marque avec un exemplaire de Gela. La caractéristique de l'impression digitale correspondant à la jonction inférieure de l'anse et son profil pourraient suggérer au contraire l'appartenance de cet exemplaire au type Solokha I. Pour le type, Lawall 1995, p. 220-221, fig. 91-92.

Chronologie : non déterminable.

Bibliographie : Desantis 1989, p. 104-107, 118, n 16, pl. III, 1 .

\section{SP36 (pl. XIV)}

Lieu de découverte : Valle Lepri, Comacchio.

Inventaire $n^{\circ} 55029$.

Type : Solokha I.

État de conservation : pied complet. H. max. cons. : 4. Pied tronconique creux, à base en anneau légèrement épaissi. Diam. min./max. : 6,7.

Surface ext. et int. : rosée (7.5YR8/4).

Fracture : gris.

Inclusions : inclusions blanches, grisâtres et rougeâtres de petites dimensions. Mica.

Engobe : restes d'engobe clair en surface.

Observations et comparaisons : classée par P. Desantis comme amphore chiote. La morphologie et les proportions du pied pourraient toutefois reconduire au stade d'évolution de la fin du $\mathrm{V}^{\mathrm{e}}$-milieu du $\mathrm{IV}^{\mathrm{e}} \mathrm{s}$. av. J.-C. de la série Solokha I. Pour le type, Lawall 1995, p. 220221, fig. 90 (datable entre fin du Ve et début du IV s. av. J.-C.) ; Zeest 1960, pl. XV, 32 (datées entre la fin du Ve et le premier quart du IV s. av. J.-C. dans Brašinskij 1965 et Mancevič 1987 et de la seconde moitié du IV s. av. J.-C. dans Farmakovskiy 1914) ; Cerdá 1987, fig. 117, 519, et fig. 118, 536 (datables autour du milieu du IVe s. av. J.-C.).

Chronologie : 410-350 av. J.-C.

Bibliographie : Desantis 1989, p. 119, n² 22, pl. IV, 3.

\section{SP37 (pl. XIV)}

Lieu de découverte : Valle Lepri, Comacchio. Inventaire $\mathrm{n}^{\circ} 55022$.

Type : Solokha I.

État de conservation : pied avec une grande partie du fond de la panse et une ample portion de l'épaulement. H. max. cons. : 12,5. Pied tronconique creux, à base en anneau légèrement épaissi. Diam. min./max. : 8.

Surface ext., int. et fracture : beige rosé (7.5YR7/6).

Inclusions : inclusions blanches de petites dimensions.

Engobe : restes d'engobe blanchâtre en surface.
Observations et comparaisons : classée par P. Desantis comme amphore chiote. Ėtant donné son profil et les proportions du pied, l'exemplaire pourrait entrer dans le cadre du type Solokha I. Pour le type, Lawall 1995, fig. 88-89, 93, de dépôts de l'Agora fermés avant la fin du Ve s. av. J.-C.

Chronologie : 450-400 av. J.-C.

Bibliographie : Desantis 1989, p. 119, n² 24, pl. IV, 4.

\section{SP38 (pl. XIV)}

Lieu de découverte : Valle Lepri, Comacchio.

Inventaire $\mathrm{n}^{\circ} 55034$.

Type : Solokha I.

État de conservation : fragment de lèvre avec un court segment du col et une anse complète. H. max. cons. : 17. Lèvre à marli oblique, de section triangulaire. Diam. restituable embouchure : 15. Col évasé. Anses obliques rentrantes, en ruban épaissi, à section elliptique, avec impression digitale au niveau de la jonction inférieure. Surface ext., int. et fracture : rosée (10YR7/4).

Inclusions : argile à pâte fine et de consistance compacte, avec inclusions blanchâtres de petites dimensions. Observations et comparaisons : défini 'attique' par P. Desantis et daté de la fin du Ve s. av. J.-C. Les proportions générales et l'inclinaison de la lèvre vers l'extérieur, à peine perceptible, permettent aujourd'hui de situer l'exemplaire au début de la série Solokha I, vers le milieu du Ve s. av. J.-C. Pour le type, Lawall 1995, p. 220, fig. 88, du dépôt N 7:3, fermé vers 440 av. J.-C.

Chronologie : autour de 450 av. J.-C.

Bibliographie : Desantis 1989, p. 110, 120, n 31, pl. VI, 1.

\section{SP39 (pl. XIV)}

Lieu de découverte : Valle Pega, Comacchio.

Sporadique.

Type : Solokha I.

État de conservation : entière. Dimensions : non repérées. Lèvre à marli oblique, de section triangulaire. Col cylindrique, légèrement concave. Anses obliques rentrantes, en ruban épaissi, à section elliptique, avec une impression digitale à la jonction inférieure. Épaule fuyante. Corps ovoïde. Pied tronconique creux, à base en anneau caréné.

Observations et comparaisons : S. De Luca De Marco attribue l'exemplaire à la production chiote après confrontation avec Grace 1953, p. 101, n 147, pl. 39. Le parallèle établi avec l'exemplaire de l'Agora choisi par S. De Luca De Marco est tout à fait convaincant, mais l'amphore publiée par V. Grace se rapportait à la production 'attique'. L'exemplaire d'Athènes (pour lequel on renvoie en dernier à Lawall 1995, p. 220, fig. 88, d'un contexte fermé vers 440 a.C.) et celui de Spina sont 
maintenant attribués au type Solokha I, et plus précisément à la forme initiale de la série.

Chronologie : autour de 450 av. J.-C.

Bibliographie : De Luca De Marco 1979, p. 576, pl. III, 9.

\section{SP40 (pl. XIV)}

Lieu de découverte : Valle Pega, Comacchio.

Sporadique.

Inventaire $n^{\circ} 40550$.

Type : Solokha I.

État de conservation : entière. Dimensions non repérées. Lèvre à marli oblique, à section triangulaire, ouverte et pendante. Col cylindrique, au profil légèrement concave. Anses obliques rentrantes, en ruban épaissi, à section elliptique. Épaule arrondie. Corps ovoïde. Pied en anneau au profil concave et base épaissie.

Observations et comparaisons : définie comme chiote et attribuée aux dernières décennies du $\mathrm{IV}^{\mathrm{e}} \mathrm{s}$. av. J.-C. par S. De Luca De Marco. L'amphore est à rattacher au type Solokha I. La morphologie du pied et surtout de la lèvre, qui pend vers le bas, suggèrent l'attribution de l'exemplaire à la phase évoluée de la production de la série. Sur la base de la comparaison avec les amphores du tumulus de Solokha (Zeest 1960, pl. XIV-XVI, 32) et avec les exemplaires du type $\mathrm{N}_{1-3}$ de l'épave d'El Sec (Cerdá 1987, fig. 117-118), on propose une datation dans le courant de la première moitié du $\mathrm{IV}^{\mathrm{e}} \mathrm{s}$. av. J.-C.

Chronologie : 400-350 a.C.

Bibliographie : De Luca De Marco 1979, p. 576, pl. III, 10.

\section{Analyses des données (graphiques 5-8)}

La plus grande partie des amphores étudiées provient du petit secteur de l'habitat qui a été l'objet de recherches dans la seconde moitié du siècle dernier à Valle Lepri. Huit individus proviennent des aires des nécropoles de Valle Trebba (cat. SP2-SP4, SP13-SP14, SP28) et de Valle Pega (cat. SP39-SP40), fouillées à plusieurs reprises respectivement à partir de 1922 et de 1924 (cf. supra II, 2 § 1) Dans trois cas seulement (cat. SP2, SP4, SP28) il a été possible de remettre les amphores en contexte (respectivement les tombes 603, 267 et 642 de Valle Trebba).

Les données quantitatives des amphores de Spina actuellement publiées ne sont certes pas proportionnelles à l'importance et à la fonction du site, qui dans le courant du $\mathrm{V}^{\mathrm{e}} \mathrm{s}$. av. J.-C. joua le rôle de port principal du système fédéral étrusco-padan ${ }^{634}$. La documentation dont on dispose aujourd'hui, et qui a été ici reprise,

634. La publication très attendue du matériel qui est encore en cours d'étude et la récente reprise des fouilles de l'habitat permettront

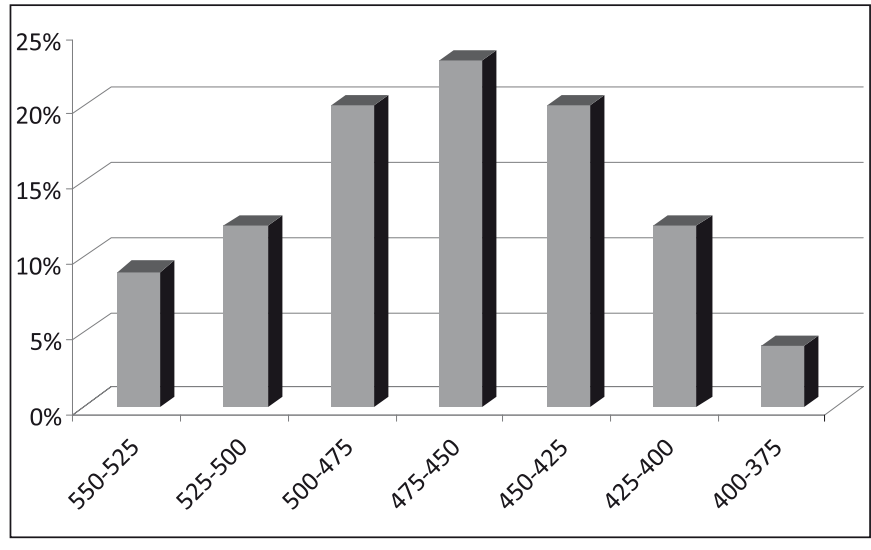

Graphique 5. Spina : tendance des importations d'amphores grecques dans la période examinée (troisième quart du $\mathrm{Vl}^{\mathrm{e}}$-début du IVe s. av. J.-C.).

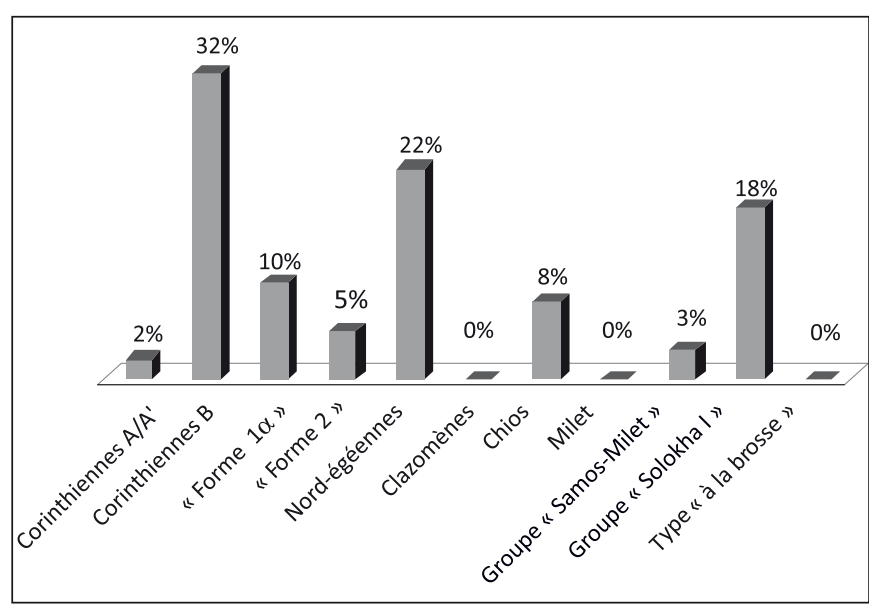

Graphique 6. Spina : données quantitatives concernant les importations des différents groupes amphoriques pendant toute la période examinée (troisième quart du VIe-début du IVe $\mathrm{s}$. av. J.-C.).

permet en tout cas de faire le point sur une situation que l'on peut considérer comme représentative des importations amphoriques parvenues jusqu'à l'aire méridionale du delta du Pô.

Faute de données provenant des contextes, il est impossible de préciser le moment où les importations amphoriques de Spina ont commencé. Au vu des données typologiques, ce moment peut être en tous cas situé dans le courant de la deuxième moitié du $\mathrm{VI}^{\mathrm{e}} \mathrm{s}$. av. J.-C., en concomitance avec le début des importations de céramique attique vers 540 av. J.-C. (Berti 1985, p. 194195, fig. 12 ; Lippolis 2000, p. 111 ; Bruni 2004, p. 84). Le pic maximal des importations amphoriques semble se situer dans le deuxième quart du $\mathrm{V}^{\mathrm{e}} \mathrm{s}$. av. J.-C., ce qui correspond à la phase d'affirmation de l'emporion

évidemment de jeter une lumière nouvelle sur la question des importations amphoriques du site. 


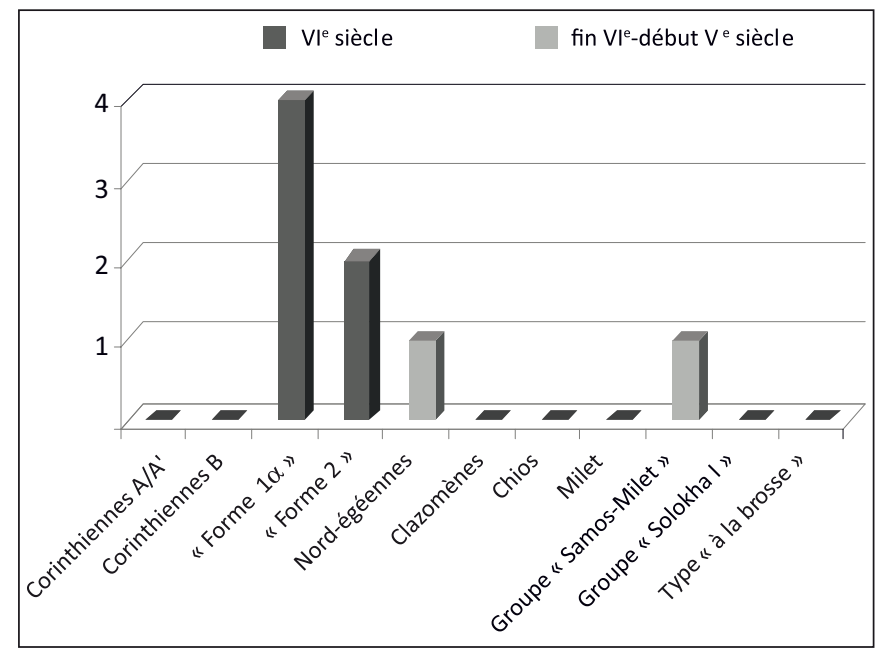

Graphique 7. Spina : données quantitatives des importations des différents groupes amphoriques entre le troisième quart du $\mathrm{Vl} \mathrm{l}^{\mathrm{e}}$ et le début du Ve s. av. J.-C.

étrusco-padan méridional. Dans le cas de Spina il n'est pas possible de proposer un parallèle avec les données sur les importations de céramique grecque décorée dans le courant du siècle (Boardman 1979 ; Giudice 2004, fig. 17-20) étant donné la diversité des contextes de provenance : presque exclusivement d'habitat pour les amphores et presque exclusivement funéraire pour la céramique attique.

Comme le montrent les données de l'habitat (Desantis 1989) et surtout des nécropoles (De Luca De Marco 1979), les importations d'amphores à Spina continuèrent au cours du IV $\mathrm{e}$ s. av. J.-C., et donc aussi après le changement des équilibres politiques de l'aire padane déterminé par l'invasion celtique des débuts du siècle ${ }^{635}$.

Dans le courant de la seconde moitié du VI ${ }^{\mathrm{e}} \mathrm{s}$. av. J.-C. les typologies les plus présentes sont constituées par les

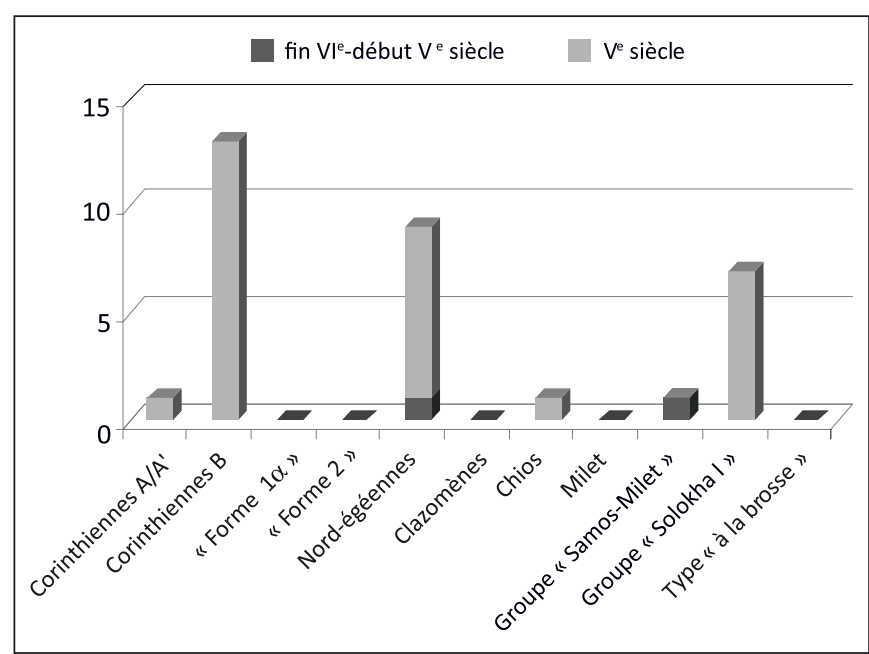

Graphique 8. Spina : données quantitatives des importations des différents groupes amphoriques entre la fin du $\mathrm{Vl}^{\mathrm{e}}$ et la fin du Ves. av. J.-C.

groupes gréco-occidentaux archaïques (« corinthien $\mathrm{B}$ archaïque » et « ionio-massaliète »). Les amphores nord-égéennes peuvent remonter probablement déjà à la période de transition du $\mathrm{VI}^{\mathrm{e}}$ au $\mathrm{V}^{\mathrm{e}} \mathrm{s}$. av. J.-C. mais sont surtout du $\mathrm{V}^{\mathrm{e}} \mathrm{s}$. av. J.-C., alors que les amphores grécoorientales du groupe Solokha I se datent dans la seconde moitié du Ve et au début du IV $\mathrm{V}^{\mathrm{e}} \mathrm{s}$. av. J.-C. Presque complètement absentes sont les amphores de Chios, celles du groupe Samos-Milet et les amphores corinthiennes A', documentées par des présences isolées. Les amphores les mieux attestées au cours du $\mathrm{V}^{\mathrm{e}} \mathrm{s}$. sont une fois de plus celles de probable production occidentale, maintenant sous la forme dite corinthienne B. Des huit amphores datables du courant du $\mathrm{V}^{\mathrm{e}} \mathrm{s}$. av. J.-C. provenant des contextes funéraires de Spina, cinq sont du type corinthien B, deux appartiennent au groupe Solokha I et une est attribuée à la production nord-égéenne.

635. Sur la continuité de la fonction économique de Spina dans les courant du IVe s. av. J.-C. : Sassatelli 1993 ; Id. 2004 ; Id. 2008. Pour la continuité des importations de céramique attique pendant le IVe s. av. J.-C. : Curti 1993 ; Guermandi 1998 ; Giudice 2004, p. 184, fig. 21 ; Govi 2006. On rappellera qu'on a fait ici le choix de ne pas traiter de cette phase chronologique qui s'écarte des problématiques historiques et économiques du système étrusco-padan centré sur Bologne/Felsina. 



\section{Chapitre 3}

\section{Le Forcello di Bagnolo San Vito (prov. de Mantoue) : l'habitat}

\section{Bilan des études}

L'habitat étrusque du Forcello ${ }^{636}$ (fig. 69) surgit sur la rive droite du fleuve Mincio aux alentours de 540 av. J.-C. et fut actif jusqu'aux invasions celtiques de 388 av. J.-C. (de Marinis, Rapi 2007, p. 34).

À partir de 1981, le site a été l'objet de diverses campagnes de fouilles mais également de sondages, de collectes de surface, de carottages et de prospections géophysiques qui ont permis d'estimer l'extension totale de l'occupation du sol à douze hectares environ (de Marinis, Rapi 2007, p. 31).

Le choix de la zone (fig. 83) fut certainement stratégique du point de vue de l'activité commerciale car l'habitat a été construit sur une aire morphologiquement surélevée dans la vallée du Mincio et séparée du paléo-lit du fleuve par une dépression de 200 mètres de large seulement. La localisation de l'habitat semble donc révéler la volonté précise de réaliser un port qui aurait consenti des communications aisées avec les ports maritimes du delta du Pô, comme Adria et Spina, grâce aux voies fluviales du Pô et d'une portion du Mincio.

En s'appuyant sur cette considération, ainsi que sur l'évaluation de l'importante quantité de matériel d'importation découvert, R. de Marinis a émis l'hypothèse que le Forcello était une tête de pont des Étrusques pour les relations commerciales avec les populations celtiques de l'Italie septentrionale et du territoire au-delà des Alpes.

En effet, outre la vaisselle de bronze provenant d'Étrurie et la céramique attique, on est frappé par le nombre d'amphores de transport grecques trouvées sur le site. On explique ce phénomène par le fait que le Forcello devait être le lieu où l'on transvasait le contenu

636. de Marinis, 1982, p. 32 ; 1984, p. 22-33 ; 1987, p. 34-37 ; 1988 , p. $211-229$; 1989, p. $27-47$; 1990 ; 1991a, p. 75-85; 1991b, p. 237-259 ; 1996, p. 330-341; 1999, p. 255-278 ; Sassatelli 1993, p. 208-210 ; de Marinis, Rapi 2005 et 2007.

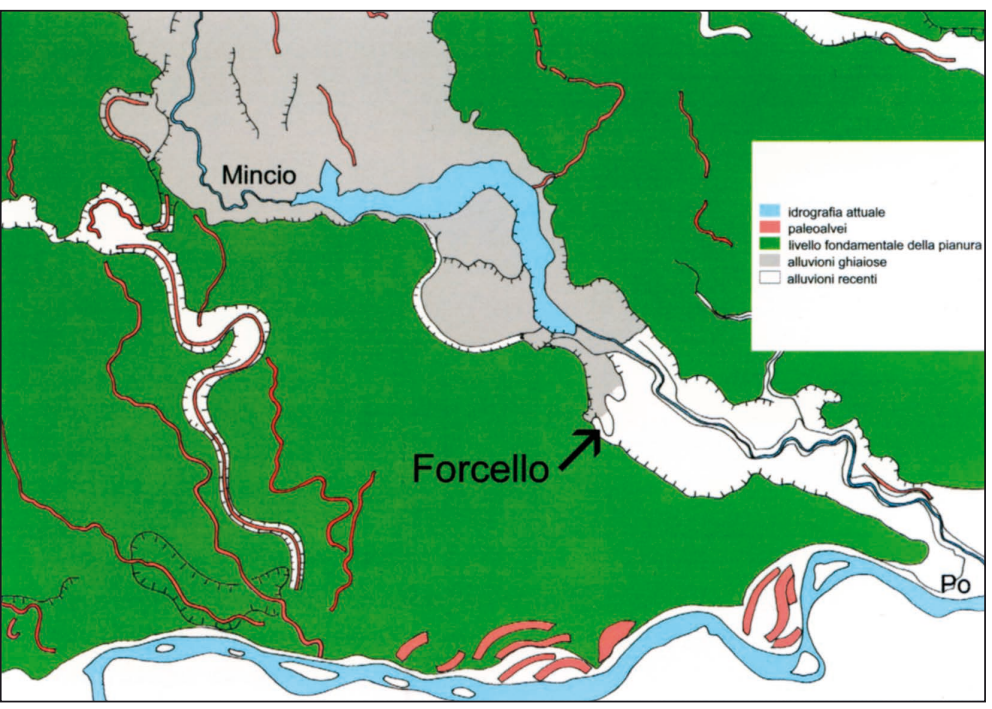

Fig. 83. Carte hydrographique de la zone du Forcello à l'époque protohistorique (d'après de Marinis, Rapi 2007, fig. 3).

des amphores, surtout du vin, dans d'autres récipients, comme les tonneaux en bois ou les outres en peau, considérés plus adaptés au transport par voie de terre. C'est la raison qui permet de supposer que les marchandises quittaient la voie fluviale, juste à la hauteur de cet habitat étrusque, pour être acheminées par une voie terrestre reliant le territoire étrusco-padan aux territoires plus septentrionaux ${ }^{637}$.

Les découvertes amphoriques du Forcello ont été récemment estimées à des milliers de fragments qui se référeraient à un peu plus d'une centaine d'exemplaires (de Marinis 2005, p. 166 ; Id. 2007, p. 159). Le mobilier, étudié par R. de Marinis, a été presqu'entièrement publié et c'est à ces publications, et notamment aux soixantedix exemplaires édités de manière analytique, que l'on fait référence ici.

Les amphores du Forcello, présentées ci-dessous dans le catalogue, sont indiquées sous le sigle «FO ».

637. Concernant cette hypothèse, voir Sassatelli 1993, p. 208-210 et de Marinis 1996, p. 340. 


\section{Catalogue}

\section{AMPHORES DE TYPE CORINTHIEN A/A'}

\section{FO1 (pl. XV)}

Secteur : entre R-S $17-18$ et R 18.

Structure : dépotoirs entre la Maison R 18 et la Maison R-S 17-18.

US : 876 .

Type : corinthienne A.

État de conservation : partie de l'embouchure, col et une anse complète. Dimensions : non repérées. Lèvre à marli oblique, de section trapézoïdale. Col cylindrique. Anses obliques, « a bastoncello », de section elliptique en haut et de section circulaire en bas.

Observations et comparaisons : R. de Marinis attribue le fragment à la production corinthienne de type A et le situe vers le milieu du $\mathrm{V}^{\mathrm{e}} \mathrm{s}$. av. J.-C., après confrontation avec Koehler 1981, fig. 1.d, pl. 98. h. La forme désignée d'une manière précise comme terme de comparaison et datée par C.G. Koehler vers le milieu du Ve s. av. J.-C. provient d'un contexte fermé autour de 420 av. J.-C. (Lawall 2005 , p. 61, et n. 21). La chronologie de ce type est maintenant comprise entre la fin du $\mathrm{VI}^{\mathrm{e}}$ et la fin du Ve $\mathrm{V}$. av. J.-C. (Lawall 1995, p. 60-61, variante A/2, fig. 9-10 ; Sourisseau 2006, type 4C).

Chronologie : vers le milieu du Ve s. av. J.-C. (?).

Bibliographie: de Marinis 2005, p. 169, fig. 89, ${ }^{\circ} 1$; Id. 2007, p. 159, fig. 109, nº 2 .

\section{FO2 (pl. XV)}

Secteur : Secteur R 18 n 5.

US : 2 .

Type : corinthienne A'.

État de conservation : fragment de lèvre avec un bref segment du col. H. max. cons. : 5,5. Lèvre à marli oblique, de section trapézoïdale. Diam. restituable embouchure : 15 .

Surface ext., int. et fracture : rose clair.

Inclusions : argile aux inclusions rouges et blanches.

Observations et comparaisons : attribué par R. de Marinis à la production corinthienne de type A'. Pour le type, datable du courant du $\mathrm{V}^{\mathrm{e}} \mathrm{s}$. av. J.-C., on renvoie à Morter, Leonard 1998, fig. 17.3. Nous en proposons une possible datation vers le milieu ou dans la seconde moitié du $\mathrm{V}^{\mathrm{e}} \mathrm{s}$. car, d'après les données fournies par R. de Marinis, le fragment le plus ancien appartenant à une amphore corinthienne A du Forcello proviendrait d'un contexte du deuxième quart ou du milieu du Ve $\mathrm{s}$. av. J.-C. (de Marinis 2007 , p. 159 , n. 8 - fragment non encore publié du secteur R-S 17, US 517, phase C). Les autres amphores du même type trouvées sur le site seraient toutes datables de la seconde moitié du siècle (de Marinis 2007, p. 159).
Chronologie : milieu ou seconde moitié du V $\mathrm{V}^{\mathrm{e}} \mathrm{s}$. av. J.-C. (?). Bibliographie : de Marinis 1988, $\mathrm{n}^{\circ}$ 267.2, p. 222, fig. 107, $\mathrm{n}^{\circ} 2$; Id. 2005, p. 169, fig. $89, \mathrm{n}^{\circ} 1$.

\section{FO3 (pl. XV)}

Secteur : Secteur R-S 18.

US : Couche arable.

Type : corinthienne A.

État de conservation : fragment de lèvre et court segment de col avec la jonction supérieure d'une anse. H. max. cons. : 7. Lèvre à marli oblique, de section trapézoïdale. Diam. restituable embouchure : 20 .

Surface ext., int. et fracture : jaune rosé clair.

Inclusions : argile aux inclusions blanches et rouges, ces dernières de grandes dimensions.

Observations et comparaisons : attribué par R. de Marinis à la production corinthienne de type A. Pour son possible encadrement chronologique on renvoie aux observations présentées dans la fiche FO2.

Chronologie : milieu ou seconde moitié du Ve s. av. J.-C. (?) Bibliographie : de Marinis 1988, $\mathrm{n}^{\circ}$ 266.3, p. 222, fig. $107, n^{\circ} 3$.

\section{FO4 (pl. XV)}

Secteur : Secteur $18 \mathrm{p} 11$.

US : 91 .

Type : corinthienne A'.

État de conservation : fragment de lèvre avec un court segment du col. H. max. cons. : 4, 5. Lèvre à marli oblique, de section trapézoïdale. Diam. restituable embouchure : 15 .

Surface ext. et int. : rouge orangé.

Fracture : rouge orangé plus intense.

Inclusions : argile aux inclusions rouges et blanches.

Observations et comparaisons : attribué par R. de Marinis à la production corinthienne de type $\mathrm{A}^{\prime}$, ce fragment vient de la phase postérieure à l'abandon, datée par les responsables de la fouille entre la seconde moitié du $\mathrm{V}^{\mathrm{e}}$ et les débuts du IV s. av. J.-C. Pour le type, Morter, Leonard 1998, fig. 17.3 (variantes datables du courant du Ve s.).

Chronologie : 450-400 av. J.-C.

Bibliographie : de Marinis 1988, $\mathrm{n}^{\circ}$ 267.1, p. 222, fig. $107, \mathrm{n}^{\circ} 1$.

\section{FO5 (pl. XV)}

US : 844 .

Type : corinthienne A'.

État de conservation : lèvre et fond attribués au même exemplaire. Dimensions non repérées. Lèvre à marli oblique, de section triangulaire.

Observations et comparaisons : attribués par R. de Marinis à la production corinthienne de type A' et datés de la seconde moitié du $\mathrm{V}^{\mathrm{e}} \mathrm{s}$. av. J.-C. probablement sur une base stratigraphique. 


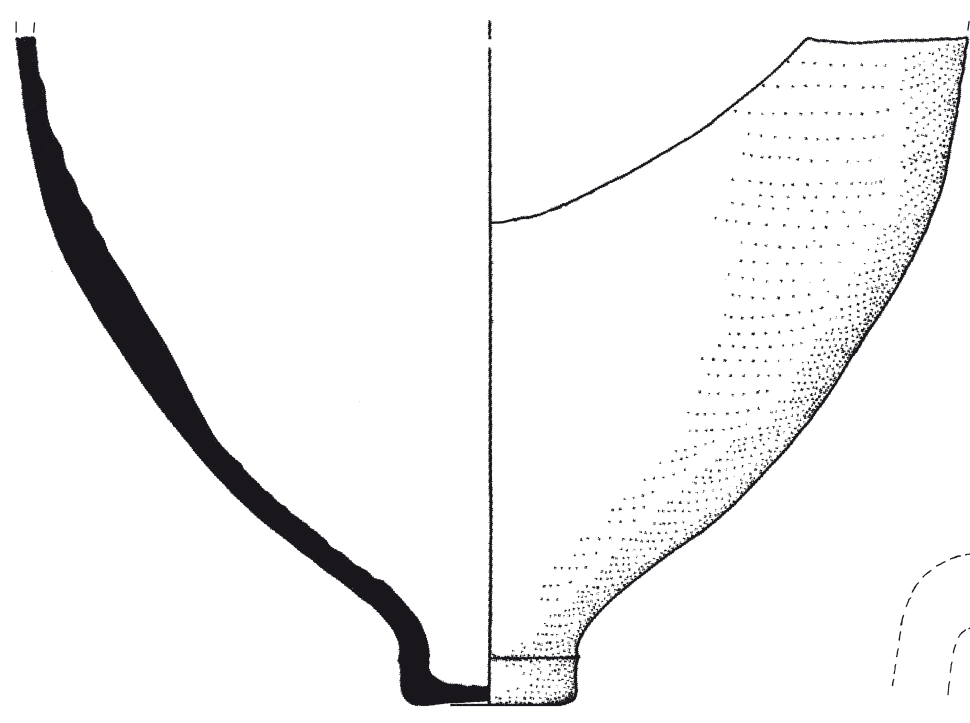

FO1

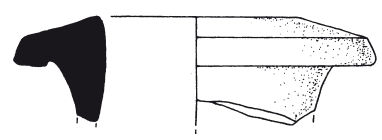

FO4

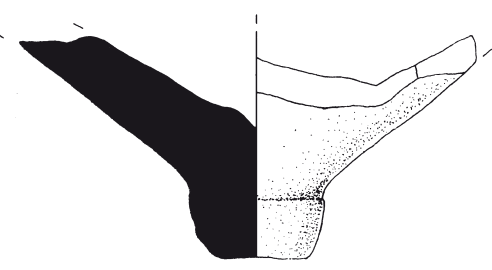

FO6

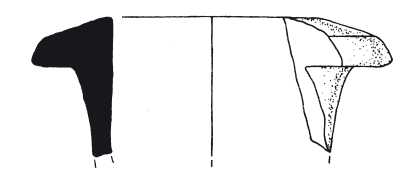

FO2

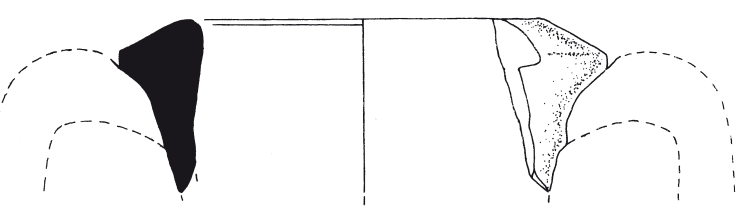

$\mathrm{FO} 3$

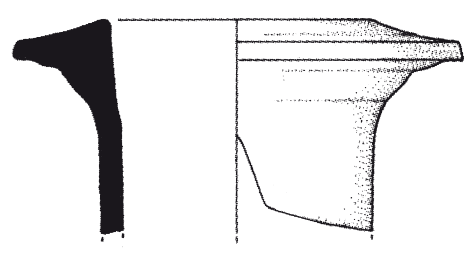

FO5
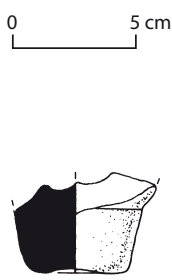

FO7

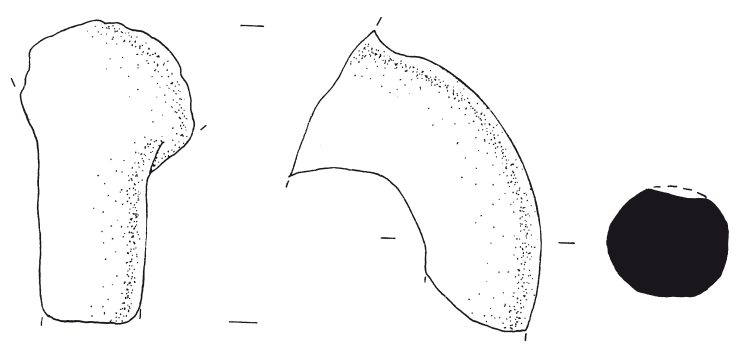

FO8

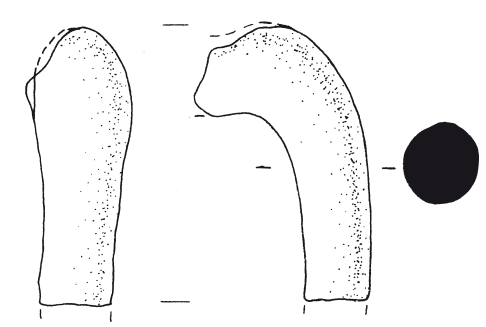

FO9

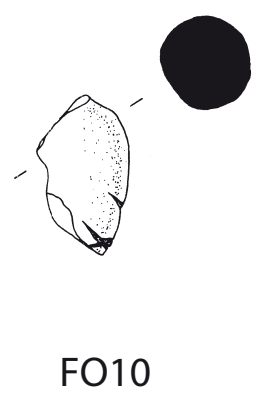

F010 
Chronologie : 450-400 av. J.-C.

Bibliographie : de Marinis 2007, p. 159, fig. 109, $\mathrm{n}^{\circ} 1$.

FO6 (pl. XV)

Secteur : Secteur V 10 r 16.

US : 2 .

Type : corinthienne A'.

État de conservation : pied avec une partie du fond. Dimensions max. fr. : 9 x 18,5. Pied en bouton, au profil caréné, plat en bas.

Surface ext., int. et fracture : rose clair.

Inclusions : argile aux inclusions blanches, grises et rouges, parfois de grandes dimensions.

Observations et comparaisons : attribué par R. de Marinis à la production corinthienne du type A. Pour la forme, Cavalier 1985, fig.4.a, datable de la première moitié du $\mathrm{V}^{\mathrm{e}} \mathrm{s}$. av. J.-C. et vraisemblablement appartenant au type A'. Le matériel livré par les nécropoles de la chora de Métaponte a montré que la morphologie de ce pied est attestée pendant tout le courant du Ve $\mathrm{s}$. av. J.-C. (Morter, Leonard 1998, fig. 17.3). L'exemplaire du Forcello pourrait être daté vers le milieu ou dans la seconde moitié du $\mathrm{V}^{\mathrm{e}} \mathrm{s}$. av. J.-C. d'après les plus récentes observations de R. de Marinis sur les amphores de ce type provenant des contextes de ses fouilles (de Marinis 2007 , p. 159). Pour ces observations on renvoie à la fiche FO2.

Chronologie : 450-400 av. J.-C. (?).

Bibliographie : de Marinis 1988, $\mathrm{n}^{\circ}$ 266. 9, p. 222, fig. $107, n^{\circ} 9$.

\section{FO7 (pl. XV)}

Type : corinthienne A'.

État de conservation : pied fragmentaire. Pied en bouton, à la surface légèrement concave en bas.

Observations et comparaisons : appartenant à une amphore corinthienne A' et génériquement datable sur une base morphologique dans le courant du Ve s. av. J.-C. (cf. Morter, Leonard 1998).

Chronologie : Ve s. av. J.-C.

Bibliographie : de Marinis 1988, fig. 107, nº 10.

\section{FO8 (pl. XV)}

Type : corinthienne A.

État de conservation : fragment d'anse, de la jonction supérieure jusqu'à la courbure. H. max. cons. : 12,5. Anse « a bastoncello », de section circulaire. H. max. cons. : 12,5 .

Surface ext., int. et fracture : jaune pâle rosé.

Inclusions : argile aux inclusions blanches et rouges, ces dernières de grandes dimensions.

Observations et comparaisons : attribuée par R. de Marinis à la production corinthienne de type $\mathrm{A}$.
Chronologie : non déterminable.

Bibliographie : de Marinis 1988, $\mathrm{n}^{\circ}$ 266.5, p. 222, fig. $107, \mathrm{n}^{\circ} 5$.

\section{FO9 (pl. XV)}

Type : corinthienne A'.

État de conservation : anse fragmentaire, privée des attaches supérieure et inférieure. H. max. cons. : 12. Anse « a bastoncello », de section circulaire.

Observations et comparaisons : attribuée par R. de Marinis à la production corinthienne de type A.

Chronologie : non déterminabile.

Bibliographie : de Marinis 1988, fig. 107, nº 6 .

\section{FO10 (pl. XV)}

Secteur : Secteur V 10 r-s 15.

US : 3 .

Type : corinthienne A'.

État de conservation : fragment d'anse, au niveau de la courbure. H. max. cons. : 6,5. Anses « a bastoncello», de section circulaire.

Surface ext. et int. : jaune pâle.

Fracture : rose clair.

Inclusions : argile aux inclusions blanches, rouges et grises de grandes dimensions.

Appareil épigraphique : trait vertical et « signe en forme de croix » (Sacchetti 2011, cat. ${ }^{\circ} 3$ ).

Observations et comparaisons : attribué par R. de Marinis à la production corinthienne de type A' .

Chronologie : non déterminable.

Bibliographie : de Marinis 1988, $\mathrm{n}^{\circ}$ 267.7, p. 223, fig. $107, n^{\circ} 7$; Sacchetti 2011, cat. $n^{\circ} 3$.

\section{AMPHORES GRÉCO-OCCIDENTALES}

\section{FO11 (pl. XVI)}

Secteur : R 18.

Structure : colmatage de défoncement de vigne.

US : 9/164.

Type : corinthienne B.

État de conservation : partie de lèvre et col avec une anse complète. Dimensions : non repérées. Lèvre en échine, légèrement ouverte. Col cylindrique, avec deux cannelures au-dessous de la lèvre. Anses verticales, en ruban épaissi, à section elliptique.

Observations et comparaisons : attribuée par R. de Marinis à la production corinthienne type $\mathrm{B}$ et confrontée avec l'exemplaire complet de la collection Bocchi d'Adria (cat. AD3) daté entre la fin du VI e et les débuts du Ve s. av. J.-C. Pour le type, Koehler 1978a, fig. 2.b, et Ead. 1981, fig. 1.a, pl. 99.c. La chronologie de cette forme, habituellement fixée au deuxième ou au troisième quart du Ve s. av. J.-C., a été récemment rehaussée sur la 
base du matériel trouvé à Olympie et à Athènes dans des contextes datables, d'après la stratigraphie, entre la fin du VI ${ }^{\mathrm{e}}$ et le début du Ve s. av. J.-C. (Gauer 1975, pl. 7, $\mathrm{n}^{\circ}$ 5, 8 ; Roberts 1986, p. 64-67, n 416-417, fig. 41, pl. 18 ; Lawall 1995, p. 72).

Chronologie : 525-480 av. J.-C.

Bibliographie: de Marinis 2005, p. 171, fig. 89, $\mathrm{n}^{\circ} 2$; Id. 2007, p. 159, fig. $109, \mathrm{n}^{\circ} 5$.

\section{FO12}

Secteur : Secteur V 11.

Type : corinthienne B.

État de conservation : partie de lèvre et col. Dimensions : non repérées. Lèvre en échine.

Observations et comparaisons : fragment attribué par $\mathrm{R}$. de Marinis à la production corinthienne de type $\mathrm{B}$ et daté des débuts du Ve s. av. J.-C. Pour le type on revoie à la fiche précédente. Considéré comme appartenant au même exemplaire de la fiche suivante.

Chronologie : début $\mathrm{V}^{\mathrm{e}} \mathrm{s}$. av. J.-C.

Bibliographie : de Marinis 1987, fig. 24 (photo); Id. 1996, p. 332-333.

\section{FO13}

Structure : défoncement de vigne.

Phase E.

Type : corinthienne B.

État de conservation : fragment de lèvre et col avec une anse complète. Dimensions : non repérées. Lèvre en échine. Anses en ruban épaissi, à section elliptique.

Observations et comparaisons : associé par R. de Marinis à l'exemplaire de la fiche précédente.

Chronologie : début Ve s. av. J.-C.

Bibliographie : de Marinis 1987, fig. 24 (photo) ; Id. 1996, p. 333.

\section{FO14}

Structure : foyer.

US : es 304.

Type : corinthienne B.

État de conservation : lèvre, col, une anse, épaulement et partie de la panse. Dimensions max. cons. : 27. Lèvre en échine. Col cylindrique au profil légèrement concave, avec un listel au-dessous de la lèvre. Anses verticales, en ruban épaissi, à section elliptique.

Surface : jaune clair.

Inclusions : fines inclusions brunes, noires et blanches.

Engobe : engobe rose pâle.

Observations et comparaisons : attribuée par R. de Marinis à la production corinthienne B, datée de 490-480 av. J.-C. et considérée par le même de Marinis comme une forme de transition de la série initiale archaïque tardive à la série de pleine époque classique de la typologie de C.G. Koehler. Aucun dessin n'ayant été publié, il est impossible d'en proposer des confrontations.

Chronologie : 490-480 av. J.-C.

Bibliographie : de Marinis 2005, p. 171-172 ; Id. 2007, p. 162-163.

\section{FO15 (pl. XVI)}

Secteur : Secteur R 18.

US : 2 .

Type : corinthienne B.

État de conservation : fragment de lèvre avec un court segment du col. H. max. cons. : 3, 5. Lèvre en échine, légèrement ouverte. Col vraisemblablement évasé, avec une cannelure ou un fin ressaut immédiatement au-dessous de la lèvre.

Surface ext., int. et fracture : jaune clair avec des nuances virant au vert.

Observations et comparaisons : attribué par R. de Marinis à la production corinthienne de type $\mathrm{B}$. Pour le type, Koehler 1978a, fig. 2.b-c, et Ead. 1981, fig. 1.a, pl. 99.c. Ce type de lèvre est attesté pendant tout le $\mathrm{V}^{\mathrm{e}} \mathrm{s}$. av. J.-C., mais ici l'absence des anses ne permet pas de circonscrire ultérieurement la chronologie.

Chronologie : Ve s. av. J.-C.

Bibliographie : de Marinis 1988, $\mathrm{n}^{\circ}$ 268.1, p. 223, fig. $109, \mathrm{n}^{\circ} 1$.

FO16

Secteur : R-S 17-18.

Structure : décharge de matériel.

US : 399 .

Type : corinthienne B.

État de conservation : fond, partie de la panse et de l'épaulement, col et une anse. Dimensions : non repérées. Observations et comparaisons : exemplaire attribué par $\mathrm{R}$. de Marinis à la production corinthienne B du milieu du Ve s. av. J.-C.

Chronologie : vers 450 av. J.-C.

Bibliographie : de Marinis 2005, p. 172 ; Id. 2007, p. 163.

\section{FO17}

Secteur : R 18 et R-S 17-18.

Structure : décharges entre les Maisons R 18 et R-S 17-18.

US : 876 .

Type : corinthienne B.

État de conservation : ample fragment du fond et de la panse. Dimensions : non repérées.

Observations et comparaisons : attribué par R. de Marinis à la production corinthienne $\mathrm{B}$ du milieu du $\mathrm{V}^{\mathrm{e}} \mathrm{s}$. av. J.-C. et confronté avec Koehler 1978a, fig. 2.b et Ead.1981, pl. 99.c.

Chronologie : vers 450 av. J.-C. 


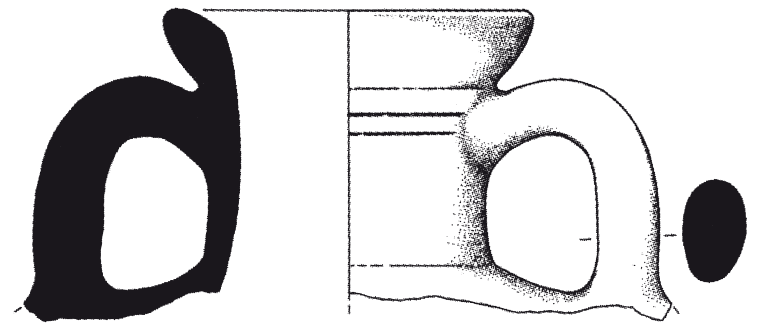

FO 11

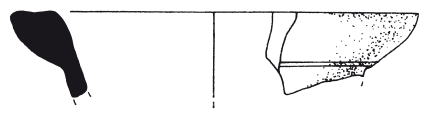

F015
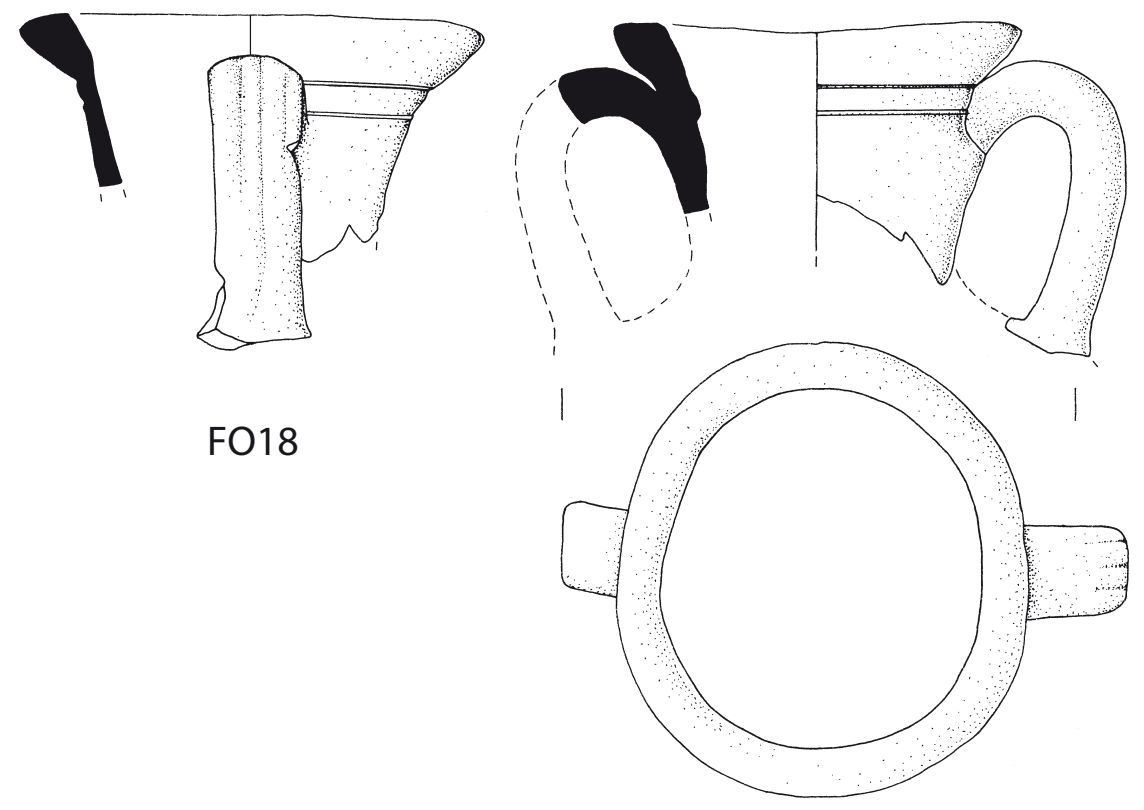

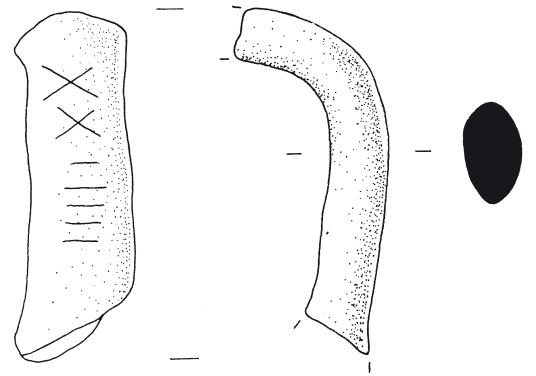

FO19

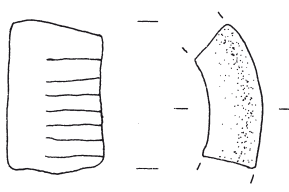

FO20 
Bibliographie : de Marinis 2005, p. 172, n. 22 ; Id. 2007, p. 163, n. 24.

\section{FO18 (pl. XVI)}

Secteur : Secteur V 10.

US : 2 .

Type : corinthienne B.

État de conservation : lèvre, partie du col, une anse complète et partie du segment supérieur de l'autre anse. H. max. cons. : 14. Lèvre en échine, ouverte. Diam. embouchure : 16,6 / 18,5. Col évasé, avec deux rainures parallèles et rapprochées immédiatement au-dessous de la lèvre. Anses en ruban épaissi, à section elliptique.

Surface ext. et int. : rose clair.

Fracture : rose intense.

Observations et comparaisons : attribuée par R. De Marinis à la production corinthienne $\mathrm{B}$ de la fin du $\mathrm{V}^{\mathrm{e}}$ ou des débuts du $\mathrm{IV}^{\mathrm{e}} \mathrm{s}$. av. J.-C. après confrontation avec Koehler 1978a, fig. 2.c., datable du dernier quart du Ve s. av. J.-C.

Chronologie : 425-400 av. J.-C.

Bibliographie : de Marinis 1988, $\mathrm{n}^{\circ}$ 268.2, p. 223, fig. 109 , n ${ }^{\circ} 2$; Id. 2005, p. 172, fig. 90 ; Id. 2007, p. 163 164, fig. 110 .

\section{FO19 (pl. XVI)}

Secteur : Secteur R 18.

US : 2 .

Type : corinthienne B.

État de conservation : anse fragmentaire, manquant des jonctions supérieure et inférieure. H. max. cons. : 14,2. Anses en ruban épaissi, à section elliptique.

Surface ext., int. et fracture : brun rosé.

Engobe : engobe jaune clair.

Appareil épigraphique : deux "signes en forme de croix » et cinq traits parallèles (Sacchetti 2011, cat. $n^{\circ} 4$ ). Observations et comparaisons : attribuée par R. de Marinis à la production corinthienne de type B.

Chronologie : non déterminable.

Bibliographie : de Marinis 1988, $\mathrm{n}^{\circ}$ 268.3, p. 223, fig. $109, n^{\circ} 3$; Sacchetti 2011 , cat. $n^{\circ} 4$.

\section{FO20 (pl. XVI)}

Secteur : Secteur R 18 o 5.

US : 2 .

Type : corinthienne B.

État de conservation : fragment d'anse au niveau de la courbure. H. max. cons. : 6,2. Anses en ruban épaissi, à section elliptique.

Surface ext., int. et fracture : brun rosé.

Engobe : engobe jaune clair.

Appareil épigraphique : sept traits parallèles (Sacchetti 2011, $\mathrm{n}^{\circ}$ 5).
Observations et comparaisons : attribuée par R. de Marinis à la production corinthienne de type $\mathrm{B}$.

Chronologie : non déterminable.

Bibliographie : de Marinis 1988, $\mathrm{n}^{\circ}$ 268.4, p. 223, fig. $109, \mathrm{n}^{\circ} 4$; Sacchetti $2011, \mathrm{n}^{\circ} 5$.

$\mathrm{FO} 21$

Secteur: R 18 f 17, RR 649.

US : 915 .

Type : corinthienne B.

État de conservation : partie de col et épaulement. Dimensions : non repérées.

Surface ext., int. et fracture : jaune.

Inclusions : pâte à la matrice très fine et avec peu d'inclusions.

Observations et comparaisons : attribuée par R. de Marinis à la production corinthienne B.

Chronologie : non déterminable.

Bibliographie : de Marinis 2005, p. 172 ; Id. 2007, p. 163.

\section{FO22 (pl. XVII)}

Secteur: R 18 i 15.

Structure : remplissage d'une fosse des phases H-I.

US : 1578 .

Type : « forme $1 \alpha »$.

État de conservation : partie inférieure d'une amphore, du fond jusqu'au milieu de la panse. Dimensions : non repérées. Fond cylindrique, au profil légèrement mouluré au niveau du plan d'appui.

Surface : beige rosé.

Inclusions : de nombreuses inclusions de quartz et de chert. Faible présence de mica.

Engobe : des traces d'engobe rougeâtre sur la surface extérieure.

Observations et comparaisons : attribuée par R. de Marinis à la production corinthienne $\mathrm{B}$ du début de la série (Koehler 1978a, fig. 2.a ; Ead. 1981, pl. 99.a) et datée vers 530-520 av. J.-C. sur une base stratigraphique. Chronologie : 530-520 av. J.-C.

Bibliographie : de Marinis 2005, p. 170-171, fig. 89, $\mathrm{n}^{\circ} 4$; Id. 2007, p. 160-162, fig. 109, $\mathrm{n}^{\circ} 3$.

\section{FO23 (pl. XVII)}

Secteur: R 18 i 15.

Structure : Maison F I.

US : 476 .

Type : « forme $1 \alpha »$.

État de conservation : entière, reconstituée à partir de fragments et restaurée. Dimensions : non repérées. Lèvre en bourrelet gonflé, ouverte. Col évasé, avec un listel audessous de la lèvre. Anses verticales, en ruban épaissi, de section elliptique. Épaule aplatie. Corps piriforme. 


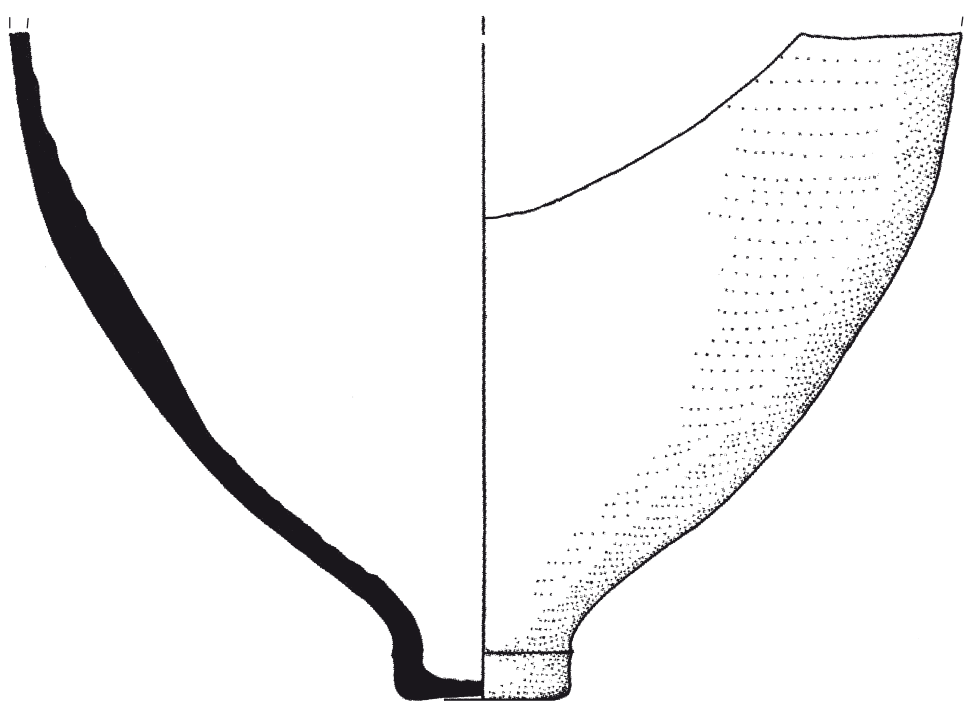

$\mathrm{FO} 22$

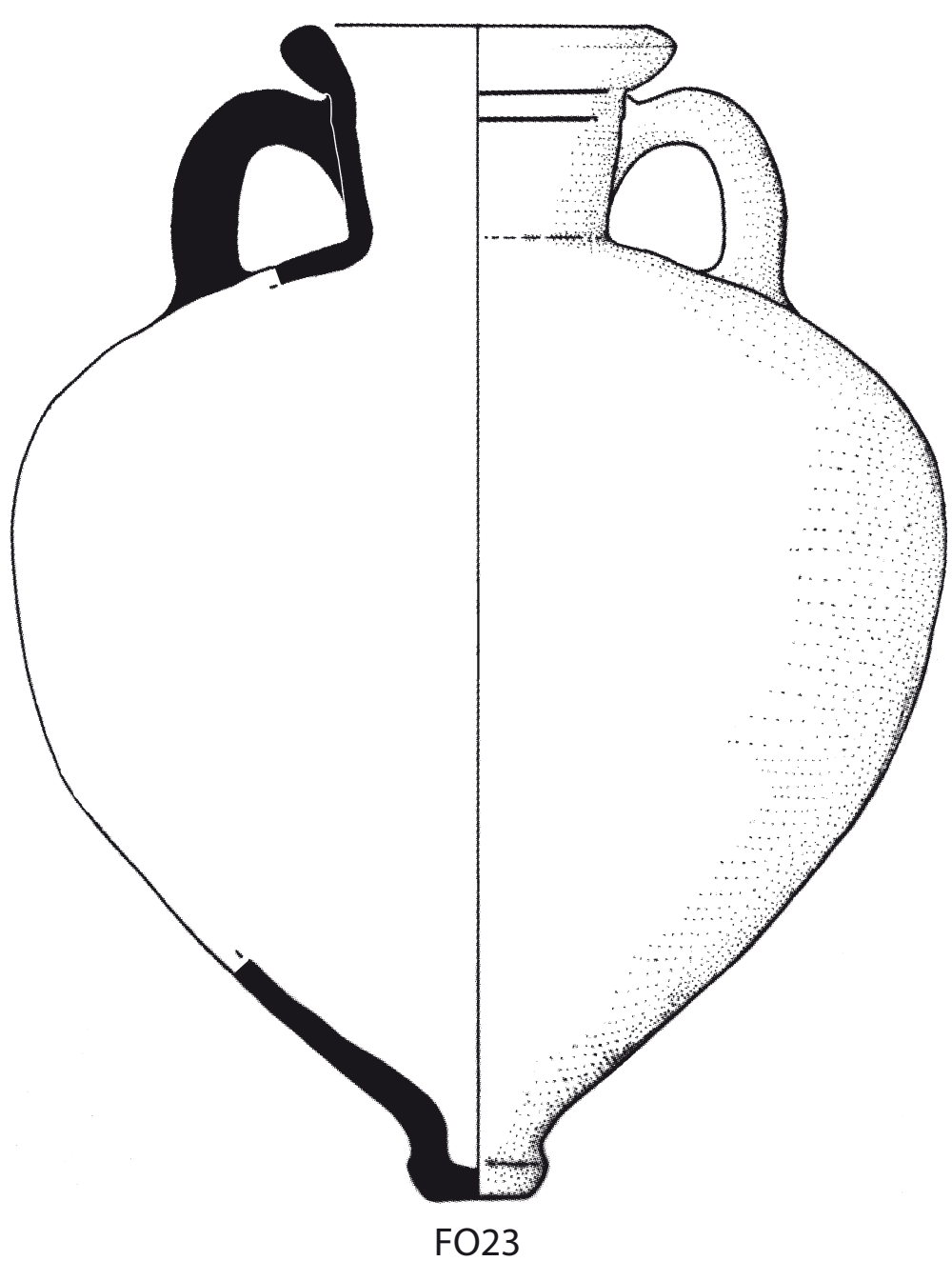

PI. XVII. Forcello. 


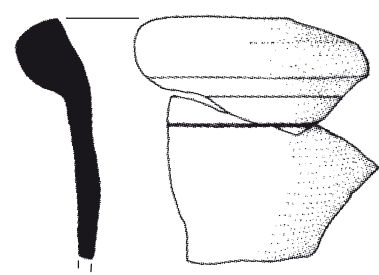

FO24

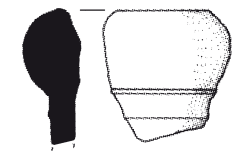

FO25
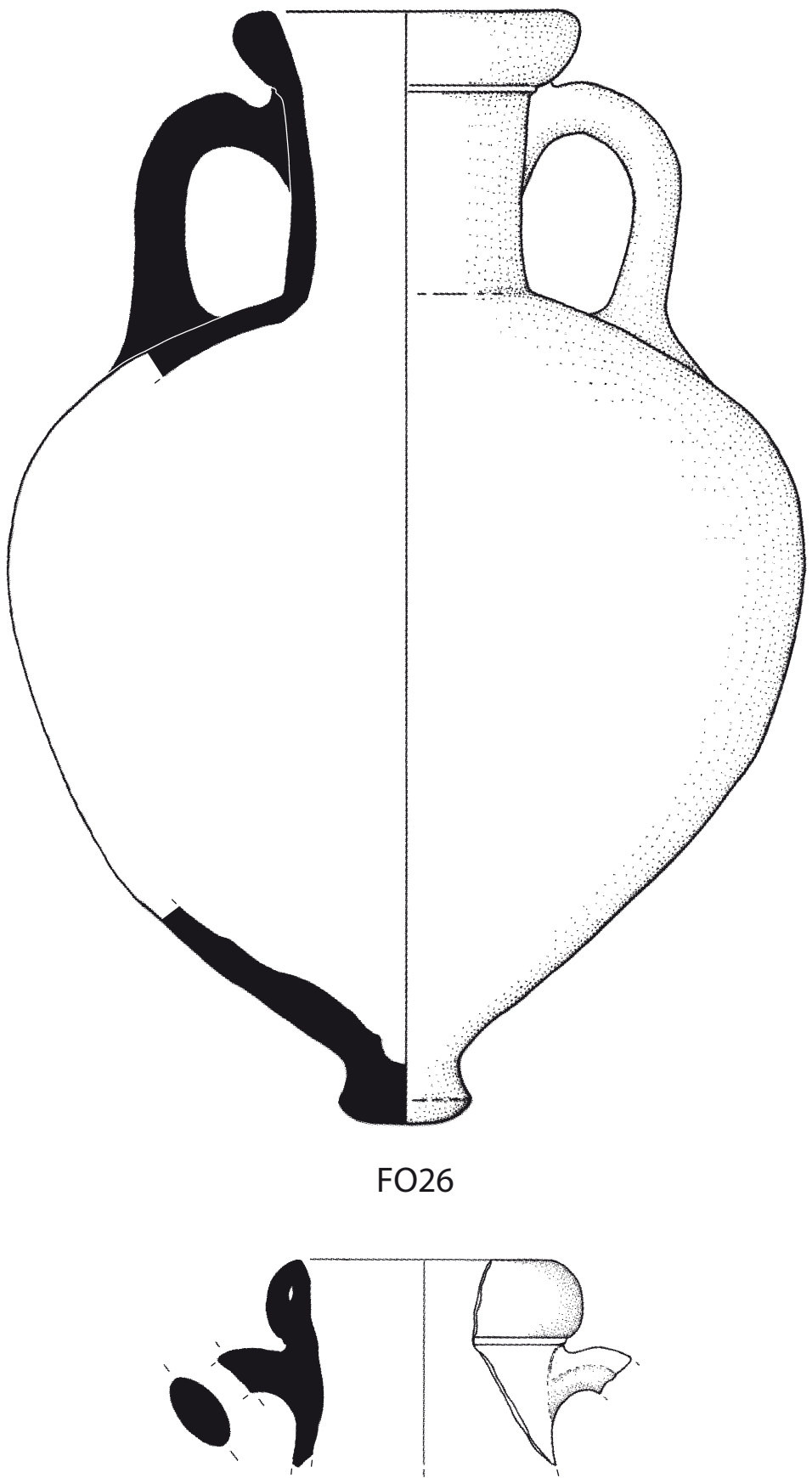

FO27

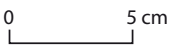

PI. XVIII. Forcello. 


$$
\begin{aligned}
& 13 \\
& 2 \\
& 2 \\
& 0
\end{aligned}
$$


Fond cylindrique, au profil mouluré au niveau du plan d'appui.

Surface : marron.

Inclusions : matrice argileuse fine avec d'abondantes inclusions de quartz microcristallin, quelques fragments rocheux constitués de quartz et de mica, et de rares inclusions de dimensions moyennes-grandes constituées de granules de silex et de quartz microcristallin.

Observations et comparaisons : attribuée par R. de Marinis à la production corinthienne de la fin du $\mathrm{VI}^{\mathrm{e}} \mathrm{s}$. av. J.-C. après confrontation avec un exemplaire de Corinthe (Koehler 1978a, fig. 2.a ; Ead. 1981, pl. 99.a). La chronologie proposée pour l'amphore en question sur la base de ce parallèle est ultérieurement renforcée par les données stratigraphiques des fouilles du Forcello (elle provient de la maison F I, détruite et scellée par un incendie à la fin du $\mathrm{V}^{\mathrm{e}} \mathrm{s}$. av. J.-C.).

Chronologie : 520-500 av. J.-C.

Bibliographie : de Marinis 2005, p. 171, fig. 89, n 5 ; Id. 2007, p. 162, fig. $109, \mathrm{n}^{\circ} 4$.

\section{FO24 (pl. XVIII)}

Secteur: V 11.

US : 5 .

Type : « forme $1 \alpha »$.

État de conservation : partie de lèvre et col. Dimensions : non repérées. Lèvre en bourrelet gonflé, légèrement ouverte. Col cylindrique, avec un listel au-dessous de la lèvre.

Observations et comparaisons : attribué par R. de Marinis à la production corinthienne de type $\mathrm{B}$ de la fin du $\mathrm{VI}^{\mathrm{e}} \mathrm{s}$. av. J.-C. sur la base de la comparaison avec l'exemplaire entier de la fiche précédente.

Chronologie : 520-500 av. J.-C.

Bibliographie : de Marinis 2005, p. 171, fig. 89, $\mathrm{n}^{\circ} 3$; Id. 2007, p. 162, fig. $109, \mathrm{n}^{\circ} 6$.

\section{FO25 (pl. XVIII)}

Secteur : R 18 u 12-13 - S 18 a 12.

US : 1378 .

Type : « forme 2 ».

État de conservation : fragment de lèvre avec un court segment du col. Dimensions : non repérées. Lèvre en bourrelet gonflé avec un fin listel à la base. Col qui présente un fin listel au-dessous de la lèvre.

Inclusions : inclusions blanches et grises parfois de grandes dimensions, et inclusions marron foncé et rouges. Observations et comparaisons : définie par R. de Marinis « de type ionio-massaliète » et datée d'après la stratigraphie entre 540 et 520 av. J.-C.

Chronologie : 540-520 av. J.-C.

Bibliographie: de Marinis 2005, p. 175, fig. 91, ${ }^{\circ} 3$; Id. 2007, p. 164-165, fig. $111, \mathrm{n}^{\circ} 3$.

\section{FO26 (pl. XVIII)}

Secteur: R 18 i 15 et k 15.

Structure : Maison F I - pièce 9.

US : 467.

Type : « forme 2 ».

État de conservation : entière, reconstituée à partir de quatre cents fragments environ et restaurée. Dimensions : non repérées. Lèvre en bourrelet gonflé, légèrement ouverte, avec un fin listel à la base. Col cylindrique au profil légèrement concave. Anses verticales, en ruban épaissi, à section elliptique. Épaule plutôt aplatie. Corps piriforme. Pied en bobine, bref, à base convexe.

Inclusions : beaucoup d'inclusions de petites dimensions de couleur sombre et inclusions rougeâtres de grandes dimensions. Mica argenté à fréquence haute.

Observations et comparaisons : attribuée par R. de Marinis au groupe « ionio-massaliète » et confrontée avec un exemplaire de l'épave de la Pointe du Dattier (Pomey, Long 1992, p. 191, fig. 3, à droite), daté autour de 525 av. J.-C., avec quelques amphores de la nécropole du lieu-dit Diana à Lipari (Cavalier 1985, fig. 5.a, de la Tombe 358 ; fig. 6.a, inv. 15034, fig. 6.b, de la Tombe 360 ) et enfin avec une amphore de la Tombe 3193 de Pontecagnano (Di Sandro 1981b, fig. 1-2, 19.8). Datée de la fin du VIe s. av. J.-C. sur la base de la stratigraphie.

Chronologie : 525-500 av. J.-C.

Bibliographie : de Marinis 2005, p. 173-174, fig. 91, $\mathrm{n}^{\circ} 1 ;$ Id. 2007, p. 164, fig. $111, \mathrm{n}^{\circ} 1$.

\section{FO27 (pl. XVIII)}

Secteur: Secteur S 18 o 12.

US : 256 .

Type : «forme 2 » ou « forme 3 ».

État de conservation : fragment de lèvre et col avec une partie du segment supérieur d'une anse. Dimensions : non repérées. Lèvre en bourrelet gonflé avec un fin listel à la base et une petite fente en forme de goutte visible en section. Col tronconique. Anses en ruban épaissi, à section elliptique.

Surface : rose clair.

Inclusions : inclusions blanches et rougeâtres. Pâte sableuse riche en mica argenté et doré.

Observations et comparaisons : attribuée par R. de Marinis au type «ionio-massaliète » et datée d'après la stratigraphie du deuxième quart du $\mathrm{V}^{\mathrm{e}} \mathrm{s}$. av. J.-C. Étant donné la chronologie déterminée sur une base stratigraphique, il pourrait s'agir d'une « forme 3 » de Sourisseau 2011, dont la lèvre présente les même caractéristiques des lèvres des amphores de « forme 2 »

Chronologie : 475-450 av. J.-C.

Bibliographie : de Marinis 1987, fig. 24 ; Id. 1996, p. 333 , fig. 6 ; Id. 2005, p. 175, fig. 91 , n 2 ; Id. 2007 , p. 164-165, fig. $111, \mathrm{n}^{\circ} 2$. 


\section{AMPHORES DE L'ÉGÉE SEPTENTRIONALE}

\section{FO28 (pl. XIX)}

Secteur : Secteur S 17 n-o 9-10.

US : 62 .

Type : Early Mendean Variant Lawall.

État de conservation : un fragment de lèvre avec un court segment de col et la jonction supérieure d'une anse. H. max. cons. : 7,5. Lèvre en coin, légèrement épaissie à l'extérieur, soulignée par un fin listel de base. Diam. restituable embouchure : 12,7. Col tronconique, au profil légèrement concave, souligné par une cannelure au-dessous de la lèvre et une fine rayure en dessous.

Surface : rose.

Inclusions : argile avec inclusions micacées.

Observations et comparaisons : R. de Marinis attribue l'exemplaire à la production de Mendé. Pour le type, Lawall 1995, fig. 37 ; pour la forme, Schmid 2001, n 4 , pl. 4 , e $\mathrm{n}^{\circ} 5$, pl. 5 , toutes les deux datables entre la fin du $\mathrm{VI}^{\mathrm{e}}$ et les débuts du Ve s. av. J.-C. ; et Monachov 1999b, fig. 17.1, attribuée à Thasos et datée de 525-475 av. J.-C. Chronologie : 525-480 av. J.-C.

Bibliographie : de Marinis 1988, $\mathrm{n}^{\circ}$ 274.5, p. 224, fig. $111, \mathrm{n}^{\circ} 5$.

\section{FO29 (pl. XIX)}

Secteur : Secteur R 18.

US : 2 .

Type : Middle Mendean Variant Lawall.

État de conservation : partie de lèvre et col, conservé jusqu'à la jonction de l'épaule, avec la partie supérieure d'une anse. Recomposée à partir de deux fragments. H. max. cons. : 11,7. Lèvre en coin, amincie et ouverte, avec une fine cannelure de base. Diam. restituable embouchure : 12. Col tronconique, au profil concave, avec une cannelure au-dessous de la lèvre et une fine rainure en dessous. Anses en ruban épaissi, de section elliptique.

Surface : brun rosé.

Inclusions : argile avec des inclusions blanches fines, de la chamotte et d'abondant mica.

Observations et comparaisons : attribuée par R. de Marinis à la production de Mendé. Les proportions du col suggèrent un possible encadrement chronologique dans le troisième quart du $\mathrm{V}^{\mathrm{e}} \mathrm{s}$. av. J.-C. Pour le type (Middle Mendean Variant), Lawall 1995, fig. 38.

Chronologie : 450-425 av. J.-C.

Bibliographie : de Marinis 1988, $\mathrm{n}^{\circ}$ 274.3, p. 224, fig. $111, \mathrm{n}^{\circ} 3$.

\section{FO30}

Secteur : R 18.

Structure : dépotoirs derrière la Maison R 18.
État de conservation : partie de pied. Dimensions : non repérées. Pied en bobine.

Observations et comparaisons : attribuée par R. de Marinis à la production de Mendé et datée du troisième ou du dernier quart du Ve s. av. J.-C. après confrontation avec des exemplaires des puits R 13 :4, H $13: 4$ et R 11 :3 de 1'Agora d'Athènes (Papadopoulos, Paspalas 1999, fig. 2-4). Le dessin n'est pas publié.

Chronologie : 450-400 av. J.-C.

Bibliographie : de Marinis 2005, p. 180-181 ; Id. 2007, p. 171-172.

\section{FO31 (pl. XIX)}

Secteur : Secteur V 10 u 17.

US : 2 .

Type : Late Mendean Variant Lawall.

État de conservation : partie de lèvre et col avec la jonction supérieure d'une anse, ainsi que deux autres fragments reconstituables du col. H. max. cons. : 20,3. Lèvre en coin, amincie et ouverte. Diam. restituable embouchure : 13. Col tronconique.

Surface ext., int. et fracture : rouge orangé.

Inclusions : argile avec de petites inclusions blanches et un abondant mica.

Engobe : traces d'engobe jaune clair.

Observations et comparaisons : attribuée par R. de Marinis à la production de Mendé. La hauteur du col et la forme aplatie de l'épaule permettent maintenant de la situer précisément dans le cadre de la production de Mendé de la fin du Ve s. av. J.-C. Pour le type, Lawall 1995, fig. 39-40 ; pour la forme, Brašinskij 1976, fig. 3. Chronologie : 425-400 av. J.-C.

Bibliographie : de Marinis 1988, $\mathrm{n}^{\circ}$ 274.1, p. 224, fig. $111, \mathrm{n}^{\circ} 1$.

\section{FO32 (pl. XIX)}

Secteur: R-S 17-18.

Structure : aire derrière les Maisons R-S 17-18.

US : 470 .

Type : Late Mendean Variant Lawall.

État de conservation : pied et partie du fond de la panse. Dimensions : non repérées. Pied en bobine, ayant la surface d'appui convexe et une profonde dépression centrale de base.

Observations et comparaisons : exemplaire attribué par R. de Marinis à la production de Mendé et daté du troisième ou du dernier quart du $\mathrm{V}^{\mathrm{e}} \mathrm{s}$. av. J.-C. après confrontation avec des exemplaires des puits $\mathrm{R} 13: 4$, H 13 :4 et R $11: 3$ de l'Agora d'Athènes (Papadopoulos, Paspalas 1999, fig. 2-4). La hauteur du pied en bobine, d'une taille moyenne, pourrait suggérer de le rapprocher plus exactement de la production de Mendé du dernier 

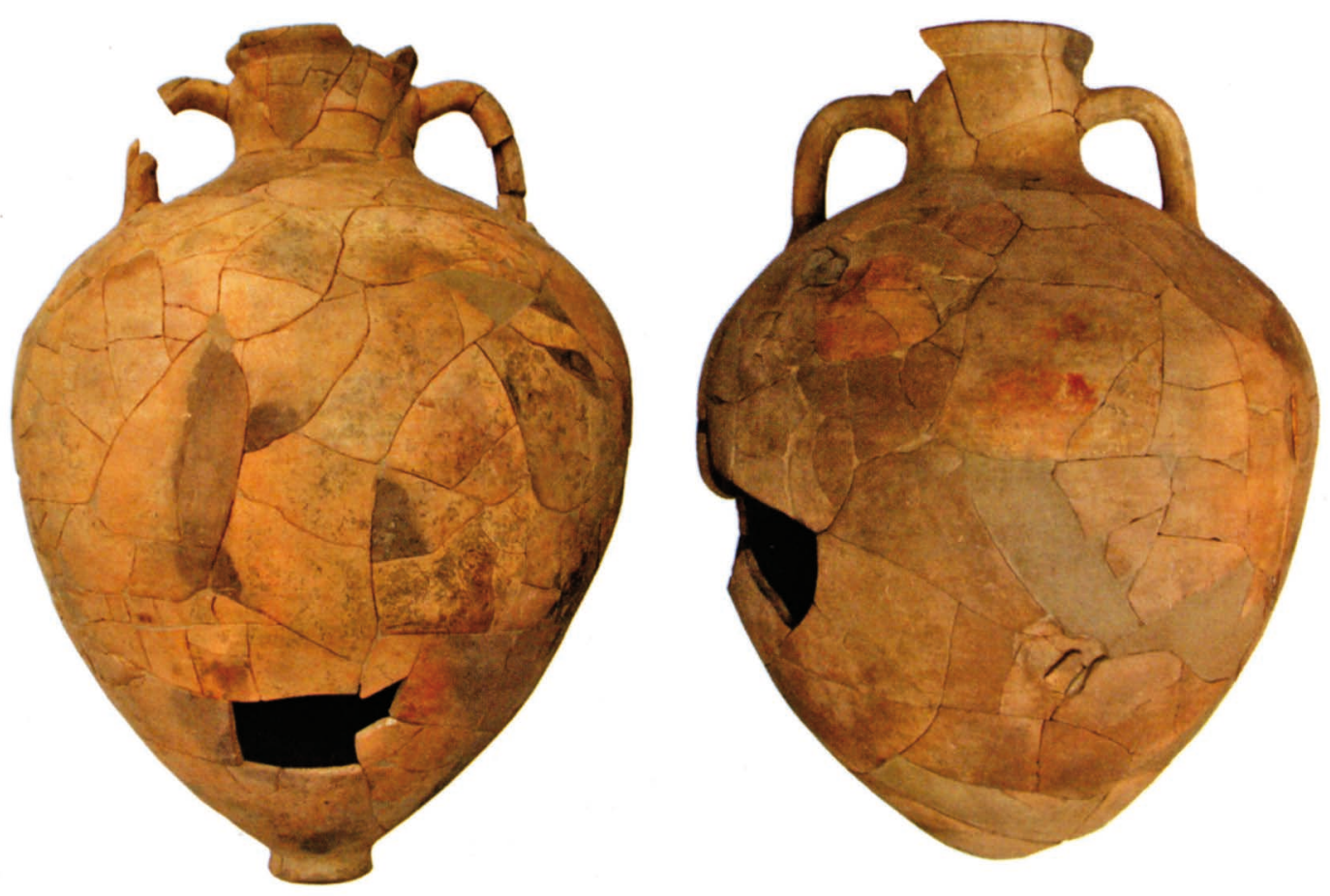

Fig. 84. Bagnolo San Vito, lieu-dit Forcello, amphores nord-égéennes. Cat. FO39-FO40 (d'après de Marinis 2007, fig. 113-114).

quart du Vé s. av. J.-C. Pour le type, Lawall 1995, fig. 39 ; et Brašinskij 1976, fig. 3.

Chronologie : 425-400 av. J.-C.

Bibliographie: de Marinis 2005, p. 180, fig. 95, $\mathrm{n}^{\circ} 1$; Id. 2007, p. 171-172, fig. $118, \mathrm{n}^{\circ} 1$.

\section{FO33 (pl. XIX)}

Secteur : R-S 18.

US : couche arable.

Type : Late Mendean Variant Lawall.

État de conservation : partie de pied. Dimensions : non repérées. Pied en bobine, à base convexe et ombiliquée. Observations et comparaisons : fragment attribué par $\mathrm{R}$. de Marinis à la production de Mendé et daté du troisième ou du dernier quart du $\mathrm{V}^{\mathrm{e}} \mathrm{s}$. av. J.-C., vu le rapprochement avec des exemplaires des puits $\mathrm{R} 13: 4$, H 13 :4 et R 11 :3 de l'Agora d'Athènes (Papadopoulos, Paspalas 1999, fig. 2-4). Les proportions du pied suggèrent de l'attribuer plus exactement à la production de Mendé du dernier quart du $\mathrm{V}^{\mathrm{e}} \mathrm{s}$. av. J.-C. Pour le type, Lawall 1995, fig. 39.

Chronologie : 425-400 av. J.-C.

Bibliographie : de Marinis 2005, p. 180-181, fig. 95, $\mathrm{n}^{\circ} 2 ;$ Id. 2007, p. 171-172, fig. $118, \mathrm{n}^{\circ} 2$.

\section{FO34 (pl. XIX)}

Secteur : Secteur R 18 h 1-2.

US : 109 .
État de conservation : fragment de lèvre et col avec la partie supérieure d'une anse. H. max. cons. : 8. Lèvre en coin, amincie et légèrement ouverte. Diam. restituable embouchure : 12,8. Anses en ruban épaissi, à section elliptique. Surface ext. et fracture : brun.

Surface int. : rouge.

Inclusions : inclusions blanches fines et chamotte.

Engobe : engobe jaune clair.

Observations et comparaisons : attribué par R. de Marinis à la production de Mendé. La morphologie de la lèvre, du type en coin, ne permet qu'un encadrement générique dans la production nord-égéenne du $\mathrm{V}^{\mathrm{e}} \mathrm{s}$. av. J.-C. (Schmid 2001).

Chronologie : non déterminable.

Bibliographie : de Marinis 1988, $\mathrm{n}^{\circ}$ 274.2, p. 224, fig. $111, \mathrm{n}^{\circ} 2$.

\section{FO35 (pl. XIX)}

Secteur : Secteur R 18 r 17.

US : 2 .

État de conservation : fragment de col avec le segment supérieur d'une anse. H. max. cons. : 10,6. Col vraisemblablement tronconique. Anses en ruban épaissi, à section elliptique.

Surface ext. et int. : orange.

Fracture : rouge vif.

Inclusions : argile avec de rares inclusions blanches, de la chamotte et un abondant mica. 


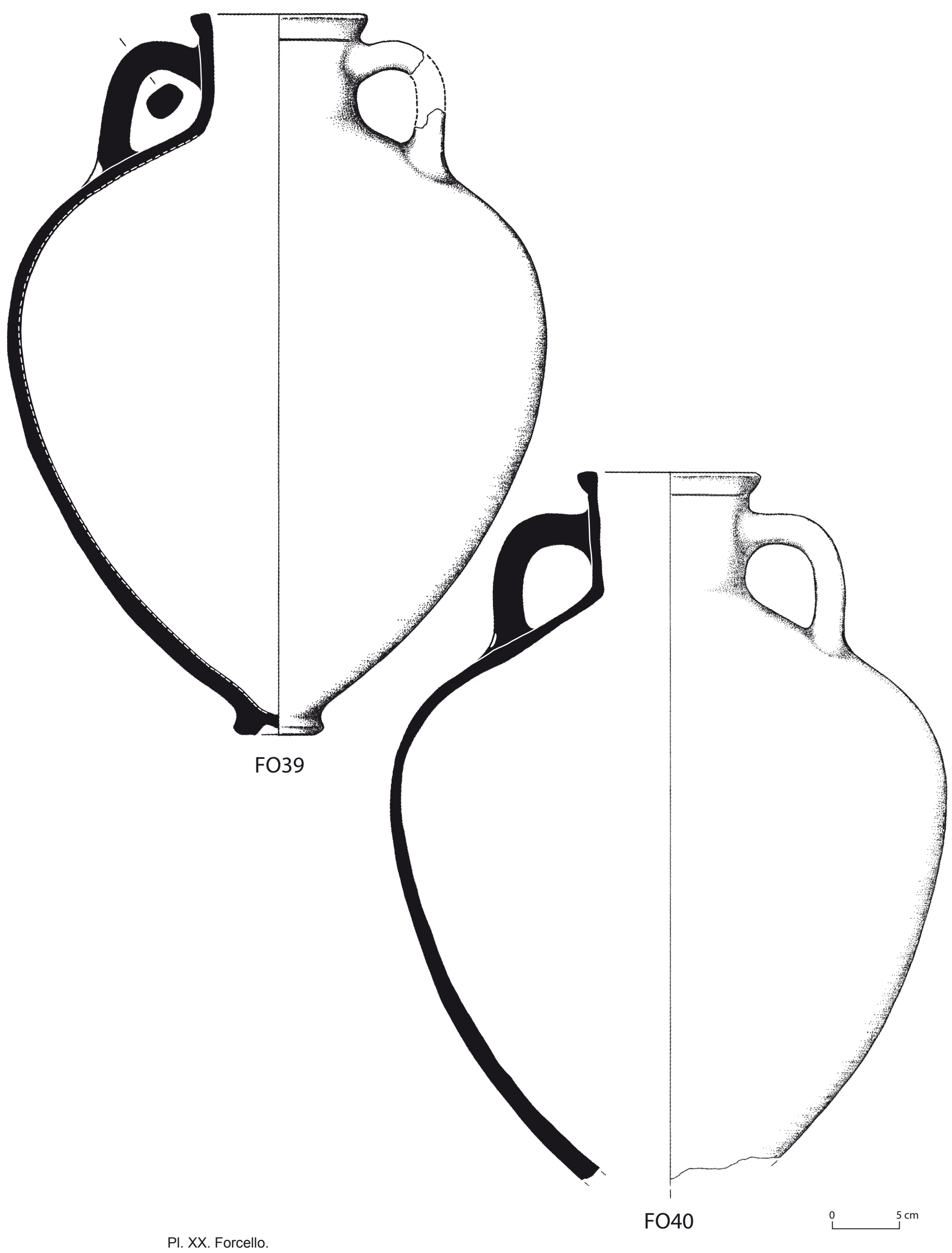


Observations et comparaisons : attribué par R. de Marinis à la production de Mendé.

Chronologie : non déterminable.

Bibliographie : de Marinis 1988, $\mathrm{n}^{\circ}$ 274.6, p. 224, fig. $111, \mathrm{n}^{\circ} 6$.

\section{FO36}

Lieu de découverte : Forcello, Bagnolo San Vito (Mn).

Secteur : Secteur R-S 18.

État de conservation : pied complet. Dimensions non repérées. Pied en bobine.

Inclusions : argile caractérisée par la présence d'un abondant mica argenté et doré.

Observations et comparaisons : attribué par R. de Marinis à la production de Mendé.

Chronologie : non déterminable.

Bibliographie : de Marinis 1989, p. 36-37; Id. 1996,p. 334.

\section{FO37}

Secteur : Secteur R 18.

État de conservation : pied complet. Dimensions : non repérées. Pied en bobine.

Observations et comparaisons : attribué par R. de Marinis à la production de Mendé.

Chronologie : non déterminable.

Bibliographie : de Marinis 1989, p. 36-37 ; Id. 1996, p. 334.

\section{FO38}

Secteur : R 17 p 11.

US : couche arable.

État de conservation : anse complète. Dimensions : non repérées. Anses en ruban épaissi, de section elliptique, avec une impression digitale au niveau des jonctions inférieures.

Surface : rougeâtre clair.

Inclusions : inclusions blanches à fréquence haute. Mica argenté.

Observations et comparaisons : attribuée par R. de Marinis à la production de Mendé.

Chronologie : non déterminable.

Bibliographie : de Marinis 2005, p. 181 ; Id.2007, p. 172.

\section{FO39 (fig. 84, à gauche; pl. XX)}

Structure : Maison N/E (= Maison F I) - pièce $\mathrm{n}^{\circ} 3$.

Type : Ring-toe Lawall.

État de conservation : amphore presque entière, reconstituée à partir de quelques centaines de fragments et restaurée. H. tot. : non repérée. Lèvre en coin, avec un fin listel à la base. Col cylindrique, au profil légèrement concave. Anses verticales, en ruban épaissi, à section elliptique, avec une impression digitale au niveau des jonctions inférieures. Épaule aplatie. Corps piriforme. Pied tronconique creux.
Surface : rouge clair.

Inclusions : pâte avec des inclusions noires et blanches. Mica argenté et doré.

Engobe : traces d'engobe blanc jaunâtre.

Observations et comparaisons : récemment attribuée par R. de Marinis à la production initiale de l'île de Thasos et datée entre 520 et 490 av. J.-C., en s'appuyant tant sur la stratigraphie que sur la confrontation avec Grandjean 1992, p. 581, Roberts 1986, n 425-427, et Monachov 1999b, fig. 17, à gauche.

Chronologie : 520-490 av. J.-C.

Bibliographie : de Marinis 1996, p. 334, fig. 9 ; Id. 2005, p. 176 , fig. $92, \mathrm{n}^{\circ} 1,94 ;$ Id. 2007 , p. 170 , fig. $113,115, \mathrm{n}^{\circ} 1$.

FO40 (fig. 84, à droite; pl. XX)

Structure : Maison S/O (= Maison F II).

Type : Ring-toe Lawall.

État de conservation : exemplaire presque complet, hormis le fond, reconstitué à partir de nombreux fragments et restauré. Dimensions : non repérées. Lèvre en coin, épaissie à l'extérieur, avec un fin ressaut à la base. Col cylindrique, au profil légèrement concave. Anses verticales, en ruban épaissi, à section elliptique, avec une impression digitale au niveau des jonctions inférieures. Épaule aplatie. Corps piriforme.

Surface : rouge clair.

Inclusions : pâte avec inclusions noires et blanches. Mica argenté et doré.

Engobe : traces d'engobe blanc-jaunâtre.

Observations et comparaisons : attribuée dans un premier temps par R. de Marinis à la production de Mendé, l'amphore est maintenant considérée comme appartenant à la production initiale de l'île de Thasos par le même spécialiste, qui la date entre 520 et 490 av. J.-C. s'appuyant à la fois sur la stratigraphie et sur la confrontation avec des exemplaires de Thasos (Grandjean 1992, p. 581), d'Athènes (Grace 1979, fig. 35 ; Roberts 1986, $\mathrm{n}^{\circ}$ 425-427) et d'Olbia (Monachov 1999b, fig. 17, à gauche).

Chronologie : 520-490 av. J.-C.

Bibliographie : de Marinis 1996, p. 334, fig. 7-8 ; Id. 2005, p. 178-179, fig. $92, \mathrm{n}^{\circ} 2,93$; Id. 2007, p. 170, fig. $114,115, \mathrm{n}^{\circ} 2$.

\section{FO41 (pl. XXI)}

Structure : Maison N/E (Maison F I) - pièce $\mathrm{n}^{\circ} 9$.

Phase F.

Type : Ring-toe Lawall.

État de conservation : amphore entièrement reconstituable à partir de plus de trois cents fragments. Dimensions : non repérées. Lèvre en coin, avec un fin listel à la base. Col cylindrique, au profil légèrement concave. Anses verticales, en ruban épaissi, de section 


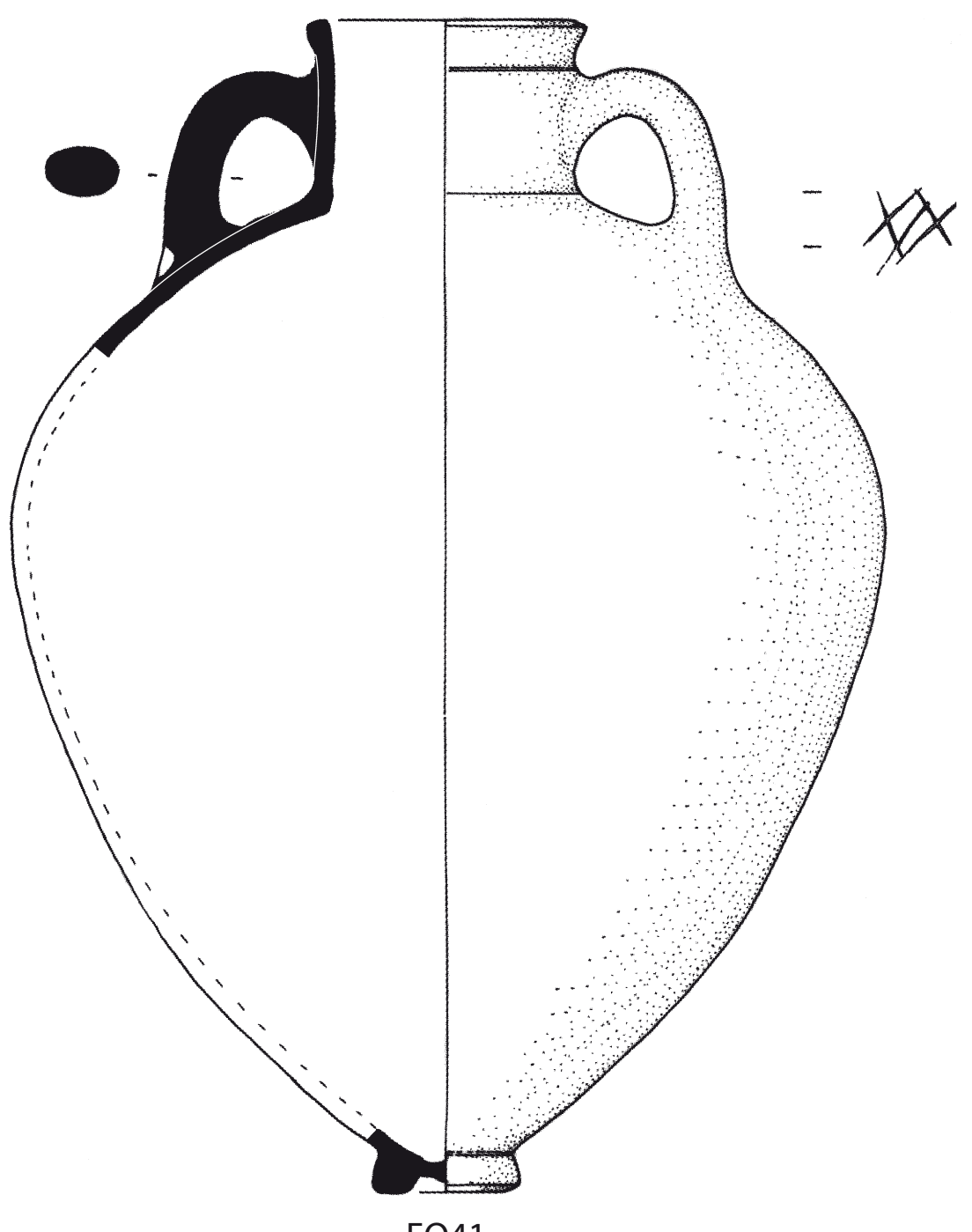

FO41

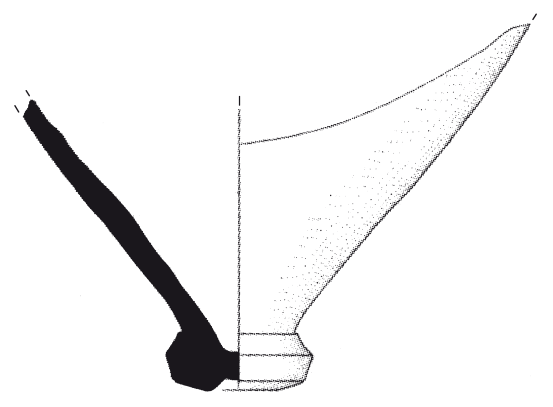

FO44

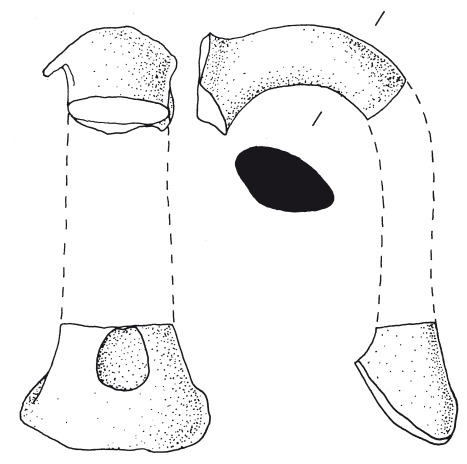

FO46

PI. XXI. Forcello. 
elliptique, avec une impression digitale au niveau de la jonction inférieure. Épaule aplatie. Corps piriforme. Pied tronconique creux.

Appareil épigraphique : sur l'épaule, deux « signes en forme de croix » avec une barre verticale interposée (Sacchetti 2011, $\mathrm{n}^{\circ}$ 6).

Observations et comparaisons : voir la fiche précédente. Chronologie : 520-490 av. J.-C.

Bibliographie : de Marinis 2005, p. 176-178; Id. 2007, p. 169-170, fig. 116 ; Sacchetti $2011, \mathrm{n}^{\circ} 6$.

\section{FO42}

Structure : Maison N/E (Maison F I) - pièce $n^{\circ} 9$.

Phase F.

Type : Ring-toe Lawall.

État de conservation : exemplaire presque entièrement reconstituable à partir de plus de deux cents fragments pour la plupart coïncidant les uns avec les autres.

Observations et comparaisons : l'exemplaire, considérablement déformé par la chaleur de l'incendie qui avait atteint la maison F I, est attribué par R. de Marinis à la production initiale de Thasos et daté, ainsi que l'exemplaire de la fiche précédente, entre 520 et 490 av. J.-C.

Chronologie : 520-490 av. J.-C.

Bibliographie : de Marinis 2005, p. 178.

\section{FO43}

Structure : Maison N/E (Maison F I) - pièce $n^{\circ} 8$.

Fase F.

Type : Ring-toe Lawall.

État de conservation : amphore presque complète, dont sont conservés quatre cents fragments environ. Dimensions : non repérées.

Appareil épigraphique : R. de Marinis mentionne la présence sur un fragment des traces d'un graffito consistant en quatre longs traits (Sacchetti 2011, cat. $n^{\circ} 7$ ).

Observations et comparaisons : on renvoie à la fiche précédente.

Chronologie : 520-490 av. J.-C.

Bibliographie : de Marinis 2005, p. 178 ; Sacchetti 2011, cat. $n^{\circ} 7$.

\section{FO44 (pl. XXI)}

Secteur : R-S 17-18.

Structure : décharge de matériel.

US : 399 .

Type : nord-égéenne ou gréco-orientale (?).

État de conservation : pied et partie du fond de la panse. Dimensions : non repérées. Pied tronconique plein, avec une dépression de base et un profil à double carène.

Surface : rouge clair.

Inclusions : inclusions micacées à fréquence haute.
Observations et comparaisons : exemplaire attribué par R. de Marinis au type «Zeest's Samian » ou au type «Zeest's Protothasian 》 (datables entre le dernier quart du $\mathrm{VI}^{\mathrm{e}}$ et le milieu du $\mathrm{V}^{\mathrm{e}} \mathrm{s}$. av. J.-C.) et considéré au début comme un produit nord-égéen (d'après Dupont 1999, p. 153) et ensuite comme un produit nord-égéen ou gréco-oriental (selon Carlson 2003). L'exemplaire du Forcello provient de l'US 399, qui contient du matériel pour la plupart appartenant au milieu ou au troisième quart du Ve s. av. J.-C.

Chronologie : vers le milieu du Ve s. av. J.-C.

Bibliographie : de Marinis 2005, p. 181, fig. 95, $\mathrm{n}^{\circ} 3$; Id. 2007, p.172, fig. $118, \mathrm{n}^{\circ} 3$ (erronément indiquée comme fig. $\left.110, \mathrm{n}^{\circ} 3\right)$.

\section{FO45}

Secteur : R-S 17-18.

Structure : décharge de matériel.

US : 399 .

État de conservation : exemplaire dont il reste une vingtaine de fragments, coïncidant en partie les uns avec les autres, appartenant au col, à une anse, à l'épaulement et à la panse. Dimensions : non repérées.

Observations et comparaisons : attribué par R. de Marinis à la production de Mendé, ou à celle de Thasos ou de son cercle. Bien qu'elle provienne de l'US 399, dont le matériel est généralement daté vers le milieu ou le troisième quart du V $\mathrm{V}^{\mathrm{e}} \mathrm{s}$. av. J.-C., l'amphore est datée par son éditeur autour de 475 av. J.-C., après confrontation avec des exemplaires appartenant au cercle de Thasos provenant de la Mer Noire (Monachov 1999b, fig. 21.1-2).

Chronologie : autour de 475 av. J.-C.

Bibliographie : de Marinis 2005, p. 180.

\section{FO46 (pl. XXI)}

Secteur: Secteur V 11 m 2-3.

US : 13 .

État de conservation : deux fragments d'anse, non reconstituables mais appartenant au même individu, correspondant aux jonctions inférieure et supérieure. H. max. cons. : 17. Anses en ruban épaissi, à section elliptique, avec une impression digitale au niveau de la jonction inférieure.

Surface int. : rose orangé.

Fracture : grise.

Inclusions : inclusions blanches et micacées.

Observations et comparaisons : l'anse, dont la fabrique a été considérée comme non identifiable par $\mathrm{R}$. de Marinis, est caractérisée par une impression digitale au niveau de la jonction inférieure qui pourrait la rapprocher de la production de l'Égée septentrionale de la fin $\mathrm{du} \mathrm{VI}{ }^{\mathrm{e}}$ ou du $\mathrm{V}^{\mathrm{e}} \mathrm{s}$. av. J.-C. Pour ce détail morphologique des amphores appartenant à la koinè nord-égéenne, on 
renvoie à l'ample documentation publiée dans Blonde, Muller, Mulliez 1991, Grandjean 1992, et Schmid 2001. Chronologie : non déterminable.

Bibliographie: de Marinis 1988, n² 275, p. 224, fig. 111, $n^{\circ} 4$.

\section{AMPHORES DE L'ÉGÉE ORIENTALE}

\section{FO47 (pl. XXII)}

Secteur : R 18 e 5.

Structure : décharge de matériel.

Type : chiote, Lambrino A 2.

État de conservation : pied et partie du fond de la panse. H. max. cons. : 11. Pied cylindrique creux, avec la base en anneau épaissi.

Inclusions : pâte riche d'inclusions blanches, à la surface rugueuse et vacuolaire.

Observations et comparaisons : classifié par R. de Marinis dans le cadre de la variété la plus ancienne du type " early bulgy », datable avant le premier quart du Ve s. av. J.-C. (de Marinis 1999, p. 266-267, fig. 7. 4-5). L'indice principal en faveur de cet encadrement serait, d'après R. de Marinis, le repli non encore accentué de la surface d'appui du pied. Cependant, la riche documentation de l'Agora d'Athènes (Lawall 1995, p. 89-92) et celle de la Mer Noire (Monachov 2003a, pl. 3-6), montrent combien, dans le but d'établir la chronologie, la variabilité du repli de la surface d'appui a peu d'importance et combien, au contraire, est plus significative la hauteur du pied, qui s'avère plus grande dans les types « Lambrino A1 et A2 » par rapport aux types suivants «swollen-necked ». L'exemplaire du Forcello pourrait donc être attribué au type « Lambrino A 2 », datable entre 510 et 490 av. J.-C. Pour le type, Lambrino 1938,p. 110-112, fig. 71-72, et Dupont 1998, fig. 23. 2, c.

Chronologie : 510-490 av. J.-C.

Bibliographie : de Marinis 1999, p. 275, fig. 11 ; Id. 2005, p. 198, fig. $99, n^{\circ} 3$ (attribuée de manière erronée à l'amphore chiote de l'US 399 - cf. FO52) ; Id. 2007, p. 186, fig. $122, \mathrm{n}^{\circ} 3$ (attribuée de manière erronée à l'amphore chiote de l'US 399 - cf. FO52).

\section{FO48 (pl. XXII)}

Secteur : Secteur V 10 t 18-19.

Structure : Décharge de matériel.

US : 2 .

Type : chiote, Swollen-necked, Early bulgy, variante C/1 Knigge-Lawall.

État de conservation : exemplaire dont n'est conservée que la partie supérieure, de la lèvre jusqu'à la jonction de l'épaule, avec une anse presque complète. H. max. cons. : 14,5. Lèvre à cordon. Diam. restituable embouchure :
11,1. Col gonflé. Anses verticales, en ruban épaissi, à section elliptique.

Surface : chamois rosé nuancé de gris.

Inclusions : inclusions blanches et micacées.

Engobe : engobe blanc.

Observations et comparaisons : le col bref et l'épaule aplatie suggèrent d'encadrer l'exemplaire dans le type « swollen-necked, early bulgy » et précisément dans la variante C/1 Knigge-Lawall, datable du premier quart du Ve s. av. J.-C. Pour le type, Lawall 1995, p. 89-90, fig. 19, 23-24 et de Marinis 1999, p. 266-268, fig. 7. 5. Attribué par R. de Marinis au même exemplaire de la fiche suivante.

Chronologie : 500-475 av. J.-C

Bibliographie : de Marinis 1988, $\mathrm{n}^{\circ} 269.5$, p. 223, fig. 109,5 ; Id. 1999, fig. 9 ; Id. 2005, p. 196, fig. 99, $\mathrm{n}^{\circ} 2$ (en haut) ; Id. 2007, p. 186, fig. 122, n² 2 (en haut).

\section{FO49 (pl. XXII)}

Secteur: Secteur V 10 t 12 s 20.

US : 2 .

Type : chiote, Swollen-necked, Early bulgy, variante C/1 Knigge-Lawall.

État de conservation : partie de panse, reconstituée à partir de fragments, et un fragment d'épaule avec la jonction inférieure d'une anse. Dimensions max. cons. : $24 \times 23,5$.

Surface ext. et fracture : chamois.

Surface int. : chamois avec des nuances d'orange.

Inclusions : inclusions blanches et micacées.

Engobe : engobe blanc.

Observations et comparaisons : attribuée par R. de Marinis au même exemplaire de la fiche précédente, à laquelle on renvoie pour les observations et les comparaisons.

Chronologie : 500-475 av. J.-C.

Bibliographie : de Marinis 1988, $\mathrm{n}^{\circ}$ 269.6, p. 223, fig. 109,$6 ; I d .2005$, p. 196 , fig. $99, \mathrm{n}^{\circ} 2$ (en bas) ; Id. 2007 , p. 186 , fig. $122, \mathrm{n}^{\circ} 2$ (en bas).

\section{FO50 (pl. XXII)}

Année de la fouille / trouvaille : 1998.

Secteur : Secteur R 19 q m1.

Sporadique.

Type : chiote, Swollen-necked, Early bulgy, variante C/1 Knigge-Lawall.

État de conservation : amphore complètement conservée jusqu'au niveau de l'épaulement. Dimensions : non repérées. Lèvre à cordon. Au-dessous de la lèvre, traces d'un fin trait rouge qui continuait peut-être le long du dos des anses. Diam. embouchure : 11,5 / 12. Col gonflé. Diam. min./max. : 14. Anses obliques rentrantes, « a bastoncello », de section circulaire. H. : 11. Épaule fuyante, au profil légèrement concave. 


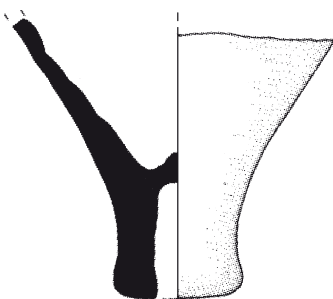

FO47
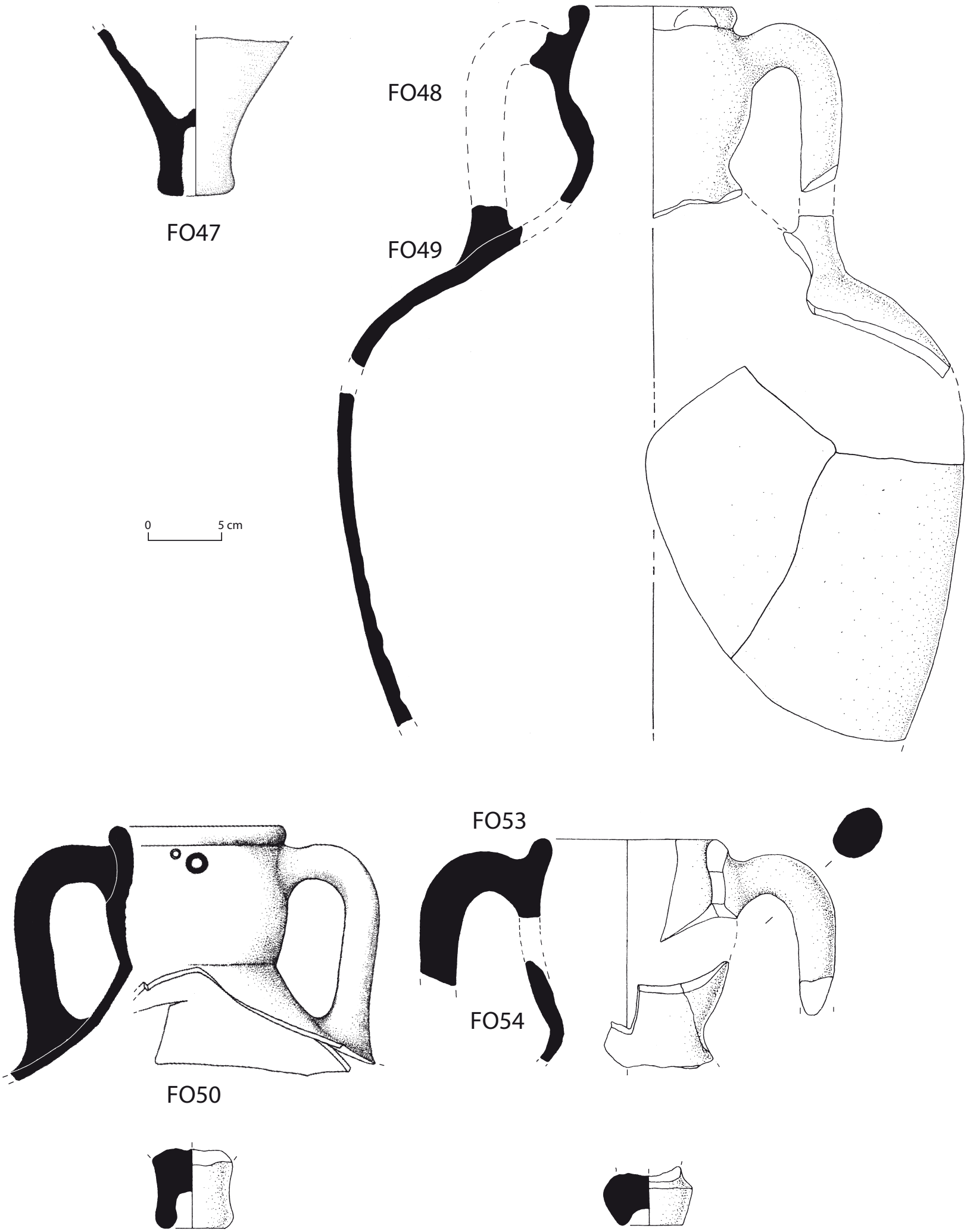

FO51

FO56

PI. XXII. Forcello. 
Surface : brun-rouge foncé (7.5YR5/6-7.5YR5/8).

Inclusions : argile avec de nombreuses inclusions blanches fines, grises et anguleuses, et de plus rares et fines inclusions rouge foncé. Mica fin et répandu. Surface rugueuse et contenant des vacuoles (classe I de Whitbread 1995, p. 139-141).

Engobe : engobe brunâtre.

Appareil épigraphique : sur le col, entre les anses, un petit cercle imprimé, et dans la partie inférieure d'une anse, une incision alphanumérique (Sacchetti 2010, p. 51-52, no 8, pl. II, 8, III, 8).

Observations et comparaisons : l'exemplaire a été encadré par R. de Marinis dans la variété la plus ancienne du type early bulgy, datable avant le premier quart du $\mathrm{V}^{\mathrm{e}} \mathrm{s}$. av. J.-C. Pour le type, Lawall 1995, p. 89-90, fig. 19, 23-24 ; et de Marinis 1999, p. 266, fig. 7.4-5.

Chronologie : 500-475 av. J.-C.

Bibliographie : de Marinis 1999, p. 260-262, 269-273, fig. $5-6,10 ;$ Id. 2005, p. 195-196, fig. 99 , n 1 ; Id. 2007, fig. $122, \mathrm{n}^{\circ} 1$; Sacchetti 2010 , p. 51-52, n 8, pl. II, 8, III, 8 .

\section{FO51 (pl. XXII)}

Sporadique.

Type : chiote, Swollen-necked, Early bulgy, variante C/1 o C/2 Knigge-Lawall.

État de conservation : pied. H. max. cons. : 5,5. Pied cylindrique creux, à base en anneau épaissi et légèrement replié vers l'intérieur.

Surface ext., int. et fracture : rose orangé.

Engobe : engobe brunâtre.

Observations et comparaisons : attribué par R. de Marinis à la production chiote et plus précisément au type «swollen-necked, early bulgy». Pour le type, datable du courant de la première moitié du Ve s. av. J.-C., Lawall 1995, fig. 25, 31 ; et de Marinis 1999, p. 266-268, fig. 7. 4 -7.

Chronologie : 500-450 av. J.-C.

Bibliographie : de Marinis 1988, $\mathrm{n}^{\circ}$ 269.9, p. 223, fig. $109, \mathrm{n}^{\circ} 9$; Id. 1999, p. 273 ; Id. 2005, p. 196.

\section{FO52}

Secteur : R-S 17-18 q 14.

Structure : décharge de matériel.

US : 399 .

Type : chiote, Swollen-necked, Early bulgy, variante C/2 Knigge-Lawall.

État de conservation : pied et partie inférieure du corps. Dimensions : non repérées. Pied cylindrique creux, épaissi, à base annulaire.

Observations et comparaisons : exemplaire attribué par $\mathrm{R}$. de Marinis à sa variante la plus récente du soustype early bulgy, datable autour de 450 av. J.-C., sur la base de la confrontation avec l'exemplaire P21971 de l'Agora. Cette variante, introduite par R. de Marinis, est difficile à distinguer de la variante $\mathrm{C} / 2$ Knigge-Lawall, datable du deuxième quart du $\mathrm{V}^{\mathrm{e}} \mathrm{s}$. av. J.-C.

Chronologie : 475-450 av. J.-C.

Bibliographie : de Marinis 1999, p. 273 ; Id. 2005, p. 198 (attribué par erreur à la fig. 99, n 3, qui se refère au contraire au pied du Secteur R 18 e 5, comme on peut le vérifier dans de Marinis 1999, p. 275, fig. 11 - cf. FO47) ; Id. 2007, p. 186 (attribué par erreur à la fig. $122, \mathrm{n}^{\circ} 3$ à cause de la même méprise de de Marinis 2005, p. 198).

\section{FO53 (pl. XXII)}

Secteur : Secteur V 11 n-o 2.

US $: 5$.

Type : chiote, Swollen-necked, Early bulgy, variante C/2 Knigge-Lawall.

État de conservation : partie de lèvre et de col avec la moitié supérieure d'une anse. H. max. cons. : 11,6. Lèvre à cordon. Diam. restituable embouchure : 11,2. Col gonflé. Anses verticales, en ruban épaissi, à section elliptique.

Surface : rose orangé.

Engobe : engobe brunâtre.

Observations et comparaisons : la morphologie et les proportions du col suggèrent de l'encadrer dans la variante C/2 Knigge-Lawall du type swollen-necked, early bulgy, datée du deuxième quart du $\mathrm{V}^{\mathrm{e}} \mathrm{s}$. av. J.-C. Pour le type, Lawall 1995, p. 90-91, fig. 20, 26-27 ; de Marinis 1999, p. 266-268, fig. 7.6. Appartenant, d'après R. de Marinis, au même individu de la fiche suivante.

Chronologie : 475-450 av. J.-C.

Bibliographie : de Marinis 1988, $\mathrm{n}^{\circ}$ 269.7, p. 223, fig. $109, \mathrm{n}^{\circ} 7$.

\section{FO54 (pl. XXII)}

Secteur : Secteur V 11 p-q 2.

Structure : décharge de matériel.

US : 2 .

Type : chiote, Swollen-necked, Early bulgy, variante C/2 Knigge-Lawall.

État de conservation : partie de col reconstituée à partir de fragments. H. max. cons. : 6,5. Col gonflé. Diam. min. /max. restituable : 14 .

Surface : rose orangé.

Engobe : engobe brunâtre.

Observations et comparaisons : attribué par R. de Marinis au même individu de la fiche précédente, à laquelle on renvoie pour les observations et les confrontations.

Chronologie : 475-450 av. J.-C.

Bibliographie : de Marinis 1988, $\mathrm{n}^{\circ}$ 269.8, p. 223, fig. $109, \mathrm{n}^{\circ} 8 ;$ Id. 2005 , p. 196. 


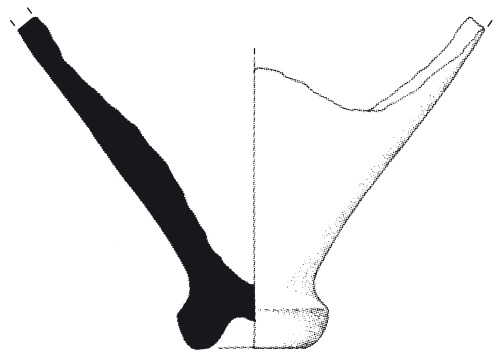

FO57

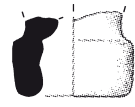

FO58
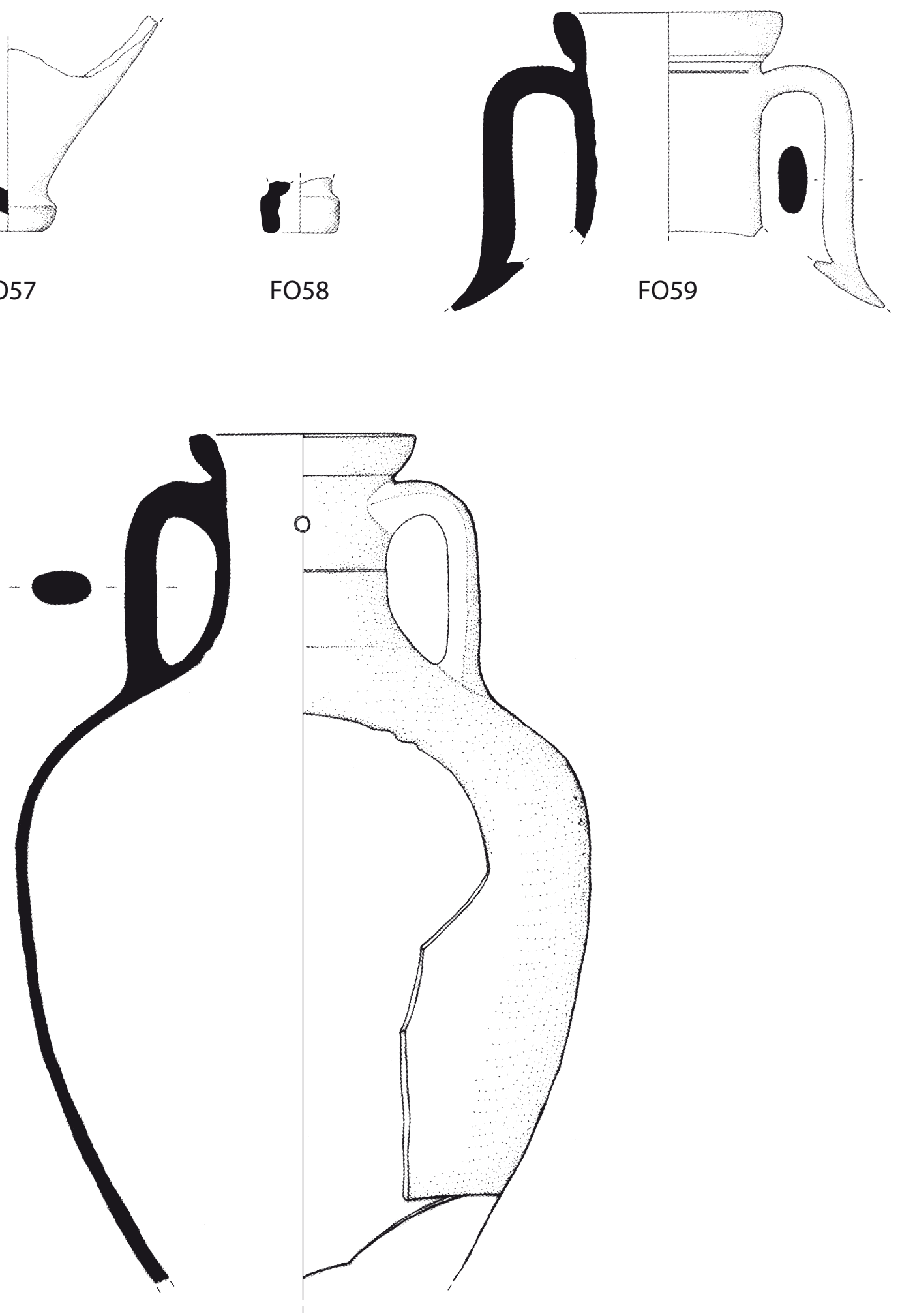

FO60

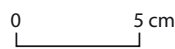

PI. XXIII. Forcello. 


\section{FO55}

Secteur : Secteur S 17.

US : 517 .

Type : chiote, Swollen-necked, Early bulgy, variante C/2 Knigge-Lawall.

État de conservation : deux fragments de lèvre, col et parties des anses. Dimensions : non repérées.

Observations et comparaisons : exemplaire classé par R. de Marinis dans le cadre de ses deux variantes plus récentes du type early bulgy, datables du deuxième quart du $\mathrm{V}^{\mathrm{e}} \mathrm{s}$. av. J.-C. et correspondant à la variante $\mathrm{C} / 2$ Knigge-Lawall. Pour le type, Lawall 1995, p. 90-91, fig. 20, 26-27 ; et de Marinis 1999, p. 267-268, fig. 7.6-7. Chronologie : 475-450 av. J.-C.

Bibliographie: de Marinis 1999,p. 273 ; Id.2005,p. 198.

\section{FO56 (pl. XXII)}

Type : chiote, Swollen-necked, Later bulgy, variante C/3 Knigge-Lawall ou Straight-neck.

État de conservation : pied incomplet. H. max. cons. : 4. Pied cylindrique creux, épaissi, à base annulaire. Diam. min./max. restituable : 6 .

Surface : rose orangé intense.

Engobe : engobe brunâtre.

Observations et comparaisons : classifié par R. de Marinis dans le cadre du type swollen-necked, later bulgy, datable du troisième quart du $\mathrm{V}^{\mathrm{e}} \mathrm{s}$. av. J.-C., ou du type à col cylindrique qui lui succède, datable du dernier quart du siècle. Pour le type, Lawall 1995, p. 91-92, fig. 21, 22, 32 ; et de Marinis 1999, p. 267-268, fig. 7. 8-9.

Chronologie : 450-400 av. J.-C.

Bibliographie : de Marinis 1988, $\mathrm{n}^{\circ} 269.10$, p. 223, fig. $109, \mathrm{n}^{\circ} 10 ;$ Id. 1999 , p. 273 ; Id. 2005, p. 197-198.

\section{FO57 (pl. XXIII)}

Secteur : R-S 17-18.

Structure : décharge de matériel.

US : 399 .

Type : Samos-Milet.

État de conservation : pied et partie du fond de la panse. Dimensions : non repérées. Pied tronconique creux, avec base en anneau épaissi et au profil caréné.

Surface : orange.

Inclusions : inclusions micacées.

Observations et comparaisons : exemplaire attribué par R. de Marinis à la production de Milet et daté entre la fin du $\mathrm{VI}^{\mathrm{e}}$ et le début du $\mathrm{V}^{\mathrm{e}} \mathrm{s}$. av. J.-C. après confrontation avec des exemplaires de l'épave de la Pointe Lequin (Long, Miro, Volpe 1992, fig. 44.1-3), dont la datation se situe vers 515 av. J.-C. Pour la forme, on renvoie à Dupont 1998, fig. 23.9, b (datée du dernier quart du $\mathrm{VI}^{\mathrm{e}}$ s. av. J.-C.), et Voigtländer 1983, fig. 27.167 (datée du VI ${ }^{\mathrm{e}}$ s. av. J.-C.).
Chronologie : fin $\mathrm{VI}^{\mathrm{e}}$-débuts $\mathrm{V}^{\mathrm{e}}$ s. av. J.-C.

Bibliographie : de Marinis 2005, p. 184, fig. 97, n $^{\circ} 1$; Id. 2007, p. 174, fig. $120, \mathrm{n}^{\circ} 4$.

\section{FO58 (pl. XXIII)}

US : 410 .

Type : Samos-Milet, S/2 Lawall.

État de conservation : fragment de pied. Dimensions : non repérées. Pied cylindrique creux à base annulaire.

Observations et comparaisons : attribué par R. de Marinis à la production de Samos. La morphologie de ce pied trouve son équivalent dans la forme datable du deuxième quart du $\mathrm{V}^{\mathrm{e}} \mathrm{s}$. av. J.-C. du type $\mathrm{S} / 2$ Lawall (Lawall 1995, p. 182-183, fig. 70-71).

Chronologie : 480-450 av. J.-C.

Bibliographie : de Marinis 2005, fig. 97, $\mathrm{n}^{\circ} 3$; Id. 2007, fig. $120, n^{\circ} 3$.

\section{FO59 (pl. XXIII)}

Secteur : R 18.

Structure : colmatage de défoncement de vigne.

US : 9/164.

Type : Samos-Milet, S/2 Lawall.

État de conservation : partie de lèvre, col et une anse complète. Dimensions : non repérées. Lèvre en amande, légèrement épaissie. Col cylindrique, avec une fine cannelure légèrement au-dessous de la lèvre. Anses verticales, en ruban épaissi, à section elliptique.

Surface : rose orangé.

Inclusions : inclusions blanches et grises. Mica fin à fréquence haute.

Observations et comparaisons : exemplaire attribué par R. de Marinis à la production de Milet. Les caractéristiques morphologiques permettent de l'encadrer dans la variante du deuxième quart du $\mathrm{V}^{\mathrm{e}} \mathrm{s}$. av. J.-C. du type $\mathrm{S} / 2$ de $\mathrm{M}$. Lawall après confrontation avec Grace 1971, fig. 3.1. Pour le type, Lawall 1995, p. 182-183, fig. 70-71.

Chronologie : 480-450 av. J.-C.

Bibliographie : de Marinis 2005, p. 182, fig. 96, n 6 ; Id. 2007, p. 173, fig. $119, \mathrm{n}^{\circ} 6$.

\section{FO60 (pl. XXIII)}

Secteur: R-S 17-18.

Structure : décharge de matériel.

US : 399 .

Type : Samos-Milet, S/1 Lawall.

État de conservation : amphore privée du fond de la panse et du pied, reconstituée à partir de fragments et restaurée. H. tot. : 57. Lèvre en amande, haute et légèrement épaissie et ouverte. Col cylindrique, avec un listel au milieu, au-dessous de la jonction supérieure des 
anses. Anses verticales, en ruban épaissi, à section elliptique. Épaule aplatie. Corps piriforme.

Surface : chamois rosé.

Fracture : rouge.

Inclusions : pâte qui contient quelques inclusions blanches et du mica fin.

Appareil épigraphique : sur le col, entre les anses, un petit cercle imprimé vraisemblablement dans le but de vérifier l'état de séchage de l'amphore (cf. Eiseman, Ridgway 1987, p. 41-42; et Carlson 2003, p. 586).

Observations et comparaisons : classifiée par R. de Marinis comme samienne et datée du deuxième quart ou du milieu $\mathrm{du} \mathrm{V}^{\mathrm{e}} \mathrm{s}$. av. J.-C. après confrontation avec l'amphore de la Tombe 73 de Fratte, datable du deuxième quart du Ve s. av. J.-C. sur la base du mobilier (Di Sandro 1981b, fig. 3.3), avec un exemplaire du puits $\mathrm{N} 7: 3$ de l'Agora d'Athènes (Grace 1971, fig. 3.1) et avec un autre provenant d'un contexte funéraire du Chersonèse (Monachov 1999b, fig. 12). On peut l'encadrer dans la variante du deuxième quart du V $\mathrm{V}$ s. av. J.-C. du type S/1 de M. Lawall. Pour le type, Lawall 1995, p. 179-180.

Chronologie : 480-450 av. J.-C.

Bibliographie : de Marinis 1990, fig. 26 ; Id. 1996, p. 335 , fig.10-11; Id. 2005 , p. 185 , fig. $96, \mathrm{n}^{\circ} 5$; Id. 2007 , p. 174 , fig. $119, \mathrm{n}^{\circ} 5$.

\section{FO61 (pl. XXIV)}

Secteur : S 17 (de Marinis 1988, p. 223, n² 271) ou S 18 (de Marinis 2005, p. 184).

Structure : Maison S 17-18.

US : 256 .

Type : Samos-Milet, S/1 Lawall.

État de conservation : partie supérieure d'amphore, de la lèvre jusqu'à l'épaule, reconstituée à partir de deux fragments. H. max. cons. : 19. Lèvre épaissie. Diam. embouchure : 14. Col cylindrique, avec un listel au milieu, au-dessous de la jonction supérieure des anses. Anses verticales, en ruban épaissi, à section elliptique. Épaule fuyante.

Surface : rouge intense.

Inclusions : argile très fine avec des inclusions de mica très fines et répandues et des inclusions blanches peu nombreuses. Quelques vacuoles.

Observations et comparaisons : classée comme samienne et datée autour du deuxième quart du $\mathrm{V}^{\mathrm{e}} \mathrm{s}$. av. J.-C. par R. de Marinis, elle provient de la phase de la maison S 17-18 qui se situe au milieu du Ve s. av. J.-C. Pour la forme, Di Sandro 1981b, fig. 3.3, correspondant à la variante du deuxième quart du $\mathrm{V}^{\mathrm{e}} \mathrm{s}$. av. J.-C. du type $\mathrm{S} / 1$ de M. Lawall. Pour le type, Lawall 1995, p. 179-180. Par rapport à ce type l'amphore du Forcello présente une lèvre plus épaissie et anguleuse qui évoque les formes Zeest's Samian.
Chronologie : 480-450 av. J.-C.

Bibliographie : de Marinis 1988, $\mathrm{n}^{\circ}$ 271, p. 223, fig. 110, $\mathrm{n}^{\circ} 1$ (indiquée comme fig. 110, $\mathrm{n}^{\circ} 2$ et rectifiée dans de Marinis 1996, p. 335) ; Id. 1996, p. 335 ; Id.2005, p. 184185 , fig. $96, \mathrm{n}^{\circ} 1 ; I d .2007$, p. 174-176, fig. $119, \mathrm{n}^{\circ} 1$.

\section{FO62 (pl. XXIV)}

Secteur : R 19.

US : 1786 et 1603 (es 1790).

Type : Samos-Milet, S/1 Lawall.

État de conservation : moitié supérieure d'une amphore reconstituée à partir d'une cinquantaine de fragments. Dimensions : non repérées. Lèvre anguleuse. Col qui présente un listel au-dessous des anses. Anses en ruban épaissi. Épaule fuyante.

Observations et comparaisons : attribuée par R. de Marinis à la production samienne, datée du deuxième quart avancé ou du milieu du Ve s. av. J.-C. et confrontée notamment avec une amphore de l'épave de Tektaş Burnu (Carlson 2003, fig. 5, en haut). On peut l'attribuer au type S/1 Lawall et la rapprocher des formes traditionnellement définies Zeest's Samian (cf. fiche précédente). Chronologie : 480-450 av. J.-C.

Bibliographie : de Marinis 2007, p. 174, fig. 120, n 1 .

\section{FO63 (pl. XXIV)}

Sporadique.

Type : Samos-Milet, S/1 Lawall.

État de conservation : partie de pied. Dimensions : non repérées. Pied cylindrique, légèrement épaissi, à base concave.

Observations et comparaisons : attribué par R. de Marinis à la production de Samos. On peut l'encadrer dans la forme du deuxième quart du $\mathrm{V}^{\mathrm{e}} \mathrm{s}$. av. J.-C. du type S/1 de M. Lawall (Lawall 1995, p. 179-180, fig. 75, en haut). Pour la forme, Di Sandro 1981b, fig. 3.3, et Dupont 1998, fig. 23.9, g.

Chronologie : 480-450 av. J.-C.

Bibliographie : de Marinis 1988, fig. 110, n 7 ; Id. 2005, fig. $97, \mathrm{n}^{\circ} 2 ; I d .2007$, fig. $120, \mathrm{n}^{\circ} 2$.

\section{FO64 (pl. XXIV)}

Secteur: R 18.

Structure : colmatage de défoncement de vigne.

US : 9/164.

Type : Samos-Milet, S/1 Lawall.

État de conservation : amphore dont il reste la partie supérieure, de la lèvre jusqu'à la jonction du col et de l'épaule, avec une partie des segments supérieurs des anses. Dimensions : non repérées. Lèvre en amande, plutôt épaissie et légèrement ouverte. Col évasé, qui présente un listel placé à mi-hauteur environ. Anses bifides. Surface : rose orangé. 


$$
\begin{aligned}
& 0 \\
& 0 \\
& 0.1
\end{aligned}
$$


Inclusions : inclusions micacées à fréquence haute.

Observations et comparaisons : attribuée par R. de Marinis à la production milésienne. L'anse bifide semble être en effet caractéristique des productions de Milet (Dupont 1998, fig. 23.7, d ; fig. 23.8, c, d, e). D'après la morphologie générale, l'exemplaire peut être vraisemblablement encadré dans la variante la plus récente du type S/1 Lawall (Lawall 1995, p. 180-181, fig. 73-74), datable de la seconde moitié du Ve s. av. J.-C.

Chronologie : 450-400 av. J.-C.

Bibliographie : de Marinis 1987, fig. 25 ; Id. 1996, p. 335 ; Id. 2005 , p. 182 , fig. $96, \mathrm{n}^{\circ} 3$; Id. 2007, p. 173 , fig. $119, \mathrm{n}^{\circ} 3$.

\section{FO65 (pl. XXIV)}

Secteur : S 17 h 16.

Structure : Maison S 17-18.

US : 57 .

Type : Samos-Milet, S/1 Lawall.

État de conservation : amphore dont il reste la partie supérieure, de la lèvre jusqu'à l'épaule. H. max. cons. : 19,2. Lèvre épaissie. Diam. embouchure : 14. Col évasé, avec un listel à mi-hauteur, immédiatement au-dessous de la jonction supérieure des anses. Anses verticales, en ruban épaissi, à section elliptique. Épaule fuyante.

Inclusions : argile fine avec des inclusions blanches très petites et denses et de la chamotte rougeâtre. Aucune inclusion micacée n'est observable.

Observations et comparaisons : attribuée par R. de Marinis à une production samienne, elle provient des niveaux de la phase récente de la maison $S$ 17-18, datables de la deuxième moitié du $\mathrm{V}^{\mathrm{e}} \mathrm{s}$. av. J.-C. L'exemplaire peut être rapproché de la variante de la deuxième moitié du $\mathrm{V}^{\mathrm{e}}$ s. av. J.-C. du type S/1 de M. Lawall. Pour le type, Lawall 1995, p. 180-181, fig. 73-74. Par rapport à ce type, l'amphore du Forcello semble présenter une lèvre plus trapue et épaissie.

Chronologie : 450-400 av. J.- C.

Bibliographie : de Marinis 1988, $n^{\circ}$ 270, p. 223, fig. 110, $\mathrm{n}^{\circ} 2$ (indiquée par erreur comme fig. $110, \mathrm{n}^{\circ} 1$ et corrigée dans de Marinis 1996, p. 335) ; Id. 1996, p. 335 ; Id. 2005 , p. 184 , fig. $96, \mathrm{n}^{\circ} 2$; Id. 2007, p. 174, fig. 119 , $\mathrm{n}^{\circ} 2$.

\section{FO66}

Secteur R 18.

US : 250 .

Type : Samos-Milet.

État de conservation : partie de col et épaule. Dimensions : non repérées. Col qui présente un listel au niveau de la jonction de l'épaule.

Observations et comparaisons : exemplaire attribué par $\mathrm{R}$. De Marinis à une fabrique samienne ou milésienne et daté de la seconde moitié du $\mathrm{V}^{\mathrm{e}} \mathrm{s}$. av. J.-C. sur la base de la stratigraphie.

Chronologie : 450-400 av. J.-C.

Bibliographie : de Marinis 1996, p. 335.

\section{FO67 (pl. XXIV)}

Secteur: R 18 s 8.

US : 250 .

Type : Samos-Milet.

État de conservation : fragment de col et épaule. Dimensions : non repérées. Col évasé, avec un listel au niveau de la jonction de l'épaule.

Surface : rouge orangé.

Inclusions : inclusions de petites dimensions constituées pour la plupart de granules de quartz.

Observations et comparaisons : attribué par R. de Marinis à la production de Milet et encadré dans la seconde moitié du Ve s. av. J.-C. sur la base de la stratigraphie.

Chronologie : 450-400 av. J.-C.

Bibliographie : de Marinis 2005, p. 182-184, fig. 96, $\mathrm{n}^{\circ} 7$; Id. 2007, p. 173-174, fig. $119, \mathrm{n}^{\circ} 7$.

\section{FO68 (pl. XXIV)}

Secteur : Secteur V 10 n 18-19 r-s 17-18.

US : 2 .

Type : Samos-Milet, S/1 Lawall.

État de conservation : fragment de lèvre et col avec les jonctions supérieures des anses. H. max. cons. : 23. Lèvre épaissie, ouverte. Diam. restituable embouchure : 16,5. Col évasé, au profil légèrement concave, portant un listel à mi-hauteur au-dessous de la jonction supérieure des anses. Anses en ruban épaissi, de section elliptique.

Surface : chamois rosé.

Fracture : rouge.

Inclusions : argile très fine avec des inclusions fines blanches et micacées.

Observations et comparaisons : exemplaire attribué par $\mathrm{R}$. de Marinis à la production samienne et daté entre la fin $d u V^{e}$ et les débuts du IV $\mathrm{s}$. av. J.-C. La confrontation la plus rapprochée indique l'appartenance à la plus récente variante du type $\mathrm{S} / 1$ de $\mathrm{M}$. Lawall, datable de la seconde moitié du V $\mathrm{V}^{\mathrm{e}}$ s. av. J.-C. (Grace 1971, fig. 3.3), mais le développement en hauteur très marqué du col suggère une datation plus basse par rapport au type et donc une chronologie allant de la fin du Ve aux débuts du IV s. av. J.-C. Pour la forme, Monachov 1999b, p. 171, fig. 14, d'une sépulture d'Olbia, dans le Chersonèse, datable sur la base du mobilier du premier tiers du IVe s. av. J.-C.

Chronologie : 410-380 av. J.-C.

Bibliographie : de Marinis 1988, p. 223-224, n² 272, fig. $110, \mathrm{n}^{\circ} 3$; Id. 1996, p. 335 ; Id. 2005, p. 184, fig. 96, $\mathrm{n}^{\circ} 4$; Id. 2007, p. 174, fig. $119, \mathrm{n}^{\circ} 4$. 


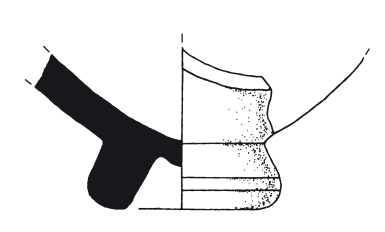

FO69
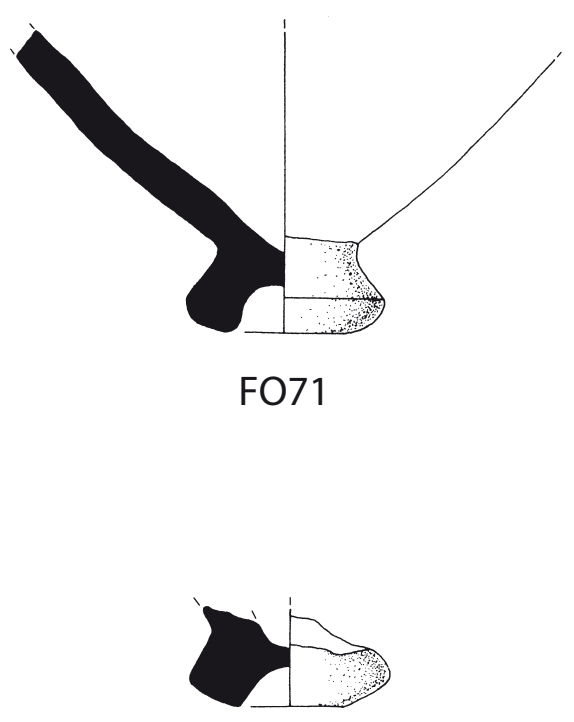

FO73

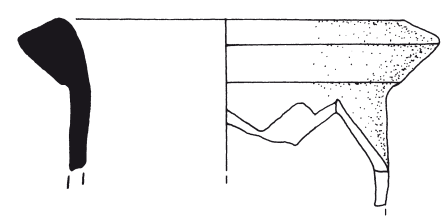

FO74

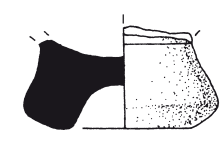

FO70

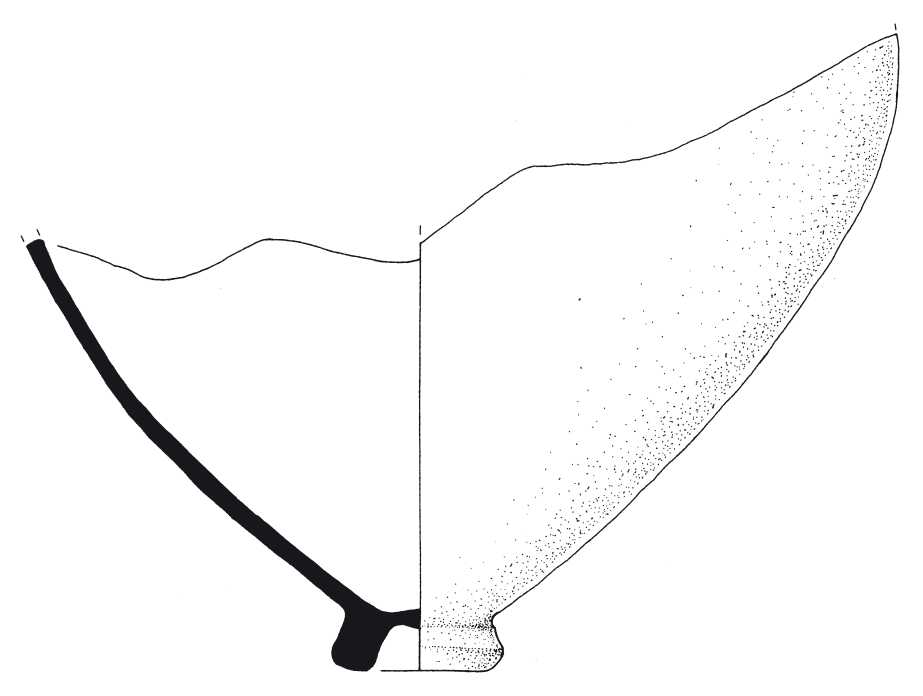

FO72

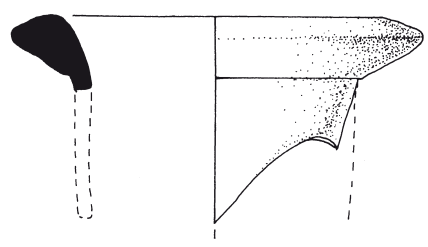

FO75

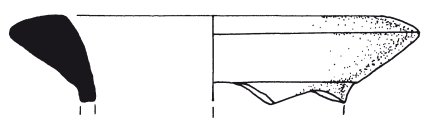

FO76

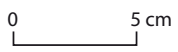

PI. XXV. Forcello. 


\section{FO69 (pl. XXV)}

Secteur : Secteur R 18 u 5.

US : 2 .

Type : Solokha I.

État de conservation : pied avec une brève partie du fond de la panse. H. max. cons. : 6,5. Pied tronconique creux, à base en anneau légèrement épaissi. Diam. : 8.

Surface : rose.

Inclusions : argile avec inclusions blanches et chamotte. Observations et comparaisons : défini d'abord attique et ensuite Solokha I par R. de Marinis. Attribuable à une amphore du groupe Solokha I de la seconde moitié du Ve s. av. J.-C. Pour le type, Grace 1953, p. 101-102 ; Ead. 1971, p. 72 ; et Lawall 1995, p. 220-221, fig. 88-89, 93. Chronologie : 450-400 av. J.-C.

Bibliographie : de Marinis 1988, $\mathrm{n}^{\circ} 273.8$, p. 224, fig. $110, \mathrm{n}^{\circ} 8 ;$ Id. 2005, p. 199, n. 133.

\section{FO70 (pl. XXV)}

Sporadique.

Type : Solokha I.

État de conservation : pied. H. max. cons. : 4. Pied tronconique creux, à base en anneau épaissi. Diam. : 8,2. Observations et comparaisons : voir la fiche précédente. Chronologie : 450-400 av. J.-C.

Bibliographie : de Marinis 1988, p. 224, $\mathrm{n}^{\circ}$ 273.9, fig. 110, n $^{\circ} 9 ; I d .2005$, p. 199 , n. 133.

\section{FO71 (pl. XXV)}

Secteur : Secteur V 10 s-t 19-20.

US : 2 .

Type : Solokha I.

État de conservation : pied et partie du fond de la panse. H. max. cons. : 12,1. Pied tronconique creux, à base en anneau épaissi. Diam. : 8.

Surface ext. : rose foncé.

Surface int. : rose saumon.

Inclusions : argile avec inclusions blanches et pierres fines.

Engobe : engobe jaune crème.

Observations et comparaisons : voir la fiche FO69.

Chronologie : 450-400 av. J.-C.

Bibliographie : de Marinis 1988, p. 224, $\mathrm{n}^{\circ}$ 273.10, fig. $110, \mathrm{n}^{\circ} 10 ; I d .2005$, p. 199, n. 133.

\section{FO72 (pl. XXV)}

Secteur : Secteur V 10 m-n 19-20.

Type : Solokha I.

État de conservation : partie inférieure d'amphore, du pied jusqu'à une grande partie de la panse. H. max. cons. : 26,2. Corps vraisemblablement ovoïde. Pied tronconique creux, à base en anneau légèrement épaissi. Diam. : 6,9.
Surface : rose orangé.

Engobe : traces d'engobe.

Inclusions : inclusions blanches, pierres fines et mica, ce dernier en quantité très faible.

Observations et comparaisons : pour le type on renvoie à la fiche FO69. D'après R. de Marinis, outre une anse (pour laquelle cf. de Marinis 2007, fig. 123.2), on pourrait l'attribuer au même individu de la fiche FO75.

Chronologie : 450-400 av. J.-C.

Bibliographie : de Marinis 1988, p. 218, 224, n 273.12, fig. $110, \mathrm{n}^{\circ} 12$; Id. 2005 , p. 199 , n. 133 ; Id. 2007, fig. $123, \mathrm{n}^{\circ} 3$ (en bas).

\section{FO73 (pl. XXV)}

Secteur: Secteur S 17 q 10.

Structure : Maison S 17.

US : 72 .

Type : Solokha I.

État de conservation : pied. H. max. cons. : 4,5. Pied tronconique creux, à base en anneau légèrement épaissi. Diam. min./max. : 7,8.

Observations et comparaisons : défini comme «attique » par R. de Marinis, il provient de la phase de la maison S 17 datée du milieu du Ve s. av. J.-C. Pour le type, Solokha I du milieu ou de la seconde moitié du Ve $\mathrm{s}$. av. J.-C., voir la fiche FO69.

Chronologie : 450-400 av. J.-C.

Bibliographie : de Marinis 1988, p. 224, $\mathrm{n}^{\circ} 273.11$, fig. $110, \mathrm{n}^{\circ} 11$.

\section{FO74 (pl. XXV)}

Secteur: Secteur V 11 p 1.

US : 2 .

Type : Solokha I.

État de conservation : fragment de lèvre et col. H. max. cons. : 7,5. Lèvre à marli oblique, de section triangulaire. Diam. restituable embouchure : 17 . Col cylindrique.

Surface : rose foncé.

Inclusions : argile fine avec des inclusions blanches. Aucune inclusion micacée n'est observable.

Observations et comparaisons : définie d'abord « attique » et ensuite «Solokha I » par R. de Marinis. La lèvre est parfaitement compatible avec le type Solokha I et son bord légèrement replié vers l'extérieur est un indice qui le place au début de la série, vers le milieu ou dans la seconde moitié du V $\mathrm{V}^{\mathrm{e}} \mathrm{s}$. av. J.-C. Pour le type, Lawall 1995, p. 220, fig. 88-92.

Chronologie : 450-400 av. J.-C.

Bibliographie : de Marinis 1988, p. 224, $\mathrm{n}^{\circ} 273.4$, fig. $110, \mathrm{n}^{\circ} 4 ; I d .2005$, p. 199, n. 131.

\section{FO75 (pl. XXV)}

Secteur : Secteur V 10 p 1, m-n 19-20. 

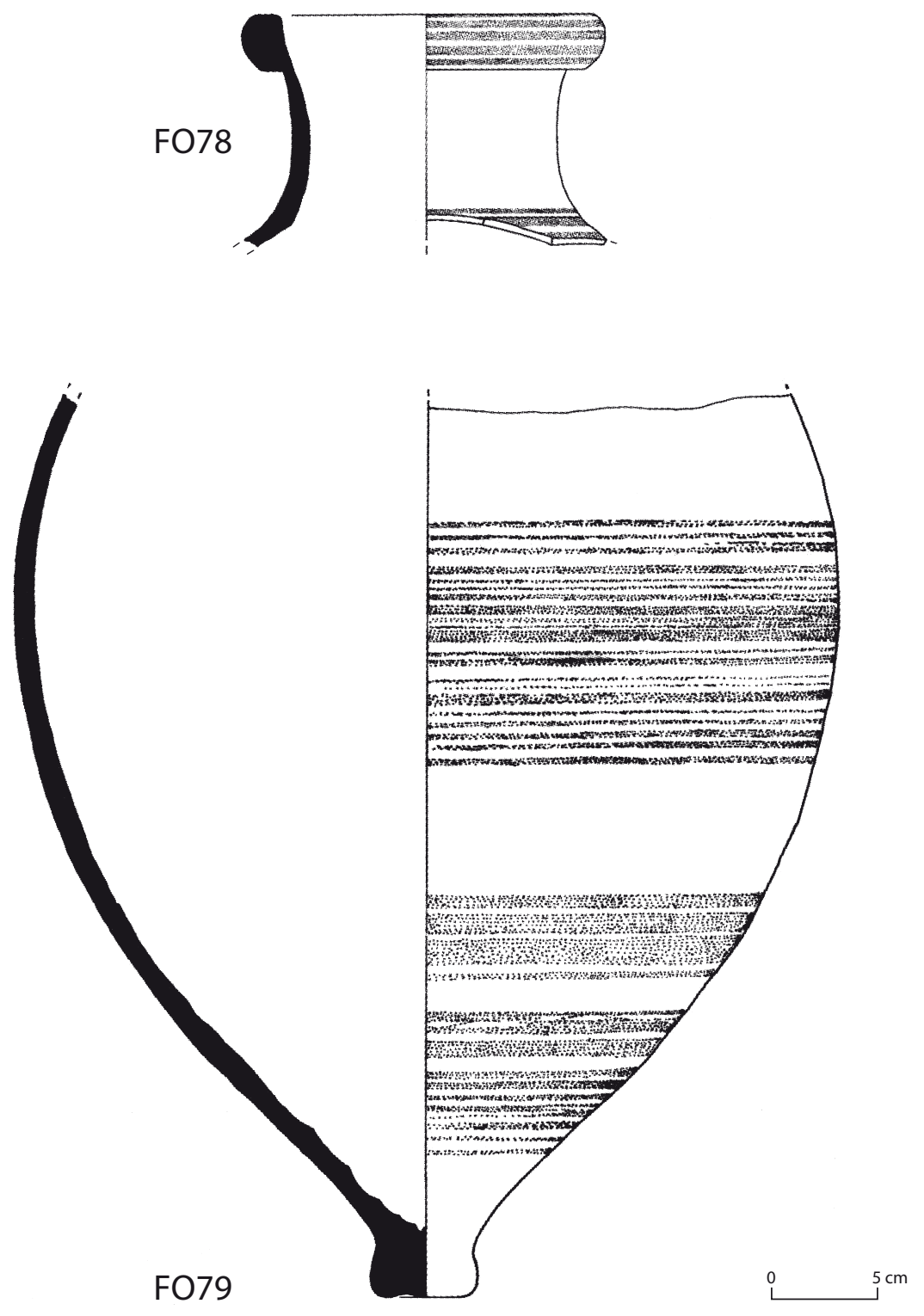

PI. XXVI. Forcello.

US : 2 .

Type : Solokha I.

État de conservation : lèvre fragmentaire avec une partie du col. H. max. cons. : 8,3. Lèvre à marli oblique, de section triangulaire. Diam. restituable embouchure : 16,7. Col cylindrique.

Surface : rose saumon.

Inclusions : argile fine avec inclusions blanches et pierres fines. Aucune inclusion micacée n'est observable. Engobe : engobe jaune clair.

Observations et comparaisons : voir la fiche précédente. R. de Marinis n'exclut pas que, ainsi qu'une anse (pour laquelle cf. de Marinis 2007, fig. 123.2), elle puisse appartenir au même individu de la fiche FO72.
Chronologie : 450-400 av. J.-C.

Bibliographie : de Marinis 1988, p. 224, $\mathrm{n}^{\circ}$ 273.5, fig. $110, \mathrm{n}^{\circ} 5 ;$ Id.2005, p. 199 , n. 131 ; Id.2007, fig. 123 , $\mathrm{n}^{\circ} 1$.

\section{FO76 (pl. XXV)}

Secteur : Secteur R-S 18.

De la couche arable.

Type : Solokha I.

État de conservation : partie de lèvre. H. max. cons. : 3,6 . Lèvre à marli oblique, de section triangulaire. Diam. restituable embouchure : 16,5.

Surface : chamois clair.

Fracture : gris clair. 
Inclusions : argile fine avec petites inclusions blanches, chamotte, pierres fines et, en très faible quantité, mica fin. Engobe : traces d'engobe.

Observations et comparaisons : on renvoie à la fiche FO74.

Chronologie : 450-400 av. J.-C.

Bibliographie : de Marinis 1988, p. 224, $\mathrm{n}^{\circ}$ 273.6, fig. $110, \mathrm{n}^{\circ} 6 ;$ Id. 2005, p. 199 , n. 131 .

\section{FO77}

US : 1058 .

Type : Solokha I.

État de conservation : fragment de lèvre.

Observations et comparaisons : voir la fiche FO74.

Chronologie : 450-400 av. J.-C.

Bibliographie : de Marinis 2005, p. 199, n. 132.

\section{AMPHORES DE TYPE «À LA BROSSE »}

FO78 (pl. XXVI)

Secteur : R $19 \mathrm{~g} 4$.

US : 1910 .

Type : Agora 1502.

État de conservation : fragment de lèvre, col et épaule. Lèvre épaissie, saillante. Col au profil concave. La lèvre et l'épaule sont peintes à coups de pinceau de couleur rouge brun. Le col est achrome.

Observations et comparaisons : exemplaire attribué par R. de Marinis au type « à la brosse », forme Agora 1503 (Sparkes, Talcott 1970, p. 192-193, 341, pl.64), traditionnellement datée du courant des vingt dernières années $\mathrm{du} \mathrm{VI}{ }^{\mathrm{e}}$ s. av. J.-C. ; son contexte de provenance correspond au moment du passage de la phase $\mathrm{F}$ du Forcello (fin $\mathrm{VI}^{\mathrm{e}} \mathrm{s}$. av. J.-C.) à la phase $\mathrm{E}$ (début $\mathrm{V}^{\mathrm{e}} \mathrm{s}$. av. J.-C.). La lèvre est particulièrement saillante et sa hauteur est modérée. Le col, au profil concave, n'est pas évasé. Ces caractéristiques pourraient suggérer un parallèle avec les amphores « à la brosse » de la forme Agora 1502 plutôt qu'avec celles de la forme Agora 1503 (dont la lèvre est plus haute et moins saillante et dont le col est évasé). Les amphores « à la brosse » de la forme Agora 1502 sont datables entre le milieu du VIe s. et 480 av. J.-C.

Chronologie : fin $\mathrm{VI}^{\mathrm{e}}$-début $\mathrm{V}^{\mathrm{e}}$ s. av. J.-C.

Bibliographie : de Marinis 2007, p. 166, fig. 112, $\mathrm{n}^{\circ} 1$.

\section{FO79 (pl. XXVI)}

Secteur : R 18 f 15.

US : 844 .

État de conservation : partie inférieure d'amphore, reconstituée à partir de quatre fragments, ainsi que d'autres fragments de paroi. Pied tronconique. Corps ovoïde. La panse présente un décor de bandes fines en vernis dilué de couleur noirâtre et rouge brun.
Observations et comparaisons : attribuée par R. de Marinis au type «à la brosse », forme Agora 1503 (Sparkes, Talcott 1970, p. 192-193, 341, pl. 64), traditionnellement datée entre 520 et 500 av. J.-C. On observera cependant que le pied en question, tronconique plein, s'éloigne du pied du type Agora 1503, dont le profil est très évasé et dont la base annulaire a un diamètre très ample. La forme de cet exemplaire ne montre pas non plus d'analogies précises avec le type Agora 1502, qui présente un pied au diamètre beaucoup plus réduit, mais toujours de type annulaire. À ma connaissance, pour cette forme d'amphore « à la brosse » il n'existe aucune confrontation possible. Le contexte de provenance (une fosse de décharge de matériel non homogène du point de vue chronologique) ne fournit aucun élément utile à sa datation. Chronologie : fin $\mathrm{VI}^{\mathrm{e}}$-début $\mathrm{V}^{\mathrm{e}}$ s. av. J.-C. (?). Bibliographie : de Marinis 2007,p. 166, fig. 112, $\mathrm{n}^{\circ} 2$.

\section{Analyse des données (graphiques 9-12)}

Les fouilles conduites à partir de 1981 à Bagnolo San Vito, lieu-dit Forcello ??, ou au Forcello, commune de Bagnolo San Vito, près de Mantoue, ont permis de documenter la présence de milliers de fragments d'amphores commerciales grecques, provenant toutes d'un contexte d'habitat. La datation de ce matériel, étudié et publié par R. de Marinis, s'appuie sur des données stratigraphiques sûres. Le nombre d'individus a été estimé à plus d'une centaine (de Marinis 2005, p. 166 ; Id. 2007,p. 159) dont plus de soixante-dix ont été édités dans différentes publications ( $c f$. supra II, $3 \S 1$ ).

La phase d'occupation du site étrusque est comprise entre 540 et les premières années du $\mathrm{IV}^{\mathrm{e}} \mathrm{s}$. av. J.-C. Les importations grecques sont des céramiques attiques datables à partir du troisième quart du $\mathrm{VI}^{\mathrm{e}} \mathrm{s}$. (de Marinis, Rapi 2007, p. 115-156).

C'est à la même période que l'on peut situer le début des importations d'amphores grecques, qui marquent cependant une forte augmentation à partir de la fin $\mathrm{du} \mathrm{VI} \mathrm{I}^{\mathrm{e}}$ et surtout dans le courant du $\mathrm{V}^{\mathrm{e}} \mathrm{s}$. av. J.-C.

Les amphores attestées dans le courant du troisième et surtout du dernier quart du $\mathrm{VI}^{\mathrm{e}} \mathrm{s}$. av. J.-C. peuvent être attribuées aux productions gréco-occidentales archaïques, dans les deux formes «corinthienne B archaïque » (ou « forme $1 \alpha »)$ et « ionio-massaliète » (ou « forme $2 »)$. On peut dater entre la fin du $\mathrm{VI}^{\mathrm{e}}$ et les débuts du $\mathrm{V}^{\mathrm{e}} \mathrm{s}$. av. J.-C. des exemplaires de production nord-égéenne et gréco-orientale (groupe Samos-Milet - cat. FO57 et peut-être déjà Chios - cat. FO47) et une amphore au moins « à la brosse » (cat. FO74).

Les importations amphoriques sont concentrées pour la plupart dans le courant du $\mathrm{V}^{\mathrm{e}} \mathrm{s}$. Un exemplaire 


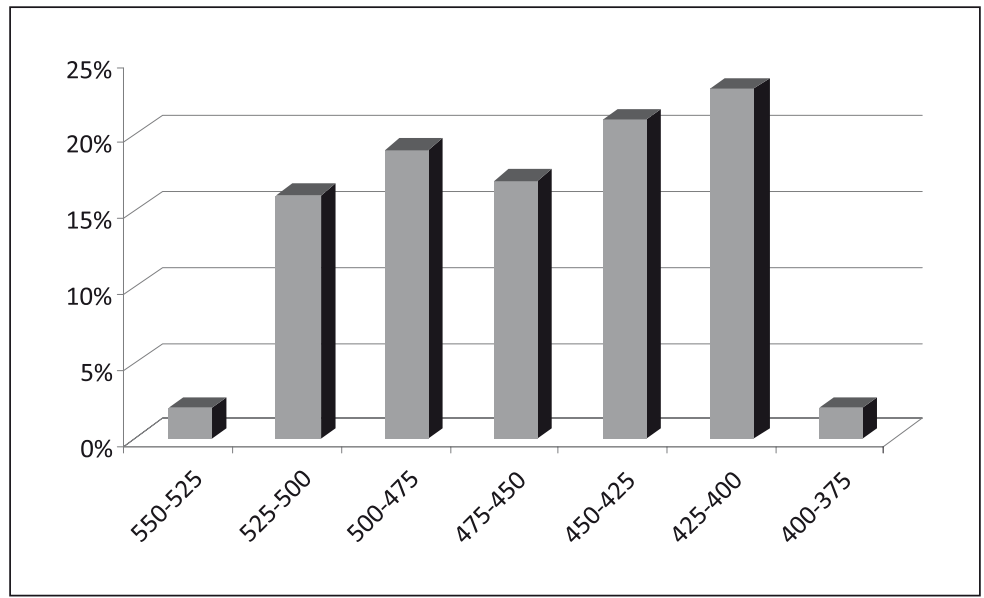

Graphique 9. Forcello : tendance des importations d'amphores grecques dans la période examinée (troisième quart du $\mathrm{Vl}^{\mathrm{e}}$-début du IV $\mathrm{s}$. av. J.-C.).

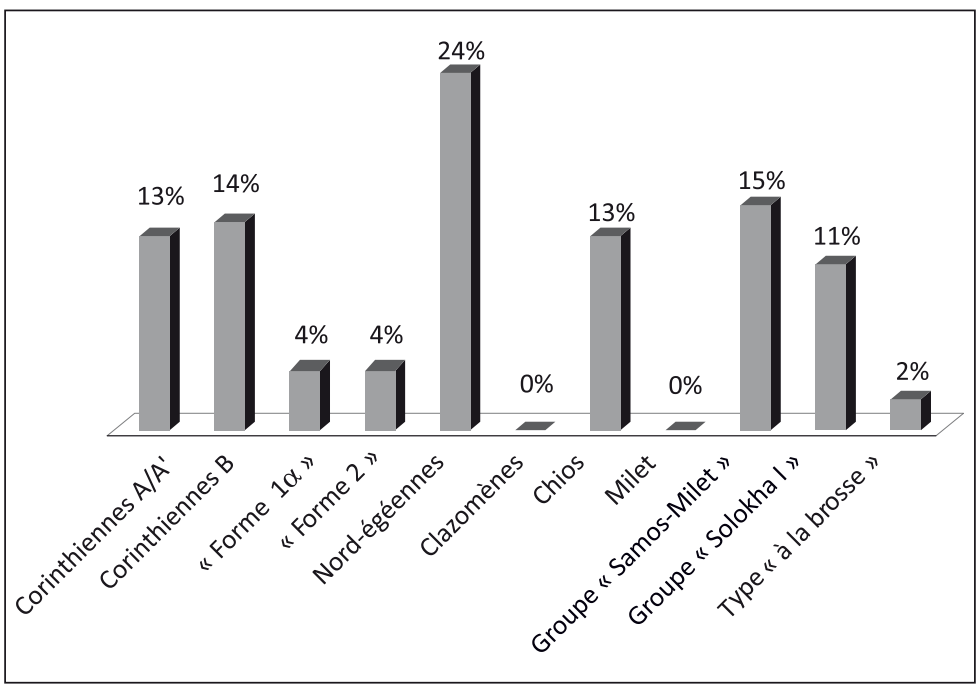

Graphique 10. Forcello : données quantitatives concernant les importations des différents groupes amphoriques dans le courant de toute la période examinée (troisième quart du $\mathrm{Vl}^{\mathrm{e}}$-début du IV ${ }^{\mathrm{e}} \mathrm{s}$. av. J.-C.).

probablement de « forme 3 » de Sourisseau sous presse) a été trouvé encore dans un contexte du deuxième quart du Vé s.av. J.-C. (cat. FO27) et du début du siècle pourrait être daté le fond d'une amphore «à la brosse » dont la forme est tout à fait singulière (cat. FO75). Les productions les plus documentées et les mieux distribuées dans le courant du siècle sont les productions gréco-orientales (Chios, Samos-Milet et le groupe Solokha I), qui sont suivies par les nord-égéennes et enfin par les amphores corinthiennes/corcyréennes $\mathrm{B}$ et par les corinthiennes
A/A' (avec un pourcentage presque équivalent). D'une manière plus détaillée, il est possible d'observer que, dans le cadre des productions gréco-orientales, celle de Chios est particulièrement documentée dans les formes datables avant le troisième quart du $\mathrm{V}^{\mathrm{e}} \mathrm{s}$. av. J.-C. (types « swollen-necked ») en se concentrant surtout dans la première moitié du siècle (types «swollen-necked early bulgy »), alors que, entre la seconde moitié du $\mathrm{V}^{\mathrm{e}}$ et le début du $\mathrm{IV}^{\mathrm{e}} \mathrm{s}$. av. J.-C., est signalée une présence importante d'amphores du groupe Solokha I. 


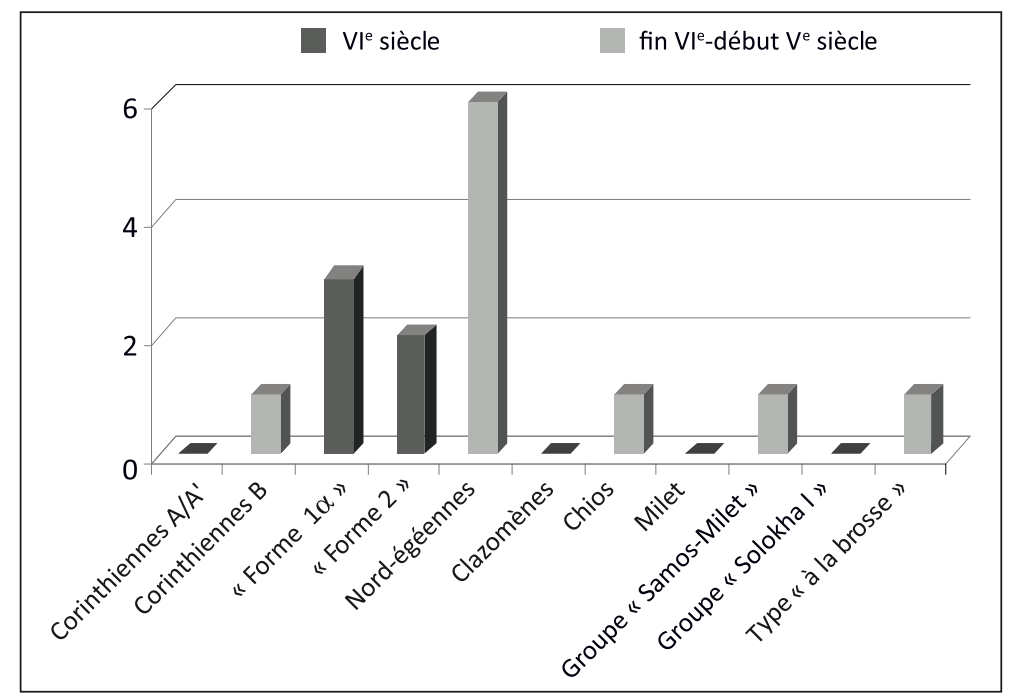

Graphique 11. Forcello: données quantitatives des importations des différents groupes amphoriques entre le troisième quart du $\mathrm{Vl}^{\mathrm{e}}$ et le début du $\mathrm{V}^{\mathrm{S}} \mathrm{s}$. av. J.-C.

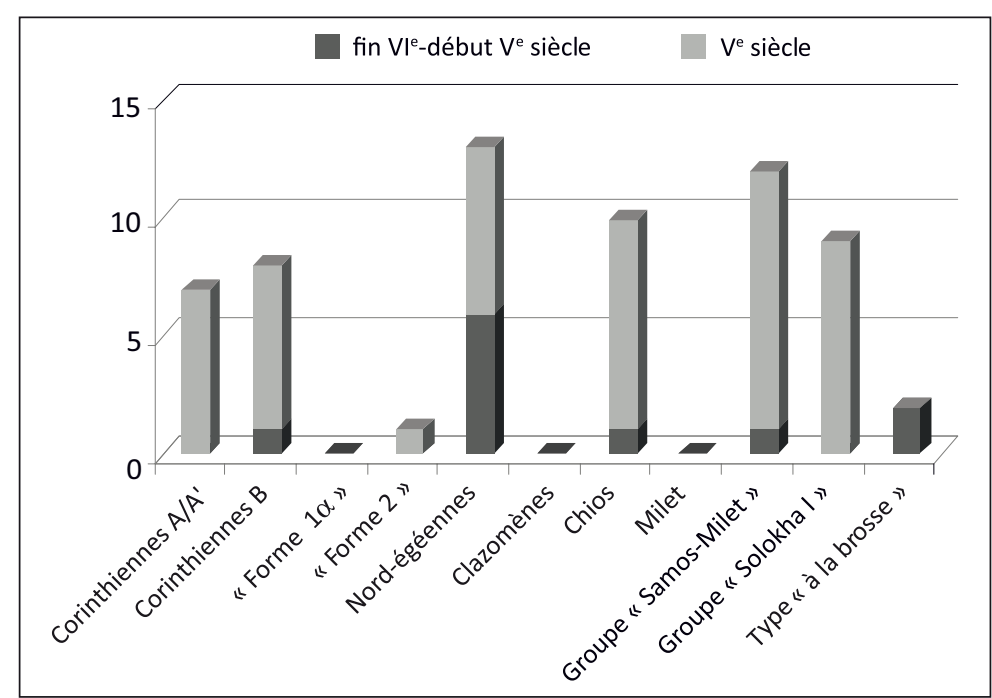

Graphique 12. Forcello : données quantitatives des importations des différents groupes amphoriques entre la fin du $\mathrm{Vl}^{\mathrm{e}}$ et la fin du $\mathrm{V}^{\mathrm{e}} \mathrm{s}$. av. J.-C. 



\section{Chapitre 4}

\section{Bologne/Felsina : l'acropole et l'habitat}

\section{Bilan des études}

Une série de fouilles réalisées dans l'aire de l'ancienne Villa Cassarini, située non loin du siège actuel de la Faculté d'Ingénierie de l'Université de Bologne, a permis de reconnaitre dans ce lieu le site de l'acropole de la ville étrusque de Bologne "Felsina, vocitata tum cum princeps Etruriae esset » (Pline l'Ancien, Nat. Hist. III, 15). de la Certosa à l'ouest et celle des Giardini Margherita, à l'est ${ }^{638}$ (fig. 69 et 85 ).

Les fouilles commencèrent entre 1906 et 1907 sous la direction d'E. Brizio, qui mourut avant d'avoir pu en publier les résultats, et furentreprises en 1963 par le Museo Civico Archeologico de Bologne ; mais ce n'est qu'en 1973, lors des travaux d'agrandissement de la Faculté d'Ingénierie, qu'il fut possible d'y réaliser une fouille

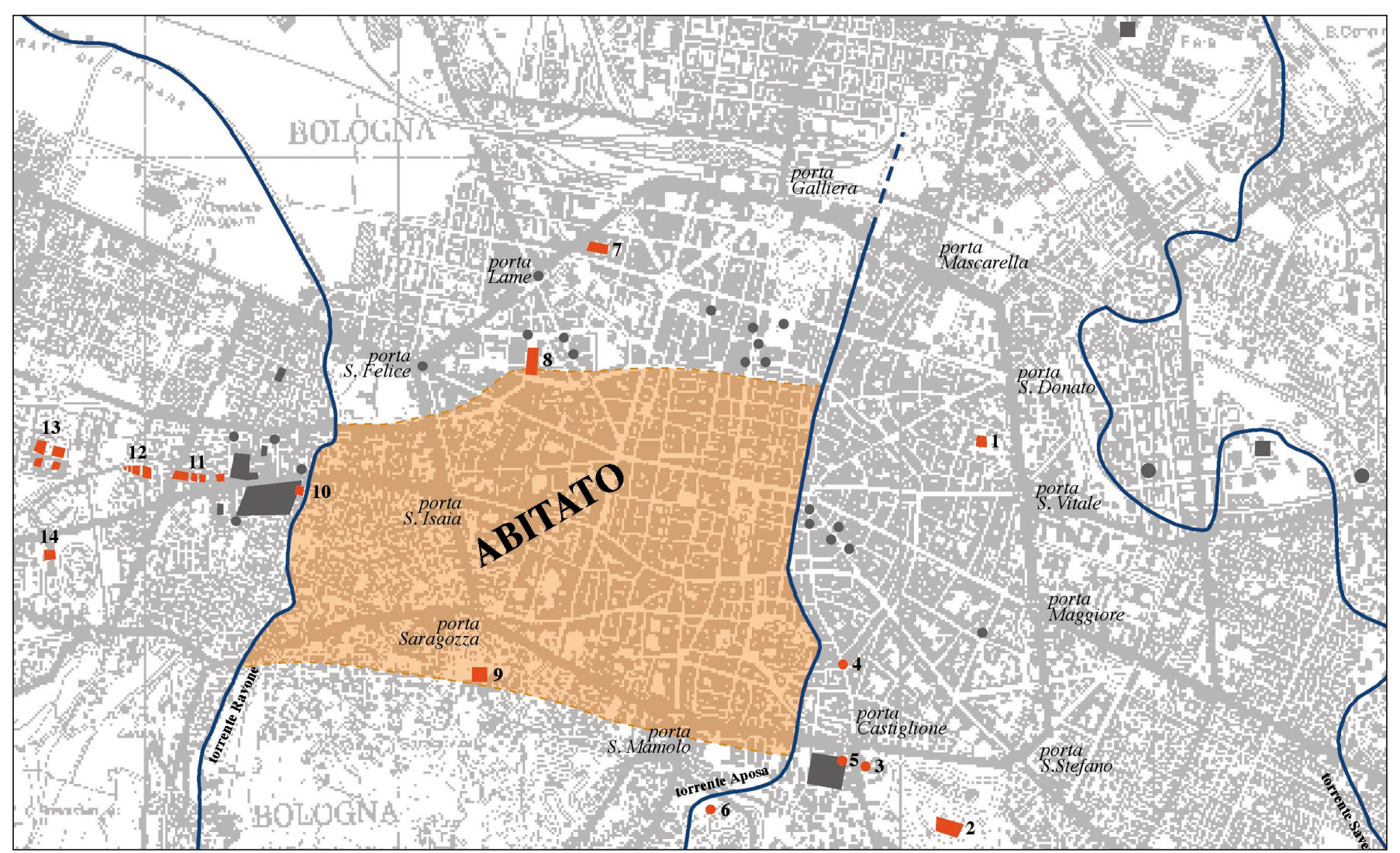

Fig. 85. Plan de la ville de Bologne. En orange le secteur concerné par l'occupation d'époque felsinienne. Au nº 9 l'acropole de Villa Cassarini (๑) Museo Civico Archeologico di Bologna).

Le terrain de l'ancienne Villa Cassarini se situe sur les premières pentes des collines, immédiatement au sud de l'habitat étrusque et presque à mi-chemin entre les deux grandes nécropoles de l'époque de Felsina : celle
638. Concernant l'identification de l'Acropole de Felsina avec les vestiges mis au jour sur les terrains de l'ancienne Villa Cassarini, voir la synthèse de C. Taglioni (Taglioni 1999, p. 63), avec bibliographie. Sur Bologne étrusque, consulter de façon générale Sassatelli, Donati 2005, avec références bibliographiques. 


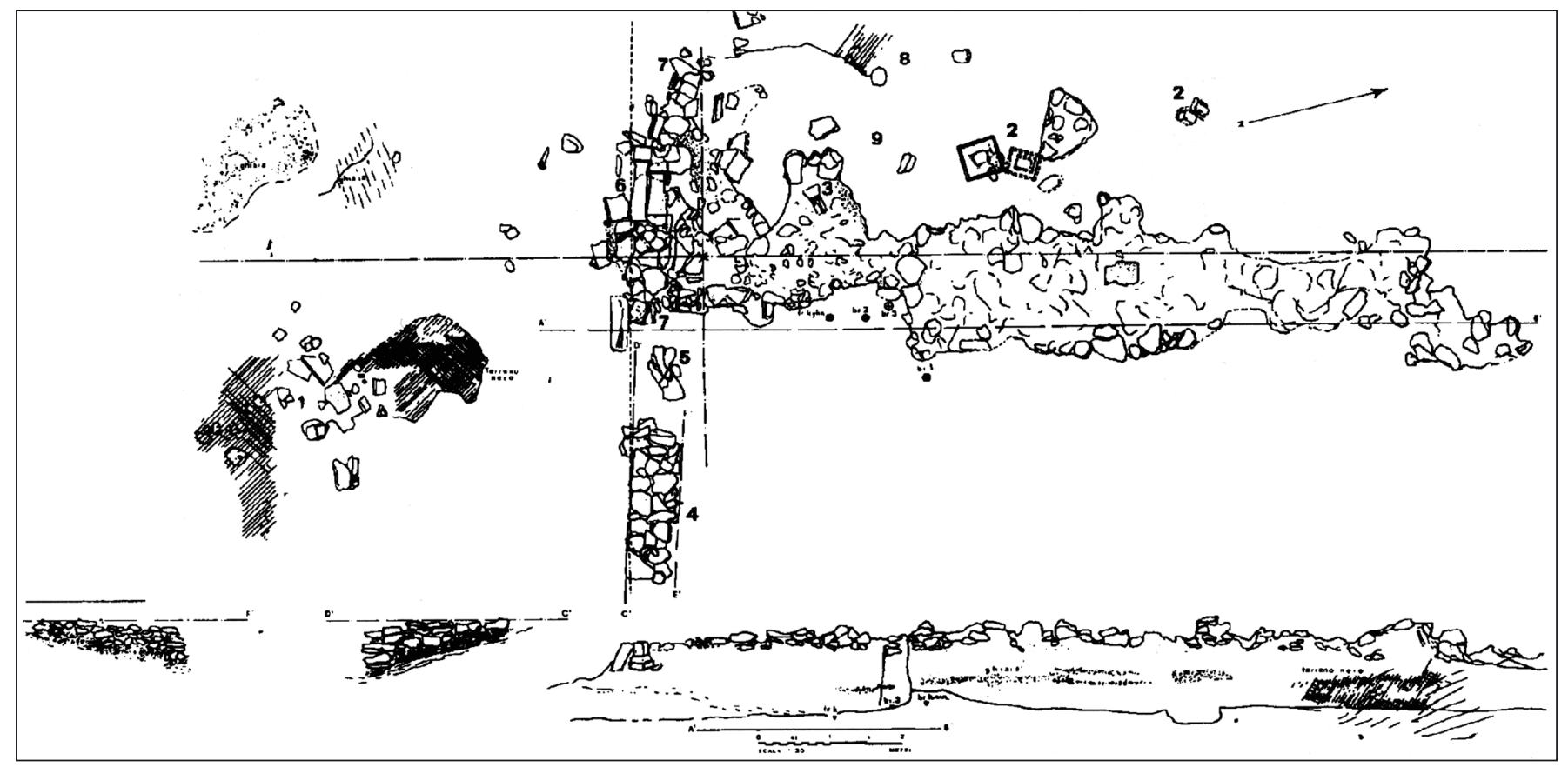

Fig. 86. Bologne, Villa Cassarini, planimétrie de la fouille Gualandi (d'après Gualandi 1975, fig. 1).

extensive ${ }^{639}$. L'intervention archéologique d'urgence fut conduite par l'Institut d'Archéologie de l'Université de Bologne en collaboration avec la Soprintendenza archeologica d'Émilie-Romagne. On releva ainsi, sur ce site, trois phases superposées d'occupation : la première se référant à une installation collinaire de l'Âge du Bronze tardif ; la seconde de l'époque villanovienne ; et la troisième, plus récente et plus consistante, relative à la phase de Felsina. Les structures attribuables à cette dernière phase se limitaient à quelques pans de murs construits en petites pierres sèches et éclats de grès, ainsi que des traces de galets du dallage routier (fig. 86); mais leur état de dégradation ne permettait d'indiquer ni le nombre, ni la planimétrie, ni l'orientation exacte des édifices dont l'agencement architectural même était loin d'être clair ${ }^{640}$. L'hypothèse de la destination sacrée de cette aire repose sur la découverte de nombreux exvoto en bronze, parmi lesquels les petits bronzes bien

639. Sur les fouilles de 1906-1907, il n'existe que quelques rapports manuscrits d'E. Brizio, conservés aux Archives du Musée Civique Archéologique de Bologne (Sassatelli 1986, note 60), dont R. Pettazzoni a publié un bref compte rendu (Pettazzoni 1917, cc. 221-308). La documentation des fouilles de ces années-là a été ensuite réexaminée par A. Ammirati et G. Morico (Ammirati, Morico 1984, p. 72-99). Pour la campagne de fouille de courte durée de 1963, voir Gualandi 1970, p. 54-58; pour l'intervention plus importante de 1973, cf. notamment Gualandi 1975, p. 316-345.

640. À propos des difficultés concernant la restitution planimétrique et architecturale des structures du sanctuaire, voir Sassatelli 1986, p. 38-41. connus représentant Héraclès avec les pommes d'or du jardin des Hespérides et Apollon avec sa lyre. Outre les ex-voto, est également attestée la présence des bases habituelles en pierre de travertin moulurées qui leur servaient de support, ainsi que celle de graffiti votifs sur des kylikes attiques à figures rouges ${ }^{641}$. L'intervention d'urgence menée en 1973, sous la direction de G. Gualandi, a fait l'objet de quelques publications, mais la majeure partie du matériel reste encore inédit.

Grâce à la disponibilité de la Soprintendenza archeologica de l'Émilie-Romagne et du Museo Civico Archeologico de Bologne, il m'a été possible d'accéder au mobilier découvert au cours des différentes fouilles sur l'aire de l'acropole de Bologne ; ce qui m'a permis de découvrir une cinquantaine de fragments d'amphores de transport grecques correspondant à pas moins d'une douzaine d'individus. Il semble qu'aucune amphore commerciale ne provienne des contextes des riches nécropoles de la phase felsinienne étudiés jusqu'à présent, dont les seuls publiés sont ceux de l'ensemble sépulcral Arnoaldi (Macellari 2002).

En ce qui concerne l'habitat d'époque étrusque (Taglioni 1999), au-dessus duquel s'élève le centre historique de Bologne, un fragment d'amphore commerciale grecque provient des fouilles de Via Santa Caterina

641. Sur les ex-voto de la Villa Cassarini, voir en général Gualandi 1974. Pour les inscriptions de caractère votif documentées dans la Villa Cassarini, Sassatelli 1986, p. 39. 
(Baldoni, Morico,Pini 2007,p. 65) entreprises entre 1988 et 1993 dans le secteur méridional de l'aire occupée par la cité de Felsina (Malnati, Violante 1995, p. 107). Trois autres individus proviennent des fouilles de Viale Aldini, sur les pentes de l'acropole de la Villa Cassarini, et ont été attribués au sanctuaire situé juste au-dessus (Calastri, Desantis 2011, p. 205-206). Il faut toutefois rappeler que nombreuses sont les fouilles menées ces dernières années dans le centre urbain de Bologne (Malnati 2011) dont le mobilier est encore entièrement inédit, ce qui rend impossible toute vérification.

Les amphores de Bologne/Felsina sont indiquées dans le catalogue qui suit par le sigle «FE».

\section{Catalogue}

\section{AMPHORES DE TYPE CORINTHIEN A/A'}

\section{FE1 (pl. XXVII)}

Lieu de découverte : Bologne, Viale Aldini. Année de la fouille / trouvaille : 2007-2008.

US : 229 .

État de conservation : fragment de lèvre. Lèvre à marli de section triangulaire.

Observations et comparaisons : attribué par les éditeurs à une amphore corinthienne de type A ou A' .

Chronologie : Ve s. av. J.-C.

Bibliographie : Calastri, Desantis 2011, p. 198, 205-206, n. 22 , fig. $6, n^{\circ} 3$.

\section{FE2 (pl. XXVII)}

Lieu de découverte : Bologne, Viale Aldini.

Année de la fouille / trouvaille : 2007-2008.

US : 229 .

État de conservation : fragment d'anse.

Observations et comparaisons : attribué par les éditeurs à une amphore corinthienne de type A ou A' .

Chronologie : non déterminable.

Bibliographie : Calastri, Desantis 2011,p. 198, 205-206, n. 22 , fig. $6, \mathrm{n}^{\circ} 2$.

\section{AMPHORES GRÉCO-OCCIDENTALES}

\section{FE3 (pl. XXVII)}

Lieu de découverte : Bologne, Villa Cassarini.

Année de la fouille / trouvaille : 1973.

Secteur : Zone D partie Nord.

Inventaire : $\mathrm{n}^{\circ} 41891$.

État de conservation : fragment de lèvre avec une partie de col conservant les traces de l'attache supérieure d'une anse. H. max. cons. : 6,4. Lèvre en échine. H. : 2,2 ; épaiss. max. : 2,4 ; diam. int./ext. restituable embouchure : 12 / 16,8. Col évasé qui présente une fine rainure immédiatement au-dessous de la lèvre. H. max. cons. : 4,2 ; diam. min./max. restituable : 11,8/ 13.

Surface ext., int. et fracture : jaune très clair (2.5YR8/ 2-2.5Y7/3).

Inclusions : inclusions blanches, grises et orange, de petites dimensions, à fréquence basse, distribuées de manière homogène. Mica argenté fin, à fréquence basse. Engobe : traces d'engobe.

Observations et comparaisons : la morphologie de la lèvre indique son appartenance à la production corinthienne du type $\mathrm{B}$ du $\mathrm{V}^{\mathrm{e}}$ s. av. J.-C. Ses dimensions réduites ne permettent pas d'en préciser ultérieurement la chronologie. Pour le type, Koehler 1978a, fig. 2.b-c ; et Ead.1981, fig. 1.a, pl. 99.c., datables entre le deuxième et le dernier quart du Ve s. av. J.-C.

Chronologie : 480-400 av. J.-C.

\section{AMPHORES DE L'ÉGÉE SEPTENTRIONALE}

\section{FE4 (pl. XXVII)}

Lieu de découverte : Bologne, Viale Aldini. Année de la fouille / trouvaille : 2007-2008.

US : 229.

Type : nord-égéenne, Late Mendean Variant Lawall.

État de conservation : partie de col et anse.

Observations et comparaisons : attribuée par les chercheurs qui l'ont publiée à une amphore de Mendé. $\mathrm{Vu}$ les proportions du col et de l'anse, je crois qu'on peut l'encadrer vraisemblablement dans la Middle Mendean Variant de Mark Lawall, datable du dernier quart du $\mathrm{V}^{\mathrm{e}} \mathrm{s}$. av. J.-C. Pour le type, Lawall 1995, fig. 39-40.

Chronologie : fin $\mathrm{V}^{\mathrm{e}} \mathrm{s}$. av. J.-C.

Bibliographie : Calastri, Desantis 2011, p. 198, 205-206, n. 21 , fig. $6, \mathrm{n}^{\circ} 1$.

\section{FE5 (pl. XXVII)}

Lieu de découverte : Bologne, Villa Cassarini.

Année de la fouille / trouvaille : 1964.

Secteur : Via Vallescura - zone A.

Inventaire : $n^{\circ} 6583$.

État de conservation : fragment de lèvre avec un court segment du col portant la trace de la jonction supérieure d'une anse. H. max. cons. : 2,3. Lèvre en échine, ouverte. H. : 2 ; épaiss. max. : $1,7$.

Surface ext. : chamois brun.

Surface int. et fracture : orange intense.

Inclusions : inclusions blanches, de petites dimensions, à fréquence haute, distribuées d'une manière homogène. Mica fin répandu.

Observations et comparaisons : la petite lèvre en échine légèrement ouverte ainsi que les caractéristiques de l'argile suggèrent de situer l'exemplaire vraisemblablement 
dans le cadre des productions nord-égéennes, peutêtre de Mendé, de la fin du $\mathrm{V}^{\mathrm{e}}$ ou du début du $\mathrm{IV}^{\mathrm{e}} \mathrm{s}$. av. J.-C. Pour le type, Lawall 1995, fig. 40 (Late Mendean variant, datable du dernier quart du $\mathrm{V}^{\mathrm{e}} \mathrm{s}$. av. J.-C.). Pour la forme de la lèvre, se reporter aussi à Eiseman 1973, fig. 1 ('Type 1C' de l'épave de Porticello); Monachov 1999b, fig. 29 ; Monachov 2003a, pl. 62, 4 (datables déjà du début du IVe s. av. J.-C.).

Chronologie : fin $V^{e}$-début IV s. av. J.-C.

\section{AMPHORES DE L'ÉGÉE ORIENTALE}

\section{FE6 (pl. XXVII)}

Lieu de découverte : Bologne, Via Santa Caterina. Année de la fouille / trouvaille : 1988-1993.

Type : Samos-Milet.

État de conservation : fragment de lèvre et col. Dimensions : non repérées. Lèvre en amande, épaissie dans sa partie supérieure. Anses en ruban épaissi, de section elliptique. Col au profil concave.

Observations et comparaisons : attribué par D. Baldoni à une amphore gréco-orientale, le fragment, provenant de la IV ${ }^{e}$ phase d'implantation d'habitat détectée dans l'aire de la Via Santa Caterina, est datable entre la fin du $\mathrm{VI}^{\mathrm{e}}$ et le milieu environ du Ve $\mathrm{S}$. av. J.-C. Il peut appartenir à une amphore du groupe Samos-Milet. La fracture du col, au niveau de la jonction de l'épaule, empêche de vérifier soit la présence du listel caractérisant les formes de fin $\mathrm{VI}^{\mathrm{e}}$-premier quart du $\mathrm{V}^{\mathrm{e}} \mathrm{s}$. des types $\mathrm{S} / 1$ et $\mathrm{S} / 3$ de $\mathrm{M}$. Lawall (Lawall 1995, p. 178-179, fig. 77 ; p. 184-185, fig. 76), soit son absence, ce qui était le cas pour la forme contemporaine du type $\mathrm{S} / 2$ (Kigge 1976, pl. 49.3 ; Lawall 1995, p. 181-182). Ses proportions permettent en tout cas de situer l'exemplaire dans le cadre des formes datables entre la fin du VIe et le premier quart du Ve $\mathrm{s}$. av. J.-C.

Chronologie : 525-480 av. J.-C.

Bibliographie : Baldoni, Morico, Pini 2007, p. 65, fig. 13, 83 .

\section{FE7 (pl. XXVII)}

Lieu de découverte : Bologne, Villa Cassarini.

Année de la fouille / trouvaille : 1973.

Secteur : Zone D, partie Nord.

Inventaire : $\mathrm{n}^{\circ} 41974$.

Type : Solokha I.

État de conservation : fragment de lèvre. H. max. cons. : 3,6. Lèvre à marli oblique, de section triangulaire, ouverte. H. : 3 , 1 ; épaiss. max. : 2 ; diam. int./ext. restituable embouchure : $12,8 / 16,4$.

Surface ext., int. et fracture orange (7.5YR6/6).

Inclusions : inclusions blanches, brun gris et orange, de dimensions petites et moyennes, à fréquence moyennebasse, distribuées d'une manière homogène. Écailles de mica doré, de dimensions petites et moyennes, à fréquence moyenne-haute. Surface vacuolaire.

Engobe : traces d'engobe crème.

Observations et comparaisons : attribuable à une amphore du groupe Solokha I du début de la série (seconde moitié du Ve s. av. J.-C.). Pour le type, Lawall 1995, p. 220-221, fig. 89-90, respectivement du dépôt $S 16: 1$ de l'Agora d'Athènes, fermé vers 410 av. J.-C., et du dépôt H 13 :4, fermé vers 390 av. J.-C.

Chronologie : 450-400 av. J.-C.

\section{FE8 (pl. XXVII)}

Lieu de découverte : Bologne, Villa Cassarini.

Année de la fouille / trouvaille : 1973.

Secteur : Sondage 1.

Inventaire : $n^{\circ} 41956$.

Type : Solokha I.

État de conservation : embouchure complète, reconstituée à partir de quatre fragments, et une petite portion du col. H. max. cons. : 4. Lèvre à marli oblique, de section triangulaire, ouverte. H. : 2,1 ; épaiss. max. : 2,2 ; diam. int./ext. embouchure : 12,6 / 16,6. Col évasé. H. max. cons. : 2 ; diam. min./max. restituable : $10 / 11,1$.

Surface ext. et int. : rose clair (5YR7/4).

Fracture : rose orange (5YR7/6).

Inclusions : inclusions blanches de petites dimensions, à fréquence basse, distribuées d'une manière homogène, et inclusions de couleur brun gris et orange de dimensions petites et moyennes, à fréquence moyenne-basse, distribuées d'une manière homogène. Mica argenté fin, à fréquence basse.

Engobe : engobe jaune clair, compact et très homogène. Observations et comparaisons : voir la fiche précédente. Chronologie : 450-400 av. J.-C.

\section{FE9 (pl. XXVII)}

Lieu de découverte : Bologne, Villa Cassarini.

Année de la fouille / trouvaille : 1973.

Secteur: Secteur 1. Sondage 1. Prof. $1.09 \mathrm{~m}$.

Inventaire : $\mathrm{n}^{\circ} 41945$.

Type : Solokha I.

État de conservation : embouchure, conservée sur presque la moitié de sa circonférence, reconstituée à partir de trois fragments : H. max. cons. : 3,6. Lèvre à marli oblique, de section triangulaire. À la base du bord, un fin listel. H. : 2,1 ; épaiss. max. : 2,8 ; diam. int./ext. restituable embouchure : 12,4 / 17,2.

Surface ext. et int. : orange chamois (7.5YR6/6).

Fracture : orange brun (5YR5/6).

Inclusions : argile assez dépurée avec inclusions blanches et brunes (chamotte) de petites dimensions, à fréquence moyenne. Mica fin argenté et doré à fréquence moyenne. Vacuoles. 


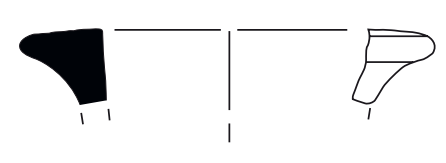

FE1

échelle inconnue

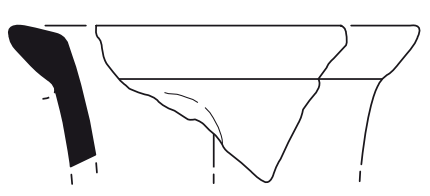

FE3

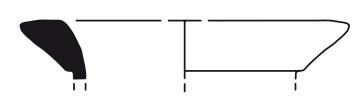

FE5

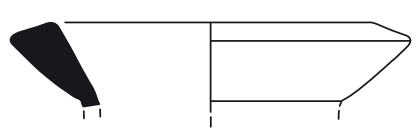

FE7

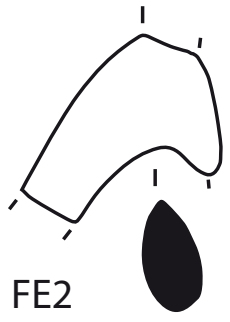

échelle inconnue

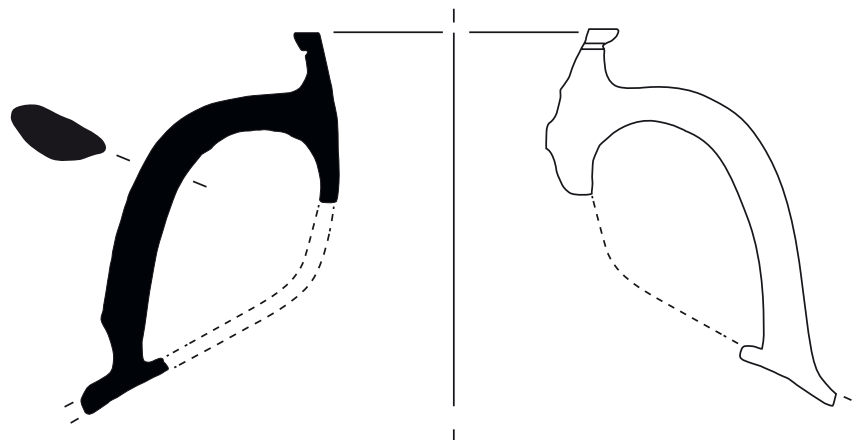

FE4

échelle inconnue

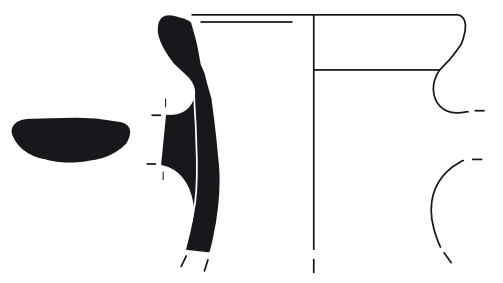

FE6

échelle inconnue

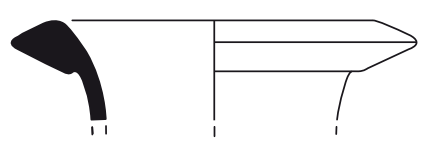

FE8

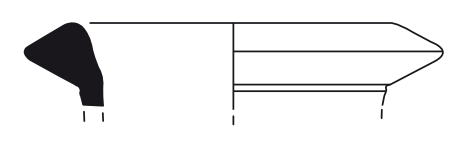

FE9

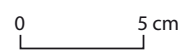

PI. XXVII. Felsina. 
Observations et comparaisons : voir la fiche FE7.

Chronologie : 450-400 av. J.-C.

\section{FE10 (pl. XXVIII)}

Lieu de découverte : Bologne, Villa Cassarini.

Année de la fouille / trouvaille : 1973.

Secteur : Secteur D au Sud.

Inventaire : $\mathrm{n}^{\circ} 41615$.

Type : Solokha I.

État de conservation : partie de lèvre, reconstituée à partir de quatre fragments, et de col, reconstitué à partir de trois fragments, un fragment de col avec la jonction de l'épaule, un fragment de l'épaule avec la jonction inférieure de l'anse, et 7 fragments de paroi, non reconstituables mais presque certainement appartenant au même individu. H. max. cons. : 17. Lèvre à marli oblique, de section triangulaire, ouverte. H. : 2,4 ; épaiss. max. : 2,3 ; diam. int./ext. restituable embouchure : 14,2 / 17,2. Col cylindrique. H. max. cons. : 12 ; diam. min./max. : 12. Anses en ruban épaissi, à section elliptique, avec une impression digitale au niveau de la jonction inférieure. H. max. cons. : 4,5 ; épaiss. max. : 4 x 4,5. Épaule arrondie. Larg. max. cons. : 7,5.

Surface ext., int. et fracture : beige (10YR8/4-10YR7/4). Inclusions : inclusions blanches de petites dimensions, à basse fréquence, distribuées d'une manière homogène et inclusions de couleur gris brun et noisette de dimensions petites et moyennes, à fréquence moyenne-basse, distribuées d'une manière homogène. Mica fin argenté.

Engobe : traces d'engobe mince, blanchâtre.

Observations et comparaisons : pour le type, au col allongé, Lawall 1995, p. 220-221, fig. 90 (du dépôt H 13:4 de l'Agora d'Athènes, fermé vers 390 av. J.-C.). Pour la forme, on renvoie à cat. SP34.

Chronologie : 410-390 av. J.-C.

\section{FE11 (fig. 87 ; pl. XXVIII)}

Lieu de découverte : Bologne, Villa Cassarini.

Année de la fouille / trouvaille : 1973.

Secteur: Sondage 1.

Inventaire : $\mathrm{n}^{\circ} 41955$.

Type : Solokha I.

État de conservation : partie de lèvre, col et une anse complète, reconstitués à partir de 11 fragments. H. max. cons. : 17,5. Lèvre à marli oblique, de section triangulaire, ouverte. H. : 2,1 ; épaiss. max.: 2,3 ; diam. int./ext. restituable embouchure : 12,8/16,8. Col évasé. H. max. cons. : 10 ; diam. min./max. restituable : 10,3 / 11,3. Anses verticales, en ruban épaissi, à section elliptique. H. : 15 ; épaiss. $\max .: 2,3 \times 4,3$.

Surface ext., int.et fracture orange (7.5YR6/6-7.5YR7/6). Inclusions : inclusions blanches, brun gris et orange, de dimensions petites et moyennes, à fréquence

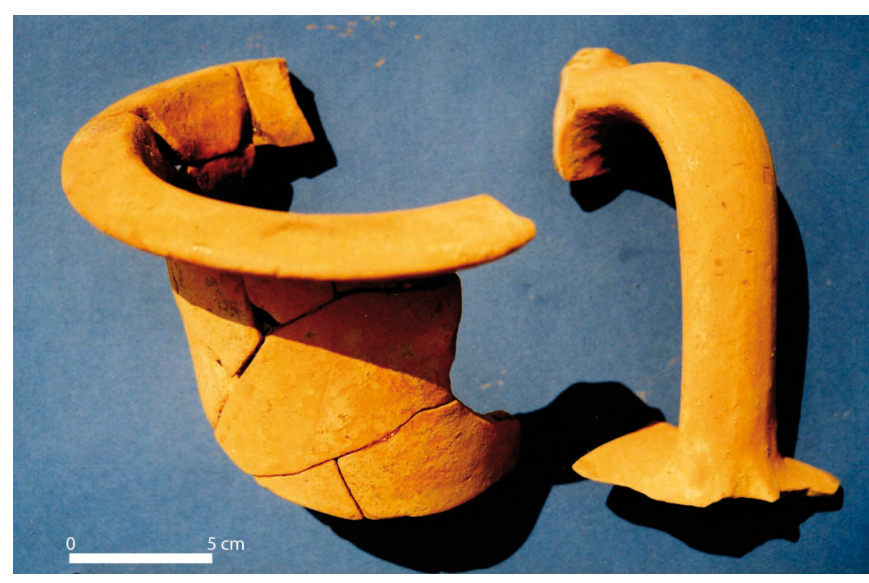

Fig. 87. Bologne, Villa Cassarini, col d'amphore du groupe Solokha I. Cat. FE11 (photo F. Sacchetti).

moyenne-basse, distribuées d'une manière homogène. Mica doré fin, à fréquence basse. Surface vacuolaire.

Engobe : traces d'engobe mince, couleur crème.

Observations et comparaisons : pour le type, voir la fiche précédente.

Chronologie : 410-390 av. J.-C.

\section{FE12 (pl. XXVIII)}

Lieu de découverte : Bologne, Villa Cassarini.

Année de la fouille / trouvaille : mars 1907.

Secteur : « zone du temple».

Inventaire : $\mathrm{n}^{\circ} 4317$.

Type : Solokha I.

État de conservation : pied et partie inférieure de la panse, reconstitués à partir de deux fragments. H. max . cons. : 11. Pied tronconique à base annulaire. H. : 3,3. Diam. max. : 7,5.

Surface ext. et int. : jaunâtre.

Fracture : rose chamois.

Engobe : traces d'engobe clair.

Inclusions : argile dépurée, avec inclusions blanches et noirâtres de dimensions petites et moyennes, à fréquence moyenne-basse. Vacuoles.

Observations et comparaisons : attribuable à une amphore du type Solokha I du début de la série. Pour le type, Lawall 1995, p. 220-221, fig. 89-90, 93 (datables du courant de la seconde moitié du Ve s. av. J.-C.). Sur le fond extérieur, on observe une concentration d'inclusions, qui est fréquente dans ce type d'amphores aussi bien de l'Italie du nord (cf. cat. FE13, MA104, MA105, MA106), que de l'Agora d'Athènes (Lawall 1995, p. 220-221 et notes 5, 8-9, fig. 93). Sur la surface extérieure du pied on remarque des zones noircies sans doute à cause de phénomènes post-dépositionnels.

Chronologie : 450-400 av. J.-C. 


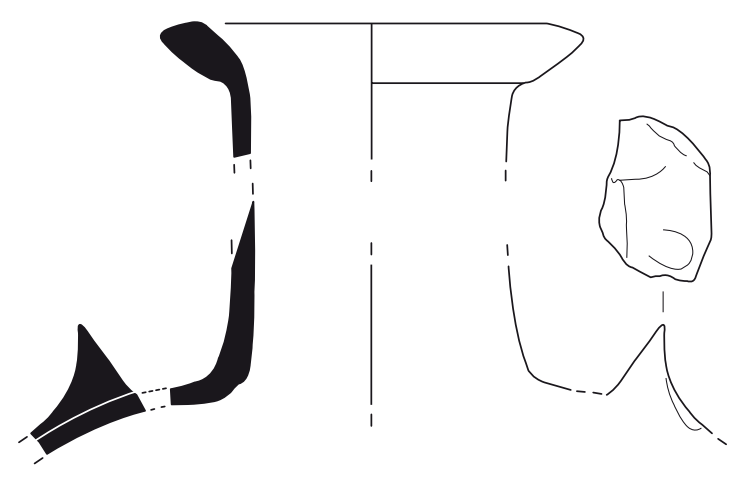

FE10

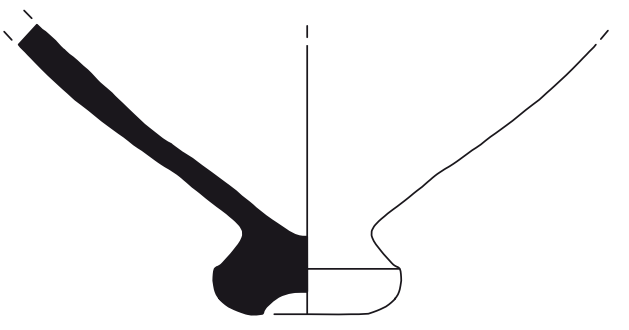

FE12

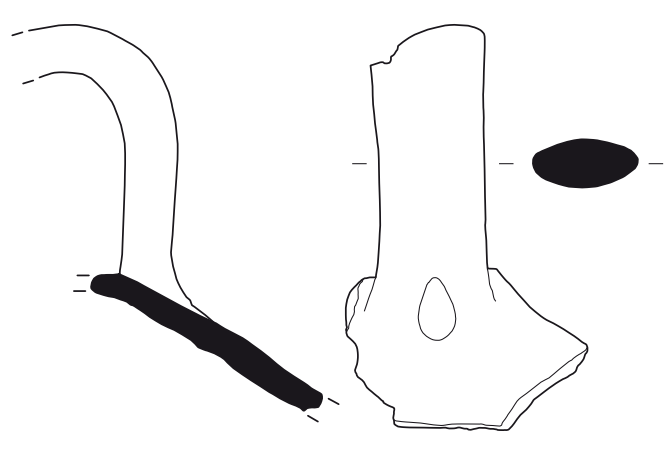

FE15

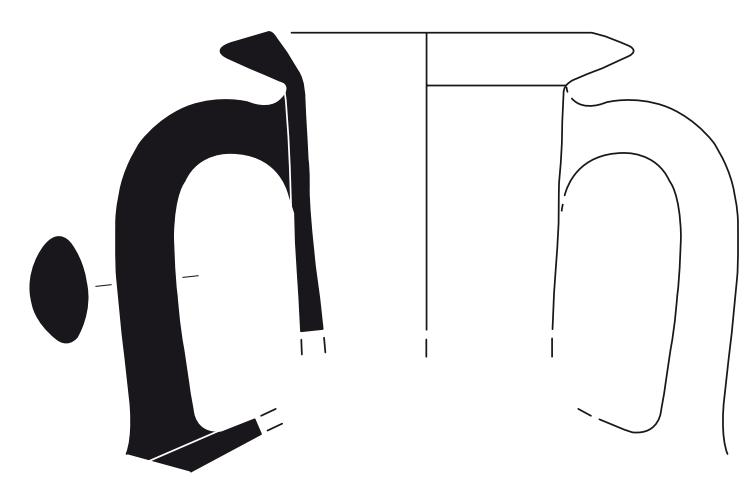

FE11

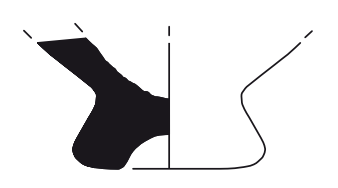

FE13

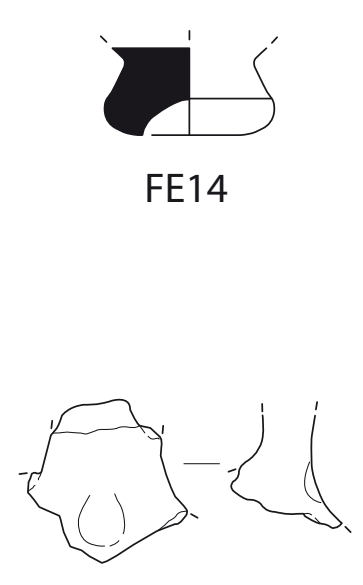

FE16 


\section{FE13 (pl. XXVIII)}

Lieu de découverte : Bologne, Villa Cassarini.

Année de la fouille / trouvaille : 1912 (?).

Secteur : « zone du temple».

Inventaire : $n^{\circ} 4379$.

État de conservation : pied et une petite partie du fond de la panse. H. max. cons. : 5. Pied tronconique à base en anneau. H. : 3. Diam. max. : 7,9.

Surface ext. et int. : chamois.

Fracture : orange.

Engobe : traces d'engobe blanchâtre.

Inclusions : argile dépurée, avec inclusions blanches et noirâtres de dimensions petites et moyennes, à fréquence basse.

Observations et comparaisons : pour le type, on renvoie à la fiche précédente. Comme dans l'exemplaire qui précède, ici aussi on remarque sur le fond extérieur une concentration d'inclusions.

Chronologie : 450-400 av. J.-C.

\section{FE14 (pl. XXVIII)}

Lieu de découverte : Bologne, Villa Cassarini.

Année de la fouille / trouvaille : 1912 (?).

Secteur : «zone du temple».

Inventaire : $n^{\circ} 4380$.

Type : Solokha I.

État de conservation : pied. H. max. cons. : 4,3. Pied tronconique à base en anneau. H. : 3. Diam. max. : 7,7 Surface ext. et int. : brun noirâtre.

Fracture : gris.

Inclusions : argile dépurée, avec inclusions blanches et noirâtres de dimensions petites et moyennes, à fréquence basse. Mica fin à fréquence basse particulièrement concentré sur le fond extérieur.

Observations et comparaisons : pour le type, voir cat. FE9. La couleur de la pâte céramique (virant au gris) de cet exemplaire est sans doute le résultat de phénomènes post-dépositionnels et plus exactement de la permanence dans un milieu humide, comme semble le confirmer la présence d'incrustations calcaires en surface.

Chronologie : 450-400 av. J.-C.

\section{FE15 (pl. XXVIII)}

Lieu de découverte : Bologne, Villa Cassarini.

Année de la fouille / trouvaille : 1964.

Secteur : Via Vallescura - II carrière. Zone I - couche B. Inventaire : $n^{\circ} 4820$.

Type : Solokha I.

État de conservation : anse, conservée de la courbure jusqu'à l'attache inférieure, brève partie de l'épaule et deux amples fragments de paroi (inventaire $n^{\circ} 4819$ ), qu'il n'est pas possible de reconstituer mais qui appartiennent sans doute au même individu. H. max. cons. :
16. Anses en ruban épaissi, à section elliptique, avec une impression digitale à la base. H. : 10,5 ; épaiss. : 4,4 x 2,2. Surface ext. et int. : beige rosé.

Fracture : orange chamois.

Inclusions : argile dépurée, avec inclusions blanches et noirâtres de dimensions petites et moyennes, à fréquence basse. Rares et fines écailles de mica.

Observations et comparaisons : attribuable à une amphore du groupe Solokha I par la particularité de l'impression digitale à la base de la jonction inférieure. La hauteur encore modérée de l'anse suggère son encadrement chronologique avant la fin du $\mathrm{V}^{\mathrm{e}}$ s. av. J.-C. Pour le type, Lawall 1995, p. 220-221, fig. 91-92 (des dépôts de l'Agora d'Athènes R $13: 1$ et $\mathrm{B} 13: 6$, fermés vers 400 av. J.-C.).

Chronologie : 450-400 av. J.-C.

\section{FE16 (pl. XXVIII)}

Lieu de découverte : Bologne, Villa Cassarini.

Année de la fouille / trouvaille : 1973.

Secteur : Secteur D au nord du mur dit 'mur B'.

Inventaire : $n^{\circ} 42064$.

Type : Solokha I.

État de conservation : fragment d'anse, au niveau de la jonction inférieure, avec impression digitale. H. max. cons. : 4,5. Anses en ruban épaissi, à section elliptique, avec impression digitale à la base.

Surface ext., int. et fracture orange noisette (7.5YR7/6-10YR6/4).

Inclusions : argile dépurée, avec inclusions blanches et noirâtres de dimensions petites et moyennes, à fréquence basse, distribuées d'une manière non homogène. Mica argenté de dimensions petites et moyennes, à fréquence très basse. Vacuoles.

Observations et comparaisons : étant donné la caractéristique de l'impression digitale à la base de l'attache inférieure, le fragment est probablement à attribuer à une amphore du groupe Solokha I. Pour le type, voir la fiche précédente.

Chronologie : non déterminable.

\section{Analyse des données}

Les amphores commerciales provenant des fouilles de Bologne que nous avons eu la possibilité d'étudier ne peuvent pas être considérées comme représentatives, du point de vue de la quantité, des importations qui ont dû réellement parvenir dans la ville et du rôle de caput Etruriae qui lui est reconnu aussi par les sources littéraires de l'Antiquité.

Cela dépend sans aucun doute de l'état de la documentation et des fouilles du site, qui a connu une continuité 
d'occupation depuis l'Antiquité jusqu'à nos jours et pour lequel les recherches ont porté presque exclusivement sur les aires des nécropoles ${ }^{642}$. Une confirmation de la coutume qui semble caractériser les cultures grecques ou d'influence culturelle grecque à partir du $\mathrm{VI}^{\mathrm{e}} \mathrm{s}$. tardif et pendant le courant du Ve s. av. J.-C. (Colonna 1985) vient de la constatation qu'aucune tombe parmi les milliers de cette phase fouillées à Bologne n'a restitué d'amphores commerciales dans son mobilier. La documentation amphorique de Bologne vient exclusivement du petit secteur d'habitation récemment fouillé dans la Via Santa Caterina (Baldoni, Morico, Pini 2007), où, sur la base de ce qui a été publié, on ne peut signaler qu'un seul individu (cat. FE6), et de l'aire de l'acropole de Villa Cassarini, d'où j'ai pu repérer une cinquantaine de fragments attribuables à douze individus (cat. FE3, FE5, FE7-FE16). À la même aire de l'acropole on peut également rattacher les trois fragments d'amphores provenant des fouilles de Viale Aldini, dans le secteur de l'habitat situé sur les pentes de la Villa Cassarini (cat. FE1-FE2, FE4). Les amphores qui proviennent de l'aire de l'acropole ont été trouvées à l'occasion de différentes interventions de fouille (en 1907, en 1912, en 1964 et surtout en 1973) sur lesquelles on dispose d'une documentation particulièrement problématique ne permettant pas l'utilisation des contextes pour déterminer la datation des amphores. Compte tenu des typologies documentées, parmi lesquelles les amphores du groupe Solokha I dans la forme initiale de la série (cat. FE7-FE16) sont prédominantes, je pense que le matériel que j'ai pu sélectionner se réfère à un contexte datable entre la deuxième moitié du $V^{e}$ et le début du $I^{e} \mathrm{~s}$. av. J.-C., en conformité avec cette typologie amphorique qui n'a pas fait son apparition avant le milieu du $\mathrm{V}^{\mathrm{e}} \mathrm{s}$. av. J.-C. Sont enfin documentées la production nordégéenne, représentée par un seul individu, datable des années de transition entre le $\mathrm{V}^{\mathrm{e}}$ et le $\mathrm{IV}^{\mathrm{e}} \mathrm{s}$. (cat. FE5), et la production occidentale des amphores « corinthiennes B », elle aussi documentée par un unique individu, dont la forme est génériquement datable entre le deuxième et le dernier quart du $\mathrm{V}^{\mathrm{e}} \mathrm{s}$. av. J.-C. (cat. FE3). La seule amphore provenant de l'habitat est attribuable au groupe Samos-Milet et peut être datée, sur la base aussi du contexte, entre la fin du $\mathrm{VI}^{\mathrm{e}}$ et le début du $\mathrm{V}^{\mathrm{e}} \mathrm{s}$. av. J.-C. (cat. FE6). Les trois fragments trouvés dans le secteur d'habitat situé sur les flancs de l'acropole, mais considérés comme provenant de l'acropole, sont à rattacher au type corinthien $\mathrm{A} / \mathrm{A}$ ' du $\mathrm{V}^{\mathrm{e}}$ s. av. J.-C. (cat. FE1-FE2) et à la production nordégéenne de la fin de ce siècle (cat. FE4).
642. Pour l'habitat étrusque de Bologne : Taglioni 1999 (où sont rassemblées toutes les données disponibles jusqu'à cette date); Baldoni, Morico, Pini 2007 ; Calastri, Desantis 2011 (pour la publication des fouilles plus récentes). Les nécropoles étrusques de la ville ont été étudiées à partir de la seconde moitié du $\mathrm{XIX}^{\mathrm{e}} \mathrm{s}$. et ont fait l'objet de plusieurs Thèses de Doctorat, mais elles restent fondamentalement inédites à l'exception de la nécropole Arnoaldi (Macellari 2002). Pour une vision d'ensemble des connaissances concernant Bologne étrusque, on renvoie à Sassatelli, Donati 2005, avec bibliographie, et Malnati 2011. 



\section{Chapitre 5}

\section{Marzabotto (prov. de Bologne) : l'habitat}

\section{Bilan des études}

Le centre étrusque de Marzabotto a été qualifié d'habitat « carovaniero » (Cristofani 1985, p. 94) ou mieux d' « itinerario » (Lippolis 2005, p. 146) en vertu de sa position particulière.

Édifié à proximité de la ligne de faîte des Apennins dans la vallée du Reno (fig. 69), d'importance stratégique pour les rapports entre l'Étrurie padane et l'Étrurie tyrrhénienne, le site de Marzabotto a dû jouer un rôle fondamental surtout vers Bologne d'une part et vers le terroir de Fiesole de l'autre ${ }^{643}$.

Situé sur une ample terrasse naturelle (Pian di Misano) qui domine le fleuve Reno, le site qui s'étend sur vingtcinq hectares est connu pour ses vestiges archéologiques depuis le $\mathrm{XVI}^{\mathrm{e}} \mathrm{s}$. et dès les premières décennies du XIX ${ }^{\mathrm{e}} \mathrm{s}$. a fait l'objet de nombreuses prospections et découvertes. À partir de la deuxième moitié du siècle dernier, la Soprintendenza archeologica de l'Émilie-Romagne, l'Université de Bologne, l'École française de Rome et les Universités de Ratisbonne et de Bonn ${ }^{644} \mathrm{y}$ ont mené des fouilles de type extensif, dont certaines sont encore en cours aujourd'hui (fig. 88).

Marzabotto représente un rare exemple de fouille extensive d'un habitat étrusque de vastes dimensions. En effet, bien que l'aire de la ville n'ait pas fait l'objet d'une enquête exhaustive, elle est connue dans son ensemble et nombreux sont les secteurs qui, à ce jour, ont été fouillés ou bien ont fait l'objet de sondages prospectifs. Outre les fouilles de l'acropole et des deux nécropoles, qui ont surtout suscité l'intérêt des premiers fouilleurs,

643. La position stratégique de Marzabotto sur cette ligne directrice a été récemment reconfirmée par les découvertes de Prato-Gonfiente, site étrusque sur le versant méridional de la ligne de faîte des Apennins (Poggesi et al. 2005).

644. En ce qui concerne la vaste bibliographie sur Marzabotto, cf. Sassatelli, Brizzolara 1991 (complet mais mis à jour jusqu'à 1989) ; et plus récemment Massa-Pairault 1997 ; Vitali, Brizzolara, Lippolis 2001 ; Culti, forma urbana e artigianato a Marzabotto, Bentz, Reusser 2004a ; Id. 2008 ; Id. 2011, tous avec références bibliographiques. différentes zones de l'habitat ont été fouillées au cours des « anciennes » mais aussi des nouvelles fouilles ${ }^{645}$.

La tradition des études sur Marzabotto veut qu'à une première phase d'occupation étrusque du site, caractérisée par des cabanes éparses et appelée «Marzabotto I », succède une deuxième phase, appelée « Marzabotto II », mieux connue archéologiquement, et caractérisée par un programme d'urbanisation de l'espace selon un plan orthogonal complexe. La première phase, préurbaine, commence au milieu (Sassatelli 1989, p. 29-30) ou peu après le début du VI ${ }^{\mathrm{e}}$ s. av. J.-C. (Malnati 1987 ; Malnati, Manfredi 1991, p. 132). La vaste littérature relative à ce site fait démarrer la deuxième phase, et par conséquent le moment de la «fondation urbaine» de «type colonial» de Marzabotto, au début du Ve s. av. J.-C. Néanmoins une proposition récente, fondée sur le réexamen de ce que les recherches stratigraphiques de certaines zones ont révélé, fait remonter ce moment aux environs de 540 av. J.-C. (Lippolis 1998, p. 215 ; Id. 2005 , p. 146, 153). C'est autour de cette date qu'auraient été réalisées la répartition générale de l'aire, la construction des premières structures publiques et l'organisation des activités artisanales complexes liées au travail des métaux. L'aménagement des espaces internes aux insulae, où les premières constructions à usage privé en pierre sont datées de 520-510 av. J.-C., aurait été mise en place progressivement et caractérisée par de multiples restructurations (Massa-Pairault 1997, p. 197-201 ; Lippolis 2005, p. 146, 153) à propos desquelles on n'exclut pas, pour les phases les plus anciennes, l'emploi de matériaux périssables (Lippolis 1998, p. 215).

Parallèlement à ces considérations d'ordre urbanistique et structurel, et pour corroborer l'hypothèse qu'une phase de grand changement s'amorce vers 540 av. J.-C., on a fait appel à la chronologie du premier grand groupe

645. La définition d' " anciennes fouilles », désormais entrée dans l'usage commun, est adoptée depuis longtemps pour désigner de façon générale toutes les campagnes de fouilles intervenues avant la Seconde Guerre mondiale. Pour une liste détaillée des campagnes de fouilles effectuées à Marzabotto, voir Lippolis 2005, p. 153-157 («regesto degli interventi di scavo»), avec références bibliographiques mises à jour en 2003. Pour les interventions successives, cf. Bentz, Reusser 2004a et Id. 2011. 


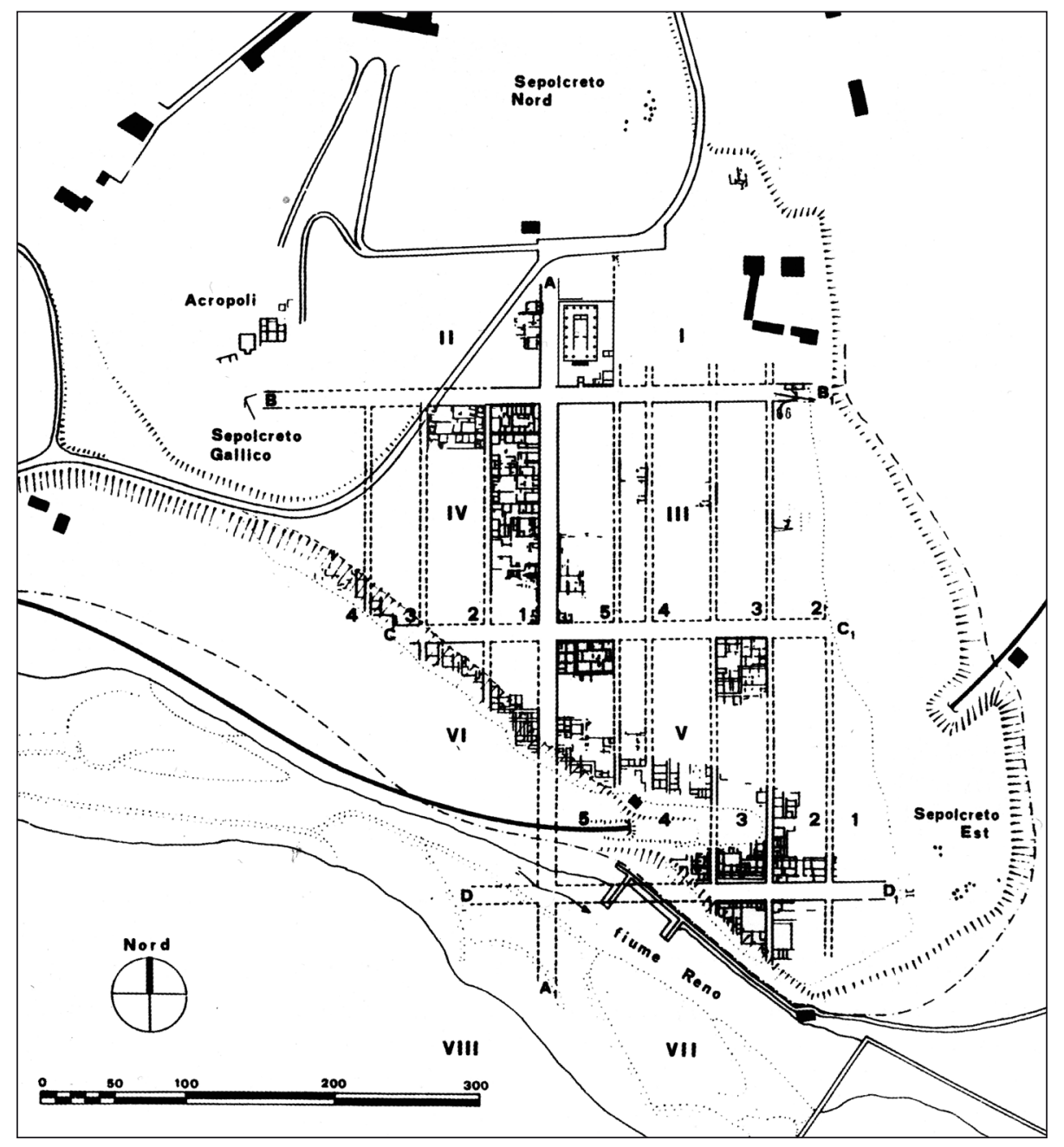

Fig. 88. Planimétrie de la cité étrusque de Marzabotto avec l'emplacement de zones fouillées (DAO Stéphane Renault d'après Marzabotto. La casa 1 della Regio IV-Insula 2, Bologna, 2010).

de céramiques d'importation, pour la plupart de production attique, datables du milieu du $\mathrm{VI}^{\mathrm{e}}$ s. av. J.-C. ${ }^{646}$. Cette donnée, connue depuis longtemps et désormais confirmée par les recherches récentes (Bentz, Reusser 2004a ; Baldoni 2008 ; Id. 2009), est apparue comme un « indice de la création d'un circuit commercial stable et d'une perspective de comportement économique et social, réellement innovatrice par rapport à la phase précédente » (Lippolis 2000, p. 103). Avant le milieu $\mathrm{du} \mathrm{VI}^{\mathrm{e}} \mathrm{s}$. av. J.-C., les seules céramiques d'importation grecque connues consistent en une kotyle et un aryballe globulaire du Corinthien Moyen et en un aryballe en

646. Parmi les importations de céramiques grecques de cette phase, outre les productions attiques, a été également identifié un fragment de céramique à figures noires de production chalcidienne, se référant au Groupe des Amphores Inscrites (570-530 av. J.-C.), et daté, au vu de la stratigraphie, des environs de 540 av. J.-C. (Lippolis 2000, p. $110, \mathrm{n}^{\circ} 5.9$, fig. 12 , avec bibliographie). couronne du Corinthien Moyen ou Tardif (Bermond Montanari 1987 ; Lippolis 2000, p. 109, $\mathrm{n}^{\circ}$ 5.1, 5.3, 5.4, fig. 4-6, 8-10). En l'état actuel de nos connaissances, il semble qu'il ne s'agisse que d'attestations épisodiques d'une fréquentation commerciale de l'habitat étrusque plutôt que d'un système d'échanges organisés ${ }^{647}$. En tout état de cause, le deuxième quart du $\mathrm{VI}^{\mathrm{e}} \mathrm{s}$. av. J.-C. peut être, pour le moment, considéré comme un terminus post

647. Étant donné que l'on ignore les contextes de découverte de ces produits corinthiens, comme d'ailleurs de la plupart du matériel mis au jour au cours des fouilles «anciennes », leur provenance de Marzabotto a longtemps été sujette à caution, à commencer déjà par E. Brizio (Brizio 1886). Bien qu'actuellement ces positions du passé soient obsolètes (Sassatelli 1989, p. 30-32 ; Lippolis 2000, p. 102), la difficulté de référer ces objets à une présence stable persiste chez les partisans de la chronologie «basse » de l'occupation et de la fondation de Marzabotto ; et la propension à les expliquer par une simple fréquentation du site considéré comme point de passage des marchandises et des influences culturelles est encore à l'ordre du jour. 
quem par rapport à l'arrivée des produits grecs sur le site des Apennins.

Après les débuts du IV $\mathrm{e}$. av. J.-C., le site étrusque pâtit des conséquences de l'invasion celtique de l'Étrurie padane de 388 av. J.-C. (Tite-Live, V, 35) qui désorganise le système politico-économique mis en place par les Étrusques de la zone. La ville subit une occupation celtique relativement limitée (D. Vitali, dans Vitali, Brizzolara, Lippolis 2001), dont la documentation archéologique est actuellement fort modeste. Au cours du IV ${ }^{\mathrm{e}}$ s., on peut noter des traces de restructurations internes aux insulae (Regio V, Insula 5, Maison 1) et la dernière phase d'occupation est caractérisée par la céramique à vernis noir avec décor à estampilles produite à Volterra dans le dernier quart du IVe s. av. J.-C. (Malnati, Violante 1995, p. 103-106). Donc, à la suite de cette invasion historique, les échanges entre les deux versants de l'Apennin, grâce aussi à Marzabotto, ne semblent pas cesser jusqu'au dernier quart du $\mathrm{IV}^{\mathrm{e}} \mathrm{s}$. Pourtant, les importations de céramique grecque semblent s'arrêter avant la fin du deuxième quart du IVe s. av. J.-C. (Baldoni 2009, p. 243244, 246). Ce sont alors sans doute les Celtes qui jouent le rôle d'intermédiaires entre l'Étrurie tyrrhénienne et l'Étrurie padane, interrompant les échanges le long de la vallée du Reno vers Bologne (Sassatelli 1989, p. 83), ou du moins réduisant le rôle central détenu précédemment par la Felsina princeps Etruriae.

C'est grâce à un seul exemplaire presque complet qui semble avoir été trouvé sous le niveau de la route de la Plateia A, devant la Maison 6 de la Regio IV ${ }^{648}$ (cat. MA1, fig. 89) que la présence d'amphores commerciales grecques était connue à Marzabotto. L'amphore, de production corinthienne de type $\mathrm{A}$, a été considérée à tort comme une sorte d'unicum dans le cadre de la ville.

Certaines recherches du début des années 1970, consacrées à la céramique de production locale, notamment celles entreprises par P. Sandri sur le matériel de la Regio IV, Insula 1, Maison $4^{649}$ et par B. Bouloumié sur la Regio IV, Insula 1 , Maison $3^{650}$, avaient déjà révélé la

648. Pour cette information, je remercie Sergio Sani, technicien des fouilles et inspecteur honoraire de la Surintendance, présent au moment de la découverte fortuite de l'amphore.

649. Sandri 1972, notamment p. 336, $\mathrm{n}^{\circ} 18$, fig. 3.18 (correspondant à la « forme 8 » de Sandri, dont Ibid.,p. 327) et p. 339, n 41, fig. 8.41. Les deux fragments ont été attribués par P. Sandri à des « amphores à vin ou à huile » et sont, tous deux, considérés aujourd'hui comme appartenant à la production nord-égéenne.

650. Bouloumié 1976, notamment p. 98-99, pl. I.713135 (pied d'amphore de production corinthienne), I.13212 (pied d'amphore nord-égéenne, probablement de Mendé), non identifiées par le chercheur mais reconnues toutefois comme des amphores, auxquelles viendront s'ajouter une lèvre d'amphore corinthienne $A$, (voir pl. I.113135), et quelques fragments d'anses visibles sur la même planche. La forme 7 de Sandri (pour laquelle cf. Sandri 1972, p. 327 , présence d'amphores commerciales (cat. MA44, 49). Ce matériel semble cependant ne pas avoir attiré l'attention des personnes qui se sont occupées de la matière, pas même à titre de comparaison ou de citation, et qui ont privilégié l'amphore corinthienne A exposée au Musée national étrusque «P. Aria » (Colonna 1985, n. 29 ; Sassatelli 1990, p. 89-90 et n. 111 ; Cattaneo Cassano 1995, p. 381, 391 et n. 8). D'autres fragments ont ensuite été publiés dans le cadre de la publication des fouilles menées par l'École française de Rome (Massa-Pairault 1997, p. 185-186, pour lesquels cat. MA76, 83-88, 112).

Au cours de ces recherches et grâce à la disponibilité de la Soprintendenza archeologica de l'Émilie-Romagne, j'ai pu procéder à un examen de tous les dépôts du Musée national étrusque «P. Aria » et revoir l'ensemble des objets archéologiques conservés et retrouvés à l'occasion des « anciennes » et des nouvelles fouilles. L'enquête a permis la récupération de milliers de tessons d'amphores grecques attribuables à environ cent soixante individus distincts dont 131/143 (nombre minimum/maximum) sont édités dans la présente publication. Je ne traiterai pas analytiquement les amphores provenant du Temple de la Regio I, Insula 5, encore en cours de fouille ${ }^{651}$.

En ce qui concerne l'abondant matériel du site, je n'ai malheureusement pas pu disposer des données relatives aux contextes de fouille qui, quand elles existent, n'ont pas encore été publiées. Les datations proposées ici sont donc exclusivement fondées sur la typologie.

Les amphores de Marzabotto sont identifiées par le sigle «MA » et proviennent des secteurs urbains suivants :

Regiones I, II, III, IV - Plateia A ${ }^{652}$. Regio I, Insula 5 - Temple urbain ${ }^{653}$. Regio II, Insula 1 - Four ${ }^{654}$.

fig. 3.17) qui, selon l'opinion de B. Bouloumié, (Bouloumié 1976, p. 99) serait à attribuer à une amphore de production étrusque semblable à celles restituées par l'épave d'Antibes, est, en revanche, reconnue par P. Sandri comme une forme de production locale sans pertinence avec les catégories d'amphores commerciales.

651. Dans le cas de la Regio I, Insula 5 les données à ma disposition sont à jour jusqu'aux campagnes de fouilles 1999-2005 et ont été prises en compte dans ma Thèse de Doctorat (Sacchetti 2005).

652. La Plateia A a été fouillée à plusieurs reprises et dans des secteurs différents. Dans ce cas précis, on se réfère aux fouilles effectuées par P. E. Arias en 1953, sur toute son extension (Arias 1953 ; Id. 1954).

653. Pour le Temple urbain de Marzabotto, toujours en cours de fouilles menées par le Département d'archéologie de l'Université de Bologne, cf. Verger, Kermorvant 1994 ; Sassatelli et al. 2004 ; Sassatelli, Govi 2005.

654. Arias 1954 ; Saronio 1965 ; Iscrizioni e graffiti, p. 57-64, avec bibliographie complémentaire. 
Regio IV - Insula 1 - Maisons 1, 2, 3, 4, 6 655.

Regio IV - Insula 2 - Maison $1{ }^{656}$.

Regio V - Insula $3^{657}$.

«Anciennes fouilles » $\mathbf{6 5 8}$.

Regio $\mathrm{V}$ - Insula 4 - « niveau du $\mathrm{VI}^{\mathrm{e}}{ }^{659}$.

Regio V - Insula 4-5 - Sporadiques au-dessus de la galerie Ouest ${ }^{660}$.

Regio V - Insula 5 (secteur Nord) - Habitation et structures de production et Plateia $\mathrm{A}^{\mathbf{6 6 1}}$.

Regio V-Insula 5 (secteur central) - Fonderie et Plateia $\mathrm{A}^{662}$.

Regiones V et VII - Puits de la Plateia $\mathrm{D}^{663}$.

Regio VII - Insula 2 - Puits près de la Plateia $\mathrm{D}^{664}$.

655. Pour les maisons de la Regio IV, Insula 1 : Arias 1951, p. 221234 ; Id. 1953 ; Mansuelli 1962, p. 14-27 ; Id. 1962a ; Id. 1963, p. 44-62 ; Sandri 1972, p. 319-340 ; Bouloumié 1976, p. 95-140; Durante, Gervasini 1987, p. 316-325; Iscrizioni e graffiti, p. 71-89; Bentz, Reusser 2004a ; Id. 2011.

656. Sassatelli et al. 2004, avec bibliographie précédente ; Brizzolara, Baldoni, Succi 2005.

657. Pairault Massa 1978 ; Iscrizioni e graffiti,p. 137-153 ; MassaPairault 1997.

658. Les questions soulevées par la conservation des objets découverts au cours des fouilles du XIX ${ }^{\mathrm{e}}$ siècle à Marzabotto sont assez complexes. Exposés depuis 1886 dans cinq salles de la Villa Aria, ils furent transférés dans la métairie de Pian di Misano qui fut détruite par les bombardements en 1944. Les objets rescapés, pour la plupart très endommagés, furent installés dans le Musée construit entre 1949 et 1950, restructuré en 1958 et agrandi selon son aménagement actuel, en 1979. Ces péripéties ont évidemment compromis toute tentative de reconstitution de la provenance exacte de ces pièces qui, hormis quelques cas rares, sont à répertorier de façon générale comme provenant de l'aire des « fouilles anciennes ». Pour la bibliographie concernant les fouilles du XIX ${ }^{\mathrm{e}}$ siècle, cf. Lippolis 2005, p. 153-157 et Baldoni 2009, p. 17-22, avec références.

659. Au sujet du niveau dit «niveau du VI ${ }^{\mathrm{e}}$ », cf. Mansuelli 1965, p. 241-247 ; Mansuelli et al. 1970, p. 53-71 ; Saletti 1970, p. 279283 ; Malnati 1987, p. 125-137 ; Iscrizioni e graffiti, p. 157-167.

660. En coïncidence avec l'ouverture du tunnel occidental de la galerie ferroviaire qui traverse le plateau dans son secteur sud-oriental, et donc aux environs de l'aire des Insula 4 et 5 de la Regio V, quelques fragments d'amphores ont été retrouvés fortuitement. Malheureusement, ces derniers sont hors contexte et l'on ignore les circonstances exactes de leur découverte.

661. Malnati 1990, p. 133-134 ; Id. 1991, p. 390-392 ; Iscrizioni e graffiti, p. 169-177; Malnati, Violante 1995, p. 103-106.

662. Pour la «fonderie» de Marzabotto, voir Gentili 1968, p. 116117 ; Mansuelli et al. 1970, p. 53-71 ; Sassatelli 1990, p. 69-74 ; Iscrizioni e graffiti, p. 179-188; Locatelli 2005.

663. En ce qui concerne l'exploration de la route de la Plateia D et de la découverte du puits central, voir Tripponi 1967 ; Gentili 1978 ; Iscrizioni e graffiti, p. 189-190.

664. Des interventions récentes et complètement inédites de la Surintendance Archéologique de l'Émilie-Romagne - notamment aux fins de vider le puits découvert dans le secteur de tête de l'Insula 2, voisin de la Plateia $\mathrm{D}$ - ont conduit à la récupération de nouveau matériel dont un fragment de pied d'une amphore de transport grecque.

\section{Catalogue}

\section{AMPHORES DE TYPE CORINTHIEN A/A'}

\section{MA1 (fig. 89 ; pl. XXIX)}

Secteur: Regio IV, Insula 1.

Structure : Plateia A, devant la Maison 6.

Inventaire : $\mathrm{n}^{\circ} 1018$.

Type : corinthienne A, qu'on peut rapprocher des types 1 et 3 de Camarine-Rifriscolaro.

État de conservation : amphore presque entière, à laquelle il ne manque que le fond. Reconstituée à partir de nombreux fragments et restaurée. H. max. cons. : 95. Lèvre à marli horizontal, de section trapézoïdale, qui présente une légère cannelure à l'endroit de sa plus grande expansion. H. : 4 ; épaiss. max. : 7 ; diam. int./ext. embouchure : $20 / 35$. Col tronconique. H. : 18 ; diam. min./max. : $28 / 32$. Anses obliques légèrement ouvertes,

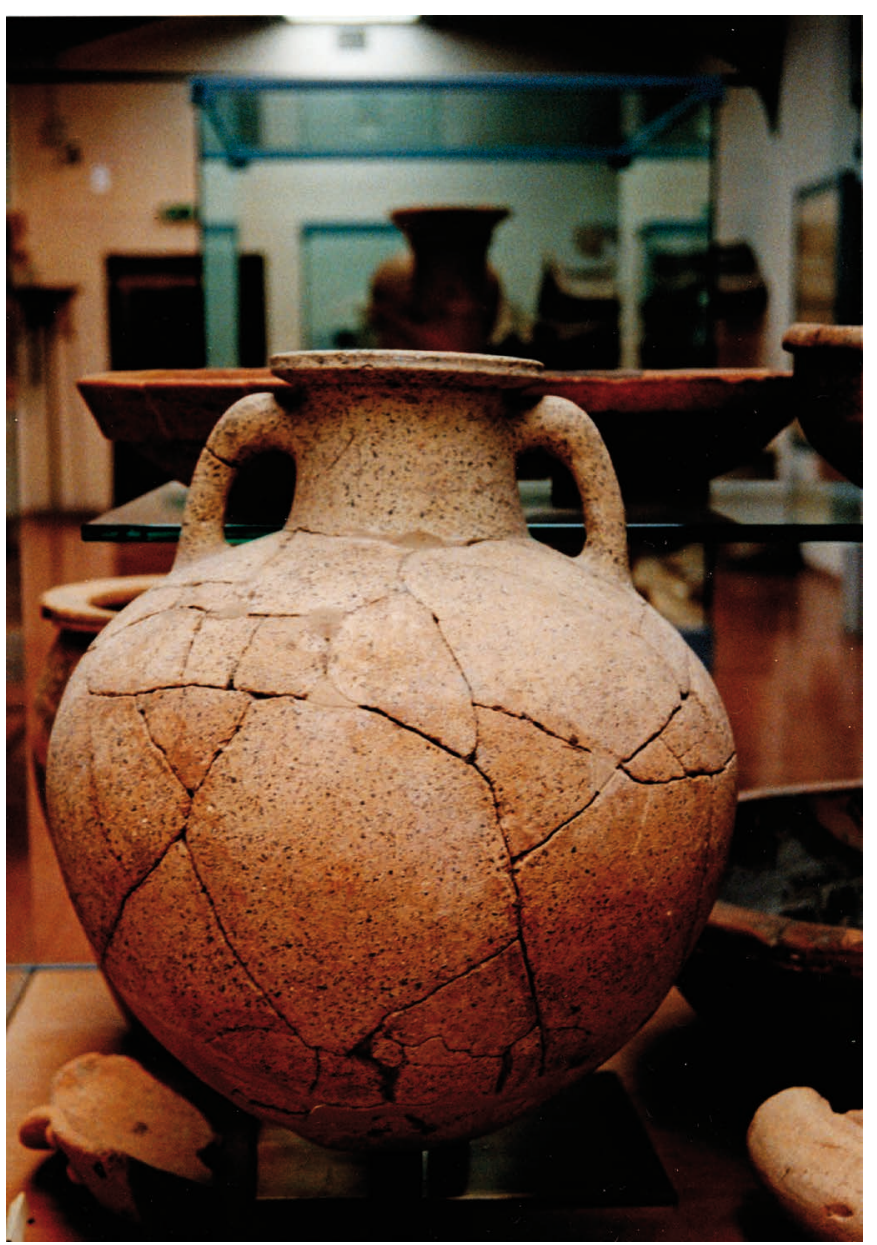

Fig. 89. Marzabotto, Museo Nazionale Etrusco P. Aria, inv. 1018. Amphore de type corinthien A en provenance de la Regio IV, Insula I. Cat. MA1 (photo F. Sacchetti). 
« a bastoncello », de section circulaire. H. : 25 ; épaiss. max. : 5. Épaulement arrondi. Larg. max. : 15. Corps globulaire. H. : 73 .

Surface ext., int. et fracture : beige clair (2.5YR8/ 2-2.5YR7/3).

Inclusions : inclusions blanches de dimensions petites et moyennes, à fréquence moyenne, distribuées d'une manière homogène, inclusions orange de dimensions petites et moyennes, à fréquence basse, distribuées d'une manière non homogène et inclusions gris brun de dimensions moyennes et grandes, à fréquence très haute. Appareil épigraphique : sur le col, près de la jonction supérieure d'une des anses, lettre grecque san incisée avant cuisson (Sacchetti 2010, p. 52-54, n 9).

Observations et comparaisons : attribuée par G. Sassatelli à la production corinthienne $\mathrm{A}$ et datée entre la fin du $\mathrm{VI}^{\mathrm{e}}$ et le début du $\mathrm{V}^{\mathrm{e}} \mathrm{s}$. av. J.-C. sans que des parallèles soient proposés. Pour le type de lèvre et pour les anses, on trouve un parallèle très proche dans Rizzo 1990, p. 55, III.1, fig. 345 (du Tumulus « della Speranza» de Cerveteri, daté sur la base du mobilier entre la fin du premier et le deuxième quart du VII ${ }^{\mathrm{e}} \mathrm{s}$. av. J.-C.). Pour la forme générale, on renvoie à Koehler 1979, p. 97-98, pl. $3, \mathrm{n}^{\circ} 20, \mathrm{pl} .4, \mathrm{n}^{\circ} 21$ (datée de la fin du premier quart du $\mathrm{VI}^{\mathrm{e}} \mathrm{s}$. av. J.-C.). L'exemplaire en question se distingue par ses dimensions remarquables. Après la récente publication d'une nouvelle typo-chronologie pour les amphores corinthiennes A d'époque archaïque qui prend en compte les contextes de la nécropole de Camarine, la chronologie de cette amphore, pour laquelle j'avais déjà suggéré une datation dans le courant de la première moitié du $\mathrm{VI}^{\mathrm{e}}$ s. av. J.-C. s'appuyant notamment sur la confrontation avec Koehler 1979, pl. 3, n 20, peut être précisée ultérieurement. On peut en effet rapprocher l'exemplaire de Marzabotto aussi bien du type 3 de Camarine-Rifriscolaro (Sourisseau 2006, p. 137-138, note 42 , fig. 4 à gauche), auquel renvoie l'évolution du corps à la forme sphérique, que du type 1 (Sourisseau 2006 , p. 135, fig. 2 à gauche), que rappelle la partie supérieure de l'amphore, aux anses se détachant encore de la lèvre (à section trapézoïdale) et au col court. Le type 3 de Camarine-Rifriscolaro, correspondant à Koehler 1979, pl 3, n 20 (Sourisseau 2006, note 42), est daté entre 580/570 et 550/540 av. J.-C. Le type 1 de Camarine-Rifriscolaro, correspondant au stade évolutif de la morphologie des amphores corinthiennes A traditionnellement attribué au VII ${ }^{\mathrm{e}}$ s. av. J.-C. (Koehler 1979, pl. 1, $\mathrm{n}^{\circ} 1-4$ ), est maintenant daté entre le deuxième quart du VII ${ }^{\mathrm{e}}$ et le premier quart du $\mathrm{VI}^{\mathrm{e}} \mathrm{s}$. av. J.-C. sans qu'il soit possible de définir à l'heure actuelle un ultérieur affinement chronologique (Sourisseau 2006, p. 142). L'exemplaire en question pourrait appartenir à une phase de transition du type 1 au type 3 de Camarine-Rifriscolaro.
Chronologie : 580-540 av. J.-C.

Bibliographie : G. Sassatelli, in Mansuelli et al. 1982, p. 65, fig. 61 ; Sassatelli 1990, p. 89-90 et n. 111 ; G. Sassatelli, dans Iscrizioni e graffiti, p. 89, $\mathrm{n}^{\circ} 121$, pl. XVa, 121 ; Sacchetti 2010, p. 52-54, n 9, fig. 4, pl. I, 9.

\section{MA2 (pl. XXIX)}

Secteur: Regio IV, Insula 1.

Structure : Maison 2. Q au sud de la cour.

Inventaire : $\mathrm{n}^{\circ} 2$ caisse 1 .

Type : corinthienne A.

État de conservation : portion de lèvre avec une brève partie du col. H. max. cons. : 3,1. Lèvre à marli horizontal, de section trapézoïdale. H. : 2 ; épaiss. max. : 4,2 ; diam. int./ext. restituable embouchure : 12,4 / 20,6. H. max. cons. : 2 ; diam. min./max. restituable : 14,2.

Surface ext., int. et fracture : jaune beige (10YR8/ 4-10YR7/4).

Inclusions : nombreuses inclusions blanches, orange (mudstone) et noirâtres, de dimensions petites, moyennes et grandes, à fréquence haute.

Observations et comparaisons : pour le type, Koehler 1978a, fig. 1.b, et Koehler 1981, pl. 98.f, datable entre le milieu du VI ${ }^{\mathrm{e}}$ et le début du V $\mathrm{V}^{\mathrm{e}}$ s. av. J.-C. (Lawall 1995, p. 59-60, variante A/1; Sourisseau 2006, type 4A et 4B). Pour la morphologie de la lèvre, Boss 1993, p. 334. L61, fig. 526, L61 ; et Di Sandro 1986, pl. 4, sg. 54.

Chronologie : milieu VIe-début Ve s. av. J.-C.

\section{MA3 (pl. XXIX)}

Année de la fouille / trouvaille : 1964.

Secteur : Regio II, Insula 1.

Structure : Four. Q C4C5-B4B5

Inventaire : $\mathrm{n}^{\circ} 6726$.

Type : corinthienne A.

État de conservation : partie de lèvre. H. max. cons. : 2,3. Lèvre à marli horizontal, de section trapézoïdale. H. : 1,4 ; épaiss. max. : 4,1 ; diam. int./ext. restituable embouchure : 11,8 / 19,6.

Surface ext., int. et fracture : rose orange (7.5YR7/4).

Inclusions : inclusions orange de dimensions grandes, moyennes et petites, à fréquence moyenne, inclusions blanches de dimensions moyennes et petites, à fréquence moyenne, et inclusions noirâtres de petites dimensions, à fréquence basse.

Observations et comparaisons : on peut l'encadrer à l'intérieur de la production corinthienne de type A par sa forme et sa pâte céramique. La morphologie de la lèvre, dont la surface supérieure est presque plate, suggère une datation avant la fin du $\mathrm{VI}^{\mathrm{e}}$ ou le début du $\mathrm{V}^{\mathrm{e}} \mathrm{s}$. av. J.-C. Pour le type, on renvoie à la fiche précédente.

Chronologie : milieu VI'-début Ve s. av. J.-C. 


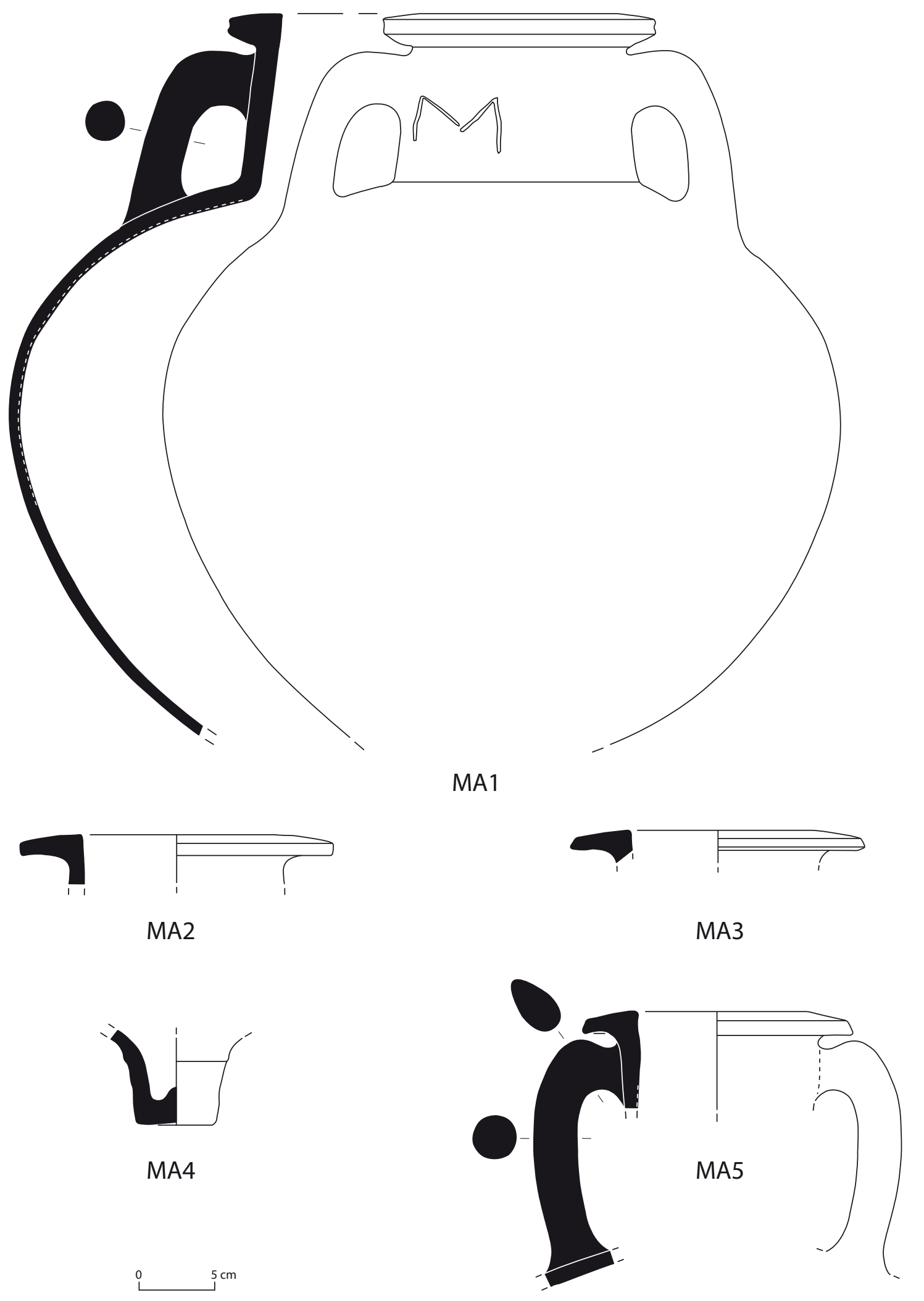

PI. XXIX. Marzabotto. 


\section{MA4 (pl. XXIX)}

Secteur : Regio V, Insulae 4-5.

Sporadique. Au-dessus de la galerie Ouest.

Inventaire : $\mathrm{n}^{\circ}$ 58409/53.

Type : corinthienne A.

État de conservation : fond complet jusqu'à la jonction de la panse. H. max. cons. : 6,2. Fond cylindrique, avec un fin listel au niveau de la jonction de la panse. H. : 5 ; diam. min./max. : 5,3 / 6,9.

Surface ext., int. et fracture : orange (10YR7/6-10YR7/4). Inclusions : inclusions orange de dimensions petites, moyennes et grandes, à fréquence moyenne, inclusions blanches de dimensions petites et moyennes, à fréquence basse, et inclusions grises de dimensions moyennes et grandes, à fréquence moyenne.

Observations et comparaisons : par sa morphologie et sa pâte on peut l'attribuer au type A de Corinthe. Pour le type, Koehler 1978a, fig. 1.b, actuellement datable entre la deuxième moitié du $\mathrm{VI}^{\mathrm{e}}$ et le début du $\mathrm{V}^{\mathrm{e}} \mathrm{s}$. av. J.-C. (Lawall 1995, p. 59-60, variante A/1 ; Sourisseau 2006, type 4B). Pour la forme, d'une manière assez ponctuelle, Boss 1993, p. 334, L65, fig. 526. L65. Le fond en question présente un détail morphologique peu courant qui consiste en une saillie au niveau de la jonction de la partie inférieure de la panse. On remarque la même caractéristique dans des amphores corinthiennes A de la nécropole de Camarine datables dans le courant de la deuxième moitié du $\mathrm{VI}^{\mathrm{e}}$ ou du début du $\mathrm{V}^{\mathrm{e}} \mathrm{s}$. av. J.-C. (Sourisseau 2006, fig. 8, type 4B) et de quelques contextes de l'Agora d'Athènes datables au plus tard du premier quart du $\mathrm{V}^{\mathrm{e}} \mathrm{s}$. (matériel inédit en cours d'étude par Mark Lawall).

Chronologie : milieu VI'e-débuts Ve s. av. J.-C.

\section{MA5 (pl. XXIX)}

Année de la fouille / trouvaille : 1964.

Secteur : Regio II, Insula 1.

Structure : Four. Q E1E2-D1D2.

Inventaire : $\mathrm{n}^{\circ} 2871-2875$.

Type : corinthienne A.

État de conservation : partie de lèvre, col et une anse entière, reconstitués à partir de fragments, ainsi que de nombreux fragments de parois sans doute appartenant au même individu mais non reconstituables. H. max. cons. : 18,5. Lèvre à marli horizontal, de section trapézoïdale. H. : 1,6 ; épaiss. max. : 3,7 ; diam. int./ext. restituable embouchure : 10,8 / 17,9. Col cylindrique. H. : 6,5 ; diam. min./max. restituable : 12,8. Anses « $a$ bastoncello $\gg$ de section elliptique dans la partie supérieure et circulaire dans la partie inférieure. H. : 15,5 ; épaiss. max. : 2,9/2,2 x 4,2.

Surface ext. : rose $(5 \mathrm{YR} 7 / 3)$.

Surface int. : rose (5YR7/3).
Surface ext., int. et fracture : rose (5YR7/3).

Inclusions : inclusions brunes, blanches et noirâtres de dimensions petites, moyennes et grandes, à fréquence moyenne-haute.

Observations et comparaisons : à classifier dans le cadre du type A de Corinthe par sa morphologie et sa pâte céramique. Pour le type, Koehler 1978a, fig. 1.c ; Ead. 1981, fig. 1.d. La chronologie de ce type est actuellement comprise entre la fin du $\mathrm{VI}^{\mathrm{e}}$ et la fin du $\mathrm{V}^{\mathrm{e}} \mathrm{s}$. av. J.-C. (Lawall 1995, p. 60-61, variante A/2 ; Sourisseau 2006, type 4C). La position des attaches supérieures des anses, et notamment la séparation entre leur courbure et la lèvre, pourrait suggérer une datation avant la fin du $\mathrm{VI}^{\mathrm{e}}$ ou au début du $\mathrm{V}^{\mathrm{e}}$ s. av. J.-C.

Chronologie : fin $\mathrm{VI}^{\mathrm{e}}$-début $\mathrm{V}^{\mathrm{e}}$ s. av. J.-C.

\section{MA6 (pl. XXX)}

Secteur : Regio IV, Insula 1.

Structure : Maison 3. Espace destiné à la cour.

Inventaire : $\mathrm{n}^{\circ} 63$ caisse 17.

Type : corinthienne A.

État de conservation : fragment de lèvre avec un court segment du col. H. max. cons. : 5,2. Lèvre à marli horizontal, de section trapézoïdale. H. : 2,5 ; épaiss. max. : 3,9 ; diam. int./ext. restituable embouchure : $11 / 18,1$. Col cylindrique. H. max. cons. : 2,5 ; diam. min./max. restituable : 12,2 / 13 .

Surface ext., int. et fracture : jaune beige (10YR7/410YR8/4).

Inclusions : inclusions orange (mudstone), brunes et blanches, de dimensions petites, moyennes et grandes, à fréquence haute.

Observations et comparaisons : pour le type, à lèvre presque horizontale, Koehler 1978a, fig. 1.c ; et Ead. 1981, fig. 1.d, attribuables au type A de Corinthe et datables entre la fin du $\mathrm{VI}^{\mathrm{e}}$ et le $\mathrm{V}^{\mathrm{e}}$ s. av. J.-C. (Lawall 1995, p. 60-61, variante A/2 ; Sourisseau 2006, type 4C). Pour la forme, à la surface inférieure moulurée, Morselli, Tortorici 1985, fig. 7.1, et Di Sandro 1986, pl. 3, sg. 56. Chronologie : fin $\mathrm{VI}^{\mathrm{e}}-\mathrm{V}^{\mathrm{e}}$ s. av. J.-C.

\section{MA7 (pl. XXX)}

Année de la fouille / trouvaille : 1988.

Secteur : Regio V, Insula 5.

Structure : Maison 1.

US : $119=120$.

Type : corinthienne A.

État de conservation : partie de lèvre, ébréchée au point de sa plus grande expansion, et une brève partie du col qui garde les traces de la jonction supérieure d'une anse. H. max. cons. : 4,1. Lèvre à marli horizontal, de section trapézoïdale. H. : 2,3 ; épaiss. max. : 3,6 ; diam. int./ext. restituable embouchure : $11 / 18,2$. 
Surface ext., int. et fracture : beige (10YR7/4).

Inclusions : inclusions blanchâtres de dimensions moyennes et grandes, à fréquence haute, et inclusions orange brun (mudstone) de petites, moyennes et surtout grandes dimensions, à fréquence haute.

Observations et comparaisons : pour le type on renvoie à la fiche précédente.

Chronologie : fin $\mathrm{VI}^{\mathrm{e}}-\mathrm{V}^{\mathrm{e}} \mathrm{s}$. av. J.-C.

\section{MA8 (pl. XXX)}

Secteur : Regio IV, Insula 1.

Structure : Maison 3. Espace destiné à la cour.

Inventaire : $\mathrm{n}^{\circ} 18$ caisse 9 .

Type : corinthienne A.

État de conservation : partie de fond. H. max. cons. : 3,3. Fond tronconique caréné. H. max. cons. : 3,3 ; diam. min./max. restituable : $8,3 / 9,1$.

Surface ext., int. et fracture : jaune beige (10YR8/4).

Inclusions : inclusions blanches, orange (mudstone) et grisâtres de petites, moyennes et grandes dimensions, à fréquence haute.

Observations et comparaisons : attribuable au type A de Corinthe par sa pâte et sa forme. Pour le type, Koehler 1978a, fig. 1.c ; et Ead. 1981, fig. 1.d, datable entre la fin du VI e et le courant du Ve s. av. J.-C. (Lawall 1995, p. 60-61, variante A/2 ; Sourisseau 2006, type 4C). Pour la morphologie on trouve des parallèles de Caeré (Boss 1993, fig. 526. L68).

Chronologie : fin $\mathrm{VI}^{\mathrm{e}}-\mathrm{V}^{\mathrm{e}} \mathrm{s}$. av. J.-C.

\section{MA9 (pl. XXX)}

Année de la fouille / trouvaille : 1953.

Secteur : Îlots sur la Plateia A.

Type : corinthienne A.

État de conservation : partie de fond sans la base d'appui. H. max. cons. : 4,3. Fond cylindrique. H. max. cons. : 4,3 ; diam. min./max. restituable : 5 / 6 .

Surface ext., int. et fracture : beige (7.5YR8/3-10YR8/3). Inclusions : inclusions orange et brunes de dimensions grandes et moyennes, à fréquence moyenne, et inclusions noirâtres et blanches, de dimensions petites et moyennes, à fréquence haute.

Observations et comparaisons : pour le type, corinthien A, actuellement datable entre le milieu du $\mathrm{VI}^{\mathrm{e}}$ et dans le courant du Ve s. av. J.-C., Koehler 1978a, fig. 1.b-c ; Ead. 1981, fig. 1.d.

Chronologie : fin $\mathrm{VI}^{\mathrm{e}}-\mathrm{V}^{\mathrm{e}}$ s. av. J.-C.

\section{MA10 (pl. XXX)}

Année de la fouille / trouvaille : 1964.

Secteur : Regio II, Insula 1.

Structure : Four. Q D2D3-C2C3.

Inventaire : $\mathrm{n}^{\circ} 5120$.
Type : corinthienne A'.

État de conservation : pied, reconstitué à partir de 2 fragments, et partie inférieure de la panse, reconstituée à partir de 99 fragments. H. max. cons. : 16. Fond cylindrique creux. H. : 5,5 ; diam. min./max. : 4,8 / 6,3.

Surface ext. : beige rosé (5YR7/3).

Surface int et fracture : orange rosé (2.5YR7/6).

Inclusions : inclusions blanches et noirâtres de petites dimensions à fréquence basse. Vacuoles.

Observations et comparaisons : attribuable à une amphore corinthienne du type A' par les caractéristiques de la pâte céramique et par la morphologie du pied. Les exemplaires provenant des nécropoles de la chora de Métaponte (Morter, Leonard 1998, fig. 17.3) témoignent du fait que ce type de fond est documenté pendant tout le $\mathrm{V}^{\mathrm{e}} \mathrm{s}$. av. J.-C.

Chronologie : fin $\mathrm{VI}^{\mathrm{e}}-\mathrm{V}^{\mathrm{e}}$ s. av. J.-C.

\section{MA11 (fig. 90 ; pl. XXX)}

Année de la fouille / trouvaille : 1961.

Secteur : Regio V, Insula 4.

'Niveau du VI ${ }^{\mathrm{e}}$ '

Type : corinthienne A'.

État de conservation : partie supérieure d'amphore, reconstituée à partir de nombreux fragments et restaurée. H. max. cons. : 21,5. Lèvre à marli oblique, de section trapézoïdale. H. : 3 ; épaiss. max. : 4,5 ; diam. int./ext. embouchure : $10 / 17,7$. Col tronconique, avec une rainure. H. 14 ; diam. min./max. : 11,3/13. Anses obliques, rentrantes, «a bastoncello », de section circulaire dont le diamètre, plus grand dans la partie supérieure, s'amincit dans la partie inférieure. H. : 16,4 ; épaiss. min./max. : 3 / 3,2. Épaule. Larg. max. cons. : 8.

Surface ext., int. et fracture : jaunâtre (2.5Y7/3-2.5Y7/4).

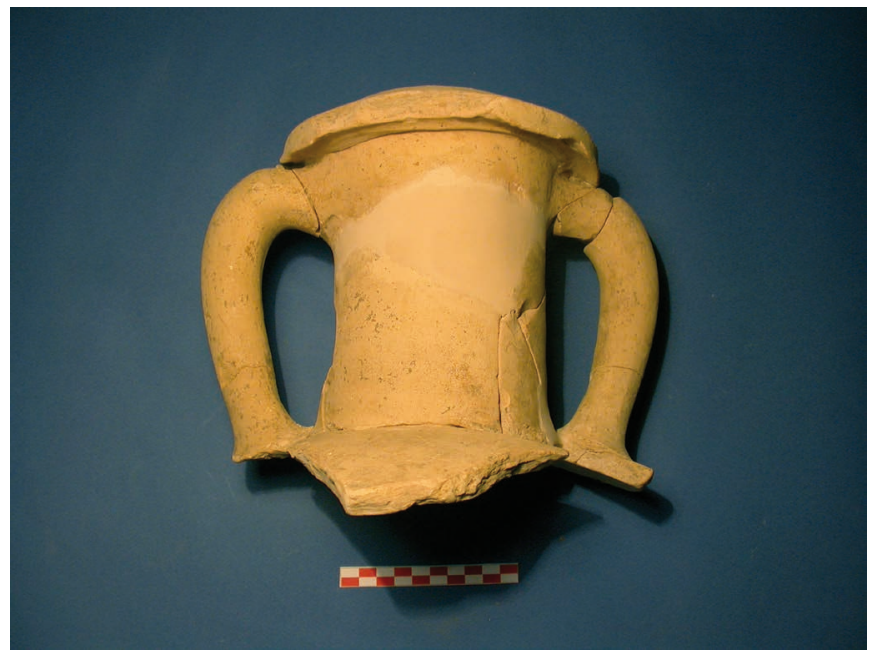

Fig. 90. Marzabotto, amphore de type corinthien A' en provenance de la Regio V, Insula IV, Strato del VI. Cat. MA11 (photo F. Sacchetti). 


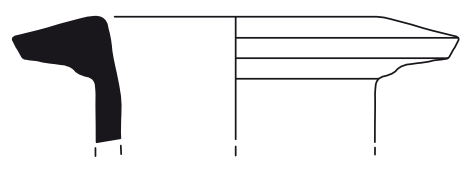

MA6

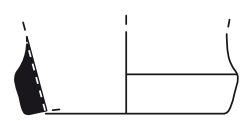

MA8

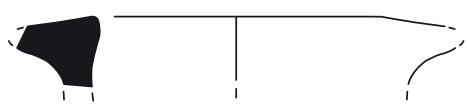

MA7

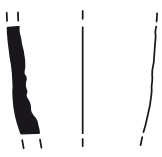

MA9

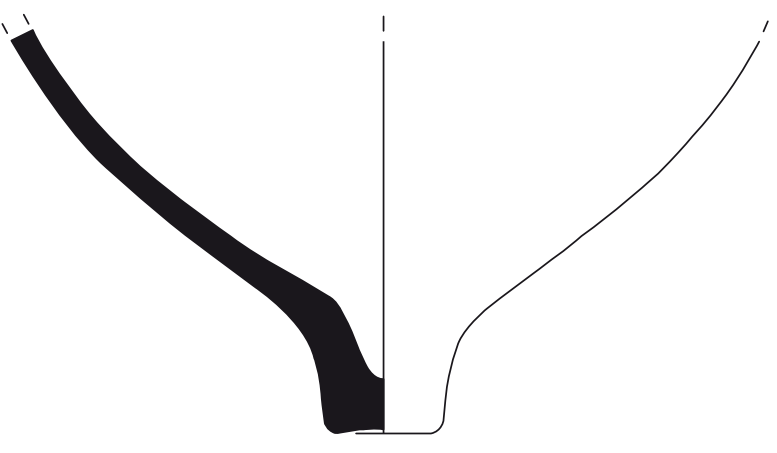

MA10

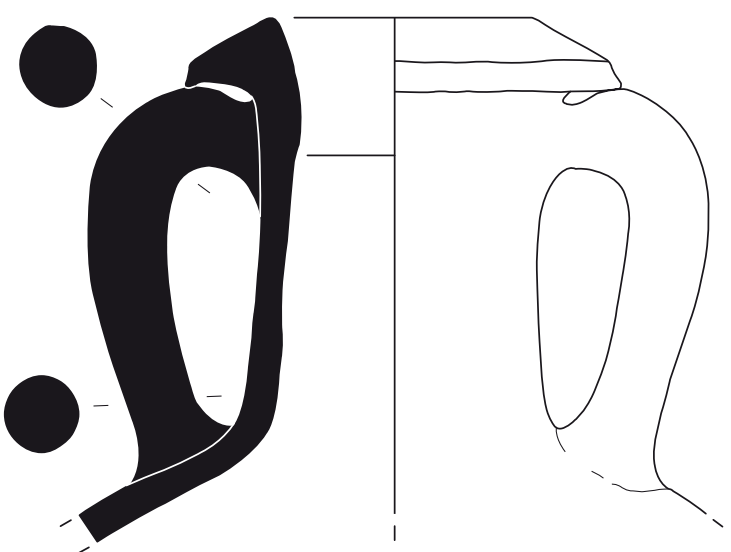

MA11

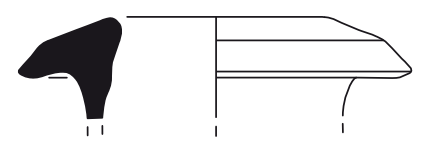

MA13

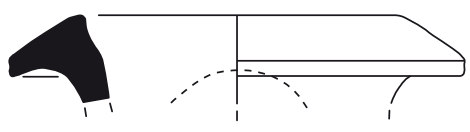

MA15

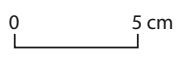


Inclusions : inclusions blanches, grises et brunes, de petites dimensions à fréquence basse.

Observations et comparaisons : sa forme et sa pâte suggèrent son attribution à une amphore corinthienne de type A'. Pour le type, Koehler 1978a, fig. 1.d ; Morter, Leonard 1998, fig. 17.3 (variantes attestées pendant tout le Ve s. av. J.-C.). Pour la forme, Di Sandro 1986, pl. 6, cat. $\mathrm{n}^{\circ}$ 57, 59. Si les indications relatives au contexte de provenance de cet exemplaire de Marzabotto sont exactes, sa datation vers la fin du $\mathrm{VI}^{\mathrm{e}} \mathrm{s}$. sera préférable. En effet, la couche dite 'niveau du VI ${ }^{\mathrm{e}}$ ' semble s'être formée dans le troisième quart du $\mathrm{VI}^{\mathrm{e}} \mathrm{s}$. av. J.-C. (Mansuelli 1965 ; Lippolis 2000, p. 103) mais le matériel provenant de ce contexte est inédit.

Chronologie : fin $\mathrm{VI}^{\mathrm{e}}-\mathrm{V}^{\mathrm{e}} \mathrm{s}$. av. J.-C.

\section{MA12 (pl. XXX)}

Année de la fouille / trouvaille : 1994.

Secteur : Regio V, Insula 5.

Structure : Plateia A, devant la Maison 1.

US : 814 .

Type : corinthienne A'.

État de conservation : embouchure presque complète, reconstituée à partir de 3 fragments, et de nombreux fragments de panse probablement appartenant au même individu, mais non reconstituables. H. max. cons. : 4,2. Lèvre à marli oblique, de section trapézoïdale. H. : 3,1 ; épaiss. max. : 3,4 ; diam. int./ext. restituable embouchure : $10,5 / 16,1$.

Surface ext., int. et fracture : beige très clair (10YR8/3). Inclusions : inclusions blanches de petites dimensions, à fréquence basse, inclusions grises, de dimensions petites et moyennes, à fréquence moyenne, et inclusions brun orange de dimensions petites et moyennes, à fréquence moyenne.

Observations et comparaisons : attribuable à une amphore corinthienne de type A'. Pour le type, Koehler 1978a, fig. 1.d (datable entre le dernier quart du Ve et le début du IV s. av. J.-C.), et Morter, Leonard 1998, fig. 17.3 (variantes datables du courant du Ve s. av. J.-C.). Chronologie : fin $\mathrm{VI}^{\mathrm{e}}-\mathrm{V}^{\mathrm{e}}$ s. av. J.-C.

\section{MA13 (pl. XXX)}

Secteur : Regio V, Insulae 4-5.

Sporadique. Au-dessus de la galerie Ouest.

Inventaire : $\mathrm{n}^{\circ}$ 58485/61.

Type : corinthienne A'.

État de conservation : partie de lèvre. H. max. cons. : 4. Lèvre à marli oblique, de section trapézoïdale. H. : 2,5; épaiss. max. : 3,8 ; diam. int./ext. restituable embouchure : 8,6/ 16 .

Surface ext., int. et fracture : orange clair (5YR7/6).

Inclusions : inclusions blanchâtres, brunes et orange (mudstone) de dimensions petites et moyennes, à fréquence moyenne.

Observations et comparaisons : attribuable à une amphore corinthienne de type A'. Pour le type, on renvoie à la fiche précédente.

Chronologie : fin $\mathrm{VI}^{\mathrm{e}}-\mathrm{V}^{\mathrm{e}} \mathrm{s}$. av. J.-C.

\section{MA14 (pl. XXX)}

Année de la fouille / trouvaille : 1964.

Secteur : Regio II, Insula 1.

Structure : Plateia A, face à Q F-E du Four.

Inventaire : $\mathrm{n}^{\circ} 4605$.

Type : corinthienne A'.

État de conservation : partie de lèvre. H. max. cons. : 4,2.

Lèvre à marli oblique, de section trapézoïdale. H. : 2,1 ; épaiss. max. : 3,7 ; diam. int./ext. Restituable embouchure : $10 / 17,3$.

Surface ext., int. et fracture : beige (10YR7/3).

Inclusions : inclusions brunes, blanches et noirâtres de dimensions petites et moyennes, à fréquence moyenne.

Observations et comparaisons : on renvoie à la fiche MA12.

Chronologie : fin $\mathrm{VI}^{\mathrm{e}}-\mathrm{V}^{\mathrm{e}}$ s. av. J.-C.

\section{MA15 (pl. XXX)}

Année de la fouille / trouvaille : 1953.

Secteur : Îlots sur la Plateia A.

Type : corinthienne A'.

État de conservation : portion de lèvre avec une courte partie du col qui garde la trace de l'attache supérieure d'une anse. H. max. cons. : 3,5. Lèvre à marli oblique, de section trapézoïdale, légèrement ouverte. H. : 2,5 ; épaiss. max. : 3,8 ; diam. int./ext. restituable embouchure : $13 / 18,5$.

Surface ext., int. et fracture : beige (7.5YR8/3-10YR8/3). Inclusions : inclusions orange et brunes de dimensions grandes et moyennes, à fréquence moyenne, et inclusions noirâtres et blanches, de dimensions petites et moyennes, à fréquence moyenne-haute.

Observations et comparaisons : on renvoie à la fiche MA12.

Chronologie : fin $\mathrm{VI}^{\mathrm{e}}-\mathrm{V}^{\mathrm{e}}$ s. av. J.-C.

\section{MA16 (pl. XXXI)}

Année de la fouille / trouvaille : 1953.

Secteur : Îlots sur la Plateia A.

Type : corinthienne A'.

État de conservation : lèvre fragmentaire avec une brève partie du col. H. max. cons. : 5,7. Lèvre à marli oblique, de section trapézoïdale. H. : 2,8 ; épaiss. max. : 1,6 ; larg. max. cons. embouchure : 4. Col. H. max. cons. : 2,9 ; larg. max. cons. : 4.

Surface ext.,int. et fracture : beige (7.5YR8/3-10YR8/3). 
Inclusions : inclusions orange et brunes de dimensions grandes et moyennes, à fréquence moyenne, et inclusions noirâtres et blanches, de dimensions petites et moyennes, à fréquence moyenne-haute.

Observations et comparaisons : on renvoie à la fiche MA12. Chronologie : fin $\mathrm{VI}^{\mathrm{e}}-\mathrm{V}^{\mathrm{e}}$ s. av. J.-C.

\section{MA17 (pl. XXXI)}

Année de la fouille / trouvaille : 1994.

Secteur : Regio V, Insula 5.

Structure : Maison 1.

US : 365 .

Type : corinthienne A'.

État de conservation : portion de lèvre avec une brève partie du col. H. max. cons. : 4,7. Lèvre à marli oblique, de section trapézoïdale. H. : 3,8 ; épaiss. max. : 3 ; diam. int./ext. restituable embouchure : 10,8 / 16,1.

Surface ext., int. et fracture : beige clair (10 YR8/3).

Inclusions : inclusions blanchâtres de moyennes et grandes dimensions, à fréquence basse, et inclusions orange brun de petites et moyennes dimensions, à fréquence moyenne-haute. Absence de mudstone de grande dimension.

Engobe : traces d'engobe fin orange.

Observations et comparaisons : on renvoie à la fiche MA12. Chronologie : fin $\mathrm{VI}^{\mathrm{e}}-\mathrm{V}^{\mathrm{e}}$ s. av. J.-C.

\section{MA18 (pl. XXXI)}

Année de la fouille / trouvaille : 1970.

Secteur : Regio V, Insula 2 / Regio VII, Insula 2.

Structure : Puits de la Plateia D.

Inventaire : $\mathrm{n}^{\circ} 225475$.

Type : corinthienne A'.

État de conservation : partie de lèvre et de col avec la jonction supérieure d'une anse. H. max. cons. : 8,5. Lèvre à marli oblique, de section trapézoïdale. H. : 2,3 ; épaiss. max. : 4,3 ; diam. int./ext. restituable embouchure : 10 / 18,2. Col cylindrique. H. max. cons. : 6,5 ; diam. min./max. restituable : 12,4 .

Surface ext., int. et fracture : beige très clair (10YR8/2). Inclusions : inclusions blanches de dimensions petites et moyennes, à fréquence haute, inclusions orange brun de dimensions petites et moyennes, à fréquence basse, et inclusions noirâtres de petites dimensions, à fréquence basse.

Observations et comparaisons : on renvoie à la fiche MA12.

Chronologie : fin $\mathrm{VI}^{\mathrm{e}}-\mathrm{V}^{\mathrm{e}}$ s. av. J.-C.

\section{MA19 (pl. XXXI)}

Année de la fouille / trouvaille : 1994.

Secteur : Regio V, Insula 5.

Structure : Plateia A, devant la Maison 1.

US : 814 .
Type : corinthienne A'.

État de conservation : amphore presque entière mais non complètement reconstituable. La lèvre a pu être reconstituée à partir de deux fragments, les anses seulement en partie, grace à deux et trois fragments ; il reste aussi le fond de la panse avec la jonction du pied et de nombreux fragments de parois non assemblables. Lèvre à marli oblique, de section trapézoïdale. H. : 3,4 ; épaiss. max. : 3,7 ; diam. int./ext. embouchure : 11,3 / 17,2. Col cylindrique. Anses « a bastoncello », de section circulaire. H. : 20 ; épaiss. max. : 3,6.

Surface ext., int. et fracture : beige noisette (7.5YR6/6). Inclusions : inclusions blanches de dimensions petites et moyennes, à fréquence moyenne, distribuées d'une manière homogène, inclusions brun orange (mudstone) de moyennes et surtout grandes dimensions, à fréquence moyenne, distribuées d'une manière homogène.

Observations et comparaisons : amphore corinthienne de type A'. Pour le type, Koehler 1978a, fig. 1.d (datable entre le dernier quart du $\mathrm{V}^{\mathrm{e}}$ et le début du IV $\mathrm{V}^{\mathrm{e}} \mathrm{s}$. av. J.-C.), et Morter, Leonard 1998, fig. 17.3 (variantes datables du courant du Ve s. av. J.-C.). Le pied de la fiche qui suit appartient probablement au même individu et sa morphologie permet de proposer pour cet exemplaire une datation dans le courant de la seconde moitié du V $\mathrm{V}$. av. J.-C.

Chronologie : milieu Ve-débuts IV ${ }^{\mathrm{e}}$ s. av. J.-C.

\section{MA20 (pl. XXXI)}

Année de la fouille / trouvaille : 1994.

Secteur: Regio V, Insula 5.

Structure : Plateia A, devant la Maison 1.

US : 814 .

Type : corinthienne A'.

État de conservation : pied, peut-être complet, mais dont la base est très endommagée. H. max. cons. : 3,2 . Pied en bouton, arrondi en bas. H. max. cons. : 3 ;. diam. min./ $\max$. : 3,7 / 4,2.

Surface ext., int. et fracture : beige noisette (7.5YR6/6). Inclusions : inclusions blanches de dimensions petites et moyennes, à fréquence moyenne, distribuées d'une manière homogène et inclusions brun orange de dimensions moyennes et grandes, à fréquence moyenne, distribuées d'une manière homogène.

Observations et comparaisons : attribuable à une amphore corinthienne de type A'. Pour le type du pied, Koehler 1978a, fig. 1.d (datable entre le dernier quart du Ve et les débuts du IV $\mathrm{e}$ s. av. J.-C.), et Morter, Leonard 1998, fig. 17.3 (variantes datables du courant de la seconde moitié du $\mathrm{V}^{\mathrm{e}} \mathrm{s}$. av. J.-C.). Appartenant probablement au même individu de la fiche précédente.

Chronologie : milieu Ve-début IV e s. av. J.-C.

MA21 (pl. XXXI) 


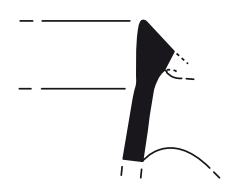

MA16

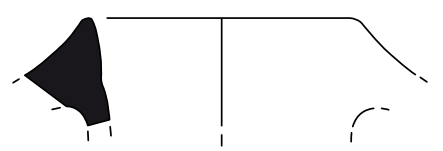

MA17

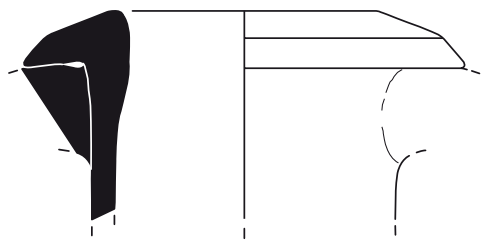

MA18
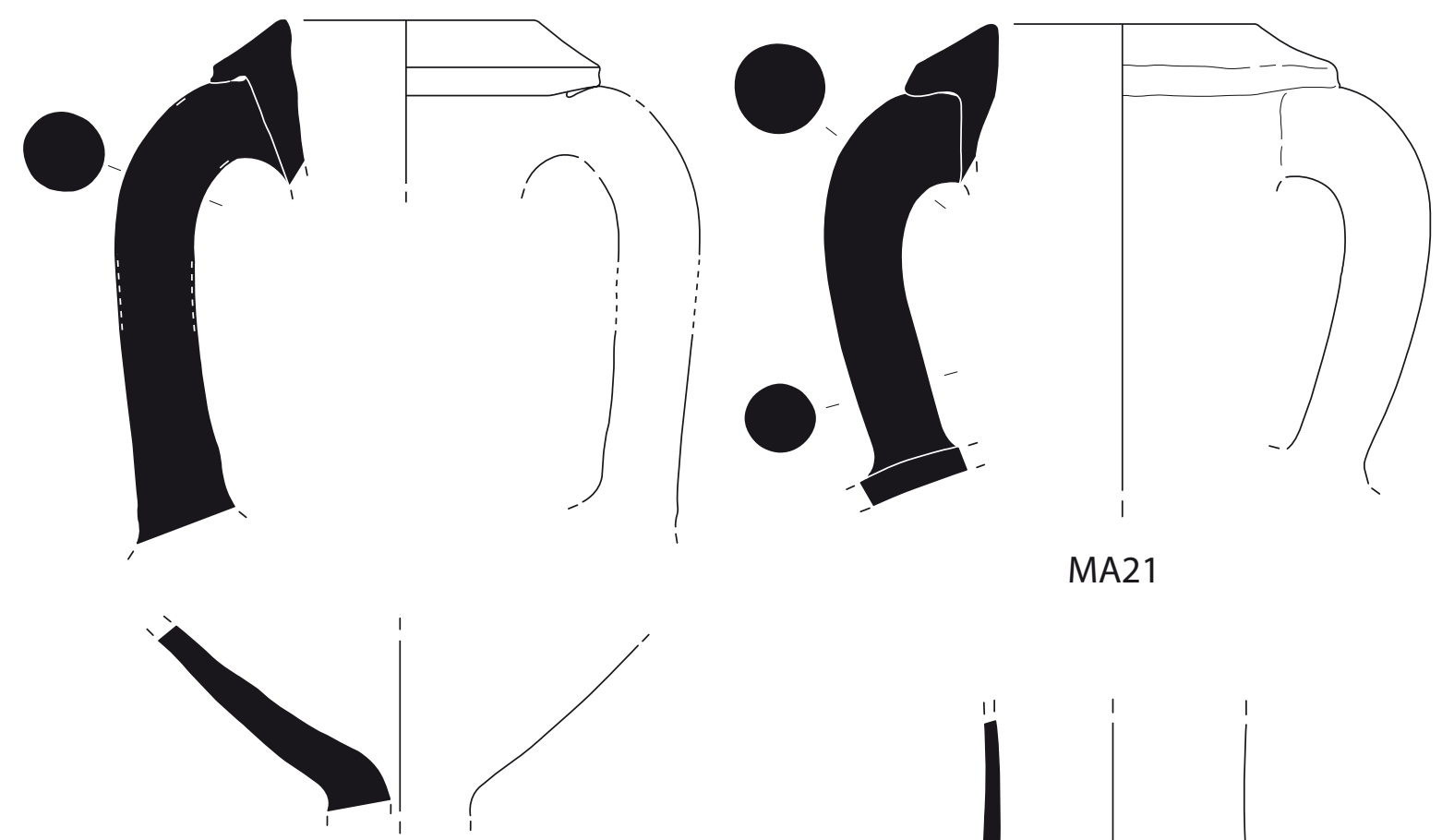

MA21
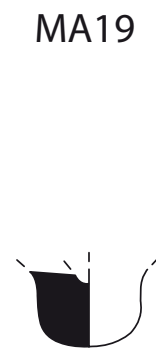

MA20

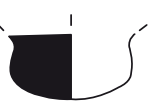

MA23
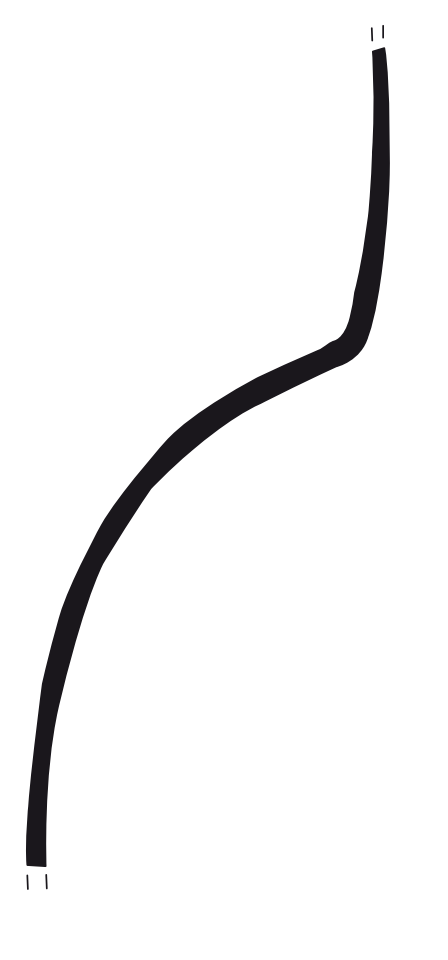

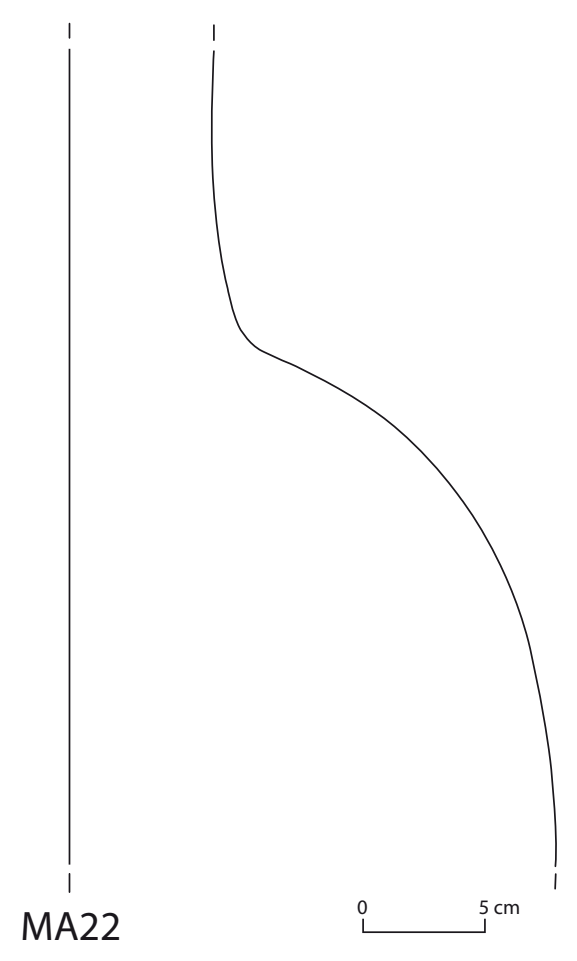

PI. XXXI. Marzabotto. 
Année de la fouille / trouvaille : 1994.

Secteur : Regio V, Insula 5.

Structure : Plateia A, devant la Maison 1.

US : 814 .

Type : corinthienne A'.

État de conservation : lèvre complète avec une brève partie de col, reconstituées à partir de 12 fragments, et les deux anses complètes, dont une reconstituée à partir de 2 fragments. Restaurée. H. max. cons. : 21. Lèvre à marli oblique, de section trapézoïdale. H. : 3,2 ; épaiss. max. : 4 ; diam. int./ext. embouchure : 11,8/19,2. Anses obliques rentrantes, «a bastoncello », de section circulaire d'un diamètre différent, plus petit dans la partie inférieure et plus grand dans la partie supérieure. H. : 17,5 ; épaiss. max. : $3 / 3,8$.

Surface ext., int. et fracture : orange (2.5YR5/8).

Inclusions : inclusions blanches et brunes de dimensions petites et moyennes, à fréquence basse, distribuées d'une manière homogène et inclusions grises de dimensions petites, moyennes et grandes, à fréquence moyennehaute, distribuées d'une manière homogène. Absence de mudstone de grandes dimensions.

Engobe : traces d'engobe orange fin.

Observations et comparaisons : amphore corinthienne de type A'. Pour le type, Koehler 1978a, fig. 1.d (datable entre le dernier quart du $\mathrm{V}^{\mathrm{e}}$ et le début du IV $\mathrm{IV}^{\mathrm{e}} \mathrm{s}$. av. J.-C.), et Morter, Leonard 1998, fig. 17.3 (variantes datables du courant du Ve s. av. J.-C.). Attribuable au même exemplaire de la fiche suivante, dont l'épaule fuyante (pour laquelle cf. Koehler 1978a, fig. 1.d) suggère une possible limitation de son arc chronologique à la seconde moitié du Ves. av. J.-C.

Chronologie : milieu Ve-début IVe s. av. J.-C.

\section{MA22 (pl. XXXI)}

Année de la fouille / trouvaille : 1994.

Secteur : Regio V, Insula 5.

Structure : Plateia A, devant la Maison 1.

US : 814 .

Type : corinthienne A'.

État de conservation : amphore dont il reste une grande partie du col et du corps, et dont il ne manque que le fond. Reconstituée à partir de nombreux fragments et restaurée en deux parties qu'on ne peut pas assembler parfaitement. H. max. cons. : 59. Col tronconique. H. max. cons. : 12 ; diam. min./max. restituable : 11,6/ 14. Épaule arrondie. Larg. : 11. Corps ovoïde. H. max. cons. : 43 ; diam. $\max$. : 40.

Surface ext., int. et fracture : orange (2.5YR5/8).

Inclusions : inclusions blanches et brunes de dimensions petites et moyennes, à fréquence basse, distribuées d'une manière homogène, et inclusions grises de petites, moyennes et grandes dimensions, à fréquence moyenne-haute, distribuées d'une manière homogène. Absence de mudstone de grandes dimensions.

Engobe : traces d'engobe orange fin.

Observations et comparaisons : appartenant au même exemplaire de la fiche précédente, à laquelle on renvoie pour les observations et les comparaisons.

Chronologie : milieu Ve-débuts IV ${ }^{\mathrm{e}}$ av. J.-C.

\section{MA23 (pl. XXXI)}

Secteur : Regio IV, Insula 1.

Structure : Maison 3.

Inventaire : $\mathrm{n}^{\circ} 15$ caisse 17 .

Type : corinthienne A'.

État de conservation : pied. H. max. cons. : 2,6. Pied en bouton, arrondi dans la partie inférieure.

Surface ext.,int.etfracture:orange(7.5YR6/6-7.5YR6/8). Inclusions : inclusions blanches et brunes de dimensions petites, à fréquence moyenne-basse.

Observations et comparaisons : attribuable à une amphore corinthienne de type A'. Pour le type du pied, Koehler 1978a, fig. 1.d (datable entre le dernier quart du Ve et le début du IV ${ }^{\mathrm{e}}$ s. av. J.-C.), et Morter, Leonard 1998, fig. 17.3 (variantes datables du courant de la seconde moitié du Ve s. av. J.-C.).

Chronologie : milieu Ve-début IVe s. av. J.-C.

\section{MA24 (pl. XXXII)}

Année de la fouille / trouvaille : 1988.

Secteur : Regio V, Insula 5.

Structure : Plateia A, devant la Maison 1. Jalon 12 ou 14.

Type : corinthienne A.

État de conservation : fragment de lèvre. H. max. cons. : 4,4. Lèvre à marli oblique, de section trapézoïdale, avec une fine cannelure au niveau de sa plus grande expansion. H. : 3,4 ; épaiss. max. : 3,1 ; diam. int./ext. restituable embouchure : 11,6/16,3.

Surface ext. et int. : beige clair (10YR8/6).

Fracture : beige (10YR7/6).

Inclusions : inclusions blanches et grises de dimensions petites et moyennes, à fréquence moyenne, distribuées d'une manière homogène, et inclusions brun orange (mudstone) de dimensions petites, moyennes et grandes, à fréquence haute, distribuées d'une manière homogène. Observations et comparaisons : pour le type, Koehler 1978a, fig. 1.e. L'obliquité très marquée de la surface de la lèvre suggère pour cet exemplaire un possible encadrement chronologique entre la fin du $\mathrm{V}^{\mathrm{e}}$ et le début du $\mathrm{IV}^{\mathrm{e}} \mathrm{s}$. av. J.-C.

Chronologie : fin Ve-début IV s. av. J.-C.

MA25

Année de la fouille / trouvaille : 1971. 
Secteur : Regio V, Insula 5.

Structure : Fonderie. Q2 Aire F-H (coupe).

Type : corinthienne A.

État de conservation : partie de lèvre en mauvaises conditions. Dimensions max. fr. : 2,5 x 5. Lèvre à marli oblique, de section trapézoïdale. H. max. cons. : 2,5 ; larg. max. cons. embouchure : 5 .

Surface ext., int. et fracture : jaune orange (2.5Y8/ 3-2.5Y8/4).

Inclusions : inclusions blanches, orange, brunes et grises, de petites, moyennes et grandes dimensions, à fréquence haute.

Observations et comparaisons : le mauvais état de conservation et les dimensions réduites ne permettent pas de suggérer un encadrement chronologique. Son appartenance à un exemplaire d'amphore corinthienne A a été proposée sur la base de la pâte caractéristique. Chronologie : non déterminable.

\section{MA26 (pl. XXXII)}

Année de la fouille / trouvaille : 1988.

Secteur : Regio V, Insula 5.

Structure : Maison 1, Pièce II.

US : 123 .

Type : corinthienne A.

État de conservation : partie inférieure d'une anse avec la jonction de l'épaule. Dimensions max. fr. : 5,3 x 5 . Anse « a bastoncello », de section circulaire. H. max. cons. : 3,5; épaiss. max. : 3,5.

Surface ext., int. et fracture : beige clair (10 YR8/3).

Inclusions : inclusions blanchâtres de dimensions moyennes et grandes, à fréquence basse, et inclusions brun orange (mudstone) de petites, moyennes et surtout grandes dimensions, à fréquence haute.

Observations et comparaisons : attribuable à une amphore de production corinthienne de type A sur la base du type caractéristique de la pâte.

Chronologie : non déterminable.

\section{MA27 (pl. XXXII)}

Année de la fouille / trouvaille : 1989.

Secteur : Regio V, Insula 5.

Structure : Maison 1.

US : 180 .

Type : corinthienne A.

État de conservation : anse presque complète, où manquent les deux attaches inférieure et supérieure. $\mathrm{H}$. max. cons. : 11,7. Anse « a bastoncello », de section circulaire dans la partie inférieure et elliptique verticale au niveau de la jonction supérieure. H. max. cons. : 11,7 ; épaiss. max. : $3 / 2,2$ x 4,1.

Surface ext., int. et fracture : beige (10YR7/4).

Inclusions : inclusions blanchâtres de dimensions moyennes et grandes, à fréquence basse, et inclusions brun orange (mudstone) de dimensions petites, moyennes et surtout grandes, à fréquence haute.

Observations et comparaisons : attribuable à la production corinthienne de type A sur la base de la pâte céramique et de la morphologie.

Chronologie : non déterminable.

\section{MA28}

Secteur : Regio IV, Insula 1.

Structure : Maison 1.

Sporadique.

Inventaire : $\mathrm{n}^{\circ} 46$ caisse 1 .

Type : corinthienne A.

État de conservation : partie d'anse en mauvais état de conservation. H. max. cons. : 5. Anse « a bastoncello». H. max. cons. : 5 ; épaiss. max. : 3,5.

Surface ext., int. et fracture : jaune beige (10YR8/ 4-10YR7/4).

Inclusions : inclusions brun orange (mudstone) et inclusions grisâtres de dimensions petites, moyennes et grandes, à fréquence moyenne-haute, et inclusions blanchâtres de petites dimensions, à fréquence basse.

Observations et comparaisons : sur la base de la pâte, on peut l'attribuer sans aucun doute à la production corinthienne de type A, mais son état de conservation et ses dimensions réduites empêchent de proposer tout encadrement chronologique.

Chronologie : non déterminable.

\section{MA29 (pl. XXXII)}

Année de la fouille / trouvaille : 1953.

Secteur : Îlots sur la Plateia A.

Type : corinthienne A'.

État de conservation : anse presque complète. H. max. cons. : 12,4. Anse «a bastoncello », de section circulaire ayant un diamètre plus petit en bas et plus grand dans sa partie haute. H. max. cons. : 12,4 ; épaiss. min./max. : $2,5 / 3,5$.

Surface ext.,int. et fracture : beige (7.5YR8/3-10YR8/3). Inclusions : inclusions orange et brunes de dimensions grandes et moyennes, à fréquence moyenne, et inclusions noirâtres et blanches, de dimensions petites et moyennes, à fréquence moyenne-haute.

Observations et comparaisons : attribuable au type corinthien A'.

Chronologie : non déterminabile.

\section{MA30 (pl. XXXII)}

Année de la fouille / trouvaille : 1953.

Secteur : Îlots sur la Plateia A.

Type : corinthienne A'.

État de conservation : anse presque complète. H. max. 
cons. : 12,3. Anse «a bastoncello », de section circulaire ayant un diamètre plus petit en bas et plus grand dans sa partie haute. Épaiss. min./max. : 2,4 / 3,4.

Surface ext., int. et fracture : beige (7.5YR8/3-10YR8/3). Inclusions : inclusions orange et brunes de dimensions moyennes et grandes, à fréquence moyenne, et inclusions noirâtres et blanches, de dimensions petites et moyennes, à fréquence moyenne-haute.

Observations et comparaisons : attribuable au type corinthien A'.

Chronologie : non déterminable.

\section{MA31 (pl. XXXII)}

Année de la fouille / trouvaille : 1994.

Secteur : Regio V, Insula 5.

Structure : Plateia A, devant la Maison 1.

US : 814 .

Type : corinthienne A'.

État de conservation : anse complète, depuis l'attache inférieure presque jusqu'à l'attache supérieure. H. max. cons. : 15,6. Anses « a bastoncello », de section circulaire d'un diamètre différent, plus petit dans la partie inférieure et plus grand dans la partie supérieure. Épaiss. $\min . / \max$. : 3,1/3,8.

Surface ext., int. et fracture : beige (7.5YR7/4).

Inclusions : inclusions blanches de dimensions petites, moyennes et grandes, à fréquence moyenne, distribuées d'une manière homogène, et inclusions noires et brun orange de dimensions petites et moyennes, à fréquence moyenne, distribuées d'une manière uniforme. Absence de mudstone de grandes dimensions.

Engobe : traces d'un fin engobe orange.

Observations et comparaisons : attribuable au type corinthien A'.

Chronologie : non déterminable.

\section{MA32 (pl. XXXII)}

Année de la fouille / trouvaille : 1994.

Secteur : Regio V, Insula 5.

Structure : Maison 1.

US : 316 .

Type : corinthienne A'.

État de conservation : anse presque complète, reconstituée à partir de deux fragments. H. max. cons. : 17, 5 ; épaiss. max. : 3, 2. Anses obliques rentrantes, «a bastoncello », de section circulaire.

Surface ext., int. et fracture : orange rosé (7.5YR7/6).

Inclusions : inclusions blanches et gris noir de dimensions petites et moyennes, à fréquence basse, et inclusions brun rougeâtre de dimensions petites et moyennes, à fréquence moyenne, distribuées d'une manière non homogène. Absence de mudstone de grandes dimensions. Engobe : traces d'engobe orange, fin.
Observations et comparaisons : attribuable à une amphore corinthienne de type A' étant donné sa pâte céramique et la caractéristique de l'anse à section circulaire de diamètre constant.

Chronologie : non déterminable.

\section{AMPHORES GRÉCO-OCCIDENTALES}

\section{MA33 (pl. XXXII)}

Secteur: Regio IV, Insula 1.

Structure : Maison 4. Q12A

Inventaire : $\mathrm{n}^{\circ} 29$ caisse 1 .

Type : corinthienne B.

État de conservation : pied incomplet, avec une petite partie du fond de la panse, reconstitué à partir de 4 fragments. H. max. cons. : 4,2. Pied en bouton, creux à l'intérieur. H. : 1,8 ; diam. min./max. : 4 / 4,6.

Surface ext., int. et fracture : jaune verdâtre $(2.5 \mathrm{Y} 7 /$ 4-2.5Y8/4).

Inclusions : pâte assez dépurée, avec quelques inclusions de petites dimensions grisâtres et blanches. Vacuoles.

Engobe : traces d'engobe à l'intérieur.

Observations et comparaisons : la morphologie du pied, de type tronconique bref et creux à l'intérieur, ainsi que le profil du fond de la panse, ample et bien distinct du pied, relient ce fragment à une amphore corinthienne de type $\mathrm{B}$ du deuxième ou du troisième quart $\mathrm{du}^{\mathrm{e}} \mathrm{s}$. av. J.-C. Pour le type, Koehler 1978a, fig. 2.b ; Ead. 1981, fig. 1.a, pl. 99.c. Pour la forme, Di Sandro 1981b, fig. 1, datée du milieu du $\mathrm{V}^{\mathrm{e}} \mathrm{s}$. av. J.-C.

Chronologie : 480-425 av. J.-C.

\section{MA34 (pl. XXXII)}

Année de la fouille / trouvaille : 1964.

Secteur : Regio II, Insula 1.

Structure : Plateia A-côté E, en face de Q DC du Four. Inventaire : $\mathrm{n}^{\circ} 8614$.

Type : corinthienne B.

État de conservation : partie de pied et fond de la panse. H. max. cons. : 4. Pied en bouton, légèrement creux à l'intérieur. H. max. cons. : 2 ; diam. min./max. : 4,9 / 6,9. Surface ext. et int. : orange rouge $(5 \mathrm{YR} 7 / 6)$.

Fracture : jaune (10YR8/4).

Inclusions : inclusions orange, brunes et blanches, de petites dimensions, à fréquence basse.

Observations et comparaisons : voir la fiche précédente. Chronologie : 480-425 av. J.-C.

\section{MA35 (pl. XXXII)}

Année de la fouille / trouvaille : 1953.

Secteur : Îlots sur la Plateia A.

Type : corinthienne B.

État de conservation : partie inférieure d'amphore. $\mathrm{H}$. 


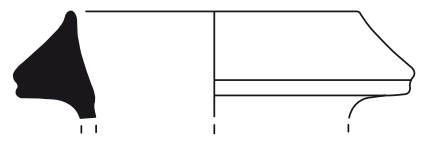

MA24

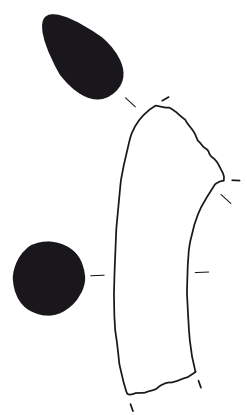

MA27

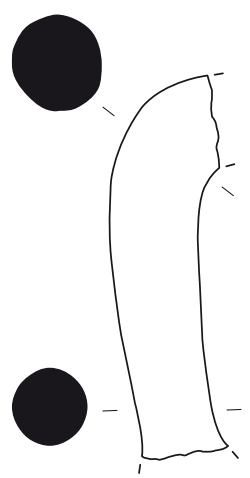

MA31

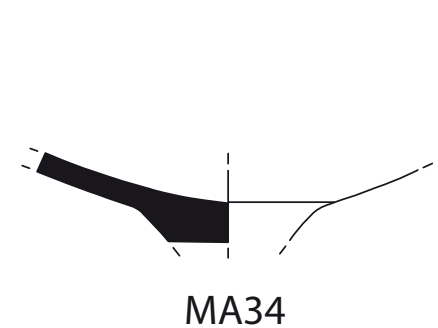

MA34
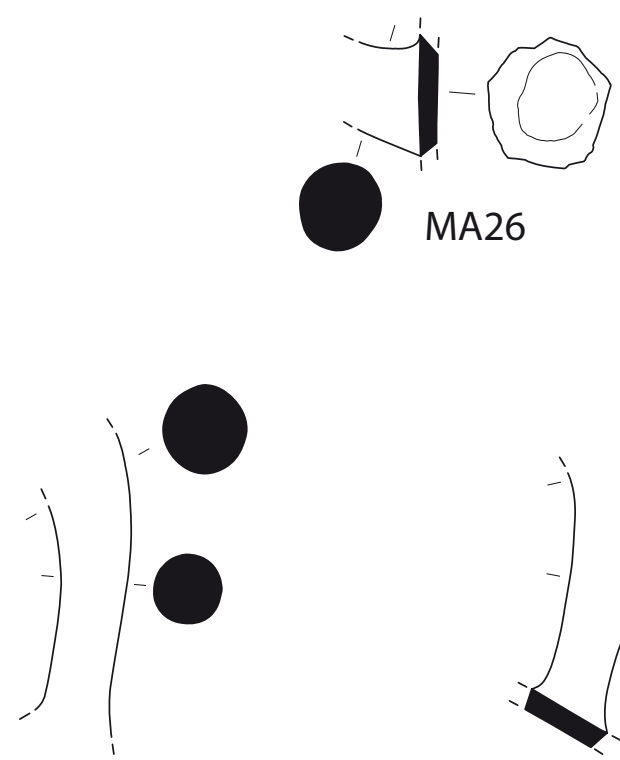

MA29

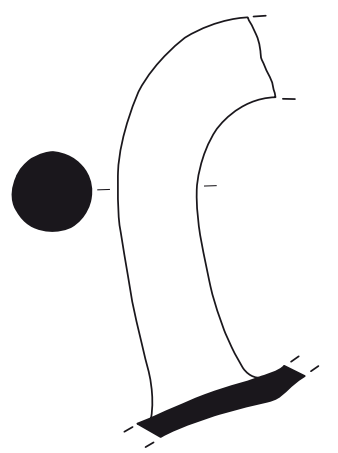

MA32

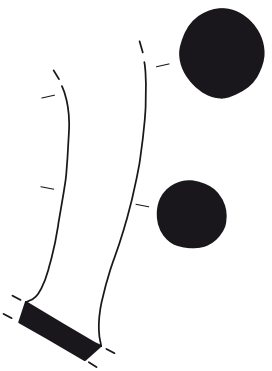

MA30

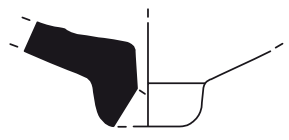

MA33

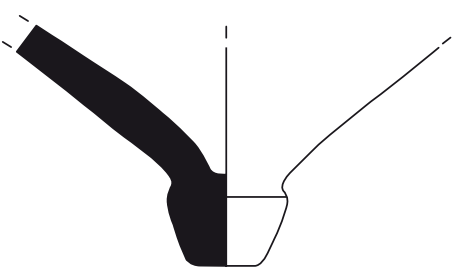

MA35

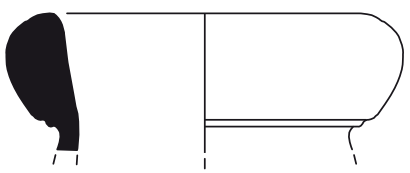

MA37

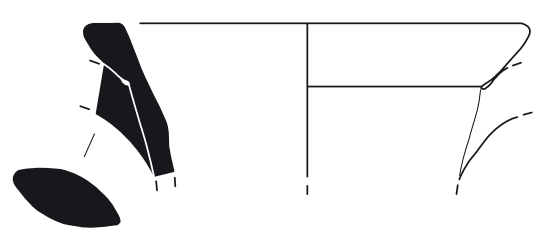

MA36

PI. XXXII. Marzabotto. 
max. cons. : 9,8. Corps. H. max. cons. : 6, 4 ; diam. max. restituable : 17,2. Pied en bouton, épaissi et à base plate. H. : 3,4 ; diam. min./max. : 3 / 4,8.

Surface ext., int. et fracture : orange rosé (2.5YR7/ 4-2.5YR8/3).

Inclusions : inclusions blanches et noires de petites dimensions, à fréquence moyenne-haute.

Observations et comparaisons : le type de pied, tronconique bref, rattache l'exemplaire à la production corinthienne $\mathrm{B} d u \mathrm{~V}^{\mathrm{e}} \mathrm{s}$. av. J.-C. La partie conservée du fond de la panse se distingue nettement du pied, comme c'est le cas pour les exemplaires du deuxième et du troisième quart du V $\mathrm{V}^{\mathrm{e}}$ s. av. J.-C. (Koehler 1978a, fig. 2.b ; Ead. 1981, fig. 1.a, pl. 99.c.), mais son profil convexe pas trop prononcé rappelle plutôt le type de la fin du $\mathrm{V}^{\mathrm{e}} \mathrm{s}$. av. J.-C. (Koehler 1978a, fig. 2.c).

Chronologie : 425-400 av. J.-C.

\section{MA36 (pl. XXXII)}

Secteur : Regio IV, Insula 1.

Structure : Maison 4. Q13.

Inventaire $: \mathrm{n}^{\circ} 15$ caisse 1 .

Type : corinthienne B.

État de conservation : fragment de lèvre avec un bref segment de col et l'attache supérieure d'une anse. $\mathrm{H}$. max. cons. : 6,5. Lèvre en échine, légèrement ouverte. H. : 2,5 ; épaiss. max. 1,7 ; diam. int./ext. restituable embouchure : 15,2 / 18,2. Col évasé, avec une rainure immédiatement au-dessous de la lèvre. H. max. cons. : 4 ; diam. min./max. restituable : 12,4 / 14,2. Anses en ruban épaissi, à section elliptique. $H$. max. cons. : 3,5 ; épaiss. max. : 4,7 x 2.

Surface ext., int. et fracture : jaunâtre (2.5Y8/2-2.5Y7/3). Inclusions : inclusions jaunâtres (chamotte), brunes et orange de dimensions petites et moyennes, à fréquence moyenne. Vacuoles.

Observations et comparaisons : la morphologie de la lèvre, du type en échine, ainsi que celle des anses surélevées dont la courbure s'écrase au-dessous de la lèvre, suggèrent une attribution à la production corinthienne de type B du dernier quart du $\mathrm{V}^{\mathrm{e}} \mathrm{s}$. av. J.-C. Pour le type, Koehler 1978a, fig. 2.c.

Chronologie : 425-400 av. J.-C.

\section{MA37 (pl. XXXII)}

Année de la fouille / trouvaille : 1971.

Secteur : Regio V, Insula 5.

Structure : Four. Q2 Aire F-H (coupe).

Type : « forme 2 ».

État de conservation : partie de lèvre. H. max. cons. : 5,7. Lèvre en bourrelet gonflé, avec un fin listel de base. H. : 4,6 ; épaiss. max. : 2,5 ; diam. int./ext. restituable embouchure : 11,2 / 15,6.
Surface ext., int. et fracture : beige (10YR8/3-10YR7/4). Inclusions : inclusions blanches, grises, orange et rougeâtres, de petites dimensions, à fréquence moyenne. Écailles de mica argenté, doré et cuivré, de dimensions petites et moyennes, à fréquence moyenne. Pâte dure à la surface rugueuse.

Observations et comparaisons : pour le type, Py 1980 , p. 5-6, notamment fig. 3.3 (de Nîmes), type Py 1 datable entre la seconde moitié du $\mathrm{VI}^{\mathrm{e}}$ et le début du $\mathrm{V}^{\mathrm{e}} \mathrm{s}$. av. J.-C. ; pour la forme, Semeraro 1997, $\mathrm{n}^{\circ} 172 \mathrm{c}$, fig. 47. 172c. Des lèvres du même type proviennent de Spina (cat. SP19) et du Forcello (cat. FO26 et FO27). La pâte céramique de cet exemplaire suggère de le rapprocher de la production de Locres.

Chronologie : milieu VI'-début Ve s. av. J.-C.

\section{AMPHORES DE L'ÉGÉE SEPTENTRIONALE}

\section{MA38 (pl. XXXIII)}

Année de la fouille / trouvaille : 1988.

Secteur : Regio V, Insula 5.

Structure : Plateia A, devant la Maison 1.

US : 3 B3-A3.

Type : Ring-toe Lawall.

État de conservation : pied avec un court segment du fond de la panse. H. max. cons. : 3,5. Pied tronconique creux, à base annulaire. H. : 2 ; diam. min./max. : $6 / 7$. Surface ext., int. et fracture : beige orange (7.5YR7/6). Inclusions : inclusions blanches, brunes et grises de petites dimensions, à fréquence basse, distribuées d'une manière homogène. Écailles de mica argenté et doré de petites dimensions, à fréquence basse, distribuées d'une manière homogène.

Observations et comparaisons : pour le type, datable entre la fin du VI ${ }^{\mathrm{e}}$ et les débuts du Ve $\mathrm{s}$. av. J.-C., Lawall 1995, p. 157-158, 163-166, fig. 65, (du dépôt E 14 :5 de l'Agora d'Athènes fermé vers 480 av. J.-C.) ; pour la forme, Schmid 2001, p. $148,152, n^{\circ} 4$, note 22 , pl. 4 (datée vers le début du $\mathrm{V}^{\mathrm{e}} \mathrm{s}$. av. J.-C.).

Chronologie : 525-480 av. J.-C.

\section{MA39 (pl. XXXIII)}

Année de la fouille / trouvaille : 1971.

Secteur : Regio V, Insula 5.

Structure : Four. Espace destiné à la cour.

Inventaire : $n^{\circ} 63 / 52$.

Type : Ring-toe Lawall.

État de conservation : pied complet. H. max. cons. : 3 . Pied tronconique, à base en anneau épaissi. H. : 3 ; diam. $\min . / \max$. : 6,2 / 6,9.

Surface ext., int. et fracture : beige orange (7.5YR7/ 6-7.5YR7/8).

Inclusions : inclusions blanches, de dimensions petites 
et moyennes, à fréquence basse, et inclusions grises et orange de petites dimensions, à fréquence basse. Mica argenté fin, à fréquence basse. Vacuoles.

Observations et comparaisons : le profil du pied renvoie à la production nord-égéenne de la fin du $\mathrm{VI}^{\mathrm{e}}$ ou du début du Ve s. av. J.-C. Pour la forme, Lawall 1995, p. 157-158, 163-166, fig. 51, datée sur la base du contexte (dépôt E $14: 5$ de l'Agora) de 480 av. J.-C. au plus tard; et Schmid 2001, p. 148, 152, n ${ }^{\circ}$ 5, note 23, pl. 5, datée entre la fin du VI e et le début du Ve $\mathrm{s}$. av. J.-C.

Chronologie : 525-480 av. J.-C.

\section{MA40 (pl. XXXIII)}

Secteur : Regio IV, Insula 1.

Structure : Maison 6.

Défoncement de vigne.

Inventaire : $n^{\circ} 36$ caisse 11 .

État de conservation : partie de lèvre et de col, reconstitué à partir de 2 fragments. H. max. cons. : 4,6. Lèvre en biseau, avec une rainure à la base, tant à l'int. qu'à l'ext. H. : 1,5 ; épaiss. max. : 1,3 ; diam. int./ext. restituable embouchure : 9,4 / 11,9. Col cylindrique. H. max. cons. : 3,1 ; diam. min./max. restituable : 10,5.

Surface ext., int. et fracture : orange foncé (5YR6/6).

Inclusions : inclusions brunes, grises et blanches, de dimensions petites, à fréquence basse.

Observations et comparaisons : attribuable à la production nord-égéenne. La morphologie de la lèvre, au caractère archaïsant à cause de son grossissement supérieur, pourrait suggérer une datation entre le dernier quart du $\mathrm{VI}^{\mathrm{e}}$ et le premier quart du Ve s. av. J.-C. Pour le type, Schmid 2001, n ${ }^{\circ} 4$, pl. 4, et $\mathrm{n}^{\circ} 5$, pl. 5, l'une et l'autre datables entre la fin du VI ${ }^{\mathrm{e}}$ et le début du $\mathrm{V}^{\mathrm{e}} \mathrm{s}$. av. J.-C. ; pour la forme, Monachov 1999b, fig. 17.1, attribuée à Thasos et datée de 525-475 av. J.-C.

Chronologie : 525-480 av. J.-C.

\section{MA41 (pl. XXXIII)}

Secteur : Regio IV, Insula 1.

Structure : Maison 4. Q10.

Inventaire : $\mathrm{n}^{\circ} 49$ caisse 4 .

État de conservation : partie de col avec le segment supérieur d'une anse. H. max. cons. : 5,5. Anses en ruban épaissi, de section elliptique, avec une arête centrale. $\mathrm{H}$. max. cons. : 5 ; épaiss. max. : 4,3 x 2,1.

Surface ext., int. et fracture : orange (2.5YR5/6).

Inclusions : inclusions blanches de dimensions petites, moyennes et grandes, à fréquence moyenne-haute, et inclusions grisâtres de dimensions petites et moyennes, à fréquence moyenne. Vacuoles.

Observations et comparaisons : vu le détail morphologique de l'arête centrale, l'anse en question peut être attribuée à la production nord-égéenne d'époque archaïque tardive. Pour l'hypothèse récente qui considère cette caractéristique comme typique des exemplaires du début de série de la production thasienne, Spagnolo 2003, p. 624 et note 96, pl. III.2.

Chronologie : fin $\mathrm{VI}^{\mathrm{e}}$-début $\mathrm{V}^{\mathrm{e}} \mathrm{s}$. av. J.-C.

\section{MA42 (pl. XXXIII)}

Secteur: Regio IV, Insula 1.

Structure : Maison 4. Q12A.

Inventaire : $n^{\circ} 27$ caisse 1 .

État de conservation : segment inférieur d'une anse, reconstitué à partir de 2 fragments. H. max. cons. : 8,5. Anses en ruban épaissi, de section elliptique, avec une arête centrale et une impression digitale à l'emplacement de l'attache inférieure. H. max. cons. : 8 ; épaiss. $\max .: 3,9 \times 2,4$.

Surface ext., int. et fracture : orange intense (2.5YR5/ 6-2.5YR5/8).

Inclusions : inclusions blanches de dimensions moyennesgrandes, à fréquence moyenne, et inclusions brunes et grisâtres de dimensions moyennes-petites, à fréquence moyenne-basse. Vacuoles.

Observations et comparaisons : attribuable probablement à la production nord-égéenne (peut-être Thasos) d'époque tardo-archaïque. Pour le type d'anse, avec arête centrale, Spagnolo 2003, p. 624 et note 96, pl. III.2 ; et Johnston 1990, ${ }^{\circ}$ 131-132, 177, fig. 12. Pour l'impression digitale appliquée en correspondance de son attache inférieure, constituant une autre caractéristique des fabriques nord-égéennes, Blondé, Muller, Mulliez 1991 ; Grandjean 1992 ; et Schmid 2001, avec de nombreux exemplaires datés entre la fin du $\mathrm{VI}^{\mathrm{e}}$ et le $\mathrm{V}^{\mathrm{e}} \mathrm{s}$. av. J.-C.

Chronologie : fin VI'-début Ve s. av. J.-C.

\section{MA43 (pl. XXXIII)}

Secteur: Regio V, Insula 5.

Structure : Fonderie.

Inventaire : $\mathrm{n}^{\circ} \mathrm{D} 16-21 \mathrm{a} / 1 / 8$.

Type : Disc-toe Lawall.

État de conservation : partie du fond et de la panse. $\mathrm{H}$. max. cons. : 8,5. Corps. H. max. cons. : 6,5 ; largh. max. cons. : 14,4. Pied tronconique plein, à base légèrement convexe et ombiliquée. H. : 2,1 ; diam. min./max. : 5,5 / 5,9 .

Surface ext. : orange (7.5YR6/6-7.5YR6/8).

Surface int. : orange rosé (5YR6/6-5YR6/8).

Fracture : gris $(2.5 \mathrm{Y} 5 / 1)$.

Inclusions : inclusions blanches de dimensions petites et moyennes, à fréquence moyenne-haute, distribuées d'une manière homogène. Inclusions noires de petites dimensions, à fréquence basse, distribuées d'une manière non homogène. Écailles de mica argenté, de dimensions petites et moyennes, à fréquence moyenne-haute. 
Observations et comparaisons : attribuable à la production nord-égéenne. Pour le pied, du type discoïdal, daté entre 480 et 440 av. J.-C., Lawall 1995, p. 158-159, 166-167, fig. 58, 66, respectivement du dépôt F $19: 4$ de l'Agora, fermé autour de 450 av. J.-C., et du dépôt Q 12 :3, fermé autour de 480 av. J.-C. ; pour la forme, Schmid 2001, p. 148,156 , note $24, \mathrm{pl} .6, \mathrm{n}^{\circ} 6$, datée autour de $460-450$ av. J.-C. ; et Spagnolo 2003, pl. III. 6, 11. Sur le fond intérieur on remarque un résidu consistant de résine.

Chronologie : 480-440 av. J.-C.

\section{MA44 (pl. XXXIII)}

Secteur : Regio IV, Insula 1.

Structure : Maison 4.

Sporadique.

Inventaire : $n^{\circ} 24-25$ caisse 13 .

État de conservation : fragment de lèvre et col avec le segment supérieur d'une anse. H. max. cons. : 5,9. Lèvre en biseau, de section quadrangulaire. H. : 1,2 ; épaiss. max. : 1 ; diam. int./ext. restituable embouchure : 10,4 / 12,1. Col cylindrique, avec une rainure légère au-dessous de la lèvre, au niveau de la jonction des anses. $\mathrm{H}$. max. cons. : 4,5; diam. min./max. restituable : 11 . Anses en ruban épaissi, de section elliptique. H. max. cons. : 3,5 ; épaiss. max. : 4,9 x 1,6.

Surface ext., int. et fracture : chamois rosé (7.5YR7/4). Inclusions : inclusions brunes, grises et blanchâtres, de petites dimensions, à fréquence moyenne. Vacuoles.

Observations et comparaisons : le type de lèvre et l'écrasement latéral typique des attaches supérieures des anses, permettent d'attribuer l'exemplaire à la production nordégéenne. Les lèvres des productions nord-égéennes, définies «en biseau» présentent de nombreuses variantes morphologiques. La petite lèvre de section quadrangulaire constitue un caractère archaïsant. Pour la forme à section quadrangulaire, Schmid 2001, $\mathrm{n}^{\circ} 6, \mathrm{pl}$. 6 , datable du deuxième quart du $\mathrm{V}^{\mathrm{e}} \mathrm{s}$. av. J.-C.

Chronologie : 475-450 av. J.-C.

Bibliographie : Sandri 1972, fig. 3, 18 (pour le dessin).

\section{MA45 (pl. XXXIII)}

Secteur : Regio V, Insulae 4-5.

Sporadique. Au-dessus de la galerie Ouest.

Inventaire : $\mathrm{n}^{\circ}$ 58424/61.

Type : Middle Mendean Variant Lawall.

État de conservation : pied entier, jusqu'à la jonction du fond de la panse. H. max. cons. : 5,8. Pied court en bobine, à base convexe et ombiliquée. H. : 5,5; diam. $\min . / \max .:$ : 4,9 / 6,9.

Surface ext. et int. : jaunâtre (10YR7/4-10YR7/6).

Fracture : rougeâtre (10R5/6).

Inclusions : inclusions blanches de dimensions petites et moyennes, à fréquence haute, et inclusions grises et noirâtres, de petites dimensions, à fréquence haute. Petites écailles de mica argenté.

Observations et comparaisons : le type de pâte et l'évasement de la base suggèrent son attribution à la production de Mendé. La hauteur encore modérée du pied, du type en bobine, permet de préciser son appartenance à la Middle Mendean Variant de M. Lawall. Pour le type, Lawall 1995, p. 121-122, fig. 38 ; pour la forme, Bonghi Jovino 1982, pl. 122.4 ; Papadopoulos, Paspalas 1999, fig. 2 ; et de manière ponctuelle, Monachov 1999b, fig. 27.4-5, datables, vu le contexte, de 450-425 av. J.-C. Chronologie : 450-425 av. J.-C.

\section{MA46 (pl. XXXIII)}

Secteur : Regio IV, Insula 1.

Structure : Maison 3. Espace destiné à la cour.

Inventaire : $\mathrm{n}^{\circ} 16$ caisse 17.

Type : Stem-toe, variante 2 Lawall.

État de conservation : pied. H. max. cons. : 9,1. Pied en bobine allongé, à base convexe et ombiliquée. Diam. $\min . / \max .:$ : 4,8 / 7,5.

Surface ext. et int. : orange (5YR6/6-5YR6/8).

Fracture : mauve (10R5/2-10R5/3).

Inclusions : inclusions blanches de petites dimensions, à fréquence basse. Mica distribué sur toute la surface.

Observations et comparaisons : pour le type, Lawall 1995, p. 141-143, fig. 53, datable du courant de la deuxième moitié du Ve s. av. J.-C. ; pour la forme, Zeest 1960, pl. 6.16 ; Grandjean 1992, fig. 11.73 ; et Monachov 1999b, fig. 23.1, datée du troisième quart du $\mathrm{V}^{\mathrm{e}} \mathrm{s}$. av. J.-C. et attribuée au « Cercle de Thasos ».

Chronologie : 450-400 av. J.-C.

\section{MA47 (pl. XXXIII)}

Année de la fouille / trouvaille : 1994.

Secteur : Regio V, Insula 5.

Structure : Maison 1.

US : 316 .

Type : Late Mendean Variant Lawall.

État de conservation : pied, légèrement ébréché, avec partie du fond de la panse. H. max. cons. : 13,6. Pied en bobine allongé, à base convexe et ombiliquée. H. : 9,3 ; diam. min./max. : 5,3 / 8,4.

Surface ext., int. et fracture : orange (7.5YR6/6).

Inclusions : inclusions blanches, de dimensions petites et moyennes, à fréquence basse et moyenne, distribuées d'une manière assez homogène, et inclusions gris noir, de dimensions petites, à fréquence basse et moyenne, distribuées d'une manière homogène. Écailles de mica argenté de dimensions petites et moyennes, à fréquence moyenne. Engobe : engobe blanc, fin, visible aussi bien à l'intérieur qu'à l'extérieur.

Observations et comparaisons : la morphologie du pied, 


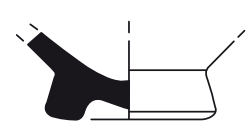

MA38

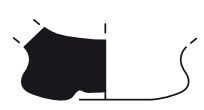

MA39

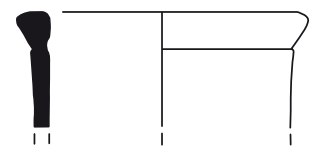

MA40

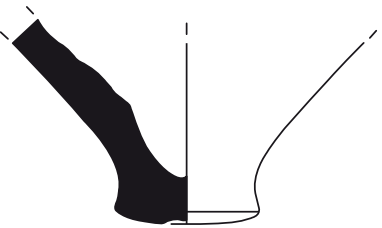

MA43

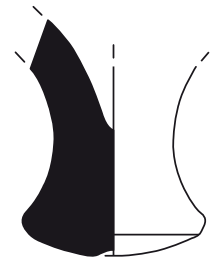

MA46

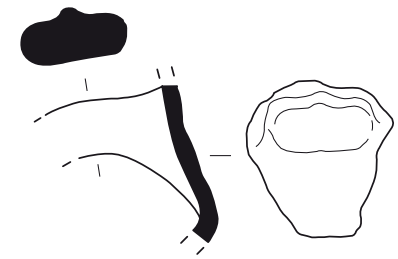

MA41

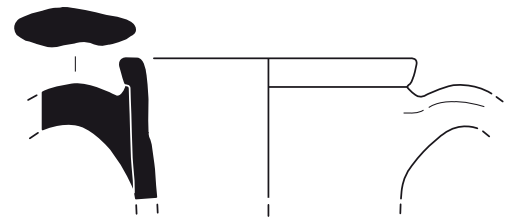

MA44

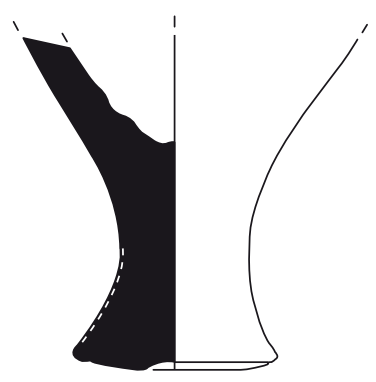

MA47

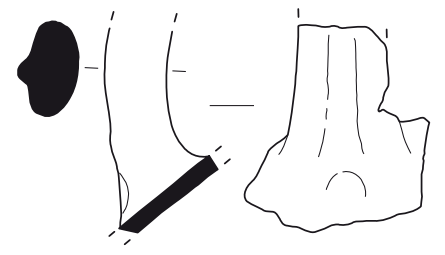

MA42

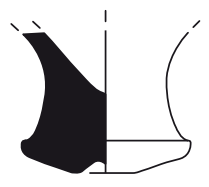

MA45

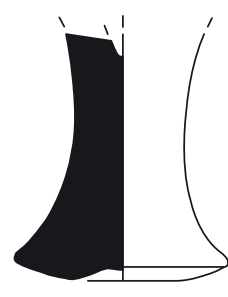

MA48 
du type en bobine de hauteur moyenne, renvoie au type Late Mendean de M. Lawall. Les caractéristiques de la pâte semblent toutefois différentes de celles de la production de Mendé. Pour le type, Lawall 1995, p. 122-123, fig. 39 ; pour la forme, Papadopoulos, Paspalas 1999, fig. 4, datables entre 425 et 400 av. J.-C.

Chronologie : 425-400 av. J.-C.

\section{MA48 (pl. XXXIII)}

Secteur : Regio IV, Insula 1.

Structure : Maison 4.

Sporadique.

Inventaire : $n^{\circ} 48$ caisse 13 .

Type : Late Mendean Variant Lawall.

État de conservation : pied complet. H. max. cons. : 10,1. Pied en bobine allongé, à base convexe et ombiliquée. H. : 9,1 ; diam. min./max. : 4,5 / 8,7.

Surface ext. et int. : orange (5YR6/6-5YR5/8).

Fracture : chamois (10YR6/4).

Inclusions : inclusions grises et blanches de dimensions petites et moyennes, à fréquence moyenne-haute. Mica fin à fréquence basse. Vacuoles.

Observations et comparaisons : attribuable à la production de Mendé. Pour le type, datable du dernier quart du Ve s. av. J.-C., Lawall 1995, p. 122-123, fig. 39 ; pour la forme, Brašinskij 1976, fig. 3.

Chronologie : 425-400 av. J.-C.

\section{MA49 (fig. $91 ;$ pl. XXXIV)}

Lieu de découverte : Marzabotto (Bo.)

Secteur : Regio IV, Insula 1.

Structure : Maison 4. Q11.

Inventaire : $n^{\circ} 5$ caisse 0 .

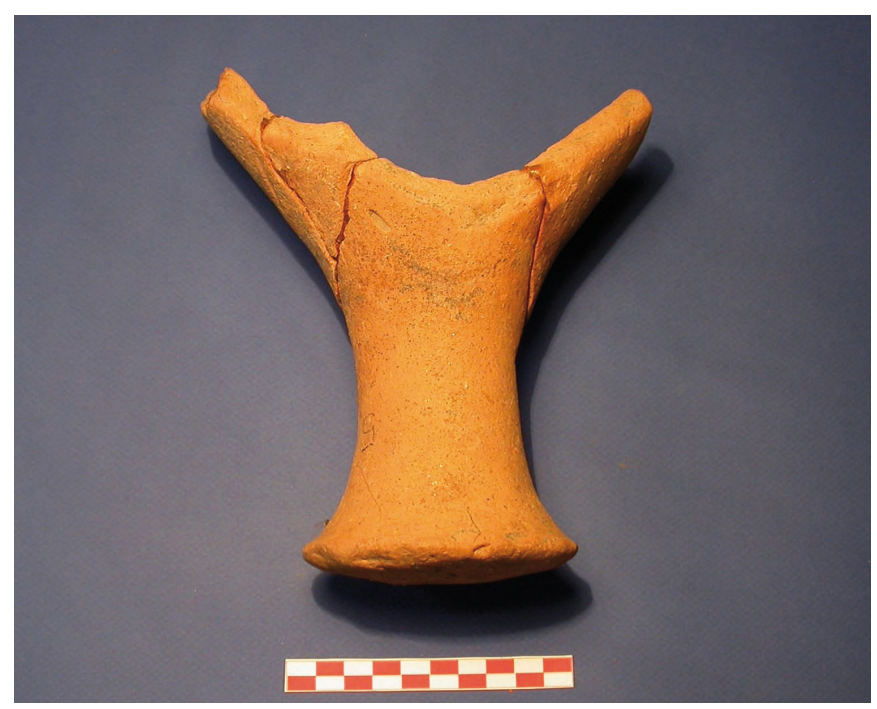

Fig. 91. Marzabotto, fond d'amphore nord-égéenne en provenance de la Regio IV, Insula I, Casa 4. Cat. MA49 (photo F. Sacchetti).
Type : Late Mendean Variant Lawall.

État de conservation : pied avec la moitié inférieure de la panse, reconstituée à partir de 6 fragments. H. max cons. : 23. Corps probablement piriforme ou biconique. H. max. cons. : 13,7 ; diam. max. : 26,4. Pied en bobine allongé, à base convexe et ombiliquée. H. : 9 ; diam. min./ $\max$. : 5,4 / 8,9.

Surface ext.,int.etfracture: chamois (7.5YR6/6-10YR6/6). Inclusions : inclusions grises et blanches de dimensions petites et moyennes, à fréquence moyenne-haute. Mica fin argenté à fréquence basse. Vacuoles.

Observations et comparaisons : attribuable à la production de Mendé. Pour le type, Lawall 1995, fig. 39 ; pour la forme, Monachov 1999b, fig. 22. 1, trouvée dans une tombe près de Steblev datée de 450 av. J.-C. et attribuée au « cercle de Thasos », peut-être Mendé.

Chronologie : 425-400 av. J.-C.

Bibliographie : Sandri 1972, fig. 8, 41 (pour le dessin).

\section{MA50 (pl. XXXIV)}

Année de la fouille / trouvaille : 1964.

Secteur : Regio II, Insula 1.

Structure : Plateia A-côté E, devant Q DC du Four.

Inventaire : $n^{\circ} 8613$.

Type : Late Mendean Variant Lawall

État de conservation : partie de pied. Pied en bobine. $\mathrm{H}$. max. cons. : 6,7 ; diam. min./max. : 6,1 / 8,6.

Surface ext. et int. : orange clair (7.5YR7/6).

Fracture : mauve rosé (5YR6/4).

Inclusions : inclusions blanches de petites dimensions, à fréquence basse, et inclusions orange de petites dimensions, à fréquence haute. Écailles de mica argenté de dimensions petites et moyennes, à fréquence basse.

Observations et comparaisons : attribuable à la production de Mendé de la fin du Ve s. av. J.-C. Pour le type, Lawall 1995, fig. 39 ; et Brašinskij 1976, fig. 3, datées du dernier quart du $\mathrm{V}^{\mathrm{e}} \mathrm{s}$. av. J.-C.

Chronologie : 425-400 av. J.-C.

\section{MA51 (pl. XXXIV)}

Secteur : Regio IV, Insula 1.

Structure : Maison 6.

Défoncement de vigne.

Inventaire : $\mathrm{n}^{\circ} 10$ caisse 6 .

Type : Biconical Lawall.

État de conservation : partie de lèvre avec une brève partie de col et le segment supérieur d'une anse, reconstitué à partir de 2 fragments. H. max. cons. : 6,9. Lèvre en biseau, légèrement ouverte. H. : 1,8 ; épaiss. max. : 1,5 ; larg. max. cons. embouchure : 3,2. Col. H. max. cons. : 5,2 ; larg. max. cons. : 5,7. Anses en ruban épaissi, à section elliptique. H. max. cons. : 4,5; épaiss. max. : 3,5 x 2 .

Surface ext.,int.etfracture: orange rosé(5YR7/6-5YR6/6). 
Inclusions : inclusions blanches de petites dimensions, à fréquence basse.

Observations et comparaisons : attribuable à la production nord-égéenne et à rapprocher du type «biconical » de M. Lawall assigné à la production de Thasos du dernier quart du Ve s. av. J.-C. Pour le type, Lawall 1995, p. 135, fig. 43-44 ; et Blondé, Muller, Mulliez 1991, fig. 3.16.

Chronologie : 425-400 av. J.-C.

\section{MA52 (pl. XXXIV)}

Année de la fouille / trouvaille : 1991.

Secteur : Regio V, Insula 5.

\section{Structure : Maison 1. Pièce XII.}

État de conservation : segment inférieur d'une anse (h. max. cons. : 7,6) et fragment d'épaule (dimensions max. fr. : 5 x 5,7) non reconstituables.

Anses en ruban épaissi, à section elliptique, avec impression digitale au niveau de la jonction inférieure. H. max. cons. : 7,6 ; épaiss. max. : 2,1 x 3,9.

Surface ext., int. et fracture : orange rougeâtre (5YR5/6). Inclusions : inclusions blanchâtres, gris noir et brunes de petites dimensions, à fréquence très basse, distribuées d'une manière homogène. Écailles de mica argenté de dimensions petites et moyennes, à fréquence très haute. Observations et comparaisons : la pâte céramique et l'impression digitale à la base de l'anse suggèrent de l'attribuer à la production nord-égéenne. Pour cette caractéristique, cf. Blondé, Muller, Mulliez 1991, avec de nombreux exemplaires datables entre la fin $\mathrm{du} \mathrm{VI}^{\mathrm{e}}$ et le $\mathrm{V}^{\mathrm{e}}$ s. av. J.-C.

Chronologie : non déterminabile.

\section{MA53 (pl. XXXIV)}

Année de la fouille / trouvaille : 1994.

Secteur : Regio V, Insula 5.

Structure : Maison 1.

US : 352 .

État de conservation : partie d'épaule portant des traces de la jonction inférieure d'une anse (dimensions max. fr. $10,4 \times 9,8)$ avec impression digitale et un fragment de paroi (dimensions max. fr. : 4,9 x 5,4) non assemblables. Surface ext., int. et fracture : beige orange (7.5YR6/6). Inclusions : inclusions blanches de dimensions petites et moyennes, à fréquence moyenne-basse distribuées d'une manière non homogène. Écailles de mica doré et argenté de dimensions petites et moyennes à fréquence moyenne. Observations et comparaisons : on renvoie à la fiche précédente.

Chronologie : non déterminabile.

\section{MA54 (pl. XXXIV)}

Année de la fouille / trouvaille : 1988.

Secteur : Regio V, Insula 5.
Structure : Plateia A, devant la Maison 1.

US : 3 .

État de conservation : partie inférieure d'une anse, depuis la jonction de l'épaule jusqu'à la courbure supérieure. H. max. cons. : 9,6. Anses en ruban épaissi, de section elliptique, avec impression digitale au niveau de la jonction inférieure. Épaiss. max. : 2 x 4,3.

Surface ext., int. et fracture : orange (2.5YR6/8).

Inclusions : inclusions de petites dimensions blanches et grises, à fréquence basse, distribuées d'une manière homogène. Écailles de mica argenté et doré de dimensions petites, à fréquence moyenne, distribuées d'une manière homogène.

Observations et comparaisons : on renvoie à la fiche MA52. Chronologie : non déterminabile.

\section{MA55 (pl. XXXIV)}

Année de la fouille / trouvaille : 1994.

Secteur : Regio V, Insula 5.

Structure : Plateia A, devant la Maison 1.

US : 814 .

État de conservation : fragment d'épaule avec l'attache inférieure d'une anse. Dimensions max. fr. : 7,4 x 9 . Anses en ruban épaissi, à section elliptique, avec impression digitale au niveau de la jonction inférieure. H. max. cons. : 3,5 ; épaiss. max. : 2,8 x 5 .

Surface ext. : beige (7.5YR7/6).

Surface int. et fracture : rosée (5YR6/4).

Inclusions : inclusions blanchâtres, orange et noires de petites dimensions, à fréquence basse, distribuées d'une manière homogène. Écailles de mica argenté de dimensions petites et moyennes, à fréquence moyenne, distribuées d'une manière homogène.

Observations et comparaisons : on renvoie à la fiche MA52. Chronologie : non déterminable.

\section{MA56 (pl. XXXIV)}

Secteur : Regio IV, Insula 1.

Structure : Maison 1.

Sporadique.

Inventaire : $\mathrm{n}^{\circ} 17$ caisse 1 .

État de conservation : anse, presque complète, manquant de l'attache supérieure. H. max. cons. : 10,4. Anses en ruban épaissi, à section elliptique, avec impression digitale au niveau de la jonction inférieure. H. max. cons. : 10,4 ; épaiss. max. : 4,2 x 1,3.

Surface ext., int. et fracture : orange (5YR7/8-5YR6/8). Inclusions : inclusions de petites dimensions blanches, noirâtres et brunes, à fréquence haute. Mica fin à fréquence basse distribué sur toute la surface.

Engobe : traces d'engobe.

Observations et comparaisons : on renvoie à la fiche MA52. Chronologie : non déterminable. 

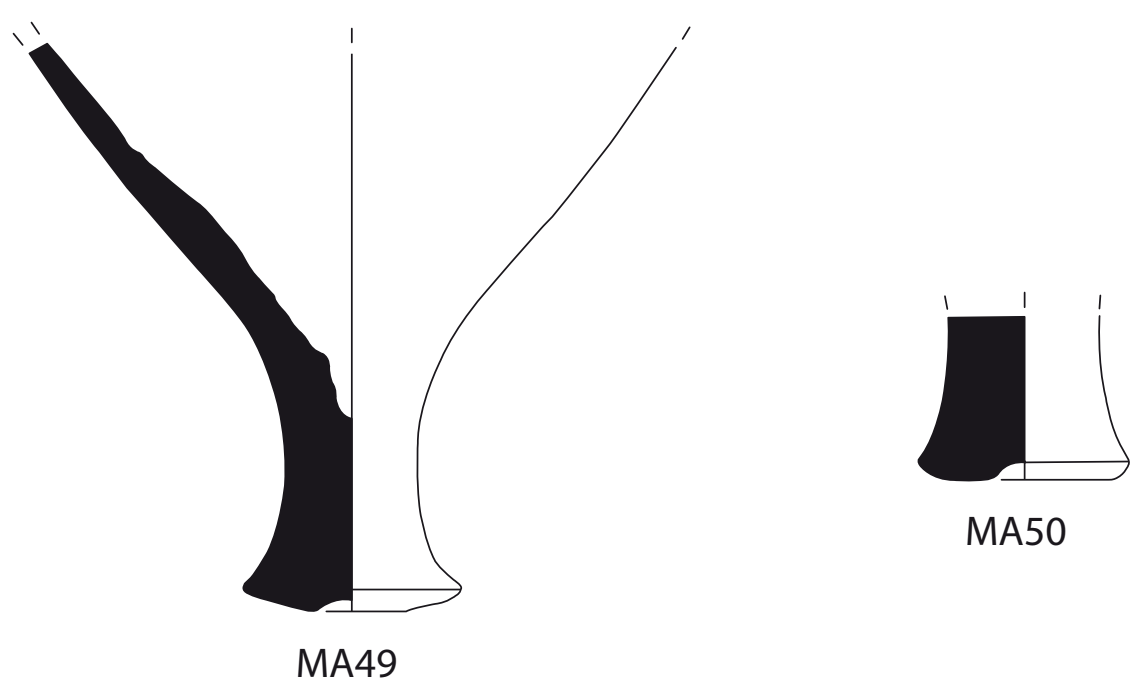

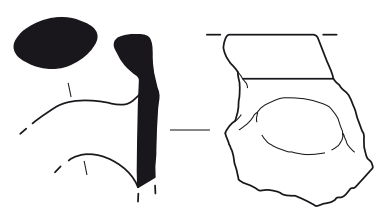

MA51

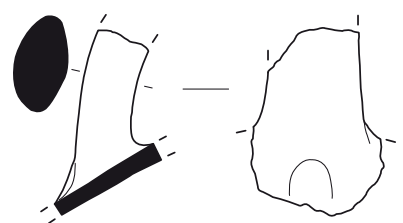

MA52

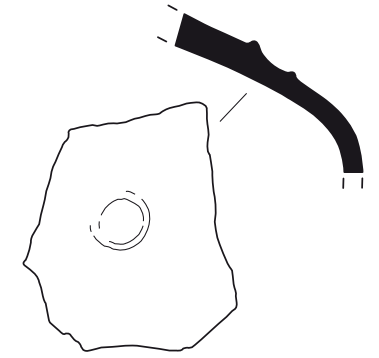

MA53

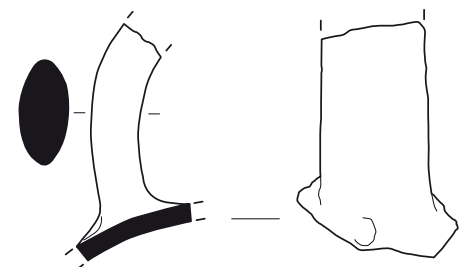

MA54

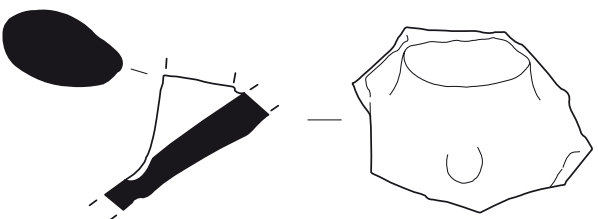

MA55

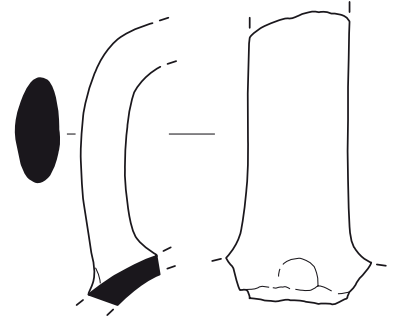

MA56

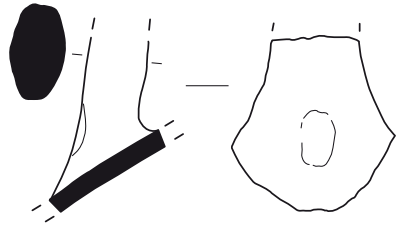

MA57

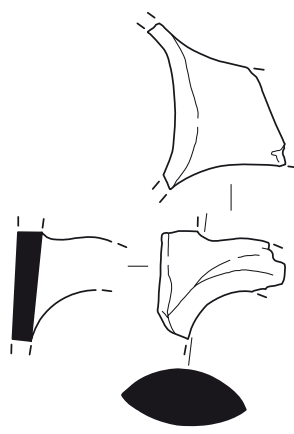

MA58 $5 \mathrm{~cm}$

PI. XXXIV. Marzabotto. 


\section{MA57 (pl. XXXIV)}

Secteur : Regio IV, Insula 1.

Structure : Maison 4. Q10.

Inventaire : $\mathrm{n}^{\circ} 65$ caisse 5 .

État de conservation : partie inférieure d'une anse, jusqu'à la jonction de l'épaule. H. max. cons. : 7,2. Anses en ruban épaissi, à section elliptique, avec impression digitale au niveau de l'attache inférieure. H. max. cons. : 7 ; épaiss. $\max .: 3,9 \times 2,2$.

Surface ext. et int. : orange jaune (5YR 6/6).

Fracture : orange foncé (5YR6/4-5YR6/5).

Inclusions : inclusions gris foncé de dimensions petites et moyennes, à fréquence moyenne, et inclusions blanc crème de dimensions moyennes-grandes, à fréquence moyenne.

Observations et comparaisons : on renvoie à la fiche MA52. Chronologie : non déterminable.

\section{MA58 (pl. XXXIV)}

Année de la fouille / trouvaille : 1964.

Secteur : Regio II, Insula 1.

Structure : Plateia A-côté E, devant Q DC du Four. Inventaire : $\mathrm{n}^{\circ} 8615$.

État de conservation : partie de col avec l'attache supérieure d'une anse. Dimensions max. fr. : 7 x 4,5. Anses en ruban épaissi, à section elliptique. H. max. cons. : 4,5 ; épaiss. max. : $3,9 \times 1,8$.

Surface ext., int. et fracture : beige jaune (10YR8/6).

Inclusions : inclusions orange, noirâtres et blanches de petites dimensions et à fréquence basse.

Observations et comparaisons : probablement attribuable à une amphore de production nord-égéenne sur la base de la pâte et de l'écrasement latéral de l'attache de l'anse.

Chronologie : non déterminable.

\section{AMPHORES DE L'ÉGÉE ORIENTALE}

\section{MA59 (pl. XXXV)}

Secteur : Regio IV, Insula 1.

Structure : Maison 3. Espace destiné à la cour.

Inventaire : $\mathrm{n}^{\circ} 40$ caisse 17.

Type : chiote, Swollen-necked, Early bulgy, variante C/1 ou C/2 Knigge-Lawall.

État de conservation : pied avec une brève partie du fond de la panse. H. max. cons. : 7,7. Pied cylindrique creux, à base en anneau légèrement épaissi. H. : 4 ; diam. min./ $\max .: 4,9 / 5,2$.

Surface ext., int. et fracture : jaune orange (7.5YR7/ 6-7.5YR6/6).

Inclusions : inclusions grisâtres, blanches et brunes de petites dimensions et à fréquence moyenne distribuées d'une manière homogène. Mica fin, argenté, à fréquence basse.
Observations et comparaisons : pour le type, datable entre le début et le milieu du Ve $\mathrm{s}$. av. J.-C., Lawall 1995, fig. 25, 31 ; Dupont 1998, fig. 23.2, d-f ; et de Marinis 1999, p. 266-268, fig. 7. 4-7. Pour la morphologie du pied, Boss 1993, p. 325-326 et note 40, fig. 522. L38, attribuée dubitativement à la production samienne ou chiote ; et Dupont 1999, fig. 30. 2, 5, datées entre 490 et 460 av. J.-C.

Chronologie : 500-450 a.C.

\section{MA60 (pl. XXXV)}

Année de la fouille / trouvaille : 1964.

Secteur : Regio II, Insula 1.

Structure : Plateia A, devant Q G-F du Four.

Inventaire : $n^{\circ} 3961$.

Type : chiote, Swollen-necked, Early bulgy, variante C/1 ou C/2 Knigge-Lawall.

État de conservation : partie de pied. H. max. cons. : 3,5. Pied cylindrique creux, à base annulaire. H. : 3,5; diam. min./max. restituable : 5,4 / 5,9.

Surface ext., int. et fracture : brun orange (5YR5/6).

Inclusions : inclusions blanches, noirâtres et brunes de dimensions petites et moyennes, à fréquence moyenne-haute.

Observations et comparaisons : la morphologie du pied renvoie à la production chiote de la première motié du $\mathrm{V}^{\mathrm{e}} \mathrm{s}$. av. J.-C. et plus exactement au type « swollen-necked, early bulgy », variante $\mathrm{C} / 1$ ou variante $\mathrm{C} / 2$. Pour le type, voir la fiche précédente. Pour la forme, Monachov 1999b, fig. 19.7-9, datées du deuxième quart du $\mathrm{V}^{\mathrm{e}} \mathrm{s}$. av. J.-C. ; et Monachov 2003a, pl. 6. 5.

Chronologie : 500-450 av. J.-C.

\section{MA61 (pl. XXXV)}

Secteur: Regio IV, Insula 1.

Structure : Maison 6.

Défoncement de vigne.

Inventaire : $\mathrm{n}^{\circ} 73$ caisse 1 .

Type : chiote, Swollen necked, Later bulgy, variante C/3

Knigge-Lawall.

État de conservation : fragment de pied. H. max. cons. :

6,3. Pied cylindrique creux, épaissi, à base annulaire.

H. : 4 ; diam. min./max. : 4,9 / 5,9.

Surface ext. et int. : orange jaunâtre (7.5YR 6/6).

Fracture : verdâtre (2.5Y5/3-2.5Y5/4).

Inclusions : inclusions blanches, noires et brunes de dimensions petites et moyennes, à fréquence moyenne-basse.

Engobe : traces d'engobe brun gris.

Observations et comparaisons : la morphologie du pied permet de situer l'exemplaire dans le cadre de la production chiote et de l'attribuer au type «swollen-necked, later bulgy ». Pour le type, datable du troisième quart du Ve s. av. J.-C., Lawall 1995, p. 91, fig. 21, 32 ; et de Marinis 1999, p. 268-269, fig. 7.8.

Chronologie : 450-425 av. J.-C. 


\section{MA62 (pl. XXXV)}

Secteur : Regio V, Insulae 4-5.

Sporadique. Au-dessus de la galerie Ouest.

Inventaire : $n^{\circ} 58405 / 15$.

Type : chiote, Swollen-necked, Later bulgy, variante C/3 Knigge-Lawall ou Straight-neck type.

État de conservation : pied complet, jusqu'à la jonction du fond de la panse. H. max. cons. : 5,8. Pied cylindrique creux, épaissi, à base annulaire. H. : 5 ; diam. min./max. : $4,2 / 5,5$.

Surface ext. et int. : orange (5YR5/6).

Fracture : rougeâtre (2.5YR6/6-2.5YR6/8).

Inclusions : inclusions blanches, grises et brunes de dimensions petites et moyennes, à fréqunce haute. Mica à fréquence basse.

Engobe : traces d'engobe crème.

Observations et comparaisons : attribuable à la variante plus récente du type chiote à col gonflé (datable du troisième quart du $\mathrm{V}^{\mathrm{e}} \mathrm{s}$. av. J.-C.) ou bien, étant donné son diamètre réduit et la hauteur de la carène latérale, au type chiote à col droit (datable du dernier quart du $\mathrm{V}^{\mathrm{e}} \mathrm{s}$. av. J.-C.). Pour le premier type, voir la fiche précédente. Pour le second, Lawall 1995, p. 91-92, fig. 22, 35. Pour la forme, Ibid. fig. 32 (variante C/3).

Chronologie : 450-400 av. J.-C.

\section{MA63 (pl. XXXV)}

Secteur : Regio IV, Insula 1.

Structure : Maison 3. Espace destiné à la cour et au puits. Inventaire : $\mathrm{n}^{\circ}$ caisse 14 .

Type : chiote, Swollen-necked.

État de conservation : fragment de col avec le segment supérieur d'une anse. H. max. cons. : 5. Col gonflé. Anses en ruban épaissi, à section elliptique. H. max. cons. : 4 ; épaiss. max. : 4 x 2.

Surface ext., int. et fracture : orange (5YR6/8-5YR 7/8). Inclusions : inclusions grisâtres et brunes de petites dimensions, à fréquence basse et inclusions blanches de petites dimensions, à fréquence moyenne. Mica absent. Vacuoles.

Observations et comparaisons : attribuable à la production chiote par les caractéristiques de la pâte et par la morphologie enflée du col. Pour le type, sûrement «swollen-necked » mais sans qu'il soit possible d'en préciser ni le sous-type, ni la variante, de Marinis 1999, p. 266-269, fig. 7.4-8, datables entre le début et le troisième quart du $\mathrm{V}^{\mathrm{e}} \mathrm{s}$. av. J.-C.

Chronologie : 500-425 av. J.-C.

\section{MA64 (pl. XXXV)}

Année de la fouille / trouvaille : 1964.

Secteur : Regio II, Insula 1.

Structure : Four. Q E-D.

Inventaire : $n^{\circ} 2045$.
Type : chiote, Swollen-necked.

État de conservation : partie de col et segment supérieur d'une anse. H. max. cons. : 5,5. Col gonflé. Anses en ruban épaissi, à section elliptique. H. max. cons. : 6 ; épaiss. $\max .: 4,2 \times 2,3$.

Surface ext. et int. : beige (7.5YR7/4).

Fracture : orange brun (7.5YR6/6).

Inclusions : inclusions blanches et brunes de dimensions moyennes et petites, à fréquence basse, et inclusions noirâtres de petites dimensions, à fréquence basse.

Observations et comparaisons : attribuable à l'un des types chiotes « swollen-necked », sans qu'il soit possible d'en préciser ni le sous-type, ni la variante. Pour le type, datable entre le début et le troisième quart du $\mathrm{V}^{\mathrm{e}} \mathrm{s}$. av. J.-C., de Marinis 1999, p. 266-269, fig. 7.4-8

Chronologie : 500-425 av. J.-C.

\section{MA65 (pl. XXXV)}

Année de la fouille / trouvaille : 1994.

Secteur : Regio V, Insula 5.

Structure : Maison 1.

US : 355 .

Type : chiote.

État de conservation : partie inférieure d'anse. Dimensions max. fr. : 7,1 x 6,1 . Anses en ruban épaissi, à section elliptique. H. max. cons. : 2,3 ; épaiss. max. : 3,3.

Surface ext. et int. : brun rouge (2.5YR5/4-2.5YR6/4).

Fracture : rougeâtre $(2.5 \mathrm{YR} 5 / 8)$.

Inclusions : inclusions blanches de dimensions petites et moyennes, à fréquence moyenne, distribuées d'une manière homogène, et inclusions gris noir, de petites dimensions, à fréquence moyenne-basse, distribuées d'une manière non homogène. Mica argenté très fin, à fréquence très basse.

Engobe : traces d'engobe crème, fin.

Observations et comparaisons : les caractéristiques de la pâte suggèrent son attribution à la production de Chios. Chronologie : non déterminable.

\section{MA66 (pl. XXXV)}

Année de la fouille / trouvaille : 1988.

Secteur : Regio V, Insula 5.

Structure : Plateia A, devant la Maison 1.

US : $14=4$.

Type : Samos-Milet, S/2 Lawall.

État de conservation : pied et nombreux fragments de parois qu'il n'est pas possible d'assembler. H. max. cons. : 2,2. Pied tronconique creux, à base en anneau caréné. H. : 2 ; diam. min./max. restituable : 5,4 / 7,1.

Surface ext. : orange rougeâtre (10R5/8).

Surface int. et fracture : orange rosé (10R5/6).

Inclusions : argile dépurée, presque complètement privée d'inclusions et de mica. Inclusions brun gris de 
petites dimension, à fréquence très basse. Écailles très fines de mica doré, presque imperceptibles, à fréquence basse, diffusées d'une manière homogène.

Engobe : traces d'engobe crème, très fin.

Observations et comparaisons : pour le type, Knigge 1976, pl. 49.3, correspondant à la variante tardoarchaïque du type $\mathrm{S} / 2$ de $\mathrm{M}$. Lawall ; pour la forme, Dupont 1998, fig. 23.7, f, attribué à la production milésienne de la seconde moitié du VI ${ }^{\mathrm{e}} \mathrm{s}$. av. J.-C. et défini «conical belly later type ».

Chronologie : 525-480 av. J.-C.

\section{MA67 (pl. XXXV)}

Année de la fouille / trouvaille : 1991.

Secteur : Regio VII, Insula 2.

Structure : Puits près de la Plateia D. Q.R. 180

Inventaire : $\mathrm{n}^{\circ} 2669$.

Type : Samos-Milet, S/1 Lawall.

État de conservation : partie de pied. H. max. cons. : 4,2. Pied tronconique, à base en anneau épaissi. H. : 4 ; diam. $\min . / \max .:$ : 5,4 / 6,8.

Surface ext. et int. : beige (7.5YR7/4).

Fracture : rosé (2.5YR7/3).

Inclusions : pâte dépurée, avec inclusions blanches, grises et brunes, de petites dimensions, à fréquence basse. Mica fin, doré, à fréquence basse.

Observations et comparaisons : le pied en question peut être classé dans la forme tardo-archaïque du type $\mathrm{S} / 1$ de la classification de M. Lawall. Pour le type, Lawall 1995, fig. 77 ; et Dupont 1998, fig. 23.9, b.

Chronologie : 525-480 a.C.

\section{MA68 (pl. XXXV)}

Secteur : Regio IV, Insula 1.

Structure : Maison 3. Area F.H.T.V.

Inventaire : $\mathrm{n}^{\circ} 71323$.

Type : Samos-Milet, S/1 ou S/3 Lawall.

État de conservation : partie de col au niveau de la jonction de l'épaule.

Dimensions max. fr. : 4,4 x 6,5. Col évasé et au profil légèrement convexe, avec un listel au niveau de la jonction de l'épaule. H. max. cons. : 4,4; diam. min./max. restituable : 6,5

Surface ext. et fracture : noisette chamois (7.5YR7/ 4-7.5YR7/6).

Surface int. : orange (5YR7/8-7.5YR7/6).

Inclusions : inclusions noirâtres, brunes et blanches de petites dimensions et à fréquence basse. Mica fin et distribué sur toute la surface.

Observations et comparaisons : le profil du col, caractérisé par un fin listel de base situé en correspondance de la jonction de l'épaule, suggère son attribution à la production de Samos-Milet de la fin du $\mathrm{VI}^{\mathrm{e}}$ ou du début du Ve s. av. J.-C. En particulier pour l'emplacement du listel, en bas et non encore au milieu, voir Dupont 1982, fig. 6-7 ; Roberts 1986, fig. 4. 413, datable de 480 av. J.-C. (P24870 de l'Agora) ; et Lawall 1995, fig. 76-77, l'une et l'autre provenant du dépôt E 14 :5 de l'Agora datable vers 480 av. J.-C. L'exemplaire en question peut donc être attribué à la variante tardo-archaïque du type $\mathrm{S} / 1$ ou au type $\mathrm{S} / 3$ de M. Lawall, datables entre 525 et 480 av. J.-C.

Chronologie : 525-480 av. J.-C.

\section{MA69 (pl. XXXV)}

Année de la fouille / trouvaille : 1970.

Secteur: Regio V, Insula 2 / Regio VII, Insula 2.

Structure : Puits de la Plateia D.

Inventaire : $n^{\circ} 225504$

Type : Samos-Milet, S/1 Lawall.

État de conservation : amphore dont il reste l'embouchure complète (reconstituée à partir de 3 fragments), une anse (reconstituée à partir de 3 fragments dont l'un ne s'accorde pas parfaitement) et une partie de l'épaule et de la panse reconstituées à partir de 39 fragments de paroi. H. max. cons. : 16,3. Lèvre à tore, épaissie, légèrement ouverte. H. : 2 ; épaiss. max. : 2 ; diam. int./ext. restituable embouchure : 13,2 / 14,2. Col cylindrique. H. max. cons. : 5 ; diam. min./max. : 11,4. Anses en ruban épaissi, à section elliptique. H. max. cons. : 13,2 ; épaiss. max. : 4,2 x 2,1.

Surface ext. : brun rougeâtre (5YR5/6).

Surface int. et fracture : brun mauve (2.5YR5/6).

Inclusions : inclusions blanchâtres de dimensions moyennespetites, à fréquence haute, et inclusions noirâtres de petites dimensions, à fréquence basse. Mica fin argenté et doré, à fréquence basse. Vacuoles.

Engobe : traces d'engobe crème sur la surface intérieure. Observations et comparaisons : on peut l'attribuer au groupe Samos-Milet et la rapprocher des formes de fin $\mathrm{VI}^{\mathrm{e}}$-début $\mathrm{V}^{\mathrm{e}}$ ou du deuxième quart du $\mathrm{V}^{\mathrm{e}} \mathrm{s}$. av. J.-C. du type S/1 de M. Lawall (pour lequel cf. Lawall 1995, p. 178-180, fig. 69, 77 ; et Di Sandro 1981b, fig. 3.3).

Chronologie : 525-450 av. J.-C.

\section{MA70 (pl. XXXV)}

Année de la fouille / trouvaille : 1953.

Secteur : Îlots sur la Plateia A.

Type : Samos-Milet, S/1 Lawall.

État de conservation : partie de lèvre avec un bref segment du col. H. max. cons. : 3,8. Lèvre en amande, épaissie, légèrement ouverte. $\mathrm{H}$. : 2,4 ; épaiss. max. : 1,8 ; diam. int./ext. restituable embouchure : 11 / 12,9. Col évasé. H. max. cons. : 1,4 ; diam. min./max. restituable : 9,3 / 9,9. Surface ext., int. et fracture : chamois brun (10YR6/3 -10YR6/4). 


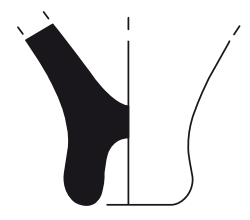

MA59

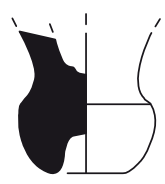

MA62

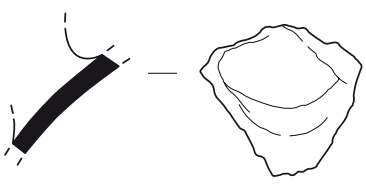

MA65

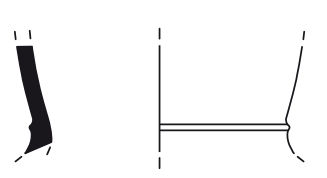

MA68

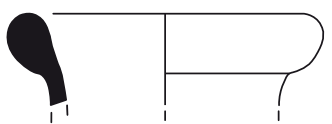

MA70

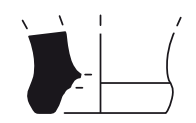

MA60

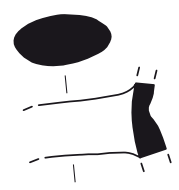

MA63

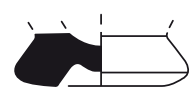

MA66

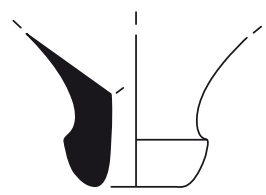

MA61

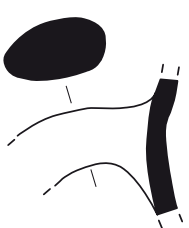

MA64

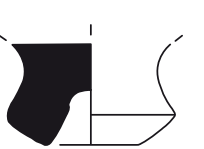

MA67

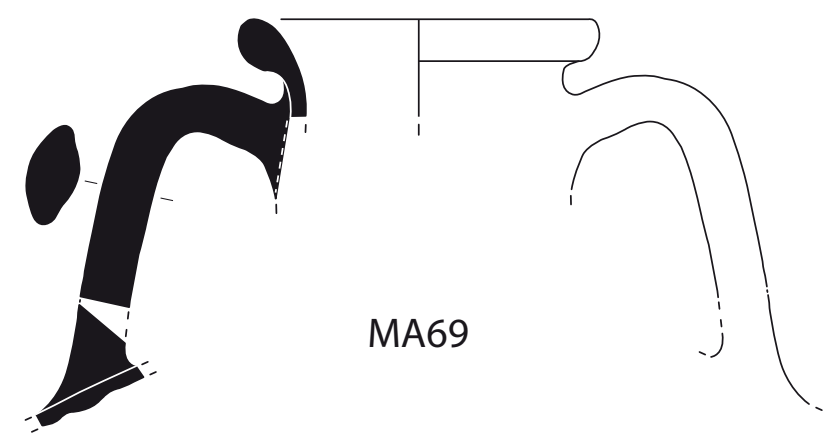

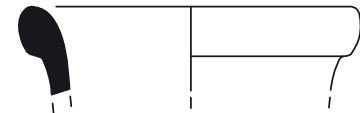

MA71

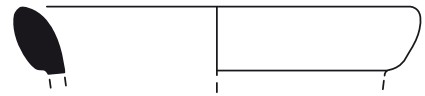

MA72

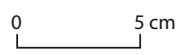

PI. XXXV. Marzabotto. 
Inclusions : inclusions blanches et orange de dimensions petites et moyennes, à fréquence moyenne, et inclusions noires de petites dimensions, à fréquence basse. Mica fin disséminé.

Observations et comparaisons : pour le type, voir la fiche précédente.

Chronologie : 525-480 av. J.-C.

\section{MA71 (pl. XXXV)}

Année de la fouille / trouvaille : 1988.

Secteur : Regio V, Insula 5.

Structure : Plateia A, devant la Maison 1.

US : 2-5-9-10-11.

Type : Samos-Milet, S/2 Lawall.

État de conservation : partie de lèvre avec un bref segment du col. H. max. cons. : 3,4. Lèvre en amande, épaissie, légèrement ouverte. H. : 2 ; épaiss. max. : 1,7 ; diam. int./ext. restituable embouchure : 13/13,8.

Surface ext., int. et fracture : orange (5YR6/6).

Inclusions : inclusions blanches de dimensions petites et moyennes, à fréquence moyenne, distribuées d'une manière homogène; inclusions brun orange et grises de petites dimensions, à fréquence basse, distribuées d'une manière homogène. Écailles de mica argenté et doré de petites dimensions, à fréquence moyenne-haute.

Observations et comparaisons : attribuable à une amphore du groupe Samos-Milet, probablement du type S/2 Lawall, mais sans aucune possibilité de distinguer entre la forme de fin $\mathrm{VI}^{\mathrm{e}}$-début $\mathrm{V}^{\mathrm{e}}$ et celle du deuxième quart du $\mathrm{V}^{\mathrm{e}} \mathrm{s}$. av. J.-C., étant donné l'absence d'autres éléments du conteneur. Pour la première forme, Knigge 1976, pl. 49. 3 ; pour la seconde, Grace 1971, fig. 3. 1 ; Lawall 1995, p. 177, 181-182, fig. 70-71 ; pour la morphologie de la lèvre, qu'on peut rapprocher des types «Zeest's Samian », voir Monachov 2003a, pl. 25. 4.

Chronologie : 525-450 a.C.

\section{MA72 (pl. XXXV)}

Secteur : Regio IV, Insula 1.

Structure : Maison 3. Espace destiné à la cour, dallage du puits.

Inventaire : $\mathrm{n}^{\circ} 115$ caisse 16 .

Type : Samos-Milet, S/2 ou S/3 Lawall.

État de conservation : fragment de lèvre. H. max. cons. : 2,3 . Lèvre en amande, légèrement épaissie. $H$. : 2,1 ; épaiss. max. : 1,6 ; diam. int./ext. restituable embouchure : 15,4/ 16. Surface ext., int. et fracture : orange chamois (5YR 6/ 6-5YR5/6).

Inclusions : inclusions noirâtres et blanches de dimensions petites, à fréquence basse. Mica fin, à fréquence basse, distribué sur toute la surface. Vacuoles.

Observations et comparaisons : attribuable à une amphore du groupe Samos-Milet. Le profil en échine pourrait renvoyer au type $\mathrm{S} / 2$ (pour lequel cf. la fiche précédente) ou au type $\mathrm{S} / 3$ de $\mathrm{M}$. Lawall (pour lequel cf. Lawall 1995, fig. 76, datable entre 525 et 480 a.C.). Pour la forme, Morselli-Tortorici 1985, fig. 8. 1, 4, de Gravisca, attribuées à Samos.

Chronologie : 525-450 av. J.-C.

\section{MA73 (pl. XXXVI)}

Année de la fouille / trouvaille : 1964.

Secteur : Regio II, Insula 1.

Structure : Four. Q F2F3-E2E3.

Inventaire : $n^{\circ} 1682$.

Type : Samos-Milet, S/2 Lawall.

État de conservation : partie de lèvre : H. max. cons. : 3,5. Lèvre en amande, légèrement épaissie et ouverte. H. : 2,1 ; épaiss. max. : 1,5 diam. int./ext. restituable embouchure : 12,6 / 13,9.

Surface ext., int. et fracture : beige (7.5YR7/4).

Inclusions : inclusions blanches de dimensions petites et moyennes, à fréquence basse, et inclusions brunes et noirâtres de petites dimensions, à fréquence moyenne.

Observations et comparaisons : pour le type (qu'on peut rapprocher des types dits «Zeest's Samian»), Knigge 1976, pl. 49.3, et Grace 1971, fig. 3. 1, correspondant aux deux formes du type $\mathrm{S} / 2$ de $\mathrm{M}$. Lawall datables entre le dernier quart du $\mathrm{VI}^{\mathrm{e}}$ et la première moitié $\mathrm{du} \mathrm{V}^{\mathrm{e}} \mathrm{s}$. av. J.-C. Pour la morphologie, Morselli-Tortorici 1985, fig. 8. 4, et Monachov 2003a, pl. 26. 3.

Chronologie : 525-450 av. J.-C.

\section{MA74 (pl. XXXVI)}

Secteur : Regio IV, Insula 1.

Structure : Maison 2. Q sud de la cour.

Inventaire : $\mathrm{n}^{\circ} 10$ caisse 3 .

Type : Samos-Milet, S/2 Lawall.

État de conservation : partie de lèvre et de col portant la trace de la jonction supérieure d'une anse. H. max. cons. : 5,6 . Lèvre épaissie, de section trapézoïdale. H. : 2 ; épaiss. max. : 1,8 ; diam. int./ext. restituable embouchure : 15,4 / 16,8. Col vraisemblablement cylindrique ou évasé. $\mathrm{H}$. max. cons. : 3,5 ; diam. min./max. restituable : 13,8.

Surfaceext.,int.etfracture: orange(7.5YR7/6-7.5YR6/6). Inclusions : pâte dépurée, presque complètement dépourvue d'inclusions. Mica fin à fréquence haute sur toute la surface.

Observations et comparaisons : pour la morphologie de la lèvre, anguleuse et assimilable aux types "Zeest's Samian », on peut l'attribuer aux formes datables entre la fin du $\mathrm{VI}^{\mathrm{e}}$ et le milieu du Ve s. av. J.-C. du type S/2 de M. Lawall. Pour ces formes, voir respectivement, Knigge 1976, pl. 49.3 ; et Grace 1971, fig. 3.1. Pour la morphologie, Dupont 1998, p. 176-177, fig. 23.8, i ; et Monachov 2003a, pl. 19. 8.

Chronologie : 525-450 av. J.-C. 


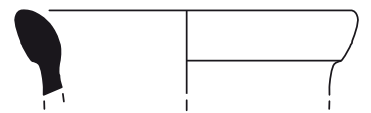

MA73

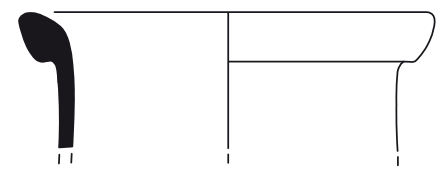

MA74

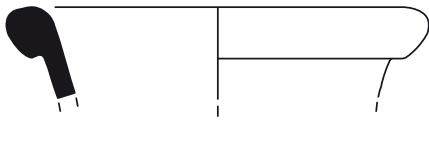

MA75

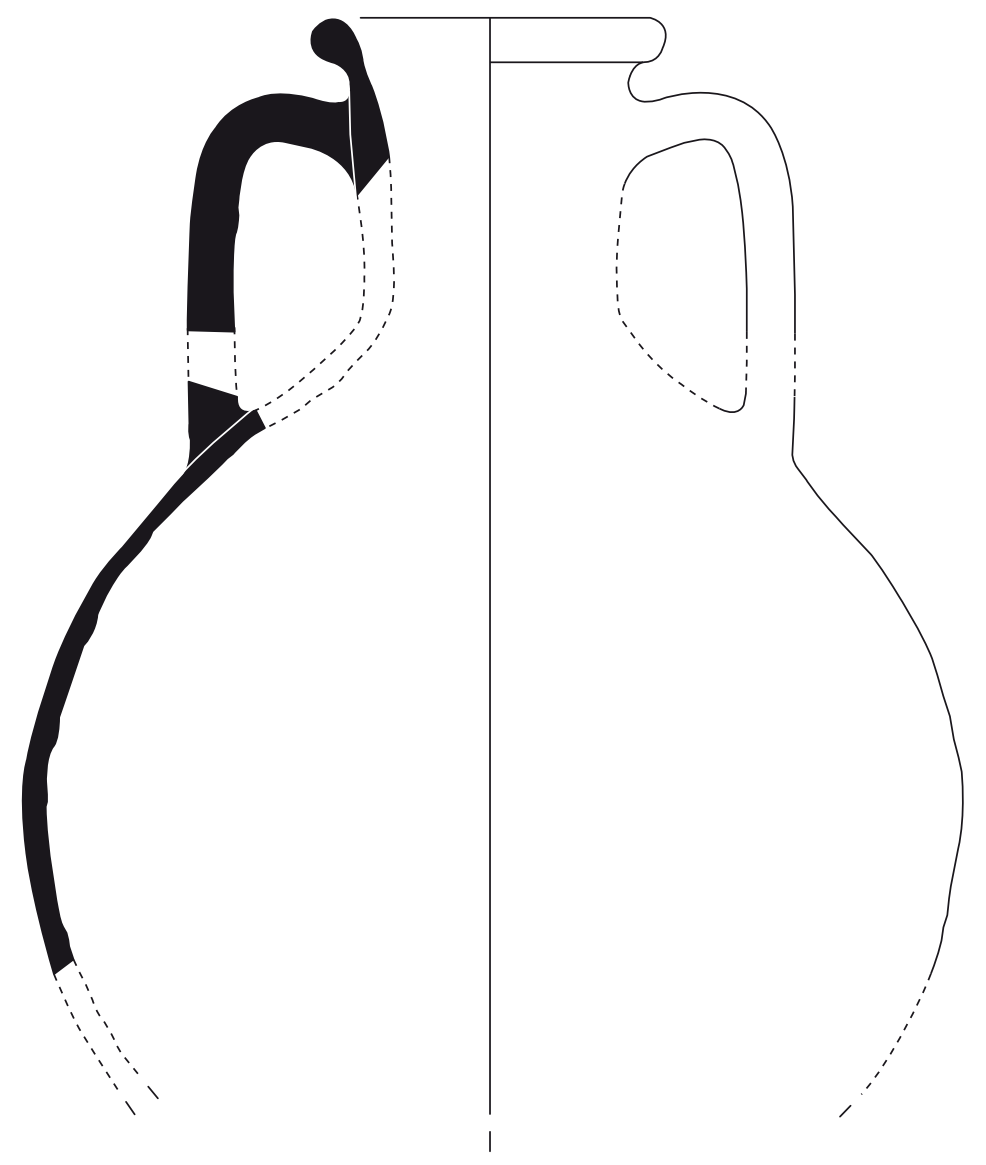

MA76

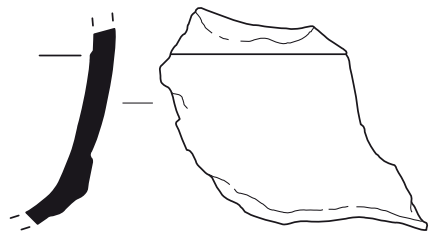

MA77

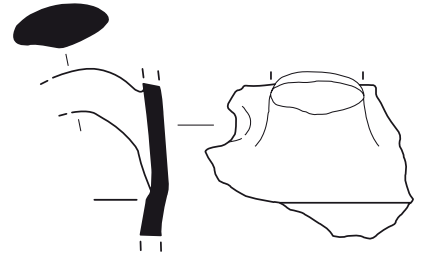

MA78 


\section{MA75 (pl. XXXVI)}

Sporadique.

Inventaire : $\mathrm{n}^{\circ} 18$ (de la caisse 23 'Sporadici').

Type : Samos-Milet, S/1 Lawall.

État de conservation : deux fragments de lèvre et court segment du col portant la trace de la jonction supérieure d'une anse. H. max. cons. : 3,7. Lèvre en amande, épaissie à l'extérieur, légèrement ouverte. H. : 2,1 ; épaiss. max. : 2 ; diam. int./ext. restituable bouche : 15 / 17,1. Col évasé. H. max. cons. : 1,1; diam. min./max. restituable : $13 / 14$. Surface ext. et int. : orange brun (5YR6/6).

Fracture : gris (GLEY 2).

Inclusions : inclusions blanches de dimensions petites et moyennes, à fréquence moyenne-basse, inclusions brun noirâtre de petites dimensions, à fréquence basse. Mica fin à fréquence basse.

Observations et comparaisons : la morphologie de la lèvre rappelle de très près le type $\mathrm{S} / 1$ du groupe SamosMilet de la classification de M. Lawall, et notamment ses deux formes les plus archaïques, pour lesquelles on renvoie à Roberts 1986, fig. 4.413, et Di Sandro 1981b, fig. 3.3.

Chronologie : 525-450 av. J.-C.

\section{MA76 (pl. XXXVI)}

Secteur : Regio V, Insula 3.

Structure : rigole entre w et w'.

Type : Samos-Milet, S/1 Lawall.

État de conservation : amphore presque entière, reconstituée à partir de fragments, manquant de la partie inférieure de la panse et de l'attache inférieure de l'anse. Dimensions : non repérées. Lèvre épaissie, Légèrement ouverte. Col évasé. Anses verticales, en ruban épaissi, à section elliptique. Épaule fuyante. Corps vraisemblablement globulaire ou piriforme.

Surface ext., int. et fracture : rougeâtre orange.

Inclusions : inclusions grisâtres et blanchâtres de petites dimensions, à fréquence moyenne. Mica.

Observations et comparaisons : attribuée à Samos par Fr.-H. Massa-Pairault et confrontée notamment avec de Marinis 1988, fig. 110, 2 (cat. FO61). Par la forme ovoïde du corps l'exemplaire peut être rapproché de la variante du type $\mathrm{S} / 1$ Lawall datée du deuxième quart du Ve s. av. J.-C. (Lawall 1995, p. 179-180 ; Di Sandro 1981b, fig. 3.3).

Chronologie : 480-450 av. J.-C.

Bibliographie : Fr.-H. Massa-Pairault, in Massa-Pairault 1997, p. 185-186, pl. XIX.

\section{MA77 (pl. XXXVI)}

Secteur : Regio IV, Insula 1.

Structure : Maison 4. Q12A.

Inventaire : $\mathrm{n}^{\circ} 28$ caisse 1 .
Type : Samos-Milet, S/1 Lawall.

État de conservation : partie de col, reconstituée à partir de 2 fragments, depuis la jonction de l'épaule jusqu'à l'attache supérieure des anses. H. max. cons. : 9. Col tronconique, avec un listel situé dans sa partie médiane. H. max. cons. : 9 ; largh. max. cons. : 11. Surface ext. et int. : chamois (7.5YR6/6). Fracture : orange (5YR5/8-5YR 5/6). Inclusions : inclusions blanches, de dimensions petites et moyennes, à fréquence moyenne, et inclusions brunes et grisâtres, de petites dimensions, à fréquence basse. Mica à fréquence haute.

Observations et comparaisons : le listel du col est typique des formes du groupe Samos-Milet et sa position médiane permet de situer l'exemplaire dans le cadre des formes du deuxième quart (Di Sandro 1981b, fig. 3.3) ou de la seconde moitié du V ${ }^{\mathrm{e}}$ s. av. J.-C. (Grace 1971, p. 76, fig. 3.3) du type S/1 de M. Lawall.

Chronologie : 480-400 av. J.-C.

\section{MA78 (pl. XXXVI)}

Secteur : Regio IV, Insula 1.

Structure : Maison 3. Espace destiné à la cour. Inventaire : $\mathrm{n}^{\circ} 37$ caisse 17.

Type : Samos-Milet, S/1 Lawall.

État de conservation : partie de col avec le segment supérieur d'une anse. Col qui présente un listel placé immédiatement au-dessous de la jonction supérieure des anses. H. max. cons. : 6,2 ; larg. max. cons. : 8,1. Anses en ruban épaissi, à section elliptique. H. max. cons. : 3,8 ; épaiss. max. : 3,9 x 1,7 .

Surface ext. et int. : orange (7.5YR6/6).

Fracture : orange intense (7.5YR6/8)

Inclusions : inclusions brunes et noirâtres de petites dimensions, à fréquence basse, et inclusions blanches de petites dimensions, à fréquence moyenne-haute. Vacuoles.

Observations et comparaisons : le listel placé immédiatement au-dessous de la jonction supérieure de l'anse caractérise les amphores du groupe Samos-Milet, attribuées par M. Lawall à son type $S / 1$ et datables entre le deuxième quart et la fin du $\mathrm{V}^{\mathrm{e}} \mathrm{s}$. av. J.-C. (cf. fiche précédente).

Chronologie : 480-400 av. J.-C.

\section{MA79 (pl. XXXVII)}

Année de la fouille / trouvaille : 1988.

Secteur : Regio V, Insula 5.

Structure : Plateia A devant la Maison 1.

US : $14=4$.

Type : Samos-Milet, S/1 Lawall.

État de conservation : amphore dont n'est conservée que la moitié supérieure jusqu'au départ de l'épaule, reconstituée à partir de 12 fragments et restaurée. Anses 
manquantes. H. max. cons. : 19. Lèvre en amande, haute et légèrement épaissie, ouverte. H. : 3,2 ; épaiss. max. : 1,4 ; diam. int./ext. restituable embouchure : 11,4 / 13,7. Col tronconique avec un listel médian situé au-dessous des attaches des anses. H. max. cons. 11,5 ; diam. min./ max. restituable : $9 / 11,2$. Anses en ruban épaissi, à section elliptique. H. max. cons. : 3 ; épaiss. max. : $2,2 \mathrm{x}$ 4,2. Épaule fuyante. Larg. max. cons. : 5,5.

Surface ext. : orange rougeâtre (10R5/8).

Surface int. et fracture : orange rosé (10R5/6).

Inclusions : argile dépurée, presque complètement dépourvue d'inclusions et de mica. Inclusions brun gris de petites dimensions, à fréquence très basse. Écailles très fines de mica doré, à fréquence basse, distribuées d'une manière homogène.

Engobe : engobe crème.

Observations et comparaisons : attribuable au type S/1 de M. Lawall. Pour le type, Lawall 1995, fig. 73, et Grace 1971, p. 76, fig. 3. 3. Pour la forme, Lawall 1995, fig. 74, provenant d'un dépôt de l'Agora daté vers 410 av. J.-C., et Dupont 1998, fig. 23.9, d, attribuée à la production milésienne de la première moitié du $\mathrm{V}^{\mathrm{e}} \mathrm{s}$. av. J.-C. (type 'high lip'). La pâte céramique de cet exemplaire étant identique à celle de l'exemplaire de la fiche suivante, trouvé dans le même niveau et correspondant à la moitié inférieure d'une amphore du même type, on peut avancer l'hypothèse que les deux parties appartenaient à un seul individu.

Chronologie : 450-400 av. J.-C.

\section{MA80 (pl. XXXVII)}

Année de la fouille / trouvaille : 1988.

Secteur : Regio V, Insula 5.

Structure : Plateia A, devant de la Maison 1.

US : $14=4$.

Type : Samos-Milet, S/1 Lawall.

État de conservation : amphore dont est conservée la moitié inférieure jusque sous l'épaule, reconstituée à partir de nombreux fragments et restaurée. Il est impossible d'assembler plusieurs autres fragments de la panse qui se sont conservés. H. max. cons. : 45. Corps ovoïde. H. max. cons. : 45 ; diam. max. : 32,6. Pied cylindrique plein, épaissi, à base ombiliquée. H. : 4,1 ; diam. min./max. : 4,8 / 6 .

Surface ext. : orange rougeâtre (10R5/8).

Surface int. et fracture : orange rosé (10R5/6).

Inclusions : argile dépurée, presque complètement dépourvue d'inclusions et de mica. Inclusions brun gris de petites dimensions, à fréquence très basse. Écailles très fines de mica doré, à fréquence basse, distribuées d'une manière homogène.

Engobe : engobe crème.

Observations et comparaisons : pour la forme du pied, Dupont 1998, fig. 23.9, g, attribué à la production milésienne du milieu du $\mathrm{V}^{\mathrm{e}}$ s. av. J.-C. (type «thickened rim »). Appartenant sans doute au même individu de la fiche précédente.

Chronologie : 450-400 av. J.-C.

\section{MA81 (pl. XXXVIII)}

Année de la fouille / trouvaille : 1964.

Secteur : Regio II, Insula 1.

Structure : Plateia A, devant le Q F-E du Four.

Inventaire : $\mathrm{n}^{\circ} 4603$.

Type : Samos-Milet, S/1 Lawall.

État de conservation : partie de pied. H. max. cons. : 4. Pied cylindrique plein, épaissi, à base ombiliquée. H. : 3,2 ; diam. min./max. : 4,2/4,8.

Surface ext., int. et fracture : brun orange (5YR5/6).

Inclusions : inclusions noirâtres, brunes et blanches de dimensions petites, à fréquence basse.

Observations et comparaisons : pour le type S/1 Lawall, datable du courant de la seconde moitié du $\mathrm{V}^{\mathrm{e}} \mathrm{s}$. av. J.-C., Lawall 1995, fig. 73. Pour la forme, Dupont 1998, fig. 23.9, g.

Chronologie : 450-400 av. J.-C.

\section{MA82 (pl. XXXVIII)}

Année de la fouille / trouvaille : 1964.

Secteur : Regio II, Insula 1.

Structure : Four. Q E2E3-D2D3.

Inventaire : $\mathrm{n}^{\circ} 3156$.

Type : Samos-Milet, S/1 Lawall.

État de conservation : partie de pied. H. max. cons. : 2,6. Pied cylindrique plein, épaissi, à base ombiliquée. Diam. min./max. restituable : 5 .

Surface ext., int. et fracture : brun orange (5YR5/6).

Inclusions : inclusions noirâtres, brunes et blanches de petites dimensions, à fréquence basse.

Observations et comparaisons : voir la fiche précédente. Chronologie : 450-400 av. J.-C.

\section{MA83 (pl. XXXVIII)}

Secteur : Regio V, Insula 3.

Inventaire : $\mathrm{n}^{\circ} 71-38$ a.

Type : Samos-Milet (?).

État de conservation : fragment de lèvre. Lèvre épaissie, légèrement ouverte.

Surface : orange.

Observations et comparaisons : Fr.-H. Massa-Pairault pense qu'on peut comparer cette lèvre à cat. MA76 (qu'elle attribue à Samos) mais aussi à Sandri 1972, $\mathrm{n}^{\circ} 18$ (cat. MA44, maintenant attribuée à la production nord-égéenne). Exemplaire appartenant au même individu de la fiche qui suit.

Chronologie : non déterminable.

Bibliographie : Fr.-H. Massa-Pairault, in Massa-Pairault 1997, p. 186, pl. I, 6 a. 

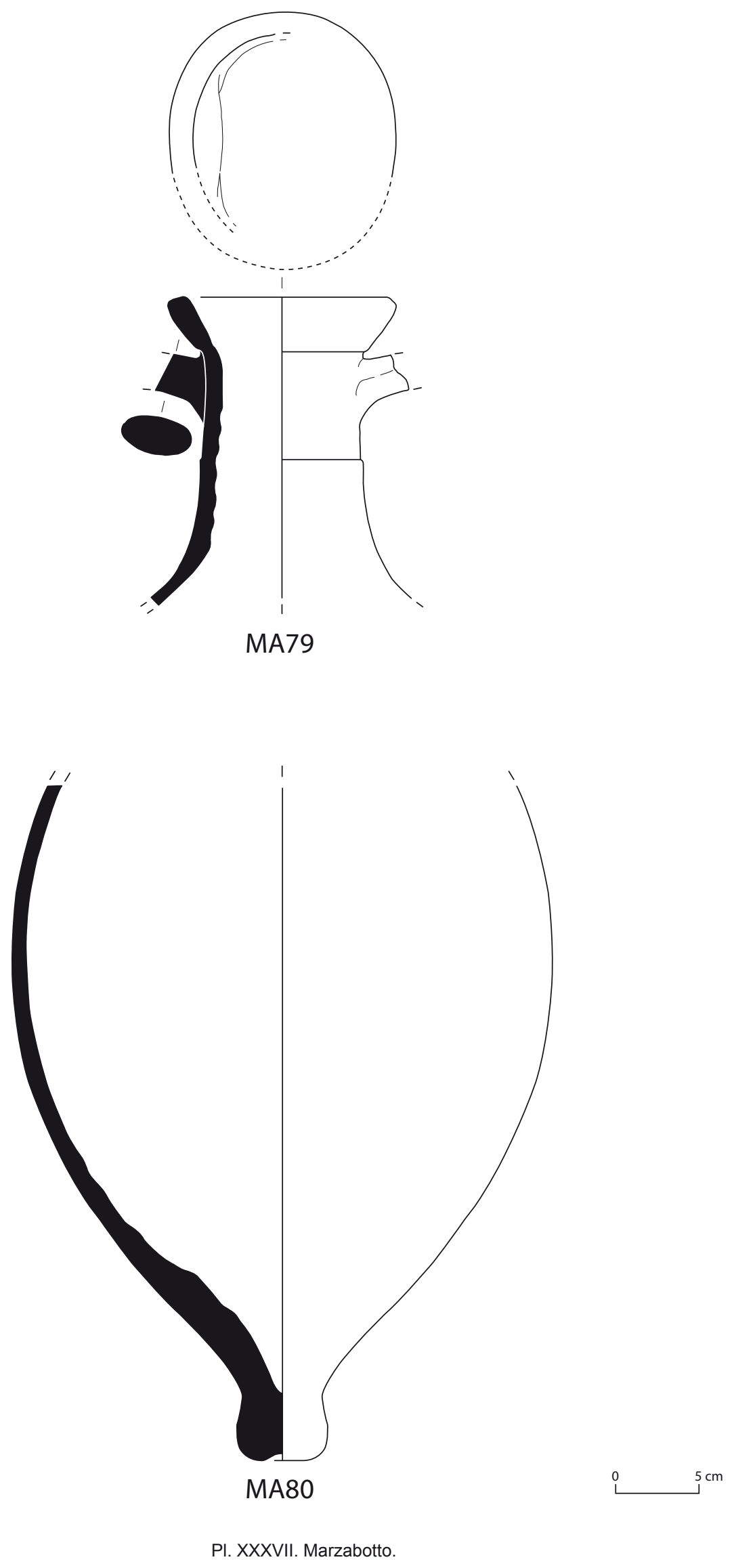


\section{MA84 (pl. XXXVIII)}

Secteur : Regio V, Insula 3.

Inventaire : $\mathrm{n}^{\circ} 71-38 \mathrm{~b}$.

Type : Samos-Milet (?).

État de conservation : fragment d'anse. Anse en ruban épaissi, à section elliptique.

Surface : orange.

Observations et comparaisons : associé par Fr.-H.

Massa-Pairault à la lèvre de la fiche précédente.

Chronologie : non déterminable.

Bibliographie : Fr.-H. Massa-Pairault, in Massa-Pairault 1997, p. 186, pl. I, 6 b.

\section{MA85 (pl. XXXVIII)}

Lieu de découverte : Marzabotto (Bo)

Secteur : Regio V, Insula 3.

Inventaire : $\mathrm{n}^{\circ} 71-51 \mathrm{D}$.

Type : Samos-Milet (?).

État de conservation : fragment de lèvre. Lèvre épaissie, légèrement ouverte.

Surface : orange.

Observations et comparaisons : Fr.-H. Massa-Pairault le rapproche de la lèvre de la fiche MA83.

Chronologie : non déterminable.

Bibliographie : Fr.-H. Massa-Pairault, in Massa-Pairault 1997, p. 186, pl. III, 8 a.

\section{MA86 (pl. XXXVIII)}

Secteur : Regio V, Insula 3.

Inventaire : $n^{\circ} 71-51 \mathrm{D}$.

Type : Samos-Milet (?).

État de conservation : fragment d'anse. Anse « a bastoncello», de section circulaire.

Surface : orange.

Observations et comparaisons : associé par Fr.-H. Massa-Pairault à la lèvre de la fiche précédente.

Chronologie : non déterminable.

Bibliographie : Fr.-H. Massa-Pairault, in Massa-Pairault 1997, p. 186, pl. III, 8 b.

\section{MA87 (pl. XXXVIII)}

Secteur : Regio V, Insula 3.

Inventaire : $\mathrm{n}^{\circ} 71-42 \mathrm{~b}$.

Type : Samos-Milet (?).

État de conservation : fragment de lèvre. Lèvre en amande, légèrement épaissie.

Observations et comparaisons : d'après Fr.-H. MassaPairault le fragment, qu'elle attribue à une amphore commerciale, appartient au même individu de la fiche suivante. La morphologie de la lèvre, du type en amande légèrement épaissie, pourrait rappeler les formes du groupe Samos-Milet ; mais, faute d'un examen visuel, on ne peut avoir aucune certitude.
Chronologie : non déterminable.

Bibliographie : Fr.-H. Massa-Pairault, in Massa-Pairault 1997, p. 186, pl. I, 10 b.

\section{MA88 (pl. XXXVIII)}

Secteur: Regio V, Insula 3.

Inventaire : $\mathrm{n}^{\circ} 71-42 \mathrm{a}$.

Type : Samos-Milet (?).

État de conservation : partie d'anse. Anse en ruban épaissi, de section elliptique.

Observations et comparaisons : Fr.-H. Massa-Pairault attribue cette anse au même individu de la fiche précédente.

Chronologie : non déterminable.

Bibliographie : Fr.-H. Massa-Pairault, in Massa-Pairault 1997, p. 186, pl. I, 10 a.

\section{MA89 (pl. XXXVIII)}

Année de la fouille / trouvaille : 1988.

Secteur : Regio V, Insula 5.

Structure : Plateia A, devant la Maison 1.

US : 8 .

État de conservation : col, non complet et reconstitué à partir de 8 fragments, ainsi que de nombreux fragments de la panse qu'il n'est pas possible d'assembler. H. max. cons. : 11 4. Col évasé. Diam. min./max. restituable : $9,1 / 10,6$.

Surface ext., int. et fracture : orange (5YR6/8).

Inclusions : inclusions blanches de dimensions petites et moyennes, à fréquence basse, distribuées d'une manière homogène, et inclusions brunes et grises de petites dimensions, à fréquence très basse, distribuées d'une manière homogène. Mica très fin, argenté, à fréquence basse.

Observations et comparaisons : les conditions de l'exemplaire ne permettent pas de l'encadrer sur une base morphologique, mais l'analyse visuelle de la pâte pourrait suggérer une provenance gréco-orientale.

Chronologie : non déterminable.

\section{MA90 (pl. XXXVIII)}

Année de la fouille / trouvaille : XIX secolo.

Secteur : Regio V, Insulae 3-4.

Inventaire : $\mathrm{n}^{\circ} 1604$.

État de conservation : amphore commerciale dont il reste la partie centrale d'une anse, jusqu'à sa courbure. H. max. cons. : 10. Anses en ruban épaissi, de section elliptique. H. max. cons. : 10 ; épaiss. max. : 4,4 x 2,5.

Surface ext. et int. : beige orange (7.5YR6/6-7.5YR6/4). Fracture : brun foncé (7.5YR5/3-7.5YR4/3).

Inclusions : inclusions grises, brunes et blanches, de petites dimensions, à fréquence moyenne-haute. Mica fin diffusé. Engobe : traces d'engobe.

Apparat épigraphique : lettre grecque chi incisée avant cuisson (Sacchetti 2010, p. 54-55, cat. $\mathrm{n}^{\circ} 10$ ). 
Observations et comparaisons : attribuée par E. Govi à une amphore de table, l'anse appartient plus probablement à une amphore commerciale pour laquelle toute proposition d'encadrement typologique et chronologique s'avère douteux. On se limitera à signaler sa possible origine gréco-orientale sur la base des caractéristiques de la pâte.

Chronologie : non déterminable.

Bibliographie : E. Govi, in Iscrizioni e graffiti, $\mathrm{n}^{\circ} 48$, p. 40-41, pl. VIa, 48 ; Sacchetti 2010, p. 54-55, cat. $\mathrm{n}^{\circ} 10, \mathrm{pl}$. II, 10.

\section{MA91 (pl. XXXVIII)}

Année de la fouille / trouvaille : 1992.

Secteur : Regio V, Insula 5.

Structure : Maison 1.

US : Q I, 1 .

État de conservation : partie supérieure d'une anse avec la jonction du col. H. max. cons. : 3. Anses en ruban épaissi, à section elliptique. H. max. cons. : 3 ; épaiss. $\max .: 3,7 \times 2,2$.

Surface ext., int. et fracture : beige clair (10YR7/4).

Inclusions : inclusions blanches, à fréquence basse, inclusions grises de petites dimensions, à fréquence moyenne, et inclusions brun orange de petites dimensions, à fréquence basse, distribuées d'une manière assez homogène. Fines écailles de mica argenté à fréquence basse.

Observations et comparaisons : étant donné le mauvais état de conservation du fragment et surtout l'absence de détails mophologiquement distinctifs, on en propose, sur la base de la pâte, une attribution générique à la production gréco-orientale.

Chronologie : non déterminable.

\section{MA92 (pl. XXXVIII)}

Année de la fouille / trouvaille : 1988.

Secteur : Regio V, Insula 5.

Structure : Plateia A, devant la Maison 1.

US : 2-5-9-10-11.

État de conservation : partie de col avec la jonction supérieure d'une anse. Dimensions max. fr. : 6 x 6,3. Anses en ruban épaissi, à section elliptique. H. max. cons. : 4 ; épaiss. max. : 1,7 x 5,2.

Surface ext. et int. : beige (7.5YR7/6).

Fracture : orange rosé (5YR6/6).

Inclusions : inclusions blanches, brunes et grises de petites dimensions, à fréquence basse, distribuées d'une manière homogène. Écailles de mica argenté de petites dimensions, à fréquence basse, diffusées d'une manière homogène.

Engobe : engobe crème, mince sur la surface extérieure. Observations et comparaisons : attribuable à la production gréco-orientale sur la base de la pâte. Chronologie : non déterminable.

\section{MA93 (pl. XXXVIII)}

Année de la fouille / trouvaille : 1990.

Secteur: Regio V, Insula 5.

Structure : Plateia A, devant la Maison 1.

US : 802 .

Type : Solokha I.

État de conservation : partie de lèvre et de col, reconstituée à partir de 2 fragments, ainsi que de nombreux fragments de parois de panse probablement appartenant au même individu mais dont l'assemblage s'avère impossible. H. max. cons. : 7,6. Lèvre à marli oblique, de section triangulaire, à la surface supérieure aplatie. H. : 2,9 ; épaiss. max. : 2,5 ; diam. int./ext. restituable embouchure : 14 / 17,9. Anses en ruban épaissi, à section elliptique. H. max. cons. : 5 ; épaiss. max. : 2,2 x 4,3.

Surface ext. et int. : orange clair (5YR6/6).

Fracture : orange (5YR6/8).

Inclusions : argile très dépurée. Inclusions blanches et grises de petites dimensions, à fréquence basse, distribuées d'une manière homogène. Peu de mica, très fin, argenté, diffusé en surface.

Observations et comparaisons : attribuable à une amphore du groupe Solokha I. Pour le type, Lawall 1995, p. 220, fig. 88, du dépôt N $7: 3$ de l'Agora fermé vers 440 av. J.-C. La morphologie de la lèvre, à la surface supérieure plate et hozontale, caractérise les exemplaires situés en tête de série et datés vers le milieu du V ${ }^{\mathrm{e}} \mathrm{s}$. av. J.-C.

Chronologie : vers 450 av. J.-C.

\section{MA94 (pl. XXXVIII)}

Secteur : Regio IV, Insula 1.

Structure : Maison 4. Q12A.

Inventaire : $n^{\circ} 49$ caisse 9.

Type : Solokha I.

État de conservation : partie de lèvre. H. max. cons. : 3. Lèvre à marli oblique, de section triangulaire, légèrement ouverte. H. : 2,4 ; épaiss. max. : 2,5 ; diam. int./ext. restituable embouchure : 12,4 / 16,5.

Surface ext., int. et fracture : brun chamois (10YR5/3).

Inclusions : inclusions grisâtres et blanches de petites dimensions, à fréquence basse.

Observations et comparaisons : pour le type, Lawall 1995, p. 220, fig. 88, du dépôt N $7: 3$ de l'Agora, fermé autour de 440 av. J.-C. ; pour la forme, Desantis 1989, $n^{\circ} 31$, pl. IV.1 (cat. SP38), de Spina. On en propose une datation vers le milieu du $\mathrm{V}^{\mathrm{e}} \mathrm{s}$. av. J.-C. étant donné la surface supérieure de la lèvre à peu près horizontale, caractéristique morphologique décrite pour les exemplaires du début de la série du groupe Solokha I.

Chronologie : vers 450 av. J.-C. 


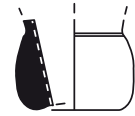

MA81

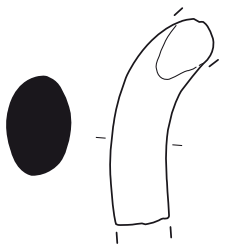

MA84

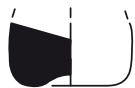

MA82

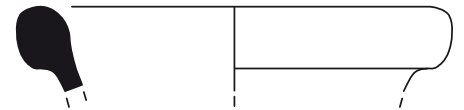

MA83

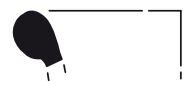

MA85

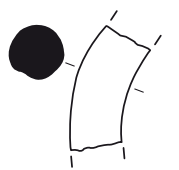

MA86

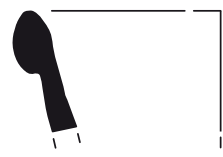

MA87

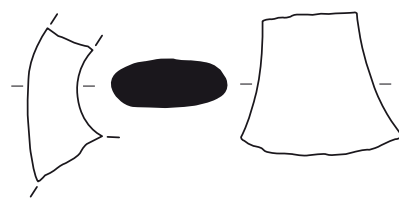

MA88

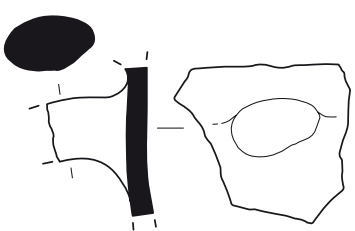

MA91

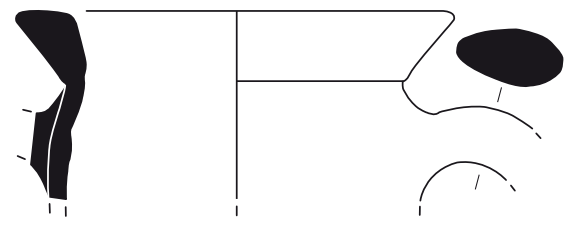

MA93

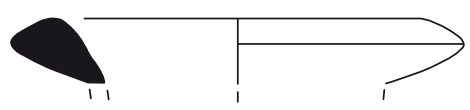

MA95
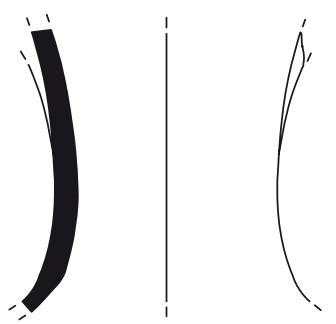

MA89

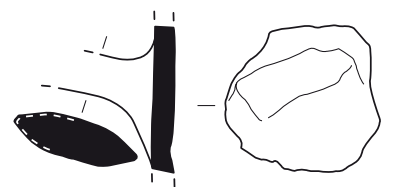

MA92

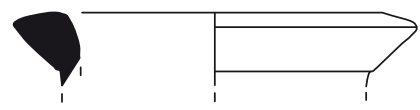

MA94

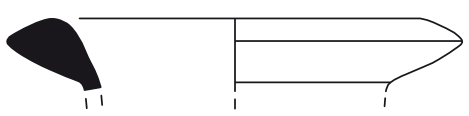

MA96 


\section{MA95 (pl. XXXVIII)}

Année de la fouille / trouvaille : 1964.

Secteur : Regio II, Insula 1.

Structure : Four. Q B-A.

Inventaire : $n^{\circ} 7476$.

Type : Solokha I.

État de conservation : partie de lèvre : H. max. cons. : 2,6. Lèvre à marli oblique, de section triangulaire. H. : 2,6 ; épaiss. max. : 3,1 ; diam. int./ext. restituable embouchure : $14,6 / 18,5$.

Surface ext., int. et fracture : chamois (10YR6/4).

Inclusions : inclusions blanches, grises et orange de petites dimensions et à fréquence basse. Mica fin argenté à fréquence moyenne.

Observations et comparaisons : pour le type, Lawall 1995, p. 220-221, fig. 88-90. Le repli, encore très léger, de la lèvre suggère une datation dans la seconde moitié du Ve s. av. J.-C. (Lawall 1995, p. 220-221, fig. 91-92, respectivement des dépôts R 13 :1 e B 13 :6 de l'Agora, datables vers 400 av. J.-C.).

Chronologie : 450-400 av. J.-C.

\section{MA96 (pl. XXXVIII)}

Année de la fouille / trouvaille : 1964.

Secteur : Regio II, Insula 1.

Structure : Four. Q C-B.

Inventaire : $n^{\circ} 5638$.

Type : Solokha I.

État de conservation : partie de lèvre : H. max. cons. : 3 . Lèvre à marli oblique, de section triangulaire. H. : 2,6 ; épaiss. max. : 3,1 ; diam. int./ext. restituable embouchure : $14,6 / 18,5$.

Surface ext., int. et fracture : chamois (10YR6/4).

Inclusions : inclusions blanches, grises et orange de petites dimensions, à fréquence basse. Mica fin argenté à fréquence moyenne.

Observations et comparaisons : attribuable à une amphore du groupe Solokha I et datable du courant de la seconde moitié du $\mathrm{V}^{\mathrm{e}} \mathrm{s}$. av. J.-C. Pour le type et la chronologie, voir la fiche précédente.

Chronologie : 450-400 av. J.-C.

\section{MA97 (pl. XXXIX)}

Année de la fouille / trouvaille : 1964.

Secteur : Regio II, Insula 1.

Structure : Four. Q C-B.

Inventaire : $n^{\circ} 5594$.

Type : Solokha I.

État de conservation : partie de lèvre : H. max. cons. : 3,2. Lèvre à marli oblique, de section triangulaire. H. : 2,8; épaiss. max. : 3 ; diam. int./ext. restituable embouchure : 14,4 / 18,5 .

Surface ext., int. et fracture : chamois (10YR6/4).
Inclusions : inclusions blanches, grises et orange de petites dimensions et à fréquence basse. Mica fin argenté à fréquence moyenne.

Observations et comparaisons : voir cat. MA95.

Chronologie : 450-400 av J.-.C.

\section{MA98 (pl. XXXIX)}

Secteur : Regio IV, Insula 1.

Structure : Maison 4. Q11.

Inventaire : $\mathrm{n}^{\circ} 6$ caisse 0 .

Type : Solokha I.

État de conservation : amphore dont il reste la moitié supérieure, depuis l'embouchure jusqu'à l'épaule, reconstituée à partir de fragments. De nombreux fragments de parois appartiennent vraisemblablement au même individu, mais il est impossible de les assembler. H. max. cons. : 23. Lèvre à marli oblique, de section triangulaire, légèrement ouverte. H. : 2,6 ; épaiss. max. : 3,2 ; diam. int./ext. restituable embouchure : 13,4 / 17,8. Col cylindrique, au profil légèrement concave. H. : 15 ; diam. min./ max. : 11. Anses verticales, en ruban épaissi, de section elliptique, avec une impression digitale au niveau de la jonction inférieure. H. : 13,5 ; épaiss. max. : 4,1 x 2,3. Épaule aplatie. Larg. : 9,5.

Surface ext., int. et fracture : orange chamois (7.5YR6/ 6-7.5YR7/6).

Inclusions : inclusions grisâtres et brunâtres de petites dimensions, à fréquence basse, et inclusions blanches, de dimensions petites et moyennes, à fréquence moyenne-basse. Observations et comparaisons : attribuable à une amphore du groupe Solokha I. Par la forme déjà allongée du col on propose son encadrement chronologique au moment de transition du $\mathrm{V}^{\mathrm{e}}$ au $\mathrm{IV}^{\mathrm{e}} \mathrm{s}$. av. J.-C. Pour le type, Lawall 1995, p. 220-221, fig. 90, du dépôt H 13 :4 de l'Agora d'Athènes, fermé autour de 390 av. J.-C.

Chronologie : 410-390 av. J.-C.

\section{MA99 (fig. 92 ; pl. XXXIX)}

Secteur : Regio V, Insulae 4-5.

Sporadique. Au-dessus de la Galerie Ouest.

Inventaire : $\mathrm{n}^{\circ} 58480 / 85$.

Type : Solokha I.

État de conservation : amphore dont il reste la partie inférieure, épaulement exclu, reconstituée à partir de nombreux fragments et restaurée. H. max. cons. : 37 . Corps vraisemblablement ovoïde. H. max. cons. : 33 ; diam. max. : 39,8. Pied tronconique, à base en anneau épaissi. H. : 3,2 ; diam. min./max. : 6 / 8,2.

Surface ext., int. et fracture : jaune (2.5Y7/3-2.5.Y7/4). Inclusions : inclusions blanches, grises et brunes de petites dimensions, à fréquence basse. Mica presque absent. Observations et comparaisons : attribuable à une amphore du groupe Solokha I et datable du courant de la seconde 


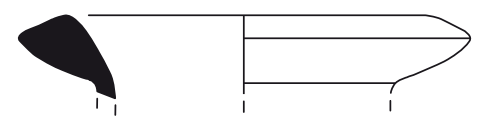

MA97
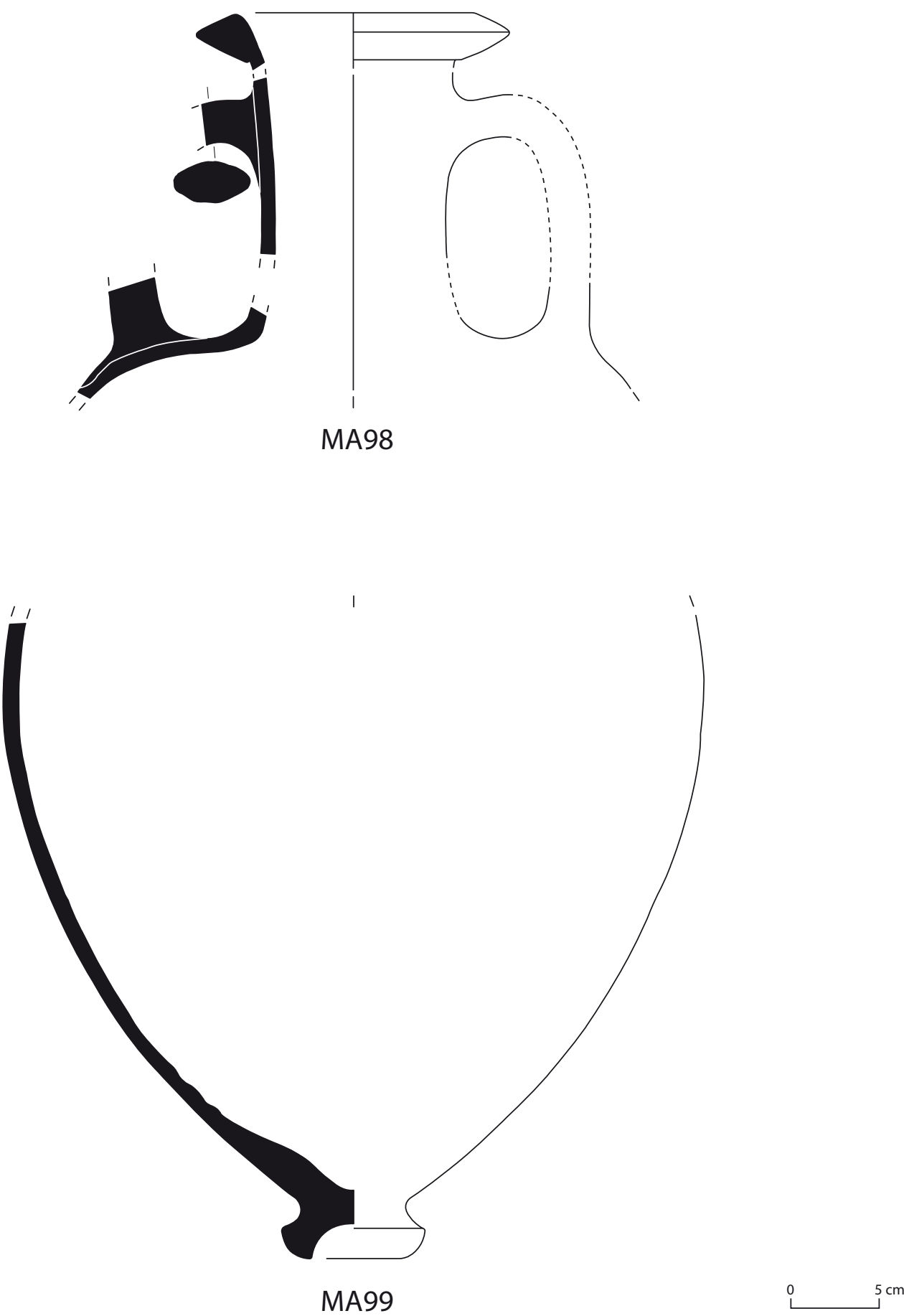

PI. XXXIX. Marzabotto. 


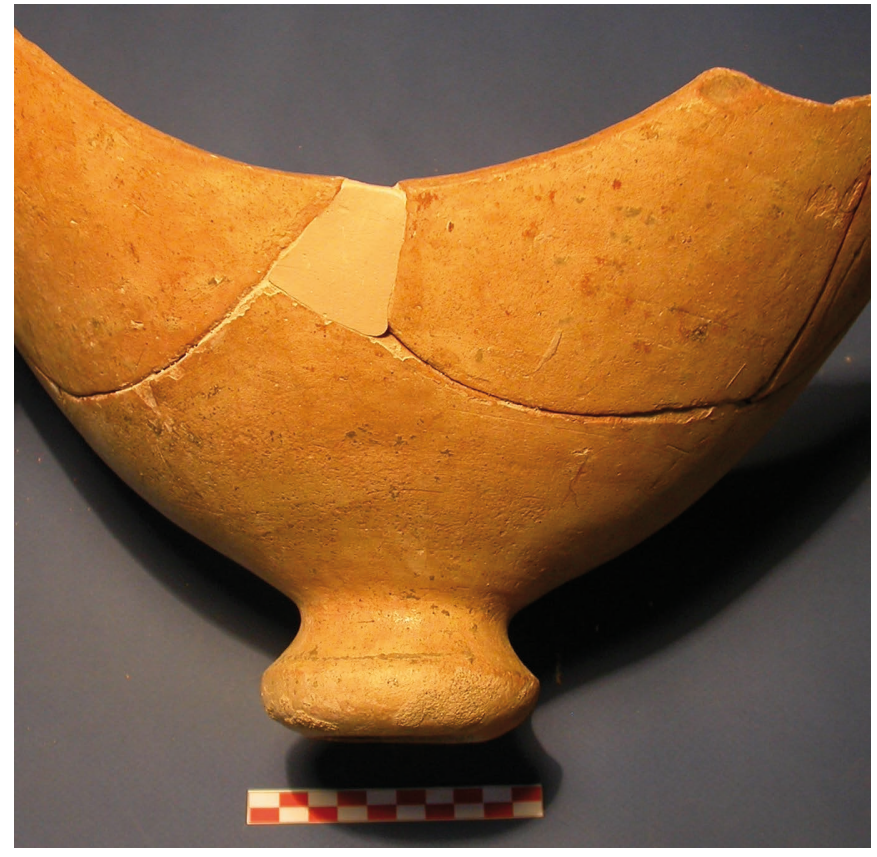

Fig. 92. Marzabotto, découverte isolée, inv. 58480/85. Fond d'amphore du groupe Solokha I. Cat. MA99 (photo F. Sacchetti).

moitié du Ve ou des débuts du IV $\mathrm{s}$. av. J.-C. Pour le type, Lawall 1995, p. 220-221, fig. 88-90, 93 (du dépôt N 7:3 de l'Agora, fermé vers 440 av. J.-C., du dépôt S 16:1, fermé vers 410 av. J.-C., et du dépôt H 13:4, fermé vers 390 av. J.-C.).

Chronologie : 450-400 av. J.-C.

\section{MA100 (pl. XL)}

Année de la fouille / trouvaille : 1964.

Secteur : Regio II, Insula 1.

Structure : Four. Q C3C4-B3B4.

Inventaire : $\mathrm{n}^{\circ} 6489$.

Type : Samos-Milet.

État de conservation : pied complet et partie inférieure de la panse reconstituée à partir de 72 fragments. $\mathrm{H}$. max. cons. : 16. Corps ovoïde. H. max. cons. : 12 ; diam. max. : 20,4. Pied tronconique creux, à base en anneau légèrement épaissi. H. : 2,4; diam. min./max. : 5 / 6,2. Surface ext., int. et fracture : brun rougeâtre (5YR5/6). Inclusions : inclusions blanches de dimensions petites et moyennes, à fréquence basse, et inclusions noirâtres de petites dimensions, à fréquence basse. Mica fin, argenté et doré, à fréquence moyenne.

Observations et comparaisons : attribuable à la production du groupe Samos-Milet et datable entre la fin $\mathrm{VI}^{\mathrm{e}}$ et le début du Ve s. av. J.-C. sur la base de la comparaison avec les exemplaires de l'épave de la Pointe Lequin (Long, Miro, Volpe 1992, fig. 44.1-3), daté vers 515 av. J.-C. Pour la forme, voir également Dupont 1998, fig. 23.9, b (datée du dernier quart du $\mathrm{VI}^{\mathrm{e}} \mathrm{s}$. av. J.-C.), et Voigtländer 1983, fig. 27.167 (datée du VI ${ }^{\mathrm{e}}$ s. av. J.-C.). Chronologie : fin $\mathrm{VI}^{\mathrm{e}}$-début $\mathrm{V}^{\mathrm{e}} \mathrm{s}$. av. J.-C.

\section{MA101 (pl. XL)}

Année de la fouille / trouvaille : 1994.

Secteur: Regio V, Insula 5.

Structure : Plateia A, devant la Maison 1.

US : 814

Type : Solokha I.

État de conservation : pied complet et partie du fond de la panse reconstituée à partir de 2 fragments. H. max. cons. : 13,5. Pied tronconique creux, à base en anneau épaissi. H. : 3,3 ; diam. min./max. : 5,9 / 7,6.

Surface ext. : orange (10R5/8).

Surface int.et fracture : orange rosé (10R5/6).

Inclusions : inclusions blanches, grises et brunes de petites dimensions, à fréquence basse, distribuées d'une manière homogène. Écailles de mica argenté et doré de petites dimensions, à fréquence basse.

Observations et comparaisons : on renvoie à cat. MA99. Chronologie : 450-400 av. J.-C.

\section{MA102 (pl. XL)}

Année de la fouille / trouvaille : 1994.

Secteur : Regio V, Insula 5.

Structure : Maison 1.

US : 351 .

Type : Solokha I.

État de conservation : pied complet. H. max. cons. : 3,4. Pied tronconique creux, à base en anneau légèrement épaissi. Diam. min./max. : 6,3 / 8,5.

Surface ext., int. et fracture : beige clair (10YR7/4).

Inclusions : inclusions blanches de dimensions petites et moyennes, à fréquence basse, inclusions grises de petites dimensions, à fréquence basse, et inclusions brun orange de dimensions petites et moyennes distribuées d'une manière non homogène et concentrées sur le fond. Fines écailles de mica argenté, à fréquence basse.

Observations et comparaisons : on renvoie à cat. MA99. Chronologie : 450-400 av. J.-C.

\section{MA103 (pl. XL)}

Année de la fouille / trouvaille : 1988.

Secteur: Regio V, Insula 5.

Structure : Plateia A devant la Maison 1.

US : 10 .

Type : Solokha I.

État de conservation : pied, complet mais à la surface inférieure ébréchée, avec une brève partie du fond de la panse. H. max. cons. : 5,4. Pied tronconique creux, à base en anneau épaissi. H. : 3,5; diam. min./max. : $6,4 / 8,3$. 
Surface ext. et int. : beige orange (7.5YR7/6).

Fracture : orange $(2.5 \mathrm{YR} 5 / 6)$.

Inclusions : inclusions blanches, brunes et grises de petites dimensions, à fréquence basse, distribuées d'une manière homogène. Écailles de mica argenté de petites dimensions, à fréquence basse, distribuées d'une manière homogène.

Observations et comparaisons : on renvoie à cat. MA99. Chronologie : 450-400 av. J.-C.

\section{MA104 (pl. XL)}

Année de la fouille / trouvaille : 1994.

Secteur : Regio V, Insula 5.

Structure : Plateia A, devant la Maison 1.

US : 814 .

Type : Solokha I.

État de conservation : pied complet, reconstitué à partir de 4 fragments. H. max. cons. : 3,7. Pied tronconique creux, à base en anneau épaissi. H. : 3,1 ; diam. min./ $\max .: 6,1 / 7,4$.

Surface ext., int. et fracture : orange intense (5YR6/ 8-5YR5/8).

Inclusions : inclusions blanches de dimensions petites et moyennes, à fréquence basse, distribuées d'une manière homogène, et inclusions grises et brunes de petites dimensions, à fréquence basse, distribuées d'une manière homogène. Écailles de mica argenté et doré de petites dimensions, à fréquence basse.

Engobe : engobe crème, fin.

Observations et comparaisons : vu sa morphologie, on peut l'assigner à la série initiale du groupe Solokha I. Pour le type, datable de la seconde moitié du $\mathrm{V}^{\mathrm{e}} \mathrm{s}$. av. J.-C., Lawall 1995, p. 220-221, fig. 88-90. Dans le creux central de la surface extérieure du pied on remarque une concentration de dégraissants et un relief central, ce dernier étant visible éaglement dans le fond intérieur. L'une et l'autre de ces caractéristiques sont décrites pour plusieurs exemplaires de la forme Solokha I des contextes de l'Agora d'Athènes datables avant la fin du $\mathrm{V}^{\mathrm{e}} \mathrm{s}$. av. J.-C. (Lawall 1995, p. 220-221 et notes 5, 8-9, fig. 93). Sur d'autres amphores du groupe Solokha I de Bologne et de Marzabotto il a été également possible de remarquer ces particularités (cf. cat. FE9, FE10, MA105, MA106).

Chronologie : 450-400 av. J.-C.

\section{MA105 (pl. XL)}

Année de la fouille / trouvaille : 1964.

Secteur : Regio II, Insula 1.

Structure : Four. Q D2D3-C2C3.

Inventaire : $\mathrm{n}^{\circ} 5121$.

Type : Solokha I.

État de conservation : pied, extrêmement fragmentaire, et 14 fragments de parois de la panse vraisemblablement appartenant au même individu, bien qu'il soit impossible de les assembler. Pied probablement tronconique creux, à base en anneau légèrement épaissi. H. max. cons. : 2 ; diam. min./max. restituable : 5,4 / 7,9.

Surface ext. et int. : beige jaune (10YR8/6).

Fracture : orange rosé (5YR6/6).

Inclusions : inclusions orange, noirâtres et blanches de petites dimensions et à fréquence basse.

Observations et comparaisons : pour le type, Lawall 1995, fig. 88-90, 93. Malgré l'état fragmentaire du pied en question, l'hypothèse d'attribution au type Solokha I est corroborée, non seulement par la partie du profil conservée, mais également par la présence d'un résidu sableux dans le creux extérieur du pied. Cette accumulation de dégraissants est caractéristique des amphores Solokha I de la seconde moitié du Ve s. av. J.-C. (cf. la fiche précédente).

Chronologie : 450-400 av. J.-C.

\section{MA106 (pl. XL)}

Année de la fouille / trouvaille : 1953.

Secteur : Îlots sur la Plateia A.

Type : Solokha I.

État de conservation : pied. H. max. cons. : 4. Pied tronconique creux, à base en anneau épaissi. H. max. cons. : 3,7; diam. min./max. : 5,1/7,8.

Surface ext., int. et fracture : chamois brun (10YR6/ 3-10YR6/4).

Inclusions : inclusions blanches de dimensions petites, moyennes et grandes, à fréquence moyenne, inclusions orange de dimensions petites et moyennes, à fréquence moyenne, et inclusions noires de petites dimensions, à fréquence basse.

Observations et comparaisons : pour le type, Lawall 1995, p. 220-221, fig. 88-90. On signale la présence d'un résidu sableux dans la pâte, très évident dans le creux extérieur du pied, qui a été reconnu comme l'une des caractéristiques de plusieurs amphores Solokha I datables de la seconde moitié du Ve s. av. J.-C. (cf. MA104).

Chronologie : 450-400 av. J.-C.

\section{MA107 (pl. XL)}

Année de la fouille / trouvaille : 1992.

Secteur : Regio V, Insula 5.

Structure : Maison 1.

US : 319 .

Type : Solokha I.

État de conservation : partie de col et épaule reconstitués à partir de 5 fragments. H. max. cons. : 11,7. Col tronconique. H. max. cons. : 9 ; diam. min./max. restituable : 10,9 / 13,9. Épaule aplatie. Larg. max. cons. : 6.

Surface ext. et int. : beige orange (7.5YR6/6). 
Fracture : beige (7.5YR7/6).

Inclusions : argile dépurée, presque entièrement dépourvue d'inclusions. Inclusions brun orange et inclusions grises de petites dimensions, à fréquence basse, distribuées d'une manière homogène.

Observations et comparaisons : sans doute attribuable à une amphore du groupe Solokha I. L'absence des éléments morphologiques les plus caractérisants, ainsi que la lèvre ou le pied, nous empêche de déterminer son encadrement chronologique.

Chronologie : non déterminable.

\section{MA108 (pl. XL)}

Année de la fouille / trouvaille : 1994.

Secteur : Regio V, Insula 5.

Structure : Plateia A, devant la Maison 1.

US : 814

Type : Solokha I.

État de conservation : partie supérieure d'une anse, jusqu'à sa courbure, avec un bref segment du col et de nombreux fragments de paroi de la panse qu'il est impossible d'assembler. Anses en ruban épaissi, à section elliptique. H. max. cons. : 4,5 ; épaiss. max. : $2,2 \mathrm{x}$ 4,2 .

Surface ext. et int. : orange intense (5YR6/8).

Fracture : orange rosé (2.5YR6/8).

Inclusions : inclusions blanches de dimensions petites et moyennes, à fréquence basse, distribuées d'une manière homogène et inclusions brunes et grises de petites dimensions, à fréquence basse, distribuées d'une manière homogène. Mica très fin, argenté, à fréquence basse, distribué d'une manière homogène.

Observations et comparaisons : pour la forme et la pâte, on renvoie à cat. MA93, attribuable à une amphore Solokha I du début de la série. Sur la base de cette confrontation on propose d'encadrer l'anse en question au même type. Appartenant paut-être au même individu de la fiche qui suit.

Chronologie : non déterminable.

\section{MA109 (pl. XL)}

Année de la fouille / trouvaille : 1994.

Secteur : Regio V, Insula 5.

Structure : Plateia A, devant la Maison 1.

US : 814 .

Type : Solokha I.

État de conservation : partie supérieure d'une anse, jusqu'à sa courbure, et de nombreux fragments de paroi de la panse qu'il est impossible d'assembler. Anses en ruban épaissi, à section elliptique. H. max. cons. : 7 ; épaiss. max. : 2,2 x 4,2.

Surface ext. et int. : orange intense (5YR6/8).

Fracture : orange rosé (2.5YR6/8).
Inclusions : inclusions blanches de dimensions petites et moyennes, à fréquence basse, distribuées d'une manière homogène, et inclusions brunes et grises de petites dimensions, à fréquence basse, distribuées d'une manière homogène. Mica argenté très fin, à fréquence basse, distribué d'une manière homogène.

Observations et comparaisons : atttibuable peut-être au même individu de la fiche précédente, à laquelle on renvoie pour les observations.

Chronologie : non déterminable.

MA110 (pl. XL)

Année de la fouille / trouvaille : 1953.

Secteur : Îlots sur la Plateia A.

Type : Solokha I.

État de conservation : partie d'une anse. H. max. cons. : 12. Anses en ruban épaissi, à section elliptique. Épaiss. $\max .: 2 \times 4,1$.

Surface ext., int. et fracture : beige rosé (7.5YR8/3).

Inclusions : inclusions blanches et noirâtres de petites dimensions, à fréquence très basse.

Observations et comparaisons : attribuable peut-être à une amphore du type Solokha I.

Chronologie : non déterminable.

MA111 (pl. XL)

Année de la fouille / trouvaille : 1964.

Secteur : Regio II, Insula 1.

Structure : Four. Q D-C.

Inventaire : $\mathrm{n}^{\circ} 4686$.

Type : Solokha I.

État de conservation : partie de col avec la jonction supérieure d'une anse. H. max. cons. : 6. Anses en ruban épaissi, à section elliptique. H. max. cons. :1,2 ; épaiss. $\max$ : : 4,6 x 2,8.

Surface ext., int. et fracture : chamois (10YR6/4).

Inclusions : inclusions blanches, grises et orange de petites dimensions et à fréquence basse. Mica fin argenté à fréquence moyenne.

Observations et comparaisons : l'état de conservation insuffisant du fragment et l'absence d'éléments morphologiquement caractérisants de l'amphore appellent à beaucoup de prudence dans sa définition typo-chronologique ; cependant les caractéristiques de la pâte céramique, très proches de celles des fragments qui font l'objet des fiches MA95, MA96 e MA97, tous attribuables au type Solokha I, autorisent à rapprocher aussi l'exemplaire en question de cette production.

Chronologie : non déterminable. 


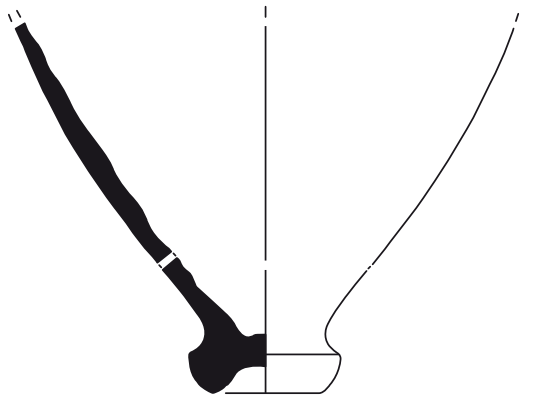

MA100

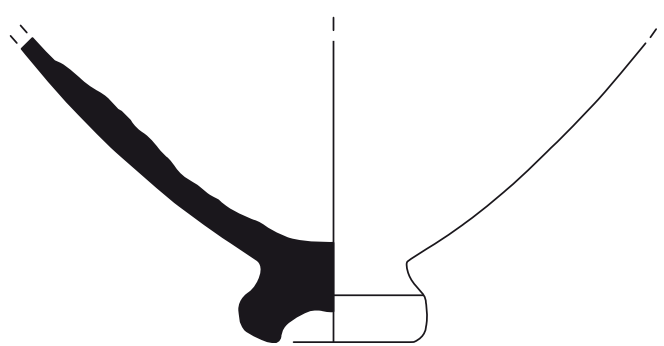

MA101

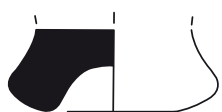

MA102

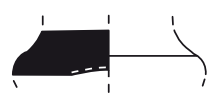

MA105

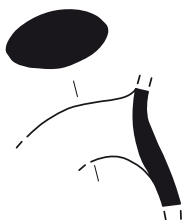

MA108

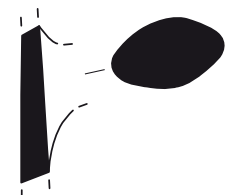

MA111

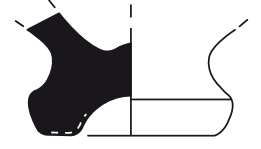

MA103

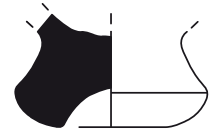

MA106

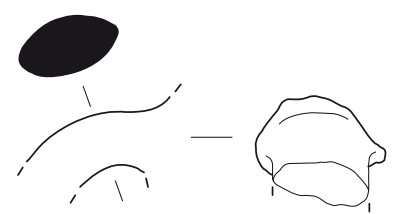

MA109
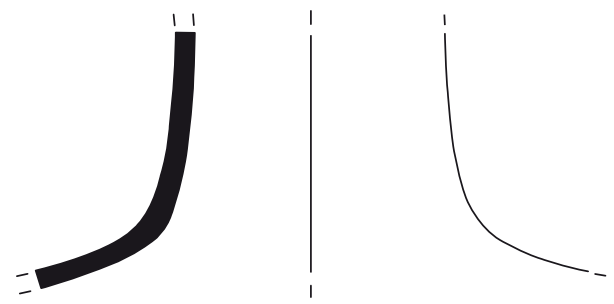

MA107

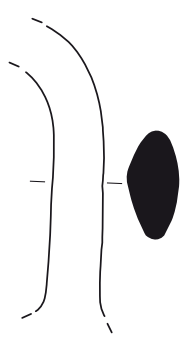

MA110
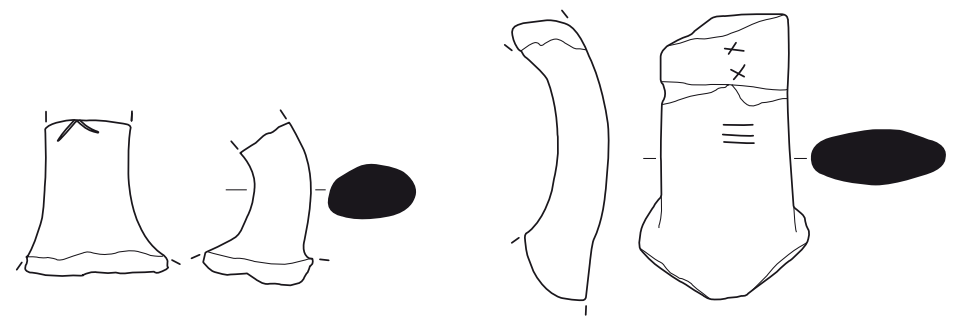

MA113 $5 \mathrm{~cm}$

PI. XL. Marzabotto. 


\section{AMPHORES DE PRODUCTION NON IDENTIFIÉE ${ }^{665}$}

MA112 (pl. XL)

Secteur : Regio V, Insula 3.

Inventaire : $\mathrm{n}^{\circ} 73-15$.

État de conservation : segment d'anse, depuis la jonction inférieure jusqu'au début de sa courbure. Dimensions : non repérées. Anses en ruban épaissi, à section elliptique. Surface ext., int. et fracture : rosée.

Apparat épigraphique : Fr.-H. Massa-Pairault et D. Briquel signalent la présence d'un graffito incomplet, consistant dans un "signe en forme de croix » (Sacchetti 2011, $\mathrm{n}^{\circ}$ 8, p. 250, pl. I, 8).

Observations et comparaisons : attribué par les éditeurs à une amphore commerciale.

Chronologie : non déterminable

Bibliographie : Fr.-H. Massa-Pairault, in Massa-Pairault 1997, p. 186, pl. VII, 5 ; D. Briquel, in Massa-Pairault $1997, \mathrm{n}^{\circ} 48$, p. 152 ; D. Briquel, in Iscrizioni e graffiti, $\mathrm{n}^{\circ} 239$, p. 149, pl. XXX, 239 ; Sacchetti 2011, p. 250, $\mathrm{n}^{\circ} 8$, pl. I, 8 .

\section{MA113 (pl. XL)}

Année de la fouille / trouvaille : 1988.

Secteur : Regio V, Insula 5.

Structure : Plateia A, devant la Maison 1.

US : 27 .

État de conservation : segment d'anse, depuis la jonction inférieure au niveau de l'épaule, jusqu'à sa courbure supérieure, reconstitué à partir de deux fragments. H. max. cons. : 8,5. Anses en ruban épaissi, à section elliptique. Épaiss. max. : 2 x 5 .

Surface ext., int. et fracture : beige rosé.

Inclusions : argile dépurée, avec de petites inclusions.

Apparat épigraphique : L. Malnati y voit un graffito numéral étrusque consistant dans deux « signes en forme de croix » et trois traits parallèles (Sacchetti 2011, cat. $\mathrm{n}^{\circ}$ 9, p. 250, pl. I, 9).

Observations et comparaisons : l'état du fragment ne permettent d'identifier avec précision ni le type d'amphore ni le centre de production, alors que la datation des dernières décennies du $\mathrm{VI}^{\mathrm{e}} \mathrm{s}$. av. J.-C. a été proposée par L. Malnati sur une base stratigraphique.

Chronologie : 525-500 av. J.-C.

Bibliographie : L. Malnati, dans Iscrizioni e graffiti, $n^{\circ}$ 288, p. 177, pl. XXXVI, 288 ; Johnston 2004, p. 784,

665. Parmi les objets livrés par les fouilles de la ville de Marzabotto, ont été trouvés vingt-quatre fragments d'anses appartenant à des amphores commerciales de production non identifiable. On fournit ci-dessous une fiche détaillée uniquement de deux exemplaires qui se distinguent par la présence de graffiti. pl. G. 3, fig. 4, n 200 ; Sacchetti 2011, cat. $n^{\circ} 9$, p. 250, pl. I, 9.

\section{AMPHORES DU TYPE CORINTHIEN A/A'}

\section{MA114 (pl. XLI)}

Année de la fouille / trouvaille : 1998.

Secteur : Regio IV, Insula 2.

Structure : Casa 1.

US : 407 .

Inventaire $n^{\circ}: 4087$

État de conservation : pied et partie inférieure de la panse. H. max. cons. : 13,5. Pied en forme de bouton, plein à l'intérieur, aplati à la base. H. : 3,5; diam. min./ $\max .: 2,7 / 4,5$.

Surface ext., int. et fracture : jaune-rose (7.5YR8/ 4-10YR8/4).

Inclusions : inclusions de couleur blanc, gris, orange (mudstone) et brun, de petites, moyenne et grande taille, très fréquentes.

Observations et comparaisons : pour le type, Koehler 1978a, fig. 1.d ; et Koehler 1981, fig. 1.b, daté à la deuxième moitié du $\mathrm{V}^{\mathrm{e}}$ ou au début du $\mathrm{IV}^{\mathrm{e}} \mathrm{s}$. av. J.-C. Pour la forme, Di Sandro 1986, tav. 6, sg. 68. Les tessons de céramique attique découverte dans le même contexte sont datables du troisième ou quatrième quart du V $\mathrm{V}^{\mathrm{e}}$ s. (Brizzolara-Baldoni-Succi 2005). Au même individu appartient sans doute le col d'amphore inv. $\mathrm{n}^{\circ} 1998.407 / 4088$.

Chronologie : 450-375 av. J.-C.

Bibliographie : de Marinis 2010, $\mathrm{n}^{\circ}$ 161, p. 84, fig. 77.161.

\section{MA115 (pl. XLI)}

Année de la fouille / trouvaille : 1996.

Secteur : Regio IV, Insula 2.

Structure : Casa 1.

US $: 1$.

Inventaire $\mathrm{n}^{\circ}: 2778$.

État de conservation : partie de la lèvre et du col. H. max. cons. : 7. Lèvre en marli, oblique, a section trapézoïdale. H. : 2,7 ; épais. max. : 2,7 ; diam. int./ext. embouchure : $7,6 / 12,3$

Surface ext., int. et fracture : beige (10YR8/4-10YR7/4). Inclusions : inclusions de couleur blanchâtre, gris et brun, de petite, moyenne et grande taille à fréquence moyennehaute et inclusions de couleur orange (mudstone) de moyenne et grande taille à haute fréquence.

Observations et comparaisons : pour le type, Koehler 1978a, fig. 1.d-e, respectivement A' et A; pour la forme, précisément Di Sandro 1986, pl., 6, sg. 57.

Chronologie : 450-375 av J.-C.

Bibliographie : de Marinis 2010, n 160, p. 84, fig. 76.160 . 


\section{AMPHORES GRÉCO-OCCIDENTALES}

\section{MA116 (pl. XLI)}

Année de la fouille / trouvaille : 1989.

Secteur: Regio IV, Insula 2.

Structure : Casa 1.

US : 1Q11A.

Inventaire $\mathrm{n}^{\circ}: 1475$.

Type : corinthienne B.

État de conservation : fragment de pied lacunaire au niveau de la base. H. max. cons. : 2,7. Pied en forme de bouton, creux à l'intérieur. H. max. cons. : 2,7 ; diam. 4,5. Surface ext., int. et fracture : beige (10YR7/4).

Inclusions : inclusions de couleur gris et brun, de petite taille et à faible fréquence.

Observations et comparaisons : bien que fort fragmentaire et abimé, il s'agit d'un pied de type tronconique avec dépression centrale intérieure, ce qui caractérise les amphores de type corinthien $B$ entre le deuxième et le dernier quart du Ve s. av. J.-C. Pour ce type, Koehler 1978a, fig. 2.b-c ; et Ead. 1981, fig. 1.a.

Chronologie : 480-400 av. J.-C.

Bibliographie : de Marinis 2010, n 159, p. 84, fig. 76.159 .

\section{MA117 (pl. XLI)}

Année de la fouille / trouvaille : 1989.

Secteur : Regio IV, Insula 2.

Structure : Casa 1 .

US : 66 .

Inventaire $\mathrm{n}^{\circ}: 1566$.

Type : gréco-occidentale, forme $1 \alpha$.

État de conservation : partie de la lèvre et du col. H. max. cons. : 6,5. Lèvre en bourrelet, penchée vers l'extérieur. H. : 2,9 ; épais. max. : 1,7 ; diam. int./ext. embouchure : 12,2 / 14,4. Col cylindrique avec cannelure en dessous de la lèvre. H. : 3,5 ; diam. min./max. : 11,9.

Surface ext., int. et fracture : beige clair (10YR8/ 3-10YR8/4).

Inclusions : pâte épurée avec inclusions de couleur gris et orange de petit taille et à faible fréquence.

Observations et comparaisons : pour la morphologie, voir précisément, Semeraro 1997, fig. 208. 942a (de Otranto, datée sur la base du contexte à la fin du $\mathrm{VI}^{\mathrm{e}} \mathrm{s}$. av. J.-C. ; et Ceka 1986, tav. III, 5 (en provenance de Belsh, datée sur la base du contexte à la première moitié du VI ${ }^{\mathrm{e}}$ s. av. J.-C.).

Chronologie : 550-490 av. J.-C.

Bibliographie : Sassatelli, Brizzolara 1990, p. 36, tav. XIIa (pour l'image); de Marinis 2010, n 157, p. 84, fig. 76.157.

\section{MA118 (pl. XLI)}

Année de la fouille / trouvaille : 1989.

Secteur : Regio IV, Insula 2.
Structure : Casa 1 .

US : 65 .

Inventaire $\mathrm{n}^{\circ}: 4336$.

Type : gréco-occidentale, forme 2.

État de conservation : pied restauré par 5 fragments. H. max. cons. : 7,5. Pied cylindrique caréné. H. : 2,5; diam. $\min . / \max .: 6,9 / 7,3$.

Surface ext., int. et fracture : rose (10YR8/4).

Inclusions : inclusions de couleur blanc de petite taille, à haute fréquence, et inclusions grises et brunes de petite taille, à fréquence moyenne. Mica de couleur argent, or et cuivre en écailles de petite et moyenne taille à haute fréquence.

Observations et comparaisons : pour la forme voir, Di Sandro 1986, p. 30, tav. 4, sg. 65, comparée avec Py 1980, fig. 1, tipo 1C ; et Tigano 2002, p. 71, en provenance de la Tomba 41 de la nécropole de Milazzo, datée au dernier tiers du VI ${ }^{\mathrm{e}} \mathrm{s}$. av. J.-C.

Chronologie : 540-500 av. J.-C.

Bibliographie : de Marinis 2010, $\mathrm{n}^{\circ}$ 158, p. 84, fig. 76.158.

\section{AMPHORES DE L'ÉGÉE SEPTENTRIONALE}

MA119 (pl. XLI)

Année de la fouille / trouvaille : 1994.

Secteur : Regio IV, Insula 2.

Structure : Casa 1.

US : 1Q12B.

Inventaire $\mathrm{n}^{\circ}: 977$.

État de conservation : fragment d'anse correspondant à la partie inférieure. H. max. cons. : 6,5. Anse à section elliptique avec nervure centrale et empreinte digitale en correspondance du point de jonction inférieur. H. max. cons. : 6,5 ; épais. max. : 3,9 x 2,7.

Surface ext., int. et fracture : orange-brun (10R5/8).

Inclusions : inclusions de couleur blanc de taille moyenne, à moyenne fréquence, et inclusions grises et brunes de petite taille, à faible fréquence. Mica en écailles de petite et moyenne taille. Vacuoles.

Observations et comparaisons : pour la morphologie de l'anse, avec nervure centrale, voir Spagnolo 2003, p. 624 et n. 96, pl. III.2, attribuée à la première production amphorique de Thasos.

Chronologie : 525-500 av. J.-C.

Bibliographie : de Marinis 2010, n $^{\circ}$ 134, p. 78, fig. 73.134 .

\section{MA120 (pl. XLI)}

Année de la fouille / trouvaille : 1989.

Secteur : Regio IV, Insula 2.

Structure : Casa 1.

US : 64.

Inventaire $\mathrm{n}^{\circ}: 4134$. 
État de conservation : anse complète. H. max. : 14,2. Anse à section elliptique, avec nervure centrale et empreinte digitale. H. max. cons. : 14 ; épais. max. : $4 \times 2,7$.

Surface ext., int. et fracture: orange-brun(10R6/8-10R5/8). Inclusions : inclusions de couleur blanc de petite et moyenne taille, à fréquence moyenne, et inclusions de couleur brun de petite taille, à fiable fréquence. Mica fin et diffus.

Observations et comparaisons : pour ce type, attribué à production tardo-archaïque de Thasos, voir Spagnolo 2003, p. 624 et n. 96, pl. III.2.

Chronologie : 525-500 av. J.-C.

Bibliographie : de Marinis 2010, $\mathrm{n}^{\circ}$ 135, p. 78, fig. 73.135.

\section{MA121 (pl. XLI)}

Année de la fouille / trouvaille : 1989.

Secteur : Regio IV, Insula 2.

Structure : Casa 1 .

US : 51 .

Inventaire $\mathrm{n}^{\circ}: 2830$.

Type : Stem-toe, variante 2 Lawall.

État de conservation : pied manquant de la surface de base. H. max. cons. : 9,8. Pied en bobine. H. max. cons. : 8,5 ; diam. min./max. : 5,7 / 7 .

Surface ext., int. et fracture : orange-rose (7.5YR7/ 4-7.5YR7/6).

Inclusions : inclusions de couleur blanc, orange, gris et noirâtre, de petite taille, à faible fréquence.

Observations et comparaisons : bien que lacunaire au niveau de la surface de base, ce pied en forme de bobine renvoie à production nord-égéenne de la deuxième moitié du Ve s. av. J.-C. Pour ce type voir Lawall 1995, fig. 53.

Chronologie : 450-400 av. J.-C.

Bibliographie : de Marinis 2010, $\mathrm{n}^{\circ}$ 140, p. 79, fig. 74.140.

\section{MA122 (pl. XLI)}

Année de la fouille / trouvaille : 1998.

Secteur : Regio IV, Insula 2.

Structure : Casa 1.

US : 400-7.

Inventaire $\mathrm{n}^{\circ}: 827$.

Type : Biconical Lawall.

État de conservation : partie de lèvre et de col. H. max. cons. : 4. Lèvre en coin, penchée vers l'extérieur. H. : 1,5 ; épais. max. : 1,4 ; diam. int./ext. embouchure : 10,4 / 12,2. Col cylindrique. H. max. cons. : 2,3 ; diam. $\min . / \max .: 10,4$.

Surface ext., int. et fracture : rougeâtre (10R5/6-10R5/8). Fracture : rougeâtre (10R5/6-10R5/8).
Inclusions : pâte plutôt épurée avec inclusions de couleur blanc et gris-noir de petite taille, à faible fréquence. Mica fin.

Observations et comparaisons : pour le type, «biconical », Lawall 1995, p. 135, fig. 43-44, daté du dernier quart du $\mathrm{V}^{\mathrm{e}}$ s. av. J.-C. ; pour la forme de la lèvre, Monachov 1999, fig. 11.6-7, les deux attribuées à Thasos et datées de la deuxième moitié du $\mathrm{V}^{\mathrm{e}} \mathrm{s}$. av. J.-C. sur la base du contexte. Probablement appartenant au même individu du fragment de la fiche suivante, non jointif.

Chronologie : 425-400 av. J.-C.

Bibliographie : de Marinis 2010, $\mathrm{n}^{\circ}$ 132, p. 78, fig. 73.132.

\section{MA123 (pl. XLI)}

Année de la fouille / trouvaille : 1998.

Secteur : Regio IV, Insula 2.

Structure : Casa 1.

US : 400-7.

Inventaire $\mathrm{n}^{\circ}: 828$.

Type : Biconical Lawall.

État de conservation : parti de lèvre et de col. H. max. cons. : 4. Lèvre en coin, penchée vers l'extérieur. H. : 1,5 ; épais. max. : 1,4 ; diam. int./ext. embouchure : 10,4 / 12,2. Col cylindrique. H. max. cons. : 2,3 ; diam. min./ max. : 10,4 .

Surface ext., int. et fracture : rougeâtre (10R5/6-10R5/8). Inclusions : pâte assez épurée avec inclusions de couleur blanc et gris-noir de petite taille, à faible fréquence. Mica fin diffus.

Observations et comparaisons : sans doute appartenant au même individu du fragment de la fiche précédente, à laquelle on renvoie.

Chronologie : 425-400 av. J.-C.

Bibliographie : de Marinis 2010, n 132, p. 78, fig. 73.132 .

\section{MA124 (pl. XLI)}

Année de la fouille / trouvaille : 1989.

Secteur : Regio IV, Insula 2.

Structure : Casa 1.

US : 1

Inventaire $\mathrm{n}^{\circ}: 1411$.

État de conservation : partie inférieure d'anse. H. max. cons. : 5,7. Anse à section elliptique avec empreinte digitale en correspondance du point de jonction. H. max. cons. : 5,7 ; épais. max. : $3,9 \times 1,9$.

Surface ext. : orange (5YR6/6-5YR7/6).

Surface int. : orange (5YR6/6-5YR7/6).

Fracture : orange (5YR6/6-5YR7/6).

Inclusions : inclusions de couleur blanc, de moyenne taille, à haute fréquence, et inclusions de couleur noirâtre et orange-rose, de petite taille. Mica fin à faible fréquence. 


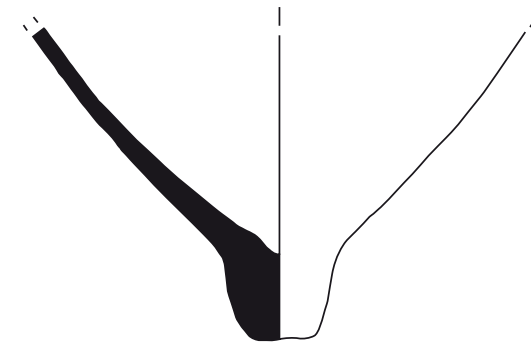

MA114

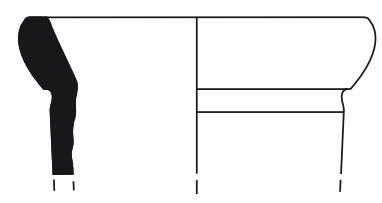

MA117

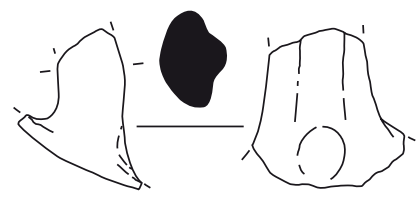

MA119

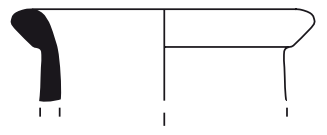

MA122

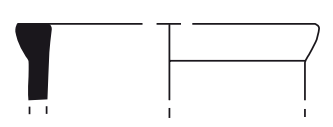

MA125

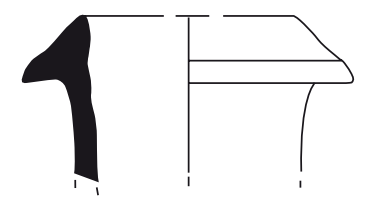

MA115

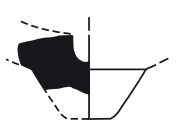

MA116

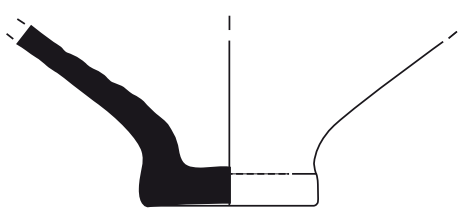

MA118
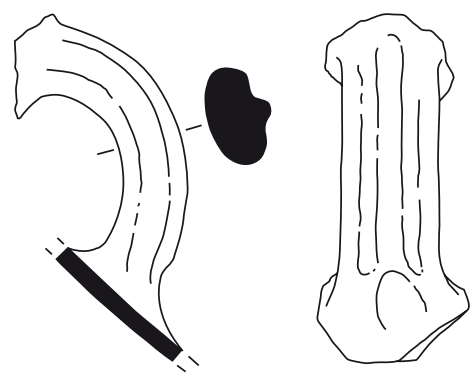

MA120

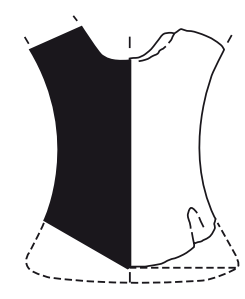

MA121

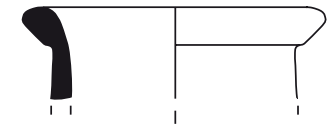

MA123

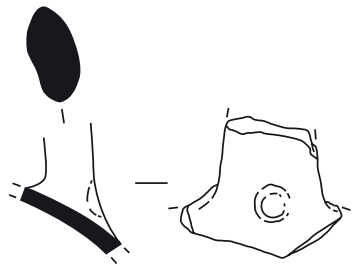

MA124

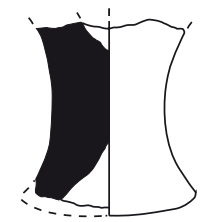

MA127 $5 \mathrm{~cm}$

PI. XLI. Marzabotto. 
Observations et comparaisons : attribué à production nord-égéenne sur la base de la pâte et du détail morphologique de l'empreinte digitale à la base. Comme il le montre la riche documentation recueilli dans Blondé, Muller, Mulliez 1991, Grandjean 1992, et Schmid 2001. Chronologie : non définissable.

Bibliographie : de Marinis 2010, n 136, p. 78, fig. 73.136.

\section{MA125 (pl. XLI)}

Année de la fouille / trouvaille : 1991.

Secteur : Regio IV, Insula 2.

Structure : Casa 1.

US : 210 .

Inventaire $\mathrm{n}^{\circ}: 3438$.

État de conservation : partie de la lèvre et du col. Dim. max. cons. : 3,6 x 5,8. Lèvre en coin. H. : 1,5 ; épais. max. : 1,4; diam. int. / ext. embouchure : 14,8 / 17,4.

Surface ext., int. et fracture : orange (5YR6/6).

Inclusions : inclusions de couleur blanc et noir de petite taille, à faible fréquence. Mica fin, argenté, à faible fréquence.

Observations et comparaisons : pour le type, Schmid 2001, Kat. Nr. 4, Abb 4, et Kat. Nr. 5, Abb. 5, datables entre la fin du $\mathrm{VI}^{\mathrm{e}}$ et le début du $\mathrm{V}^{\mathrm{e}}$ s. av. J.-C. ; pour la forme, Roberts 1986, fig. 43, d"un dépôt l'Agora d'Athènes datable entre la fin du $\mathrm{VI}^{\mathrm{e}}$ et le 480 av. J.-C. Une datation entre la fin $\mathrm{du} \mathrm{VI}^{\mathrm{e}}$ et la première moitié $\mathrm{du} \mathrm{V}^{\mathrm{e}}$ s. av. J.-C. est confortée par la présence, dans le même contexte, d'un fragment de cratère attique à f.r. datable entre 480 et 460 av. J.-C. (Brizzolara, Baldoni, Succi 2005).

Chronologie : 525-480 av. J.-C.

Bibliographie : de Marinis 2010, $\mathrm{n}^{\circ}$ 143, p. 81.

\section{MA126 (pl. XLI)}

Année de la fouille / trouvaille : 1988.

Secteur : Regio IV, Insula 2.

Structure : Casa 1.

US : 22.

Inventaire $\mathrm{n}^{\circ}: 3135$.

Type : Double-banded rim type.

État de conservation : partie de la lèvre et du col. H. max. cons. : 7,7; h. de la lèvre : 2,4; larg. max. cons. : 6,6. Surface : rougeâtre (MSC 5.5YR 6/7).

Inclusions : inclusions de couleur rougeâtre, brun et noir à haute fréquence.

Observations et comparaisons : pour le type, Johnston 1990 ; Lawall 1995, p. 132-135, fig. 42.

Chronologie : fin VI ${ }^{\mathrm{e}} \mathrm{s}$. av. J.-C.

Bibliographie : de Marinis 2010, n 133, p. 78, fig. 73.133.

MA127 (pl. XLI)

Année de la fouille / trouvaille : 1996.
Secteur : Regio IV, Insula 2.

Structure : Casa 1.

US : 386 .

Inventaire $\mathrm{n}^{\circ}: 2273$.

Type : nord-égéenne. Stem-toe, variante 2 Lawall.

État de conservation : pied fragmentaire. H. max. cons. :

8 ; diam. ric. base : 7,3.

Surface : orange (MSC 5YR 6/8).

Inclusions : nombreuses inclusions blanches et mica. Vacuoles.

Observations et comparaisons : pied en forme de bobine renvoyant à la production nord-égéenne de la deuxième moitié du Ve s. av. J.-C. Pour ce type, Lawall 1995, fig. 53.

Chronologie : deuxième moitié du V $\mathrm{V}^{\mathrm{e}} \mathrm{S}$. av. J.-C.

Bibliographie : de Marinis 2010, n 141, p. 79, fig. 74.141.

\section{AMPHORES DE L'ÉGÉE ORIENTALE}

MA128 (pl. XLII)

Année de la fouille / trouvaille : 1997.

Secteur : Regio IV, Insula 2.

Structure : Casa 1.

US : 360 .

Inventaire $\mathrm{n}^{\circ}: 2429$.

Type : Samos-Milet. S/1 Lawall.

État de conservation : pied. H. max. cons. : 4. Pied tronconique, au profil en carène. H. : 2 ; diam. min. / $\max$. : 4,4 / 5,2.

Surface ext.,int.et fracture: orangeclair(5YR7/8-5YR6/8). Inclusions : inclusions de couleur blanc, jaunâtre et orange, de petite taille et faible fréquence.

Observations et comparaisons : pour le type, Lawall 1995 , p. 177-179, fig. 178 ; pour la morphologie, qui se rapproche des types «Zeest's Samian », Zeest 1960, pl. I.3, n. 2, 70 ; et Dupont 1998, fig. 23.12, h. Une datation entre la fin du $\mathrm{VI}^{\mathrm{e}}$ et le début du Ve est confirmé par le donné stratigraphiques. De l'US 360 viennent, en effet, une lekythos et un skyphos à f.n., une coupe à v.n. attiques datés entre la fin du $\mathrm{VI}^{\mathrm{e}}$ et les premières années du Ve s. av. J.-C. (cf. Brizzolara, Baldoni, Succi 2005).

Chronologie : 525-480 av. J.-C.

Bibliographie : de Marinis 2010, n 139, p. 79, fig. 74.139.

\section{MA129 (pl. XLII)}

Année de la fouille / trouvaille : 1991.

Secteur : Regio IV, Insula 2.

Structure : Casa 1.

US : 204 .

Inventaire $\mathrm{n}^{\circ}: 4100$.

Type : Samos/Milet. S/2 Lawall.

État de conservation : partie de la lèvre et du col. H. max. cons. : 9. Lèvre en petit bourrelet. H. : 1,7 ; épais. max. : 
1,1 ; diam. int. / ext. embouchure : 12,4 / 13,6. Col évasé avec ressaut en dessous de la lèvre.

Surface ext., int. et fracture : orange (5YR7/8-7.5YR7/6). Inclusions : pâte plutôt épurée avec inclusions de couleur blanc, noir, gris et brun de petite taille à faible fréquence. Mica doré en écailles de petite et moyenne taille, à fréquence moyenne.

Observations et comparaisons : pour le type, Grace 1971, fig. 3. 1, correspondent à la variante du type $\mathrm{S} / 2$ Lawall datables entre le deuxième quart et le milieu du $\mathrm{V}^{\mathrm{e}}$ s. av. J.-C. (Lawall 1995, p. 182-183).

Chronologie : 480-450 av. J.-C.

Bibliographie: de Marinis 2010, n $^{\circ}$ 148, p. 82, fig.75.148.

\section{MA130 (pl. XLII)}

Année de la fouille / trouvaille : 1994.

Secteur : Regio IV, Insula 2.

Structure : Casa 1.

US : 1Q8B.

Inventaire $\mathrm{n}^{\circ}: 556$.

Type : Samos-Milet.

État de conservation : partie de la lèvre. $\mathrm{H}$. max. cons. : 4,2. Lèvre en amande. H. : 2,4; épais. max. : 1,5; diam. int. / ext. embouchure : 14 / 15,5.

Surface ext., int. et fracture : orange-beige (7.5YR7/4).

Inclusions : pâte assez épurée. Inclusions de couleur blanc, brune et noir de petite taille, à faible fréquence. Mica fin doré.

Observations et comparaisons : attribué au groupe Samos-Milet pour la morphologie et pour la pâte. Pour la forme voir Morselli, Tortorici 1985, fig. 8.1, définie samienne, et Monachov 2003, tav. 25.6.

Chronologie : 525-480 av. J.-C.

Bibliographie : de Marinis 2010, nº 145, p. 81, fig.74.145.

\section{MA131 (pl. XLII)}

Année de la fouille / trouvaille : 1993.

Secteur : Regio IV, Insula 2.

Structure : Casa 1 .

US : 1

Inventaire $n^{\circ}: 462$.

Type : Samos-Milet.

État de conservation : partie de la lèvre. H. max. cons. : 3. Lèvre en amande avec subtile cannelure à la base. H. : 2,5 ; épais. max. : 1,4 ; diam. int./ext. embouchure : 5,5. Surface ext., int. et fracture : orange (5YR6/6-5YR7/6). Inclusions : inclusions de couleur noir, brun-orange et blanc de petite taille, à fréquence moyenne. Mica fin, doré, à fréquence moyenne.

Observations et comparaisons : la morphologie de la lèvre, à la section en amande, renvoie à la production du groupe Samos-Milet entre la deuxième moitié $\mathrm{du} \mathrm{VI}^{\mathrm{e}}$ et la première moitié du $\mathrm{V}^{\mathrm{e}} \mathrm{s}$. av. J.-C. (Dupont
1998, fig. 23.9, a-c). Pour la forme, Morselli, Tortorici 1985, fig. 8. 1, 4, en provenance de Gravisca, définies samiennes, et Monachov 2003, pl. 24. 6. Sans doute référable au même individu du fragment de la fiche suivante (fragment non jointif).

Chronologie : 525-450 av. J.-C.

Bibliographie : de Marinis 2010, $\mathrm{n}^{\circ}$ 147,p. 82, fig.75.147.

\section{MA132 (pl. XLII)}

Année de la fouille / trouvaille : 1988.

Secteur : Regio IV, Insula 2.

Structure : Casa 1 .

US : 22 .

Inventaire $n^{\circ}: 3453$.

Type : Samos-Milet.

État de conservation : fragment de la lèvre. H. max. cons. : 3,5. Lèvre en amande avec légère cannelure en dessous. H. : 2,6 ; épais. max. : 1,5 ; diam. int./ext. embouchure : 4,1 .

Surfaceext.,int.etfracture:noisette(7.5YR6/4-7.5YR5/4). Inclusions : inclusions de couleur blanc de taille petite et moyenne, à moyenne fréquence, et inclusions de couleur noir et brun de petite taille, à moyenne fréquence. Mica fin doré à moyenne fréquence. Vacuoles.

Observations et comparaisons : Référable, sans doute, au même individu du fragment de la fiche précédente (non jointif).

Chronologie : 525-450 av. J.-C.

Bibliographie : de Marinis 2010, $\mathrm{n}^{\circ}$ 147, p. 82, fig.75.147.

\section{MA133 (pl. XLII)}

Année de la fouille / trouvaille : 1994.

Secteur : Regio IV, Insula 2.

Structure : Casa 1.

US : 1Q8B.

Inventaire $\mathrm{n}^{\circ}: 519$.

Type : Samos-Milet.

État de conservation : partie de la lèvre. H. max. cons. : 3,5 . Lèvre en amande, avec léger grossissement, supérieurement aplati. H. : 2,9 ; épais. max. : 1,6 ; diam. int./ ext. embouchure : 3,3 .

orange (7.5YR6/6).

Fracture : orange (7.5YR6/6).

Inclusions : pâte épurée avec inclusions de couleur blanc taille moyenne-petite, à faible fréquence, et inclusions de couleur gris et brun, de petite taille, à faible fréquence. Mica fin doré. Vacuoles.

Observations et comparaisons : pour le type, Dupont 1998, fig. 23.9, a-c, datables entre la deuxième moitié $\mathrm{du} \mathrm{VI}^{\mathrm{e}}$ et le milieu du Ve ; pour la forme, avec aplatissement de la partie supérieure, Monachov 1999a, fig. 12, renvoyée au cercle se Samos et datée du deuxième quart du Ve s. av. J.-C. 
Chronologie : 525-450 av. J.-C.

Bibliographie : de Marinis 2010, $\mathrm{n}^{\circ}$ 146, p. 81-82, fig.75.146.

\section{MA134 (pl. XLII)}

Année de la fouille / trouvaille : 1994.

Secteur : Regio IV, Insula 2.

Structure : Casa 1.

US : 328 .

Inventaire $\mathrm{n}^{\circ}: 1180$.

Type : Samos-Milet. S/2 Lawall.

État de conservation : partie de la lèvre et du col. H. max. cons. : 3,5. Lèvre en amande, avec léger grossissement, supérieurement aplati. H. : 2 ; épais. max. : 1,8; diam. int./ext. embouchure : $11 / 12,3$.

Surface ext., int. et fracture : orange (5YR6/8).

Inclusions : pâte plutôt épurée avec inclusions de couleur gris, brun et blanc de petite taille, à faible fréquence. Mica fin à faible fréquence.

Observations et comparaisons : pour le type, Knigge 1976, pl. 49.3 ; et Grace 1971, fig. 3. 1, correspondent aux variantes du type $\mathrm{S} / 2$ Lawall datables entre la fin du $\mathrm{VI}^{\mathrm{e}}$ et le milieu du Ve s. av. J.-C. (Lawall 1995, p. 181-183); pour l'aplatissement supérieure de la lèvre, Monachov 1999a, fig. 12, attribuée au « cercle de Samos » et datée au deuxième quart du $\mathrm{V}^{\mathrm{e}} \mathrm{s}$. av. J.-C. Du même contexte, l'US 328, vient un tesson de stemless cup datable entre 450 et 430 av. J.-C. (Brizzolara, Baldoni, Succi 2005). Chronologie : 525-450 av. J.-C.

Bibliographie : de Marinis 2010, n 149, p. 82, fig. 75.149.

\section{MA135 (pl. XLII)}

Année de la fouille / trouvaille :

Secteur : Regio IV, Insula 2.

Structure : Casa 1.

US : 352 .

Inventaire $\mathrm{n}^{\circ}: 1763$.

Type : Samos-Milet. S/2 Lawall.

État de conservation : partie de la lèvre et du col. H. max. cons. : 3. Lèvre en amande, avec léger grossissement, supérieurement aplati. H. : 2 ; épais. max. : 1,9 ; diam. int./ext. embouchure : 11/14,8.

Surface ext., int. et fracture : orange (5YR6/6-5YR6/8). Inclusions : pâte assez épurée avec inclusions de couleur gris, brune et blanc de petite taille, à faible fréquence. Mica fin à faible fréquence.

Observations et comparaisons : pour le type Knigge 1976, pl. 49.3 ; et Lawall 1995, fig. 70-71 ; pour l'aplatissement de la lèvre, caractéristique des types samiens de Zeest, Monachov 2003, pl. 25.7.

Chronologie : 525-450 av. J.-C.

Bibliographie : de Marinis 2010, $\mathrm{n}^{\circ} 150$, p. 82, fig. 75.150 .

\section{MA136 (pl. XLII)}

Année de la fouille / trouvaille : 1991.

Secteur: Regio IV, Insula 2.

Structure : Casa 1.

US : 210 .

Inventaire $n^{\circ}: 3437$.

Type : Samos-Milet. S/2 Lawall.

État de conservation : partie de la lèvre. H. max. cons. :

3,6. Lèvre en amande, avec léger grossissement, supérieurement aplati. H. : 2,5 ; épais. max. : 1,5 ; diam. int./ext. embouchure : 4,5 .

Surface ext. : beige (7.5YR7/6).

Surface int. et fracture : orange (5YR5/8-5YR6/8).

Inclusions : inclusions de couleur blanc, gris et brun de petite taille, à faible fréquence. Vacuoles.

Observations et comparaisons : pour la lèvre, que l'on peut assigner aux deux premières variantes du type $S / 2$ Lawall, cf. Knigge 1976, pl. 49.3 ; et Grace 1971, fig. 3. 1. Du même contexte, l'US 210, vient un fragment de cratère attique à figures rouges datable entre 480 et 460 av. J.-C. (Brizzolara, Baldoni, Succi 2005).

Chronologie : 525-450 av. J.-C.

Bibliographie : de Marinis 2010, $\mathrm{n}^{\circ}$ 144, p. 81, fig. 74.144.

\section{MA137 (pl. XLII)}

Année de la fouille / trouvaille : 1996.

Secteur : Regio IV, Insula 2.

Structure : Casa 1.

US : 1Q21B.

Inventaire $\mathrm{n}^{\circ}: 2425$.

Type : Samos-Milet. S/2 Lawall.

État de conservation : partie de la lèvre. H. max. cons. : 3,7. Lèvre en amande, grossi. H. : 2,3 ; épais. max. : 1,6 ; diam. int./ext. embouchure : 3,4 .

Surface ext., int. et fracture : beige (10YR7/4-10YR6/4). Inclusions : inclusions de couleur blanc et brun de petite taille, à faible fréquence. Mica fin doré.

Observations et comparaisons : pour le type, Knigge 1976, pl. 49.3 ; et Lawall 1995, fig. 70-71 ; pour la forme, Morselli, Tortorici 1985, fig. 8. 1. Datable entre le dernier quart du $\mathrm{VI}^{\mathrm{e}}$ et le milieu du $\mathrm{V}^{\mathrm{e}} \mathrm{s}$. av. J.-C.

Chronologie : 525-450 av. J.-C.

Bibliographie : de Marinis 2010, $\mathrm{n}^{\circ}$ 142, p. 81, fig. 74.142 .

\section{MA138 (pl. XLII)}

Année de la fouille / trouvaille : 1996.

Secteur : Regio IV, Insula 2.

Structure : Casa 1.

US : 1Q21B.

Inventaire $\mathrm{n}^{\circ}: 2426$.

Type : Samos-Milet. S/1 Lawall. 
État de conservation : pied. H. max.cons. : 4,7.Pied de forme cylindrique, plein, grossi, à la surface de base concave.

H. : 3,2 ; diam. min./max. : 4,4/5,2.

Surface ext., int. et fracture : brun-rougeâtre (5YR6/ 6-5YR5/6).

Inclusions : inclusions blanches et grises de petite taille, à faible fréquence. Mica fin doré à haute fréquence. Vacuoles.

Observations et comparaisons : le type de pied renvoie à la production du groupe Samos-Milet du milieu ou de la deuxième moitié du $\mathrm{V}^{\mathrm{e}} \mathrm{s}$. av. J.-C. (Dupont 1998, fig. 23.9, $\mathrm{g})$. Pour ce type on renvoie à un fragment en provenance de l'Agora d'Athènes datable sur la base du contexte (dépôt F 19 :4) du milieu du V V s. av. J.-C. (Lawall 1995, fig. 75), et à un exemplaire également de l'Agora (P27531 du dépôt $S \mathrm{~S} 16: 1)$ datables du dernier quart du $\mathrm{V}^{\mathrm{e}} \mathrm{s}$. av. J.-C. (Grace 1971, p. 76, fig. 3.3 ; Lawall 1995, fig. 73). La morphologie du pied en permet un encadrement dans la variante plus récente du type $\mathrm{S} / 1$ de $\mathrm{M}$. Lawall.

Chronologie : $450-400$ av. J.-C.

Bibliographie : de Marinis 2010, n 155, p. 83, fig. 75.155 .

MA139 (pl. XLII)

Année de la fouille / trouvaille : 1995.

Secteur : Regio IV, Insula 2.

Structure : Casa 1 .

US : 1 Q20.

Inventaire $\mathrm{n}^{\circ}: 291$.

Type : Solokha I.

État de conservation : partie du pied. H. max. cons. : 6,4. Pied tronconique creux avec base en anneau. H. : 3 ; diam. $\min . / \max .: 6,5 / 7,8$.

Surfaceext.,int.etfracture:orange-rose(5YR7/4-5YR7/6).

Inclusions : pâte épurée. Inclusions blanches de petite taille, à faible fréquence, visible surtout en section. Inclusions noires de petite taille, à fréquence moyenne, uniformément diffusées. Mica argenté à faible fréquence. Engobe : engobe blanchâtre plutôt épais.

Observations et comparaisons : pour le type, Lawall 1995, p. 220-221, fig. 88-90, 93 ; pour la forme, Slaska 1978, pl. 95.7, de Gravisca ; Pancrazzi 1982, p. 336, fig. 2.16, de Pise ; Maggiani, Paribeni 1990, p. 145, n. 25, fig. 78.25, de Casa Baldi di Pozzi ; Massa 1993, p. 349, n. 4, de Pise ; Boss 1993, fig. 522. L26, L27, de Caere ; Scotti 1999, tav. 89. 1, de Tarquinia.

Chronologie : 450-400 av. J.-C.

\section{MA140 (pl. XLII)}

Année de la fouille / trouvaille : 1996.

Secteur : Regio IV, Insula 2.

Structure : Casa 1 .

US : 386 .

Inventaire $\mathrm{n}^{\circ}: 1716$.
Type : Solokha I.

État de conservation : pied. H. max. cons. : 5. Pied tronconique creux, avec surface d'appui en anneau. H. : 2,5 ; diam. min./max. : 5,5/6,4.

Surface ext., int. et fracture : orange-brun (5YR6/ 6-5YR5/6).

Inclusions : inclusions de couleur blanc de taille petite et moyenne, à faible fréquence, et inclusions de couleur noir de petite taille, à fréquence moyenne. Mica doré, fin, à haute fréquence.

Engobe : traces d'engobe de couleur crème sur la surface extérieure.

Observations et comparaisons : pour le type, datable dans le courant de la deuxième moitié du $\mathrm{V}^{\mathrm{e}} \mathrm{s}$. av. J.-C., Lawall 1995, p. 220-221, fig. 88-90, 93.

Chronologie : 450-400 av. J.-C.

Bibliographie : de Marinis 2010, ${ }^{\circ} 153$, p. 82, fig. 75.153 .

MA141 (pl. XLII)

Année de la fouille / trouvaille : 1995.

Secteur : Regio IV, Insula 2.

Structure : Casa 1.

US : 303 .

Inventaire $\mathrm{n}^{\circ}: 1351$.

Type : Solokha I.

État de conservation : partie du pied correspondant à la moitié de la circonférence. H. max. cons. : 3. Pied tronconique creux avec surface de base en anneau et au profil caréné. H. : 3 ; diam. min./max. : 6,1/7,1.

Surfaceext.,int.et fracture: orange clair(5YR7/6-5YR6/6). Inclusions : inclusions de couleur blanc de taille moyenne et petite, à faible fréquence, et inclusions de couleur gris et orange de petite taille, à fréquence moyenne. Mica doré en écailles de petite et moyenne taille, à fréquence moyenne.

Observations et comparaisons : pour le type, Lawall 1995, p. 220-221, fig. 88-90, 93, daté dans le courant de la deuxième moitié du Ve $\mathrm{s}$. av. J.-C. Du même contexte, l'US 303 , vient un fragment de cratère attique à vernis noir de datation incertaine (Brizzolara, Baldoni, Succi 2005).

Chronologie : 450-400 av. J.-C.

Bibliographie : de Marinis 2010, n 154,p. 82, fig. 75.154 .

\section{MA142}

Année de la fouille / trouvaille : 1995.

Secteur : Regio IV, Insula 2.

Structure : Casa 1.

US : 1 .

Inventaire $\mathrm{n}^{\circ}: 199$.

Type : Samos-Milet. S/1 ou S/2 Lawall.

État de conservation : fragment de la lèvre. H. max. cons. : 3,7 ; larg. 3,2 ; épais. : 2.

Surface : orange (MSC 5YR 6/8). 


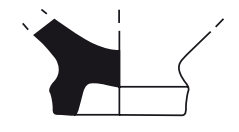

MA128

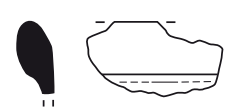

MA131

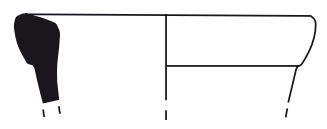

MA134

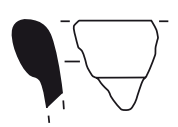

MA137

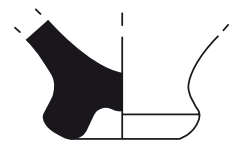

MA140

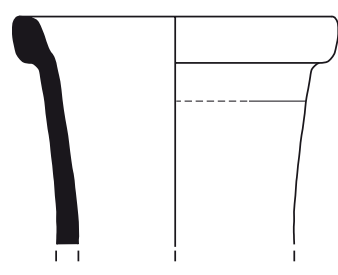

MA129

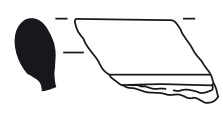

MA132

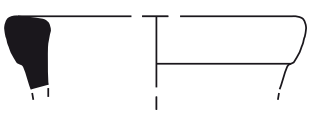

MA135

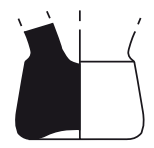

MA138

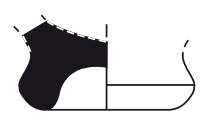

MA141

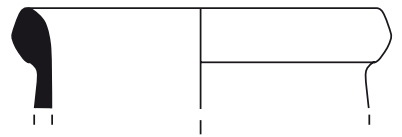

MA130

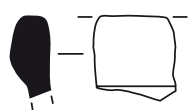

MA133

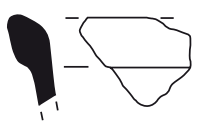

MA136

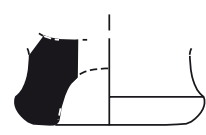

MA139

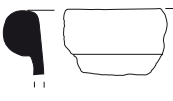

MA143

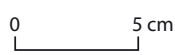


Observations et comparaisons : pour le type, cf. Lawall 1995. Chronologie : non définissable.

Bibliographie : de Marinis 2010, n 151, p. 82.

\section{MA143 (pl. XLII)}

Année de la fouille / trouvaille : 1992.

Secteur : Regio IV, Insula 2.

Structure : Casa 1 .

US : 237 .

Inventaire $n^{\circ}: 2381$.

Type : Samos-Milet.

État de conservation : fragment de la lèvre. H. max. cons. : 2,7 ; larg. 4,$2 ;$ h. de la lèvre : 1,8 ; épais. : 1,4.

Surface : beige claire (MSC 7.5YR 7/4).

Engobe : traces d'engobe jaune-blanchâtre.

Chronologie : non définissable.

Bibliographie : de Marinis 2010, ${ }^{\circ}$ 152, p. 82, fig. 75.152.

\section{Analyses des données (graphiques 13-16)}

Les amphores de Marzabotto proviennent des secteurs d'habitat et des zones artisanales, des sondages effectués le long des rues (plateiai) et de l'aire sacrée récemment fouillée dans l'aire urbaine (cf. supra II, 5 $\S 1$ ) Aucune amphore n'est signalée sur l'acropole, où les recherches principales ont été effectuées au XIX ${ }^{\mathrm{e}}$ s., lorsque ce type d'objet, qui ne présentait aucun intérêt pour les fouilleurs, n'était pas conservé (Vitali, Brizzolara, Lippolis 2001) ${ }^{666}$. De même, les mobiliers des 295 tombes des nécropoles (Marchesi 2005), tout comme c'est le cas de la voisine Bologne, n'ont restitué aucune amphore commerciale. La documentation de Marzabotto, ainsi que celle du Forcello proviennent donc exclusivement de quartiers d'habitation et fournit l'occasion d'analyser le phénomène commercial des importations amphoriques au moment de la plus grande prospérité de l'Étrurie padane. Il faut cependant souligner que, à la différence de ce qui se passe pour le Forcello, l'histoire des fouilles de Marzabotto, site prospecté à plusieurs reprises et par différentes équipes pendant plus d'un siècle et demi de recherches archéologiques, est telle que dans la plupart des cas on n'a pas pu se servir des données stratigraphiques pour la datation des amphores ${ }^{667}$. Au-delà du débat sur la chronologie des différentes phases d'occupation du site (Marzabotto I

666. Les interventions plus récentes (Lippolis 2005) effectuées dans l'aire déjà intéressée par les fouilles du $\mathrm{XIX}^{\mathrm{e}} \mathrm{s}$. n'ont de toute façon restitué aucun fragment amphorique.

667. Comme il va ressortir d'une manière évidente de la consultation du catalogue, le matériel que j'ai pu étudier provient de nombreuses interventions réalisées dans la zone surtout dans le courant du siècle dernier pour lesquelles, exception faite pour le cas de la Regio V, et II), le terminus post quem pour l'arrivée d'importations grecques peut être fixé au deuxième quart du $\mathrm{VI}^{\mathrm{e}} \mathrm{s}$. av. J.-C., époque à laquelle remontent une kotyle et un aryballe globulaire du Corinthien Moyen et un aryballe en couronne du Corinthien Moyen ou Tardif (Bermond Montanari 1987 ; Lippolis 2000, p. 109, $\mathrm{n}^{\circ}$ 5.1, 5.3, 5.4, fig. 4-6, 8-10).

C'est sans doute à la période des premières importations grecques, dans le deuxième quart du $\mathrm{VI}^{\mathrm{e}} \mathrm{s}$. (cf. supra, p. 212) qu'il convient de faire remonter la plus ancienne amphore commerciale grecque actuellement documentée à Marzabotto, elle aussi de production corinthienne et attribuable au type A (cat. MA1). Ce cas semble actuellement isolé ${ }^{668}$ mais il s'inscrit sans difficulté dans le cadre des premières importations corinthiennes, arrivées sur le site probablement à travers les cols des Apennins (Lippolis 2000, p. 102). C'est dans le courant de la deuxième moitié, plus probablement vers la fin du $\mathrm{VI}^{\mathrm{e}}$ s. av. J.-C., que les attestations d'amphores deviennent plus consistantes, en conformité avec les données fournies par la céramique décorée grecque, à ce moment-là de production attique (Locatelli 1997 ; Lippolis 2000, p. 103 ; Benz, Reusser 2004b ; Brizzolara, Baldoni, Succi 2005 ; Baldoni 2008 ; Id. 2009, p. 243-246). Les données relatives à la céramique attique suggèrent de dater autour de 540 av. J.-C. une première phase qui marque un changement net dans le comportement social et économique ainsi que la création d'un système d'échanges commerciaux stables

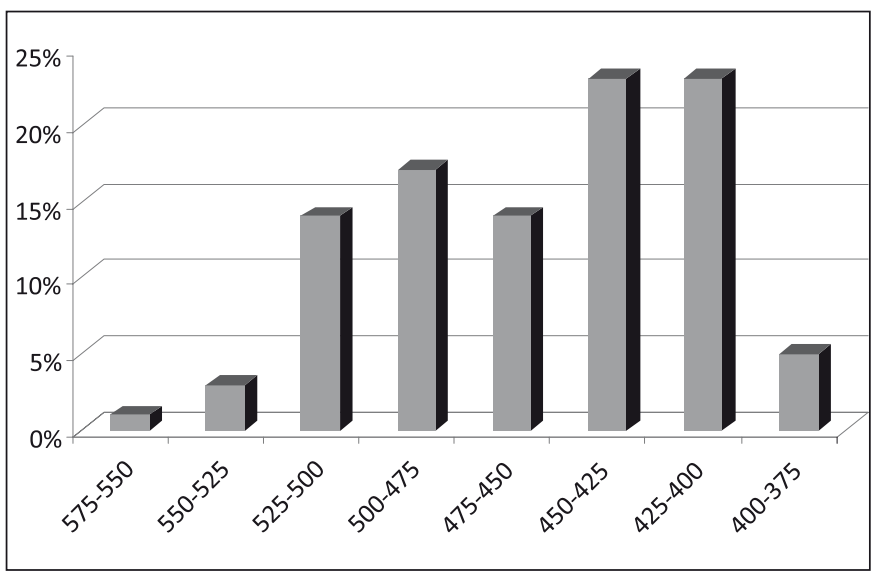

Graphique 13. Marzabotto : tendance des importations d'amphores grecques dans la période étudiée (deuxième-troisième quart du $\mathrm{Vl}^{\mathrm{e}}$-début du $\mathrm{IV}^{\mathrm{e}} \mathrm{S}$. av. J.-C.).

Insula III, n'ont été publiées que des relations préliminaires. Pour ces considérations, (cf. supra II, 5 § 1).

668. Je rappelle que nos connaissances sur la phase la plus archaïque de Marzabotto, interceptée uniquement dans quelques secteurs pour la plupart inédits, sont encore très lacunaires (cf. supra II, 5 § 1). 

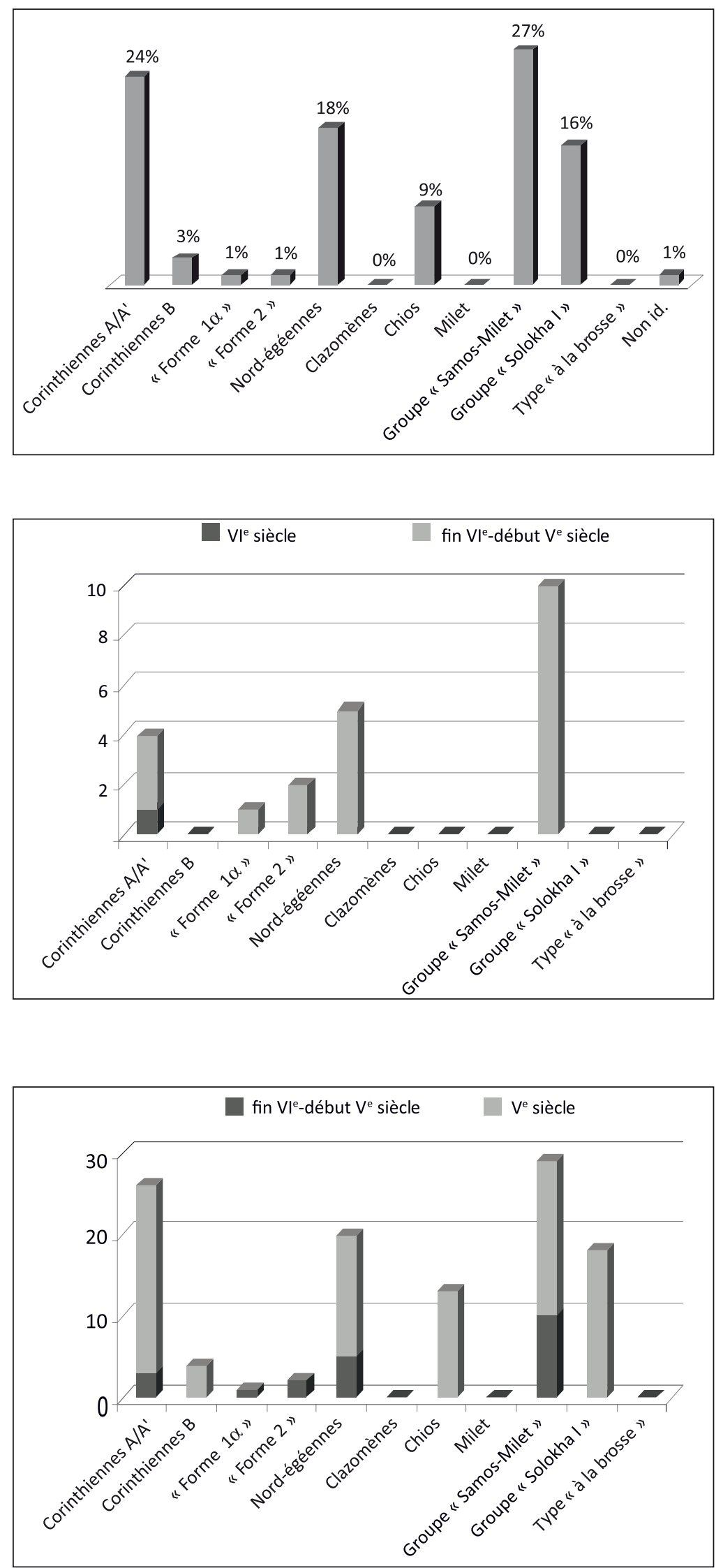

Graphique 14. Marzabotto : données quantitatives concernant les importations des différents groupes amphoriques dans le courant de toute la période examinée (deuxième-troisième quart du $\mathrm{Vl}^{\mathrm{e}}$-début du $\mathrm{IV}^{\mathrm{e}} \mathrm{s}$. av. J.-C.).

Graphique 15. Marzabotto : données quantitatives des importations des différents groupes amphoriques entre le deuxième-troisième quart du $\mathrm{Vl}^{\mathrm{e}}$ et le début du Ve s. av. J.-C.
Graphique 16. Marzabotto : données quantitatives des importations des différents groupes amphoriques entre la fin du Vle et la fin du $\mathrm{V}^{\mathrm{e}} \mathrm{s}$. av. J.-C. 
(Lippolis 1998 ; Id. 2000, p. 103 ; Id. 2005) et de situer dans le dernier quart du siècle un accroissement des importations ${ }^{669}$. Entre la seconde moitié du $\mathrm{VI}^{\mathrm{e}}$ et les débuts du $V^{e}$ s. av. J.-C., les importations d'amphores concernent les productions gréco-orientales et nordégéennes, alors qu'on relève un pourcentage minime pour les importations gréco-occidentales archaïques (une amphore de 'forme $1 \alpha$ ' et deux de 'forme 2') attestées à Marzabotto ${ }^{670}$. Pendant le Ve $\mathrm{s}$. av. J.-C. les productions gréco-orientales (groupe Samos-Milet, Chios et groupe Solokha I) continuent d'être les mieux attestées. Viennent ensuite les amphores corinthiennes du type A/A' et les nord-égéennes. Un nombre tout à fait réduit de fragments peut se rapporter à des amphores corinthiennes de type $\mathrm{B}$, attribuables à la production corinthienne/corcyréenne et peut-être à d'autres ateliers occidentaux (cat. MA33-MA36). Dans le détail, on peut observer que les amphores chiotes sont concentrées dans le courant de la première moitié ou au plus tard avant le troisième quart du $\mathrm{V}^{\mathrm{e}} \mathrm{s}$. av. J.-C., leur documentation étant limitée presque exclusivement aux types 'swollen-necked' ${ }^{671}$. Une considération analogue peut être faite pour les amphores du groupe Samos-Milet, pour lequel il est cependant plus délicat d'opérer une distinction chronologique à l'intérieur du siècle basée sur les données typologiques. Dans le courant de la seconde moitié du $\mathrm{V}^{\mathrm{e}} \mathrm{s}$., toujours dans le cadre des productions gréco-orientales, les amphores du groupe Solokha I sont au contraire bien documentées dans les formes initiales de la série. Exception faite pour l'exemplaire du type A' provenant du «niveau du $\mathrm{VI}^{\mathrm{e}} »$ (cat. MA11), il n'est pas possible de préciser ultérieurement la chronologie des nombreuses amphores corinthiennes $\mathrm{A} / \mathrm{A}^{\prime}$ dans la phase comprise entre la fin du $\mathrm{VI}^{\mathrm{e}}$ et le $\mathrm{V}^{\mathrm{e}} \mathrm{s}$. av. J.-C, période pendant laquelle leur forme ne semble pas subir des variations chronologiquement significatives. Pour ce qui concerne ces formes amphoriques, je me limiterai donc à en souligner le pourcentage particulièrement important. Encore plus intéressant s'avère le rapport quantitatif entre les amphores corinthiennes $\mathrm{A} / \mathrm{A}^{\prime}$ et les quelques amphores corinthiennes de type B. À Marzabotto, en effet, leur proportion réciproque est inversée par rapport à celle qu'on observe dans les autres sites du secteur géographique analysé.

Il est difficile de préciser le moment où le commerce d'amphores grecques à Marzabotto a été interrompu. Les données qu'on a pu tirer de la céramique attique suggèrent une certaine continuité dans le flux des importations de céramique décorée grecque jusqu'à la première moitié du IV $\mathrm{e}$ s. av. J.-C., malgré une diminution prévisible après l'invasion celtique des débuts du siècle (Locatelli 1997 ; Massa-Pairault 1997 ; Benz, Reusser 2004b ; Brizzolara, Baldoni, Succi 2005 ; Baldoni 2009, p. 243-246). Dans le cas des amphores, leur datation s'appuyant exclusivement sur une base typologique ne permet pas dans la plupart des cas de distinguer les formes de fin $\mathrm{V}^{\mathrm{e}}$ de celles du $\mathrm{IV}^{\mathrm{e}} \mathrm{s}$. initial ou de la première moitié du $\mathrm{IV}^{\mathrm{e}} \mathrm{s}$. av. J.-C.

L'impression générale qu'on en reçoit est de toute façon celle d'un afflux assez constant de marchandises dans le courant de tout le $V^{\mathrm{e}} \mathrm{s}$., d'un probable développement dans le courant de la seconde moitié du siècle et d'une diminution après l'invasion celtique.

669. Comme on l'a déjà remarqué (Baldoni 2009, p. 249, n. 40), les connaissances actuelles sur les importations attiques à Marzabotto dans le courant du $\mathrm{VI}^{\mathrm{e}} \mathrm{s}$. av. J.-C. doivent être considérées comme partielles, vu l'absence d'éditions exhaustives du matériel des contextes plus archaïques connus jusqu'ici.

670. Pour les amphores de forme $1 \alpha$, cf. cat. MA117; pour celles de forme 2, cf. cat. MA37 (de possible production locrienne) et MA118. 671. Outre les amphores chiotes insérées dans le catalogue (cat. MA59-MA65), d'autres individus attribuables aux formes «swollennecked» viennent de l'aire du temple de la Regio I, Insula I qui est encore en cours de fouille et dont les matériels n'a pas été inséré ici par les raisons déjà exposées (cf. supra II,. 5 § 1), mais ils ont été pris en considération dans Sacchetti 2005. 



\section{Conclusions}

L e développement du commerce dans la vallée du Pô et dans le reste de l'Italie du nord entre le $\mathrm{VI}^{\mathrm{e}}$ et le $\mathrm{V}^{\mathrm{e}} \mathrm{s}$. av. J.-C. est un thème central du débat scientifique de ces dernières années à propos du rôle des Étrusques dans le système des échanges. L'attention des chercheurs s'est en effet concentrée non seulement sur l'organisation socio-politique, mais également sur les potentialités économiques et sur les rapports internes de la région, dans l'effort de restituer le cadre général des dynamiques et des itinéraires commerciaux. Ce cadre se présente dans son complexe très articulé, l'Étrurie padane se proposant comme le carrefour des intérêts commerciaux de différents groupes ethniques dès une époque très ancienne.

Le territoire padan fut traversé dès l'époque villanovienne par un important itinéraire commercial ${ }^{672}$. À la suite de l'insertion progressive des partenaires commerciaux grecs, dans le courant de la première moitié $\mathrm{du} \mathrm{VI}^{\mathrm{e}}$ s. av. J.-C., le rôle de centre des intérêts économiques et culturels exercé depuis toujours par la région atteignit son acmé après le milieu du siècle et pendant le siècle suivant ${ }^{673}$. Dans cette période, les échanges se sont totalement réorganisés sous l'effet du changement des conditions politico-économiques internationales qui toucha d'un côté le monde grec, et de l'autre le monde celtique, en impliquant l'intermédiaire étrusque. La manifestation la plus éclatante du phénomène consista dans la nouvelle physionomie fédérale prise par la région étrusco-

672. Pour la situation historico-économique et les contacts culturels attestés dans la région padane entre le $\mathrm{IX}^{\mathrm{e}}$ et le $\mathrm{VI}^{\mathrm{e}} \mathrm{s}$. av. J.-C. nous renvoyons à Sassatelli 1986, p. 11-36 et Id. 1990, p. 53-58 (pour Bologne); Id. 1996 et Id. 1999, en particulier p. 71-87 (pour Verucchio). Pour une synthèse récente cf. Morigi Govi, Marchesi 2000 et Sassatelli, Donati 2005.

673. Pour une synthèse de la situation des importations et des contacts commerciaux et culturels de la région padane au moment de l'intensification des échanges avec le monde grec, nous renvoyons à Sassatelli 1993, notamment p. 180-182, avec références bibliographiques précédentes. Plus récemment G. Sassatelli lui-même (Id. 1998, notamment p. 36-38; Id. 1999, notamment p. 71-87; Id. 2008) et d'autres auteurs sont revenus sur le sujet avec une remise en discussion du problème ou la mise à jour du tableau des découvertes : Bruni 1994 ; Bruni 1998 ; Bonomi 2000a ; Lippolis 2000 ; Bermond Montanari 2003 ; Bonomi 2003 ; Colonna 2003 ; Harari 2003. padane dans la diffusion du modèle urbain à travers la nouvelle fondation de Bologne/Felsina, et dans la fondation ou l'implication d'autres cités et habitats, tels Adria, Spina, Marzabotto, et le Forcello. Cette réorganisation doit être examinée en liaison avec la nécessité de soutenir et de renforcer des activités commerciales de plus en plus complexes et désormais décidément projetées vers la Côte Adriatique septentrionale ${ }^{674}$. Que le développement et l'organisation du territoire et des centres urbains aient été intimement liés au progrès des activités commerciales est prouvé par le fait que les centres principaux ont été fondés dans des endroits stratégiques le long des routes qui reliaient Felsina, princeps de la dodécapole padane, aux points-clés des principales routes commerciales qui traversaient son territoire : la route orientale, qui dans les villes adriatiques d'Adria et de Spina voyait se mélanger plusieurs composantes ethniques, et notamment les éléments étrusque et grec ; la route méridionale, qui à travers la ligne de faîte des Apennins contrôlée par le centre de Marzabotto conduisait à l'Étrurie tyrrhénienne et surtout à la zone minière ; et enfin la route septentrionale, qui à travers l'axe Pô-Mincio et le port fluvial du Forcello garantissait les liaisons avec les populations de l'Italie nord-occidentale et du monde celtique transalpin. Ce système complexe et articulé permet à la région étrusco-padane de s'insérer pleinement dans les parcours commerciaux les plus importants de la fin $\mathrm{du} \mathrm{VI}^{\mathrm{e}}$ et du Ves. av. J.-C.

Dans les trente dernières années, la reprise des fouilles archéologiques de quelques centres parmi les plus importants de la région étrusco-padane, tels Adria, Spina et Marzabotto, et la découverte de nouveaux sites, comme le Forcello, et San Basilio, ont permis de reconsidérer sous une lumière nouvelle certains aspects déjà traités dans le passé, de fournir un tableau beaucoup plus complexe qu'on ne l'imaginait des modalités d'occupation

674. Sur le floruit de l'Étrurie padane vers le milieu du VI ${ }^{\mathrm{e}} \mathrm{s}$. av. J.-C. et la naissance d'un système fédéral lié, d'un côté au développement du phénomène urbain, de l'autre au nouvel intérêt des Étrusques padans pour l'Adriatique, cf. Sassatelli 1990, p. 58-69 ; Id. 1993, p. 183-189 ; Malnati 1996, p. 184-185 (avec proposition 'à la hausse' en termes chronologiques); Sassatelli 1999, p. 87-93. 
du territoire padan, notamment de l'aire du delta, et ont enfin contribué à porter au premier plan des problématiques jamais affrontées auparavant.

Parmi ces dernières, une place tout à fait évidente est occupée par les amphores de transport, catégorie d'importations négligée jusqu'ici par les études traditionnelles à cause des difficultés d'identification et de classification, Ces lacunes étaient particulièrement évidentes entre la seconde moitié du VI et les débuts du $\mathrm{IV}^{\mathrm{e}}$ s. av. J.-C, période où se développe l'Étrurie padane. Les lacunes d'un cadre historico-économique traditionnellement reconstitué sur la base exclusive de céramiques de table et de biens de prestige, en excluant complètement les conteneurs destinés au transport et au commerce de biens de première nécessité, sont tout à fait claires et ont été depuis longtemps dénoncées de plusieurs côtés ${ }^{675}$.

L'examen des sources littéraires et archéologiques montre que, mises à part les matières premières comme les métaux, l'objet principal des échanges dans l'aire padane était les produits de l'agriculture et de l'élevage ${ }^{676}$. Certaines de ces marchandises, à cause de leur nature périssable, ne peuvent évidemment être étudiées que de manière indirecte, à travers l'analyse des récipients utilisés pour les transporter, et principalement les amphores destinées au commerce de l'huile et surtout du vin, qui constituaient sans aucun doute les produits d'échange privilégiés des emporoi grecs.

Comme en témoigne la documentation du secteur centro-méridional de la péninsule italienne, leur importation en Occident commence au moins à la fin du VIII ${ }^{\mathrm{e}} \mathrm{s}$. av. J.-C. Mais ce n'est qu' au début du VI ${ }^{\mathrm{e}}$ qu'elle touche aussi le marché septentrional, pour le conquérir à partir de la seconde moitié du siècle. Cette évolution est la conséquence, non seulement de la réorganisation de l'Étrurie padane qu'on vient d'examiner, mais aussi des nouveaux choix de politique agraire mis en place en Grèce sous Pisistrate. Ces choix, comme cela est bien connu, privilégièrent les productions de type intensif comme la vigne et l'olivier, dont les dérivés connurent un surplus de production destiné à l'exportation, et provoquèrent l'abandon progressif des cultures extensives de blé et de céréales, qu'on allait maintenant chercher dans d'autres marchés comme celui du riche arrière-pays padan.

Selon une opinion courante, le vin grec qui arrivait aux emporia de la côte septentrionale de l'Adriatique ne devait être destiné qu'en partie à la consommation locale, son ultime et principale destination étant vraisemblablement

675. Vallet, Villard 1961, notamment p. 312 ; Vallet 1962. Sur l'argument sont ensuite revenus à plusieurs reprises d'autres auteurs (Martelli 1985, p. 177 ; Ead. 1989, p. 784 ; Sassatelli 1993, p. 190). 676. Sassatelli 1993, notamment p. 183, 211-213, avec bibliographie ; Id. 2008, avec mise à jour des références bibliographiques. le marché celtique au-delà des Alpes ${ }^{677}$. À l'appui de cette hypothèse, plusieurs arguments ont été énoncés : avant tout la diffusion de céramiques attiques et de vases en bronze étrusques, destinés à la consommation du vin, dans le territoire de Golasecca et les centres celtiques transalpins ${ }^{678}$; ensuite les attestations littéraires et archéologiques relatives à une production vinicole dans l'aire de la Haute Adriatique au moins à partir du Ve s. ${ }^{679}$; enfin et surtout, l'absence ou la faible diffusion des amphores vinaires grecques dans les sites de l'Italie du nord en dehors des localités considérées comme des lieux de transit pour ces marchandises vers le monde transalpin, c'est-à-dire les ports où elles faisaient escale comme Spina et Adria, ou les centres d'où l'on imagine que la redistribution vers le nord avait lieu, comme le Forcello. Sur la base du tableau de diffusion des amphores grecques tracé il y a quelques années on pouvait en effet observer leur présence presque exclusive dans des localités situées sur les voies d'eau : dans l'aire du delta, comme à Adria, à San Basilio et à Spina ; ou bien le long des itinéraires fluviaux qui allaient vers le nord, comme à Le Balone, sur une branche du Pô, et au Forcello, à la confluence entre le Pô et le Mincio. En dehors de ces parcours fluviaux, les attestations étaient rares, limitées aux trois localités de Poviglio, Marzabotto et sans doute Este, et tout à fait isolées, chaque site comptant une unique présence amphorique ${ }^{680}$. Donc, les centres étrusques au sud du Pô comme Bologne et Marzabotto, la région des lacs lombards et la région paléo-vénète et rhétique et enfin les pays au-delà des Alpes, bien qu'insérés dans le circuit commercial de la céramique attique, semblaient être exclus de celui des amphores. Celles-ci, attestées le long de la côte, semblaient arrêter leur parcours en direction nord au Forcello. Selon l'hypothèse qui

677. Pour l'hypothèse, amplement acceptée ensuite (cf. Sassatelli 1990 , p. $89-90$; Id. 1993 , p. 209), nous renvoyons principalement à R. de Marinis qui, ayant développé cette idée à la suite des abondantes découvertes d'amphores du Forcello (de Marinis 1988, notamment p. 211-213), l'a ensuite soutenue à plusieurs reprises (dernièrement, Id. 1996, p. 340-341, et Id. 2007, avec références bibliographiques précédentes).

678. Cf. dernièrement les cartes de répartition de Pape 2000.

679. Les plus anciens pépins de raisin cultivé découverts jusqu'ici en Italie septentrionale semblent pouvoir être datés du $\mathrm{V}^{\mathrm{e}} \mathrm{s}$. av. J.-C. Il s'agit des découvertes de l'habitat rhéto-euganéen d'Archi di Castelrotto (Nisbet 1987). Du IV e s. av. J.-C. est datable l'édifice rhétique de Nomi, au lieu-dit Bersaglio, avec les restes probablement d'un tonneau carbonisé en association avec un égouttoir et un gobelet (Marzatico 1995, p. 523-529 ; Ciurletti 1996, p. 454-455). D'après P. Desantis, une production locale de vin entre la fin du IV et le début du III ${ }^{\mathrm{e}}$ s. av. J.-C. serait indirectement attestée aussi par la découverte de quelques rebuts de cuisson d'amphores à Spina, témoignages d'une production locale de conteneurs pour le transport (Desantis 1989, p. 116-117, et n. 75-78).

680. Voir les cartes de diffusions dressées par M.C. Cattaneo et R. de Marinis (Cattaneo Cassano 1995, pl. VII ; Cattaneo, de Marinis 1996, fig. 5). Dernièrement voir aussi de Marinis 2007, p. 157. 
fut alors avancée, au Forcello, à l'endroit où le Mincio devenait plus difficilement navigable, la route fluviale fut abandonnée pour une route terrestre conduisant à la zone de piémont et aux défilés des Alpes ; pour ce faire, on a jugé qu'il devait être plus pratique de transvaser le vin dans des outres de peau et des tonneaux en bois ${ }^{681}$.

Dans ces conteneurs, le vin devait ainsi être redistribué dans le territoire interne, aussi bien auprès des populations celtiques transalpines, considérées comme les principaux destinataires de cette marchandise, qu'auprès des populations paléo-vénètes, rhétiques et du territoire de Golasecca en deçà des Alpes. Dans ces territoires, la consommation du vin est documentée par la diffusion de la vaisselle à boire, importée ou de production locale ${ }^{682}$.

Quelques voix plus prudentes, ou même discordantes, ont récemment mis en garde contre des reconstructions des dynamiques historico-commerciales basées sur des données encore partielles. Elles mettaient en avant une série d'arguments qui pouvaient contredire le cadre ainsi dessiné et suggérer une plus grande participation de l'aire étrusco-padane tout entière non seulement au commerce mais également à la consommation du vin grec, ou même contester que les produits alimentaires grecs qui arrivaient aux ports de la Haute Adriatique ${ }^{683}$ fussent destinés aux régions au nord des Alpes.

L'étude d'ensemble des amphores grecques dans le territoire compris entre les Apennins et les Alpes permet aujourd'hui de nourrir ce débat. La contribution fournie par le matériel inédit des fouilles anciennes et récentes permet de combler certaines lacunes ou d'en proposer une

681. La possibilité d'un transvasement du vin des amphores dans des conteneurs de matière périssable pour affronter des trajets sur terre, déjà proposée d'une manière générale par G. Colonna (Colonna 1985, p. 276), a été soutenue pour le cas spécifique du Forcello par R. de Marinis (de Marinis 1988, p. 213) qui a prétendu que la résine de pin d'Alep qu'on étalait sur la paroi interne des amphores grecques devait stabiliser les caractéristiques chimico-physiques du vin en lui permettant, non seulement d'affronter sans problèmes le transport sur de longues distances, mais aussi de ne pas tourner une fois transvasé dans un conteneur différent d'une amphore (en dernier, de Marinis 1996, p. 340, avec bibliographie précédente). Sur ce sujet, nous renvoyons à Lawall 2000, note 18 avec bibliographie.

682. Cf. Pape 2000, avec cartes de diffusion et aussi Ciurletti 1996, où sont répertoriées des iconographies relatives à la consommation du vin dans des productions artisanales locales comme celle de l'art des situles.

683. Je fais allusion à la position plus modérée de M. Gras (Gras 1998, p. 60-61), qui tout en étant persuadé que la destination du vin grec qui arrivait à Spina se trouvait au-delà des Alpes, ne semble pas écarter l'hypothèse de son utilisation de la part des cités de l'Étrurie padane, et à celle plus nette de D. Vitali (Vitali 1998, p. 267-270) qui avance quelques arguments en faveur de l'exclusion d'un destinataire transalpin en ce qui concerne le vin et l'huile grecs qui débarquaient dans les ports de la Haute Adriatique. explication possible. L'analyse globale des importations du territoire permet de sortir des limites de chaque site pour considérer la région dans son ensemble.

Une première observation générale concerne donc la carte de diffusion des amphores grecques importées dans ce secteur de la péninsule italienne entre le $\mathrm{VI}^{\mathrm{e}}$ et les débuts du IVe s. av. J.-C. (fig. 69 et 95 ).

Sur la base de la nouvelle carte, il est évident que l'impression de vide général ou de défaut de documentation dans les sites principaux de l'aire padane, Adria, San Basilio, Bologne et Marzabotto, a été conditionnée par l'état des études et des publications.

En effet, la diffusion des amphores commerciales dans le territoire padan est assez capillaire, concernant tous les principaux sites étrusques. La quantité d'individus documentés dans les différents sites peut être considérée comme proportionnelle aux aires d'habitat explorées suivant les méthodes modernes de la fouille stratigraphique. La présence inattendue d'amphores dans les centres situés à l'intérieur, loin des parcours commerciaux qui menaient aux Alpes, témoigne de la totale implication du marché padan dans l'importation de produits alimentaires de la Grèce, non seulement pour leur acheminement vers d'autres destinations mais aussi pour leur consommation locale. Tant la région septentrionale au-delà du Pô (ce à quoi on s'attendait), que le secteur padan central et apenninique, étaient en effet pleinement intéressés par le circuit commercial qui acheminait les produits alimentaires (et non seulement artisanaux) d'origine méditerranéenne dans les principaux centres contrôlés dans la région par les Étrusques.

Il faudra par conséquent redimensionner l'idée que les marchandises grecques arrivées en Italie du nord, et notamment le vin, étaient presque exclusivement destinées au marché transalpin.

Fondamentalement, le commerce des céramiques grecques décorées, amplement diffusées dans le territoire avec une nette préférence pour les formes utilisées pour la consommation du $v{ }^{684}$, ne devait pas être séparé de celui des produits consommés à l'occasion des cérémonies d'origine grecque que les peuples étrusques de l'aire padane ont adoptées à partir de la deuxième moitié du $\mathrm{VI}^{\mathrm{e}}$ s. av. J.-C.

La caractéristique que semblent en effet partager les sites de diffusion des amphores de l'Italie septentrionale est leur participation au système fédéral étrusco-padan.

684. Voir en dernier Baldoni 2009, p. 246-249, qui traite de la situation spécifique de Marzabotto, mais qui ne fournit ni de comparaisons ni de références bibliographiques concernant la situation des autres sites étrusco-padans. 

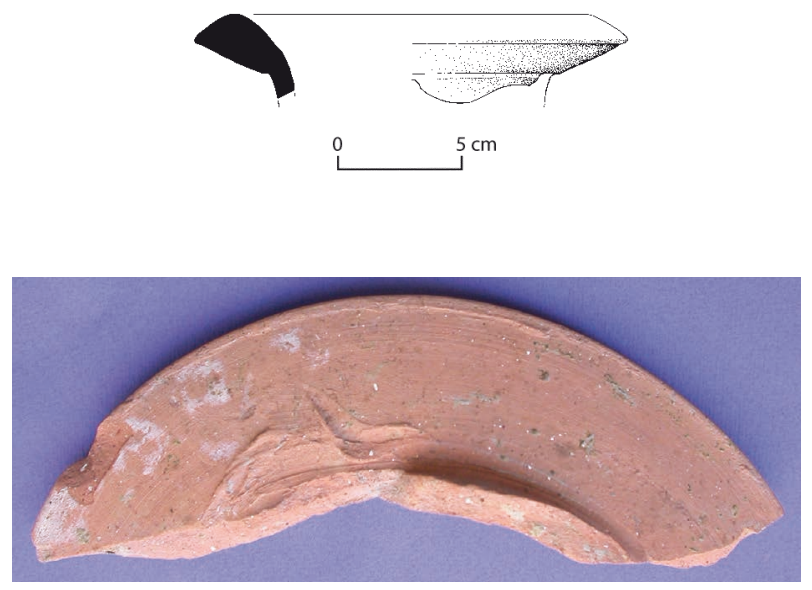

Fig. 93. Padoue, Via San Francesco (Palazzo Zabarella), lèvre d'amphore du groupe Solokha I

(๑) Soprintendenza per i Beni Archeologici per il Veneto).

En l'état actuel de nos connaissances, il semblerait que l'aire paléo-vénète ainsi que l'aire celtique de la culture de Golasecca se soient tenues plutôt en marge de ce phénomène. En ce qui concerne l'aire paléo-vénète, il n'y a actuellement qu'une seule amphore commerciale grecque documentée à Padoue (fig. 93) ${ }^{685}$, où le problème de la faible présence de céramique grecque décorée a été également longtemps débattu ${ }^{686}$. Je n'ai trouvé, en revanche, aucune confirmation concernant la possible présence d'une amphore corinthienne $\mathrm{B}$ signalée à Este ${ }^{687}$.

Pour ce qui concerne l'aire de la culture de Golasecca, un fragment de panse appartenant à une amphore commerciale grecque a été trouvé dans l'habitat de Brescia ${ }^{688}$. Les seuls autres fragments d'amphores commerciales que je connaisse provenant de cette zone viennent de l'habitat de Briccola de Castelletto Ticino, mais ils sont à attribuer à la production étrusque et sont datables entre la fin du VII ${ }^{\mathrm{e}}$ et le milieu du $\mathrm{VI}^{\mathrm{e}}$ s. av. J.-C. ${ }^{689}$.

685. Bonomi 2003, fig. 4. Il s'agit d'une lèvre d'amphore du groupe Solokha I datable du courant de la seconde moitié du Ve s. av. J.-C. et provenant des fouilles de Via San Francesco-Palazzo Zabarella, dont l'étude m'a été confiée par Simonetta Bonomi. Pour le type, cf. Lawall 1995, p. 220-221, fig. 88-92.

686. Bonomi 2004, avec bibliographie précédente.

687. La présence d'une amphore grecque, corinthienne B pour la précision, à Este a été signalée de manière dubitative par R. de Marinis qui ne fournit pas d'indications ultérieures à ce propos (de Marinis 1996, fig. 5, p. 340).

688. Carta archeologica della Lombardia. V. Brescia. La città, Modena 1996, II, p. 43. Il s'agirait d'une amphore identifiée comme « attique » ou «samienne » sur la base de la pâte céramique. Il pourrait donc également être question d'un fragment d'amphore du groupe Solokha I (cf. supra I, $5 \S 4$, pour les dénominations utilisées en littérature pour définir les amphores de cette série).

689. Gambari 1993, p. 128, n. 8-9 (type Py 3).
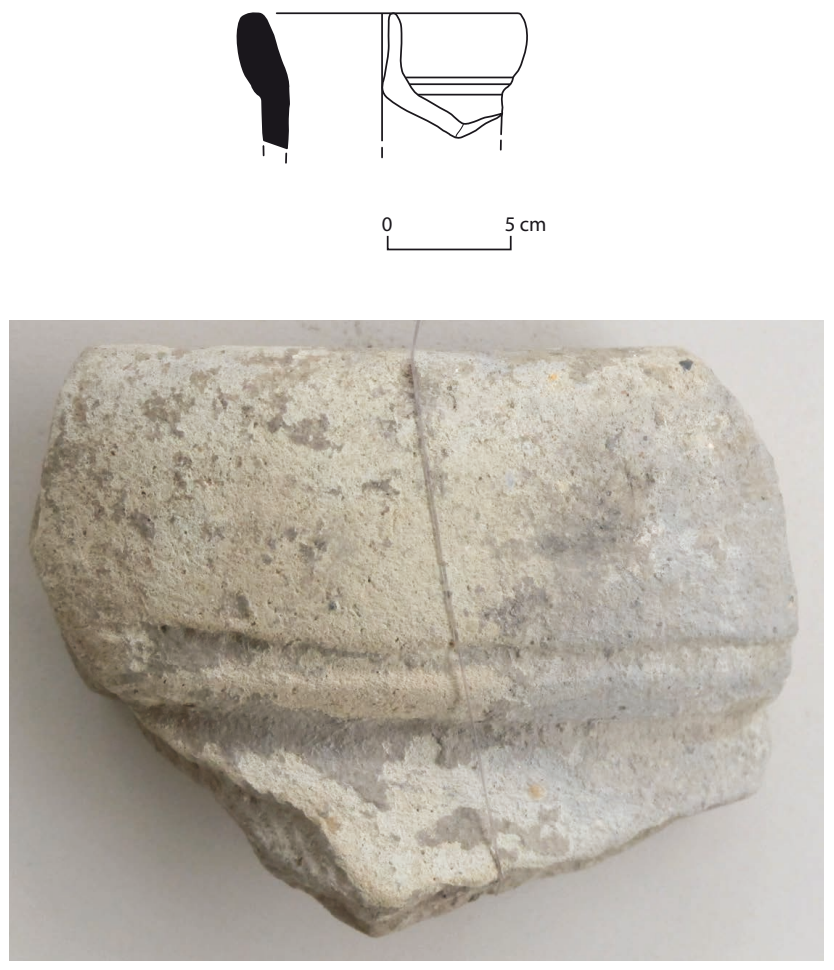

Fig. 94. Reggio Emilia, Civici Musei, lèvre d'amphore gréco-occidentale de « forme 2 » ou « 3 » en provenance de Poviglio, lieu-dit Case Carpi (photo F. Sacchetti).

Pour revenir au secteur étrusco-padan, où sont concentrées les découvertes, la diffusion des amphores ne semble pas, d'après nos connaissances actuelles, avoir atteint la zone à l'Ouest du territoire de Mantoue, et notamment du site du Forcello. La trouvaille occasionnelle d'un fragment d'amphore grecque à Case Carpi de Poviglio, dans l’Émilie occidentale (fig. 94), est actuellement tout à fait isolée ${ }^{690}$.

Donc, le circuit de distribution des amphores semble avoir touché fondamentalement les centres principaux de l'Étrurie padane : Adria-San Basilio, Spina, Bologne, Marzabotto, le Forcello et Verucchio ${ }^{691}$.

690. Macellari 1990,p. 265-266, pl. LXXIX, 2. Il s'agit d'un fragment de lèvre d'amphore de 'forme 2' avec une lèvre de type Py 3 (Py 1980, p. 8, fig. 5) datable du courant de la première moitié du Ve $\mathrm{s}$. av. J.-C. 691. Les amphores grecques de Verucchio, provenant de contextes d'habitat du V $V^{\mathrm{e}} \mathrm{s}$. av. J.-C., ont été analysées dans le cadre d'une Thèse de l'École de Spécialisation de l'Université de Bologne (Paola Poli, Verucchio. Pian del Monte. Casa del Podere Parma. Le ceramiche attiche e i materiali di importazione, Università degli Studi di Bologna, a.a. 1998/1999), dont la publication est en cours de préparation. Les amphores de Verucchio ne pouvaient donc pas être prises en considération dans le cadre de ce volume, mais je juge important d'en signaler au moins l'existence et je voudrais remercier Paola Poli et 


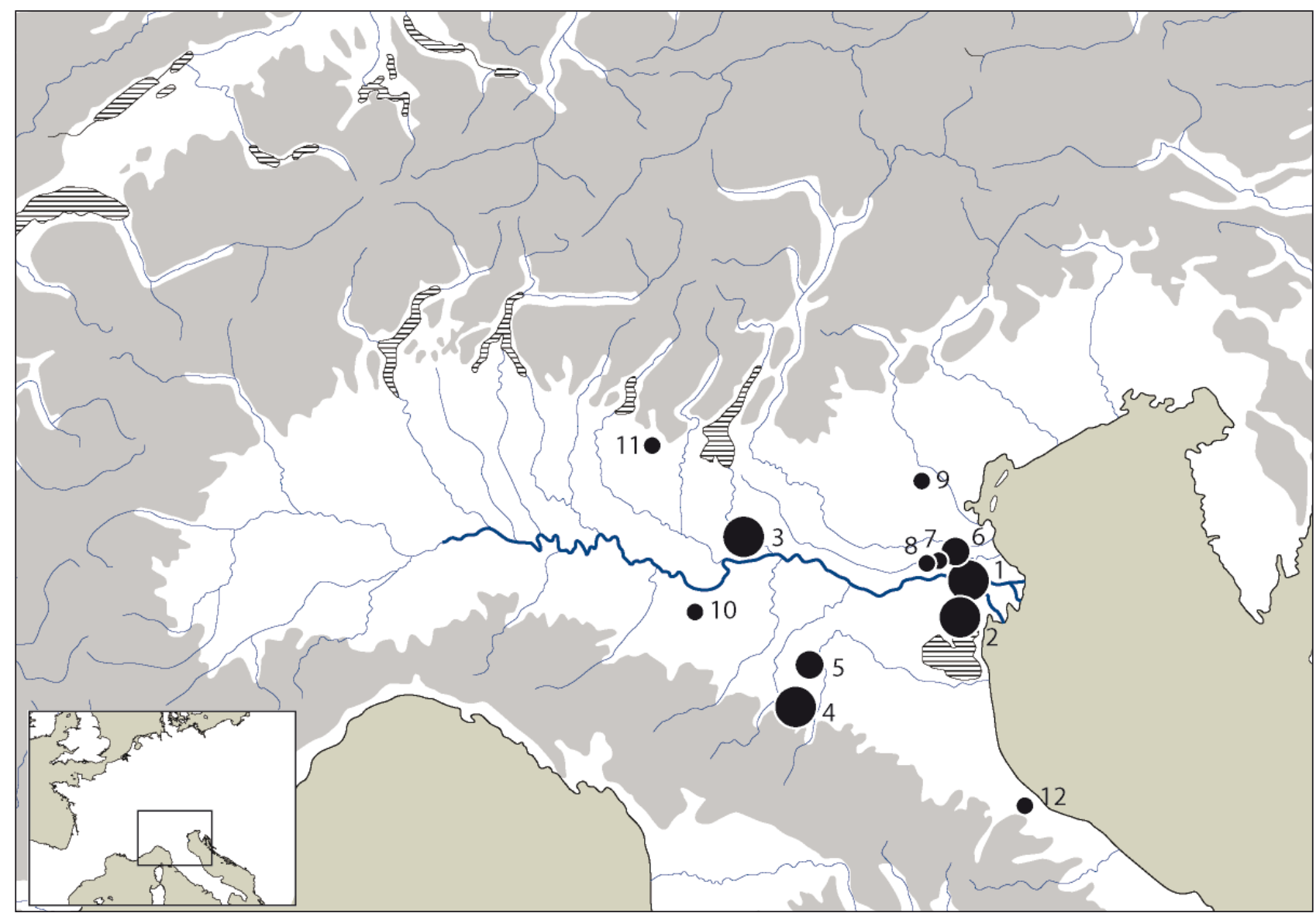

Fig. 95. Carte de répartition des amphores commerciales grecques dans le territoire compris entre les Apennins et les Alpes. Nombre d'amphores supérieur à 30 individus (points de grande taille) : 1. San Basilio; 2. Spina ; 3. Forcello; 4. Marzabotto. Nombre d'amphores compris entre 15 et 30 individus (points de taille moyenne) : 5. Bologne ; 6. Adria. Nombre d'amphores inférieur à 15 individus (points de petite taille) : 7. San Cassiano ; 8. Le Balone ; 9. Padoue ; 10. Poviglio ; 11. Brescia ; 12. Verucchio. (F. Sacchetti).

Quant aux contextes de provenance des amphores, il s'agit presque exclusivement de zones d'habitat. Treize amphores uniquement sur les 324/342 individus ${ }^{62}$ pris en considération dans ce volume proviennent de contextes funéraires. Huit d'entre elles viennent des nécropoles de Spina et cinq d'Adria et de son territoire. En l'état actuel des connaissances on ne peut que se limiter à observer un phénomène qu'il est très difficile d'interpréter. En effet, d'une part, les études sur le rituel funéraire de l'Étrurie padane sont encore insuffisantes. D'autre part, la coutume de l'offrande de vin dans ces quelques tombes du Nord de l'Italie ne peut être reliée ni à la culture étrusque,

Patrizia von Eles (Soprintendenza per i Beni Archeologici de l'Émilie-Romagne) de m'en avoir donné l'autorisation.

692. Dans ce calcul, outre les individus présentés dans les catalogues des sites (cf. supra p. 106 et 114, tableaux 1-2) j'ai aussi considéré le fragment de Padoue et celui de Case Carpi de Poviglio mentionnés ci-dessus. compte tenu du manque total d'amphores dans les milliers de tombes connues à Bologne et Marzabotto (cf. supra p. 209 et p. 261), ni à la culture grecque, étant donné la rareté d'amphores dans les contextes funéraires des cultures grecques entre la fin du VI ${ }^{\mathrm{e}} \mathrm{s}$. et le courant du Ve s. av. J.-C.

On se limitera donc à mettre en évidence que ces présences constituent des exceptions dans le rituel funéraire de l'Étrurie padane et que ces exceptions se concentrent dans les zones portuaires du delta du Pô. On serait donc tenté de penser que cette particularité dépend d'une situation propre aux sites étrusques « de frontière », vu le caractère multiethnique, bien connu et typique des ports, qui distingue les deux centres du delta du Pô.

Une observation complémentaire concernant ces découvertes est que la presque totalité des amphores faisant partie de mobiliers funéraires est de production gréco-occidentale. Dix des treize amphores provenant de contextes funéraires appartiennent en effet au type corinthien B 
(cat. AD3-AD6 ; LB2 ; SP2-SP4 ; SP13-SP14) (fig. 72, $\mathbf{7 3}, \mathbf{7 9}, \mathbf{8 0}$ ), deux au groupe gréco-oriental Solokha I (cat. SP39-SP40) et une est de production nord-égéenne (cat. SP 28). Leur chronologie est comprise entre les débuts du $V^{e}$ et les débuts du IV ${ }^{\mathrm{e}} \mathrm{s}$. av. J.-C.

Du point de vue du niveau chronologique, on a pu établir que le début des importations d'amphores devait se situer dans le courant du deuxième quart du VI $\mathrm{e}$. av. J.-C. et qu'il concerne l'aire septentrionale du delta du Pô, notamment Adria et San Basilio ${ }^{693}$. Ce n'est qu'après le milieu et surtout à partir de la fin du siècle qu'on peut en enregistrer la diffusion dans le reste du territoire étrusco-padan, excepté sans doute un cas isolé documenté à Marzabotto (cf. supra p. 214-215, cat. MA1).

Les amphores attestées dans le Nord de l'Italie ne concernent que les productions du monde grec. À l'heure actuelle, les productions phénico-puniques ou étrusques, ne sont pas documentées dans ce territoire, à l'exception d'une amphore étrusque de type Py 3 , produite à Caere entre la fin du VII et le milieu du VI ${ }^{\mathrm{e}}$ s. av. J.-C., trouvée dans l'habitat de Briccola de Castelletto Ticino, dans l'aire de la culture de Golasecca ${ }^{694}$.

En revanche, presque toutes les séries amphoriques connues dans le monde grec sont attestées dans le Nord de l'Italie. En l'état actuel des recherches, seules manquent les amphores de Marseille, parmi les productions de l'Occident grec, et les amphores laconiennes et de Lesbos parmi les productions de la Grèce propre et de l'Est. L'absence également des amphores SOS peut sans doute s'expliquer par des raisons chronologiques. En effet la forme SOS Late II, la plus récente, est datable entre le dernier quart du $\mathrm{VII}^{\mathrm{e}}$ et le premier quart du VI ${ }^{\text {e }}$ s. a.C. ${ }^{695}$. Par conséquent, l'interruption de sa production se situe juste avant les plus anciennes importations d'amphores dans le secteur nord-adriatique et bien avant l'épanouissement du système économique étrusco-padan engendrant la floraison des échanges avec le monde grec.

Il est assez difficile de quantifier pour les différents sites les importations des diverses macro-aires de productions (Grèce d'Occident, Grèce de l'Est, Égée septentrionale, Grèce propre) à cause de l'insuffisance de la documentation sur les ports d'arrivée des

693. Cf. Sacchetti 2012.

694. Identification et détermination de F.M. Gambari (Gambari 1993, p. 128, notes 8-9). Les autres amphores étrusques connues en Italie septentrionale concernent Gênes et la Ligurie.

695. Johnston \& Jones 1978 et Rizzo 1990. marchandises méditerranéennes et de l'absence d'un site emporique représentatif.

En effet, l'habitat étrusque d'Adria, le port le plus septentrional et le plus anciennement actif, étant donné que sa documentation remonte à la première moitié $\mathrm{du} \mathrm{VI} \mathrm{V}^{\mathrm{e}}$ s ${ }^{696}$, se trouve en dessous de la ville moderne. Seules les anciennes fouilles de 1878 du Pubblico Giardino et celles récentes et en partie inédites de via San Francesco et de via Ex Riformati ont pu mettre au jour des couches du $\mathrm{VI}^{\mathrm{e}}$ et du $\mathrm{V}^{\mathrm{e}} \mathrm{s}$. av. J.-C., mais sur des surfaces réduites ${ }^{697}$. L'habitat de Spina, actif à partir de 540 av. J.-C. ${ }^{698}$, a été découvert dans les années 1970 à l'occasion des travaux d'assainissement des marais de Valle Lepri, du côté de Comacchio. Il n'a été exploré que par quelques sondages et il a été fouillé sur une surface également très limitée : une bande de terrain de $200 \mathrm{~m}$ x $20 \mathrm{~m}$ dont on prévoyait l'élimination pour des exigences d'assainissement ${ }^{699}$. À ces deux ports s'ajoute aujourd'hui le site côtier de San Basilio, fouillé sur une surface de $103 \mathrm{~m}^{2}$ grâce à l'ouverture de deux tranchées de $7 \mathrm{~m} \times 2 \mathrm{~m}$ et de $8,9 \mathrm{~m} \times 2 \mathrm{~m}^{700}$.

Mais nos réflexions sont également conditionnées par le manque d'homogénéité des contextes du territoire padan. Dans les seuls cas du Forcello et de Marzabotto, deux sites de l'intérieur, il est possible de suivre le comportement économique en s'appuyant sur des données provenant de l'exploration de vastes secteurs d'habitat entre le milieu du $\mathrm{VI}^{\mathrm{e}}$ et les débuts du $\mathrm{IV}^{\mathrm{e}} \mathrm{s}$. av. J.-C., données témoignant donc de toute la période considérée dans cette étude.

Pour cet arc chronologique, les documentations archéologiques de Bologne et de Spina sont de type presque exclusivement funéraire et les amphores provenant de secteurs limités d'habitat n'ont qu'une valeur indicative. En plus, à Bologne, le secteur qui a restitué le plus grand nombre d'amphores est celui de l'acropole de la cité étrusque, c'est-à-dire un contexte de sanctuaire qui ne reflète pas nécessairement les situations réelles de la consommation de l'habitat et où, comme on l'a suggéré (cf. supra p. 209), le contexte d'où proviennent les amphores est probablement limité à la seconde moitié du Ves. av. J.-C. En ce qui concerne l'aire septentrionale

696. Bonomi 2000a.

697. Pubblico Giardino (Donati, Parrini 1999) ; via San Francesco (Bonomi, Camerin, Tamassia 2003). Cette dernière fouille n'a pas atteint les niveaux les plus anciens. La fouille de via Ex Riformati, très récente et qui a atteint les niveaux du $\mathrm{VI}^{\mathrm{e}} \mathrm{s}$. a.C., est encore inédite. Je remercie S. Bonomi pour avoir bien voulu me confier l'étude des amphores en provenance de ces contextes.

698. Lippolis 2000, p. 111 ; Bruni 2004, p. 84.

699. Patitucci Uggeri, Uggeri 1993, en particulier p. 24-25.

700. Salzani, Vitali 2003. 
du delta du Pô, les secteurs d'habitat qui ont fait l'objet d'enquêtes dans le courant des dernières années et sur lesquels il a été possible de faire une recherche extensive, même sur des surfaces réduites, concernent surtout les phases d'époque archaïque (Adria-Via Ex Riformati et San Basilio), tandis que les recherches conduites sur les contextes du milieu et de la seconde moitié du $\mathrm{V}^{\mathrm{e}} \mathrm{s}$. ont été jusqu'ici plus limitées (Adria-Via San Francesco).

En général, on peut de toute façon observer quelques tendances partagées par tous les sites dans le secteur du delta du Pô, septentrional et méridional.

Dans cette aire les productions amphoriques les mieux attestées dans le courant du $\mathrm{VI}^{\mathrm{e}} \mathrm{s}$. av. J.-C. sont les productions grecques d'Occident, attribuables en particulier au groupe « corinthien B archaïque » (ou « forme $1 \alpha \gg$ ) et datables de la seconde moitié du siècle.

Les premières importations de la Grèce de l'Est remontent au deuxième quart du $\mathrm{VI}^{\mathrm{e}} \mathrm{s}$. av. J.-C. et vers la fin du siècle semblent commencer les importations de l'Égée du nord et de rare amphores du type « à la brosse ». Dans le courant du Ve s. av. J.-C., les productions les mieux documentées dans ce secteur sont encore gréco-occidentales, désormais avec le type corinthien B évolué, toujours suivies par les productions de la Grèce de l'Est et celles d'Égée du nord. Les amphores corinthiennes $\mathrm{A} / \mathrm{A}^{\prime}$ sont très rares et limitées au milieu ou à la seconde moitié du siècle.

Au V $V^{\mathrm{e}}$ s., les deux sites les mieux documentés, le Forcello et surtout Marzabotto, présentent une situation particulière, différente de celle du delta, puisque les amphores les mieux attestées sont celles de la Grèce de l'Est. À l'intérieur des productions gréco-orientales, on peut observer une concentration d'amphores de Chios et du groupe Samos-Milet avant le troisième quart du $\mathrm{V}^{\mathrm{e}} \mathrm{s}$. On a l'impression que la place occupée dans le marché adriatique par ces productions a été remplacé dans la seconde moitié du $\mathrm{V}^{\mathrm{e}}$ et au début du $\mathrm{VI}^{\mathrm{e}} \mathrm{s}$. av. J.-C. par le groupe Solokha I ${ }^{701}$. La production de cette forme amphorique était probablement partagée par plusieurs centres de l'aire égéenne orientale et méridionale, ceux-là mêmes qui avaient participé à la production des amphores du groupe Samos-Milet. L'incidence remarquable des amphores attribuables au groupe Solokha I dans l'Italie septentrionale est à souligner. Il faut en effet rappeler que ce groupe est connu presque exclusivement grâce à des découvertes effectuées dans l'aire égéenne et datables, sauf pour Athènes, surtout dans le courant du

701. En se limitant à l'aire occidentale de la Méditerranée, un passage analogue peut être remarqué à Vélia (Gassner 2005, notamment p. 43).
IV ${ }^{\mathrm{e}}$ s. av. J.-C. ${ }^{702}$, alors qu'il semble pratiquement absent dans la Méditerranée occidentale (cf. supra I, 5 § 4.4). Les découvertes du Nord de l'Italie témoignent donc du grand intérêt exercé par cette zone au cours de la deuxième moitié du $\mathrm{V}^{\mathrm{e}} \mathrm{s}$. av. J.-C. et de sa totale implication dans les circuits commerciaux de longue distance liés à la Mer Égée.

Dans le courant du Ve s. av. J.-C., dans les deux sites, on trouve également bien documentées les amphores de l’Égée du nord. L'identification des différents centres de production de cette aire est encore très difficile, mais la morphologie et la pâte des amphores de Mendé, bien connue par son vin, sont assez caractéristiques. On peut ainsi noter que, tant à Marzabotto qu'au Forcello, les importations de Mendé semblent se concentrer dans la deuxième moitié du $\mathrm{V}^{\mathrm{e}} \mathrm{s}$. av. J.-C. (formes Middle et Late Mendean) ${ }^{703}$.

On notera une certaine différence entre les deux sites pour les amphores grecques d'Occident, pratiquement absentes à Marzabotto, aussi bien au $\mathrm{VI}^{\mathrm{e}}$ (« forme $1 \alpha »$ et « forme 2 ») qu'au $\mathrm{V}^{\mathrm{e}} \mathrm{s}$. (type corinthien B), tandis qu'elles sont bien documentées dans toute l'aire du delta et au Forcello. Sur ce site, en effet, au VI ${ }^{\mathrm{e}}$ s., les amphores de production occidentale archaïque couvrent la moitié environ des importations et dans le courant du $\mathrm{V}^{\mathrm{e}} \mathrm{s}$. les amphores du type corinthien B en représentent encore un bon pourcentage.

Ce pourcentage du Forcello est à peu près équivalent à celui des amphores de type corinthien A/A', bien représentées dans la phase plus récente de l'habitat, mais totalement absentes des contextes antérieurs au deuxième quart du Ve s. av. J.-C. (de Marinis 2007, p. 159).

Il en va de même pour le secteur du delta, septentrional et méridional, où la présence de ce type amphorique, tout comme au Forcello, est datable du milieu ou du courant de la seconde moitié du Ve $\mathrm{S}$. av. J.-C.

À Marzabotto, en revanche, on remarque un fort pourcentage d'amphores de type corinthien A/A', dans des formes et des contextes de pleine époque archaïque.

Ce qui distingue donc Marzabotto du reste de l'aire examinée est, d'une part, la considérable quantité d'amphores corinthiennes $\mathrm{A} / \mathrm{A}$ ' et, de l'autre, la faible présence des amphores de production occidentale, aussi bien au $\mathrm{VI}^{\mathrm{e}}$ que dans le courant du V'e s. av. J.-C.

702. Pour les découvertes d'amphores de ce groupe hétérogène dans la forme du IV'e s. av. J.-C., voir en dernier Demesticha 2011, p. 44-45, avec bibliographie. Pour les découvertes en milieu égéen datables encore du V s. av. J.-C., cf. Lawall 1995, p. 218-233 (Athènes).

703. Observations personnelles pour Marzabotto ; observations de R. de Marinis pour le Forcello - cf. catalogue supra et en dernier de Marinis 2007, p. 172. 
Les dénominations utilisées conventionnellement pour ces productions occidentales étant souvent trompeuses, il n'est pas superflu de rappeler une fois de plus qu'une partie de ces productions pouvait être localisée dans l'Est de la Mer Ionienne ou le Sud de l'Adriatique, aussi bien au VI ${ }^{\mathrm{e}}$ qu'au $\mathrm{V}^{\mathrm{e}}$ s. av. J.-C. ${ }^{704}$.

Pour ce qui concerne l'époque archaïque, c'est dans cette zone qu'il faut situer la fabrique identifiée par R.F. Docter sur la base des analyses pétrographiques du matériel de Butrint, encore inédit (cf. supra I, 2 § $1.1)^{705}$. Des recherches ultérieures pourront peut-être déterminer si les amphores de la cité de l'Albanie méridionale sont attribuables au même atelier corcyréen actif aux époques classique et hellénistique (KourkoumélisRodostamos 1988 ; Preka-Alexandri 1992) ou bien à une autre fabrique à rechercher sur la terre ferme d'en face.

D'après un examen visuel, le matériel de l'Albanie présente des pâtes céramiques semblables à celles de plusieurs exemplaires de l'aire étudiée dans ce volume, mais ce n'est qu'avec la poursuite des recherches dans cette direction que l'on pourra identifier l'origine des amphores gréco-occidentales d'époque archaïque si bien attestées dans la Haute Adriatique et déterminer le rôle éventuel de Corcyre et du secteur ionien oriental / adriatique méridional dans leur commerce (Sacchetti 2012).

En ce qui concerne la forme dite corinthienne B du V $\mathrm{V}^{\mathrm{e}}$ s. av. J.-C., attribuée dans un premier temps à Corinthe, les lieux de production restent pour l'instant difficiles à préciser. Il existe deux exceptions, limitées uniquement au $\mathrm{V}^{\mathrm{e}} \mathrm{s}$. av. J.-C. : l'atelier identifié dans le quartier artisanal de Corcyre (Corfou) et la fabrique qui a été attribuée à Apollonia, dans l'Illyrie méridionale (Mano 1971 ; Desy 1982 ; Whitbread 1995, p. 283), qui pourrait avoir été en activité dès ce moment-là (Ceka 1986, p. 89). L'examen visuel des pâtes des amphores corinthiennes B des fouilles de Corcyre a cependant fait penser que la fabrique corcyréenne aurait pu être flanquée d'autres productions, à rechercher peut-être en Épire (Kourkoumélis-Rodostamos 1988, p. 113), où 1 'on connaît maintenant les amphores corinthiennes $\mathrm{B}$ de forme évoluée étudiées à Butrint par R.F. Docter et définies comme « local production from CorfùButrint » (Docter sous presse). De plus en plus d'indices viennent appuyer l'hypothèse d'une concentration de

704. Cf. respectivement supra I, $1 \S 2.2$ et I, $2 \S 1.1$.

705. Docter sous presse. Cette contribution, bien qu'elle soit encore inédite, a été déjà mentionnée aussi par d'autres chercheurs (cf. Gassner 2003b, p. 185, n. 67 ; Ead. 2003a, p. 96, n. 18). J'exprime ici tous mes remerciements à R.F. Docter et à C. Neeft qui m'ont donné la possibilité de la lire. la production du type corinthien B dans l'aire comprise entre la basse Adriatique et la MerIonienne.

La variété des pâtes qu'on peut parfois observer dans les amphores du type corinthien B de l'Italie du nord suggère de ne pas exclure qu'elles proviennent de sites différents, qu'on ne peut pas actuellement préciser, mais qui s'insérerait dans le même problématique que les amphores « corinthiennes B archaïques » (ou « forme $1 \alpha »)$. Les exemplaires de l'une et de l'autre série que j'ai pu analyser dans la Haute Adriatique sembleraient présenter des pâtes très semblables.

Marzabotto semble partiellement en marge de ce système. Ici les productions occidentales sont rares et les premières importations d'amphores, encore isolées, pourraient être liées aux routes apenniniques qui ont véhiculé sur le site avant le milieu du $\mathrm{VI}^{\mathrm{e}} \mathrm{s}$. les céramiques méso- et tardo-corinthiennes déjà mentionnées (cf. p. 212), la céramique étrusco-corinthienne (Lippolis 2000, p. 102-103, 109, nº 5.2, 5.5) et le bucchero d'importation (Malnati, Macellari 1989, p. 30-31) ${ }^{706}$, à moins d'envisager la mise en place de rapports d'échanges précoces avec le secteur septentrional du delta du Pô (Adria et San Basilio) pour une époque où la zone littorale est encore mal connue. C'est au contraire aux relations avec l'aire du delta, et notamment avec son secteur méridional (Spina), qu'il faudra probablement rattacher les amphores grecques arrivées en plus grand nombre sur le site de l'Apennin entre la fin du VI ${ }^{\mathrm{e}}$ et le début du IV $\mathrm{s}$. av. J.-C. Dans cette phase, les liens commerciaux entre Marzabotto et Spina sont justifiés par le nouveau rôle joué par les deux cités dans la restructuration de l'Étrurie padane de la part de Bologne (Sassatelli 1990) et ils ont été récemment mis en relief par l'analyse de la céramique décorée (Lippolis 2000, p. 103-106 ; Baldoni 2009, p. 245).

Je ne peux toutefois m'empêcher d'observer que la grande quantité d'amphores corinthiennes A/A' (datables exclusivement sur une base typologique) et la faible quantité d'amphores de production occidentale, tant du VI ${ }^{\mathrm{e}}$ que du $\mathrm{V}^{\mathrm{e}} \mathrm{s}$., semblent distinguer Marzabotto du reste de l'aire padane et le rapprocher plutôt de ce que nous savons de Pise et d'autres sites trans-apenniniques du versant étrusco-tyrrhénien septentrional ${ }^{707}$. Il n'est

706. Ces publications ont également souligné l'anomalie du comportement de Marzabotto par rapport à la cité de Bologne même pendant cette phase historique, ainsi que ses contacts privilégiés et directs avec l'aire trans-apenninique et avec le territoire de l'Émilie centro-occidentale, qui du moins dans sa phase archaïque entretient à son tour des contacts directs avec l'aire tyrrhénienne.

707. À Pise, les productions occidentales archaïques sont documentées par quelques fragments attribuables à la « forme 2 ». On n'a pas de témoignages, au contraire, du groupe «corinthien B archaïque » (ou « forme $1 \alpha »$ ) ni des amphores corinthiennes B du Ve s. av. J.-C. 
pas à exclure que Marzabotto, encore au $\mathrm{V}^{\mathrm{e}} \mathrm{s}$., ait pu bénéficier de relations directes et privilégiées avec l'aire tyrrhénienne grâce à sa position sur les routes reliant les deux versants des Apennins. Cette observation devra un jour être reconsidérée à la lumière de la publication des contextes fouillés au cours du XX ${ }^{\mathrm{e}} \mathrm{s}$. à Marzabotto ${ }^{708}$, de l'avancement souhaité des recherches sur le delta padan méridional (notamment Spina) ainsi que de la publication des amphores de Prato-Gonfienti ${ }^{709}$. La proximité géographique et culturelle de ce nouveau site étrusque découvert sur l'autre versant de l'Apennin permettra de mieux définir la position de Marzabotto le long des routes qui reliaient l'Étrurie tyrrhénienne à l'Étrurie padane $^{710}$.

Les amphores corinthiennes A et A' entre la fin du $\mathrm{VI}^{\mathrm{e}}$ et le $\mathrm{V}^{\mathrm{e}} \mathrm{s}$. av. J.-C. sont en revanche bien documentées. Pour les amphores de Pise : Pancrazzi 1982 ; Ead. 1993 ; Pancrazzi 1993 ; Massa 1993 ; Bonamici 1996. Pour les amphores des sites mineurs du versant tyrrhénien septentrional, cf. Maggiani 1990b ; Bonamici 1996 (San Rocchino de Massarosa ) ; Maggiani, Paribeni 1990 (Casa Baldi de Pozzi, Seravezza) ; Ciampoltrini 1994 (Fossa Nera de Porcari).

708. La publication du matériel datant, provenant de ces contextes, permettra sans doute de préciser la chronologie des amphores corinthiennes A/A' génériquement datables entre la fin du VI ${ }^{\mathrm{e}}$ et le $\mathrm{V}^{\mathrm{e}} \mathrm{s}$. sur une base exclusivement morphologique.

709. Cf. Poggesi et al. 2005, p. 272, où l'on fait mention de la découverte de fragments d'amphores étrusques du type Py 4 et de «typologies gréco-orientales ».

710. Sur les rapports apenniniques entre l'Étrurie tyrrhénienne et la vallée du Reno, et donc Marzabotto, cf. Maggiani 1985 ; Ciampoltrini, Notini 1987 ; Malnati, Macellari 1989 ; Millemaci 1999 ; Id. 2001 ; Poggesi et al. 2005, p. 273). 


\section{RÉFÉRENCES BIBLIOGRAPHIQUES}

Abbate Edlmann, Giachi 1989 : ABBATE EDLMANN (M.L.), GIACHI (G.) - I legni di un relitto navale recuperato presso l'Isola del Giglio. StEtr, 55, 1987-1988 (1989), p. 235-243.

Adriatico greco : Dall'Adriatico greco all'Adriatico veneziano. Archeologia e leggenda troiana. Atti dell'Incontro di Studio, Venezia, 10-11 novembre 1997. Rome, 2000 (Hesperia. Studi sulla grecità d'occidente, 12, 2000).

Alaminos et al. 1991 : ALAMINOS (A.), OJUEL (M.), SANMARTí (J.), SANTACANA (J.) - algunas observaciones sobre el comercio colonial en la costa central y meridional de Catalunya en época arcaica. In : Presencia de material etrusco, p. 275-294.

Albanese Procelli 1997a : ALBANESE PROCELLI (R.M.) - Échanges dans la Sicilie archaïque. Amphores commerciales, intermédiaires et redistribution en milieu indigène. $R A, 1997 / 1$, p. 3-25.

Albanese Procelli 1997b : ALBANESE PROCELLI (R.M.) - Appunti sulla distribuzione delle anfore commerciali nella Sicilia arcaica. Kokalos, 42, 1996 (1997), p. 91-137.

Albanese Procelli 2000 : ALBANESE PROCELLI (R.M.) - Contenitori da derrate nella Sicilia arcaica e classica. Per una definizione dell'evidenza. In : Die Ägäis und das westliche Mittelmeer. Beziehungen und Wechselwirkungen 8. bis 5. Jh. v. Chr. Akten des Symposions, Wien 24. bis 27 Marz 1999. Vienne, 2000, p. 479-485.

Albanese Procelli 2003 : ALBANESE PROCELLI (R.M.) - Anfore commerciali dal centro indigeno della Montagna di Ramacca (Catania). In : FIORENTINI (G.), CALTABIANO (M.), CALDERONE (A.) dir., Archeologia del Mediterraneo. Studi in onore di Ernesto De Miro, Rome, 2003, p. 37-50.

Alberti 1986 : ALBERTI (L.) - Per una interpretazione paleoambientale dei trovamenti de 'Le Balone' fra i comuni di Rovigo e Arquà Polesine. In : Introduzione storica alla lettura della carta catastale del Retratto del Garzon. Itinerari e documenti per una storia della bassa padovana, Stanghella, 1986, p. 317-324 (Quaderni del Gruppo Bassa Padovana, 7, parte I).

Albore Livadie 1985 : ALBORE LIVADIE (C.) - L'épave étrusque du Cap d'Antibes. RStLig, 33, 1967, p. 300-326.

Albore Livadie 1985 : ALBORE LIVADIE (C.) - La situazione in Campania. In : Commercio etrusco arcaico, p. 127-154.

Alfieri 1959a : ALFIERI (N.) - Spina e le nuove scoperte, problemi archeologici e urbanistici. In : Spina e l'Etruria padana. Atti del I Convegno di Studi Etruschi, Ferrara, 8-11 Settembre 1957. Florence, 1959, p. 25-44.

Alfieri 1959b : ALFIERI (N.) - Problemi di Spina. In : Cisalpina. Atti del Convegno sull'attività archeologica nell'Italia settentrionale, Varenna, 9-15 Giugno 1958. Milan, 1959, vol. I, p. 89-102.

Alfieri 1981 : ALFIERI (N.) - Strabone e il delta del Po. Padusa, n.s., 17, 1981, p. 3-11.

Alfieri 1987 : ALFIERI (N.) - Spina. La nascita di un emporio adriatico. In : Formazione della città in Emilia Romagna, II, p. 177-179.

Alfieri 1988 : ALFIERI (N.) - Spina polis hellenis. In : Formazione della città preromana, p. 283-288.

Alfieri 1993 : ALFIERI (N.) - La ricerca e la scoperta di Spina. In : Spina, p. 3-18
Alto e Medio Adriatico : L'Alto e Medio Adriatico tra VI e V secolo a.C Atti del Convegno Internazionale, Adria, 19-21 marzo 1999. Pise - Rome 2003 (Padusa, n.s., 38, 2002, éd. 2003).

Ammirati, Morico 1984 : AMMIRATI (C.), MORICO (G.) - L'abitato preistorico di villa Cassarini (Bologna). Scavi del 1906. EmPrerom, 9-10, 1981-1982 (1984), p. 72-99.

Amphores de Marseille grecque : BATS (M.) dir. - Les amphores de Marseille grecque. Chronologie et diffusion (IVe-Ier s. av. J. -C.). Actes de la table-ronde de Lattes (11 mars 1989). Lattes - Aix-en-Provence, 1990 (Études Massaliètes 2).

Anderson 1954 : ANDERSON (J.K.) - Excavations on the Ridge of Kofinà in Chios. BSA, 49, 1954, p. 123-182.

Antico Polesine : L'antico Polesine. Testimonianze archeologiche e paleoambientali. Catalogo della Mostra, Adria - Rovigo, febbraio-novembre 1986. Padoue, 1986.

Aquilué Abadías et al. 2000 : AQUILUÉ ABADÍAS (X.A.), CASTANYER I MASOLIVER (P.), SANTOS RETOLAZA (M.), TREMOLADA I TRILLA (J.) - Les ceràmiques gregues arcaiques de la Palaià Polis d'Empòrion. In : Ceràmiques jònies, p. 285-346.

Arcelin 1990 : ARCELIN (P.) - La diffusion des amphores massaliètes en Provence occidentale. In : Amphores de Marseille grecque, p. 191-205.

Archeologia dell'Alto Adriatico : L'Archeologia dell'Alto Adriatico dalla Preistoria al Medioevo. Atti del Convegno Internazionale, Ravenna, 7-9 giugno 2001. Florence, 2003.

Arias 1951 : ARIAS (P.E.) - Considerazioni sulla città etrusca a Pian di Misano (Marzabotto). AttiMemBologna, n.s., IV, 1951, p. 221-234.

Arias 1953 : ARIAS (P.E.). FA, VIII, 1953, p. 168-169, n 2198

Arias 1954 : ARIAS (P.E.). FA, IX (1954), p. 214, n 2904.

Arribas et al. 1987 : ARRIBAS (A.), TRíAS (M.GL.), CERDá (D.), DE HOZ (J.) - El barco de El Sec. Estudio de los materiales, Mallorca, 1987.

ARV2 : BEAZLEY (J.D.) - Attic red-figure vase-painters, Oxford 1963 ( $2^{e}$ éd.).

Aurigemma 1936 : AURIGEMMA (S.) - Il R. Museo di Spina in Ferrara, Ferrara, $1936^{2}$.

Aurigemma 1960 : AURIGEMMA (S.) - Scavi di Spina. La necropoli di Spina in Valle Trebba, I, Rome, 1960.

Aurigemma 1965 : AURIGEMMA (S.) - Scavi di Spina. La necropoli di Spina in Valle Trebba, II, Rome 1965.

Avram 1989 : AVRAM (A.) - Wo sind die Amphoren vom Typ 'Solokha I‘ hergestellt worden ? Dacia, 33, 1989, p. 247-252.

Bacci, Tigano 1999 : BACCI (G.M.), TIGANO (G.) dir. - Da Zancle a Messina. Un percorso archeologico attraverso gli scavi, I, Palerme, 1999.

Bacci, Tigano 2002 : BACCI (G.M.), TIGANO (G.) dir. - Da Zancle a Messina. Un percorso archeologico attraverso gli scavi, II, 2, Messine, 2002.

Baldoni 1984 : BALDONI (D.) - Ceramica d'importazione a Spina : un gruppo di vasi corinzi. Musei Ferraresi, 12, 1982 (1984), p. 27-58.

Baldoni 2008 : BALDONI (V.) - Nuovi dati sulle prime importazioni attiche a figure nere di Marzabotto. Hesperia. Studi sulla grecità di Occidente, 22, 2008, p. 33-49. 
Baldoni 2009 : BALDONI (V.) - La ceramica attica dagli scavi ottocenteschi di Marzabotto, Bologne, 2009.

Baldoni, Morico, Pini 2007 : BALDONI (D.), MORICO (G.), PINI (L.) - Materiali per una stratigrafia abitativa di Felsina : lo scavo Bolognese di via Santa Caterina. In : Genti nel Delta da Spina a Comacchio: uomini, territorio e culto dall'antichità all'Alto Medioevo. Catalogo della mostra, Comacchio, 16 dicembre 2006-14 ottobre 2007. Ferrare, p. 49-107.

Barone et al. 2002 : BARONE (G.), IOPPOLO (S.), MAJOLINO (D.), MiGLIARDO (P.), SANNINO (L.), SPAGNOLO (G.), TIGANO (G.) - Contributo delle analisi archometriche allo studio delle ceramiche provenienti dagli scavi di Messina. Risultati preliminari. In : Bacci, Tigano 2002, p. 87-117.

Barra Bagnasco 1989b : BARRA BAGNASCO (M.) - Aspetti di vita quotidiana a Locri Epizefiri. In : BARRA BAGNASCO (M.) dir., Locri Epizefiri, III, Cultura materiale e vita quotidiana, Florence, 1989, p. 5-40.

Barra Bagnasco 1989a : BARRA BAGNASCO (M.) - Lo scavo. In : BARRA BAGNASCO (M.), Locri Epizefiri, II, Gli isolati 12 e I3 dell'area di Centocamere, Florence, 1989, p. 7-65.

Barra Bagnasco 1992 : BARRA BAGNASCO (M.) - Le anfore. In : BARRA BAGNASCO (M.) dir., Locri Epizefiri, IV, Lo scavo di Marasà Sud. Il sacello tardo-arcaico e la 'casa dei leoni', Florence, 1992, p. 205-240.

Barra Bagnasco 1993 : BARRA BAGNASCO (M.) - Due tipi di anfore di produzione locrese. Klearchos. Bollettino dell'Associazione amici del Museo nazionale di Reggio Calabria, 125-128, 1990 (1993), p. 29-48.

Barra Bagnasco 1995 : BARRA BAGNASCO (M.) - Anfore Locresi: documenti a favore di una produzione locale tra VI e IV sec. a.C. In : Estudis sobre ceràmica antiga. Actes del simposi sobre ceràmica antiga, Barcelona, 1993. Barcelone, 1995, p. 77-81.

Bats 1990a : BATS (M.) - Postface. In : Amphores de Marseille grecque, p. 289-291.

Bats 1990b : BATS (M.) - La diffusion des amphores massaliètes en Provence orientale. In: Amphores de Marseille grecque, p. 207-213.

Bats 1990c : BATS (M.) - En guise d'ouverture : quelques pistes de recherche. In : Amphores de Marseille grecque, p. 9-12.

Beazley 1951 : BEAZLEY (J.D.) - The development of Attic blackfigure, Londres, 1951.

Benoît 1955 : BENOÎT (F.) - Amphores grecques d'origine ou de provenance marseillaise. RStLig, 21, 1955, p. 32-43.

Benoît 1965 : BENOÎT (F.) - Recherches sur l'hellénisation du Midi de la Gaule, Aix-en-Provence 1965.

Bentz, Reusser 2004a : BENTZ (M.), REUSSER (C.) dir. - Attische Vasen in etruskischem Kontext. Funde aus Häusern und Heiligtümern, Munich, 2004 (Beihefte zum Corpus Vasorum Antiquorum, Deutschland, Band II).

Bentz, Reusser 2004b : BENTZ (M.), REUSSER (C.) - Keramik aus Marzabotto, Haus IV I, 2 - die alten Grabungen. In : Bentz, Reusser 2004a, p. 35-44.

Bentz, Reusser 2008 : BENTZ (M.), REUSSER (C.) - Marzabotto. Planstadt der Etrusker, Mainz am Rhein, 2008.

Bentz, Reusser 2011 : BENTZ (M.), REUSSER (C.) - Das Haus der Hippokampen in Marzabotto. In : BENTZ (M.), REUSSER (C.) dir. Etruskisch-italische und römisch-republikanische Häuser, Wiesbaden, 2011, p. 105-116.

Bereti 1992 : BERETI (V.) - Amfora transporti te zbuluara ne vendbanimin e Treportit. Iliria, 22/1-2, 1992, p. 129-147.

Berlingò 1986 : BERLINGÒ (I.) - La necropoli arcaica di Policoro in Contrada Madannelle. In : Siris-Polieion. Fonti letterarie e nuova documentazione archeologica. Atti dell'Incontro di Studi, Policoro, 8-10 giugno 1984. Galatina, 1986, p. 117-127.
Berlingò 1993 : BERLINGÒ (I.) - Le necropoli di Siris. BA, 22, 1993 p. 1-21.

Berlingò 1995 : BERLINGÒ (I.) - Importazioni corinzie nella Siritide. In : Corinto e l'Occidente, I, p. 417-430.

Bermond Montanari 1987 : BERMOND MONTANARI (G.) - [notices du catalogue]. In : Malnati 1987, p. 129-130.

Bermond Montanari 2003 : Bermond montanari (G.) - La circolazione della ceramica greca nell'Adriatico settentrionale durante il VI sec. a.C. In : Alto e Medio Adriatico, p. 29-33.

Berti 1985 : BERTI (F.) - Spina. L'abitato alla luce degli ultimi scavi. In : Romagna tra VI e IV secolo, p. 189-196.

Berti 1993 : BERTI (F.) - Appunti per Valle Trebba. In : Spina, p. 33-45.

Bertucchi 1990 : BERTUCCHI (G.) - Les amphores massaliètes à Marseille : les différentes productions. In : Amphores de Marseille grecque, p. 15-20.

Bertucchi 1992 : BERTUCCHI (G.) - Les amphores et le vin de Marseille. $V I^{e}$ s. avant J.-C.-II ${ }^{e}$ s. après J.-C., Paris, 1992 (RANarb Supplément 25).

Biers 1980 : BIERS (W.R.) - The Archaeology of Greece. An Introduction, Ithaque - Londres, 1980.

Blondé, Muller, Mulliez 1991 : BLONDÉ (F.), MULLER (A.), MULLIEZ (D.) - Le comblement d'un puits public à Thasos, 5. Le matériel amphorique. $B C H, 115 / 2,1991$, p. 213-242.

Boardman 1967 : BOARDMAN (J.) - Excavations in Chios 1952-1955. Greek Emporio, Londres, 1967 (BSA Suppl. 6).

Boardman 1970 : BOARDMAN (J.) - Greek gems and finger rings. Early Bronze Age to Classical, Londres, 1970.

Boardman 1979 : BOARDMAN (J.) - The Athenian Pottery Trade. The Classical Period. Expedition, 21/4, 1979, p. 33-39.

Boitani 1985 : BOITANI (F.) - Cenni sulla distribuzione delle anfore da trasporto arcaiche nelle necropoli dell'Etruria meridionale. In : Commercio etrusco arcaico, p. 23-25.

Bon, Bon 1957 : BON (A.M.), BON (A.) - Les timbres amphoriques de Thasos, Paris, 1957.

Bonamici 1989 : BONAMICI (M.) - Contributo a Pisa arcaica. In : Secondo Congresso Internazionale Etrusco, II, p. 1135-1147.

Bonamici 1996 : BONAMICI (M.) - ApÕ dû Ant...ou Turrhno^ œqnoj... (Ps. Skyl., 5). Contributo alle rotte arcaiche nell'Alto Tirreno. StEtr, 61, 1995 (1996), p. 3-43.

Bonghi Jovino 1982 : BONGHI JOVINO (M.) - La necropoli preromana di Vico Equense, Cava dei Tirreni, 1982.

Bonghi Jovino, Donceel 1969 : BONGHI JOVINO (M.), DONCEEL (R.) La necropoli di Nola preromana, Naples, 1969.

Bonomi 1988 : BONOMI (S.) - La ceramica greca di Adria. In : Etruschi a nord del Po, II, p. 67-83.

Bonomi 1991 : BONOMI (S.) - Corpus Vasorum Antiquorum. Italia, LXV, Adria, Museo Archeologico Nazionale, Roma 1991.

Bonomi 1995 : BONOMI (S.) - Adria (Rovigo). StEtr, 60, 1995, p. 509-510.

Bonomi 1997 : BONOMI (S.) - Adria : la necropoli di Cà Cima. Beni Culturali e Ambientali in Polesine, 1, 1997, p. 31-34.

Bonomi 2000a : BONOMI (S.) - Ceramiche d'importazione nel Veneto prima del 550 a.C. In : Adriatico greco, p. 119-123.

Bonomi 2000b : BONOMI (S.) - Ceramica attica da corredi tombali del IV sec. a.C. di Adria. In : SABATTINI (B.) dir., La céramique attique du $I V^{e}$ siècle en Méditerranéenne occidentale. Actes du Colloque International, Arles, 1995. Napoli, 2000, p. 93-98.

Bonomi 2003 : BONOMI (S.) - Recenti rinvenimenti archeologici nell'Alto Adriatico tra la fine del VII e il IV sec. a.C. : nuovi dati. In : Archeologia dell'Alto Adriatico, p. 140-145.

Bonomi 2004 : BONOMI (S.) - Padova : nuovi dati sulle importazioni di ceramica greca. In : Greci in Adriatico, p. 211-214. 
Bonomi, Camerin, Tamassia 2002 : BONOMI (S.), CAMERIN (N.), TAMASSIA (K.) dir. - Etruschi adriati, Rovigo, 2002.

Bonomi, Camerin, Tamassia 2003 : BONOMI (S.), CAMERIN (N.), TAMASSIA (K.) - Adria, Via San Francesco, Scavo 1994 : materiali dagli strati arcaici. In : Alto e Medio Adriatico, p. 201-213.

Börker 1986 : BÖRKER (C.) - Die Herkunft der Schiffsbug-Stempel. In : Recherches sur les amphores, p. 473-478.

Boss 1993 : BOSS (M.) - Die Transportamphoren. In : CRISTOFANI (M.) dir., Caere, 3.2, Lo scarico arcaico della Vigna Parrocchiale, Roma, 1993, p. 319-348.

Bouloumiè 1976 : BOULOUMIÈ (B.) - La céramique locale de Marzabotto : définition de quelques groupes. MEFRA, 88/1, 1976, p. 95-140.

Bouloumiè 1982a : BOULOUMIÈ (B.) - Saint-Blaise (Fouilles H. Rolland). L'habitat protohistorique. Les céramiques grecques, Aix-enProvence, 1992.

Bouloumiè 1982b : BOULOUMIÈ (B.) - L'épave étrusque d'Antibes et le commerce en Méditerranée occidentale au VIe siècle av. J-C., Marburg, 1982.

Bouloumiè 1982c : BOULOUMIÈ (B.) - Saint-Blaise et Marseille au VI ${ }^{\mathrm{e}}$ siècle av. J. C. L'hypothèse étrusque. Latomus, 41, 1982, p. 74-91.

Boulter 1953 : BOULTER (C.) - Pottery of the Mid-Fifth Century from a Well in the Athenian Agora. Hesperia, 22/2, 1953, p. 59-115.

Bound 1985 : BOUND (M.) - Una nave mercantile di età arcaica all'isola del Giglio. In : Commercio etrusco arcaico, p. 65-70.

Braccesi 1977 : BRACCESI (L.) - Grecità adriatica, Bologne 1977 (2éd.).

Braccesi, Coppola 1993 : BRACCESI (L.), COPPOLA (A.) - I Greci descrivono Spina. In : Spina, p. 71-79.

Brašinskij 1965 : BRAŠINSKIJ (I.B.) - Novye dokumenty k datirovke kurganov skiphskoy plemennoy znati severnogo Prichernomor'ya. Eirene, 4 , 1965, p. 89-110.

Brašinskij 1976 : BRAŠINSKIJ (I.B.) - Amphori Mendi. In : SOKOL'SKII (N.I.), BRAŠINSKIJ (I.B.), GORBUNOVA (K.S.), SCHULTZ (P.N.) dir., Khudozhestvennaya kul'tura i arkheologiya antichnogo mira. Sbornik pamiati B.V. Pharmakovskogo, Moscou, 1976, p. 67-74.

Brašinskij 1980 : BRAŠINSKIJ (I.B.), Grečeskiy keramičeskiy import na Nižnem Donu v v-iii vv. do n.e. [The ceramic imports into the Lower Don from the 5th to the 3rd centuries BC], Leningrad, 1980.

Brašinskij 1984 : BRAŠINSKIJ (I.B.), Metody issledovanija antičnoj torgovli (na primere severnogo Pričernomor'ja), Leningrad, 1984.

Brašinskij, Marčenko 1984 : BRAŠINSKIJ (I.B.), MARČENKO (K.K.) - Elisavetovskoje. Skythische Stadt im Don-Delta, Munich 1984 (Materialien zur Allgemeinen und Vergleichenden Archäologie 27).

Brizio 1886 : BRIZIO (E.), Guida alle antichità della villa e del Museo Etrusco di Marzabotto, Bologne, 1886.

Brizzolara, Baldoni, Succi 2005 : BRIZZOLARA (A.M.), BALDONI (V.), SUCCI (V.), Anticipazioni sulla ceramica attica della casa di testa della Regio IV, insula 2. In : Culti, forma urbana e artigianato a Marzabotto, p. 167-190.

Broneer 1938 : BRONEER (O.) - Excavations on the North Slope of the Acropolis, 1937. Hesperia, 7, 1938, p. 161-263.

Bruni 1993 : BRUNI (S.) dir., Pisa. Piazza Dante : uno spaccato della storia pisana. La campagna di scavo 1991, Pontedera, 1993.

Bruni 1994 : BRUNI (S.) - L'Etruria tirrenica e il territorio del Delta del Po. Appunto su una kotyle da San Basilio di Ariano Polesine. Padusa, n.s., 30, 1994, p. 187-193.

Bruni 1998 : BRUNI (S.) - Un problematico documento per la storia della frequentazione dell'area spinetica prima di Spina. Appunti sulle rotte adriatiche in età arcaica. In : Spina e il delta padano, p. 203-223.

Bruni 2004 : BRUNI (S.) - Spina e la ceramica greca. Alcune considerazioni. In : BERTI (F.), HARARI (M.) dir, Storia di Ferrara, II. Spina tra archeologia e storia, Ferrare 2004, p. 78-116.
Bruschetti 1993 : BRUSCHETTI (P.) dir., Corciano. Testimonianza archeologiche, Pérouse, 1993.

Buchner, Ridgway 1993 : BUCHNER (G.), RIDGWAY (D.) Pithecoussai, I, Tombe 1-723. Scavate dal 1952 al 1961, Rome, 1993.

Bulle 1934 : BULLE (H.) - Ausgrabungen bei Aphionia auf Corfu. AM, 59, 1934, p. 147-240.

Cabrera, Rouillard 2003 : CABRERA (P.), ROUILLARD (P.) - L'épave d'El Sec, dans la baie de Palma de Majorque (milieu di IVe siècle av. J.-C.). In : ROUILLARD (P.), VERBANCK-PIÉRARD (A.) dir., Le vase grec et ses destins, Monaco, 2003, p. 125-131.

Calastri, Desantis 2011 : CALASTRI (C.), DESANTIS (P.) - Lo scavo di viale Aldini. In : CURINA (R.), MALNATI (L.), NEGRELLI (C.), PINI, (L.) dir., Alla ricerca di Bologna antica e medievale, Florence, 2011, p. 191-208.

Carlson 2003 : CARLSON (D.N.) - The Classical Greek Shipwreck at Tektaş Burnu. AJA, 107/4, 2003, p. 581-600.

Carlson, Lawall 2007 : CARLSON (D.N.), LAWALL (M.L.) - Towards a Typology of Erythraian Amphoras. Skyllis, 7, 2005-2006 (2007), p. 32-39.

Campbell 1938 : CAMPBELL (M.) - A well of the black-figure period at Corinth. Hesperia, 7, 1938, p. 557-611.

Camerin 1993 : CAMERIN (N.) - Alcune tipologie tombali. In : Spina, p. $267-272$.

Cattaneo Cassano 1995 : CATTANEO CASSANO (A.C.) - Anfore greche da trasporto del VI-V sec. a.C. in Italia settentrionale. StClOr, 43, 1993 (1995), p. 381-398.

Cattaneo 1996 : CATTANEO (A.C.) - Adria e S. Basilio: le anfore greche da trasporto. In : Cattaneo, de Marinis 1996, p. 317-329.

Cattaneo, de Marinis 1996 : CATTANEO (A.C.), DE MARINIS (R.C.) - Le anfore greche da trasporto di Adria, S. Basilio e del Forcello di Bagnolo S. Vito e il commercio del vino nell'Italia padana all'epoca della colonizzazione etrusca. In : FORNI (G.), SCIENZA (A.) dir., 2500 anni di cultura della vite nell'ambito alpino e cisalpino, Trente, 1996, p. 317-347.

Cavagnera 1995 : CAVAGNERA (L.) - Anfore commerciali. In : Ricerche archeologiche all'Incoronata di Metaponto, 3, L'oikos greco del saggio S. Lo scavo e i reperti, Milan, 1995, p. 41-55.

Cavalier 1985 : CAVALIER (M.) - Les amphores du VI ${ }^{\mathrm{e}}$ au IVe siècle dans les fouilles de Lipari, Naples, 1985 (Cahiers du Centre Jean Bérard XI).

Ceka 1983 : CEKA (N.) - Processi di trasformazione nell'Illiria del Sud durante il periodo arcaico. In : Forme di contatto e processi di trasformazione nelle società antiche. Atti del Convegno, Cortona, 24-30 maggio 1981. Pise - Rome, 1983, p. 203-218.

Ceka 1986 : CEKA (N.) - Amfora antike nga Margëlliçi. Iliria, 16/2, 1986, p. 71-98.

Ceràmiques jònies : CABRERA BONET (P.) SANTOS RETOLAZA (M.) dir., Ceràmiques jònies d'època arcaica : centres de producció i comercialització al Mediterrani occidental. Actes de la Taula rodona celebrada a Empúries, els dies 26 al 28 de maig de 1999. Barcelone, 2000 [Copyright 2001].

Cerchiai 1981 : CERCHIAI (L.) - Un corredo arcaico da Pontecagnano. AIONArch, 3, 1981, p. 29-53.

Cerdá 1987 : CERDÁ (D.) - Las anforas de la nave de El Sec. In : Arribas et al. 1987, p. 401-499.

Cerdá 1989 : CERDÁ (D.) - La ceramica antica de barniz negro y las anforas. In : ROUILLARD (P.), VILLANUEVA-PUIG (M.-L.) dir., Grecs et Ibères au IVe siècle av. J.-C. Commerce et iconographie. Actes de la Table Ronde, Bordeaux, 16-17-18 décembre 1986. Paris, 1989, p. 51-96.

Charlin, Gassend, Lequément 1979 : CHARLIN (G.), GASSEND (J.-M.), LEQUÉMENT (R.) - L'épave antique de la baie de Cavalière (Le Lavandou, Var). Archaeonautica, 2, 1978 (1979), p. 9-93.

Ciampoltrini 1994 : CIAMPOLTRINI (G.) - Aspetti dell'insediamento etrusco nella Valle del Serchio: il V secolo a.C. StEtr, 59, 1993 (1994), p. 59-85. 
Ciampoltrini, Notini 1987 : CIAMPOLTRINI (G.), NOTINI (P.) - Un insediamento etrusco nell'Alta Valle del Serchio. StEtr, 53, 1985 (1987), p. 65-75.

Cibecchini 2007 : CIBECCHINI (F.) - The unsolved Question of the Greco-italic Amphorae. Some Solutions from Shipwrecks. Skyllis, 7, 20052006 (2007), p. 50-58.

Cisneros Garcia et al. 2000 : CISNEROS GARCIA (I.), SUÁREZ PADILLA (J.), MAYORGA MAYORGA (J.) - Cerámicas griegas arcaicas en la Bahía de Mílaga. In : Ceràmiques jònies, p. 189-205.

Ciurletti 1996 : CIURLETTI (G.) - La vitivinicoltura nel Trentino Alto Adige fra preistoria ed età romana. Contributi archeologici. In : FORNI (G.), SCIENZA (A.) dir., 2500 anni di cultura della vite nell'ambito alpino e cisalpino, Trente, 1996, p. 441-470.

Colonna 1974 : COLONNA (G.) - I Greci d'Adria. RStorAnt, 4, 1974, p. 1-21.

Colonna 1985 : COLONNA (G.) - Anfore da trasporto arcaiche: il contributo di Pyrgi. In : Commercio etrusco arcaico, p. 5-18.

Colonna 1993 : COLONNA (G.), La società spinetica e gli altri ethne. In : Spina, p. 131-143.

Colonna 2003 : COLONNA (G.) - L'Adriatico tra VIII e inizi V sec. a.C. con particolare riguardo al ruolo di Adria. In : Archeologia dell'Alto Adriatico, p. 146-175.

Commercio etrusco arcaico : Il commercio etrusco arcaico, Actes du colloque de Rome (5-7 décembre 1983), Rome, 1985. Atti dell'Incontro di Studio, Roma 1983. Rome, 1985 (Quaderni del Centro di studio per l'archeologia etrusco-italica 9).

Commerci e produzione : Commerci e produzione in età antica nella fascia costiera fra Ravenna e Adria. Atti della Giornata di Studio, Ferrara, 21 giugno 2001. Ferrare, 2002.

Condurachi 1954 : CONDURACHI (E.), Histria, I, Bucuresti, 1954.

Condurachi 1966 : CONDURACHI (E.), Histria, II, Bucuresti, 1966.

Cook, Dupont 1998 : COOK (R.M.), DUPONT (P.) - East Greek Pottery, Londres, 1998.

Corchia, Pancrazzi, Tagliente 1982 : CORCHIA (R.), PANCRAZZI (O.), TAGLIENTE (M.) - Cavallino, settore C dell'abitato. Fondo 'Aiera Vecchia'. Relazione preliminare. StAnt, 3, 1982, p. 5-61.

Corinto e l'Occidente : Corinto e l'Occidente. Atti del XXXIV Convegno di Studi sulla Magna Grecia, Taranto, 7-11 ottobre 1994. Tarente, 1995, I-II.

Cristofani 1985 : CRISTOFANI (M.) - Gli Etruschi del mare, Firenze, 1985.

Cristofani 1995 : CRISTOFANI (M.) - Novità sul commercio etrusco arcaico : dal relitto del Giglio al contratto di Pech Maho. In : Italy in Europe: economic Relations 700. BC-AD 50. Sixteenth British Museum Classical Colloquium, London, 8-10 December 1992, Londres, 1995, p. 131-137.

Culti, forma urbana e artigianato a Marzabotto : SASSATELLI (G.), GOVI (E.) dir. - Culti, forma urbana e artigianato a Marzabotto. Nuove prospettive di ricerca. Atti del Convegno di Studi, Bologna, 3-4 giugno 2003. Bologne, 2005.

Curti 1993 : CURTI (F.) - Contributo allo studio dei contatti commerciali tra Spina ed Atene nel IV secolo a.C.: la ceramica figurata attica. In : Studi sulla necropoli di Spina, p. 135-154.

Cygielman, De Tommaso 1998 : CYGIELMAN (M.), DE TOMMASO (G.) - Castiglione della Pescaia-Punta Ala. In : POGGESI (G.), RENDINI (P.) dir., Memorie sommerse. Archeologia subacquea in Toscana. Catalogo della Mostra, Monte Argentario - Porto Santo Stefano, 1997. Pitigliano, 1998, p. 91-123.

d'Agostino, Gastaldi 2002 : D'AGOSTINO (B.), GASTALDI (P.) Ricerche archeologiche a Pale (Cefalonia). ASAtene, 80/1, 2002, p. 123-181.

d'Agostino, Soteriou 1998 : D'AGOSTINO (B.), SOTERIOU (A.) Campania in the framework of the earliest Greek colonization in the West. In :
BATS (M.), D’AGOSTINO (B.) dir., Euboica. L'Eubea e la presenza euboica in Calcidica e in Occidente. Atti del Convegno Internazionale, Napoli, 13-16 novembre 1996. Naples, 1998, p. 355-368 (AIONArch Quaderno 12).

D'Andria 1995 : D'ANDRIA (F.) - Corinto e l'Occidente: la costa adriatica. In : Corinto e l'Occidente, I, p. 457-508.

D'Ercole 2006 : D'ERCOLE (M.C.) - Itinerari e scambi nell'Adriatico preromano (VIII-V sec. a.C.). In : KURILIC (A.), TASSAUX (F.) dir., Les routes maritimes de l'Adriatique romaine. Actes de la table ronde, Zadar 2001. Bordeaux - Zadar, 2006, p. 91-106.

De Caro, Gialanella 1998 : DE CARO (S.), GIALANELLA (C.) Novità pitecusane. L'insediamento di Punta Chiarito a Forio d'Ischia. In : BATS (M.), D'AGOSTINO (B.) dir., Euboica. L'Eubea e la presenza euboica in Calcidica e in Occidente. Atti del Convegno Internazionale, Napoli, 13-16 novembre 1996. Naples, 1998, p. 337-353 (AIONArch Quaderno 12).

De Luca De Marco 1979 : DE LUCA DE MARCO (S.) - Le anfore commerciali della necropoli di Spina. MEFRA 91/2, 1979, p. 571-600.

De Maria, Gjongecaj 2007 : DE MARIA (S.), GJONGECAJ (S.) dir., Phoinike IV. Rapporto preliminare sulle campagne di scavi e ricerche 20042006, Bologna, 2007.

De Marinis 1982 : DE MARINIS (R.C.) - Bagnolo S. Vito, loc. Forcello. NotALomb, 1982, p. 32.

De Marinis 1987 : DE MARINIS (R.C.) - Bagnolo S.Vito, loc. Forcello. Scavi nell'abitato etrusco. NotALomb, 1987, p. 34-37.

De Marinis 1988 : DE MARINIS (R.C.) - Le anfore greche da trasporto. In : Etruschi a nord del Po, I, p. 211-229.

De Marinis 1989 : DE MARINIS (R.C.) - Problemi e prospettive della ricerca protostorica nel mantovano. In : Gli Etruschi a nord del Po. Atti del Convegno di Studi, Mantova, 4-5 ottobre 1986. Mantoue, 1989, p. 27-47.

De Marinis 1990 : DE MARINIS (R.C.) - Bagnolo S. Vito, loc. Forcello. NotALomb, 1988-1989 (1990), p. 38-40.

De Marinis 1991a : DE MARINIS (R.C.) - L'abitato etrusco del Forcello : opere di difesa e di drenaggio e importanza delle vie fluviali. In : BERGAMINI (M.) dir., Gli Etruschi maestri d'idraulica, Venezia, 1991, p. 75-85.

De Marinis 1991b : DE MARINIS (R.C.) - La stratigrafia dell'abitato del Forcello di Bagnolo S. Vito e i rapporti cronologici con le culture dell'area circumalpina. In : Miscellanea etrusca e italica in onore di Massimo Pallottino, p. 237-259 (ArchCl, XLIII/1, 1991).

De Marinis 1994 : DE MARINIS (R.C.) - L'anfora corinzia della tomba 1. In : Balone. Insediamento etrusco presso un ramo de Po, Padoue, 1994, p. $75-80$.

De Marinis 1996 : DE MARINIS (R.C.) - Il Forcello e il commercio delle anfore da trasporto greche nell'Etruria padana. In: Cattaneo, De Marinis 1996, p. 330-347.

De Marinis 1999 : DE MARINIS (R.C.) - Anfore chiote dal Forcello di Bagnolo S. Vito (Mantova). In : CASTOLDI (M.) dir., Koina. Miscellanea di studi archeologici in onore di Piero Orlandini, Milan, 1999, p. 255-278.

De Marinis 2005 : DE MARINIS (R.C.) - Le anfore greche da trasporto. In : de Marinis, Rapi 2005, p. 165-201.

De Marinis 2007 : DE MARINIS (R.C.) - Le anfore greche da trasporto. In : de Marinis, Rapi 2007, p. 157-189.

De Marinis 2010 : DE MARINIS (R.C.) - Anfore greche da trasporto. In : GOVI (E.), SASSATELLI (G.) dir., Marzabotto. La casa 1 della Regio IV-Insula 2. 2, I materiali, Bologne, 2010, p. 77-88.

De Marinis, Rapi 2005 : DE MARINIS (R.C.), RAPI (M.) dir. L'abitato etrusco del Forcello di Bagnolo S. Vito (Mantova). Le fasi di età arcaica, Mantoue, 2005.

De Marinis, Rapi 2007 : DE MARINIS (R.C.), RAPI (M.) dir. L'abitato etrusco del Forcello di Bagnolo S. Vito (Mantova). Le fasi di età arcaica, Florence, 2007. 
De Min 1984 : DE MIN (M.) - Adria antica. In : Il Veneto nell'antichità, II, Vérone, p. 809-830.

De Min 1988 : DE MIN (M.) - L'abitato arcaico di S. Basilio. In : Etruschi a nord del Po, II, p. 84-91.

De Min, Iacopazzi 1986 : DE MIN (M.), IACOPAZZI (E.) - L'abitato arcaico di S. Basilio di Ariano Polesine. In : Antico Polesine, p. 171-184.

Demesticha 2011 : DEMESTICHA (S.) - The 4th-Century-BC Mazotos Shipwreck, Cyprus : a preliminary report. IntJNautA, 40/1, 2011, p. 39-59.

Desantis 1989 : DESANTIS (P.) - Anfore commerciali dall'abitato di Spina dal V al III sec. a.C. : appunti preliminari. In : Gli Etruschi a nord del Po. Atti del Convegno di Studi, Mantova, 4-5 ottobre 1986, Mantoue, 1989, p. 103-127.

Desantis 1993 : DESANTIS (P.) - Le anfore commerciali della necropoli di Spina: Valle Trebba. In : Studi sulla necropoli di Spina, p. 155-180.

Desantis 1996 : DESANTIS (P.) - Anfore commerciali ed altri particolari vasi vinari nei corredi funebri della necropoli di Spina-Valle Trebba dal V al III sec. a.C. In : FORNI (G.), SCIENZA (A.) dir., 2500 anni di cultura della vite nell'ambito alpino e cisalpino, Trente, 1996, p. 346-372.

Desy 1982 : DESY (Ph.) - À propos d'une première synthèse sur les amphores corinthiennes. AntCl, 51, 1982, p. 285-290.

Desy, De Paepe 1990 : DESY (Ph.), DE PAEPE (P.) - Torre San Giovanni (Ugento): les amphores commerciales hellénistiques et républicaines. StAnt, 6, 1990, p. 187-233.

Di Sandro 1981a : DI SANDRO (N.) - Appendice. Le anfore 'massaliote' in Campania. In : Cerchiai 1981, p. 49-53.

Di Sandro 1981b : DI SANDRO (N.) - Appunti sulla distribuzione delle anfore commerciali greche in Campania tra l'VIII sec. e il 273 a.C. AIONArch, 3, 1981, p. 1-14.

Di Sandro 1986 : DI SANDRO (N.) - Le anfore arcaiche dallo scarico Gosetti, Pithecusa, Naples, 1986 (Cahiers du Centre Jean Bérard XII).

Di Stefano 1995 : DI STEFANO (G.) - Il relitto di Punta Braccetto (Camarina), gli emporia e i relitti di età arcaica lungo la costa meridionale della Sicilia. Kokalos, 39-40, 1993-1994 (1995), p. 111-133.

Docter 1991 : DOCTER (R.F.) - Athena vs Dionysos. Reconsidering the contents of SOS amphorae. BABesch, 66, 1991, p. 45-49.

Docter 2000 : Docter (R.F.) - East Greek fine Wares and Transport Amphorae of the 8th-5th Century BC from Carthage and Toscanos. In : Ceràmiques jònies, p. 63-88.

Docter 2007 : Docter (R.F.) - Archaische Transportamphoren. In : NIEMEYER (H.G.), DOCTER (R.F.), SCHMIDT (K.), BECHTOLD (B.) dir., Karthago. Die Ergebnisse der Hamburger Grabung unter dem Decumanus Maximus, II, Mainz am Rheim, 2007, p. 616-662 (Hamburger Forschungen zur Archäologie 2).

Docter sous presse : DOCTER (R.F.) - Amphorae and Pithoi. In : HADZIS (K.), NANAJ (A.), NEEFT (C.W.) dir., Bouthrotos I. The pottery, sous presse.

Doğer 1986 : DOĞER (E.) - Premières remarques sur les amphores de Clazomènes. In : Recherches sur les amphores, p. 461-471.

Donati, Parrini 1999 : DONATI (L.), PARRINI (A.) - Resti di abitazione d'età arcaica ad Adria. Gli scavi di Francesco Antonio Bocchi nel Giardino Pubblico. In : Protostoria e storia del Venetorum angulus, p. 567-614.

Doulgéri-Intzessiloglou, Garlan 1990 : DOULGÉRI-INTZESSILOGLOU (A.), GARLAN (Y.) - Vin et amphores de Péparéthos et d'Ikos. BCH, 114/1, 1990, p. 362-393.

Drakidès et al. 2010 : DRAKIDÈS (D.), NANTET (E.), GRAS (M.), ESPOSITO (A.) - Échanges et circulations. In : ÉTIENNE (R.) dir., $L a$ Méditerranée au VI siècle av. J.-C., Paris, 2010, p. 91-146.

Dupont 1982 : DUPONT (P.) - Amphores commerciales archaïques de la Grèce de l'Est. In : I Focei dall'Anatolia all'Oceano, p. 193-209.
Dupont 1983 : DUPONT (P.) - Classification et détermination de provenance des céramiques grecques orientales archaïques d'Istros. Rapport préliminaire. Dacia, 27, 1983, p. 19-43.

Dupont 1986 : DUPONT (P.) - Naturwissenschaftliche Bestimmung der archaische Keramik Milets. In : Milet 1899-1980. Ergebnisse, Probleme und Perspektiven einer Ausgrabung. Kolloquium (Frankfurt am Main, 1980), Tübingen, 1986, p. 57-71 (Istanbuler Mitteilungen. Beihefte, 31).

Dupont 1998 : DUPONT (P.) - Archaic East Greek Trade Amphoras. In : Cook, Dupont 1998, p. 142-191.

Dupont 1999 : DUPONT (P.) - La circulation amphorique en Mer Noire à l'époque archaïque. Spécificité et problèmes. In : Production et commerce, p. 143-161.

Dupont 2000 : DUPONT (P.) - Amphores samiennes archaïques : sources de confusion et questionnement. In : Ceràmiques jònies, p. 57-61.

Dupont 2002 : DUPONT (P.) - Amphores vinaires et vases à boire : reconsidération du binôme samien archaïque. In : KACHARAVA (D.D.), FAUDOT (M.), GENY (E.) dir., Autour de la mer Noire. Hommage à Otar Lordkipanidzé, Besançon, 2002, p. 65-70.

Dupont 2007 : DUPONT (P.) - Diffusion des amphores commerciales de type milésien dans le Pont archaïque. In : Frühes Ionien, eine Bestandsaufnahme (Panionion-Symposion, Güzelçamli, 1999), Mainz am Rhein, 2007, p. 621-630 (Milesische Forschungen 5).

Dupont 2010 : DUPONT (P.) - Les amphores 'samiennes' et 'protothasiennes' de Zeest : la piste nord-ionienne ? In : INAISHVILI (N.), KASSAB (D.) dir., Patabs I. Production and Trade of Amphorae in the Black Sea. Actes de la Table Ronde internationale de Batoumi et Trabzon, 27-29 Avril 2006. Paris, 2010, p. 39-43 (Varia Anatolica XXI).

Eiseman 1973 : EISEMAN (C.J.) - Amphoras from the Porticello Shipwreck (Calabria). IntJNautA, 2, 1973, p. 13-23.

Eiseman 1975 : EISEMAN (C.J.) - Classical inkpots. AJA, 79, 1975, p. 374-375.

Eiseman 1979 : EISEMAN (C.J.) - The Porticello shipwreck : lead isotope. IntJNautA, 8, 1979, p. 339-340.

Eiseman, Ridgway 1987 : EISEMAN (C.J.), RIDGWAY (B.) - The Porticello shipwreck : a Mediterranean merchant vessel of 415-385 B.C., College Station, 1987.

Empereur, Garlan 1987 : EMPEREUR (J.-Y.), GARLAN (Y.) - Bulletin archéologique: amphores et timbres amphoriques (1980-1986). REG, 100, 1987, p. 58-109.

Empereur, Garlan 1992 : EMPEREUR (J.-Y.), GARLAN (Y.) - Bulletin archéologique : amphores et timbres amphoriques (1987-1991). REG, 105, 1992, p. 176-220.

Empereur, Garlan 1997 : EMPEREUR (J.-Y.), GARLAN (Y.) - Bulletin archéologique : amphores et timbres amphoriques (1992-1996). REG, 110, 1997, p. 161-209.

Empereur, Picon 1986 : EMPEREUR (J.-Y.), PICON (M.) - à la recherche des fours d'amphores. In: Recherches sur les amphores, p. 103-126.

Etruria mineraria : NEPPI MODONA (A.) dir., L'Etruria mineraria. Atti del XII Convegno di Studi Etruschi e Italici, Firenze - Populonia Piombino, 16-20 Giugno 1979. Florence, 1981.

Etruschi a nord del Po : DE MARINIS (R.C.) dir. - Gli Etruschi a nord del Po, I-II. Catalogo della Mostra, Manova, 1986-1987. Udine, 1988 (2éd.).

Etruschi e il Mediterraneo : Gli Etruschi e il Mediterraneo : commerci e politica. Atti del XIII Convegno internazionale di Studi sulla Storia e l'Archeologia dell'Etruria, Orvieto 2005. Rome, 2006 (AnnFaina XIII).

Etruscorum ante quam Ligurum : PARIBENI (E.) dir., Etruscorum ante quam Ligurum. La Versilia tra VII e III secolo a.C., Catalogo della Mostra, Pietrasanta, 1989. Pontedera, 1990

Farmakovskiy 1914 : FARMAKOVSKIY (B.V.) - Archäologische Funde in Jahre 1913. AA, 1914, p. 260-290. 
Farnsworth 1964 : FARNSWORTH (M.) - Greek pottery : a mineralogical study. AJA, 68, 1964, p. 221-228.

Farnsworth 1970 : FARNSWORTH (M.) - Corinthian pottery : technical studies. AJA, 74, 1970, p. 9-20.

Farnsworth, Perlman, Asaro 1977 - FARNSWORTH (M.), PERLMAN (I.), ASARO (F.) - Corinth and Corfu. A neutron Activation Study of their Pottery. AJA, 81, 1977, p. 45-469.

Fernández, Gomez, Ribera 1988 : FERNÁNDEZ (A.), GOMEZ (C.), RIBERA (A.), Las amphoras griegas, etruscas y fenico-punicas en las costas del Pais Valenciano. In : Flotte e commercio greco, p. 317-333.

Fiorentini 1990 : FIORENTINI (G.) - La nave di Gela e osservazioni sul carico residuo. QuadAMess, 5, 1990, p. 25-39.

Flotte e commercio greco : Flotte e commercio greco, cartaginese ed etrusco nel Mar Tirreno. Atti del Simposio Europeo, Ravello, gennaio 1987. Court-Saint-Etienne, 1988 (Pact, 20).

Focei dall'Anatolia all'Oceano : I Focei dall'Anatolia all'Oceano. Atti del Colloquio, Napoli, dicembre 1981), Naples, 1982 (PP, 37).

Formazione della città in Emilia Romagna : La formazione della città in Emilia Romagna: prime esperienze urbane attraverso le nuove scoperte archeologiche. Catalogo della Mostra, Bologna, 26 Settembre 1987-24 Gennaio 1988, I-III, Bologna, 1987.

Formazione della città preromana : La formazione della città preromana in Emilia Romagna. Atti del Convegno di Studi, Bologna - Marzabotto, 7-8 Dicembre 1985. Imola, 1988.

Fortunelli 2007 : FORTUNELLI (S.) - Gravisca. Scavi nel santuario greco. Il deposito votivo del santuario settentrionale, Bari, 2007.

Freschi 1991 : FRESCHI (A.) - Note tecniche sul relitto greco arcaico di Gela. In : IV rassegna di archeologia subacquea. IV premio Franco Papò. Atti del Convegno di Studi, Giardini Naxos, 13-15 ottobre 1989, Messina, 1991, p. 201-210.

Gailledrat, Taffanel, Taffanel 2002 : GAILLEDRAT (E.), TAFFANEL (O.), TAFFANEL (J.), dir. - Le Cayla de Mailhac (Aude). Les niveaux du premier âge du Fer (VIe-Ve s. av. J.-C.), Lattes, 2002 (Monographies d'Archéologie Méditerranéenne 12).

Gailledrat, Solier 2004 : GAILLEDRAT (E.), SOLIER (Y.) dir. L'établissement côtier de Pech Maho (Sigean, Aude) aux VIe-Ve s. av. J.-C. (fouilles 1959-1979), Lattes, 2004 (Monographies d'Archéologie Méditerranéenne 19).

Gambari 1993 : GAMBARI (F.M.) - Il bucchero etrusco nei contesti piemontesi della prima età del Ferro. In : Produzione artigianale ed esportazione nel mondo antico. Il bucchero etrusco. Atti del Colloquio Internazionale, Milano, 10-11 maggio 1990, Milan, 1993, p. 127-134.

Garlan 1979 : GARLAN (Y.) - Koukos. In : Thasiaca, Paris, 1979, p. 213-268 (BCH Supplément 5).

Garlan 1986 : GARLAN (Y.) - Quelques nouveaux ateliers amphoriques à Thasos. In : Recherches sur les amphores, p. 201-276

Garlan 1988: GARLAN (Y.) - Vin et amphores de Thasos, Limoges, 1988.

Garlan 1989 : GARLAN (Y.) - Le comblement d'un puits public à Thasos. 2. Les timbres céramiques. BCH, 113, 1989, p. 477-480.

Garlan 1999 : GARLAN (Y.) - Les timbres amphoriques de Thasos, I, Timbres protothasiens et thasiens anciens, Paris, 1999.

Gassner 2003a : GASSNER (V.) - Economia e commercio ad Elea in età tardo-arcaica. In : GRECO (G.), Elea-Velia. Le nuove ricerche. Atti del Convegno di Studi, Napoli, 14 dicembre 2001. Pozzuoli, 2003, p. 91-100.

Gassner 2003b : GASSNER (V.) dir. - Materielle Kultur und kulturelle Identität in Elea in spätarchaisch-frühklassischer Zeit, Vienne, 2003 (Velia Studien 2).

Gassner 2005 : GASSNER (V.) - Amphoren süd- und mittelionischer Produktionen in Velia. In : BRANDT (B.), GASSNER (V.), LADSTÄTTER (S.) dir., Synergia. Festschrift für Friedrich Krinzinger, Vienne, 2005, II, p. $37-43$.
Gassner 2006 : GASSNER (V.) - Velia. La cultura materiale. In : STAZIO (A.), CECCOLI (S.) dir., Velia. Atti del XLV convegno di studi sulla Magna Grecia, Taranto - Marina di Ascea, 21-25 settembre 2005. Naples, 2006, p. 471-504.

Gauer 1975 : GAUER (W.) - Die Tongefässe aus den Brunnen unterm Stadion-Nordwall und im Südost-Gebiet, Berlin 1975 (Olympische Forschungen 8).

Gentili 1968 : GENTILI (G.V.) - Problemi e testimonianze della città etrusca di Marzabotto. Esplorazione di una fonderia di bronzo. Nota preliminare. StEtr, XXXVI, 1968, p. 116-117.

Gentili 1978 : GENTILI (G.V.) - Coperchio d'avorio con quadriga dell'Orientalizzante recente a Marzabotto. Carrobbio, 4, 1978, p. 255-262.

Georgopoulos 2004 : GEORGOPOULOS (V.) - The archaeological evidence of Coan amphorae from Kardamaina (ancient Halasarna). In : HÖGHAMMAR (K.) dr., The Hellenistic Polis of Kos. State, Economy and Culture. Proceedings of International Seminar, Uppsala, 11-13 May 2000. Uppsala, 2004, p. 129-132.

Gianfrotta 1981 : GIANFROTTA (P.A.) - Archeologia sott'acqua. Rinvenimenti sottomarini in Etruria meridionale. BA, 10, 1981, p. 69-92.

Gill 1987 : GILL (D.W.J.) - The date of the Porticello Shipwreck : some observations on the Attic bolsals. IntJNautA, 16, 1987, p. 31-33.

Giudice 2004 : GIUDICE (F.) - La ceramica attica dell'Adriatico e la rotta di distribuzione verso gli empori padani. In : Greci in Adriatico, p. 171-210.

Govi 2006 : GOVI (E.) - L'“ultima Spina”. Riflessioni sulla tarda etruscità adriatica. In : LENZI (F.) dir., Rimini e l'Adriatico nell'età delle guerre puniche. Atti del Convegno internazionale, Rimini, 2004. Bologne, 2006, p. 111-135.

Grace 1934 : GRACE (V.) - The stamped amphora handles, found in the American excavations in the Athenian Agora, 1931-1932. Hesperia, 3/3, 1934, p, 197-310.

Grace 1949 : GRACE (V.) - Standard pottery containers of the ancient Greek world. In : Commemorative Studies in Honor of Theodore Leslie Shear, Princeton 1949, p. 175-189 (Hesperia Supplement 8).

Grace 1953 : GRACE (V.) - Wine Jars. In : Boulter 1953, p. 101-110.

Grace 1961 : Grace (V.) - Amphoras and the Ancient Wine Trade, Princeton, 1961.

Grace 1963 : GRACE (V.) - Notes on the Amphoras from the Koroni Peninsula. Hesperia, 32, 1963, p. 319-334.

Grace 1971 : Grace (V.) - Samian Amphoras. Hesperia, 40/1, 1971, p. 53-95.

Grace 1979a : GRACE (V.) - Exceptional Amphora stamps. In : KOPCKE (G.), MOORE (M.B.) dir., Studies in classical art and archaeology : a tribute to Peter Heinrich von Blanckenhagen, Locust Valley, 1979, pp. 117-127.

Grace 1979b : GRACE (V.) - Amphoras and the Ancient Wine Trade, Athènes - Princeton, 1979 ( $2^{\mathrm{e}}$ éd.).

Grace 1985 : GRACE (V.) - The Middle Stoa Dated by Amphora Stamps. Hesperia, 54, 1985, p. 1-54.

Grace, Savvatianou-Petropoulakou 1970 : GRACE (V.), SAVVATIANOUPETROPOULAKOU (M.) - Les timbres amphoriques grecs. In : BRUNEAU (P.), BECH (N.), FISTER (P.) dir., L'îlot de la Maison des Comédiens, Paris, 1970, p. 277-382 (Exploration archéologique de Délos 27).

Grandjean 1992 : GRANDJEAN (J.) - Contribution à l'établissement d'une typologie des amphores thasiens. Le matériel amphorique du quartier de la porte du Silène. $B C H, 116 / 2,1992$, p. 541-584.

Gras 1978 : GRAS (M.) - Les amphores commerciales archaïques. In : Leuca, Galatina 1978, p. 171-176 (Collana dell'Istituto di archeologia e storia antica 1).

Gras 1987 : GRAS (M.) - Amphores commerciales et histoire archaïque. DialA, 5, 1987, p. 41-50. 
Gras 1988 : GRAS (M.) - L'apport des amphores à la connaissance des commerces archaïques en mer Tyrrhénienne. In : Flotte e commercio greco, p. 291-304.

Gras 1996 : GRAS (M.) - Les grands courants commerciaux. Époques archaïque et classique. In : PRONTERA (F.) dir., La Magna Grecia e il mare. Studi di storia marittima, Tarente, 1996, p. 121-144.

Gras 1998 : GRAS (M.) - Spina: aspetti commerciali. In : Spina e il delta padano, p. 57-64.

Greco, Theodorescu 1987 : GRECO (E.), THEODORESCU (D.), Poseidonia-Paestum III. Forum nord, Rome, 1987.

Greci in Adriatico : I Greci in Adriatico. Atti del Convegno, Urbino, 1999. Rome 2004 (Hesperia. Studi sulla grecità d'Occidente, 18, 2004).

Greene, Lawall, Polzer 2008 : GREENE (E.), LAWALL (M.L.), POLZER (M.E.) - Inconspicuous Consumption: The Sixth-Century B.C.E. Shipwreck at Pabuç Burnu, Turkey. AJA, 112/4, 2008, p. 685-711.

Gualandi 1970 : GUALANDI G.) - Problemi urbanistici e cronologici di Felsina alla luce degli scavi dei Giardini Margherita e della Facoltà di Ingegneria (ex Villa Cassarini). AttiMemBologna, n.s., 20, 1969 (1970), p. 47-67.

Gualandi 1974 : GUALANDI G.) - Santuari e stipi votive dell'Etruria Padana. StEtr, 42, 1974, p. 37-68.

Gualandi 1975 : GUALANDI G.) - Un santuario felsineo nell'ex Villa Cassarini (facoltà d'Ingegneria). AttiMemBologna, n.s., 24, 1973 (1975), p. $315-345$.

Guarducci 1978 : GUARDUCCI (M.) - Adria e gli Egineti. In : GASPERINI (L.) dir., Scritti storico-epigrafici in memoria di Marcello Zambelli, Rome 1978, p. 175-180.

Guermandi 1998 : GUERMANDI (M.P.) - Figure in quantità. L'analisi quantitativa della ceramica attica di Spina. In : Spina e il delta padano, p. $179-202$.

Guzzo 1993 : GUZZO (P.G.) - Ipotesi di lavoro per un'analisi dell'ideologia funeraria. In : Spina, p. 219-229.

Harari 1999 : HARARI (M.) - Un edificio tardoarcaico presso il peleoalveo di San Cassiano. In : Protostoria e storia del Venetorum angulus, p. 627-632.

Harari 2002 : HARARI (M.) - Adria da emporion a polis. In : Commerci e produzione, p. 43-58.

Harari 2003 : HARARI (M.) - Tirreno e Adriatico: mari paralleli. In : Alto e Medio Adriatico, p. 19-27.

Harari 2004a : HARARI (M.) - Note di aggiornamento sugli scavi delle Università di Pavia e di Ferrara nell'entroterra di Adria. In : Greci in Adriatico, p. 215-225.

Harari 2004b : HARARI (M.) - Ceramica attica figurata nella chora di Adria : il caso di Crespino, San Cassiano. In : Bentz, Reusser 2004a, p. 25-34.

Heron, Pollard 1988 : HERON (C.), POLLARD (A.M.) - The analysis of natural resinous material from Roman amphoras. In : SLATER (E.A.), TATE (J.O.) dir., Science and Archaeology, Oxford, 1988, p. 429-447 (B.A.R. 196).

Iscrizioni e graffiti : SaSSAtelli (G) dir., Iscrizioni e graffiti della città etrusca di Marzabotto, Imola, 1994.

Johnston 1990 : JOHNSTON (A.W.) - Aegina, Aphaia-Tempel. XIII. The storage Amphorae. AA, 1990, p. 37-64.

Johnston 1991 : JOHNSTON (A.W.) - An archaic amphora of thasian type. Hesperia, 60, 1991, p. 363-365.

Johnston 1993 : JOHNSTON (A.) - Pottery from Archaic Building Q at Kommos. Hesperia, 62/3, 1993, p. 339-382.

Johnston 2004 : JOHNSTON (A.W.) - Amphorae and text. In : Anfore e testo in età greca arcaica. Atti del Seminario dell'Università degli Studi di Milano, Milano, 26 marzo 2001. Rome, 2004, p. 735-760 (MEFRA 116/2 2004).
Johnston, Jones 1978 : JOHNSTON (A.W.), JONES (R.E.) - The 'SOS' Amphora. BSA, 73, 1978, p. 103-141.

Joncheray 1976a : JONCHERAY (J-P.) - L'épave grecque ou étrusque de Bon-Porté. CahASubaqu, 5, 1976, p. 5-36.

Joncheray 1976b : JONCHERAY (J-P.) - Au sujet des amphores grecques au large de Boulouris. Nouvelle typologie du type massaliète. CahASubaqu, 5, 1976, p. 51-57.

Jones 1986 : JONES (R.E.), Greek and Cypriot Pottery : a Review of Scientific Studies, Athènes, 1986.

Kac, Monachov 1992 : KAC (V.I.), MONACHOV (S.Ju.), Grečeskie Amfory, Saratov, 1992.

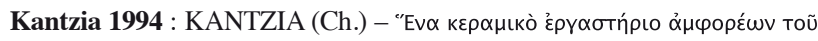

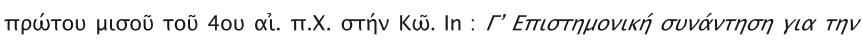

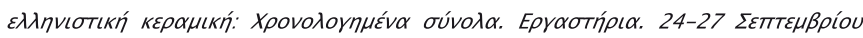

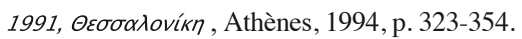

Käpitan 1973 : KÄPITAN (G.) - A Corinthian Shipwreck at Savelletri (Brindisi, Apulia, Italy). IntJNautA, 2, 1973, p. 185-186.

Käpitan 1978 : KÄPITAN (G.) - Exploration at Cape Graziano, Filicudi, Aeolian Islands. IntJNautA, 7, 1978, p. 269-277.

Katić 2004 : KATIĆ (M.) - The production of late Corinthian B amphoras in Pharos. In : L'Illyrie méridionale et l'Épire dans l'antiquité - IV. Actes du IV colloque international, Grenoble, 10-12 octobre 2002. Bresson, 2004, p. $75-80$.

Kerschner, Mommsen 2005 : KERSCHNER (M.), MOMMSEN (H) Transportamphoren milesischen Typs in Ephesos. Archäometrische und archäologische Untersuchungen zum Handel in archaischen Ionien. In : BRANDT (B.), GASSNER (V.), LADSTÄTTER (S.) dir., Synergia. Festschrift für Friedrich Krinzinger, Vienne, 2005, I, p. 119-130.

Kimmig 1983 : KIMMIG (W.) - Die griechische Kolonisation in westlichen Mittelmeergebicht und ihre Wirkung auf die Landschaften des westlichen Mitteleuropa. JbRGZM, 30, 1983, p. 5-78.

Kimmig 2000 : KIMMIG (W.) dir. - Importe und mediterrane Einflüsse auf der Heuneburg, Mainz am Rheim, 2000 (Heuneburgstudien XI).

Knigge 1976 : KNIGGE (U.) - Der Südhügel, Berlin, 1976 (Kerameikos IX).

Koehler 1978a : KOEHLER (C.G.) - Evidence around the Mediterranean for Corinthian Export of Wine and Oil. In : Beneath the waters of time. The Proceedings of the Ninth Conference on Underwater Archaeology, San Antonio, January 3-8, 1978. Austin, 1978, p. 231-239.

Koehler 1978b : KOEHLER (C.G.) - Transport Amphoras as Evidence for Trade. ANews, 8, 1978, p. 54-61.

Koehler 1979 : KOEHLER (C.G.), Corinthian A and B Transport Amphoras, Ph.D. Dissertation Princeton University, 1979.

Koehler 1981 : KOEHLER (C.G.) - Corinthian Developments in the Study of Trade in the Fifth Century. Hesperia, 50/4, 1981, p. 449-458.

Koehler 1982 : KOEHLER (C.G.) - Amphoras on Amphoras. Hesperia, 51/3, 1982, p. 284-292.

Koehler 1986 : KOEHLER (C.G.) - Handling of Greek Transport Amphoras. In : Recherches sur les amphores, p. 49-67.

Koehler 1992 : KOEHLER (C.G.) - A Brief Typology and Chronology of Corinthian Transport Amphoras. In : Kac, Monachov 1992, p. 265-279. (www.chass.utoronto.ca/amphoras/corab92.htm).

Koehler 1996 : KOEHLER (C.G.) - Wine Amphoras in ancient Greek Trade. In : McGOVERN (P.E.), FLEMING (S.J.), KATZ (S.H.), The origins and the ancient history of wine, Amsterdam, 1996, p. 223-337.

Kourkoumélis-Rodostamos 1988 : KOURKOUMÉLIS-RODOSTAMOS (D.) - Recherches archéologiques à Corfou: topographie, questions historiques, amphores de transport et commerce antique, Thèse de Doctorat en Archéologie et Histoire et Civilisations de l'Antiquité, Université de Provence (Aix - Marseille I), I-II, 1988. 
Kourkoumélis 1992 : KOURKOUMÉLIS (D.) - Corcyrean Amphoras. Enalia, 2, 1990 (1992), p. 42-47.

La Fauci 2002 : LA FAUCI (F.) - Nuove osservazioni sui relitti di Capo d'Ognina (Siracusa). ASubacq, 3, 2002, p. 335-352.

Lambrino 1938 : LAMBRINO (M.F.) - Les vases archaïques d'Histria, Bucharest, 1938.

Landolfi 1999 : LANDOLFI (M.) - Le importazioni di ceramica greca. In : I Piceni. Popolo d'Europa, Catalogo della Mostra, Francoforte - Ascoli Piceno - Teramo - Chieti - Roma, 11 dicembre 1999 - 31 gennaio 2001. Rome, 1999, p. 147-150.

Lattanzi 1989 : LATTANZI (E.) - L'attività archeologica in Calabria 1988. In : Un secolo di ricerche in Magna Grecia. Atti del XXVIII Convegno di Studi sulla Magna Grecia, Taranto, 7-12 ottobre 1988, Tarente, 1989, p. 546-563.

Lawall 1995 : LAWALL. (M.L.) - Transport Amphoras and Trademarks : Imports to Athens and Economic Diversity in the Fifth Century B.C., Ph.D. Dissertation University of Michigan, 1995.

Lawall 1998 : LAWALL. (M.L.) - Bolsals, Mendean Amphoras, and the Date of the Porticello Shipwreck. IntJNautA, 27/1, 1998, p. 16-23.

Lawall 2000 : LAWALL. (M.L.) - Graffiti, Wine Selling, and the Reuse of Amphoras in the Athenian Agora, ca. 430-400 b.c.. Hesperia, 69/1, 2000, p. 3-90.

Lawall 2005 : LAWALL. (M.L.), Negotiating Chronologies. In : HANNESTAD (L.), STOLBA (V.F.) dir., Chronologies of the Black Sea Area in the Period c. 400-100 B.C., Aarhus, 2005, p. 31-67 (Black Sea Studies, 3).

Lentini, Savelli, Blackman 2007 : LENTINI (M.C.), SAVELLI (S.), BLACKMAN (D.J.) - Amphorae from the slipways of the ancient dockyard of Naxos Sicily. Skyllis, 7, 2005-2006 (2007), p. 94-102.

Lentini, Savelli, Blackman 2009 : LENTINI (M.C.), SAVELLI (S.), BLACKMAN (D.J.) - Amphorae from the Slipways of the Ancient Dockyard of Naxos in Sicily. In : LENTINI (M.C.), Naxos di Sicilia. Ricerche nell'abitato coloniale (secoli VIII-V a.C.) e all'arsenale navale (V secolo a.C.). Scavi 2003-2006, Messine, 2009, p. 99-109.

Liou 1974 : LIOU (B.) - Note provisoire sur deux gisements grécoétrusques (Bon-Porte A et Pointe du Dattier). CahA Subaqu, 3, 1974, p. 7-20.

Liou 1975 : LIOU (B.) - Recherches sous-marines. Gallia, 33/2, 1975, p. 571-605.

Lippolis 1998 : LIPPOLIS (E.) - F.-H. Massa-Pairault (éd), Marzabotto. Recherches sur 1'Insula V, 3. Ostraka, 7/1-2, 1998, p. 211-216.

Lippolis 2000 : LIPPOLIS (E.) - Le importazioni greche in Emilia tra VII e VI secolo. In : Adriatico greco, p. 99-118.

Lippolis 2005 : LIPPOLIS (E.) - Nuovi dati sull'Acropoli e sulla forma urbana di Marzabotto. In : Culti, forma urbana e artigianato a Marzabotto, p. $139-165$.

Lo Porto 1966 : LO PORTO (F.G.) - Metaponto. Scavi e ricerche archeologiche. NSc, 1966, p. 136-231.

Lo Porto 1992 : LO PORTO (F.G.) - Metaponto (Matera). Rinvenimenti nella città antica e nel suo retroterra ellenizzato. NSc, 1988-1989 (1992), p. 299-441.

Locatelli 2005 : LOCATELLI (D.) - La «fonderia» della Regio V, Insula 5 : elementi per una definizione dell'attività produttiva. In : Culti, forma urbana e artigianato a Marzabotto, p. 213-237.

Long, Miro, Volpe 1992 : LONG (L.), MIRO (J.), VOLPE (G.) - Les épaves archaïques de la Pointe Lequin (Porquerolles, Hyères, Var). In : Marseille grecque et la Gaule, p. 199-243.

Long, Pomey, Sourisseau 2002 : LONG (L.), POMEY (P.), SOURISSEAU (J.-Chr.) dir. - Les Étrusques en mer. Épaves d'Antibes à Marseille, Aix-en Provence - Marseille, 2002.

Lordkipanidzé 1968 : LORDKIPANIDZE (O.D) - Colchis in the Early Antique Period and her relations with the Greek World. Archeologia (Warzsawa), 19, 1968, p. 15-44.
Lüscher 1996 : LÜSCHER (G.) - Der Amphorenimport in Châtillon-surGlâne (Kanton Freiburg/Schweiz). Germania, 74, 1996, p. 337-360.

Lyding Will 1982 : LYDING WILL (E.) - Greco-Italic Amphoras. Hesperia, 51/3, 1982, p. 338-356.

Macellari 1990 : MACELLARI (R.) - Poviglio-Case Carpi. Materiali da raccolte di superficie. In : Vestigia Crustunei. Catalogo della Mostra, Reggio Emilia, 1990. Reggio Emilia, 1990, p. 265-268.

Macellari 2002 : MACELLARI (R.) - Il sepolcreto etrusco del terreno Arnoaldi di Bologna (530-350 a.C.), Venise, 2002.

Maggiani 1981 : MAGGIANI (A.) - Nuove evidenze archeologiche all'isola d'Elba: i rinvenimenti di età classica ed ellenistica. In : Etruria mineraria, p. 173-192.

Maggiani 1985 : MAGGIANI (A.) - Pisa, Spina e un passo controverso di Scilace. In : Romagna tra VI e IV secolo, p. 307-319.

Maggiani 1990a : MAGGIANI (A.) - La situazione archeologica dell'Etruria settentrionale. In : Crise et transformation des sociétés archaïques de l'Italie antique au Ve siècle av. J.-C. Actes de la Table Ronde, Rome 1987. Rome, 1990, p. 23-49.

Maggiani 1990b : MAGGIANI (A.) - S. Rocchino (Massarosa). In : Etruscorum ante quam Ligurum, p. 69-96.

Maggiani 2002 : MAGGIANI (A.) - L'alfabeto etrusco nel Veneto. In : Akeo. I tempi della scrittura. Veneti antichi, strumenti e documenti. Catalogo della Mostra, Montebelluna, 3 dicembre 2001-26 maggio 2002. Cornuda, 2002, p. 55-63.

Maggiani, Paribeni 1990 : MAGGIANI (A.), PARIBENI (E.) - Pozzi, Casa Baldi (Serravezza). In : Etruscorum ante quam Ligurum, p. 136-146.

Malnati 1987 : MALNATI (L.) - Marzabotto : la fase arcaica. In : Formazione della città in Emilia Romagna, II, p. 125-137.

Malnati 1990 : MALNATI (L.) - Marzabotto (Bologna). Località Pian di Misano- Scavi 1988-1989. BA, 5-6, 1990, p. 133-134.

Malnati 1991 : MALNATI (L.) - Marzabotto. Regio V - Insula 5. StEtr, LVII, 1991, p. 390-392.

Malnati 1993 : MALNATI (L.) - Le istituzioni politiche e religiose a Spina e nell'Etruria padana. In : Spina, p. 145-177.

Malnati 1996 : MALNATI (L.) - Il fenomeno urbano nell'Italia settentrionale in età preromana. In : XIII International Congress of Prehistoric and Protohistoric Sciences. Atti del Congresso Internazionale, Forlì, 8-14 settembre 1996. Forli, 1996, p. 181-190.

Malnati 2011 : MALNATI (L.) - Bologna preromana alla luce degli ultimi scavi. In : CURINA (R.), MALNATI (L.), NEGRELLI (C.), PINI, (L.) dir., Alla ricerca di Bologna antica e medievale, Florence, 2011, p. 209-222.

Malnati, Macellari 1989 : MALNATI (L.), MACELLARI (R.) - Rubiera e la Valle del Secchia : dalla colonizzazione etrusca alla crisi politica ed economica del IV-III secolo a.C. In : Rubiera. «Principi» etruschi in val di Secchia. Catalogo della Mostra, Reggio Emilia, 1989. Reggio Emilia, 1989, p. 25-38.

Malnati, Manfredi 1991 : MALNATI (L.), MANFREDI (V.M.) - Gli Etruschi in Val Padana, Milan, 1991.

Malnati, Violante 1995 : MALNATI (L.), VIOLATE (A.) - Il sistema urbano di IV e III secolo in Emilia Romagna tra Etruschi e Celti (Plut. Vita Cam. 16, 3). In : L'Europe celtique du Ve au IIIe siècle avant J.-C. Contacts, échanges et mouvements de populations. Actes du deuxième symposium international d'Hautvillers, 8-10 octobre 1992. Sceaux, 1995, p. 97-123.

Mancevič 1987 : MANCEVIč (A.) - Kurgan Solokha, Leningrad, 1987.

Mano 1971 : MANO (A.), Nekropoli i Apollonisē - Tuma I. Iliria, 1, 1971, p. 103-207.

Mansuelli 1962 : MANSUELLI (G.A.) - La città etrusca di Misano (Marzabotto). ArtAntMod, XVII, 1962, p. 14-27.

Mansuelli 1962a : MANSUELLI (G.A.) - FA, XVII, 1962, nº 2728.

Mansuelli 1963 : MANSUELLI (G.A.) - La casa etrusca a Marzabotto. Constatazioni dei nuovi scavi. RM, LXX, 1963, p. 44-62. 
Mansuelli 1965 : MANSUELLI (G.A.) - Sulle testimonianze più antiche di Marzabotto. In : Studi in onore di L. Banti, Rome, 1965, p. 241-247.

Mansuelli et al. 1970 : CAMPAGNANO (L.), GRILLINI (A.), MANSUELLI (G.A.), SASSATELLI (G.) - Nuove scoperte dal 1958 al 1969 a Marzabotto. EmPrerom, VI, 1970, p. 53-71.

Mansuelli et al. 1982 : MANSUELLI (G.A.), BRIZZOLARA(A.M.), DE MARIA (S.), SASSATELLI (G.), Guida Alla città etrusca e al Museo di Marzabotto, Bologne, 1982.

Mantzouka 2004 : MANTZOUKA (E.) - The Transport Amphoras from a Fifth Century Shipwreck found off the Island of Alonnesos, Northern Sporades, Greece, Thesis presented to the Faculty of the Maritime Studies Program, Department of History, East Carolina University, April 2004.

Marchesi 2005 : MARCHESI (M.) - Le necropoli : dagli scavi ottocenteschi alla ricostruzione dei corredi. In : Culti, forma urbana e artigianato a Marzabotto, p. 191-212.

Marinetti 1994 : MARINETTI (A.) - La documentazione epigrafica. In : Balone. Insediamento etrusco presso un ramo de Po, Padoue, 1994, p. $81-87$.

Marseille grecque et la Gaule : BATS (M.), BERTUCCHI (G.), CONGES (G.), TRÉZINY (H.) dir. - Marseille grecque et la Gaule. Actes du Colloque International, Marseille, 18-23 novembre 1990. Lattes - Aix-enProvence, 1992 (études Massaliètes 3).

Martelli 1985 : MARTELLI (M.) - I luoghi e i prodotti dello scambio. In : Civiltà degli Etruschi, Catalogo della Mostra, Firenze, 16 Maggio -20 Ottobre 1985. Milan, 1985, p. 175-183.

Martelli 1989 : MARTELLI (M.) - La ceramica greca in Etruria : problemi e prospettive di ricerca. In : Secondo Congresso Internazionale Etrusco, II, p. 781-811.

Martelli, Nasorri 2000 : MARTELLI (A.), NASORRI (L.) - La Tomba dell'Iscrizione nella necropoli di Poggio Renzo. In : GASTALDI (P.) dir., Studi su Chiusi arcaica, p. 81-101 (AIONArch, 5).

Marzatico 1995 : MARZATICO (F.) - Nomi (loc. Bersaglio). StEtr, 60, 1994 (1995), p. 523-529.

Massa 1993 : MASSA (M.) - Anfore commerciali. In : Bruni 1993, p. $345-375$.

Massa-Pairault 1997 : MASSA-PAIRAULT (F.-H.) dir., Marzabotto. Recherches sur l'Insula V, 3, Roma, 1997 (Collection de l'École Française de Rome 228).

Mattingly 1981 : MATTINGLY (H.B.) - Coins and Amphoras. Chios, Samos and Thasos in the Fifth Century B.C. JHS, 101, 1981, p. 78-86.

Mele 1979 : MELE (A.) - Il commercio greco arcaico. Prexis ed emporie, Naples, 1979.

Mertens et al. 2004 : MERTENS (D.), DEHL-VON KAENEL (C.), HOESCH (N.), HENNING (A.), SEIFERT (A.), VOGT (S.) - Die Agora von Selinunt. RM, 110, 2003 (2004), p. 389-446.

Milanese 1987 : MILANESE (M.) - Gli scavi dell'oppidum preromano di Genova (Genova-S. Silvestro 1), Rome, 1987 (Studia Archaeologica 48).

Milanese 1990 : MILANESE (M.) - La diffusione delle anfore massaliote in Liguria. In : Amphores de Marseille grecque, p. 217-222.

Milanese, Mannoni 1986 : MILANESE (M.), MANNONI (T.) - Gli Etruschi a Genova e il commercio mediterraneo. StEtr, 52, 1984 (1986), p. 117-146.

Millemaci 1999 : MILLEMACI (G.) - Viabilità transappenninica etrusca. RTopAnt, IX, 1999, p. 121-140.

Millemaci 2001 : MILLEMACI (G.) - Viabilità transappenninica etrusca. In : La viabilità romana in Italia. Atti del III Congresso di Topografia Antica, Roma 10-11 novembre 1998, Rome, 2001, I, 121-140.

Minetti, Rastrelli 2001 : MINETTI (A.), RASTRELLI (A). - La necropoli della Palazzina nel Museo Civico di Sarteano, Sienne, 2001 (Quaderni del Sistema dei musei senesi 3).
Monachov 1999a : MONACHOV (S.JU.) - Grečeskie amfory $v$ Pričernomor'e. Tipologiia amfor vedushchih zentrov exporterov tovarov $v$ keramicesckoy tare. Katalog opredelitel [Greek amphorae in the Black Sea area : complexes of ceramic containers of VII-II BC], Saratov, 1999.

Monachov 1999b : MONACHOV (S.JU.) - Quelques séries d'amphores grecques des $\mathrm{VII}^{\mathrm{e}}-\mathrm{V}^{\mathrm{e}} \mathrm{s}$. av. n. è. au nord de la mer Noire. In : Production et commerce, p. 163-194.

Monachov 2003a : MONACHOV (S.JU.) - Grečeskie amfory $v$ Pričernomor'e. Tipologiia amfor vedushchih zentrov exporterov tovarov $v$ keramicesckoy tare. Katalog opredelitel, Saratov, 2003.

Monachov 2003b : MONACHOV (S.JU.) - Amphorae from unidentified centres in the North Aegean. In : GULDAGER BILDE (P.), HOEJTE (J.M.), STOLBA (V.F.) dir., The Cauldron of Ariantas. Studies presented to A.N. Ščeglov on the occasion of his 70th birthday, Aarhus, 2003, pp. 247-260.

Morel 1988 : MOREL (J.-P.) - Les Phocéens dans la mer Tyrrhénienne. In : Flotte e commercio greco, p. 429-455.

Morel 1990a : MOREL (J.-P.) - Les amphores massaliètes en Afrique $\mathrm{du}$ Nord et particulièrement à Carthage. In : Amphores de Marseille grecque, p. 269-272.

Morel 1990b : MOREL (J.-P.) - Remarques finales sur les amphores massaliètes. In : Amphores de Marseille grecque, p. 281-287.

Morel 1990c : MOREL (J.-P.) - Nouvelles données sur le commerce de Carthage Punique entre le VII ${ }^{\mathrm{e}}$ siècle et le $\mathrm{II}^{\mathrm{e}}$ siècle avant J.-C. In : Carthage et son territoire dans l'Antiquité. Actes du IV Colloque International, Strasbourg 1988. Paris, 1990., I, p. 67-100.

Morel 1995 : MOREL (J.-P.) - Les rapports entre la Sicile et la Gaule jusqu'au VI ${ }^{\mathrm{e}}$ siècle av. J.-C. In : VIII Congresso Internazionale di Studi sulla Sicilia Antica. Atti del Congresso, Palermo, 18-23 aprile 1993. Rome, 1995, I, 1, p. 333-361 (Kokalos, 39-40, I, 1-2-II, 1-2).

Morigi Govi, Marchesi 2000 : MORIGI GOVI (C.), MARCHESI (M.) - I principi padani: l'Orientalizzante settentrionale. In : Principi etruschi tra Mediterraneo ed Europa. Catalogo della Mostra, Bologna, 1 ottobre 2000-1 aprile 2001. Venise, 2000, p. 327-337.

Morselli, Tortorici 1985 : MORSELLI (C.), TORTORICI (E.) - La situazione di Regisvilla. In : Commercio etrusco arcaico, p. 27-40.

Morter, Leonard 1998 : MORTER (J.), LEONARD (J.R.) - Storage Amphoras. In : CARTER (J.C.) dir., The Chora of Metaponto: The Necropoleis, Austin, 1998, II, p. 731-747.

MSC : Munsell Soil Colour Charts, Baltimore, 1975.

Naso 2005 : NASO (A.) - Funde aus Milet. XIX. Anfore commerciali arcaiche a Mileto : rapporto preliminare. AA, 2005/2, p. 73-84.

Neeft 2006 : NEEFT (C.W.), Camarina e la sua ceramica corinzia. In : PELAGATTI (P.), DI STEFANO (G.), DE LACHENAL (L.) dir., Camarina 2600 anni dopo la fondazione. Nuovi studi sulla città e sul territorio. Atti del Convegno Internazionale, Ragusa, 7 dicembre 2002 e 7-9 aprile 2003. Rome, 2006, p. 77-107.

Negrioli 1924 : NEGRIOLI (A.) - Comacchio. Vasto sepolcreto etrusco scoperto in Valle Trebba. NSc, 1924, p. 279-322.

Negrioli 1927 : NEGRIOLI (A.) - Comacchio. Vasto sepolcreto etrusco scoperto in Valle Trebba (Relazione provvisoria delle campagne di scavo del 1924 e del 1925). NSc, 1927, p. 143-198.

Nickels 1983 : NICKELS (A.) - Les Grecs en Gaule: l'exemple du Languedoc. In : Modes de contacts et processus de transformation dans les sociétés anciennes. Atti del Convegno, Cortona, 24-30 Maggio 1981. Rome, 1983, p. 409-428.

Nickels 1990 : NICKELS (A.) - Les amphores 'ionio-massaliètes' et massaliètes archaïques de la région d'Agde. In : Amphores de Marseille grecque, p. 99-110.

Nickels 1995 : NICKELS (A.) - Les sondages de la rue Perben à Agde (Hérault). In : Sur les pas des Grecs, p. 59-98. 
Nielsen-Phillips 1977 : NIELSEN (E.O.), PHILLIPS (K.M.) - Bryn Mawr College Excavations in Tuscany, 1975. AJA, 81, 1977, p. 85-100.

Nikolaïdou-Patera 1986 : NIKOLAÏDOU-PATERA (M.) - Un nouveau centre de production d'amphores timbrées en Macédonie. In : Recherches sur les amphores, p. 485-490.

Nisbet 1987 : NISBET (R.) - I vegetali carbonizzati dell'insediamento dell'età del Ferro di Castelrotto (Verona). In : Prima della storia. Inediti di 10 anni di ricerche a Verona, Vérone, 1987, p. 121-130.

Oliver Foix, Gusi Jener 1991 : OLIVER FOIX (A.), GUSI JENER (F.) - Los primeros contactos comerciales mediterráneos en el Norte del Pais Valenciano (siglos VII-VI a.C.). In : Presencia de material etrusco, p. 197-213.

Pairault Massa 1978 : PAIRAULT MASSA (F.H.) - Marzabotto (Bologna). Rapport préliminaire sur six ans de recherches (1971-1976) dans l'Insula VIII (Brizio) = V, 3. NSc, 1978, p. 131-157.

Pancrazzi 1982 : PANCRAZZI (O.) - Pisa: testimonianze di una rotta greco arcaica. In : Focei dall'Anatolia all'Oceano, p. 331-342.

Pancrazzi 1993 : PANCRAZZI (O.) - Anfore commerciali dalla piazza dei Cavalieri di Pisa. In : Archeologia di Pisa. Atti della Giornata di Studio, Pisa, 16 aprile 1988. Pise, 1993, p. 45-52.

Panvini 1991 : PANVINI (R.) - L'attività delle soprintendenze di Agrigento e Caltanisetta nel campo dell'archeologia subacquea. In : IV rassegna di archeologia subacquea. IV premio Franco Papò. Atti del Convegno di Studi, Giardini Naxos, 13-15 ottobre 1989. Messine, 1991, p. 193-200.

Panvini 1993 : PANVINI (R.) - La nave greca di Gela. AViva, Avril 1993, p. 58-68.

Panvini 1997 : PANVINI (R.) - La nave greca arcaica di Gela: nuovi dati dallo scavo e ipotesi sulla rotta seguita. In : Atti del Convegno Nazionale di Archeologia Subacquea. Atti del Convegno, Anzio, 30 maggio-1 giugno 1996. Bari, 1997, p. 135-142.

Panvini 2001 : PANVINI (R.) - La nave greca arcaica di Gela e primi dati sul secondo relitto greco, Caltanisetta, 2001.

Paolucci 1992 : PAOLUCCI (G.), Testimonianze archeologiche. Nuove acquisizioni del Museo Civico di Chianciano Terme, Città di Castello, 1992.

Paolucci 2006 : PAOLUCCI (G.) - Le anfore tipo 'Tolle'. Contributo al commercio del vino nell'Etruria settentrionale interna. In : Etruschi e il Mediterraneo, p. 417-432.

Paolucci, Rasterelli 1999 : PAOLUCCI (G.), RASTRELLI (A.), Chianciano Terme, I, Necropoli della Pedata (Tombe1-21), necropoli di Via Montale (Tombe 2-4), Rome, 1999.

Papadopoulos, Paspalas 1999 : PAPADOPOULOS (J.K.), PASPALAS (S.A.) - Mendaian as Chalkidian Wine. Hesperia, 68/2, 1999, p. 161-188.

Pape 2000 : PAPE (J.) - Die attische Keramik der Heuneburg und der keramische Südimport in der Zone nördlich der Alpen während der Hallstattzeit. In : Kimmig 2000, p. 71-175.

Parker 1978 : PARKER (A.J.) - Sicilia e Malta nel commercio marittimo dell'antichità. Kokalos, 22-23, 1976-1977 (1978), II, 1, p. $622-631$.

Parker 1992 : PARKER (A.J.)-Ancient Shipwrecks of the Mediterranean \& the Roman Provinces, Oxford, 1992 (BAR International Series 580).

Parrini 1993 : PARRINI (A.) - Tombe di V secolo a.C. In : Spina, p. $273-286$.

Patitucci 1971 : PATITUCCI (S.) - Corpus Vasorum Antiquorum. Italia, XLVIII, Ferrara, Museo Archeologico Nazionale, II, Roma 1971.

Patitucci Uggeri, Uggeri 1993 : PATITUCCI UGGERI (S.), UGGERI (G.) - La topografia della città. In : Spina, p. 21-31.

Pease 1937 : PEASE (M.) - A well of the late fifth century at Corinth. Hesperia, 6, 1937, p. 257-316.

Pelagatti 1978 : PELAGATTI (P.) - Camarina (Ragusa). StEtr, 46, 1978, p. $571-574$.
Pelagatti 1982 : PELAGATTI (P.) - L'attività della Soprintendenza alle Antichità della Sicilia Orientale. Kokalos, 26-27, 1980-1981 (1982), II, 1, p. 694-730.

Pelagatti 1995 : PELAGATTI (P.) - Le anfore commerciali. In : STAZIO (A.), CECCOLI (S.) dir., Corinto e l'Occidente. Atti del XXXIV Convegno di Studi sulla Magna Grecia, Taranto, 7-11 ottobre 1994. Tarente, 1995, I, p. 403-416.

Pelagatti 2000 : PELAGATTI (P.) - Camarina nel VI e nel V secolo. Problemi di cronologia alla luce della documentazione archeologica. In : Un ponte fra l'Italia e la Grecia. Atti del Simposio in onore di Antonino di Vita, Ragusa, 13-15 febbraio 1998. Padoue, 2000, p. 173-190.

Pelagatti 2006 : In : PELAGATTI (P.) - Camarina città e necropoli. Studi e ricerche recenti. In : PELAGATTI (P.), DI STEFANO (G.), DE LACHENAL (L.) dir., Camarina 2600 anni dopo la fondazione. Nuovi studi sulla città e sul territorio. Atti del Convegno Internazionale, Ragusa, 7 dicembre 2002 e 7-9 aprile 2003. Rome, 2006, p. 45-76.

Peretto 1999 : PERETTO (R.) - Vie fluviali nell'antica area settentrionale del delta padano. In : Protostoria e storia del Venetorum angulus, p. 615-626.

Peretto, Vallicelli, Wiel-Marin 2003 : PERETTO (R.), VALLICELLI (M.C.), WIEL-MARIN (F.) - L'entroterra di Adria: conoscenze archeologiche e paleo ambientali. In : Alto e Medio Adriatico, p. 91-114.

Peristeri-Otatzi 1986 : PERISTERI-OTATZI (C.) - Amphores et timbres amphoriques d'Abdère. In : Recherches sur les amphores, p. 491-496.

Perkins, Walker 1990 : PERKINS (P.), WALKER (L.) - Survey of an Etruscan city at Doganella, in Albegna valley. BSR, 58, 1990, p. 1-143.

Pettazzoni 1917 : PETTAZZONI (R.) - Stazioni preistoriche nella provincia di Bologna. Monumenti antichi, 24, 1916 (1917), col. 221-308.

Picon 1990 : PICON (M.) - Origine d'amphores du groupe dit Solokha II trouvées en Russie. In : Doulgéri-Intzessiloglou, Garlan 1990, p. 390-393.

Poggesi et al. 2005 : POGGESI (G.), DONATI (L.), BOCCI (E.), MILlEMACI (E.), PAGNINI (L.), PALLECCHI (P.) - Prato-Gonfienti: un nuovo centro etrusco sulla via per Marzabotto. In : Culti, forma urbana e artigianato a Marzabotto, p. 267-300.

Polizzi 1997 : POLIZZI (C.) - Materiali della necropoli punica di Solunto: studi preliminari. Anfore greche da trasporto. In : Archeologia e territorio, Palerme, 1997, p. 95-103.

Polizzi 1999 : POLIZZI (C.) - Anfore da trasporto. In : VASSALLO (S.) dir., Colle Madore. Un caso di ellenizzazione in terra sicana, Palermo, 1999. p. 221-231.

Pomey et al. 1988 : POMEY (P.), LONG (L.), L'HOUR (M.), RICHEZ (F.), BERNARD (H.) - Recherches sous-marine. GalliaInf, 19871988, 1 (1988), p. 1-78.

Pomey, Long 1992 : POMEY (P.), LONG (L.) - Les premiers échanges maritimes du Midi de la Gaule du VI ${ }^{\mathrm{e}}$ au $\mathrm{III}^{\mathrm{e}}$ s. av. J.-C. à travers les épaves. In : Marseille grecque et la Gaule, p. 189-198.

Preka-Alexandri 1992 : PREKA-ALEXANDRI (K.) - A ceramic Workshop in Figareto, Corfu. In : BLONDÉ (F.), PERREAULT (J.Y.) dir., Les ateliers de potiers dans le monde grec aux époques géométrique, archaïque et classique. Actes de la Table Ronde, Athènes, 2-3 octobre 1987. Paris, 1992, p. 41-52 (BCH Supplément 23).

Presencia de material etrusco : REMESAL (J.), MUSSO (O.) dir. La presencia de material etrusco en la Península Ibérica. Mesa Redonda, Barcelona, 24-27 aprile 1990. Barcelone, 1991.

Production et commerce : GARLAN (Y.) dir.-Production et commerce des amphores anciennes en Mer Noire. Actes du colloque international, Istanbul, 25-28 mai 1994. Aix-en-Provence, 1999.

Protostoria e storia del Venetorum angulus : Protostoria e storia del Venetorum angelus. Atti del XX Convegno di Studi Etruschi e Italici, 
Portogruaro - Quarto d'Altino - Este - Adria, 16-19 ottobre 1996. Pise Rome, 1999.

Py 1980 : PY (M.) - Quatre siècles d'amphores massaliètes. Essai de classification des bords. Figlina, 3, 1978 (1980), p. 1-23.

Quantification des céramiques : ARCELIN (P.), TUFFREAULIBRE (M.) dir. - La quantification des céramiques. Conditions et protocole. Actes de la Table Ronde, Glux-en-Glanne, 7-9 avril 1998. Dijon - Quetigny, 1998 (Collection Bibracte 2).

Rastrelli 1993 : RASTRELLI (A.) - Le scoperte archeologiche di Chiusi negli ultimi anni. In : MAETZKE (G.) dir., La civiltà di Chiusi e del suo territorio. Atti del XVII Convegno di Studi Etruschi ed Italici, Chianciano Terme, 28 maggio-1 giugno 1989. Florence, 1993, p. 115-130.

Rastrelli 1997 : RASTRELLI (A.) - La tomba dipinta. In : MINETTI (A.) dir., Museo Civico Archeologico di Sarteano, Sienne, 1997, p. 76-82.

Recherches sur les amphores : EMPEREUR (J.-Y.), GARLAN (Y.) dir. - Recherches sur les amphores grecques, Actes du colloque international, Athènes, 10-12 septembre 1984. Paris, 1986 (BCH Supplément 13).

Rendini 1988 : RENDINI (P.) - Isola del Giglio : acquisizioni sul commercio. In : Flotte e commercio greco, p. 191-201.

Rhomiopoulou 1986 : RHOMIOPOULOU (C.) - Amphores de la nécropole d'Acanthe. In : Recherches sur les amphores, p. 479-483.

Rizzo 1990 : RIZZO (M.A.) dir. - Le anfore da trasporto e il commercio etrusco arcaico. I. Complessi tombali dell'Etruria meridionale, Catalogo della Mostra, Roma, 1983. Rome, 1990.

Roberts 1986 : ROBERTS (S.R.) - The Stoa Gutter Well. A Late Archaic Deposit in the Athenian Agora. Hesperia, 55/1, 1986, p. 1-74.

Roebuck 1940 : ROEBUCK (C.) - Pottery from the North Slope of the Acropolis, 1937-1938. Hesperia, 9/2, 1940, p. 141-260.

Romagna tra VI e IV secolo : La Romagna tra VI e IV secolo a.C. nel quadro della protostorica dell'Italia centrale. Atti del Convegno di Studi, Bologna, 23-24 Ottobre 1982. Bologne, 1985.

Romanus et al. 2009 : ROMANUS (K.), BAETEN (J.), POBLOME (J.), ACCARDO (S.), DEGRYSE (P.), JACOBS (P.), DE VOS (D.), WAELKENS (M.) - Wine and oil permeation in pitched and non-pitched ceramics : relation with results from archaeological amphorae from Sagalassos, Turkey. JASC, 36, 2009, p. $900-909$.

Romualdi 1993 : ROMUALDI (A.) - Necropoli di S. Cerbone. Restauro della Tomba dei Carri. StEtr, 58, 1993, p. 604-606.

Rouillard 1991 : ROUILLARD (P.) - Les Grecs et la Péninsule Ibérique du VIII' au IVe siècle avant Jésus-Christ, Paris, 1991.

Ruban 1991 : Ruban (V.V.) - Opyt klassifikatsii tak nazyvaemykh miletskikh amfor iz Nizhnego Pobuzh'ya. SovA, 2, 1991, p. 182-195.

Sabbione 1981a : SABBIONE (C.) - Reggio e Metauros nell'VIII e VII sec. a.C. ASAtene, 59, 1981, p. 275-289.

Sabbione 1981b : SABBIONE (C.) - L'area locrese. In : Il Commercio greco nel Tirreno in età arcaica. Atti del Seminario in Memoria di Mario Napoli, Salerno, 31 gennaio 1977. Salerne, 1981, p. 15-34.

Sabbione 1982 : SABBIONE (C.) - Le aree di colonizzazione di Crotone e Locri Epizefiri nell'VIII e VII sec. a.C. ASAtene, 60, 1982, p. 251-299.

Sabbione 1987 : SABBIONE (C.) - La colonizzazione greca: Metauros e Mylai. In : Lo stretto. Crocevia di culture. Atti del XXVI Convegno di Studi sulla Magna Grecia, Taranto - Reggio Calabria, 9-14 ottobre 1986. Tarente, 1987, p. 221-236.

Sacchetti 2002 : SACCHETTI (F.) - Imbarcazioni monossili e trasporti fluviali sul fiume Oglio. Problemi di cronologia e aspetti tecnici. Orizzonti, 2, 2001 (2002), p. 111-126.

Sacchetti 2005 : SACCHETTI (F.), Le anfore commerciali greche d'età tardo-arcaica e classica in Etruria padana e in Italia settentrionale, I-II, Tesi di Dottorato in Scienze Archeologiche - XVIII ciclo - Università degli Studi di Padova.
Sacchetti 2006 : SACCHETTI (F.) - Anfore commerciali greche tardoarcaiche e classiche in Etruria padana e in Italia settentrionale : la metodologia di studio e di catalogazione. Ocnus. Quaderni della Scuola di specializzazione in archeologia dell'Università di Bologna, 14, 2006, p. 293-298.

Sacchetti 2010 : SACCHETTI (F.) - Contrassegni commerciali alfabetici e alfanumerici dalle anfore greche arcaiche e classiche dell'Etruria padana. Epigraphica. Periodico Internazionale di Epigrafia, 71, 2009 (2010), p. 37-72.

Sacchetti 2011 : SACCHETTI (F.) - Graffiti commerciali numerici sulle anfore da trasporto greche d'età arcaica e classica dell'Italia settentrionale. In : LEONARDI (G.) dir., Tra protostoria e storia. Studi in onore di Loredana Capuis, p. 246-263 (Antenor Quaderni 20).

Sacchetti 2012 : SACCHETTI (F.) - Le anfore commerciali greche della fascia costiera e della chora di Adria. Padusa, n.s., 47, 2011 (2012), p. $97-149$.

Saletti 1970 : SALETTI (C.) - Problemi artistici di Marzabotto. Le impressioni figurative e decorative. In : Studi sulla città antica. Atti del Convegno di Studi sulla città etrusca e italica preromana. Bologna Marzabotto - Ferrara -Comacchio, 1966. Bologne, 1970, p. 279-283.

Salviat 1986 : SALVIAT (Fr.) - Le vin de Thasos. Amphores, vin et sources écrites. In : Recherches sur les amphores, p. 145-196.

Salviat 1990 : SALVIAT (Fr.) - Vignes et vins anciens de Maronée à Mendé.In : Recherche franco-helléniques, I, Thessalonique, 1990, p. 457-476.

Salzani 1991 : SALZANI (L.) - Rovigo, loc. Le Balone. StEtr, 57, 1991, p. 414-415.

Salzani 1994 : SALZANI (L.) - Lo scavo. In : Balone. Insediamento etrusco presso un ramo de Po, Padoue, 1994, p. 43-68.

Salzani, Peretto, Zerbinati 1988 : SALZANI (L.), Peretto (R.), ZERBINATI (E.) - Rovigo. Saggi di scavo in località Le Balone (Com. di Rovigo). QuadAVen, 4, 1988, p. 28-36.

Salzani, Vitali 1988 : SALZANI (L.), VITALI (D.) - L'abitato arcaico di San Basilio di Ariano Polesine. QuadAVen, 4, 1988, p. 37-40.

Salzani, Vitali 1991 : SALZANI (L.), VITALI (D.) - San Basilio (Comune di Ariano Polesine). StEtr, 57 (1991), p. 415-419.

Salzani, Vitali 2003 : SALZANI (L.), VITALI (D.) - Gli scavi archeologici nel podere Forzello a San Basilio di Ariano Polesine. In : Alto e Medio Adriatico, p. 115-138.

Sandri 1972 : SANDRI (P.) - Saggio preliminare sulle forme della ceramica acroma di Marzabotto. StEtr, XL (1972), p. 319-340.

Sanmartí, Castanyer, Tremolada 1990 : SANMARTÍ (E.), CASTANYER (P.), TREMOLADA (J.) - Les amphores massaliètes d'Emporion du milieu du $\mathrm{V}^{\mathrm{e}}$ au milieu du $\mathrm{IV}^{\mathrm{e}}$ s. av. J.-C. In : Amphores de Marseille grecque, p. 165-170.

Sanmartí Grego et al. 1991 : SANMARTí GREGO (E.), CASTANYER (P.), TREMOLEDA (J.), SANTOS (M.) - La presencia comercial etrusca en la Emporion arcaica, determinada a partir de las ànforas. In : Presencia de material etrusco, p. 83-94.

Sanmartí Grego et al. 1995 : SANMARTí GREGO (E.), CASTANYER (P.), TREMOLEDA (J.), SANTOS (M.) - Amphores grecques et trafics commerciaux en Méditerranée occidentale au IVe s. av. J.-C. Nouvelles données issues d'Emporion. In : Sur les pas des Grecs, p. 31-47.

Santos Retolaza 2008 : SANTOS RETOLAZA (M.) - Les àmfores gregues. In : NIETOS (X.), SANTOS (M.) dir., El vaixell grec arcaic de Cala Sant Vicenç, Barcelone, 2008, p. 125-153.

Saronio 1965 : SARONIO (P.) - Nuovi scavi nella città etrusca di Misano a Marzabotto. StEtr, XXXIII, 1965, p. 385-416.

Sassatelli 1986 : SASSATELLI (G.) - Bologna etrusca: nuovi dati e recenti acquisizioni. AttiMemBologna, n.s., 36, 1986, p. 9-56.

Sassatelli 1989 : SASSATELLI (G.), La città etrusca di Marzabotto, Casalecchio di Reno, 1989. 
Sassatelli 1990 : SASSATELLI (G.) - La situazione in Etruria padana. In : Crise et transformation des sociétés archaïques de l'Italie antique au Ve siècle av. J.-C. Actes de la Table Ronde, Rome 1987. Rome, 1990, p. 51-100.

Sassatelli 1993 : SASSATELLI (G.) - La funzione economica e produttiva: merci, scambi, artigianato. In : Spina, p. 179-217.

Sassatelli 1996 : SASSATELLI (G.) - Verucchio, centro etrusco di « frontiera ». Ocnus, 4, 1996, p. 249-271.

Sassatelli 1998 : SASSATELLI (G.) - Il commercio greco in età arcaica e classica : importazioni ceramiche nel Mediterraneo e in Etruria. In : DE MIN (M.) dir., Documenti inediti dell'Italia antica, Padoue, 1998, p. 33-39.

Sassatelli 1999 : SASSATELLI (G.) - Spina e gli Etruschi padani. In : Produzioni, merci e commerci in Altino preromana e romana. Atti del Convegno, Venezia, 12-14 Dicembre 2001. Rome, 2003, p. 71-107.

Sassatelli 2004 : Sassatelli (G.) - Gli Etruschi di Spina e la pirateria adriatica. In : BRACCESI (L.) dir., La pirateria nell'Adriatico antico. Atti del Convegno, Ravenna, 2002. Rome, 2004, p. 21-38 (Hesperia. Studi sulla grecità d'Occidente, 19, 2004).

Sassatelli 2008 : Sassatelli (G.) - Gli Etruschi nella Valle del Po. Riflessioni, problemi e prospettive di ricerca. In : La colonizzazione etrusca in Italia. Atti del XV Convegno Internazionale di Studi sulla Storia e l'Archeologia dell'Etruria, Orvieto, 2007. Rome, 2008, p. 71-114 (AnnFaina XV).

Sassatelli et al. 2004 : BRIZZOLARA (A.M.), GOVI (E.), MATTIOLI (C.), POZZI (A.), SACCHETTI (F.), SASSATELLI (G.) - La città etrusca di Marzabotto (Bologna). In : Scoprire. Scavi del Dipartimento di Archeologia. Catalogo della Mostra, Bologna, 18 maggio-18 giugno 2004. Bologne, 2004, p. 37-49.

Sassatelli, Brizzolara 1990 : SASSATELLI (G.), BRIZZOLARA (A.M.) dir. - I nuovi scavi dell'Università di Bologna nella città etrusca di Marzabtto. Catalogo della Mostra, Bologna, 1990. Bologne, 1990.

Sassatelli, Brizzolara 1991 : SASSATELLI (G.), BRIZZOLARA (A.M.) - Marzabotto. In : NENCI (G.), VALLET (G.) dir., Bibliografia topografica della colonizzazione greca in Italia e nelle isole tirreniche, IX,1991, p. 397-452.

Sassatelli, Donati 2005 : SASSATELLI (G.), DONATI (A.) dir., Storia di Bologna. Bologna nell'antichità, Bologne, 2005.

Sassatelli, Govi 2005 : SASSATELLI (G.), GOVI (E.) - Il tempio di Tinia in area urbana. In : Culti, forma urbana e artigianato a Marzabotto, p. 9-62.

Savelli 2006 : SAVELLI (S.) - Le anfore da trasporto. In : CUOZZO (M.), D’AGOSTINO (B.), DEL VERME (L.), Cuma. Le fortificazioni, 2, I materiali dai terrapieni arcaici, Naples, 2006, p. 103-126 (AIONArch Quaderno 16).

Savelli 2009 : SAVELLI (S.) - Anfore greco-occidentali in Magna Grecia: un aggiornamento sul tipo "corinzio B arcaico" e 'ionico-massaliota". In : CAMIA (F.) PRIVITERA (S.) dir., Obeloi. Contatti, scambi e valori nel Mediterraneo antico. Studi offerti a Nicola Parise, Paestum - Athènes, 2009, p. $105-130$.

Savelli sous presse : SAVELLI (S) - Le anfore da trasporto. In : GASTALDI (P.) dir., Lo scavo del Petriolo, Chiusi, sous presse.

Schmid 2001 : SCHMID (S.G.) - Eine Gruppe nordägäischer Transportamphoren. In : Die Ausgrabungen im Kerameikos. Bilanz und Perspektiven. Akten des Internationalen Symposions, Athen, 27. bis 31. Januar 1999. Mainz am Rhein, 2001, p. 143-156 (AM, 114, 2001).

Scioli, Sacchi 1992 : SCIOLI (P.), SACCHI (C.) - Anfore Commerciali. In : Ricerche archeologiche all'Incoronata di Metaponto, 2, Dal villaggio indigeno all'emporio greco. Le strutture e i materiali del saggio T, Milan, 1992, p. 63-70.

Scotti 1986 : SCOTTI (C.) - Le anfore. In : BONGHI JOVINO (M.) dir., Gli Etruschi di Tarquinia, Catalogo della Mostra, Milano, 14 aprile-26n giugno 1986. Modène, 1986, p. 169-172.
Scotti 1999 : SCOTTI (C.) - Le anfore. In : CHIARAMONTE TRERÉ (C.) dir., Tarquinia. Scavi sistematici nell'abitato. Campagne 19821988. I materiali, 1, Rome, 1999, p. 261-278 (Tarchna 2).

Secondo Congresso Internazionale Etrusco : Secondo Congresso Internazionale Etrusco. Atti del Congresso di Studi, Firenze, 26 maggio-2 giugno 1985. Florence, 1989.

Sedda 1989 : Sedda (A.F.) - Appendice. Analisi della natura del residuo rinvenuto su frammenti di anfora provenienti dall'abitato di Spina. In : Desantis 1989, p. 124-127.

Semeraro 1990 : SEMERARO (G.) - [notices du catalogue]. In : D'ANDRIA (F.), dir., Archeologia dei Messapi. Catalogo della Mostra, Lecce, 7 ottobre 1990-7 gennaio 1991. Bari, 1990.

Semeraro 1997 : SEMERARO (G.) - En neusi. Ceramica greca e società nel Salento arcaico, Lecce - Bari, 1997.

Sezgin 2004 : SEZGIN (Y.) - Clazomenian Transport Amphorae of the Seventh and the Sixth Centuries. In : MOUSTAKA (A.), ISKARLATIDOU (E.), TZANNES (M.C.), ERSOY (Y.) dir., Klazomenai, Teos and Abdera, Metropoleis and Colony. Proceedings of the International Symposium, Abdera, 20-21 October 2001. Thessalonique, 2004, p. 169-183.

Shipley 1987 : SHIPLEY (D.J.G.) - A History of Samos, 800-188 B.C., Oxford, 1987.

Slaska 1978 : SLASKA (M.) - Gravisca. Le ceramiche comuni di produzione greco-orientale. In : Les céramiques de la Grèce de l'Est et leur diffusion en Occident. Actes des Colloques Internationaux du Centre National de la Recherche Scientifique, Naples, 6-9 Juillet 1976. Paris - Naples, 1978, p. 223-230.

Slaska 1982 : SLASKA (M.) - Anfore marsigliesi a Gravisca. In : Focei dall'Anatolia all'Oceano, p. 354-359.

Slaska 1985 : SLASKA (M.) - Le anfore da trasporto a Gravisca. In : Commercio etrusco arcaico, p. 19-22.

Slaska 1986 : SLASKA (M.) - Anfore arcaiche greco-orientali e loro diffusione nel Mediterraneo. In : BOUZEK (J.) dir., Thracia pontica, 3, Les Thraces et les colonies grecques, VII-Ve s. av. n.è. Troisième Symposium International, Sozopol, 6-12 octobre 1985. Sophia, 1986, p. 54-67.

Slaska 1990 : SLASKA (M.) - Le anfore massaliote in Etruria meridionale. In : Amphores de Marseille grecque, p. 223-233.

Sonno 1997 : SONNO M.) - Rinvenimenti sottomarini lungo il litorale di Civitavecchia. ASubacq, 2, 1997, p. 75-87.

Sourisseau 1993 : SOURISSEAU (J.-Chr.) - Amphores magnogrecques. In : PY (M.) dir., Dicocer. Dictionnaire des céramiques antiques (VII s. av.n.è.-VII s. de n.è.) en Méditerranée nord-occidentale (Provence, Languedoc, Ampurdan), Lattes, 1993, p. 64-66 (Lattara 6).

Sourisseau 1997 : SOURISSEAU (J.-Chr.) - Recherches sur les amphores de Provence et de la basse vallée du Rhône aux époques archaïque et classique (fin VIIe - début VIe s. av. J.-C., I-II, 1-2, Thèse de Doctorat nouveau régime, Université de Provence (Aix - Marseille I), I-II, 1997.

Sourisseau 1998a : SOURISSEAU (J.-Chr.) - Marseille et la production des amphores 'ionio-massaliètes' en Occident : les problèmes de fabrication. In : AMOURETTI (M.-Cl.), COMET (G.) dir., Artisanat et matériaux. La place des matériaux dans l'histoire des techniques, Aix-en-Provence, 1998, p. 127-152 (Cahiers d'histoire et des techniques 4).

Sourisseau 1998b : SOURISSEAU (J.-Chr.) - Une expérience de quantification sur les amphores archaïques et classiques de Provence. Limites et difficultés. In : Quantification des céramiques, p. 47-52.

Sourisseau 2000 : SOURISSEAU (J.-Chr.) - Les importations d'amphores grecques à Marseille aux $\mathrm{VI}^{\mathrm{e}}$ et $\mathrm{V}^{\mathrm{e}} \mathrm{s}$. av. J.-C.: bilan quantitatif. In : Ceràmiques jònies, p. 137-146.

Sourisseau 2002 : SOURISSEAU (J.-Chr.) - Camarina (com. de Ragusa). Les amphores commerciales. MEFRA, 114/1, 2002, p. 547-551.

Sourisseau 2006 : SOURISSEAU (J.-Chr.) - Les amphores commerciales de la nécropole de Rifriscolaro à Camarine. Remarques préliminaires sur les 
productions corinthiennes de type A. In : PELAGATTI (P.), DI STEFANO (G.), DE LACHENAL (L.) dir., Camarina 2600 anni dopo la fondazione. Nuovi studi sulla città e sul territorio. Atti del Convegno Internazionale, Ragusa, 7 dicembre 2002 e 7-9 aprile 2003. Rome, 2006, p. 129-147.

Sourisseau 2011 : SOURISSEAU (J.-Chr.) - La diffusion de vins grecs d'Occident du VIII' au IV'e s. av. J.-C., sources écrites et documents archéologiques. In : GRECO (E.) dir., La vigna di Dioniso: vite, vino e culti in Magna Grecia. Atti del XLIX Convegno di Studi sulla Magna Grecia, Taranto, 24-28 settembre 2009. Tarente, 2011, p. 145-252.

Sourisseau, Tichit 2007 : SOURISSEAU (J.-CHR.), TICHIT (A.) Les importations. In : AUGIER (L.), BUCHSENSCHUTZ (O.) RALSTON (I.B.M.) dir., Un complexe princier de l'âge du Fer. L'habitat du promontoire de Bourges (Cher), Bourges - Tours, 2007, p. 161-166.

Spagnolo 1999 : SPAGNOLO (G.) - [notices du catalogue]. In : Tigano 1999, p. 147-155.

Spagnolo 2002 : SPAGNOLO (G.) - Le anfore da trasporto arcaiche e classiche nell'Occidente greco: nuove acquisizioni da recenti rinvenimenti a Messina. In : Bacci, Tigano 2002, p. 31-46.

Spagnolo 2003 : SPAGNOLO (G.) - Anfore da trasporto nord-egee in Occidente nel periodo arcaico e classico: l'esempio di Gela. In : FIORENTINI (G.), CALtABiAno (M.), CALDERONE (A.) dir., Archeologia del Mediterraneo. Studi in onore di Ernesto De Miro, Rome, 2003, p. 619-641.

Sparkes, Talcott 1970 : SPARKES (B.A.), TALCOTT (L.) - Black and Plain Pottery of the 6th, 5th, and 4th centuries B.C., Princeton, 1970 (The Athenian Agora XII).

Spina : Spina. Storia di una città tra Greci ed Etruschi, Catalogo della Mostra, Ferrara, 26 settembre 1993-15 maggio 1994. Ferrare, 1993.

Spina e il delta padano : REBECCHI (F.) dir. - Spina e il delta padano. Riflessioni sul Catalogo e sulla Mostra ferrarese. Atti del Convegno di Studi, Ferrara, 21 gennaio 1994. Rome, 1998 (Studia Archaeologica 90).

Stea 1997 : STEA (G.) - Anfore commerciali. In : Ricerche archeologiche all'Incoronata di Metaponto, 5, L'oikos greco del saggio H, Milan, 1997, p. 35-51.

Stea 2000 : STEA(G.) - Anfore commerciali. In : Ricerche archeologiche all'Incoronata di Metaponto, 4, L'oikos greco del grande perirrhanterion nel contesto del saggio G, Milan, 2000, p. 27-31.

Studi sulla necropoli di Spina : Studi sulla necropoli di Spina in Valle Trebba. Atti del Convegno di Studi, Ferrara, 15 ottobre 1992. Ferrare, 1993.

Sur les pas des Grecs : ARCELIN (P.), BATS (M.), GARCIA (D.) dir., Sur les pas des Grecs en Occident. Hommages à André Nickels, Lattes - Paris, 1995 (Études Massaliètes 4).

Taglioni 1999 : Taglioni (C.) - L'abitato etrusco di Bologna, Imola, 1999.

Tartari 1982 : TARTARI (F.) - Amforat e muzeut arkeologjik të Durrësit. Iliria, 12/2, 1982, p. 239-279.

Thompson 1955 : THOMPOSON (H.A.) - Activities in the Athenian Agora: 1954. Hesperia, 24, 1955, p. 50-71.

Tigano 1999 : TIGANO (G.) - Isolato S - Via Industriale - Lo scavo e i primi dati sui materiali. In : Bacci, Tigano 1999, p. 123-155.

Tigano 2002 : TIGANO (G.) dir. - Le necropoli di Mylai (VIII-I sec. a.C.), Milazzo, 2002.

Toniolo 2000 : TONIOLO (A.) - Le anfore di Adria (IV-II secolo a.C.), Sottomarina, 2000.

Torelli 1993 : TORELLI (M.) - Spina e la sua storia. In : Spina, p. 53-69.

Tripponi 1967 : TRIPPONI (A.) - L'esplorazione della porta e del settore sud-est dell'area urbana. StEtr, XXXV, 1967, p. 389-410.

Tuna et al. 1991 : TUNA (N.), EMPEREUR (J.-Y.), DOĞER (E.), $\operatorname{DESBAT}$ (A.) - Rapport sur la première campagne de la fouille francoturque de Resadiye (péninsule de Cnide) - juillet 1988. In : DES COURTILS (J.), MORETTI (J.-C.), PLANET (Fr.) dir., De Anatolia antiqua I, Paris,
1991, p. 109-166 (Bibliothèque de l'Institut française d'études anatoliennes d' Istanbul 32).

Uggeri 1991 : UGGERI (G.) - Interventi idraulici nell'Etruria padana. In : BERGAMINI (M.) dir. - Gli Etruschi maestri d'idraulica, Venise, 1991, p. 69-72.

Uggeri, Uggeri Patitucci 1974 : UGGERI (G.), PATITUCCI UGGERI (S.) - Topografia e urbanistica di Spina. StEtr, 42, 1974, p. 69-97.

Vallet 1962 : VALLET (G) - L'introduction de l'olivier en Italie centrale d'après les données de la céramique. In : Hommages à Albert Grenier, III, Bruxelles 1962, p. 1556-1563.

Vallet, Villard 1961 : VALLET (G.), VILLARD (F.) - Céramique et histoire grecque. Revue historique, 225, 1961, p. 295-318.

Van den Boom 1990 : VAN DEN BOOM (H.) - Amphoren der Heuneburg. In : Amphores de Marseille grecque, p. 263-266.

Van den Boom, Pape 2000 : VAN DEN BOOM (H.), PAPE (J.) - Die massaliotischen Amphoren. In : Kimmig 2000, p. 43-70.

Vandermersch 1986a : VANDERMERSCH (Ch.) - Monnaies et amphores commerciales d'Hipponion. À propos d'une famille de conteneurs magno-grecs du IVe siècle avant J.-C. PP, 40, 1985 (1986), p. 110-145.

Vandermersch 1986b : VANDERMERSCH (Ch.) - Productions magnogrecques et siciliotes du IV $\mathrm{s}$. avant J.-C. In : Recherches sur les amphores, p. $567-580$.

Vandermersch 1989 : VANDERMERSCH (Ch.) - Le matériel amphorique. In : TREZINY (H.) - Kaulonia, I, Naples, 1989, p. 90-109.

Vandermersch 1994 : VANDERMERSCH (Ch.) - Vins et amphores de Grande Grèce et de Sicile, IVe-IIIe s. avant J.-C., Naples, 1994.

Vanderpool 1938 : VANDERPOOL (E.) - The rectangular rock-cut shaft. The shaft and its lower fill. Hesperia, 7, 1938, p. 363-411.

Vanderpool-McCredie-Steinberg 1962 : VANDERPOOL (E.), McCREDIE (J.R.), STEINBERG (A.) - Koroni : A Ptolemaic Camp on the East Coast of Attica. Hesperia, 31, 1962, p. 26-61.

Vandiver, Koehler 1986 : VANDIVER (P.B.), KOEHLER (K.G.) Structure, processing, properties and style of corinthian transport amphoras. In : KINGERY (W.D.), LENSE (E.) dir., Ceramics and Civilization, 2, Technology and Style, Columbus, 1986, p. 173-215.

Vassallo 2003 : VASSALLO (S.) - Himera. Necropoli di Pestavecchia. Un primo bilancio sulle anfore da trasporto. Kokalos, 45, 1999 (2003), p. 329-379.

Verger, Kermovant 1994 : VERGER (S.), KERMOVANT (A.) Nouvelles données et hypothèses sur la topographie de la ville étrusque de Marzabotto. MEFRA, 106, 2, 1994, p. 1077-1094.

Villard 1960 : VILLARD (F.) - La céramique grecque de Marseille (VI ${ }^{e}$ IV siècle), Paris, 1960.

Villard 1988 : VILLARD (F.) - Des vases grecs chez les Celtes. In : Les Princes Celtes et la Méditerranée. Actes du colloque de l'École du Louvre, 1987. Paris, 1988, p. 333-341 (Rencontres de l'École du Louvre).

Vitali 1998 : VITALI (D.) - I Celti a Spina. In : Spina e il delta padano, p. 253-273.

Vitali, Brizzolara, Lippolis 2001 : VITALI (D.), BRIZZOLARA (A.M.), LIPPOLIS (E.) - L'acropoli della città etrusca di Marzabotto, Bologne Imola, 2001.

Voigtländer 1983 : VOIGTLÄNDER (W.) - Funde aus der Insula westlich des Buleuterion in Milet. IstMitt, 32, 1982 (1983), p. 30-173.

Voigtländer 1986 : VOIGTLÄNDER (W.) - Umrisse eines vor-und frühgeschichtlichen Zentrums an der karisch-ionischen Küste. AA, 1986, p. 613-667.

Wallace 1986 : WALLACE (M.B.) - Progress in Amphora Capacities Measurement. In : Recherches sur les amphores, p. 87-94.

Wallon 1979 : WALLON (D.) - Typologie de cols d'amphores massaliètes de l'oppidum de Montjean (La Môle, Var.). RANarb, 12, 1979, p. 43-54. 
Whitbread 1986 : WHITBREAD (I.K.) - The Application of Ceramic Petrology to the Study of Ancient Greek Amphorae. In : Recherches sur les amphores, p. 95-101.

Whitbread 1988 : WHITBREAD (I.K.) - Petrographic analysis of a Corinthian B amphora rim fragment. In : de Marinis 1988, p. 221-222.

Whitbread 1995 : WHITBREAD (I.K.) - Greek Transport Amphorae. A petrological and archaeological study, Athènes, 1995 (The British School at Athens. Fitch Laboratory Occasional Paper 4).

Wiel-Marin 2005 : Wiel-Marin (F.) - La ceramica attica a figure rosse di Adria : la famiglia Bocchi e l'archeologia, Padoue, 2005.

Williams 1978 : WILLIAMS (C.K.) - Corinth 1977 Forum Southwest. Hesperia, 47, 1978, p. 1-39.

Wilson 1988 : WILSON (R.J.A.) - Archaeology in Sicily 1982-1987. ARepLond, 34, 1987-1988 (1988), p. 105-150.

Young 1938 : YOUNG (R.S.) - Pottery from Seventh Century Well. Hesperia, 7, 1938, p. 412-428.

Young 1939 : YOUNG (R.S.) - Late geometric graves and a seventh century well in the Agora, Vienne 1939 (Hesperia Supplement 2).

Zecchini 1978 : ZECCHINI (M.) - Gli Etruschi all'Isola d'Elba, Portoferraio, 1978

Zecchini 2001 : ZECCHINI (M.) - Isola d'Elba : le origini, Lucques, 2001.

Zeest 1960 : ZEEST (I.B.) - Keramičeskaja tara Bospora, Materialy $i$ Issledovanija Archeologii, Moscou, 1960.

Zevi 1985 : ZEVI (F.) - La situazione nel Lazio. In : Commercio etrusco arcaico, p. 119-125. 


\section{PUBLICATIONS DU CENTRE CAMILLE JULLIAN}

http://sites.univ-provence.fr/ccj/spip.php?rubrique79

\section{BIBLIOTHÈQUE D’ARCHÉOLOGIE MÉDITERRANÉENNE ET AFRICAINE (BiAMA)}

1. Maxence Segard, Les Alpes Occidentales à l'époque romaine, Développement urbain et exploitation des ressources des régions de montagne (Gaule Narbonnaise, Italie, provinces alpines), Paris 2009, 288 p.

2. Patrick Thollard, La Gaule selon Strabon : du texte à l'archéologie (Géographie livre IV). Traduction et études, Paris 2009, $265 \mathrm{p}$.

3. Grecs et indigènes de la Catalogne à la mer Noire, actes des rencontres du programme européen Ramses (20062008), édités par Henri Tréziny, Paris 2010, 716 p.

4. Archéologie de la montagne européenne. Actes de la table ronde internationale de Gap (29 septembre-1eroctobre 2008), textes réunis par Stéfan Tzortzis et Xavier Delestre, avec la collaboration de Jennifer Greck, Paris 2010, 333 p. 5. Apollonia du Pont (Sozopol) La nécropole de Kalfata (Ve-IIIe s. av J.-C.) - Fouilles francobulgares (2002-2004), sous la direction de A. Hermary, 2010, 432 p.

6. Lucien Rivet, Recherches archéologiques au coeur de Forum Iulii - Les fouilles dans et autour du groupe cathédral de Fréjus (1979-1989), 2010, 420 p.

7. Fouilles à Marseille, la ville médiévale et moderne, sous la direction de Marc Bouiron, Françoise Paone, Bernard Sillano, Colette Castrucci et Nadine Scherrer (ÉtMassa 10), 2011, 463 p.

8. Pierre Excoffon, Ville et campagne de Fréjus romaine. La fouille préventive de «Villa Romana » 2011, $306 \mathrm{p}$.

9. Batellerie gallo-romaine : pratiques régionales et influences maritimes méditerranéennes, sous la direction de Giulia Boetto, Patrice Pomey, André Tchernia, 2011,191 p.

10. Federica Sacchetti, Les amphores grecques dans le Nord de l'Italie. Echanges commerciaux entre les Apennins et les Alpes aux époques archaïque et classique, 2012, $287 \mathrm{p}$.

11. Bernard Dedet, Une nécropole du second Âge du Fer à Ambrussum, Hérault, 2012, 282 p.

12. L'enfant et la mort dans l'Antiquité III. Le matériel associé aux tombes d'enfants, Actes du colloque international organisé à la Maison Méditerranéenne des Sciences de l'Homme (MMSH) d'Aix-en-Provence, 20-22 janvier 2011, édités par Antoine Hermary et Céline Dubois, 2012, 461 p.

\section{BiAMA Hors Collection}

André Tchernia., Les Romains et le commerce. Naples : Centre Jean Bérard ; Aix-en-Provence : Centre Camille Jullian, 2011, 439 p. (Études du CJB ; 8)

Achevé d'imprimer en février 2013 par l'imprimerie Sepec à Peronnas

Dépôt légal : mars 2013

Numéro d'imprimeur :

Imprimé en France 